U.S. Department of the Interior

U.S. Geological Survey

\title{
Selected Hydrologic Data from the Cedar Rapids Area, Linn County, Iowa, April 1996 Through March 1999
}

By Robert A. Boyd, Ronald L. Kuzniar, and Peter M. Schulmeyer

Open-File Report 99-461

Prepared in cooperation with the

CITY OF CEDAR RAPIDS, IOWA

Iowa City, Iowa

1999 


\section{U.S. Department of the Interior \\ Bruce Babbitt, Secretary}

\section{U.S. Geological Survey}

Charles G. Groat, Director

Any use of trade, product, or firm names in this publication is for descriptive purposes only and does not imply endorsement by the U.S. Government

For additional information write to:

\section{District Chief}

U.S. Geological Survey, WRD

P.O. Box 1230

Iowa City, Iowa 52240

Copies of this report can be purchased from:

\section{U.S. Geological Survey}

Branch of Information Services

Box 25286, Denver Federal Center

Denver, Colorado 80225 


\section{CONTENTS}

Abstract

Introduction

Purpose and Scope

Description of Study Area

Methods of Investigation

Well Construction and Nomenclature

Water Quality

Ground-Water Levels

Physical Properties

Selected Hydrologic Data

Water-Quality Data

Ground-Water Levels

Physcial Properties.

References Cited

\section{FIGURES}

1-3. Maps showing:

1. Location of the Cedar Rapids, Iowa municipal well fields

2. Sample collection and measurement sites in the Seminole and Northwest Well Fields, Cedar Rapids, Iowa, April 1996-March 1999

3. Sample collection and measurement sites in the East and West Well Fields, Cedar Rapids, Iowa, April 1996-March 1999

\section{TABLES}

1. Information for observation wells and sampling sites near the Cedar Rapids, Iowa, municipal well fields, April 1996 through March 1999

2. Types of data collected at sampling and measurement sites near the Cedar Rapids, Iowa, municipal well fields, April 1996 through March 1999

3. Summary of common-ion, trace-element, and nutrient concentrations in water samples collected from the Cedar Rapids, Iowa, municipal well fields and Cedar River, April 1996 through March 1999

4. Summary of pesticide concentrations in water samples collected from the Cedar Rapids, Iowa, municipal well fields, April 1996 through March 1999

5. Dissolved common-ion and trace-element concentrations in water samples collected from the Cedar Rapids, Iowa, municipal well fields and Cedar River, April 1996 through March 1999.

6. Dissolved nutrient and organic-carbon concentrations in water samples collected from the Cedar Rapids, Iowa, municipal well fields and Cedar River, April 1996 through March 1999

7. Dissolved pesticide concentrations in water samples collected from the Cedar Rapids, Iowa, municipal well fields and Cedar River, April 1996 through March 1999

8. Dissolved concentrations of ethanesulfonic-acid degradates of acetanilide herbicides in water samples collected from the Seminole Well Field and Cedar River, Cedar Rapids, Iowa, June 1998 through August 1998.

9. Dissolved concentrations of oxanilic-acid degradates of acetanilide herbicides in water samples collected from the Seminole Well Field and Cedar River, Cedar Rapids, Iowa, June 1998 through August 1998

10a. Mean daily ground-water levels in observation well CRM-1 (map ID \#1), East Well Field, Cedar Rapids, Iowa, July 1997 through March 1999

10b. Mean daily ground-water levels in observation well CRM-2 (map ID \#2), West Well Field, Cedar Rapids, Iowa, July 1997 through March 1999 


\section{CONTENTS--Continued}

10c. Mean daily ground-water levels in observation well CRM-3 (map ID \#3), Seminole Well Field, Cedar Rapids, Iowa, January 1997 through March 1999

10d. Mean daily ground-water levels in observation well CRM-4 (map ID \#4), Seminole Well Field, Cedar

Rapids, Iowa, January 1997 through June 1998

10e. Mean daily ground-water levels in observation well CRM-6 (map ID \#5) Seminole Well Field, Cedar

Rapids, Iowa, January 1997 through February 1999.

10f. Mean daily ground-water levels in observation well CRM-7 (map ID \#6), Seminole Well Field, Cedar

Rapids, Iowa, January 1997 through February 1999.

10g. Mean daily ground-water levels in observation well CRM-9 (map ID \#7), Seminole Well Field, Cedar

Rapids, Iowa, January 1997 through.February $1999 .$.

10h. Mean daily ground-water levels in observation well CRM-11 (map ID \#9), West Well Field, Cedar Rapids, Iowa, July 1997 through February 1999.

10i. Mean daily ground-water levels in observation well CRM-12 (map ID \#10), East Well Field, Cedar Rapids, Iowa, July 1997 through February 1999.

10j. Mean daily ground-water levels in observation well CRM-15 (map ID \#12), Seminole Well Field, Cedar

Rapids, Iowa, January 1997 through February 1999.

10k. Mean daily ground-water levels in observation well CRM-16 (map ID \#13), Seminole Well Field, Cedar Rapids, Iowa, January 1997 through February 1999......

101. Mean daily ground-water levels in observation well CRM-17 (map ID \#14), Seminole Well Field, Cedar

Rapids, Iowa, Novemebr 1997 through September 1998

10m. Mean daily ground-water levels in observation well CRM-18 (map ID \#15), Seminole Well Field, Cedar

Rapids, Iowa, January 1997 through February 1999

10n. Mean daily ground-water levels in observation well CRM-19 (map ID \#16), Seminole Well Field, Cedar

Rapids, Iowa, January 1997 through February 1999

10o. Mean daily ground-water levels in observation well CRM-22 (map ID \#19), Seminole Well Field, Cedar

Rapids, Iowa, February 1998 through February 1999

10p. Mean daily ground-water levels in observation well CRM-23 (map ID \#20), Seminole Well Field, Cedar

Rapids, Iowa, February 1998 through February 1999

10q. Mean daily ground-water levels in observation well CRM-24 (map ID \#21), Seminole Well Field, Cedar

Rapids, Iowa, February 1998 through February 1999.

10r. Mean daily ground-water levels in observation well CRM-25 (map ID \#22), Seminole Well Field, Cedar

Rapids, Iowa, February 1998 through March 1999

10s. Mean daily ground-water levels in observation well CRM-27 (map ID \#24), Seminole Well Field, Cedar

Rapids, Iowa, February 1998 through March 1999

11a. Mean daily specific conductance in ground water at site CRM-1 (map ID \#1), East Well Field, Cedar

Rapids, Iowa, April 1996 through June 1997.

11b. Mean daily specific conductance in ground water at site CRM-2 (map ID \#2), West Well Field, Cedar

Rapids, Iowa, April 1996 through June 1997.

11c. Mean daily specific conductance in ground water at site CRM-11 (map ID \#9), West Well Field, Cedar

Rapids, Iowa, April 1996 through June 1997.

11d. Mean daily specific conductance in ground water at site CRM-12 (map ID \#10), East Well Field, Cedar

Rapids, Iowa, April 1996 through July 1997

11e. Mean daily specific conductance in ground water at site CRM-20 (map ID \#17), Northwest Well Field,

Cedar Rapids, Iowa, February 1998 through March 1999

11f. Mean daily specific conductance in ground water at site CRM-21 (map ID \#18), Northwest Well Field,

Cedar Rapids, Iowa, February 1998 through March 1999

11g. Mean daily specific conductance in ground water at site CRM-22 (map ID \#19), Seminole Well Field,

Cedar Rapids, Iowa, February 1998 through March 1999 


\section{CONTENTS--Continued}

11h. Mean daily specific conductance in ground water at site CRM-23 (map ID \#20), Seminole Well Field, Cedar Rapids, Iowa, February 1998 through March 1999.

11. Mean daily specific conductance in ground water at site CRM-25 (map ID \#22), Seminole Well Field,

Cedar Rapids, Iowa, February 1998 through March 1999.

11j. Mean daily specific conductance in ground water at site CRM-27 (map ID \#24), Seminole Well Field,

Cedar Rapids, Iowa, February 1998 through March 1999.

$11 \mathrm{k}$. Mean daily specific conductance in the Cedar River at site SW-2 (map ID \#32), Seminole Well Field,

Cedar Rapids, Iowa, April 1996 through March 1999

12a. Mean daily pH in ground water at site CRM-1 (map ID \#1), East Well Field, Cedar Rapids, Iowa, April 1996 through June 1997.

12b. Mean daily $\mathrm{pH}$ in ground water at site CRM-2 (map ID \#2), West Well Field, Cedar Rapids, Iowa, April 1996 through July 1997 .....

12c. Mean daily pH in ground water at site CRM-11 (map ID \#9), West Well Field, Cedar Rapids, Iowa, April 1996 through June 1997.

12d. Mean daily pH in ground water at site CRM-12 (map ID \#10), East Well Field, Cedar Rapids, Iowa, April 1996 through July 1997

12e. Mean daily pH in ground water at site CRM-20 (map ID \#17), Northwest Well Field, Cedar Rapids,

Iowa, February 1998 through March 1999.

12f. Mean daily pH in ground water at site CRM-21 (map ID \#18), Northwest Well Field, Cedar Rapids, Iowa, February 1998 through March 1999.

12g. Mean daily pH in ground water at site CRM-22 (map ID \#19), Seminole Well Field, Cedar Rapids, Iowa, February 1998 through March 1999.

12h. Mean daily pH in ground water at site CRM-23 (map ID \#20), Seminole Well Field, Cedar Rapids, Iowa, February 1998 through March 1999

12i. Mean daily pH in the Cedar River at site SW-2 (map ID \#32) Seminole Well Field, Cedar Rapids, Iowa, October 1996 through March 1999

13a. Mean daily dissolved-oxygen concentration in ground water at site CRM-2 (map ID \#2), West Well

Field, Cedar Rapids, Iowa, April 1996 through June 1997

13b. Mean daily dissolved-oxygen concentration in ground water at site CRM-12 (map ID \#10), East Well

Field, Cedar Rapids, Iowa, April 1996 through July 1997.

13c. Mean daily dissolved-oxygen concentration in ground water at site CRM-20 (map ID \#17), Northwest

Well Field, Cedar Rapids, Iowa, February 1998 through March 1999

13d. Mean daily dissolved-oxygen concentration in ground water at site CRM-21 (map ID \#18), Northwest

Well Field, Cedar Rapids, Iowa, February 1998 through March 1999

13e. Mean daily dissolved-oxygen concentration in ground water at site CRM-22 (map ID \#19), Seminole

Well Field, Cedar Rapids, Iowa, February 1998 through March 1999

13f. Mean daily dissolved-oxygen concentration in ground water at site CRM-23 (map ID \#20), Seminole

Well Field, Cedar Rapids, Iowa, February 1998 through March 1999

13g. Mean daily dissolved-oxygen concentration in the Cedar River at site SW-2 (map ID \#32), Seminole

Well Field, Cedar Rapids, Iowa, April 1996 through March 1999

14a. Mean daily ground-water temperature at site CRM-1 (map ID \#1), East Well Field, Cedar Rapids, Iowa,

April 1996 through February 1999.

14b. Mean daily ground-water temperature at site CRM-2 (map ID \#2), West Well Field, Cedar Rapids, Iowa,

April 1996 through February 1999

14c. Mean daily ground-water temperature at site CRM-3 (map ID \#3), Seminole Well Field, Cedar Rapids,

Iowa, January 1997 through February 1999.

14d. Mean daily ground-water temperature at site CRM-4 (map ID \#4), Seminole Well Field, Cedar Rapids, 


\section{CONTENTS--Continued}

14e. Mean daily ground-water temperature at site CRM-6 (map ID \#5), Seminole Well Field, Cedar Rapids, Iowa, January 1997 through February 1999.

14f. Mean daily ground-water temperature at site CRM-7 (map ID \#6), Seminole Well Field, Cedar Rapids, Iowa, January 1997 through February 1999.

14g. Mean daily ground-water temperature at site CRM-9 (map ID \#7), Seminole Well Field, Cedar Rapids, Iowa, January 1997 through February 1999.

14h. Mean daily ground-water temperature at site CRM-11 (map ID \#9), West Well Field, Cedar Rapids, Iowa, April 1996 through February 1999

14i. Mean daily ground-water temperature at site CRM-12 (map ID \#10), East Well Field, Cedar Rapids, Iowa, April 1996 through February 1999

14j. Mean daily ground-water temperature at site CRM-15 (map ID \#12), Seminole Well Field, Cedar Rapids, Iowa, January 1997 through February 1999.

14k. Mean daily ground-water temperature at site CRM-16 (map ID \#13), Seminole Well Field, Cedar Rapids, Iowa, January 1997 through February 1999.

141. Mean daily ground-water temperature at site CRM-17 (map ID \#14), Seminole Well Field, Cedar Rapids, Iowa, November 1997 through September 1998.

14m. Mean daily ground-water temperature at site CRM-18 (map ID \#15), Seminole Well Field, Cedar Rapids, Iowa, February 1997 through February 1999

14n. Mean daily ground-water temperature at site CRM-19 (map ID \#16), Seminole Well Field, Cedar Rapids, Iowa, January 1997 through February 1999

140. Mean daily ground-water temperature at site CRM-20 (map ID \#17), Northwest Well Field, Cedar Rapids, Iowa, February 1998 through March 1999

14p. Mean daily ground-water temperature at site CRM-21 (map ID \#18), Northwest Well Field, Cedar Rapids, Iowa, February 1998 through March 1999

14q. Mean daily ground-water temperature at site CRM-22 (map ID \#19), Seminole Well Field, Cedar Rapids, Iowa, February 1998 through March 1999

14r. Mean daily ground-water temperature at site CRM-23 (map ID \#20), Seminole Well Field, Cedar Rapids, Iowa, February 1998 through March 1999

14s. Mean daily ground-water temperature at site CRM-24 (map ID \#21), Seminole Well Field, Cedar Rapids, Iowa, February 1998 through March 1999.

14t. Mean daily ground-water temperature at site CRM-25 (map ID \#22), Seminole Well Field, Cedar Rapids, Iowa, February 1998 through February 1999.

14u. Mean daily ground-water temperature at site CRM-27 (map ID \#24), Seminole Well Field, Cedar Rapids, Iowa, February 1998 through February 1999.

14v. Mean daily water temperature in the Cedar River at site SW-2 (map ID \#32), Seminole Well, Field, Cedar Rapids, Iowa, April 1996 through March 1999 


\title{
CONVERSION FACTORS, ABBREVIATIONS, AND VERTICAL DATUM
}

\begin{tabular}{rll}
\hline Multiply & by & To obtain \\
inch & & centimeter \\
foot & 2.54 & meter \\
square mile & 0.3048 & square kilometer \\
gallon & 2.590 & liter \\
cubic foot per second & 3.785 & cubic meter per second \\
\end{tabular}

Temperature can be converted to degrees Celsius $\left({ }^{\circ} \mathrm{C}\right)$ or degrees Fahrenheit $\left({ }^{\circ} \mathrm{F}\right)$ by using the following equations:

$$
\begin{aligned}
& { }^{\circ} \mathrm{C}=5 / 9\left({ }^{\circ} \mathrm{F}-32\right) \\
& { }^{\circ} \mathrm{F}=9 / 5\left({ }^{\circ} \mathrm{C}\right)+32
\end{aligned}
$$

\begin{abstract}
Abbreviated water-quality units used in this report: Chemical concentrations are reported in milligrams per liter (mg/L), micrograms per liter $(\mu \mathrm{g} / \mathrm{L})$, or microsiemens per centimeter $\mu \mathrm{S} / \mathrm{cm}$ at 25 degrees Celsius $\left({ }^{\circ} \mathrm{C}\right)$. Milligrams per liter expresses the concentration of chemical constituents in solution as weight (milligrams) of solute per unit volume (liter) of water.

Micrograms per liter expresses the concentration of chemical constituents in solution as weight (micrograms) of solute per unit volume (liter) of water. Microsiemens per centimeter expresses the capability of a unit volume of water to conduct an applied electrical current.
\end{abstract}

Sea level: In this report, "sea level" refers to the National Geodetic Vertical Datum of 1929 (NGVD of 1929)--a geodetic datum derived from a general adjustment of the first-order level nets of both the United States and Canada, formerly called the "Sea Level Datum of $1929 . "$ 


\title{
SELECTED HYDROLOGIC DATA FROM THE CEDAR RAPIDS AREA, LINN COUNTY, IOWA, APRIL 1996 THROUGH MARCH 1999
}

\author{
By ROBERT A. BOYD, RONALD L. KUZNIAR, and PETER M. SCHULMEYER
}

\section{ABSTRACT}

The City of Cedar Rapids, Iowa obtains its municipal water supply from four well fields along the Cedar River. The wells are completed at depths of about 60 to 80 feet in a shallow alluvial aquifer adjacent to the Cedar River. The City of Cedar Rapids and the U.S. Geological Survey have conducted a cooperative study of the groundwater flow system and water quality near the well fields since 1992. The purpose of this report is to document selected hydrologic data collected from April 1996 through March 1999. Data include the results of water-quality analyses, ground-waterlevels continuously measured with pressure transducers and data recorders, and physical properties continuously monitored using multiprobe instruments. Water-quality samples were collected from selected wells and the Cedar River to conduct periodic monitoring, to evaluate ground-water geochemistry, to assess the occurrence of pesticides and herbicide degradates in the alluvial aquifer, and to characterize water quality in shallow ground water near a wetland area in the Seminole Well Field. Types of water-quality analyses included common ions (calcium, chloride, iron, magnesium, manganese, potassium, silica, sodium, and sulfate), trace elements (boron, bromide, and fluoride), nutrients (ammonia as nitrogen, nitrite as nitrogen, nitrite plus nitrate as nitrogen, and orthophosphate as phosphorus), dissolved organic carbon, and selected pesticides and herbicide degradates. Ground-water levels in selected observation wells were continuously measured to assess temporal trends in groundwater levels in the alluvial aquifer and bedrock aquifer, to help calibrate a ground-water flow model being constructed to simulate local groundwater flow under transient conditions near the well fields, and to assess hydrologic conditions near a wetland area in the Seminole Well Field. Physical properties (specific conductance, $\mathrm{pH}$, dissolved oxygen, and water temperature) were continuously monitored to assess temporal variation and to help evaluate the interaction between the Cedar River and ground water in the alluvial aquifer.

\section{INTRODUCTION}

The City of Cedar Rapids, in Linn County, Iowa obtains its municipal water supply from four well fields (Seminole, East, West, and Northwest) along the Cedar River (fig. 1). Fifty-five municipal wells are completed at depths of about 60 to 80 feet (ft) in a shallow alluvial aquifer adjacent to the Cedar River. Twenty-three vertical wells are in the Seminole Well Field; 11 vertical wells are in the West Well Field; 19 vertical wells are in the East Well Field; and two horizontal-collector wells are in the Northwest Well Field. Adequate quantities of generally high-quality water have been obtained from the alluvial aquifer since the resource was developed in 1962. Increasing population and industrial development have increased the demand for municipal water. Cedar Rapids pumped about 8,487 million gallons (Mgal) from the alluvial aquifer in 1980 , about $9,118 \mathrm{Mgal}$ in 1990, and about 13,140 Mgal in 1997 (Bob Glass, City of Cedar Rapids Water Department, oral commun., 1998).

The City of Cedar Rapids and the U.S. Geological Survey (USGS) have conducted a cooperative study of the ground-water flow system and water quality near the well fields since 1992. Schulmeyer (1995) analyzed the influence of the Cedar River on the quality of ground water near the municipal well fields. Schnoe- 

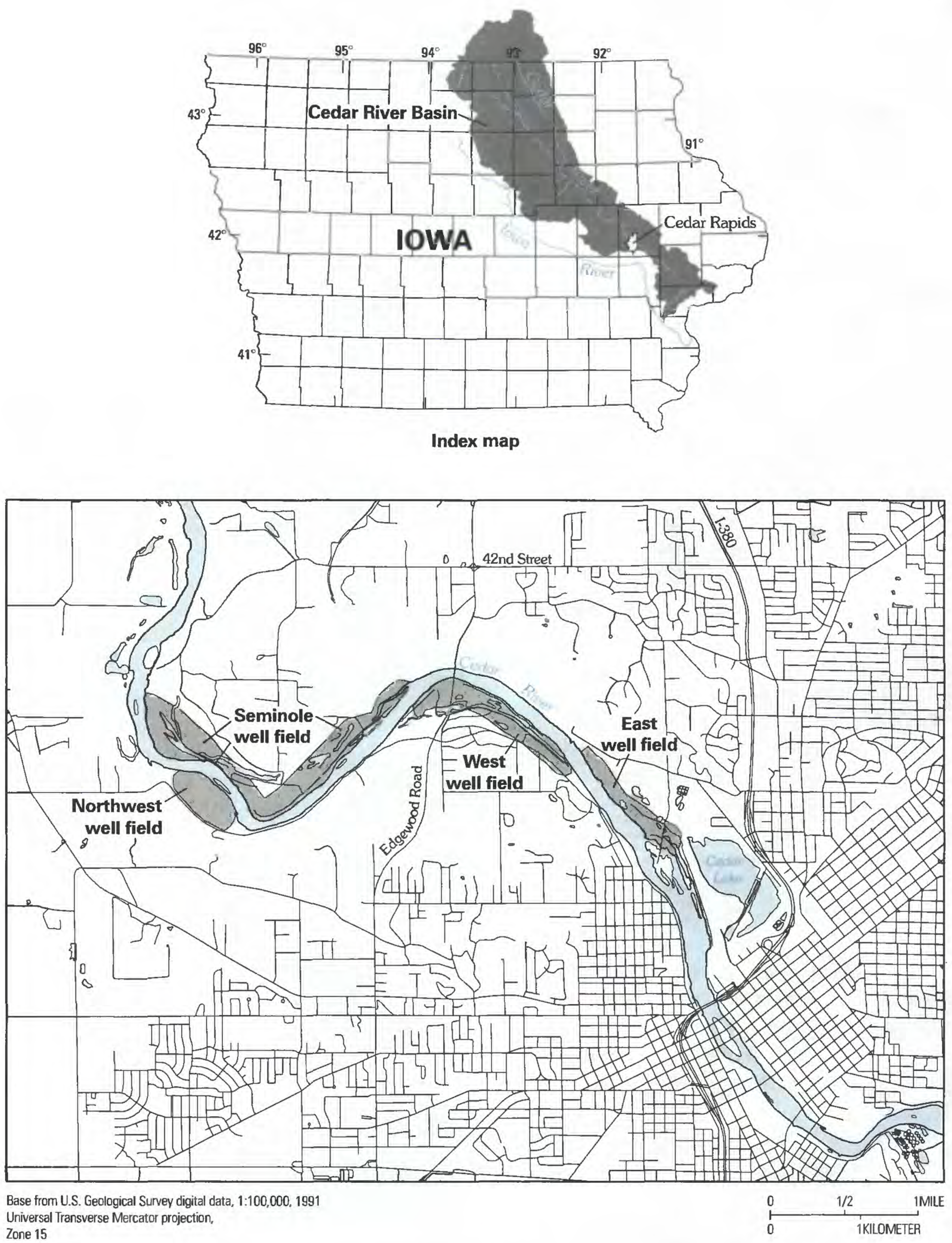

Figure 1. Location of the Cedar Rapids, lowa, municipal well fields. 
belen and Schulmeyer (1996) documented hydrogeologic data collected and compiled from October 1992 to March 1996. Schulmeyer and Schnoebelen (1998) described the hydrogeology near the municipal well fields, documented a ground-water flow model constructed to simulate regional ground-water flow under steady-state conditions and identify sources of water to the municipal well fields, and assessed temporal and spatial variations of selected water-quality constituents and properties. Boyd (1998) characterized groundwater flow near the municipal well fields with selected environmental isotopes and tracers. Boyd (1999) evaluated the occurrence and distribution of selected herbicide and herbicide degradate concentrations in the alluvial aquifer and Cedar River following springtime application of herbicides to upstream cropland.

\section{Purpose and Scope}

The purpose of this report is to document selected hydrologic data collected from April 1996 through March 1999 for the cooperative study of the Cedar Rapids municipal well fields being conducted by the City of Cedar Rapids and the USGS. Data presented in this report include the results of water-quality analyses, ground-water levels continuously measured with pressure transducers and data recorders, and physical properties continuously monitored with multiprobe instruments.

\section{Description of Study Area}

Cedar Rapids is in east-central Iowa and has a population of about 110,000 . Upland topography is characterized by rolling hills of low relief. Upstream land use in the Cedar River basin is more than 80 percent agricultural; corn and soybeans are the predominant crops. Livestock raised in the area primarily include beef and dairy cattle, hogs, and sheep (Squillace and others, 1996). Iowa has a typical continental, subhumid climate. Air temperatures generally range from $-18^{\circ} \mathrm{F}$ in winter to $100^{\circ} \mathrm{F}$ in summer. The average annual precipitation is about 36 inches, with the greatest amounts occurring from May to August (Schulmeyer and Schnoebelen, 1998). The Cedar River Basin drains an area of about 6,510 square miles upstream from the streamflow gaging station at Cedar Rapids (Cedar River at Cedar Rapids, USGS station number 05464500 ). The annual mean flow recorded at the gaging station from 1903-1998 was 3,711 cubic feet per second $\left(\mathrm{ft}^{3} / \mathrm{s}\right)$. Extreme daily mean flows in the Cedar River during this period of record were $71,500 \mathrm{ft}^{3} / \mathrm{s}$ (March 31, 1961) and $140 \mathrm{ft}^{3} / \mathrm{s}$ (November 18, 1989) (May and others, 1999).

Hydrogeologic units near the well fields consist of an unconsolidated surficial layer of glacial till, loess, and the Cedar River alluvium (alluvial aquifer), underlain by carbonate bedrock of Devonian and Silurian age (bedrock aquifer). The glacial till and loess form upland areas that bound the alluvial aquifer. The alluvial aquifer ranges in thickness from 5 to $95 \mathrm{ft}$ near the well fields. The alluvial aquifer consists of a sequence of coarse sand and gravel at the base, grading upward to fine sand, silt, and clay near the surface. The bedrock aquifer has a maximum thickness of about $700 \mathrm{ft}$ near the well fields. The bedrock aquifer primarily consists of jointed and fractured limestone and dolomite, with some interbedded chert and shale (Schulmeyer and Schnoebelen, 1998). The unconsolidated surficial layers, carbonate bedrock of Devonian and Silurian age, and deeper hydrogeologic units are described in detail by Hansen (1970), Wahl and Bunker (1986) and Schulmeyer and Schnoebelen (1998).

The alluvial aquifer near the well fields is recharged by infiltration from the Cedar River induced by pumping of municipal supply wells, infiltration of precipitation, and ground-water flow from adjacent hydrogeologic units. Recharge also can occur during periods of high river stage, when river water can move into the alluvial aquifer because of the head difference between the river and aquifer or when flood waters inundate low-lying areas adjacent to the Cedar River (Schulmeyer and Schnoebelen, 1998). In areas influenced by the pumping of municipal supply wells, the water-table gradient is from the Cedar River to the alluvial aquifer. In areas outside the influence of municipal pumping, the water-table gradient generally is toward the Cedar River, although the gradient can reverse during periods of high river stage (Schulmeyer and Schnoebelen, 1998). Results from a regional groundwater flow model constructed by Schulmeyer and Schnoebelen (1998) indicated that about 74 percent of water pumped from the alluvial aquifer is induced from the Cedar River, about 21 percent of the water is flow from adjacent and underlying hydrogeologic units, and about 5 percent of the water is recharge from infiltrating precipitation. The bedrock aquifer is recharged by downward leakage from the overlying unconsolidated 
surficial layer and by infiltrating precipitation on outcrop areas distant from the study area. The bedrock aquifer is not used as a municipal water supply but is used for private residential and industrial water supplies.

\section{METHODS OF INVESTIGATION}

Data were collected from a network of observation wells within the municipal well fields, the Cedar River, and the J-Avenue waterworks plant. The data include water-quality analyses, continuously measured ground-water levels in selected wells, and continuously monitored physical properties in selected observation wells and the Cedar River. The locations of sampling sites are shown in figure 2 and figure 3 . Well construction information for the sampling and measurement sites is listed in table 1 .

\section{Well Construction and Nomenclature}

Data were collected from small-diameter wells (0.14-inch outer-diameter tubing), 2 -inch outer-diameter observation wells, and 4-inch outer-diameter observation wells. The observation wells are named according to a convention that includes the year the well was installed, the agency identifier, USGS, the local project identifier, CRM (Cedar Rapids Municipal), and a unique incremental number (for example, 1993USGS CRM-1). Small-diameter well names also include the identifier $S D$ (for example, 1996USGS CRM-SD-60). For convenience in this report, the year and agency identifier will not be included when referring to a site name, however, the map identification number listed in table 1 will be included with the site name so the reader can easily locate the well in figure 2 and figure 3 . The observation wells were surveyed using a combination of a Global Positioning System and conventional surveying techniques to determine location (latitude and longitude) and to establish vertical control referenced to sea level. Observation-well data are stored in the USGS Ground Water Storage Information (GWSI) database. The observation wells and measurement sites were assigned a unique 15-digit site-identification number in GWSI that is based on the latitude and longitude of the site (for example, the 15-digit site-identification number for 1993USGS CRM-1 is 415949091405401). The first six digits designate the latitude ( $\left.41^{\circ} 59^{\prime} 49^{\prime \prime}\right)$ of the site, the next seven digits designate the longitude (091 $40^{\prime}$ '54") of the site, and the last two numbers (01) are sequential numbers to differentiate between individual sites at the same latitude and longitude.

Small-diameter wells were installed in the alluvial aquifer by drilling a pilot borehole with a 9-foot long, 1.25-inch outer-diameter solid-stem auger. A 3-inch long, stainless-steel sandpoint attached to 0.14-inch outer-diameter Teflon tubing was advanced inside a 0.875 -inch outer-diameter drill rod driven to the desired depth with a portable hammer drill. Teflon tubing extending above ground surface was protected with 1-inch outer-diameter polyvinyl-chloride (PVC) pipe. Two-inch and 4-inch observation wells were installed by drilling boreholes with continuous-flight, hollow-stem augers. Boreholes for 2-inch wells were installed with 4.25-inch inner-diameter augers; boreholes for 4-inch wells were installed with 6.25 -inch inner-diameter augers. Wells were completed with 2-inch or 4-inch outer-diameter PVC casing with 2.5foot or 5.0-foot long screened intervals above the bottom of the borehole. Aquifer material was allowed to collapse around the screened interval and the remaining annular space was sealed with bentonite and capped with cement and a protective cover. The observation wells at CRM-6 (map ID \#5), CRM-7 (map ID \#6), CRM-11 (map ID \#9), and CRM-12 (map ID \# 10) were installed in the bedrock aquifer using direct mudrotary drilling. Boreholes were drilled about 15 to 20 $\mathrm{ft}$ into the bedrock aquifer at each location. These wells were completed with 4-inch outer-diameter PVC casing with a 5-foot long screened interval above the bottom of the borehole. The annular space around the screened interval was filled with a washed sand pack and sealed with about $5 \mathrm{ft}$ of bentonite to the top of the bedrock surface. The remaining annular space was filled with a bentonite/cement grout and capped with cement and a protective cover.

\section{Water Quality}

Water-quality samples were collected from small-diameter wells, 2-inch and 4-inch diameter observation wells, the Cedar River at Edgewood Road (map ID \# 31), and the J-Avenue Waterworks Plant (map ID \# 30). Ground-water samples were collected with a stainless-steel submersible pump after pumping about three borehole volumes of water from 2 -inch and 4-inch observation wells and after pumping small- 


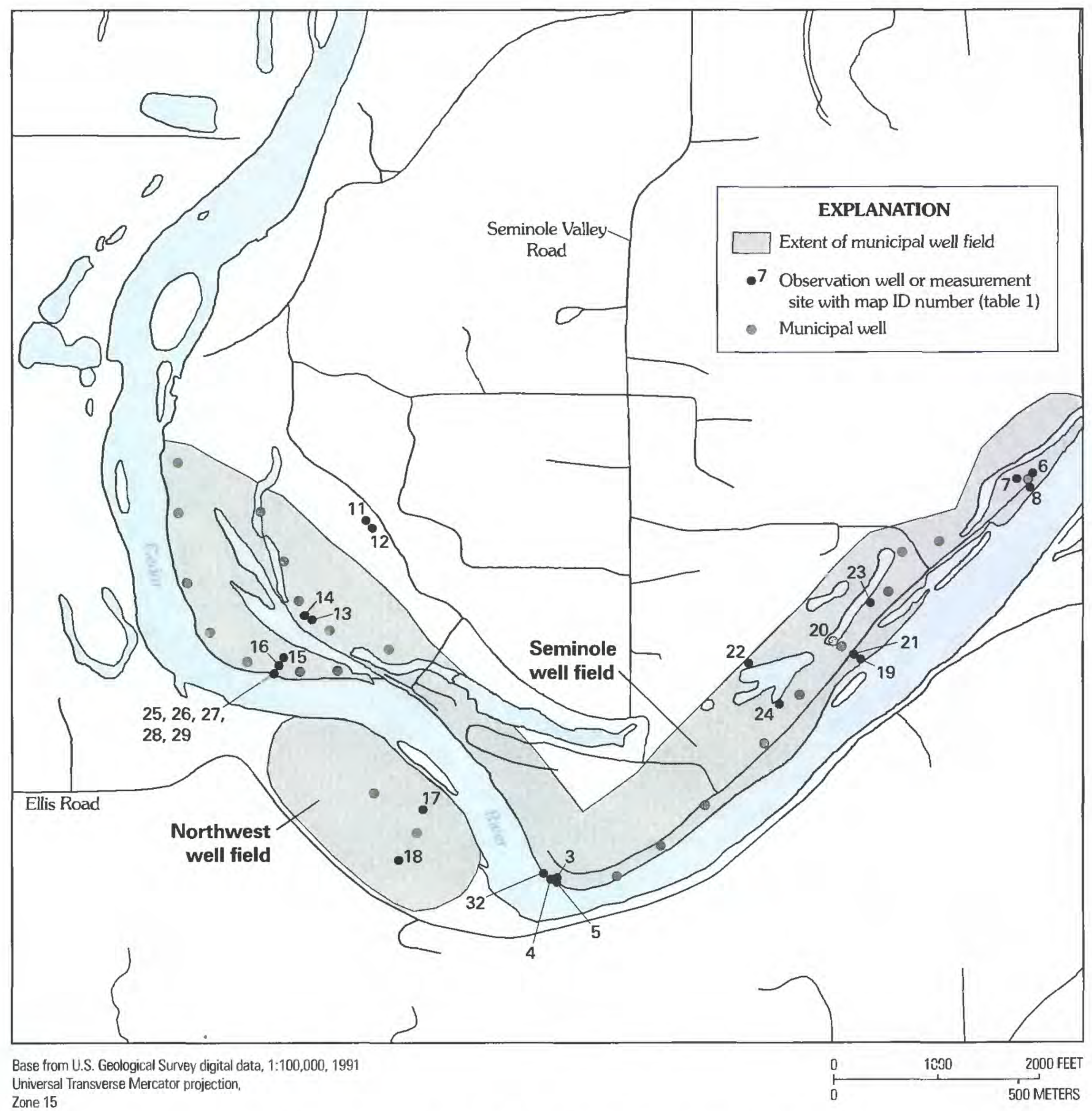

Figure 2. Sample collection and measurement sites in the Seminole and Northwest well fields, Cedar Rapids, lowa, April 1996 through March 1999. 


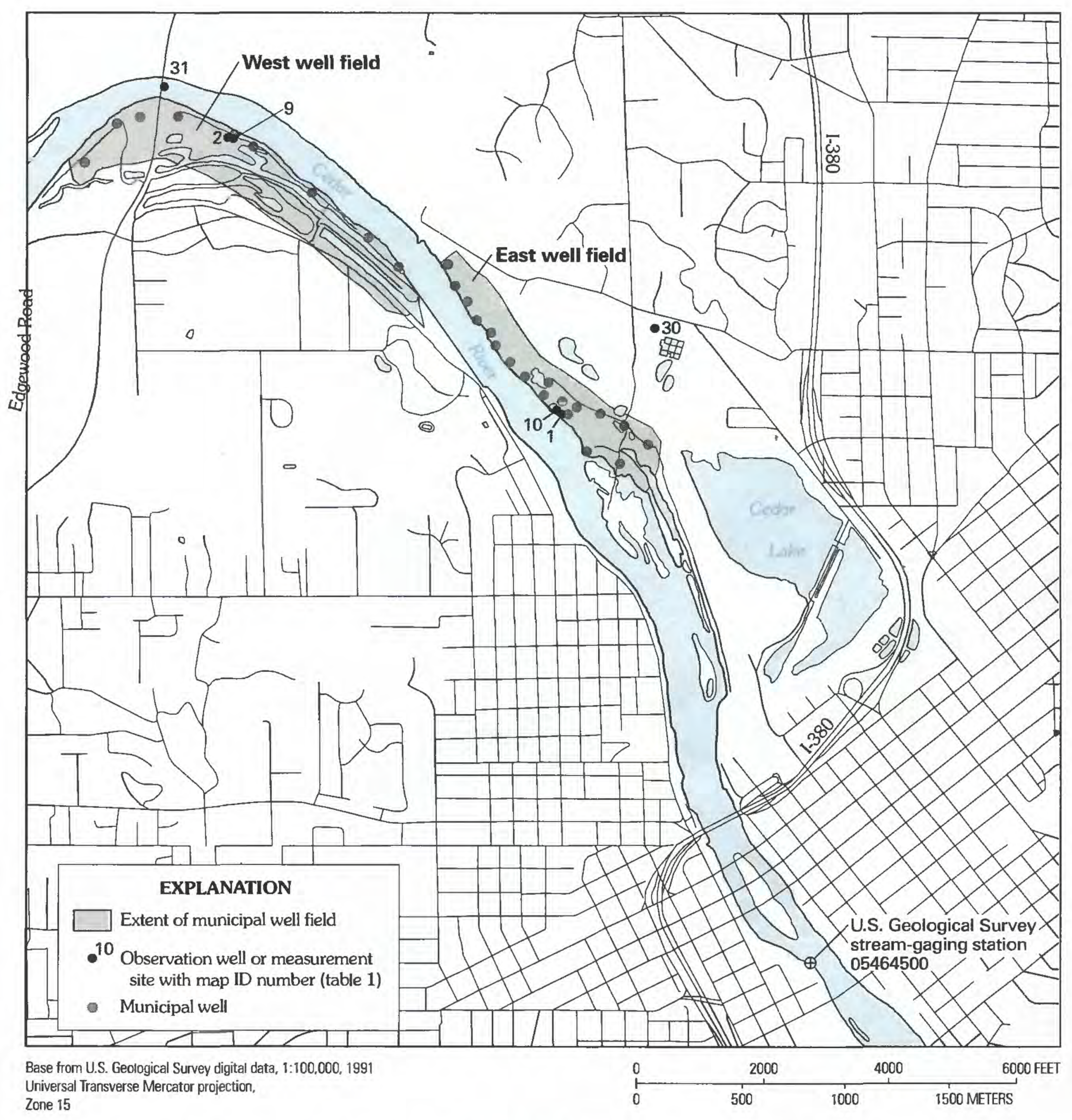

Figure 3. Sample collection and measurement sites near the East and West well fields, Cedar Rapids, lowa, April 1996 through March 1999. 
diameter wells for about 20 minutes with a peristaltic pump. Surface-water samples were collected from the Cedar River with a stainless-steel submersible pump that was extended into the river from the riverbank. Samples of untreated municipal water were collected from a tap at the J-Avenue Waterworks Plant (map ID \# 30). Dissolved inorganic-constituent samples were collected through an inline 0.45 -micrometer $(\mu \mathrm{m})$ pore-size, cellulose-fiber filter. Pesticide and herbicide degradate samples were collected through an inline $0.7-\mu \mathrm{m}$ pore-size, glass-fiber filter. Dissolved organiccarbon samples were filtered in the field immediately after collection through a $0.45-\mu \mathrm{m}$ silver-membrane filter.

Water samples were collected for analyses of common ions (calcium, chloride, iron, magnesium, manganese, potassium, silica, sodium, and sulfate); trace elements (boron, bromide, and fluoride); nutrients (ammonia as nitrogen $(\mathrm{N})$, nitrite as $\mathrm{N}$, nitrite plus nitrate as $\mathrm{N}$, and orthophosphate as phosphorus); dissolved organic carbon; and selected pesticides. Water samples were analyzed by the USGS National WaterQuality Laboratory in Arvada, Colorado using procedures documented by Fishman and Friedman (1989). Some samples collected from June through August 1998 were analyzed for selected pesticides and chloroacetanilide and triazine herbicide degradates by the USGS Organic Geochemistry Research Laboratory in Lawrence, Kansas using procedures documented by Zimmerman and Thurman (1999).

Field-replicate samples and field-blank samples were collected for quality control. A field-replicate sample or field-blank sample was collected approximately every 10 samples. Field-replicate samples are collected to assess precision of laboratory analytical methods and consistency of sample-collection and sample-handling procedures. Field-replicate samples were collected simultaneously with ground-water and surface-water samples. Field-blank samples consisted of organic-free deionized water that was passed through the pump and sampling equipment to evaluate the cleanliness of sampling equipment and sampling techniques. Quality-control sample analyses indicate minimal variability in laboratory analytical methods and that sample-collection and sample-handling techniques did not affect the water-quality samples.

\section{Ground-Water Levels}

Water levels in selected 2-inch and 4-inch observation wells were continuously measured with pressure transducers and data recorders. Data were recorded at hourly intervals. Barometric pressure was recorded at site CRM-15 (map ID \# 12) in the Seminole Well Field. Water-levels measured with pressure transducers were corrected for changes in barometric pressure. The water levels measured by pressure transducers were verified about every two weeks by manually measuring water levels in each observation well to the nearest 0.01 $\mathrm{ft}$ using either a calibrated steel tape or calibrated electric tape. Water levels were measured according to protocols documented in U.S. Office of Water-Data Coordination (1977).

\section{Physical Properties}

Dissolved oxygen, $\mathrm{pH}$, specific conductance, and water temperature were continuously monitored with multiprobe instruments installed in selected 4-inch diameter observation wells and in the Cedar River near the Seminole Well Field at site SW-2 (map ID \# 32). The multiprobe instrument used to monitor physical properties in the Cedar River was installed in a 6 -inch diameter pipe that extended from the river bank into the river. Measurements generally were recorded at 60-minute intervals and the data were retrieved at about monthly intervals. During winter months, data were retrieved from the Cedar River multiprobe less frequently because the instrument could not be retrieved when ice formed in the 6-inch diameter pipe. Measurements in the river were recorded at 3-hour intervals during winter months. The multiprobes were calibrated each time data were retrieved by using specific conductance and $\mathrm{pH}$ standards obtained from the USGS NWQL. In addition to multiprobe measurements, water temperature also was continuously monitored in 2-inch diameter observation wells equipped with pressure transducers and data recorders used to continuously measure ground-water levels.

\section{SELECTED HYDROLOGIC DATA}

The types of data and the sampling and measurement period at each site are summarized in table 2 . Water-quality analyses are listed in tables 3 through 9. Mean daily ground-water levels in observation wells 
equipped with pressure transducers and data recorders are listed in table 10. Physical properties continuously monitored in selected observation wells and the Cedar River are listed in tables 11 through 14. All reported data are stored in the USGS National Water Information System database.

\section{Water-Quality Data}

Water-quality samples were collected from selected observation wells and the Cedar River at Edgewood Road (map ID \# 31) from April 1996 to March 1999 to conduct periodic monitoring, to evaluate ground-water geochemistry, to assess the occurrence of pesticides and herbicide degradates in the alluvial aquifer, and to characterize water quality in shallow ground water near a wetland area in the Seminole Well Field. Samples collected from observation wells CRM-14 (map ID \#11), CRM-15 (map ID \#12), CRM-16 (map ID \#13), CRM-17 (map ID \#14), CRM18 (map ID \#15), CRM-19 (map ID \#16), CRM-SD-60 (map ID \#25), CRM-SD-61 (map ID \#26), CRM-SD62 (map ID \#27), CRM-SD-63 (map ID \#28), and CRM-SD-64 (map ID \#29) were used to evaluate ground-water geochemistry (samples collected in September 1996 and May 1997) and to assess the occurrence of pesticides and herbicide degradates (samples collected from June 1998 through August 1998) in the alluvial aquifer near the Seminole Well Field. Samples collected from observation wells CRM-22 (map ID \#19), CRM-23 (map ID \#20), CRM-24 (map ID \#21), CRM-25 (map ID \# 22), CRM-26 (map ID \#23), and CRM-27 (map ID \# 24) were collected to characterize water quality in shallow ground water near a wetland area in the Seminole Well Field. Samples collected from the remaining observation wells and the Cedar River at Edgewood Road (map ID \#31) primarily were collected for periodic monitoring. Common-ion, traceelement, and nutrient concentrations in water samples collected from April 1996 through March 1999 are summarized in table 3. Pesticide concentrations in water samples collected from April 1996 through March 1999 are summarized in table 4. Common-ion and trace-element concentrations in water samples are listed in table 5. Nutrient and dissolved organic-carbon concentrations in water samples are listed in table 6. Pesticide concentrations in water samples are listed in table 7. Concentrations of ethanesulfonic-acid and oxanilic-acid degradates of acetanilide herbicides in water samples collected from June 1998 to August 1998 are listed in table 8 and table 9 , respectively.

\section{Ground-Water Levels}

Mean daily ground-water levels in observation wells equipped with pressure tranducers and data recorders are listed in table 10. Data were not recorded for some time periods because of instrument malfunction or data recorders were removed during periods of flooding.

\section{Physical Properties}

Mean daily specific-conductance values are listed in table 11. Mean daily $\mathrm{pH}$ values are listed in table 12. Mean daily dissolved-oxygen concentrations are listed in table 13 . Mean daily ground-water temperatures and the mean daily water temperature in the Cedar River are listed in table 14. Water temperatures measured at sites with multiprobe instruments and in observation wells equipped with pressure transducers and data recorders are listed in table 14. The mean daily specific conductance in ground water ranged between 149 microsiemens per centimeter $(\mu \mathrm{S} / \mathrm{cm})$ at CRM-25 (map ID \#22) on April 2, 1998 and $850 \mu \mathrm{S} / \mathrm{cm}$ at CRM-1 (map ID \#1) on January 22, 1997. The mean daily specific conductance in the Cedar River at site SW-2 (map ID \#32) ranged between $202 \mu \mathrm{S} / \mathrm{cm}$ on February 23, 1997 and $651 \mu \mathrm{S} / \mathrm{cm}$ on January 1,1999 . The mean daily $\mathrm{pH}$ in ground water ranged between 6.5 at CRM-2 (map ID \#2) on April 12, 1996 and 8.5 at CRM-2 (map ID \#2) on June 10-12, 1997. The mean daily $\mathrm{pH}$ in the Cedar River at site SW-2 (map ID \#32) ranged between 7.3 on June 21, 1998 and 9.3 on October 6, 1997 and October 10-11, 1997. The mean daily dissolved-oxygen concentration in ground water ranged between 0 milligrams per liter (mg/L) at CRM-21 (map ID \#18) in June, July, October, and November 1998 and $12.6 \mathrm{mg} / \mathrm{L}$ at CRM-21 on February 27, 1999. The mean daily dissolved-oxygen concentration in the Cedar River at site SW-2 (map ID \#32) ranged between $1.7 \mathrm{mg} / \mathrm{L}$ on May 6, 1996 and $17.4 \mathrm{mg} / \mathrm{L}$ on December 4, 1996.

New multiprobe instruments were purchased in the fall of 1997 to upgrade and replace older instruments. Data were collected with the older instruments at sampling sites CRM-1 (map ID \#1), CRM-2 (map ID 
\#2), CRM-11 (map ID \#9), CRM-12 (map ID \#10) through July 1997 and in the Cedar River at site SW-2 (map ID \#32) until February 1998. All dissolvedoxygen measurements at sites CRM-1 (map ID \#1) and CRM-11 (map ID \#9) were rejected after data review because the multiprobe instruments at these sites exhibited large drift after calibration. The new multiprobe instruments were installed in February 1998 in the Northwest Well Field at sites CRM-20 (map ID \#17) and CRM-21 (map ID \#18), in the Seminole Well Field at sites CRM-22 (map ID \#19) and CRM-23 (map ID \#20), and in the Cedar River near the Seminole Well Field at site SW-2 (map ID \#32).

\section{REFERENCES CITED}

Boyd, R.A., 1998, Characterizing ground-water flow in the municipal well fields of Cedar Rapids, Iowa, with selected environmental tracers: Journal of the American Water Resources Association, vol. 34, no. 3, pp. 507-518.

Boyd, R.A., 1999, Herbicides and herbicide degradates in shallow ground water and the Cedar River near a municipal well field, Cedar Rapids, Iowa: Proceedings of the U.S. Geological Survey Toxic Substances Hydrology Program 1999 Technical Meeting.

Fishman, M.J. and Friedman, L.C., 1989, Methods for determination of inorganic substances in water and fluvial sediments (3rd ed.): U.S. Geological Survey Techniques of Water-Resources Investigations, book 5 , chapter $\mathrm{A} 1,545 \mathrm{p}$.

Hansen, R.E., 1970, Geology and ground-water resources of Linn County, Iowa: Iowa Geological Survey, WaterSupply Bulletin number 10, $66 \mathrm{p}$.

May, J.E., Gorman, J.G., Goodrich, R.D., Miller, V.E., Turco, M.J., and Linhart, S.M., 1999, Water resources data, Iowa Water Year 1998, Volume 1, Mississippi River Basin: U.S. Geological Survey Water-Data Report IA-98-1, 374 p.

Schnoebelen, D.J. and Schulmeyer, P.M., 1996, Selected hydrogeologic data from the Cedar Rapids area, Benton and Linn Counties, Iowa, October 1992 through March 1996: U.S. Geological Survey Open-File

Report 96-471, $172 \mathrm{p}$.

Schulmeyer, P.M., 1995, Effect of the Cedar River on the quality of the ground-water supply for Cedar Rapids, Iowa: U.S. Geological Survey Water-Resources Investigations Report 94-4211, 68 p.
Schulmeyer, P.M. and Schnoebelen, D.J., 1998, Hydrogeology and water quality in the Cedar Rapids area, Iowa, 1992-96: U.S. Geological Survey Water-Resources Investigations Report 97-4261, 77 p.

Squillace, P.J., Caldwell, J.P., Schulmeyer, P.M., and Harvey, C.A., 1996, Movement of agricultural chemicals between surface water and ground water, Lower Cedar River Basin, Iowa: U.S. Geological Survey WaterSupply Paper 2448, $59 \mathrm{p}$.

U.S. Office of Water-Data Coordination, 1977, National handbook of recommended methods for water-data acquisition: Reston, Virginia, Office of Water-Data Coordination, Geological Survey, U.S. Department of the Interior, chap. 2, p. 8-10.

Wahl, K.D. and Bunker, B.J., 1986, Hydrology and carbonate aquifers in southwestern Linn County and adjacent parts of Bemton, Iowa, and Johnson Counties, Iowa: Iowa Geological Survey, Water Supply Bulletin number $15,56 \mathrm{p}$.

Zimmerman, L.R. and Thurman, E.M., 1999, Method of analysis by the U.S. Geological Survey Organic Geochemistry Research Group-Determination of triazine and chloroacetanilide herbicides in water by solidphase extraction and capillary-column gas chromatography/mass spectrometry with selected ion monitoring: U.S. Geological Survey Open-File Report 98-634, 21 p. 


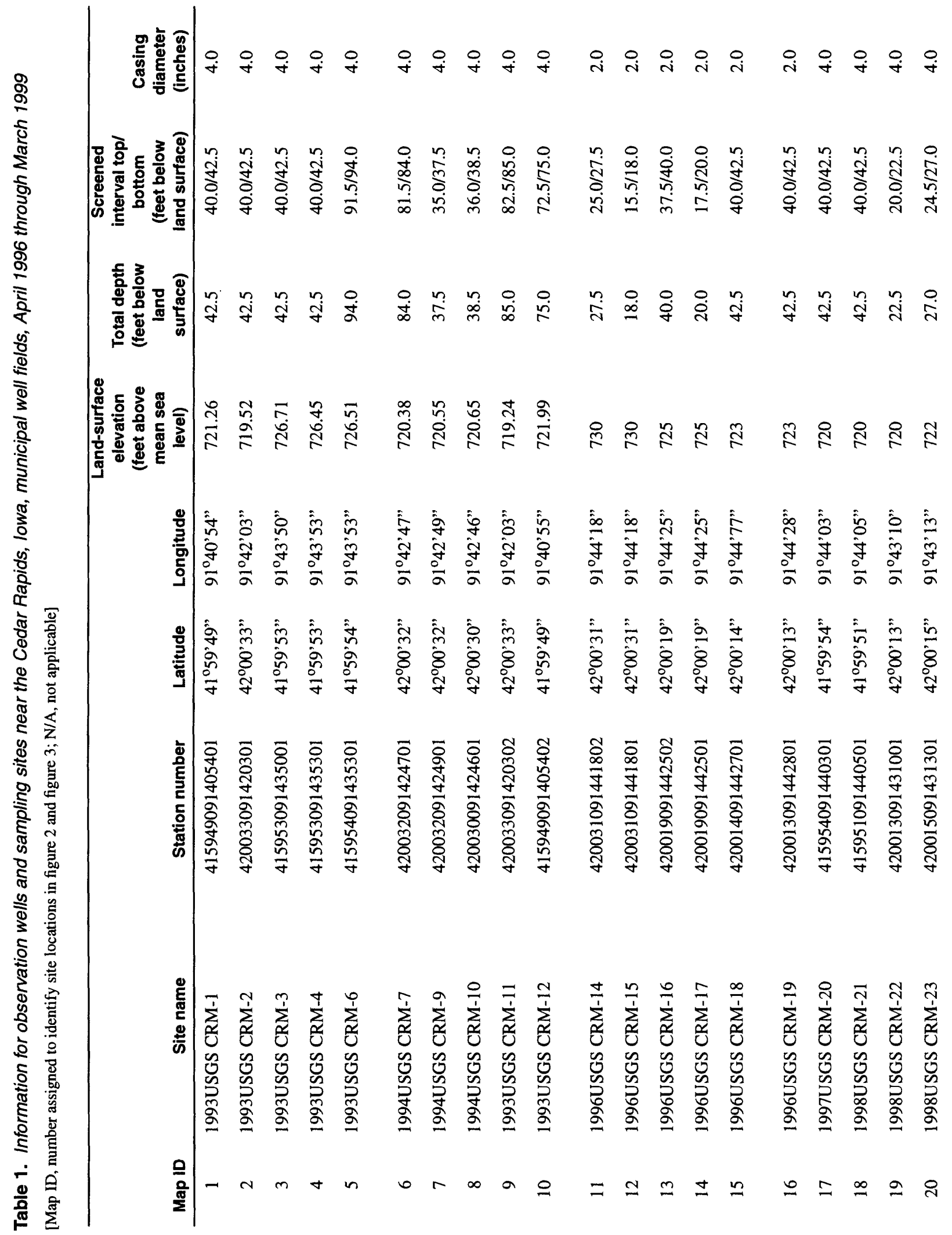




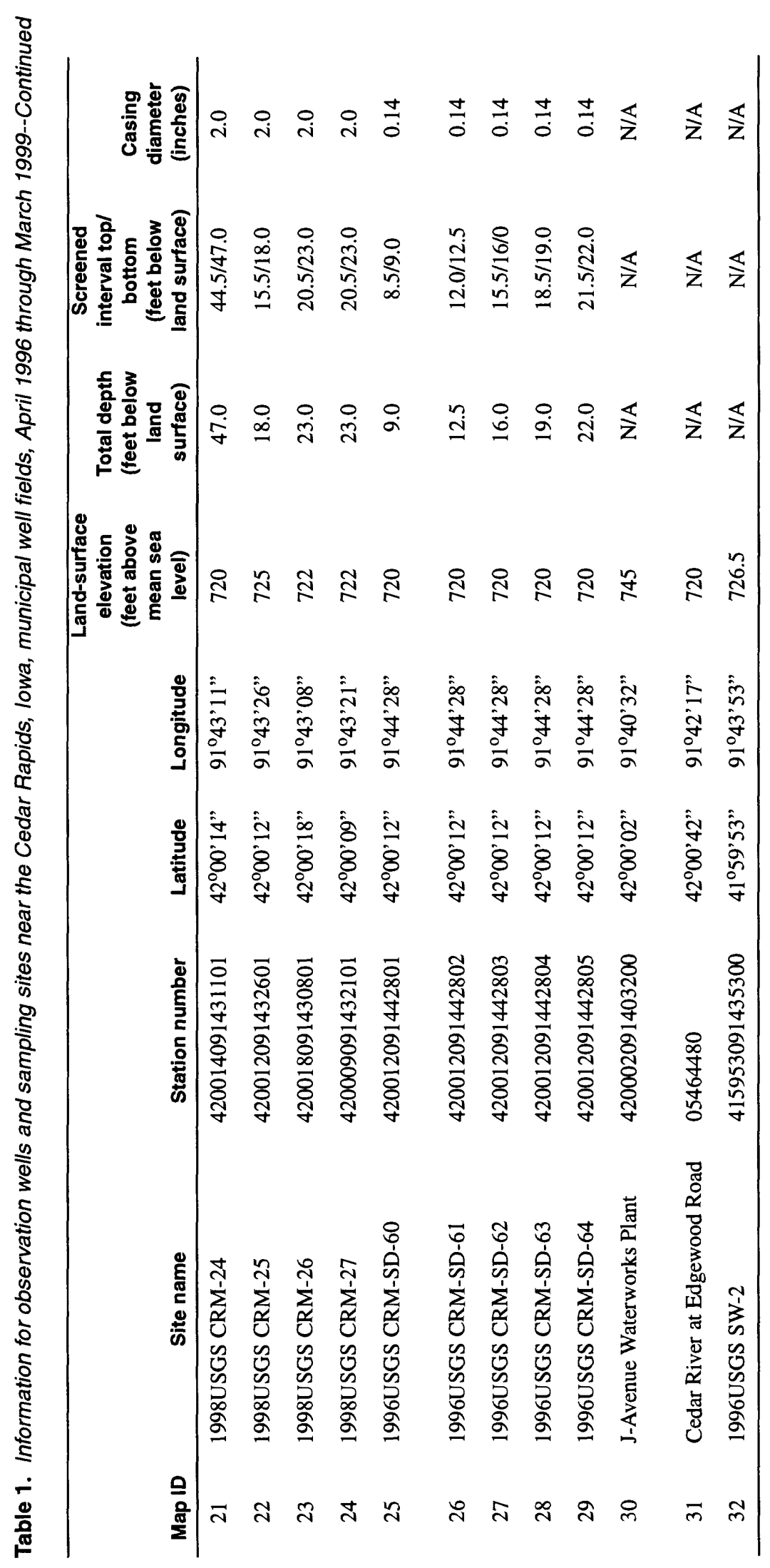




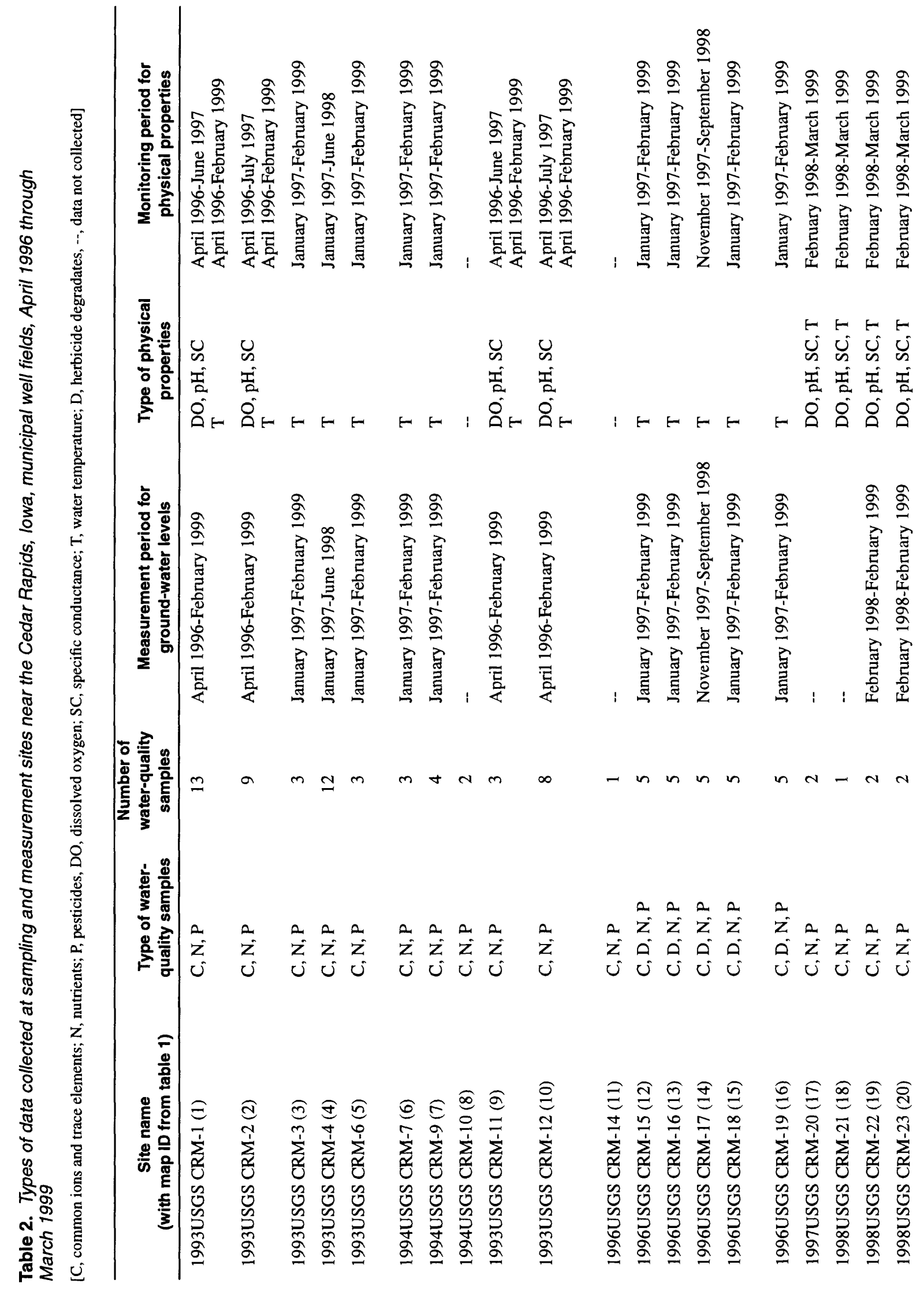




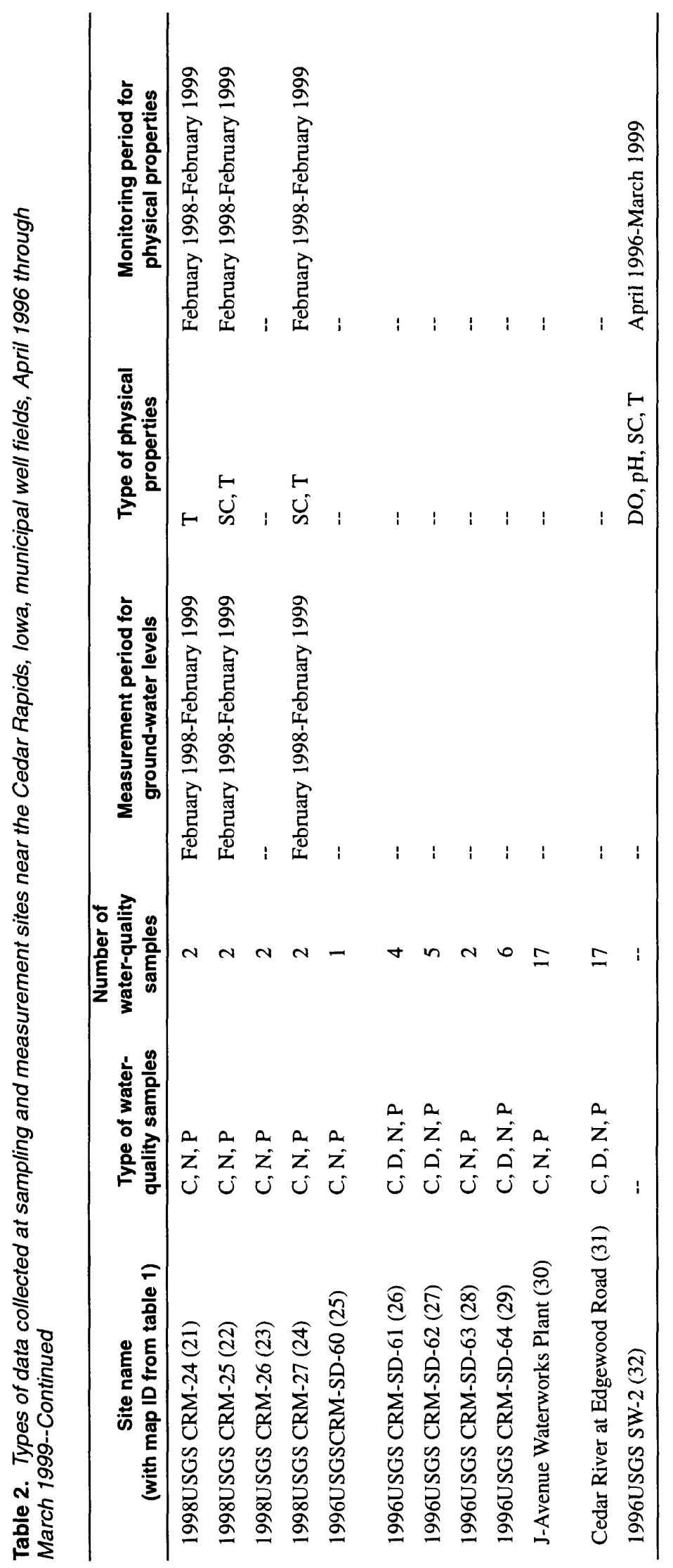


Table 3. Summary of common-ion, trace-element, and nutrient concentrations in water samples collected from the Cedar Rapids, lowa, municipal well fields and Cedar River, April 1996 through March 1999

[mg/L, milligrams per liter, $\mu \mathrm{g} / \mathrm{L}$, micrograms per liter; <, less than]

\begin{tabular}{|c|c|c|c|c|c|c|}
\hline Constituent & Units & $\begin{array}{l}\text { Sample } \\
\text { medium }\end{array}$ & $\begin{array}{c}\text { Number of } \\
\text { samples }\end{array}$ & $\begin{array}{c}\text { Mean } \\
\text { concentration }\end{array}$ & $\begin{array}{c}\text { Minimum } \\
\text { concentration }\end{array}$ & $\begin{array}{c}\text { Maximum } \\
\text { concentration }\end{array}$ \\
\hline \multicolumn{7}{|c|}{ Common ions } \\
\hline \multirow[t]{3}{*}{ Calcium } & $\mathrm{mg} / \mathrm{L}$ as $\mathrm{Ca}$ & Alluvium & 75 & 77 & 49 & 140 \\
\hline & & Bedrock & 16 & 69 & 49 & 80 \\
\hline & & Cedar River & 12 & 63 & 27 & 80 \\
\hline \multirow[t]{3}{*}{ Chloride } & $\mathrm{mg} / \mathrm{L}$ as $\mathrm{Cl}$ & Alluvium & 75 & 21 & 1.2 & 31 \\
\hline & & Bedrock & 16 & 17 & 0.50 & 34 \\
\hline & & Cedar River & 12 & 23 & 16 & 27 \\
\hline \multirow[t]{3}{*}{ Magnesium } & $\mathrm{mg} / \mathrm{L}$ as $\mathrm{Mg}$ & Alluvium & 75 & 21 & 8.4 & 46 \\
\hline & & Bedrock & 16 & 23 & 15 & 28 \\
\hline & & Cedar River & 12 & 21 & 15 & 24 \\
\hline \multirow[t]{3}{*}{ Potassium } & $\mathrm{mg} / \mathrm{L}$ as $\mathrm{K}$ & Alluvium & 75 & 3.0 & 0.80 & 8.2 \\
\hline & & Bedrock & 16 & 2.5 & 0.90 & 3.6 \\
\hline & & Cedar River & 12 & 2.5 & 1.9 & 3.5 \\
\hline \multirow[t]{3}{*}{ Silica } & $\mathrm{mg} / \mathrm{L}$ as $\mathrm{SiO}_{2}$ & Alluvium & 75 & 14 & 5.0 & 25 \\
\hline & & Bedrock & 16 & 16 & 12 & 20 \\
\hline & & Cedar River & 12 & 8.1 & 0.07 & 14 \\
\hline \multirow[t]{3}{*}{ Sodium } & $\mathrm{mg} / \mathrm{L}$ as $\mathrm{Na}$ & Alluvium & 75 & 11 & 3.2 & 24 \\
\hline & & Bedrock & 16 & 10 & 3.6 & 16 \\
\hline & & Cedar River & 12 & 10 & 5.8 & 15 \\
\hline \multirow[t]{3}{*}{ Sulfate } & $\mathrm{mg} / \mathrm{L}$ as $\mathrm{SO}_{4}$ & Alluvium & 75 & 30 & $<0.10$ & 150 \\
\hline & & Bedrock & 16 & 14 & 3.1 & 30 \\
\hline & & Cedar River & 12 & 33 & 19 & 41 \\
\hline \multicolumn{7}{|c|}{ Trace elements } \\
\hline \multirow[t]{3}{*}{ Boron } & $\mu \mathrm{g} / \mathrm{L}$ as $B$ & Alluvium & 65 & 34 & 20 & 86 \\
\hline & & Bedrock & 8 & 41 & 21 & 49 \\
\hline & & Cedar River & 10 & 29 & 22 & 35 \\
\hline \multirow[t]{3}{*}{ Bromide } & $\mathrm{mg} / \mathrm{L}$ as $\mathrm{Br}$ & Alluvium & 75 & 0.06 & $<0.01$ & 0.70 \\
\hline & & Bedrock & 16 & 0.04 & $<0.01$ & 0.10 \\
\hline & & Cedar River & 12 & 0.02 & $<0.01$ & 0.06 \\
\hline
\end{tabular}


Table 3. Summary of common-ion, trace-element, and nutrient concentrations in water samples collected from the Cedar Rapids, lowa, municipal well fields and Cedar River, April 1996 through March 1999--Continued

\begin{tabular}{|c|c|c|c|c|c|c|}
\hline Constituent & Units & $\begin{array}{l}\text { Sample } \\
\text { medium }\end{array}$ & $\begin{array}{l}\text { Number of } \\
\text { samples }\end{array}$ & $\begin{array}{c}\text { Mean } \\
\text { concentration }\end{array}$ & $\begin{array}{c}\text { Minimum } \\
\text { concentration }\end{array}$ & $\begin{array}{c}\text { Maximum } \\
\text { concentration }\end{array}$ \\
\hline \multicolumn{7}{|c|}{ Trace elements--Continued } \\
\hline \multirow[t]{3}{*}{ Fluoride } & $\mathrm{mg} / \mathrm{L}$ as $\mathrm{F}$ & Alluvium & 75 & 0.21 & $<0.10$ & 0.33 \\
\hline & & Bedrock & 16 & 0.23 & 0.10 & 0.30 \\
\hline & & Cedar River & 12 & 0.24 & 0.19 & 0.30 \\
\hline \multirow[t]{3}{*}{ Iron } & $\mu \mathrm{g} / \mathrm{L}$ as $\mathrm{Fe}$ & Alluvium & 75 & 2,460 & $<3.0$ & 20,000 \\
\hline & & Bedrock & 16 & 2,000 & 7.0 & 4,200 \\
\hline & & Cedar River & 12 & 10 & 4.4 & 32 \\
\hline \multirow[t]{3}{*}{ Manganese } & $\mu \mathrm{g} / \mathrm{L}$ as $\mathrm{Mn}$ & Alluvium & 75 & 1,200 & $<1.0$ & 7,500 \\
\hline & & Bedrock & 16 & 627 & 2.0 & 2,200 \\
\hline & & Cedar River & 12 & 14 & $<3.0$ & 60 \\
\hline \multicolumn{7}{|c|}{ Nutrients and organic carbon } \\
\hline \multirow[t]{3}{*}{ Nitrogen, ammonia } & $\mathrm{mg} / \mathrm{L}$ as $\mathrm{N}$ & Alluvium & 70 & 1.5 & $<0.015$ & 9.8 \\
\hline & & Bedrock & 11 & 2.3 & 0.05 & 4.3 \\
\hline & & Cedar River & 10 & 0.16 & $<0.015$ & 0.95 \\
\hline \multirow[t]{3}{*}{ Nitrogen, nitrite } & $\mathrm{mg} / \mathrm{L}$ as $\mathrm{N}$ & Alluvium & 70 & 0.02 & $<0.01$ & 0.19 \\
\hline & & Bedrock & 11 & $<0.01$ & $<0.01$ & $<0.01$ \\
\hline & & Cedar River & 10 & 0.03 & 0.01 & 0.09 \\
\hline \multirow[t]{3}{*}{$\begin{array}{l}\text { Nitrogen, nitrite } \\
\text { plus nitrate }\end{array}$} & $\mathrm{mg} / \mathrm{L}$ as $\mathrm{N}$ & Alluvium & 70 & 1.6 & $<0.05$ & 10.2 \\
\hline & & Bedrock & 11 & 0.04 & $<0.05$ & 0.08 \\
\hline & & Cedar River & 10 & 5.5 & 0.05 & 11.0 \\
\hline \multirow[t]{3}{*}{ Organic carbon } & $\mathrm{mg} / \mathrm{L}$ as $\mathrm{C}$ & Alluvium & 76 & 2.4 & 0.50 & 6.4 \\
\hline & & Bedrock & 16 & 1.5 & 0.40 & 2.9 \\
\hline & & Cedar River & 12 & 2.9 & 2.1 & 4.4 \\
\hline \multirow[t]{3}{*}{ Orthophosphate } & $\mathrm{mg} / \mathrm{L}$ as $\mathrm{P}$ & Alluvium & 69 & 0.06 & $<0.01$ & 0.40 \\
\hline & & Bedrock & 11 & 0.01 & $<0.01$ & 0.05 \\
\hline & & Cedar River & 10 & 0.06 & $<0.01$ & 0.15 \\
\hline
\end{tabular}


Table 4. Summary of pesticide concentrations in water samples collected from the Cedar Rapids, lowa, municipal well fields and Cedar River, April 1996 through March 1999

[All concentration in micrograms per liter; MRL, minimum reporting limit; --, not applicable]

\begin{tabular}{|c|c|c|c|c|c|c|}
\hline Constituent & $\begin{array}{l}\text { Sample } \\
\text { medium }\end{array}$ & $\begin{array}{c}\text { Number of } \\
\text { samples }\end{array}$ & $\begin{array}{c}\text { Number of } \\
\text { concentrations } \\
\text { greater than } \\
\text { MRL }\end{array}$ & MRL & $\begin{array}{c}\text { Minimum } \\
\text { concentration }\end{array}$ & $\begin{array}{c}\text { Maximum } \\
\text { concentration }\end{array}$ \\
\hline \multirow[t]{3}{*}{ Acetochlor } & Alluvium & 92 & 10 & 0.05 & 0.06 & 0.17 \\
\hline & Bedrock & 9 & 0 & & -- & -- \\
\hline & Cedar River & 15 & 4 & & 0.15 & 1.17 \\
\hline \multirow[t]{3}{*}{ Alachlor } & Alluvium & 101 & 0 & 0.05 & -- & -- \\
\hline & Bedrock & 16 & 0 & & -- & -- \\
\hline & Cedar River & 17 & 1 & & -- & 0.16 \\
\hline \multirow[t]{3}{*}{ Ametryn } & Alluvium & 101 & 0 & 0.05 & -- & -- \\
\hline & Bedrock & 16 & 0 & & -- & -- \\
\hline & Cedar River & 17 & 0 & & -- & - \\
\hline \multirow[t]{3}{*}{ Atrazine } & Alluvium & 101 & 81 & 0.05 & 0.05 & 2.7 \\
\hline & Bedrock & 16 & 8 & & 0.05 & 0.09 \\
\hline & Cedar River & 17 & 17 & & 0.05 & 8.2 \\
\hline \multirow[t]{3}{*}{ Butachlor } & Alluvium & 66 & 1 & 0.05 & -- & 0.05 \\
\hline & Bedrock & 9 & 0 & & -- & - \\
\hline & Cedar River & 10 & 0 & & -- & -- \\
\hline \multirow[t]{3}{*}{ Butylate } & Alluvium & 66 & 0 & 0.05 & -- & -- \\
\hline & Bedrock & 9 & 0 & & -- & - \\
\hline & Cedar River & 10 & 0 & & -- & -- \\
\hline \multirow[t]{3}{*}{ Bromacil } & Alluvium & 66 & 0 & 0.05 & -- & -- \\
\hline & Bedrock & 9 & 0 & & -- & -- \\
\hline & Cedar River & 10 & 0 & & -- & -- \\
\hline \multirow[t]{3}{*}{ Carboxin } & Alluvium & 66 & 0 & 0.05 & -- & -- \\
\hline & Bedrock & 9 & 5 & & 0.05 & 0.06 \\
\hline & Cedar River & 10 & 0 & & -- & -- \\
\hline \multirow[t]{3}{*}{ Cyanazine } & Alluvium & 101 & 7 & $0.20 / 0.05$ & 0.05 & 0.50 \\
\hline & Bedrock & 16 & 0 & & -- & -- \\
\hline & Cedar River & 17 & 1 & & -- & 0.74 \\
\hline
\end{tabular}


Table 4. Summary of pesticide concentrations in water samples collected from the Cedar Rapids, lowa, municipal well fields and Cedar River, April 1996 through March 1999.--Continued

\begin{tabular}{|c|c|c|c|c|c|c|}
\hline Constituent & $\begin{array}{l}\text { Sample } \\
\text { medium }\end{array}$ & $\begin{array}{l}\text { Number of } \\
\text { samples }\end{array}$ & $\begin{array}{c}\text { Number of } \\
\text { concentrations } \\
\text { greater than } \\
\text { MRL }\end{array}$ & MRL & $\begin{array}{c}\text { Minimum } \\
\text { concentration }\end{array}$ & $\begin{array}{c}\text { Maximum } \\
\text { concentration }\end{array}$ \\
\hline \multirow[t]{3}{*}{ Deethylatrazine } & Alluvium & 101 & 70 & 0.05 & 0.05 & 0.50 \\
\hline & Bedrock & 16 & 0 & & -- & -- \\
\hline & Cedar River & 17 & 16 & & 0.08 & 0.64 \\
\hline \multirow[t]{3}{*}{ Deisopropylatrazine } & Alluvium & 101 & 32 & 0.05 & 0.05 & 0.26 \\
\hline & Bedrock & 16 & 0 & & -- & -- \\
\hline & Cedar River & 17 & 10 & & 0.05 & 0.28 \\
\hline \multirow[t]{3}{*}{ Diphenamid } & Alluvium & 66 & 0 & 0.05 & -- & -- \\
\hline & Bedrock & 9 & 0 & & -- & -- \\
\hline & Cedar River & 10 & 0 & & -- & -- \\
\hline \multirow[t]{3}{*}{ Hexazinone } & Alluvium & 66 & 2 & 0.05 & 0.05 & 0.05 \\
\hline & Bedrock & 9 & 0 & & - & -- \\
\hline & Cedar River & 10 & 0 & & - & -- \\
\hline \multirow[t]{3}{*}{ Metolachlor } & Alluvium & 101 & 58 & 0.05 & 0.05 & 0.62 \\
\hline & Bedrock & 16 & 1 & & -- & 0.08 \\
\hline & Cedar River & 17 & 15 & & 0.05 & 2.45 \\
\hline \multirow[t]{3}{*}{ Metribuzin } & Alluvium & 101 & 6 & 0.05 & 0.05 & 0.07 \\
\hline & Bedrock & 16 & 0 & & - & -- \\
\hline & Cedar River & 17 & 2 & & 0.06 & 0.07 \\
\hline \multirow[t]{3}{*}{ Prometon } & Alluvium & 99 & 0 & 0.05 & - & -- \\
\hline & Bedrock & 16 & 0 & & -- & -- \\
\hline & Cedar River & 17 & 1 & & - & 0.06 \\
\hline \multirow[t]{3}{*}{ Prometryn } & Alluvium & 101 & 0 & 0.05 & -- & -- \\
\hline & Bedrock & 16 & 0 & & -- & -- \\
\hline & Cedar River & 17 & 0 & & -- & -- \\
\hline \multirow[t]{3}{*}{ Propachlor } & Alluvium & 91 & 0 & 0.05 & -- & -- \\
\hline & Bedrock & 9 & 0 & & -- & -- \\
\hline & Cedar River & 15 & 0 & & -- & -- \\
\hline \multirow[t]{3}{*}{ Propazine } & Alluvium & 101 & 0 & 0.05 & -- & -- \\
\hline & Bedrock & 16 & 0 & & - & - \\
\hline & Cedar River & 17 & 0 & & -- & -- \\
\hline
\end{tabular}


Table 4. Summary of pesticide concentrations in water samples collected from the Cedar Rapids, lowa, municipal well fields and Cedar River, April 1996 through March 1999.--Continued

\begin{tabular}{|c|c|c|c|c|c|c|}
\hline Constituent & $\begin{array}{l}\text { Sample } \\
\text { medium }\end{array}$ & $\begin{array}{c}\text { Number of } \\
\text { samples }\end{array}$ & $\begin{array}{c}\text { Number of } \\
\text { concentrations } \\
\text { greater than } \\
\text { MRL }\end{array}$ & MRL & $\begin{array}{c}\text { Minimum } \\
\text { concentration }\end{array}$ & $\begin{array}{c}\text { Maximum } \\
\text { concentration }\end{array}$ \\
\hline \multirow[t]{3}{*}{ Sicloate } & Alluvium & 66 & 0 & 0.05 & - & - \\
\hline & Bedrock & 9 & 0 & & -- & -- \\
\hline & Cedar River & 10 & 0 & & -- & -- \\
\hline \multirow[t]{3}{*}{ Simazine } & Alluvium & 101 & 0 & 0.05 & -- & -- \\
\hline & Bedrock & 16 & 0 & & -- & -- \\
\hline & Cedar River & 17 & 0 & & -- & -- \\
\hline \multirow[t]{3}{*}{ Simetryn } & Alluvium & 66 & 0 & 0.05 & -- & -- \\
\hline & Bedrock & 9 & 0 & & -- & -- \\
\hline & Cedar River & 10 & 0 & & -- & -- \\
\hline \multirow[t]{3}{*}{ Terbacil } & Alluvium & 66 & 0 & 0.05 & -- & -- \\
\hline & Bedrock & 9 & 0 & & -- & -- \\
\hline & Cedar River & 10 & 0 & & -- & -- \\
\hline \multirow[t]{3}{*}{ Terbutryn } & Alluvium & 25 & 0 & 0.05 & -- & -- \\
\hline & Bedrock & 0 & 0 & & -- & -- \\
\hline & Cedar River & 5 & 0 & & -- & -- \\
\hline \multirow[t]{3}{*}{ Trifluralin } & Alluvium & 66 & 0 & 0.05 & -- & -- \\
\hline & Bedrock & 9 & 0 & & -- & -- \\
\hline & Cedar River & 10 & 0 & & -- & -- \\
\hline \multirow[t]{3}{*}{ Vernolate } & Alluvium & 66 & 0 & 0.05 & -- & -- \\
\hline & Bedrock & 9 & 0 & & -- & -- \\
\hline & Cedar River & 10 & 0 & & -- & -- \\
\hline
\end{tabular}




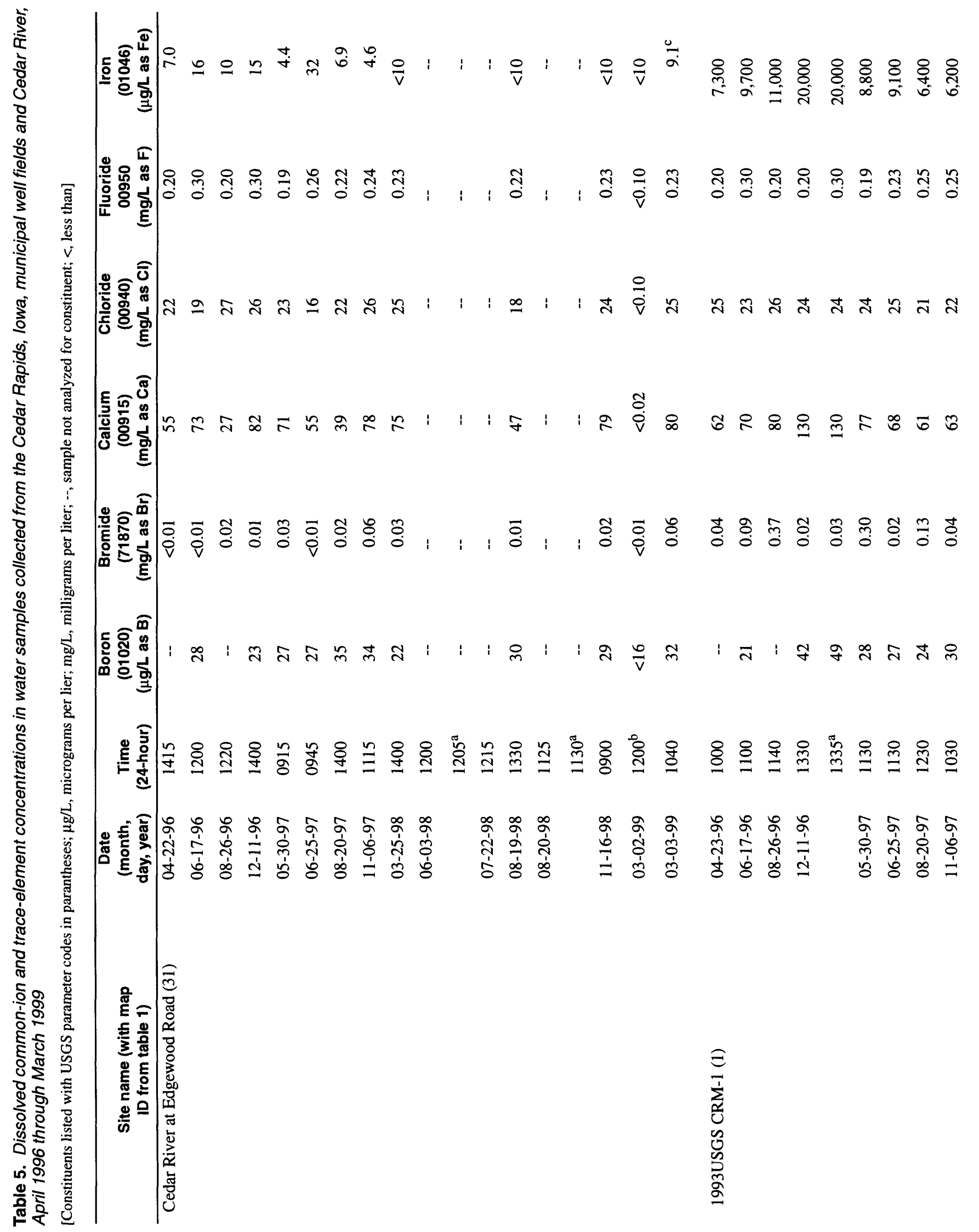




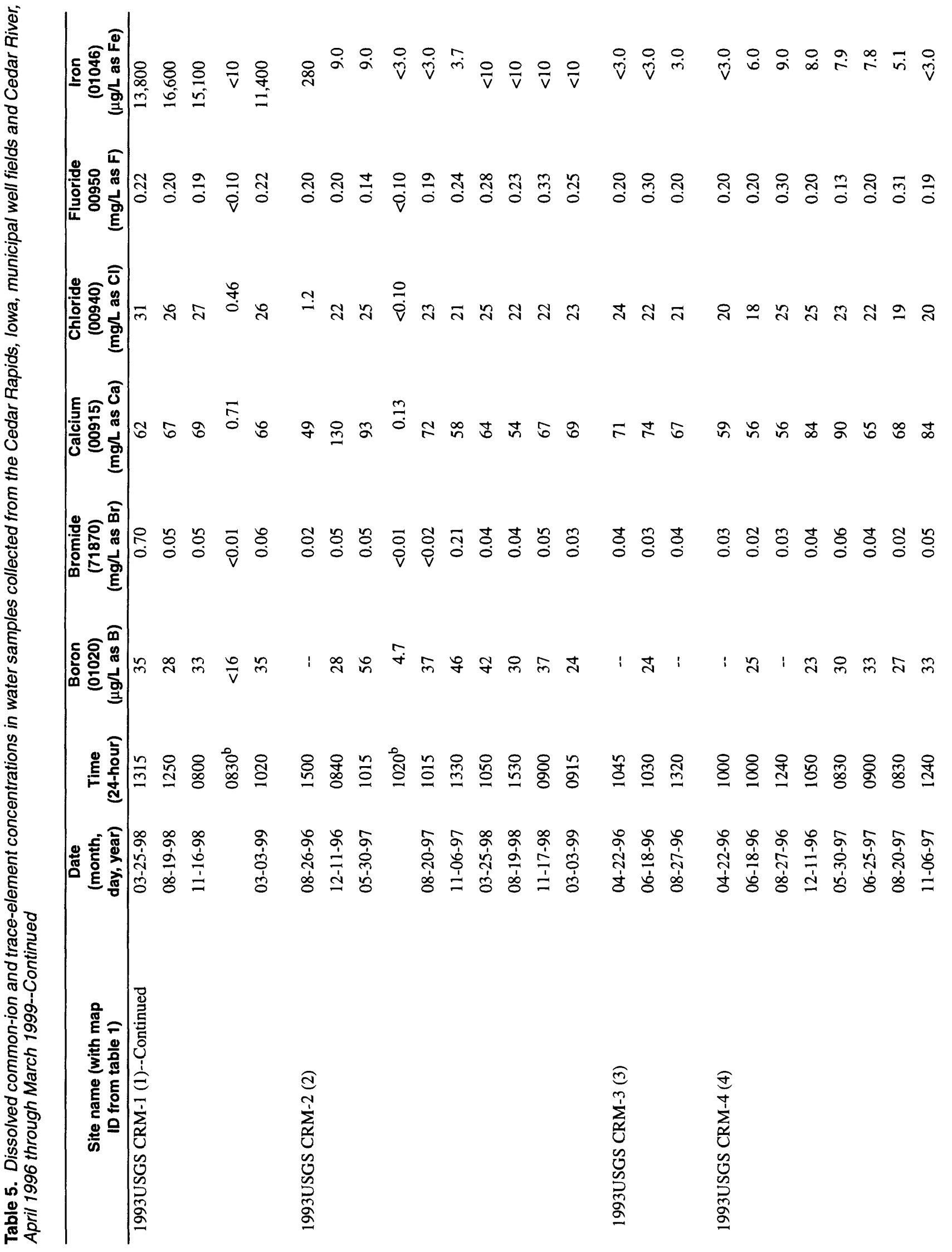




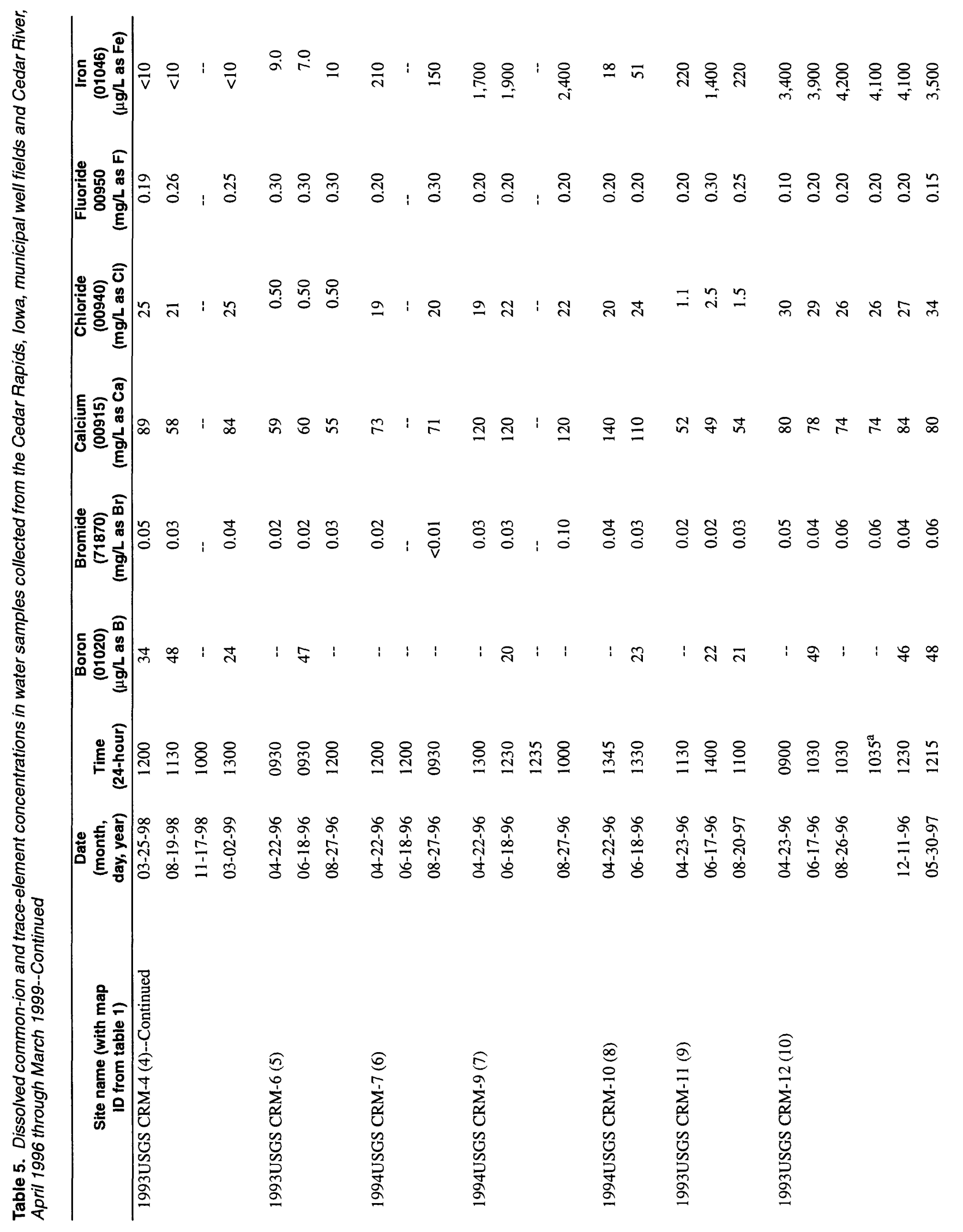




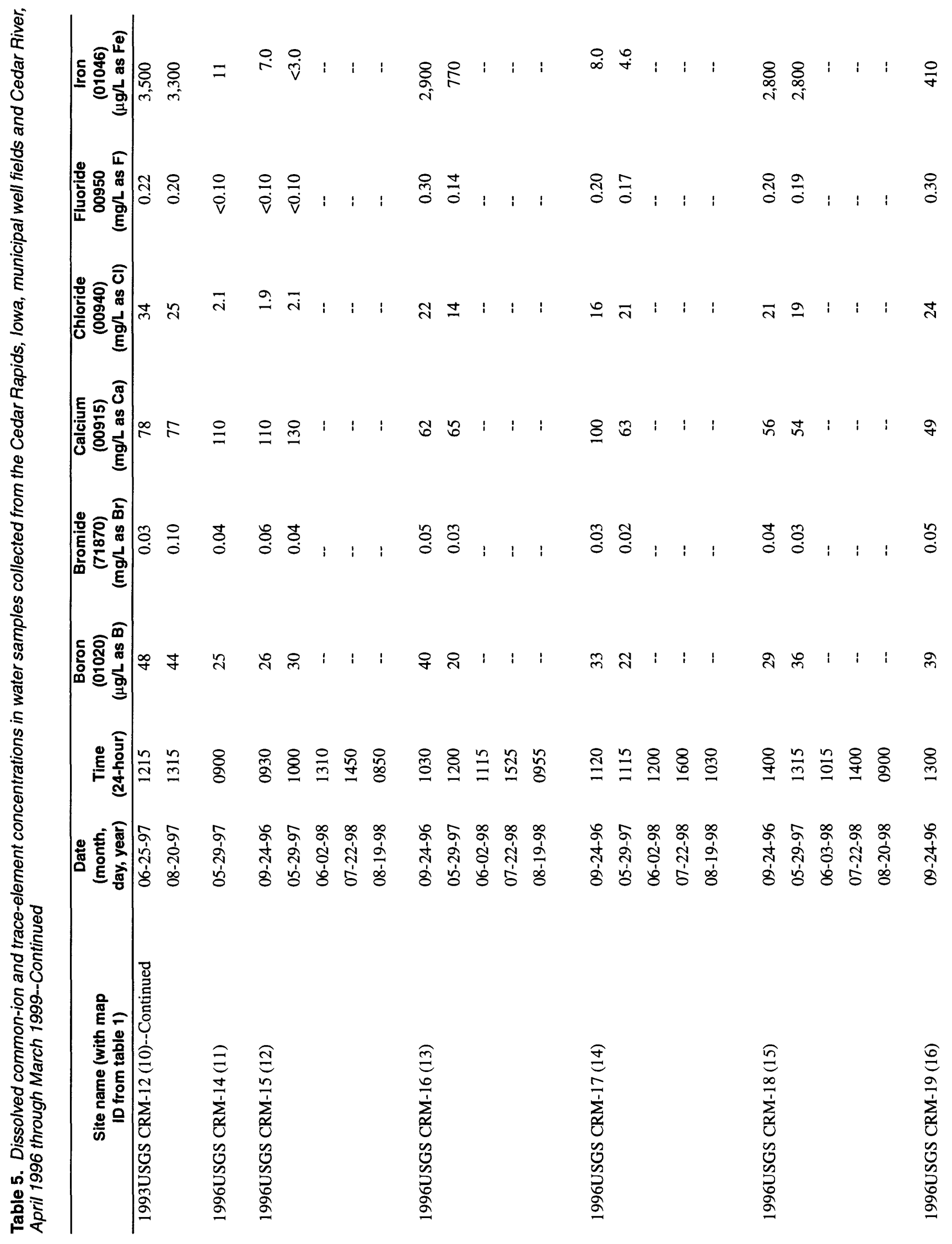




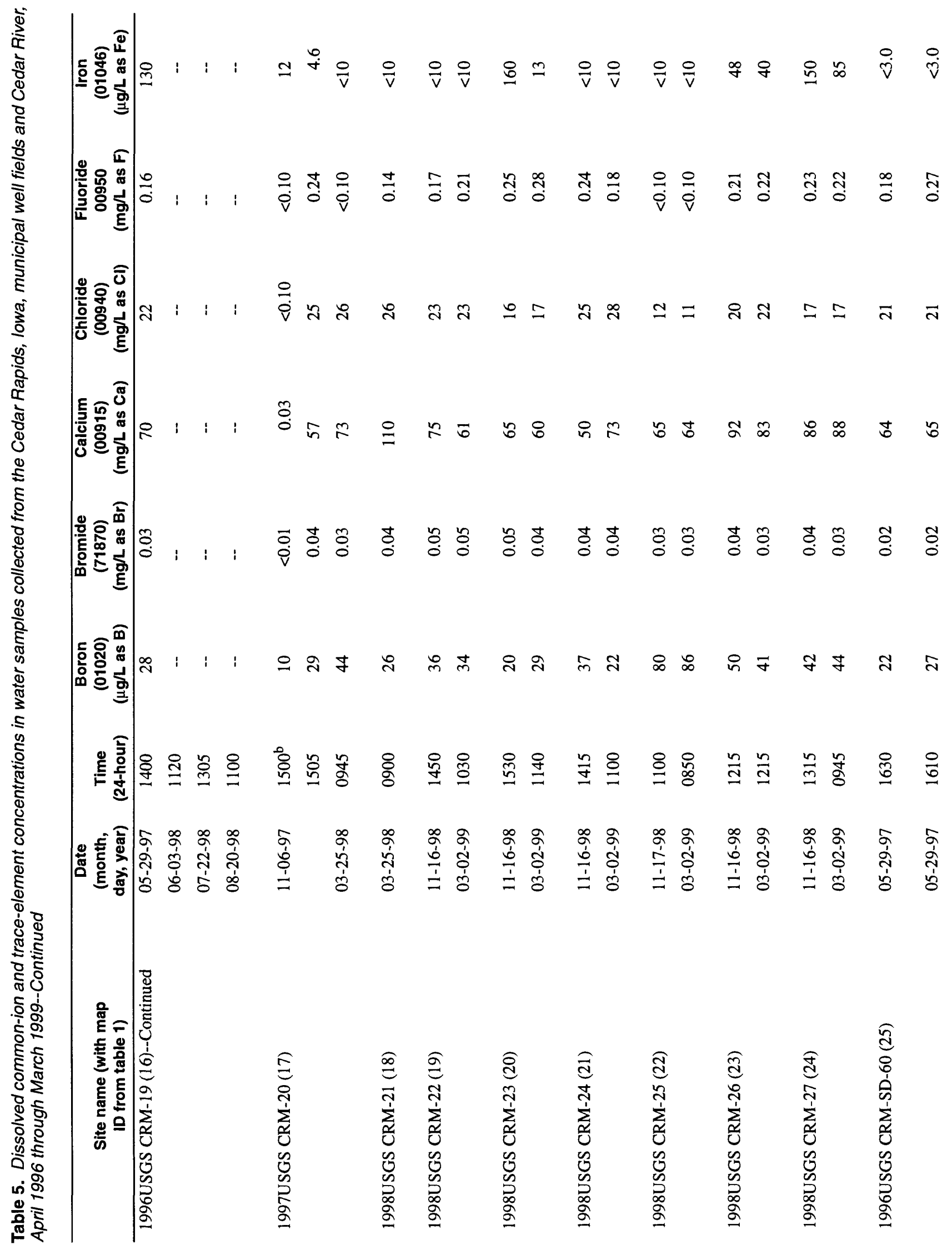




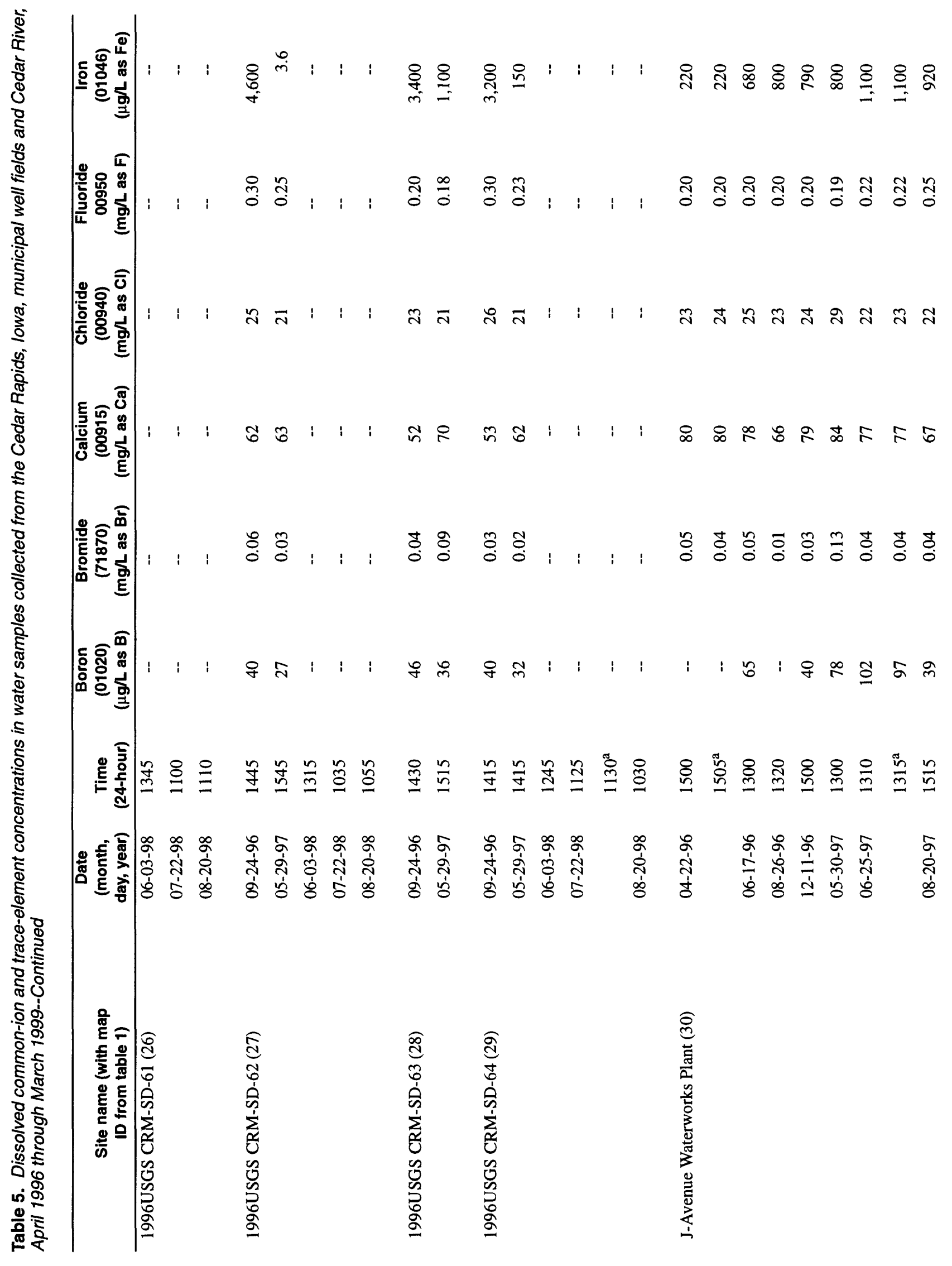




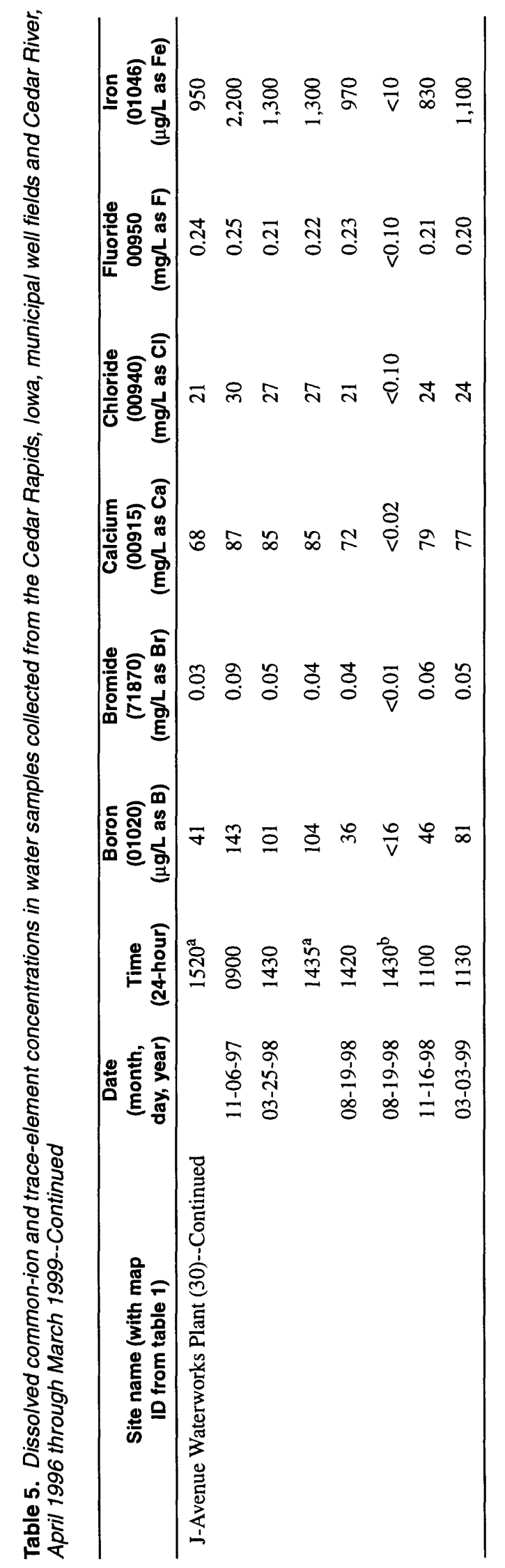




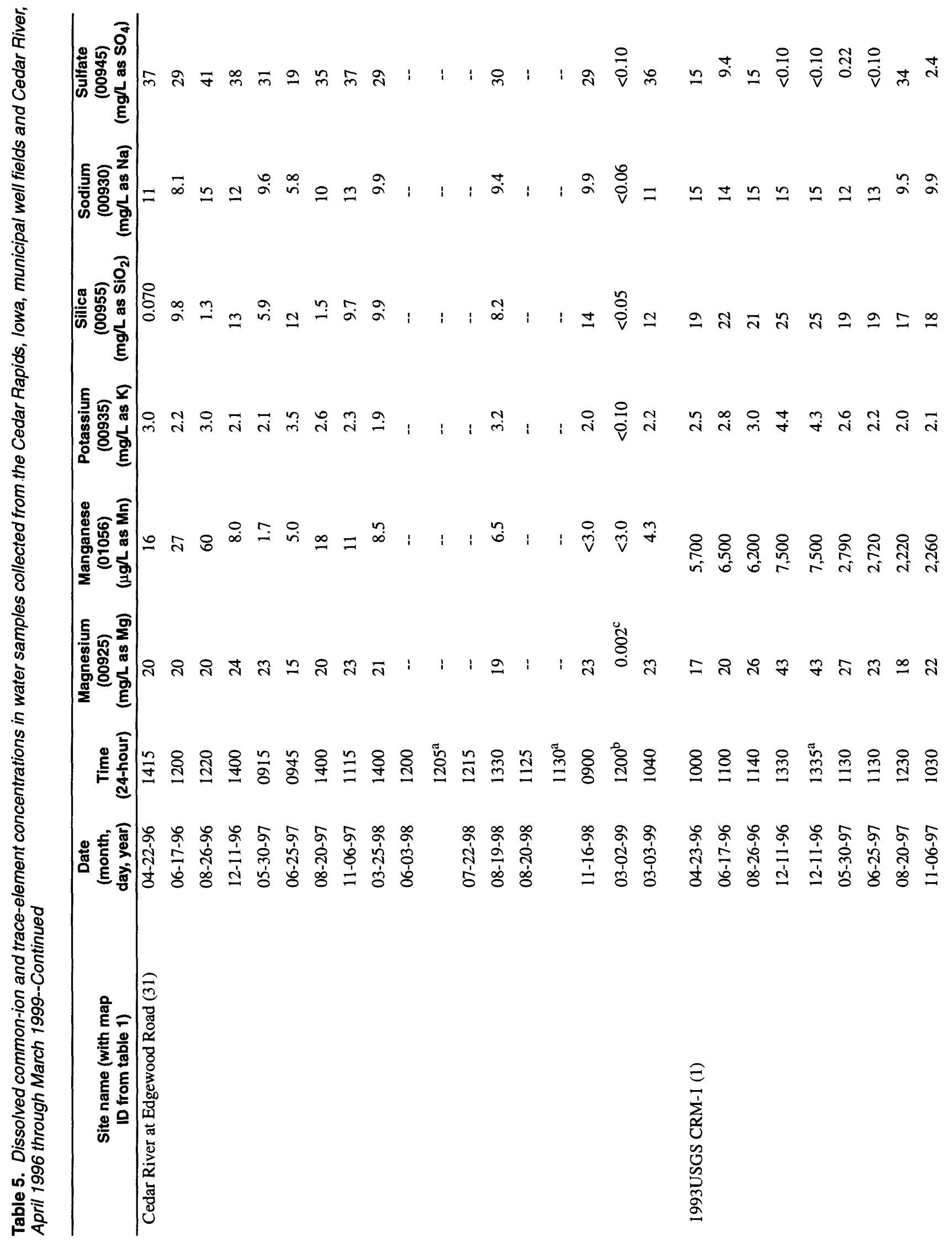




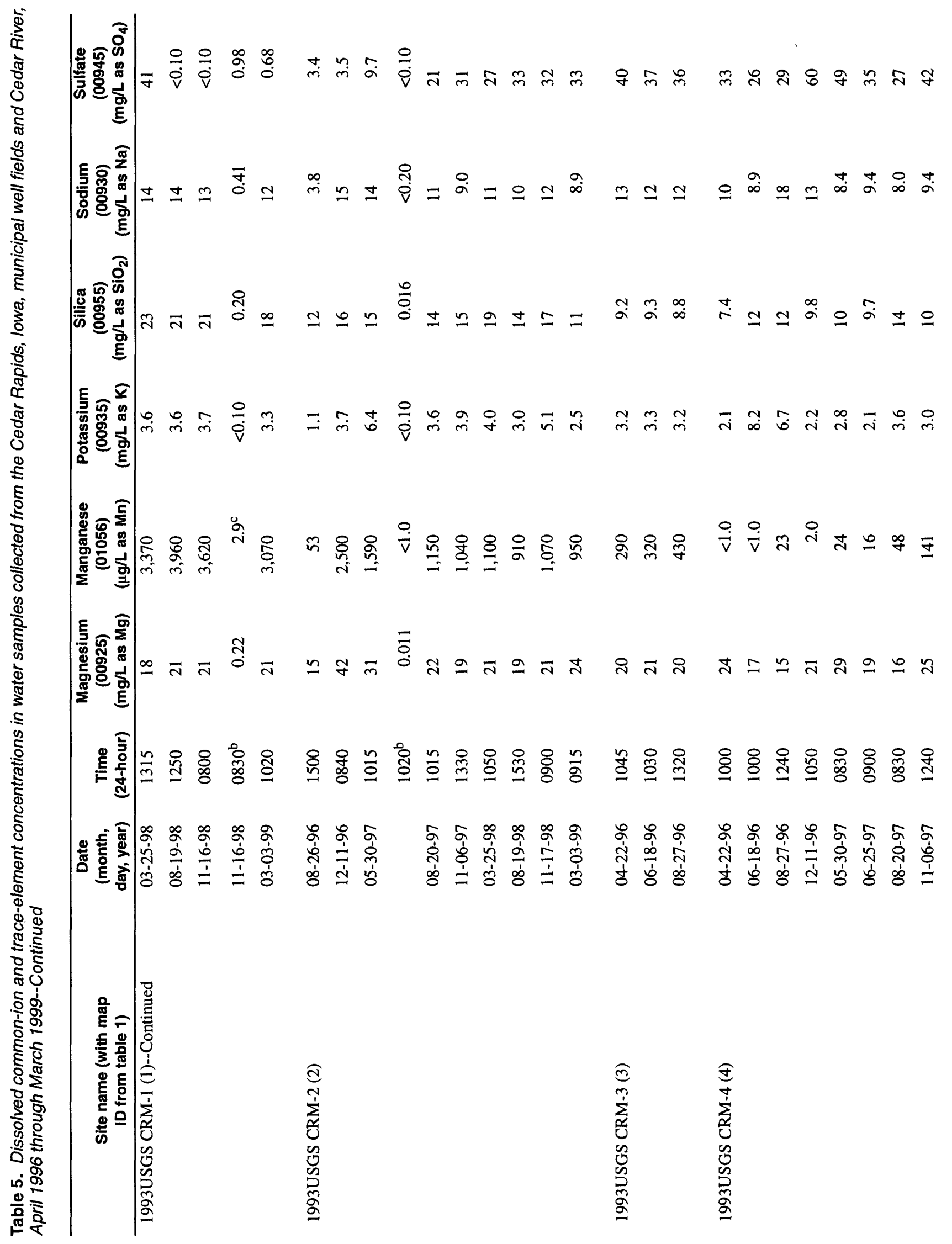




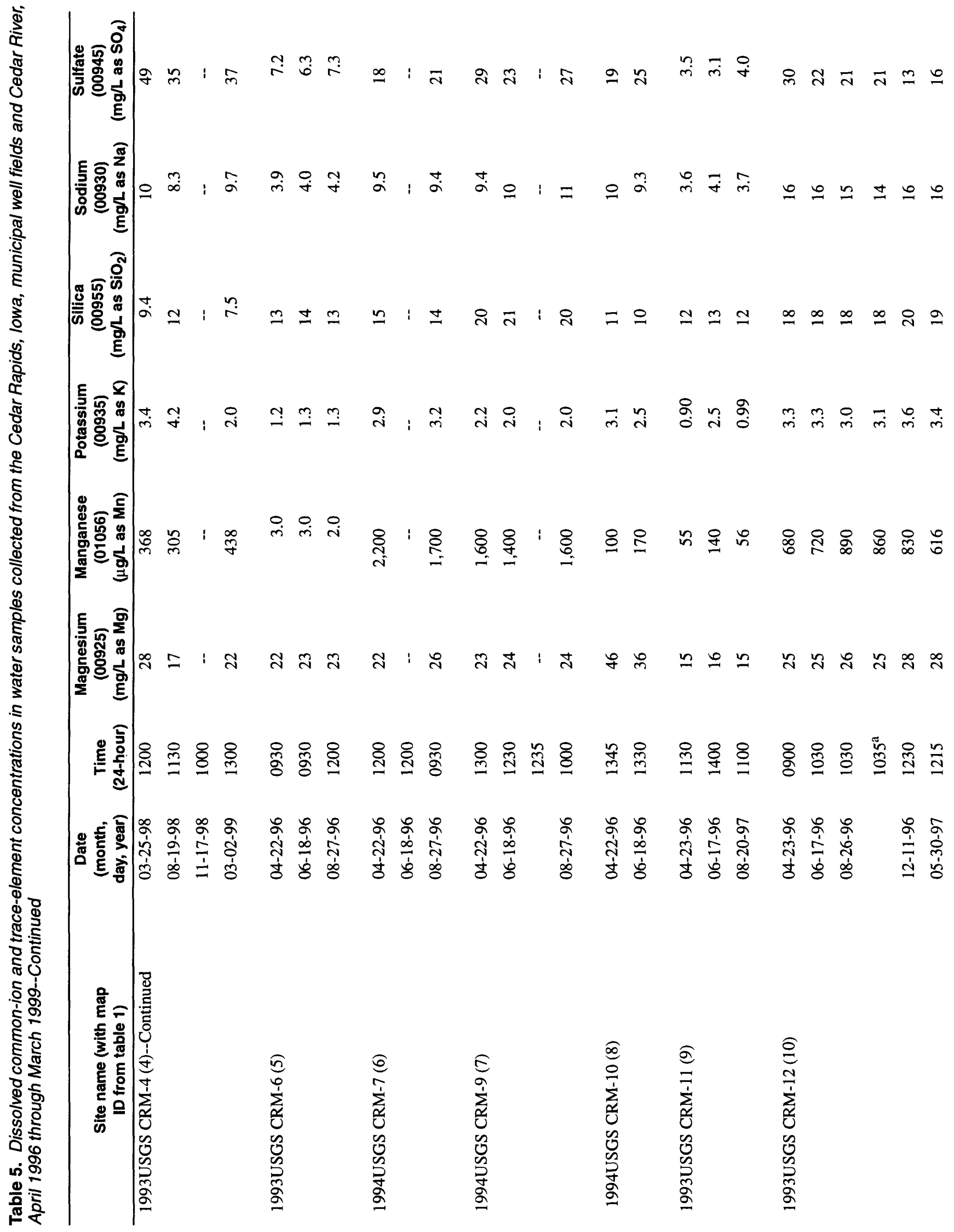




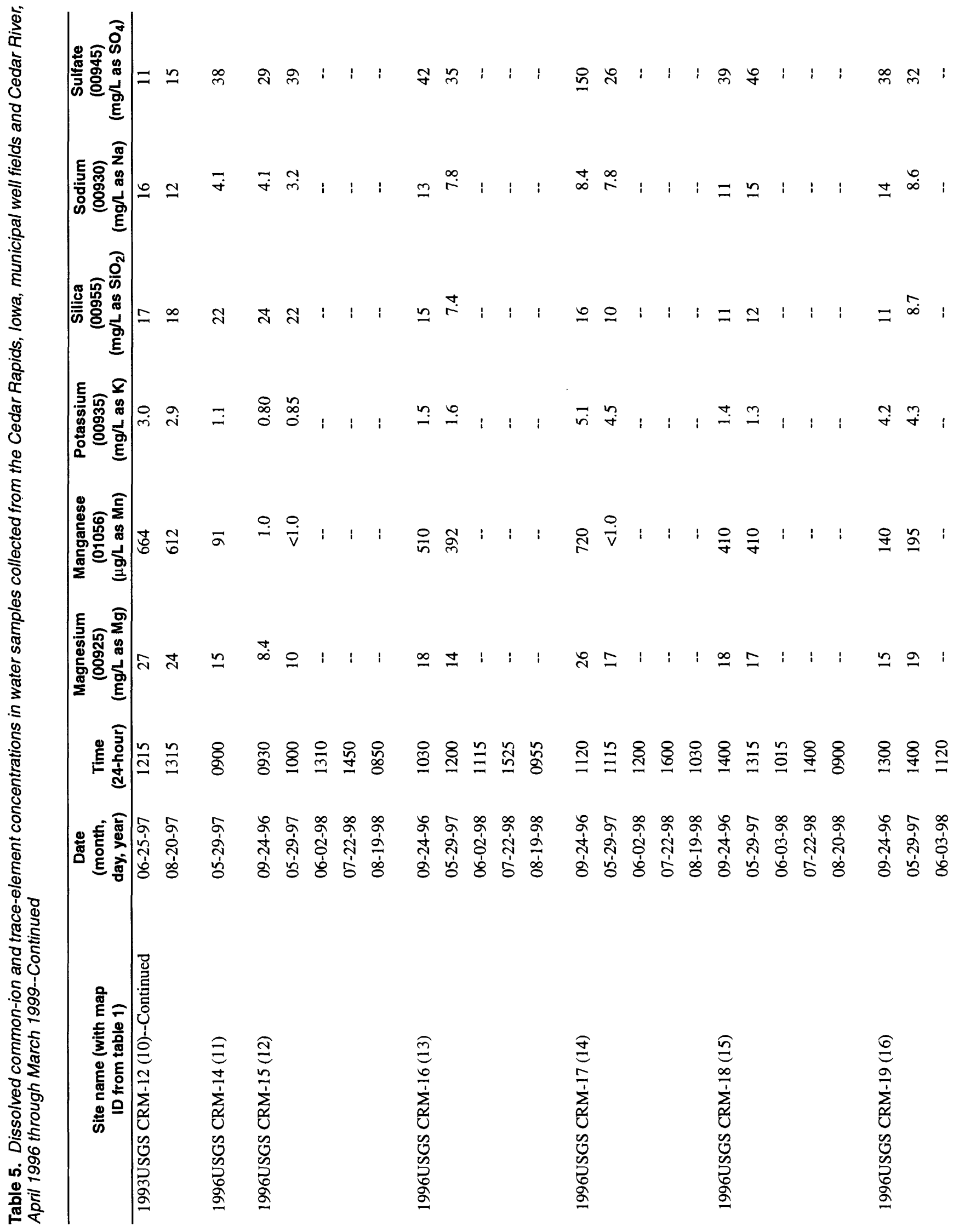




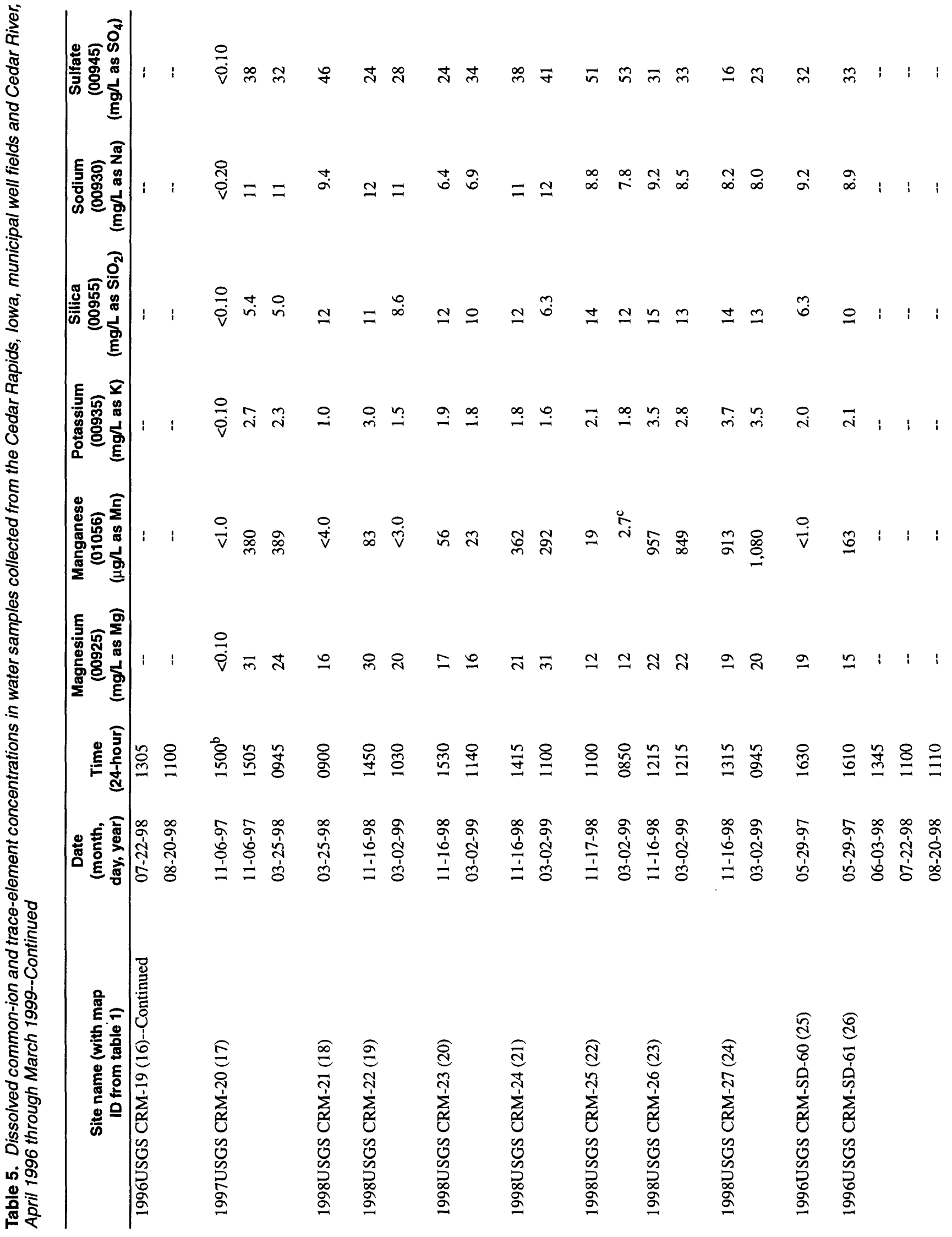




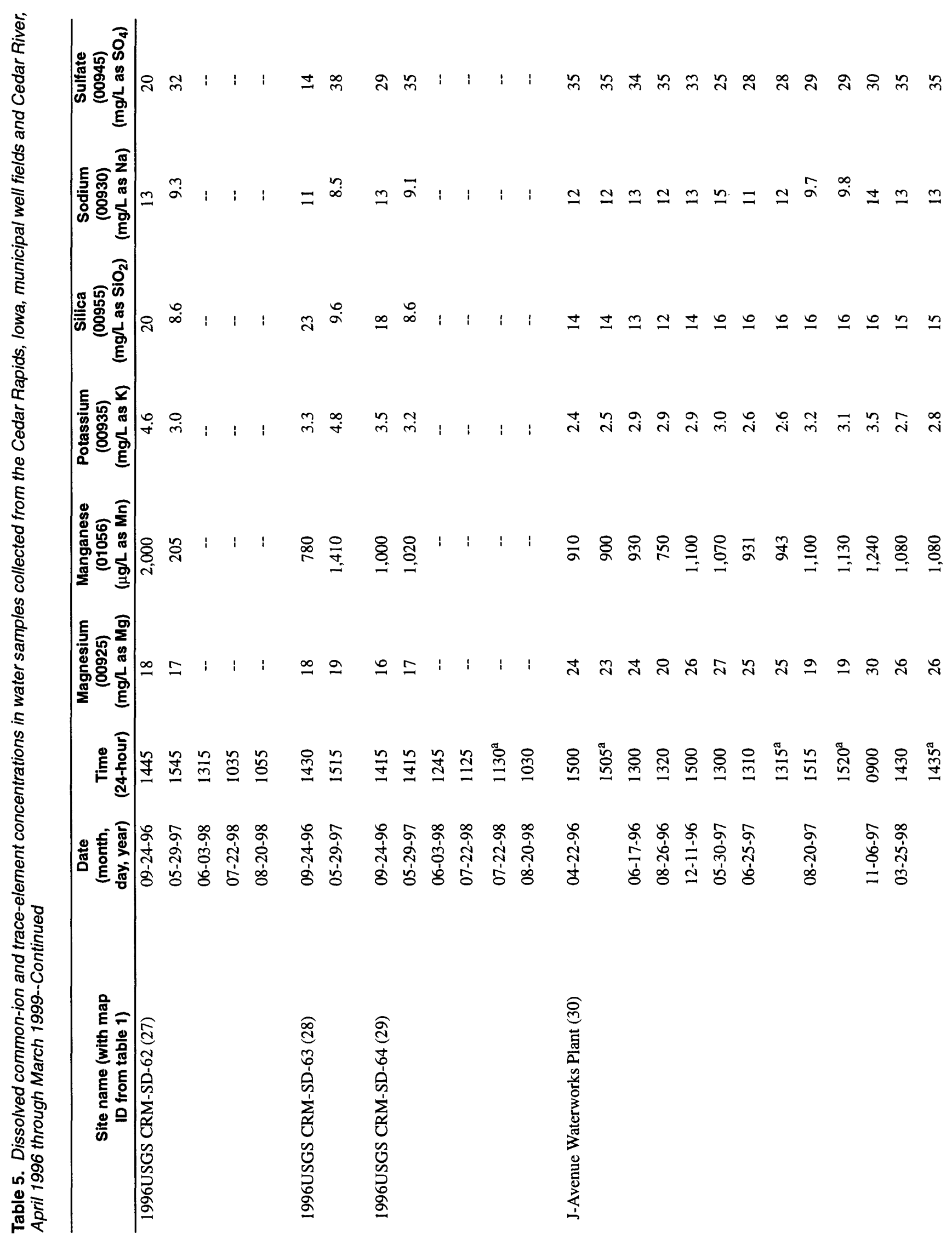

TABLES 31 


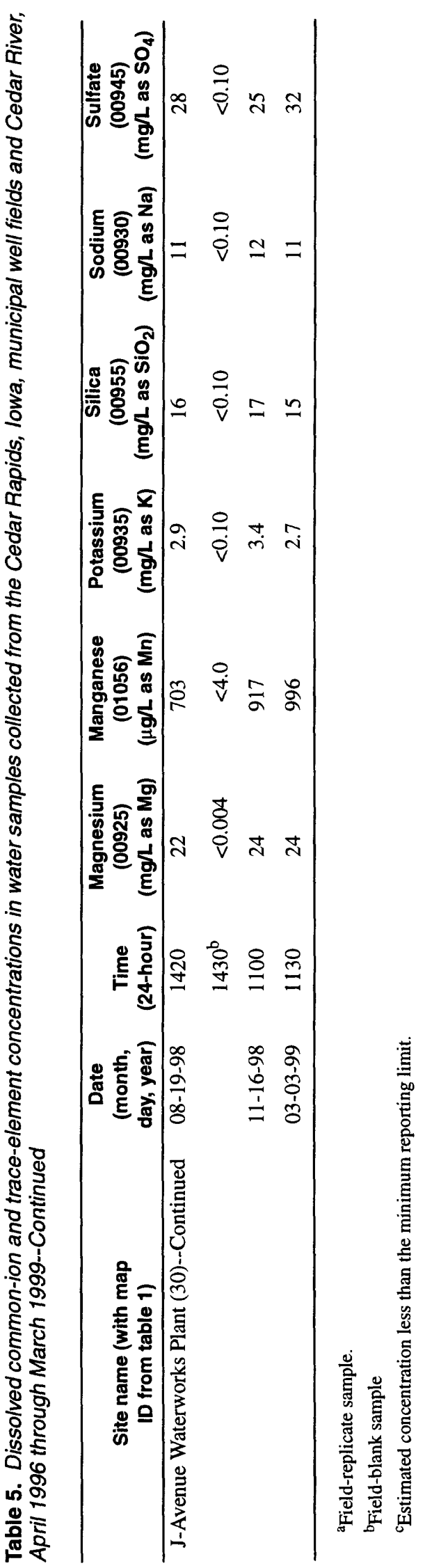




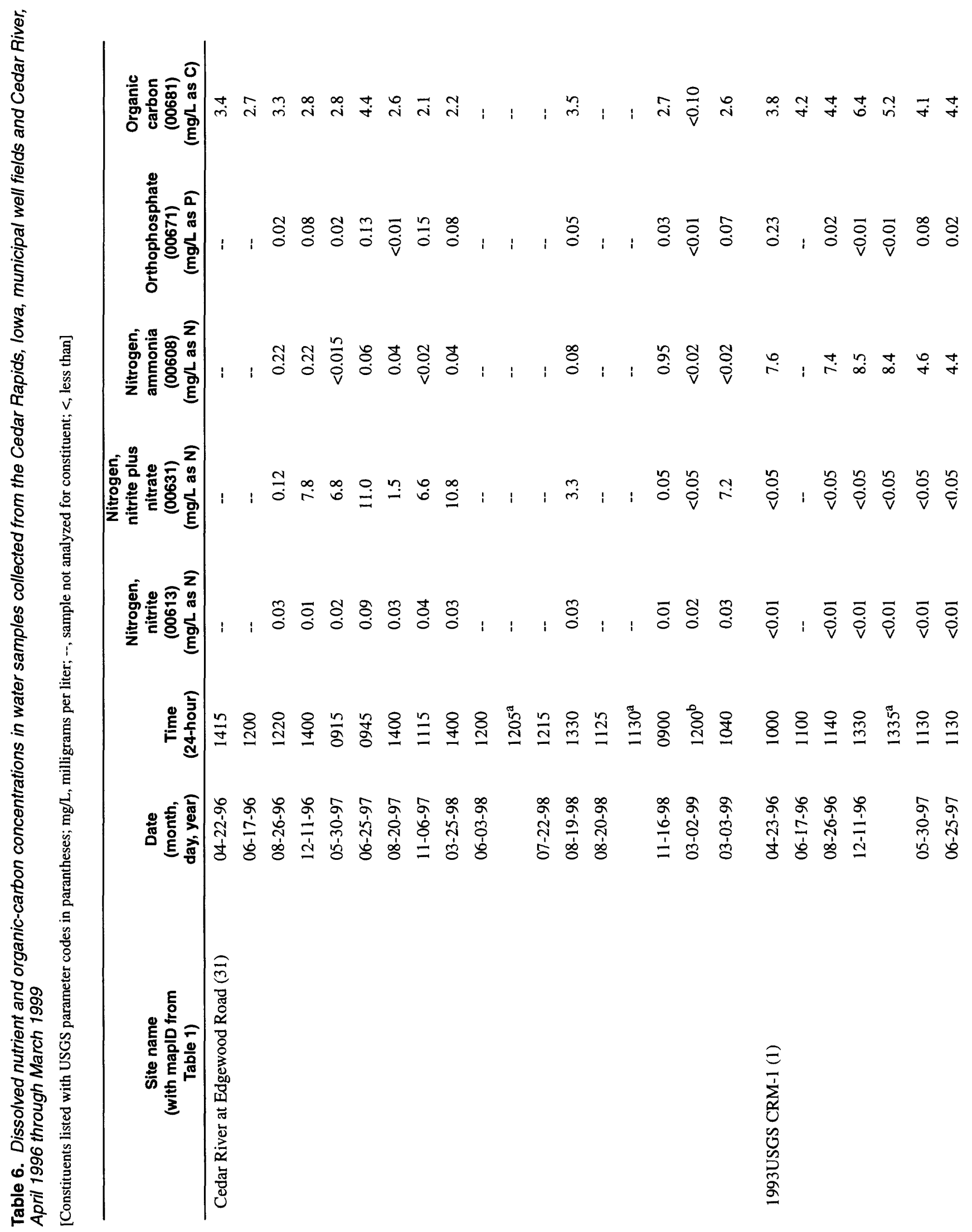




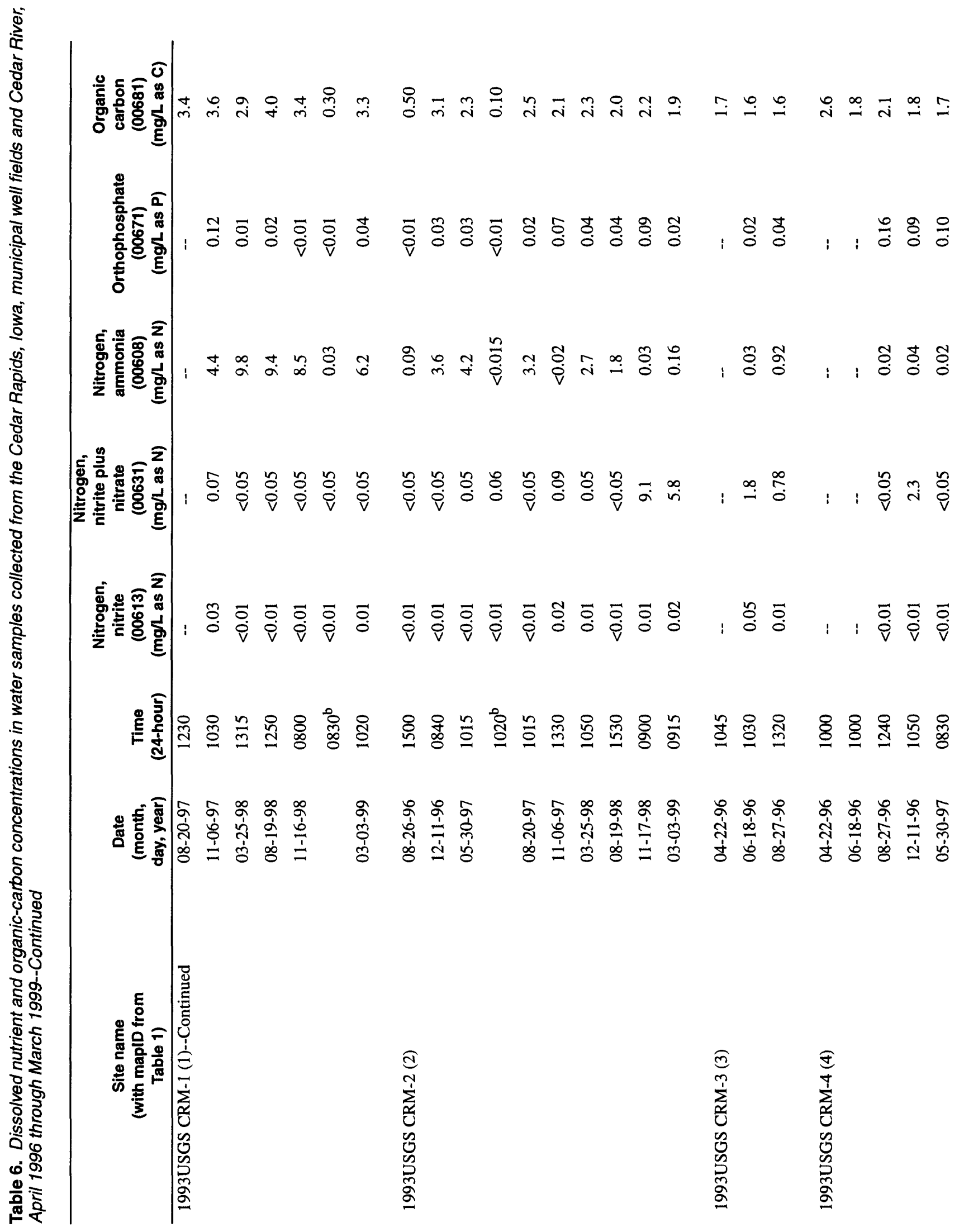




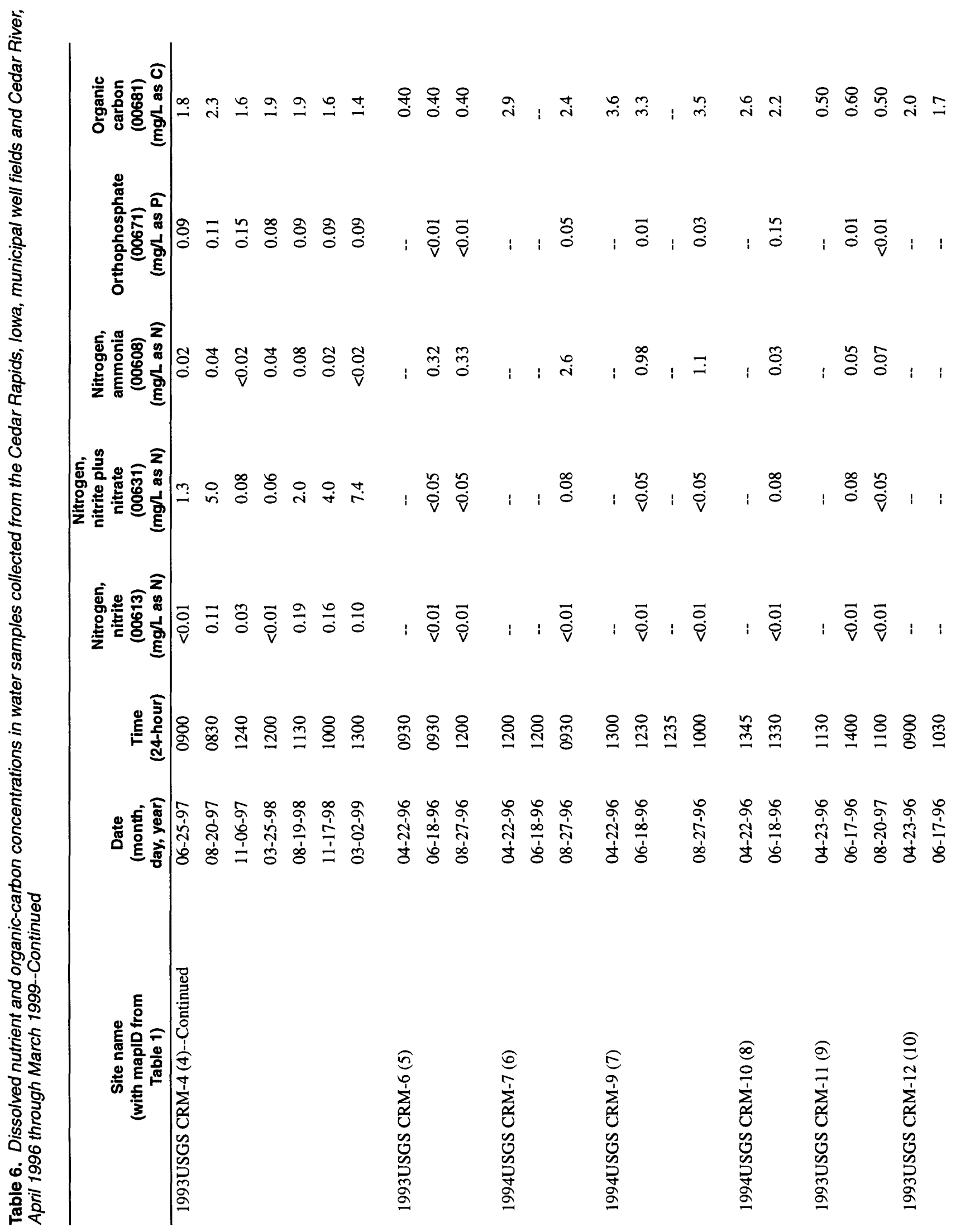




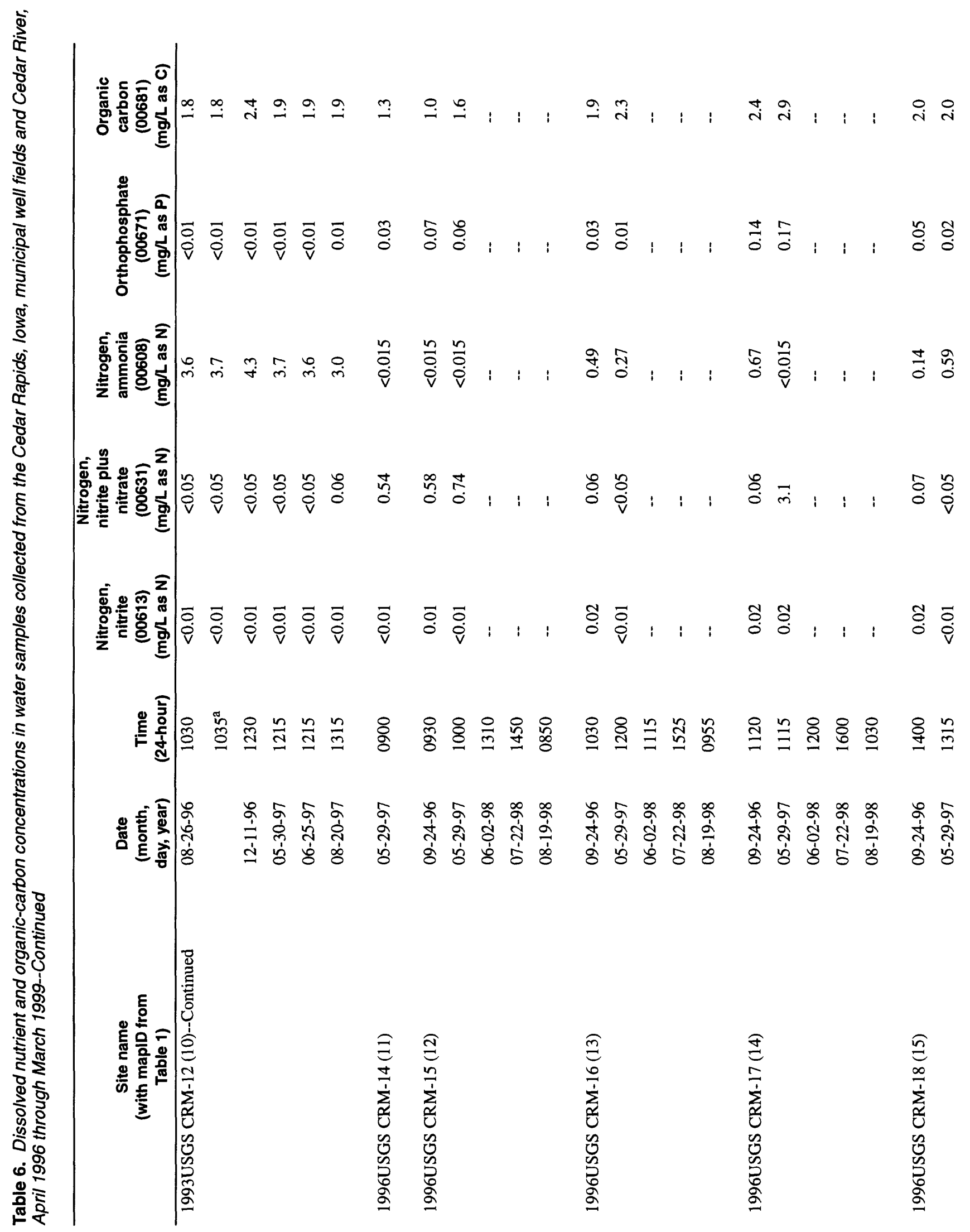




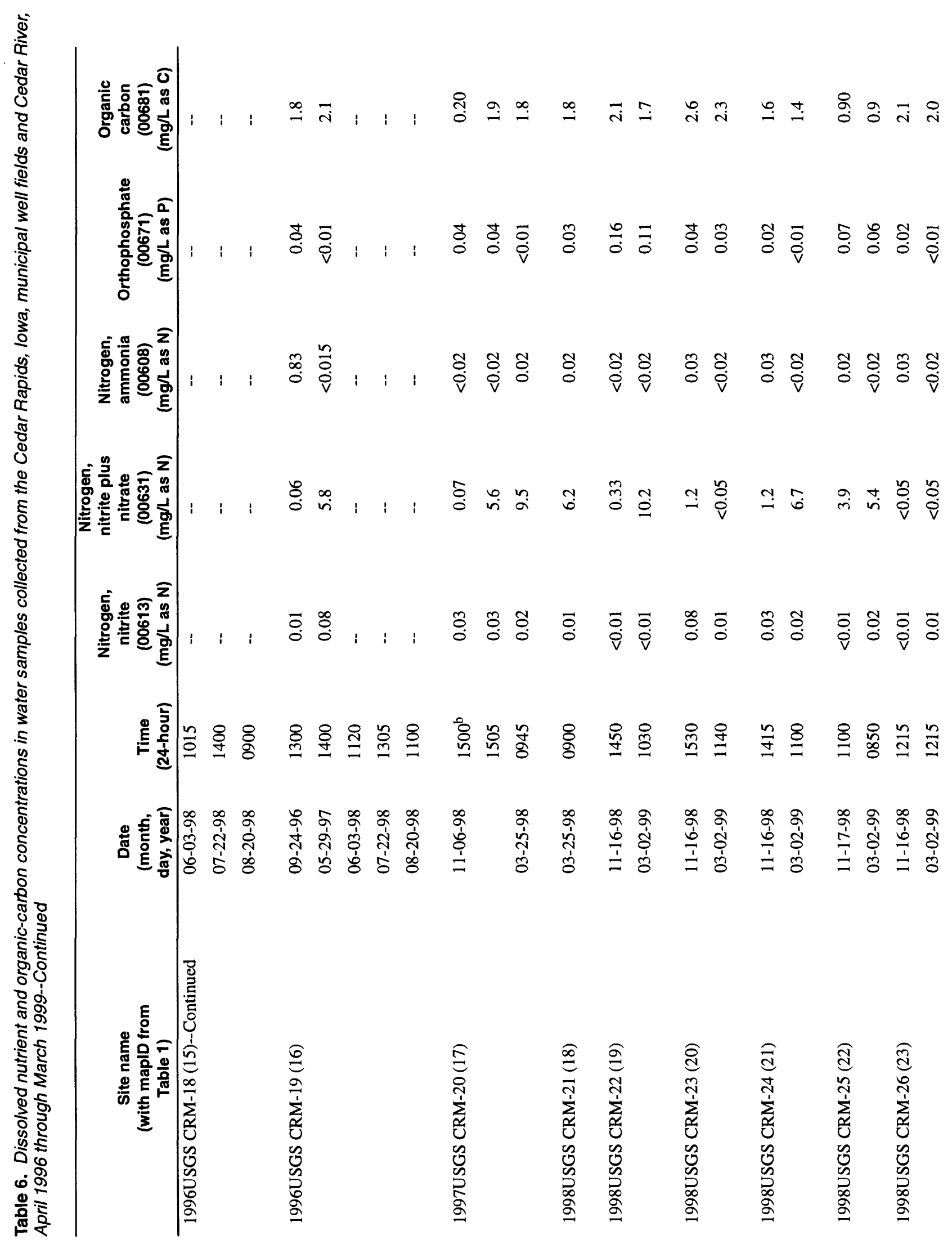




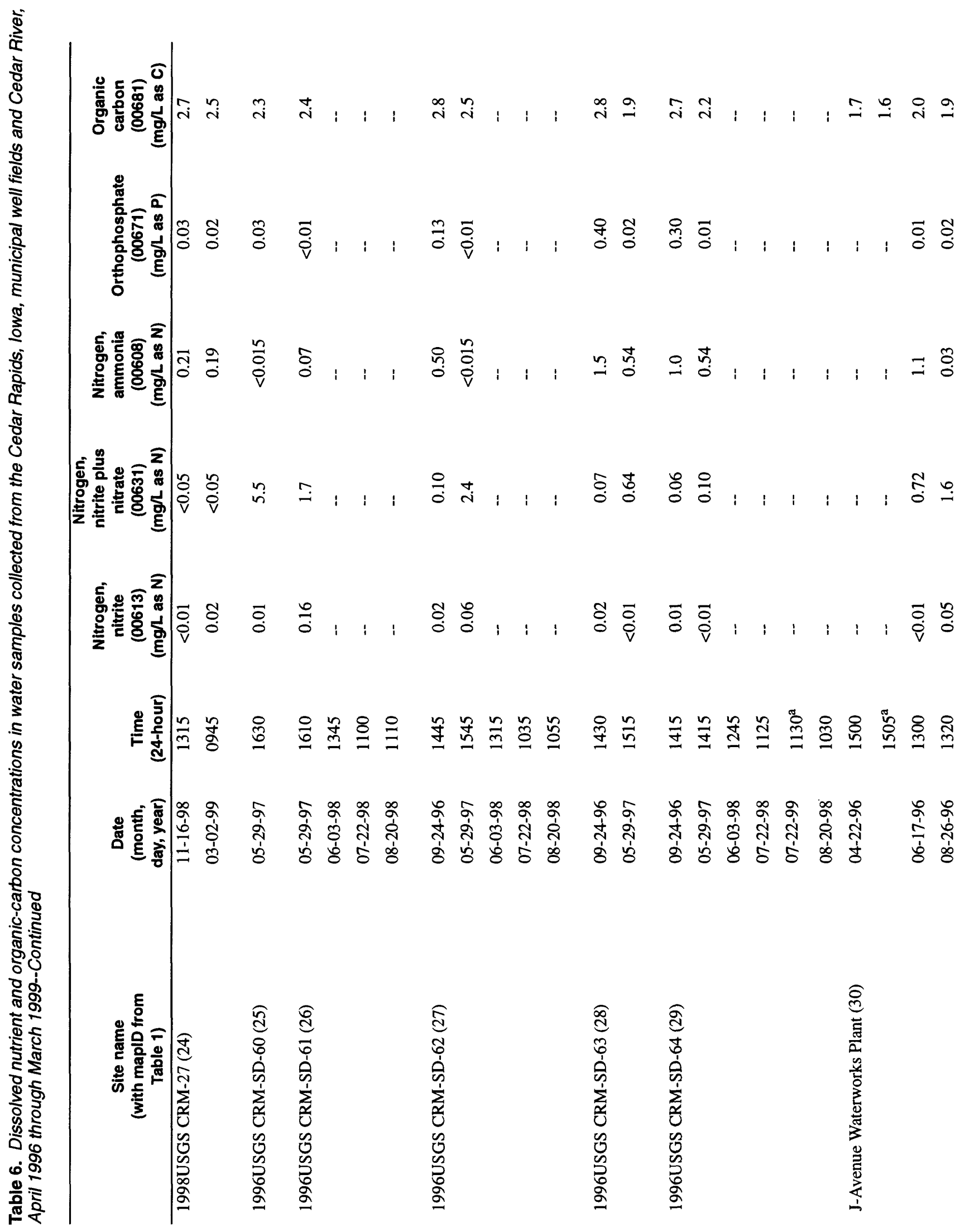




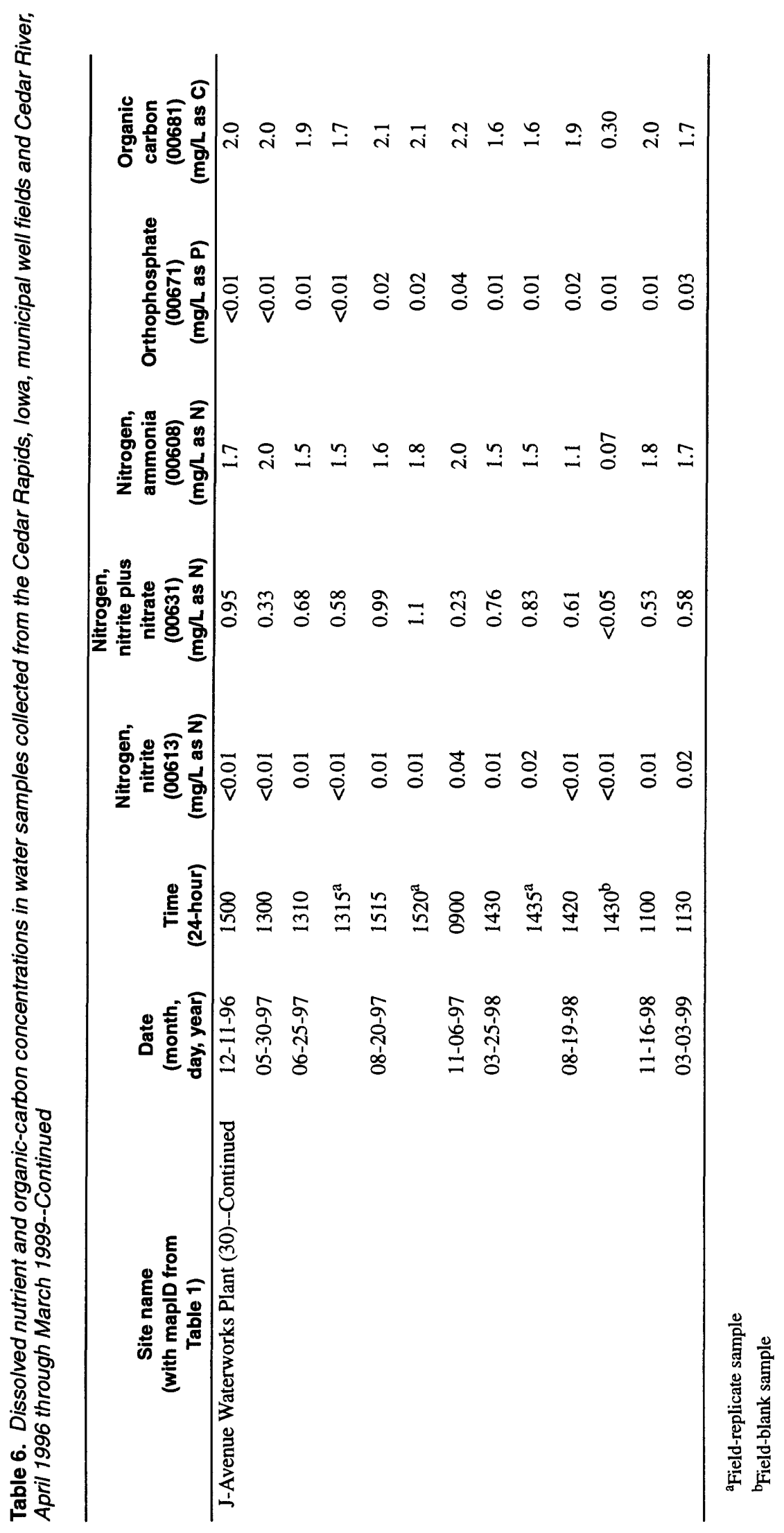




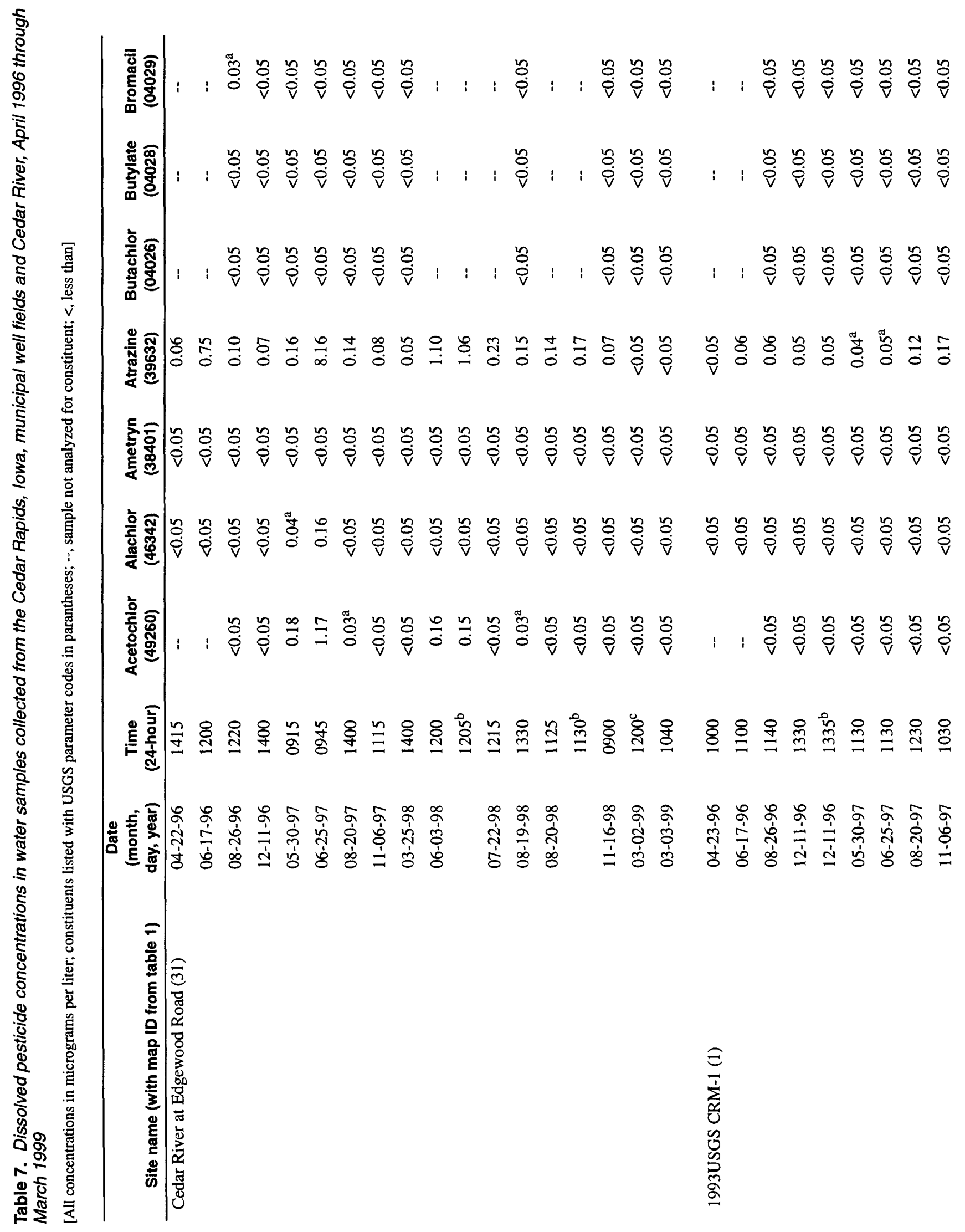




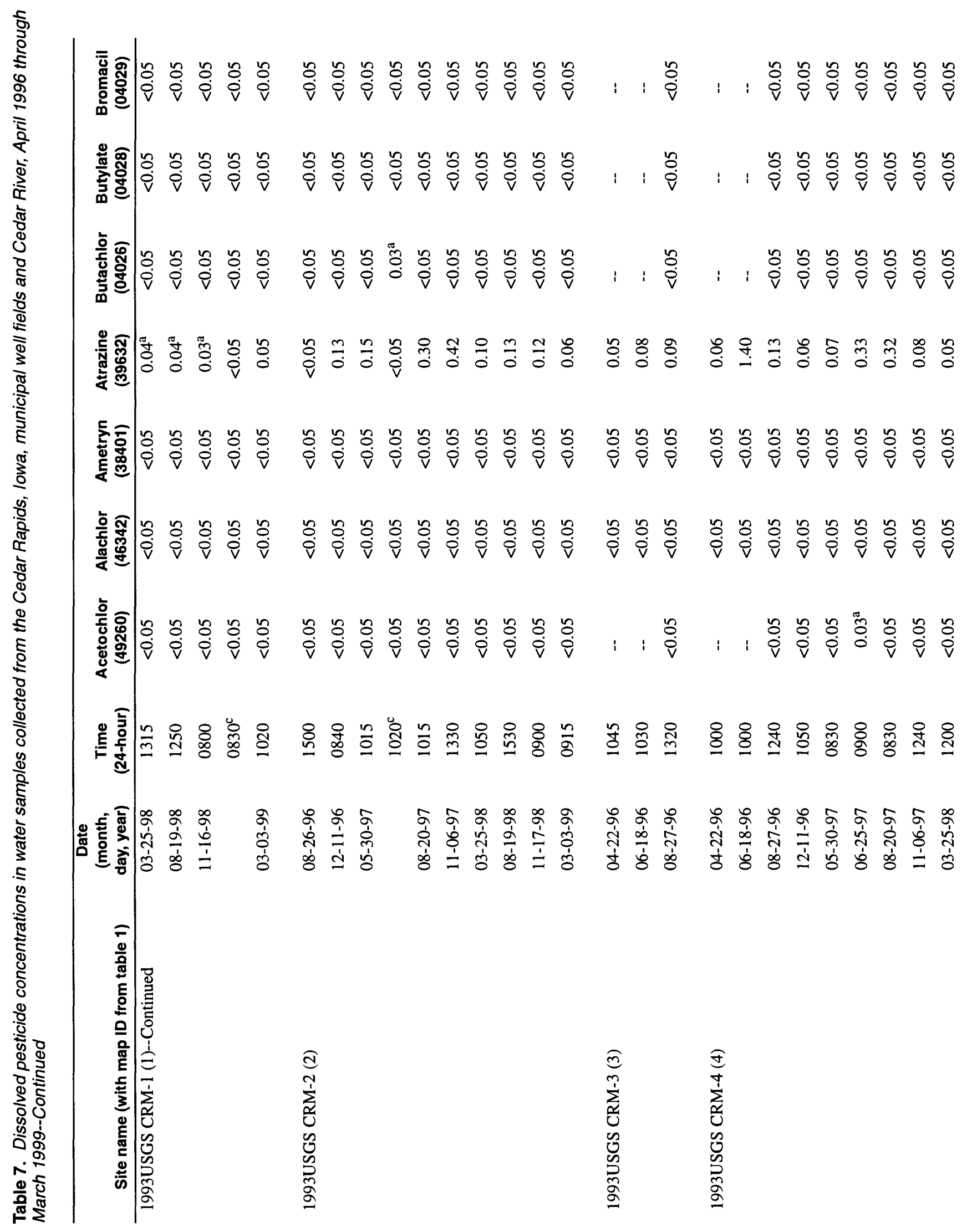




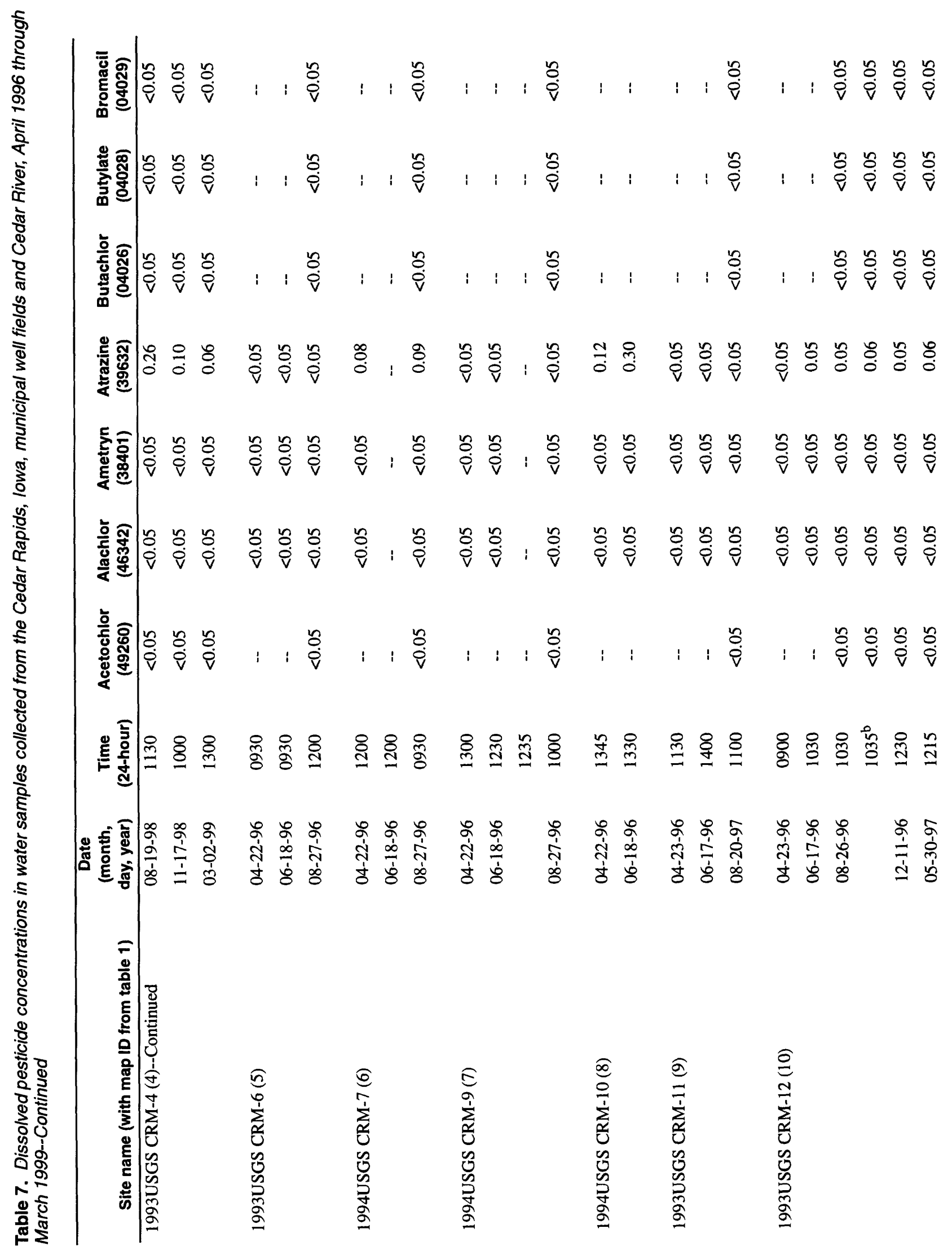




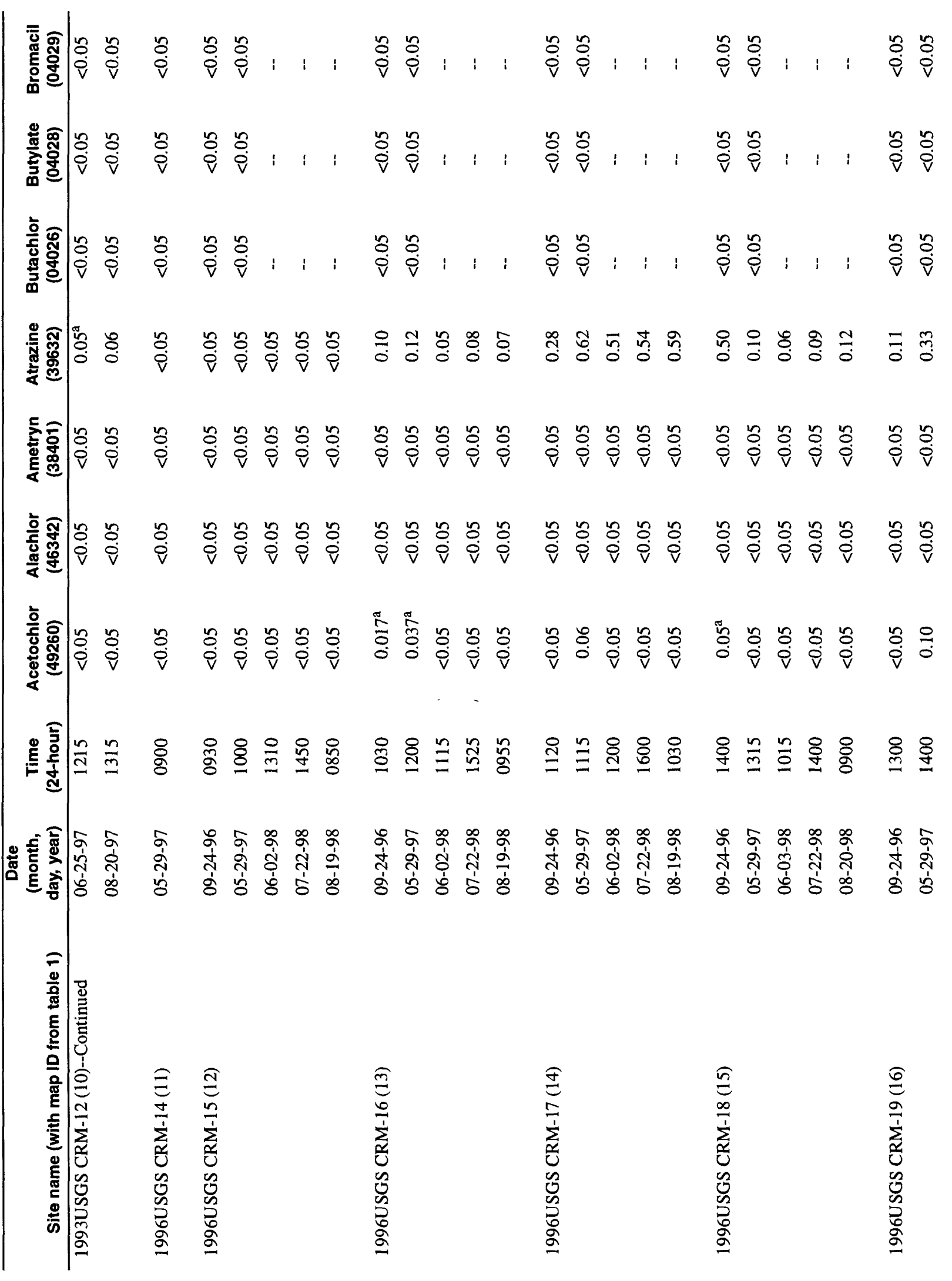




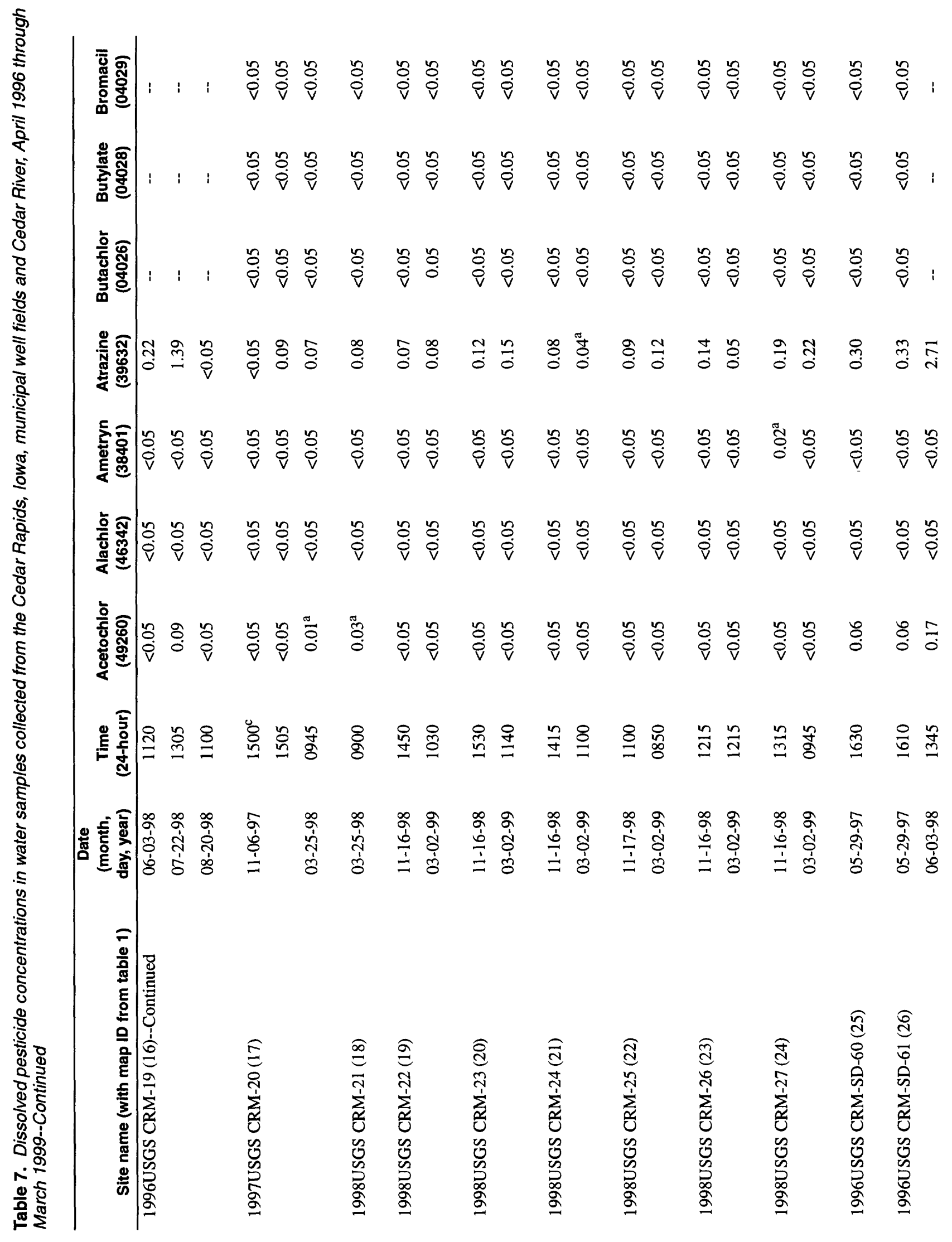




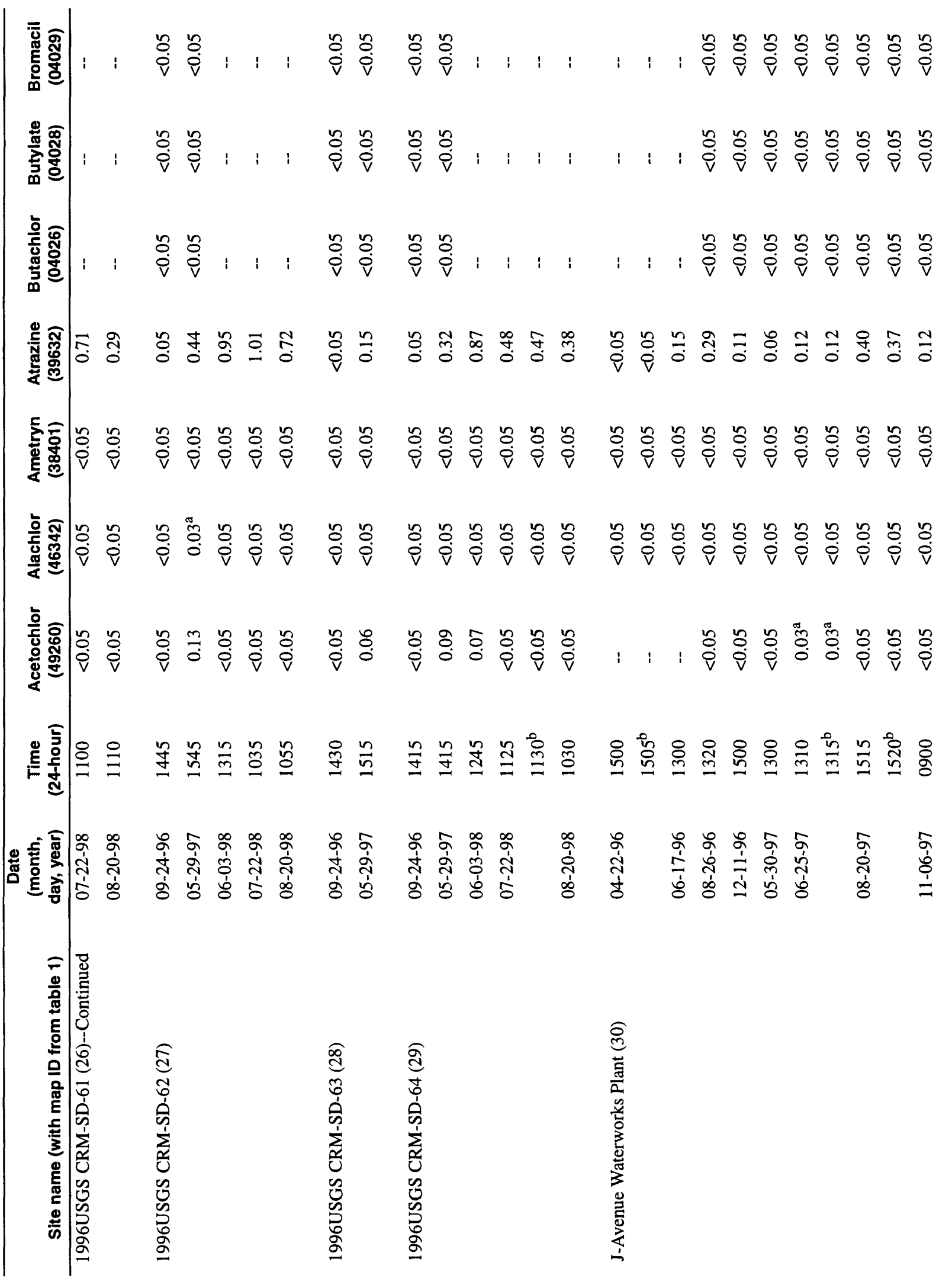




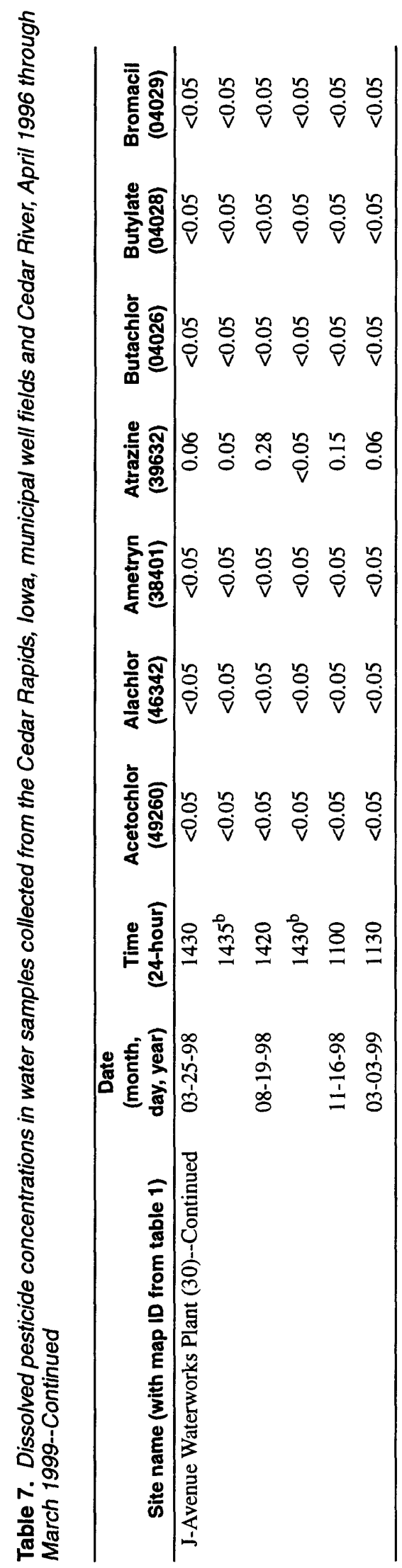




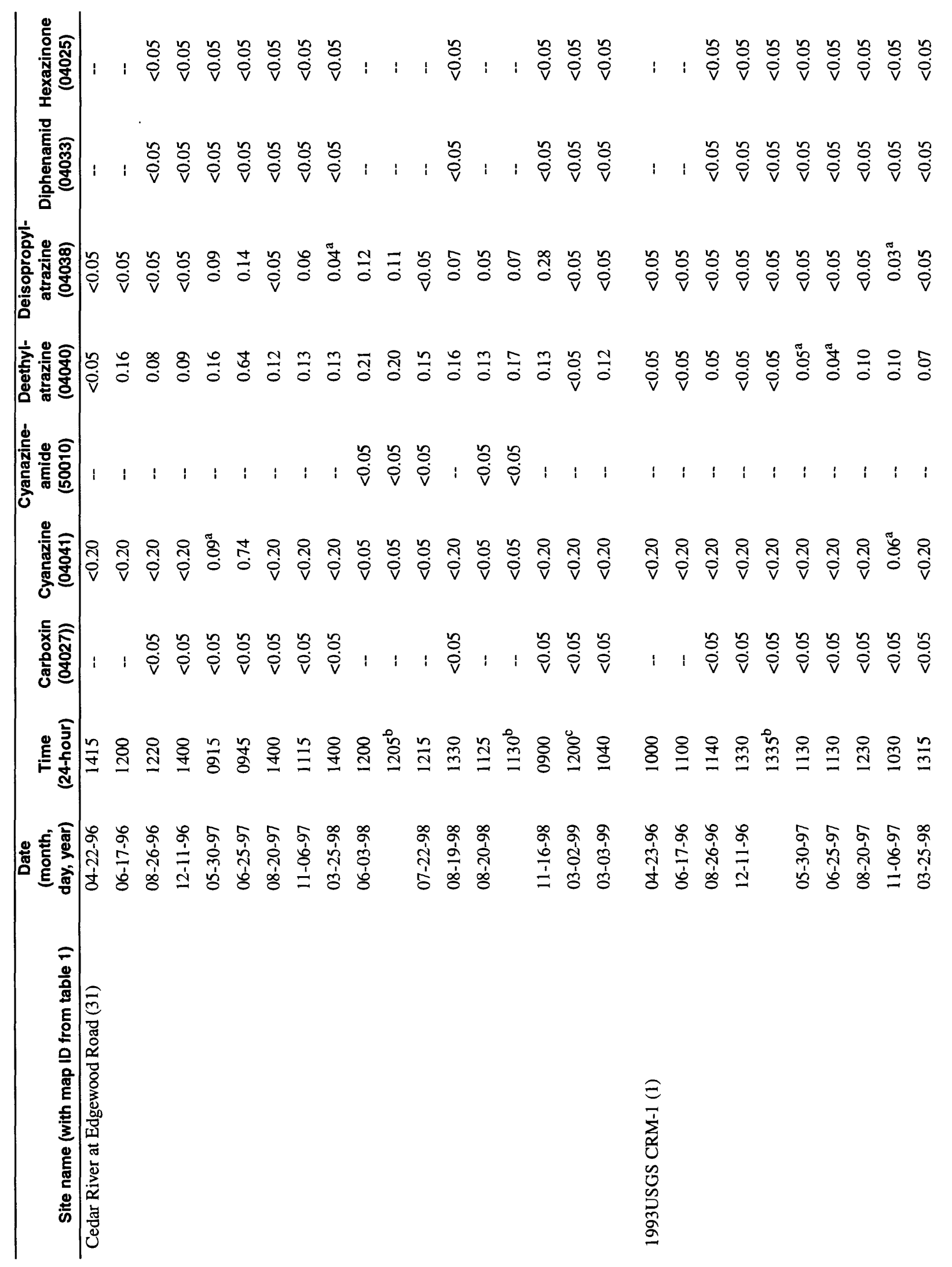




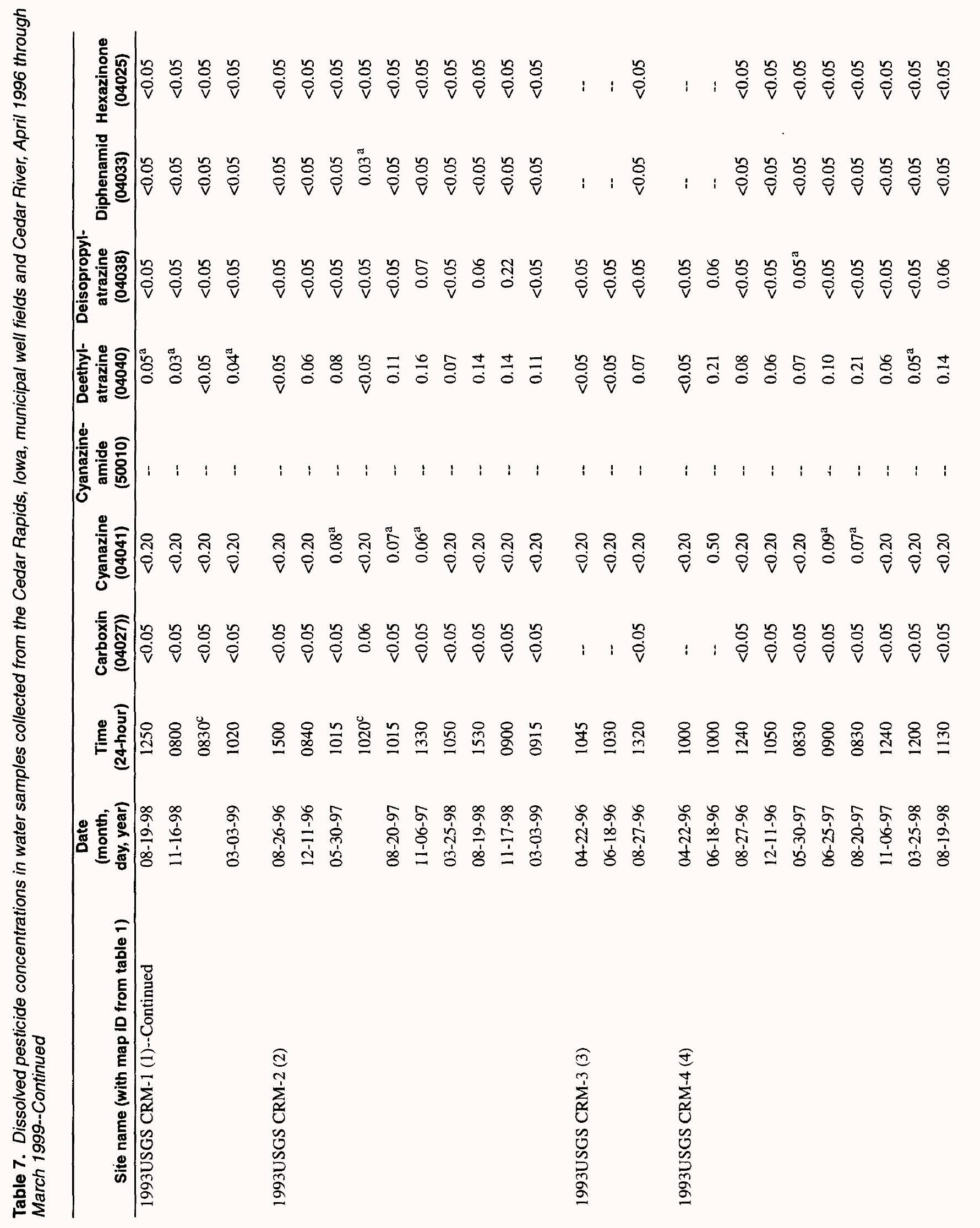




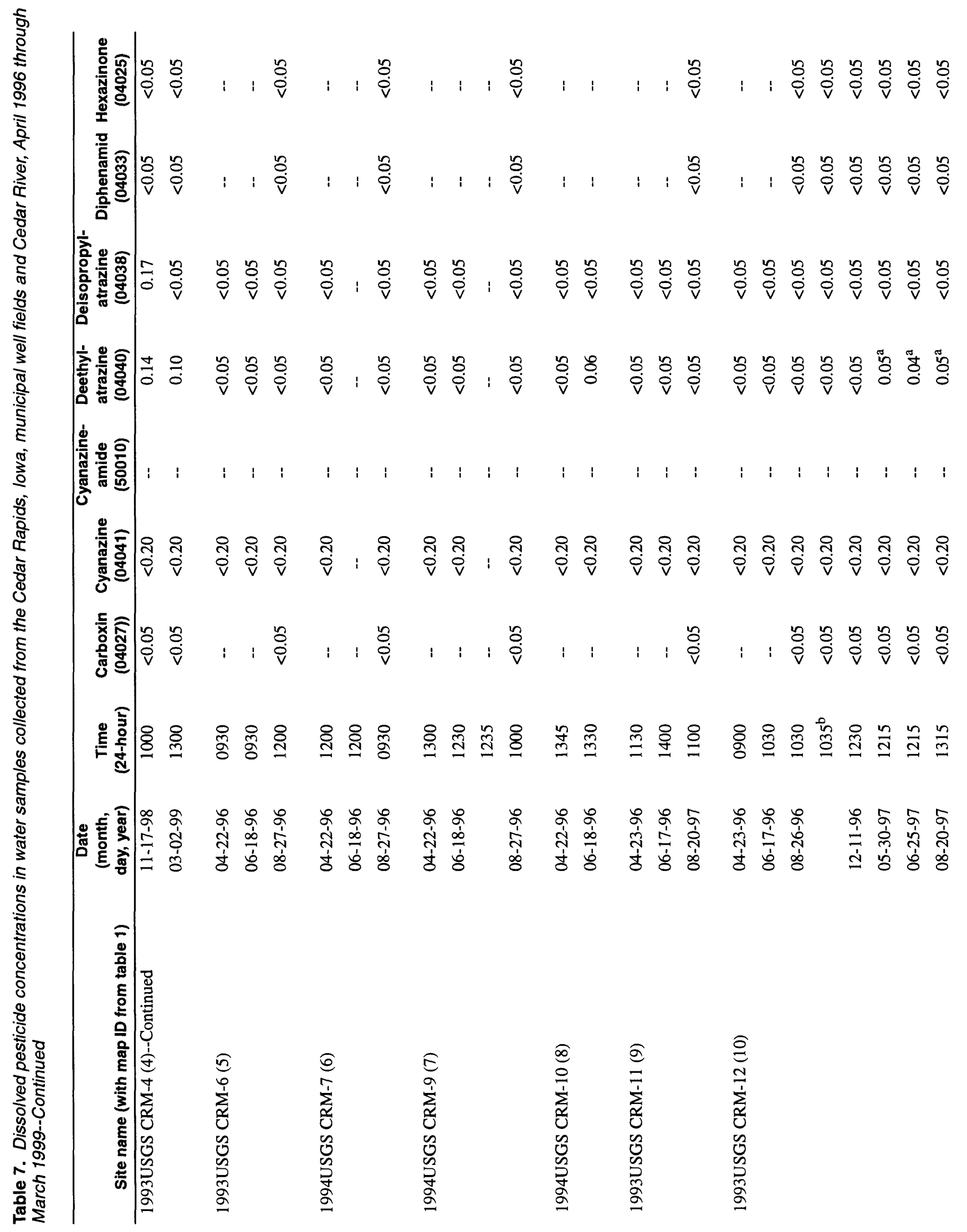




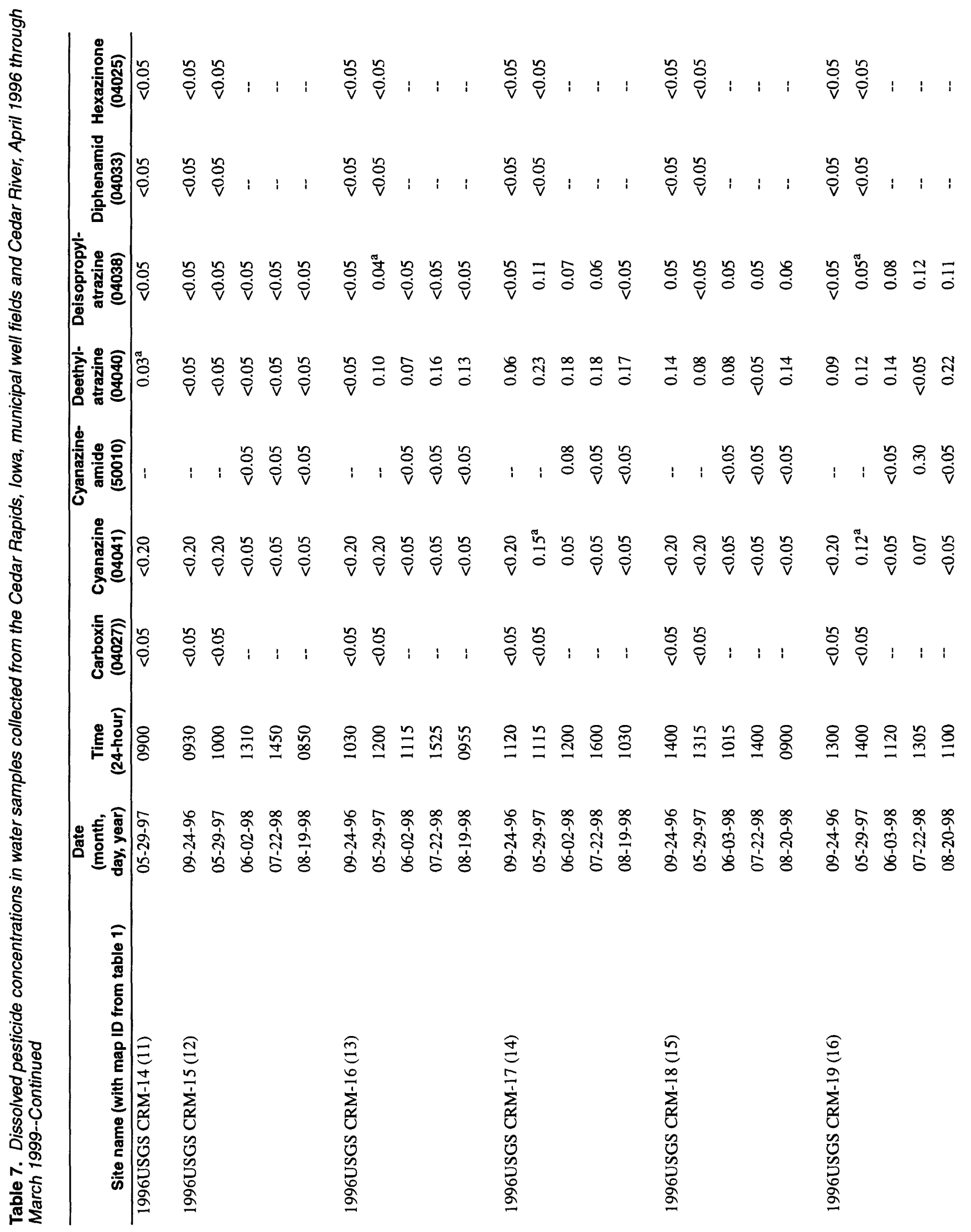




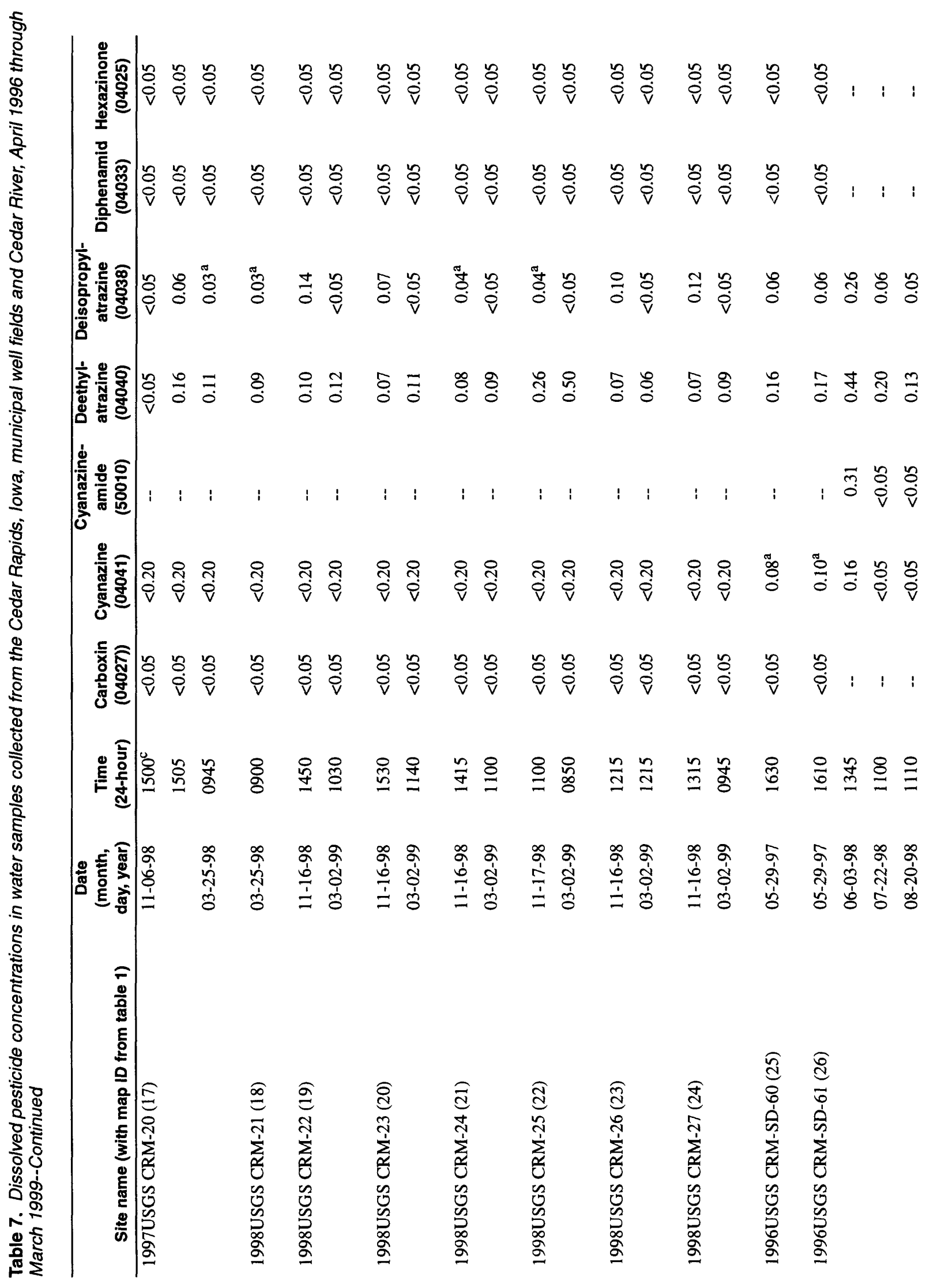




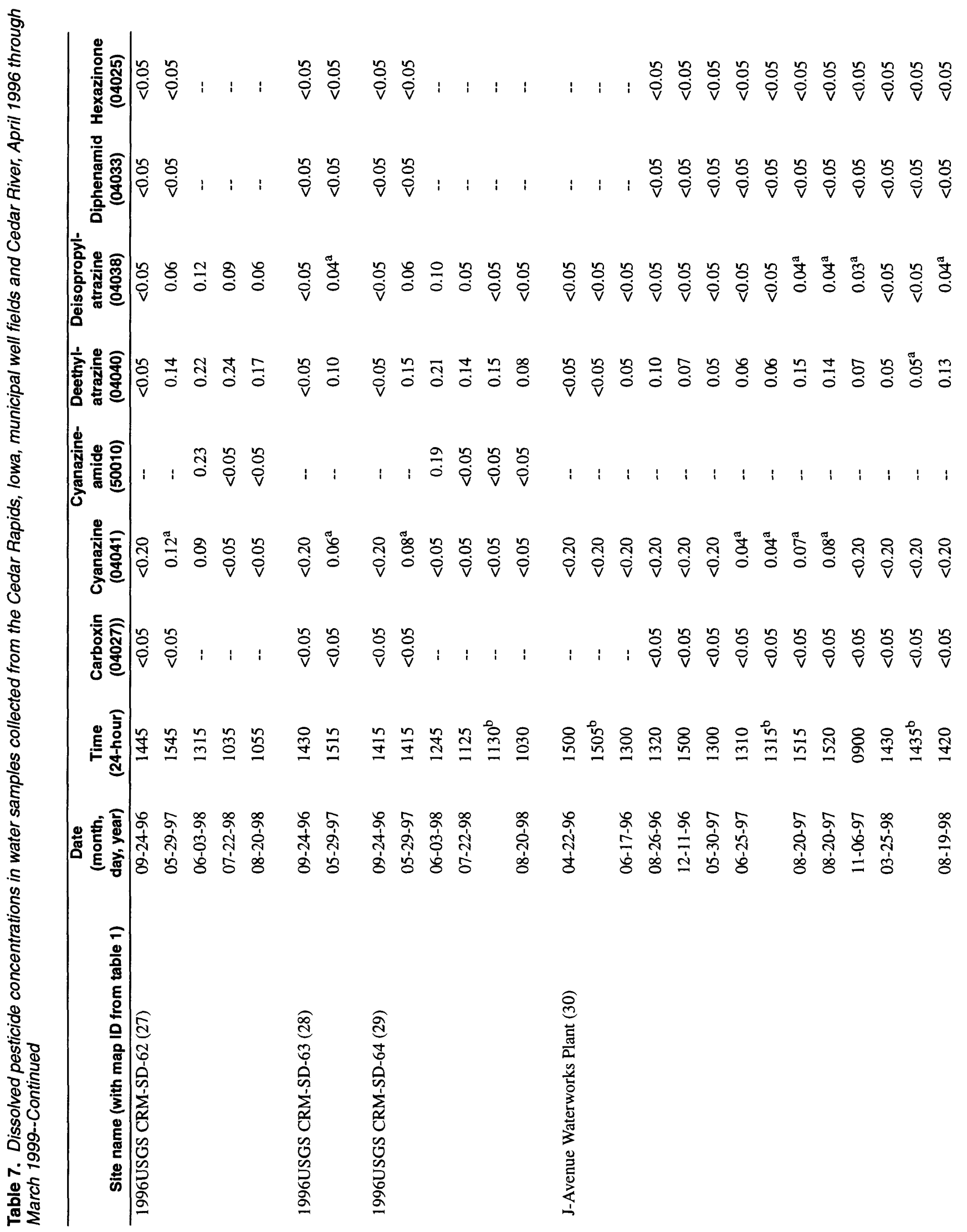




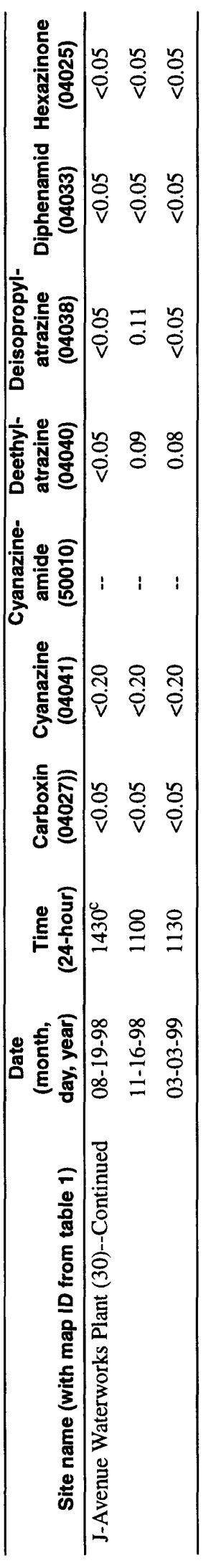

TABLES 53 


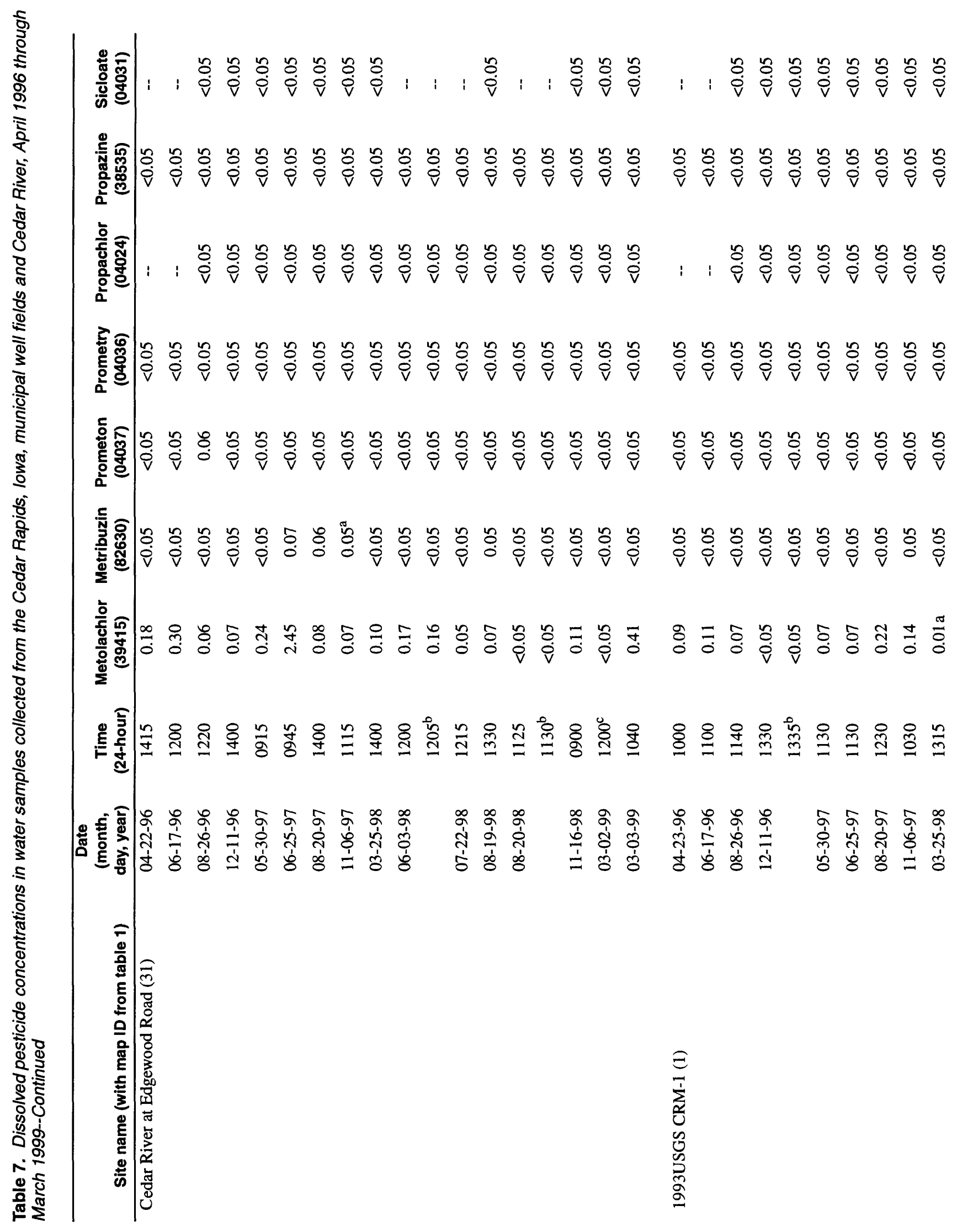




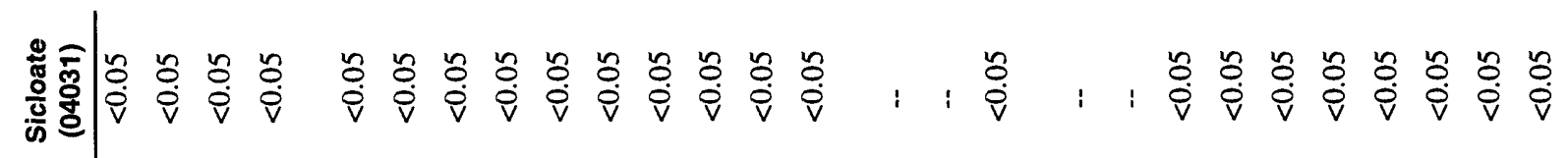

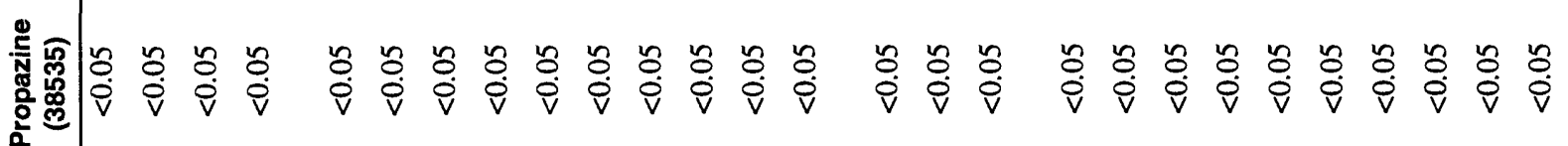

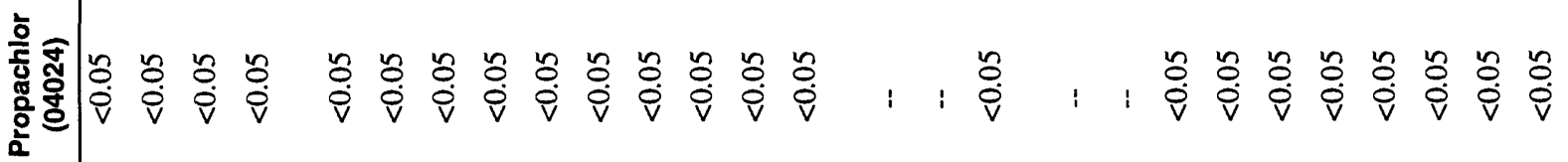

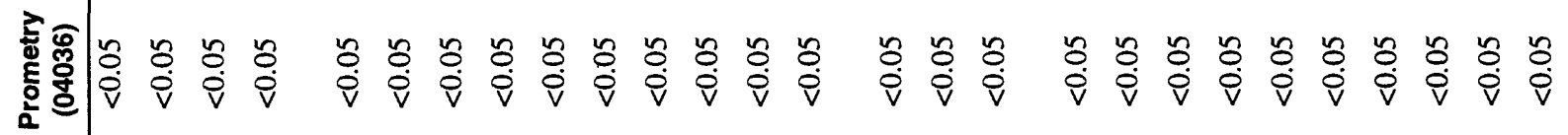

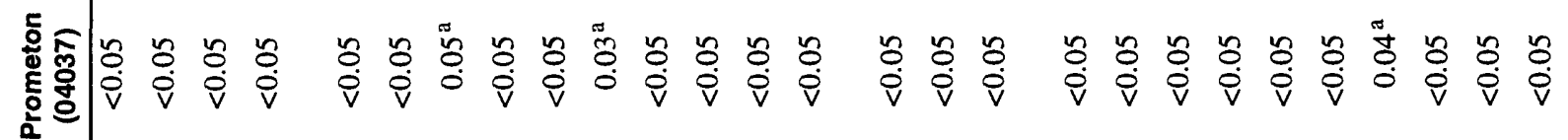

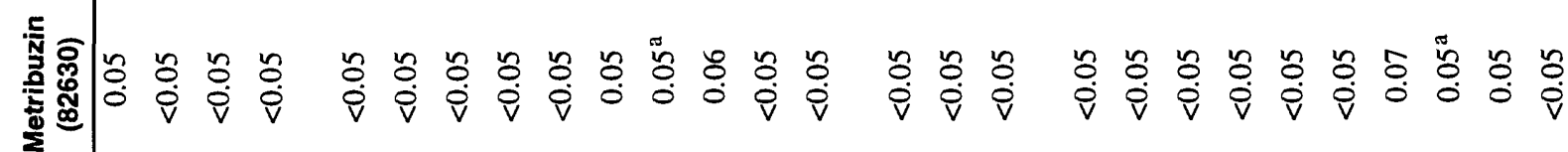

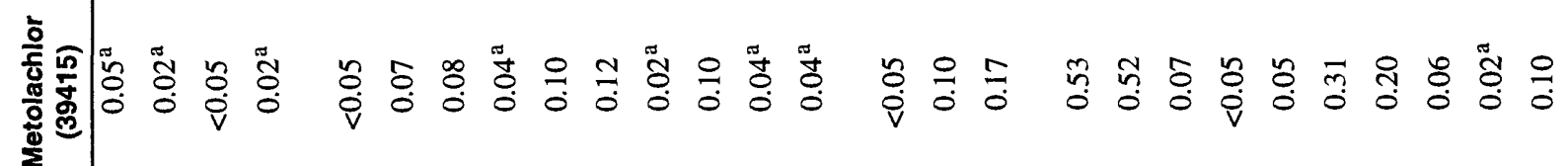

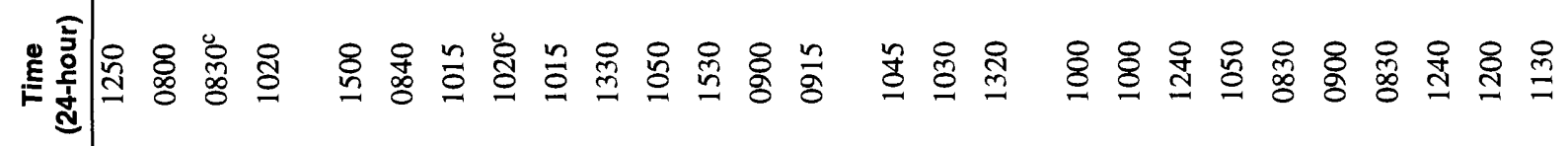

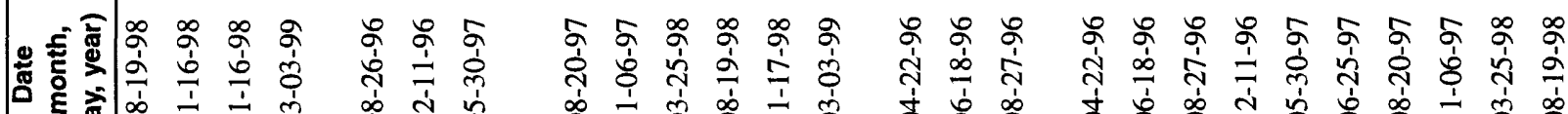

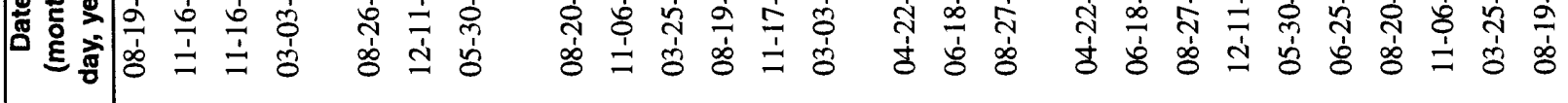




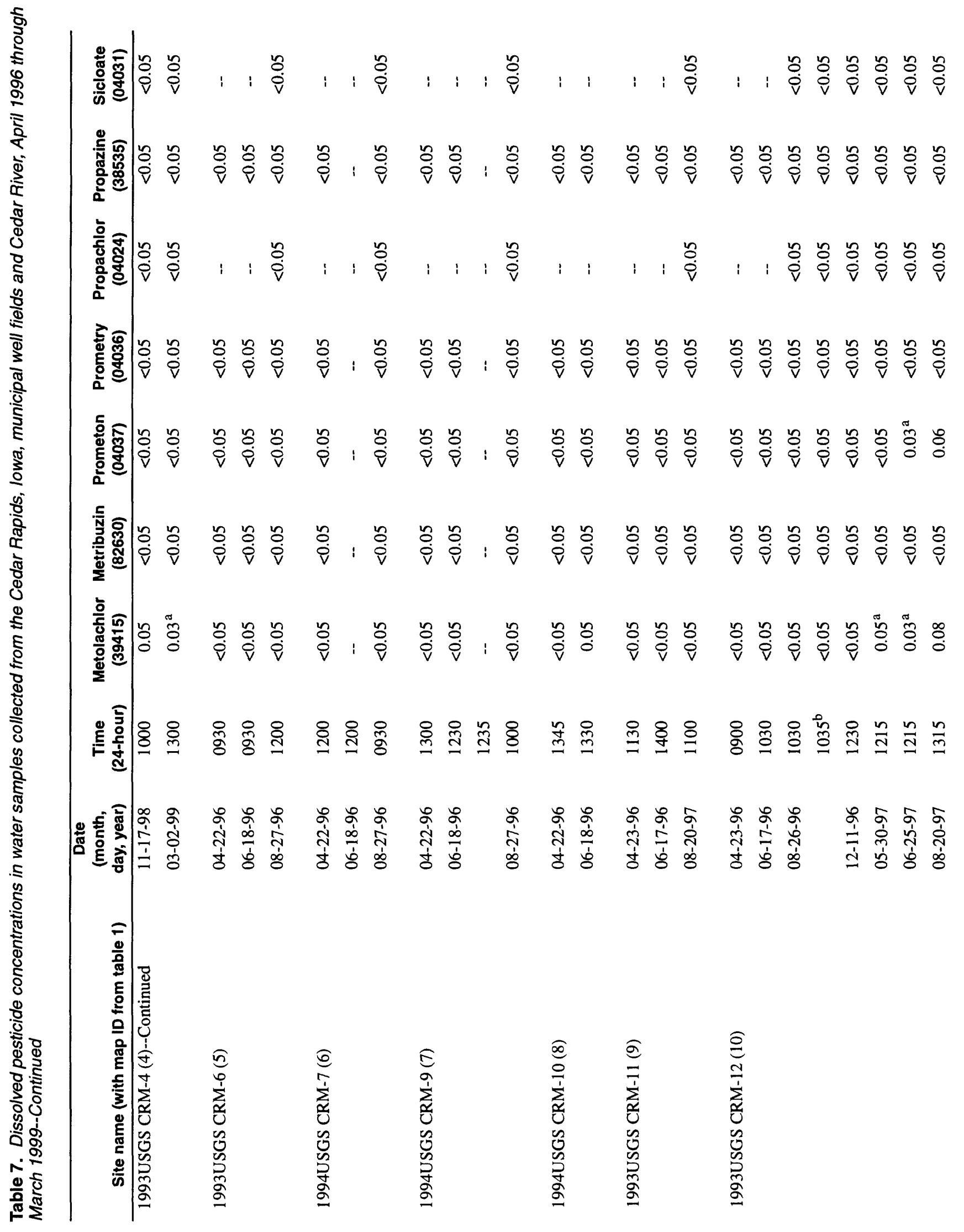




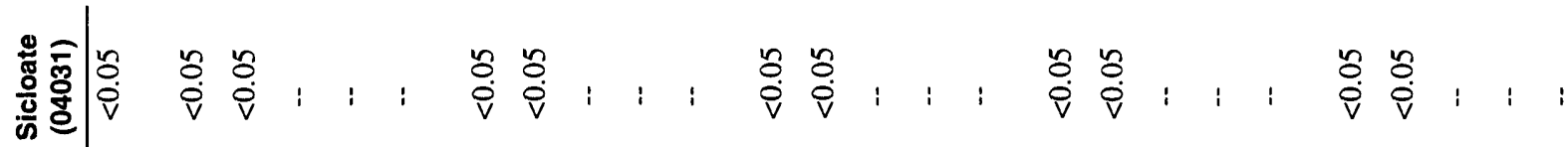

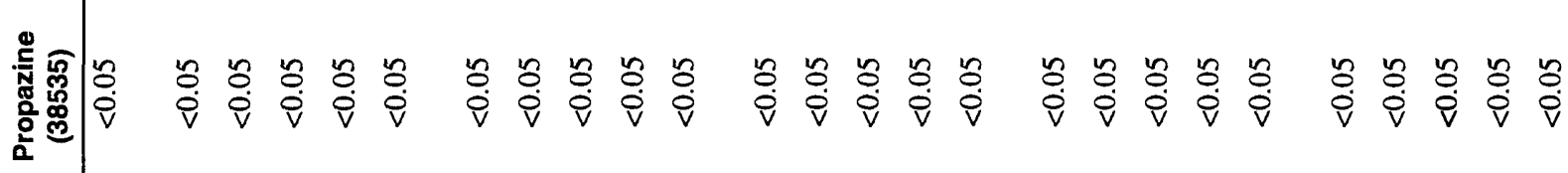

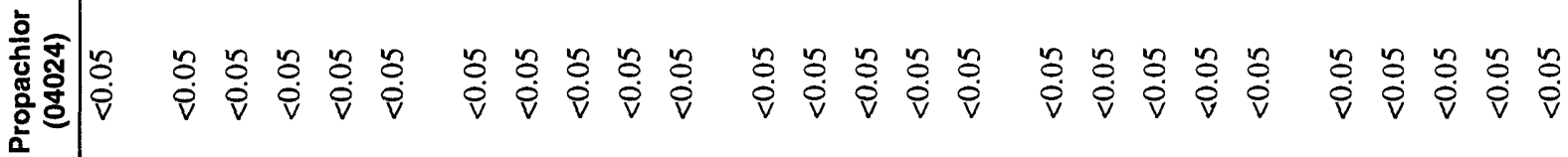

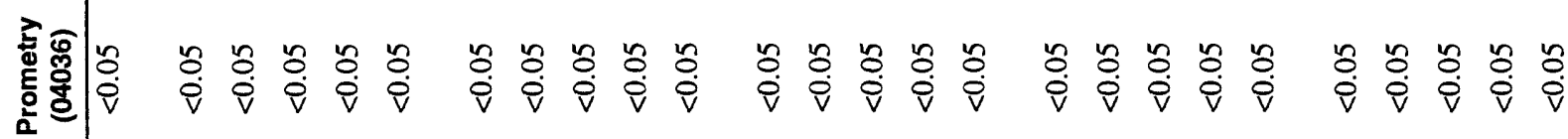

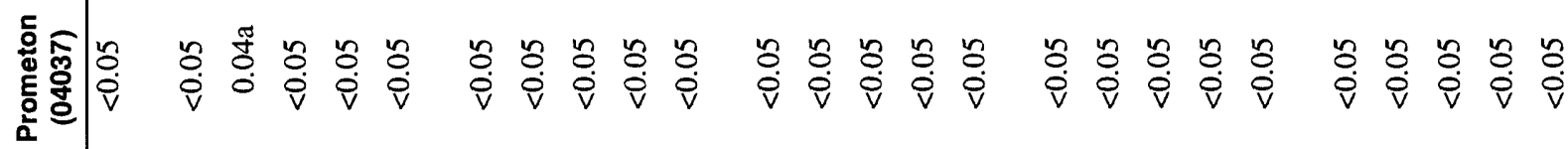

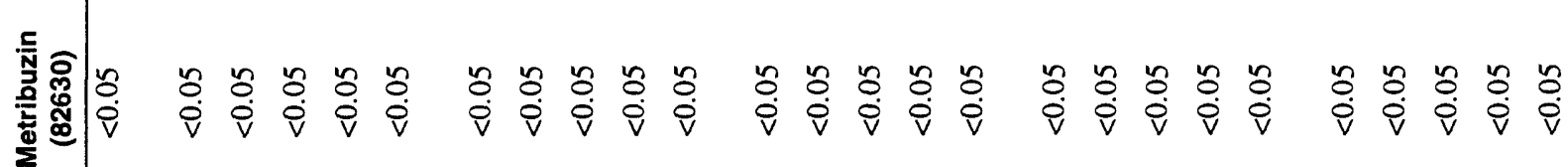

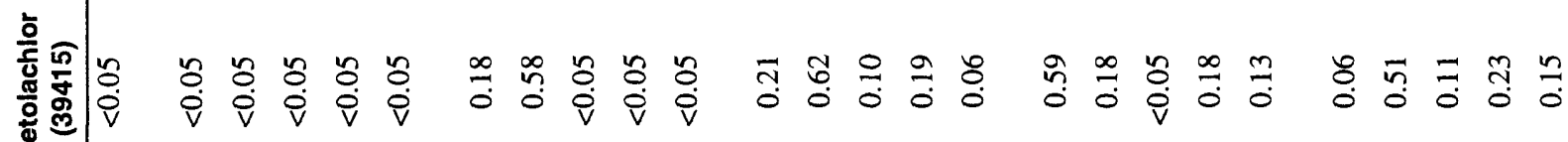

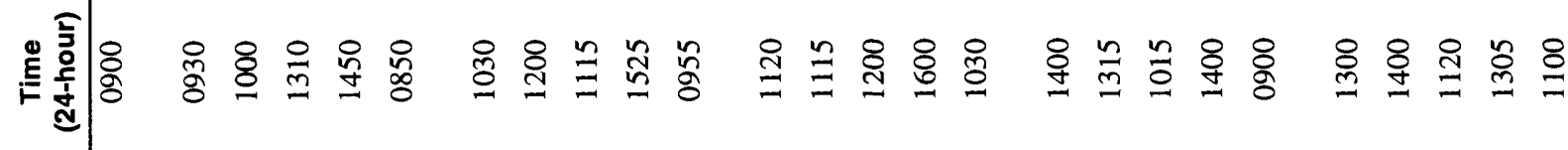

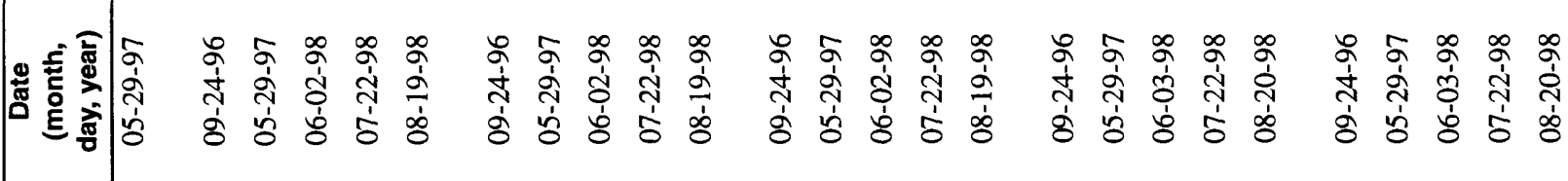

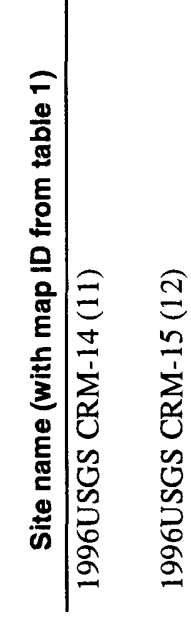

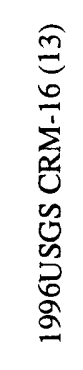

$\sqrt[n]{二}$
$\infty$
$\sum_{0}^{1}$
0
$y$
0
0
0
0
2 


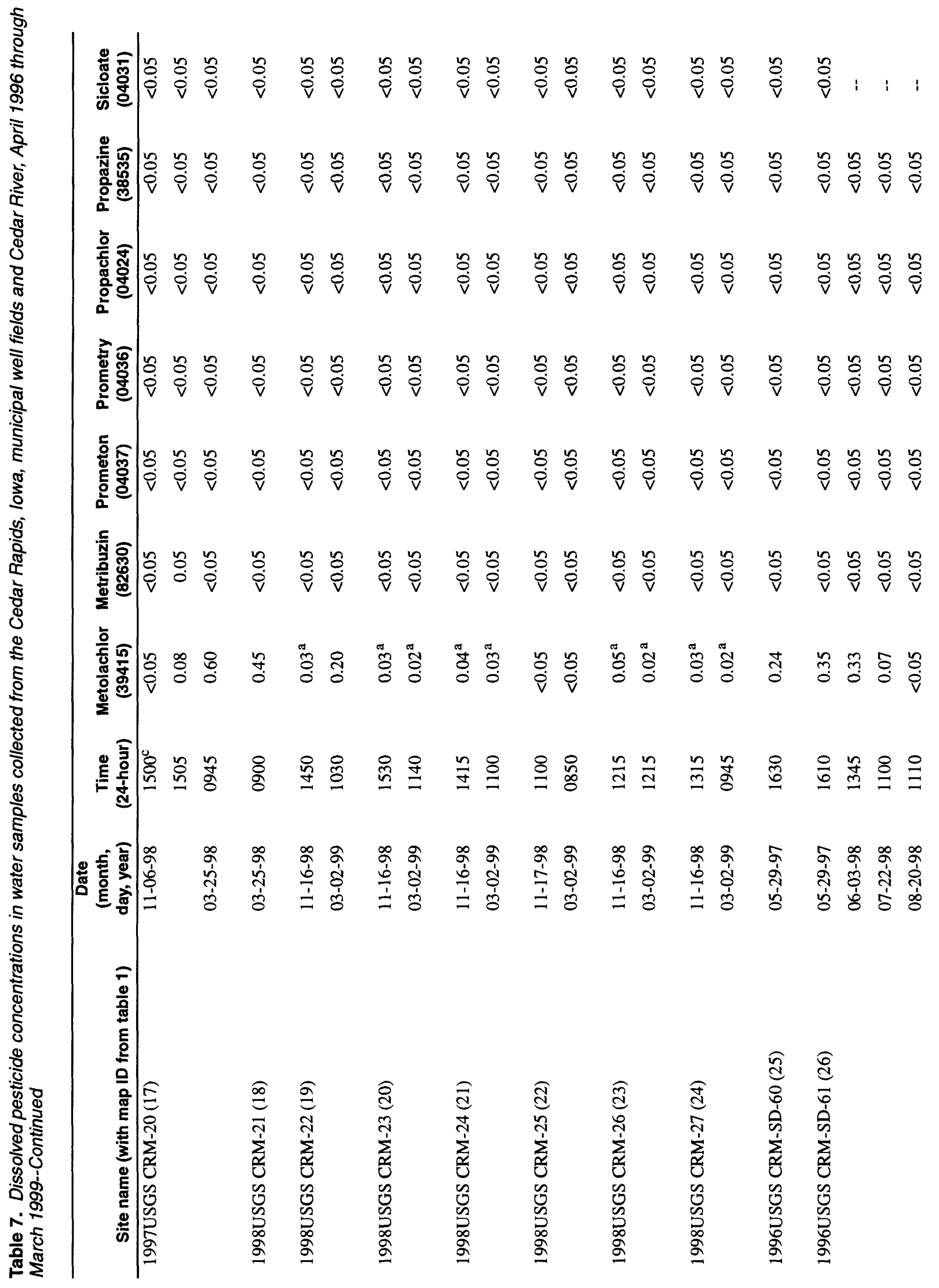




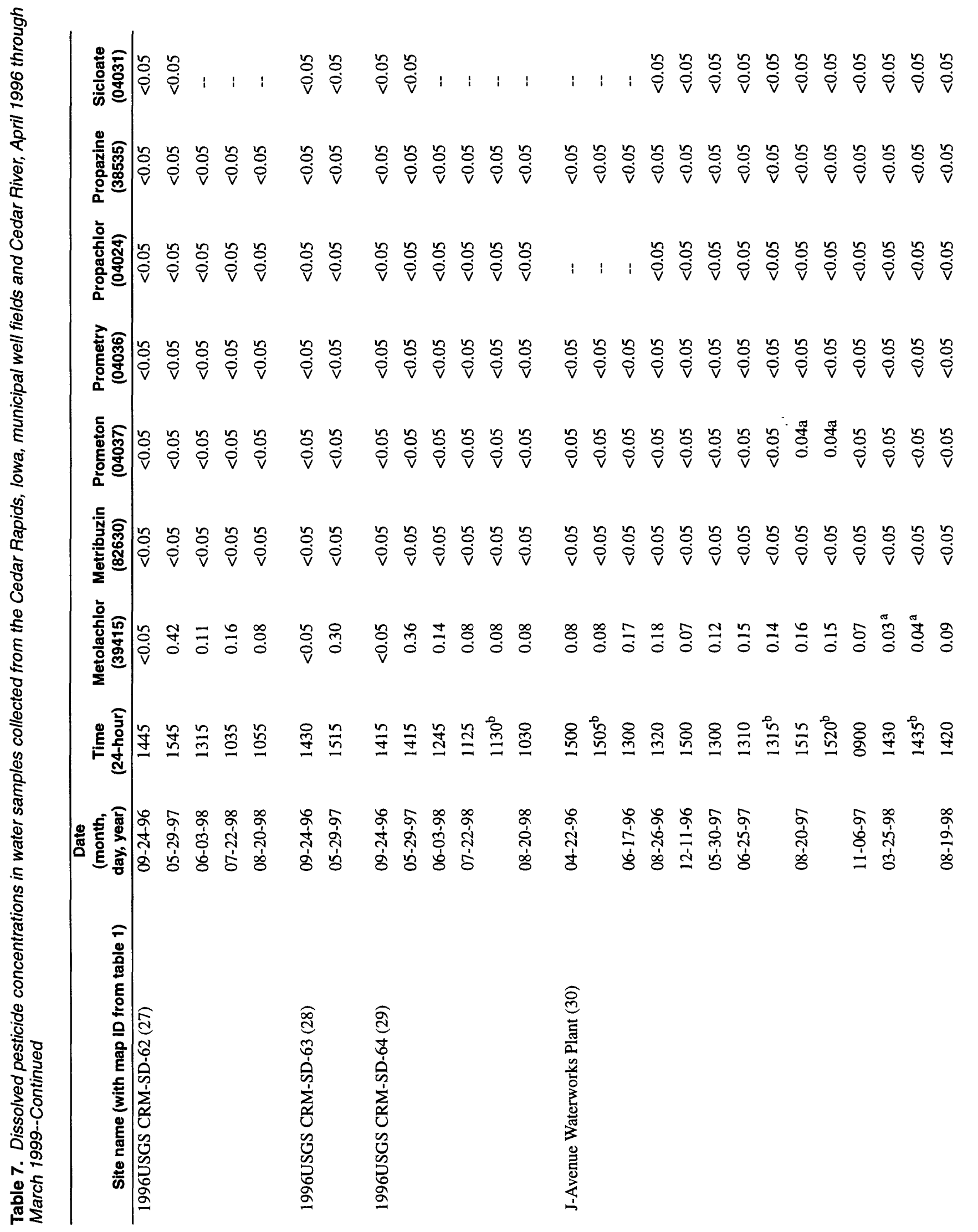




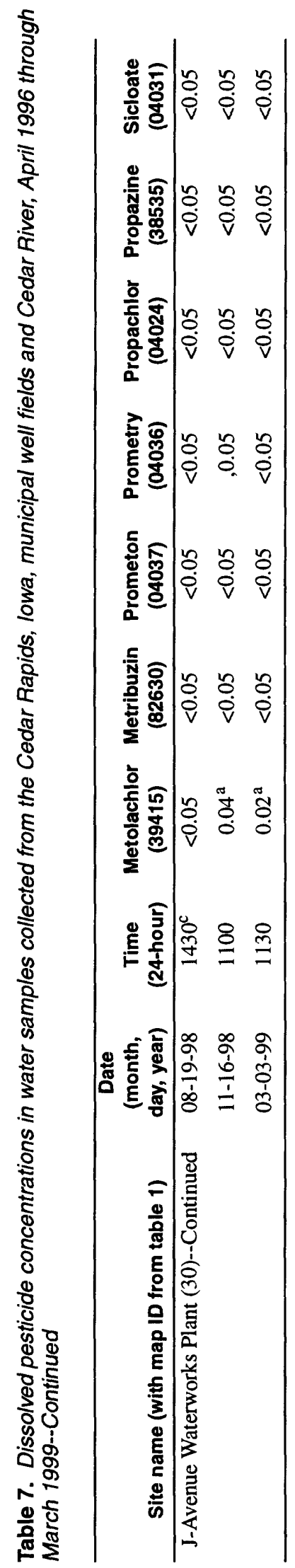

60 SELECTED HYDROLOGIC DATA FROM THE CEDAR RAPIDS AREA 


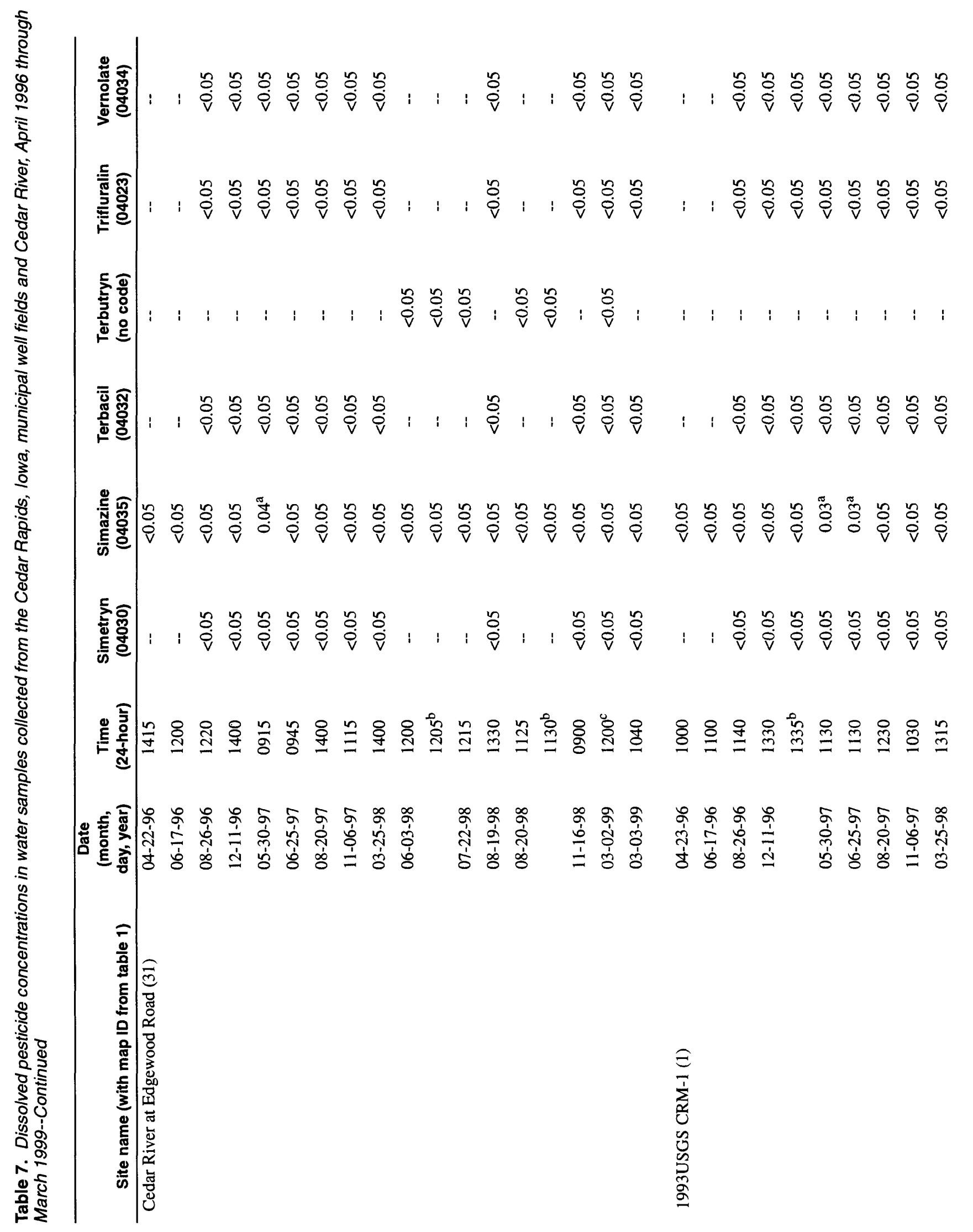




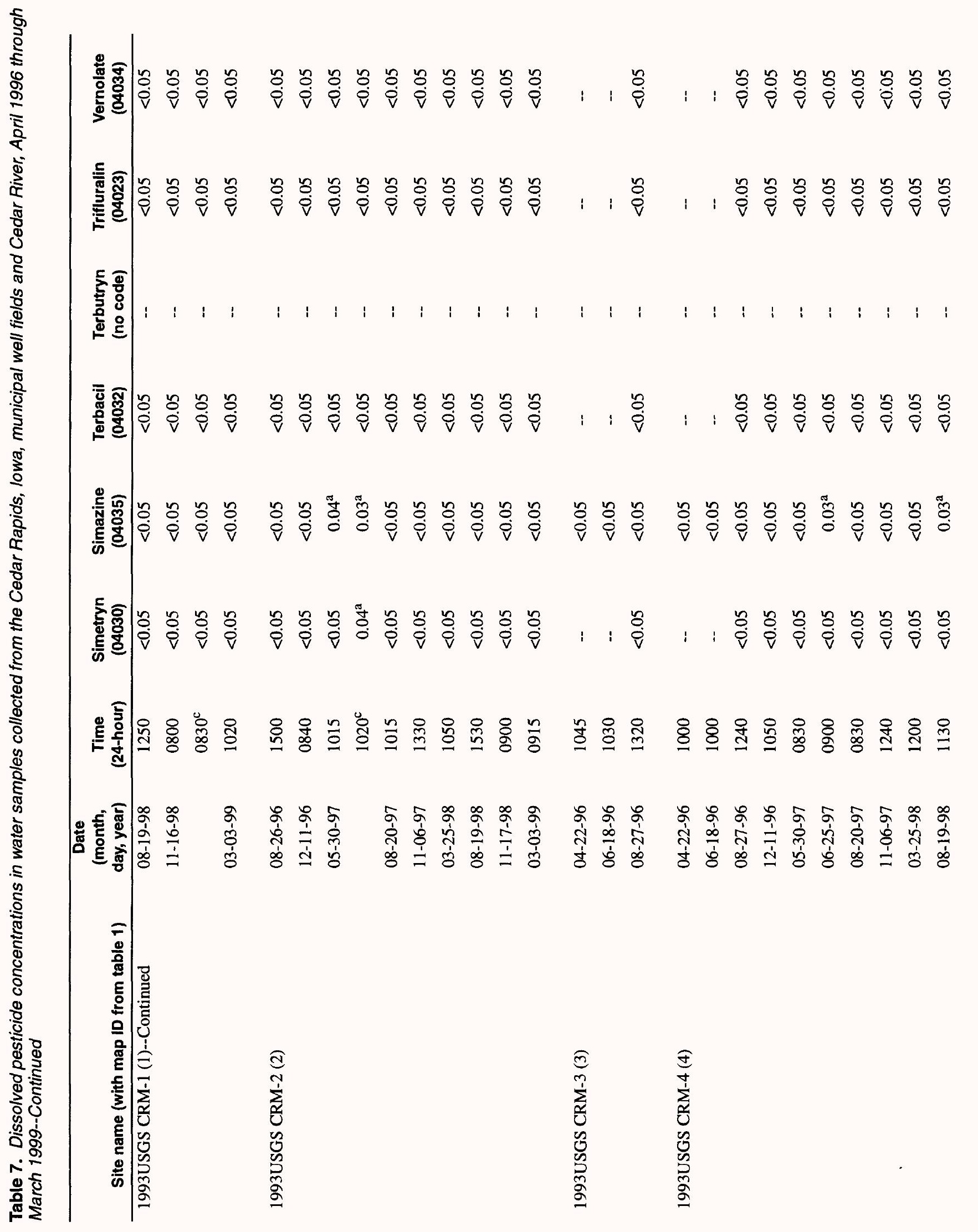




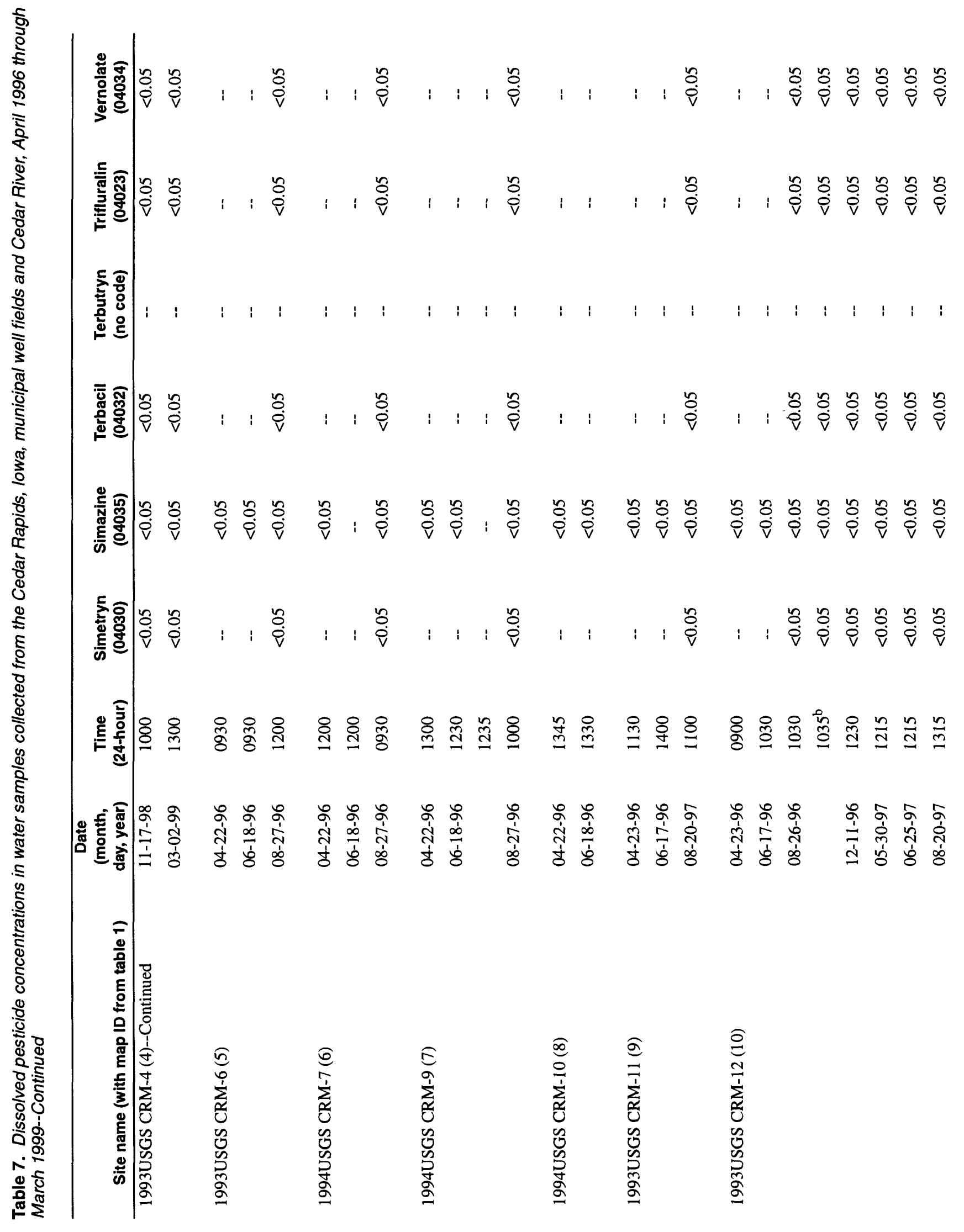




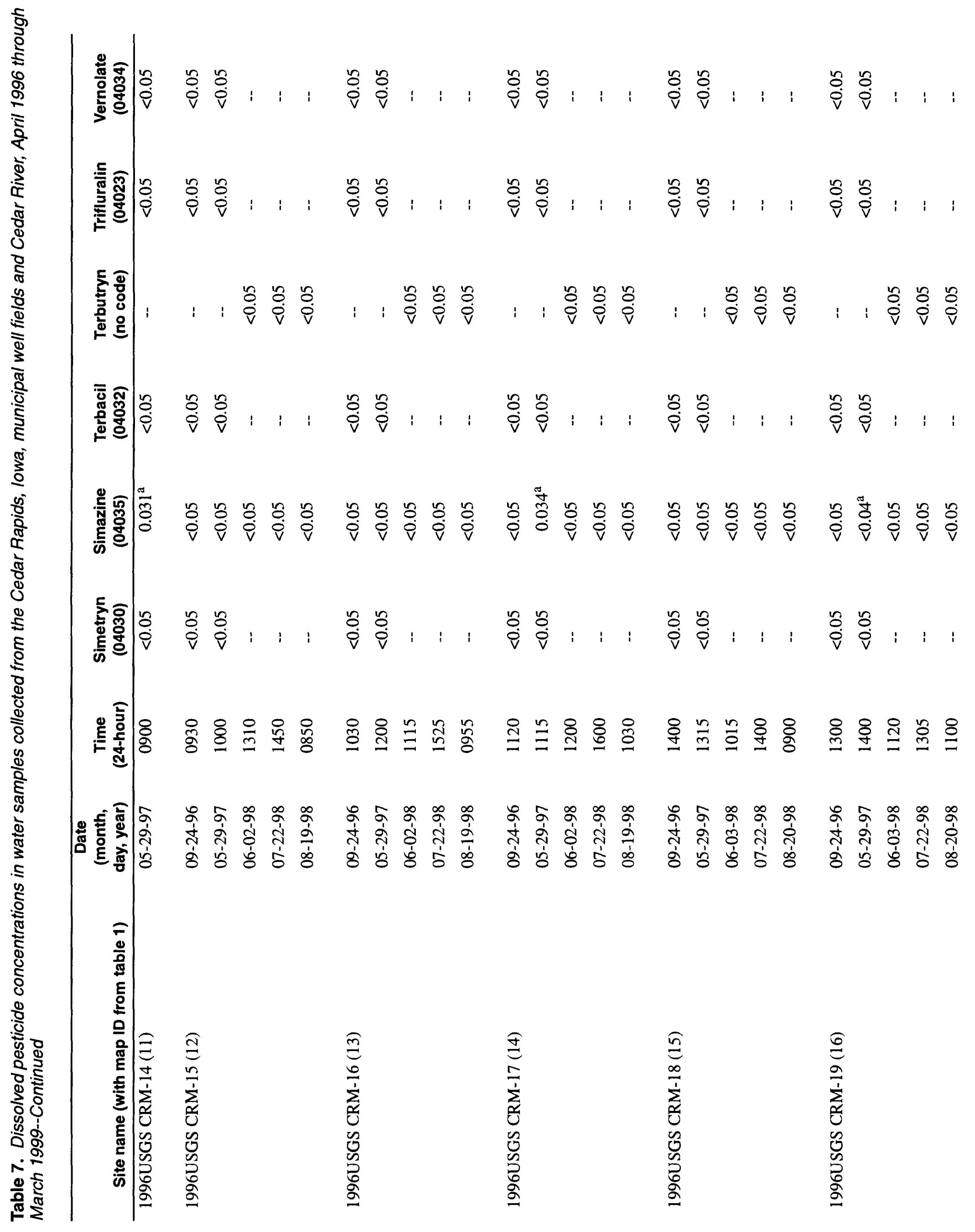




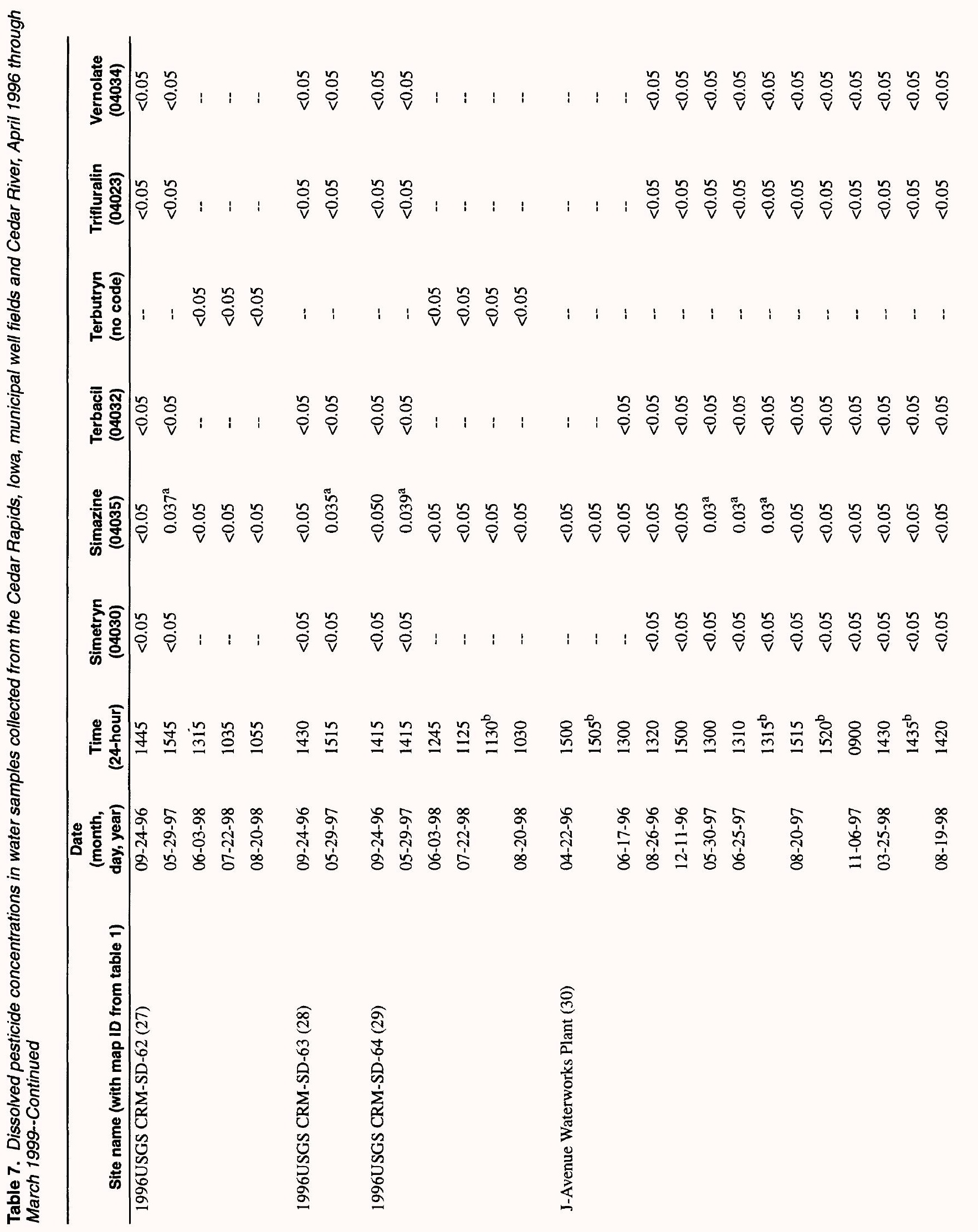




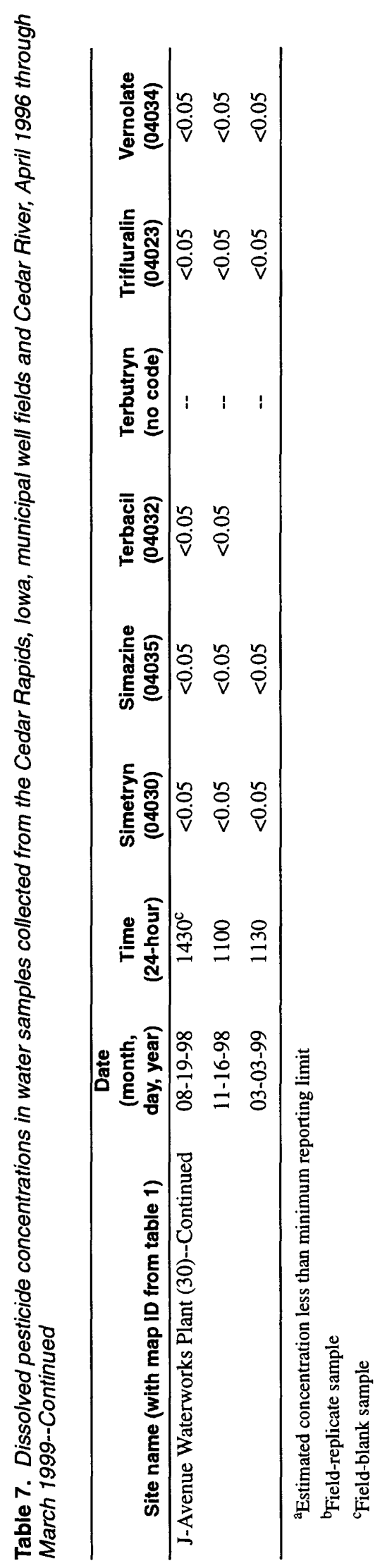


Table 8. Dissolved concentrations of ethanesulfonic-acid degradates of acetanilide herbicides and hydroxy-atrazine in water samples collected from the Seminole Well Field and Cedar River, Cedar Rapids, lowa, June 1998 through August 1998

[All concentrations in micrograms per liter; constituents listed with USGS parameter codes in parantheses; ESA, ethanesulfonic acid]

\begin{tabular}{|c|c|c|c|c|c|c|}
\hline $\begin{array}{l}\text { Site name } \\
\text { (with map ID from table 1) }\end{array}$ & $\begin{array}{c}\text { Date } \\
\text { (month, } \\
\text { day, year) }\end{array}$ & $\begin{array}{c}\text { Time } \\
\text { (24-hour) }\end{array}$ & $\begin{array}{c}\text { Acetochlor } \\
\text { ESA } \\
(61029)\end{array}$ & $\begin{array}{c}\text { Alachlor } \\
\text { ESA } \\
(50009)\end{array}$ & $\begin{array}{c}\text { Metolachlor } \\
\text { ESA } \\
(61043)\end{array}$ & $\begin{array}{l}\text { Hydroxy- } \\
\text { atrazine } \\
\text { (50355) }\end{array}$ \\
\hline \multirow[t]{5}{*}{ Cedar River at Edgwood Road (31) } & $06-03-98$ & 1200 & 1.47 & 1.38 & 5.45 & $<0.20$ \\
\hline & & $1205^{\mathrm{a}}$ & 1.50 & 1.37 & 5.31 & $<0.20$ \\
\hline & $07-22-98$ & 1215 & 1.08 & 1.96 & 4.25 & $<0.20$ \\
\hline & $08-20-98$ & 1125 & 0.90 & 2.13 & 4.15 & $<0.20$ \\
\hline & & $1130^{\mathrm{a}}$ & 1.05 & 2.28 & 4.56 & $<0.20$ \\
\hline \multirow[t]{3}{*}{ 1996USGS CRM-15 (12) } & $06-02-98$ & 1310 & $<0.20$ & $<0.20$ & $<0.20$ & $<0.20$ \\
\hline & $07-22-98$ & 1450 & $<0.20$ & $<0.20$ & 0.38 & $<0.20$ \\
\hline & $08-19-98$ & 0850 & $<0.20$ & $<0.20$ & 0.37 & $<0.20$ \\
\hline \multirow[t]{3}{*}{ 1996USGS CRM-16 (13) } & $06-02-98$ & 1115 & 0.39 & 1.02 & 2.27 & $<0.20$ \\
\hline & $07-22-98$ & 1525 & 0.85 & 1.62 & 3.92 & $<0.20$ \\
\hline & $08-19-98$ & 0955 & 1.10 & 1.21 & 3.81 & $<0.20$ \\
\hline \multirow[t]{3}{*}{ 1996USGS CRM-17 (14) } & $06-02-98$ & 1200 & 1.26 & 1.62 & 4.83 & $<0.20$ \\
\hline & $07-22-98$ & 1600 & 1.37 & 1.73 & 4.51 & $<0.20$ \\
\hline & $08-19-98$ & 1030 & 1.48 & 1.28 & 4.14 & $<0.20$ \\
\hline \multirow[t]{3}{*}{ 1996USGS CRM-18 (15) } & $06-03-98$ & 1015 & 0.71 & 1.06 & 3.09 & $<0.20$ \\
\hline & $07-22-98$ & 1400 & 1.14 & 2.19 & 5.64 & $<0.20$ \\
\hline & $08-20-98$ & 0900 & 1.39 & 2.50 & 6.49 & $<0.20$ \\
\hline \multirow[t]{3}{*}{ 1996USGS CRM-19 (16) } & $06-03-98$ & 1120 & 0.99 & 1.38 & 4.24 & $<0.20$ \\
\hline & $07-22-98$ & 1305 & 2.06 & 2.06 & 6.36 & $<0.20$ \\
\hline & $08-20-98$ & 1100 & 1.58 & 2.35 & 5.48 & $<0.20$ \\
\hline \multirow[t]{3}{*}{ 1996USGS CRM-SD-61 (26) } & $06-03-98$ & 1345 & 2.08 & 1.36 & 5.48 & 0.21 \\
\hline & $07-22-98$ & 1100 & 2.90 & 2.36 & 6.84 & $<0.20$ \\
\hline & $08-20-98$ & 1110 & 1.55 & 2.24 & 5.85 & $<0.20$ \\
\hline \multirow[t]{3}{*}{ 1996USGS CRM-SD-62 (27) } & $06-03-98$ & 1315 & 1.98 & 1.31 & 5.16 & $<0.20$ \\
\hline & $07-22-98$ & 1100 & 3.32 & 2.20 & 6.51 & $<0.20$ \\
\hline & $08-20-98$ & 1055 & 2.49 & 2.23 & 6.47 & $<0.20$ \\
\hline \multirow[t]{4}{*}{ 1996USGS CRM-SD-64 (29) } & $06-03-98$ & 1245 & 1.72 & 1.43 & 5.27 & $<0.20$ \\
\hline & $07-22-98$ & 1125 & 2.48 & 1.97 & 5.64 & $<0.20$ \\
\hline & & $1130^{\mathrm{a}}$ & 2.55 & 2.02 & 5.80 & $<0.20$ \\
\hline & $08-20-98$ & 1030 & 1.07 & 2.15 & 4.75 & $<0.20$ \\
\hline
\end{tabular}

${ }^{\mathrm{a}}$ Field-replicate sample 
Table 9. Dissolved concentrations of oxanilic-acid degradates of acetanilide herbicides in water samples collected from the Seminole Well Field and Cedar River, Cedar Rapids, lowa, June 1998 through August 1998

[All concentrations in micrograms per liter; constituents listed with USGS parameter codes in parantheses; OA,oxanilic acid]

\begin{tabular}{|c|c|c|c|c|c|}
\hline $\begin{array}{l}\text { Site name (with map } \\
\text { ID from table 1) }\end{array}$ & $\begin{array}{c}\text { Date } \\
\text { (month } \\
\text { day, year) }\end{array}$ & $\begin{array}{c}\text { Time } \\
\text { (24-hour) }\end{array}$ & $\begin{array}{c}\text { Acetochlor } \\
\text { OA } \\
(61030)\end{array}$ & $\begin{array}{c}\text { Alachlor } \\
\text { OA } \\
(61031)\end{array}$ & $\begin{array}{c}\text { Metolachlor } \\
\text { OA } \\
(61044)\end{array}$ \\
\hline \multirow[t]{5}{*}{ Cedar River at Edgewood Road (31) } & $06-03-98$ & 1200 & 0.64 & $<0.20$ & 0.92 \\
\hline & & $1205^{\mathrm{a}}$ & 0.66 & $<0.20$ & 1.04 \\
\hline & $07-22-98$ & 1215 & 0.29 & $<0.20$ & 0.62 \\
\hline & $08-20-98$ & 1125 & $<0.20$ & $<0.20$ & 0.54 \\
\hline & & $1130^{\mathrm{a}}$ & $<0.20$ & $<0.20$ & 0.56 \\
\hline \multirow[t]{3}{*}{ 1996USGS CRM-15 (12) } & $06-02-98$ & 1310 & $<0.20$ & $<0.20$ & $<0.20$ \\
\hline & $07-22-98$ & 1450 & $<0.20$ & $<0.20$ & $<0.20$ \\
\hline & $08-19-98$ & 0950 & $<0.20$ & $<0.20$ & $<0.20$ \\
\hline \multirow[t]{3}{*}{ 1996USGS CRM-16 (13) } & $06-02-98$ & 1115 & $<0.20$ & $<0.20$ & 0.45 \\
\hline & $07-22-98$ & 1525 & 0.28 & $<0.20$ & 0.76 \\
\hline & $08-19-98$ & 0955 & 0.35 & $<0.20$ & 0.82 \\
\hline \multirow[t]{3}{*}{ 1996USGS CRM-17 (14) } & $06-02-98$ & 1200 & 0.85 & $<0.20$ & 1.22 \\
\hline & $07-22-98$ & 1600 & 0.69 & 0.29 & 1.25 \\
\hline & $08-19-98$ & 1030 & 0.61 & $<0.20$ & 0.87 \\
\hline \multirow[t]{3}{*}{ 1996USGS CRM-18 (15) } & $06-03-98$ & 1015 & $<0.20$ & $<0.20$ & 0.77 \\
\hline & $07-22-98$ & 1400 & $<0.20$ & $<0.20$ & 0.89 \\
\hline & $08-20-98$ & 0900 & $<0.20$ & $<0.20$ & 0.81 \\
\hline \multirow[t]{3}{*}{ 1996USGS CRM-19 (16) } & $06-03-98$ & 1120 & 0.66 & $<0.20$ & 0.78 \\
\hline & $07-22-98$ & 1305 & 1.17 & $<0.20$ & 1.17 \\
\hline & $08-20-98$ & 1100 & 0.20 & $<0.20$ & 0.69 \\
\hline \multirow[t]{3}{*}{ 1996USGS CRM-SD-61 (26) } & $06-03-98$ & 1345 & 1.66 & $<0.20$ & 1.16 \\
\hline & $07-22-98$ & 1100 & 1.08 & 0.20 & 1.43 \\
\hline & $08-20-98$ & 1110 & 0.26 & $<0.20$ & 0.88 \\
\hline \multirow[t]{3}{*}{ 1996USGS CRM-SD-62 (27) } & $06-03-98$ & 1315 & 1.18 & $<0.20$ & 1.19 \\
\hline & $07-22-98$ & 1035 & 1.75 & 0.31 & 1.72 \\
\hline & $08-20-98$ & 1055 & 0.67 & 0.29 & 1.04 \\
\hline \multirow[t]{4}{*}{ 1996USGS CRM-SD-64 (29) } & $06-03-98$ & 1245 & 1.04 & $<0.20$ & 1.20 \\
\hline & $07-22-98$ & 1125 & 1.34 & 0.25 & 1.40 \\
\hline & & $1130^{\mathrm{a}}$ & 1.41 & 0.26 & 1.46 \\
\hline & $08-20-98$ & 1030 & $<0.20$ & $<0.20$ & 0.59 \\
\hline
\end{tabular}

${ }^{\text {a}}$ Field-replicate sample 
Table 10a. Mean daily ground-water levels in observation well CRM-1 (map ID \#1), East Well Field, Cedar Rapids, lowa, July 1997 through February 1999

[Water levels measured as feet below land surface; --, value not measured or recorded]

\begin{tabular}{|c|c|c|c|c|c|c|c|c|c|c|c|c|}
\hline Day & Jan & Feb & Mar & Apr & May & Jun & Jul & Aug & Sep & Oct & Nov & Dec \\
\hline & & & & & & 1997 & & & & & & \\
\hline 1 & -- & -- & -- & -- & -- & -- & -. & 11.22 & 9.79 & 7.55 & 20.52 & 20.08 \\
\hline 2 & -- & -- & -- & -- & -- & -- & -- & 11.05 & 8.31 & 7.25 & 22.90 & 19.97 \\
\hline 3 & -. & -- & -- & -- & -- & -- & -- & 11.24 & 6.47 & 7.38 & 20.74 & 19.76 \\
\hline 4 & -- & -- & -- & -- & -- & -- & -- & 11.13 & 5.55 & 7.19 & 20.19 & 19.88 \\
\hline 5 & -- & -- & -- & - & -- & - & -- & 11.13 & 5.10 & 7.59 & 20.59 & 20.29 \\
\hline 6 & -- & -- & -- & -- & -- & -- & -- & 11.00 & 5.59 & 7.30 & 20.48 & 20.30 \\
\hline 7 & -- & -- & -- & -- & -- & -- & -- & 10.85 & 4.24 & 7.92 & 20.42 & 20.34 \\
\hline 8 & -- & -- & -- & -- & - & -- & -- & 10.43 & 4.03 & 8.43 & 20.13 & 20.43 \\
\hline 9 & -- & -- & - & -- & -- & -- & -- & 10.31 & 3.62 & 8.62 & 19.94 & 18.85 \\
\hline 10 & -- & -- & -- & -- & -- & -- & -- & 10.38 & 3.02 & 8.42 & 20.23 & 18.21 \\
\hline 11 & -- & -- & -- & -- & -. & -- & -- & 10.39 & 2.67 & 8.11 & 20.25 & 18.62 \\
\hline 12 & -- & -- & -- & -- & - & -- & - & 9.95 & 2.81 & 8.19 & 20.06 & 18.52 \\
\hline 13 & -- & -- & -- & -- & -- & -- & -- & 9.97 & 3.47 & 7.71 & 19.73 & 18.05 \\
\hline 14 & -- & -- & -- & -- & -. & -- & -- & 9.80 & 4.05 & 7.61 & 19.87 & 17.92 \\
\hline 15 & -. & -- & -- & -- & -. & -- & -- & 10.87 & 4.76 & 7.13 & 20.27 & 17.76 \\
\hline 16 & -- & -- & -- & -- & -. & -- & -- & 9.09 & 6.43 & 6.79 & 20.44 & 17.49 \\
\hline 17 & -- & -- & -- & -- & -- & -- & -- & 8.84 & 6.66 & 7.09 & 20.42 & 17.20 \\
\hline 18 & -- & -- & -- & -- & - & - & -- & 9.33 & 6.46 & 6.94 & 20.12 & 17.76 \\
\hline 19 & -- & -- & -- & -- & -- & -- & -- & 11.16 & 6.92 & 6.88 & 20.14 & 18.06 \\
\hline 20 & -- & -- & -- & - & -- & -- & -- & 12.38 & 6.86 & 7.17 & 20.08 & 17.40 \\
\hline 21 & -- & -- & -- & -- & -- & -- & -- & 11.24 & 6.61 & 7.19 & 20.01 & 15.80 \\
\hline 22 & -- & -- & -- & - & -- & -- & -- & 10.05 & 6.67 & 7.27 & 20.06 & 15.11 \\
\hline 23 & -- & -- & -- & -. & -- & -- & -- & 9.08 & 6.94 & 7.52 & 20.27 & 14.51 \\
\hline 24 & -. & -- & -- & -- & -- & -- & 11.82 & 8.19 & 6.77 & 9.21 & 20.40 & 15.29 \\
\hline 25 & -- & -- & -- & -- & -- & -- & 11.82 & 7.56 & 6.81 & 10.04 & 19.90 & 14.47 \\
\hline 26 & -- & -- & -- & - & -- & -- & 12.42 & 7.01 & 6.96 & 10.02 & 19.98 & 14.02 \\
\hline 27 & -- & -- & -- & -- & -- & -- & 12.30 & 6.74 & 7.07 & 12.50 & 20.05 & 14.69 \\
\hline 28 & - & -- & -- & -- & -- & - & 11.88 & 7.51 & 6.94 & 14.28 & 19.93 & 16.32 \\
\hline 29 & -. & -- & -- & -- & -- & -- & 11.85 & 9.89 & 8.37 & 15.66 & 19.84 & 16.00 \\
\hline 30 & -- & -. & -. & - & -- & -- & 11.49 & 10.07 & 7.65 & 16.89 & 19.87 & 15.77 \\
\hline 31 & -- & -- & -- & -- & -- & -. & 11.40 & 11.13 & -- & 19.83 & -- & -- \\
\hline
\end{tabular}


Table 10a. Mean daily ground-water levels in observation well CRM-1 (map ID \#1), East Well Field, Cedar Rapids, lowa, July 1997 through February 1999--Continued

\begin{tabular}{|c|c|c|c|c|c|c|c|c|c|c|c|c|}
\hline Day & Jan & Feb & Mar & Apr & May & Jun & Jul & Aug & Sep & Oct & Nov & $\overline{\text { Dec }}$ \\
\hline & & & & & & 1998 & & & & & & \\
\hline 1 & -- & 24.94 & 18.98 & 17.10 & 13.44 & 13.31 & 0.24 & 2.57 & 7.08 & 6.76 & 2.35 & 2.16 \\
\hline 2 & 14.53 & 25.40 & 18.44 & 13.19 & 13.26 & 13.43 & 0.10 & 2.20 & 7.06 & 5.38 & 1.81 & 2.18 \\
\hline 3 & 14.85 & 26.13 & 18.31 & 8.44 & 11.73 & 13.08 & -0.37 & 1.58 & 6.73 & 4.78 & 1.86 & 1.97 \\
\hline 4 & 14.71 & 25.11 & 18.63 & 4.86 & 11.89 & 13.06 & -0.51 & 1.55 & 6.71 & 4.39 & 2.31 & 1.93 \\
\hline 5 & 15.03 & 24.80 & 17.62 & 2.51 & 12.82 & 13.41 & 0.51 & 1.60 & 6.91 & 3.41 & 1.93 & 1.48 \\
\hline 6 & 15.54 & 25.77 & 17.87 & 1.01 & 11.52 & 14.46 & 1.88 & 0.81 & 7.24 & 3.06 & 1.26 & 1.56 \\
\hline 7 & 17.23 & 25.30 & 17.80 & 3.00 & 11.28 & 16.66 & 2.62 & 0.28 & 6.80 & 3.16 & 0.91 & 1.94 \\
\hline 8 & 17.58 & 24.61 & 17.13 & 2.52 & 11.72 & 17.86 & 3.12 & 0.65 & 7.37 & 3.67 & 1.12 & 2.41 \\
\hline 9 & 17.89 & 24.30 & 17.18 & 1.80 & 10.54 & 18.98 & 5.46 & 0.66 & 7.16 & 3.64 & 5.84 & 2.74 \\
\hline 10 & 18.25 & 24.26 & 16.81 & 1.92 & 9.44 & 19.61 & 6.60 & 0.48 & 7.54 & 3.59 & 9.27 & 2.68 \\
\hline 11 & 19.42 & 24.26 & 17.79 & 0.34 & 9.56 & 20.58 & 5.90 & 0.93 & 7.60 & 3.56 & 7.85 & 3.26 \\
\hline 12 & 19.76 & 23.85 & 19.05 & 0.13 & 11.74 & 19.64 & 5.59 & 1.39 & 7.89 & 3.58 & 6.96 & 3.64 \\
\hline 13 & 20.26 & 23.76 & 20.01 & 1.74 & 11.47 & 18.04 & 4.81 & 1.83 & 7.97 & 2.90 & 5.69 & 3.59 \\
\hline 14 & 20.36 & 23.72 & 18.93 & 4.33 & 11.29 & 18.80 & 3.47 & 2.55 & 7.29 & 3.02 & 4.25 & 3.38 \\
\hline 15 & 20.32 & 23.45 & 18.05 & 6.97 & 11.59 & 13.36 & 3.38 & 2.93 & 6.62 & 2.55 & 3.66 & 3.18 \\
\hline 16 & 20.39 & 23.66 & 19.33 & 5.40 & 10.90 & 11.11 & 3.55 & 1.92 & 5.97 & 2.86 & 3.70 & 2.66 \\
\hline 17 & 20.42 & 25.32 & 20.19 & 2.02 & 10.15 & 11.34 & 3.44 & 1.90 & 5.35 & 2.16 & 4.90 & 2.53 \\
\hline 18 & 20.31 & 23.92 & 20.45 & 1.26 & 9.39 & 11.61 & 3.48 & 2.55 & 4.78 & 1.55 & 5.90 & 3.14 \\
\hline 19 & 20.31 & 23.89 & 20.64 & 0.44 & 9.16 & 12.78 & 3.19 & 2.18 & 4.22 & 1.37 & 6.70 & 2.33 \\
\hline 20 & 20.27 & 23.81 & 20.85 & 2.37 & 9.94 & 13.47 & 1.98 & 3.63 & 4.04 & 0.96 & 5.26 & 2.44 \\
\hline 21 & 20.38 & 23.59 & 21.07 & 3.10 & 10.22 & 13.75 & 1.46 & 3.32 & 3.63 & 1.16 & 4.51 & 2.46 \\
\hline 22 & 19.78 & 23.62 & 21.62 & 5.48 & 10.52 & 12.63 & 0.88 & 2.51 & 3.83 & 1.44 & 4.30 & 1.88 \\
\hline 23 & 19.67 & 23.74 & 22.60 & 6.52 & 10.47 & 10.61 & 0.72 & 2.66 & 3.82 & 3.22 & 4.35 & 2.22 \\
\hline 24 & 19.59 & 23.51 & 22.91 & 4.85 & 10.08 & 6.69 & 0.92 & 3.43 & 3.62 & 3.39 & 4.09 & 2.18 \\
\hline 25 & 19.75 & 23.00 & 21.39 & 2.62 & 9.56 & 2.30 & 1.02 & 4.27 & 3.80 & 3.41 & 3.57 & 2.31 \\
\hline 26 & -- & 24.21 & 19.72 & 3.63 & 9.15 & 0.00 & 0.88 & 4.20 & 3.66 & 4.31 & 3.30 & 2.12 \\
\hline 27 & 24.27 & 23.04 & 20.40 & 8.41 & 10.67 & -0.01 & 0.79 & 5.03 & 3.97 & 4.44 & 3.10 & 2.45 \\
\hline 28 & 24.34 & 19.71 & 19.06 & 8.09 & 11.74 & -0.26 & 1.03 & 5.60 & 3.99 & 3.89 & 2.77 & 3.12 \\
\hline 29 & 24.53 & -- & 16.52 & 11.08 & 12.12 & -0.37 & 1.01 & 5.89 & 5.76 & 3.67 & 2.63 & 3.83 \\
\hline 30 & 24.51 & -- & 17.66 & 12.70 & 12.91 & 0.09 & 1.26 & 6.34 & 7.82 & 2.28 & 2.16 & 4.06 \\
\hline 31 & 24.72 & -- & 20.21 & -- & 13.26 & -- & 2.80 & 6.84 & -- & 2.39 & -- & -- \\
\hline
\end{tabular}


Table 10a. Mean daily ground-water levels in observation well CRM-1 (map ID \#1), East Well Field, Cedar Rapids, lowa, July 1997 through February 1999--Continued

\begin{tabular}{|c|c|c|c|c|c|c|c|c|c|c|c|c|}
\hline Day & Jan & Feb & Mar & Apr & May & Jun & Jul & Aug & Sep & Oct & Nov & $\overline{\mathrm{Dec}}$ \\
\hline & & & & & & 1999 & & & & & & \\
\hline 1 & -. & 5.13 & -- & -- & -- & -- & -- & -- & -. & -- & -- & -- \\
\hline 2 & 5.72 & 4.91 & -- & -- & -- & - & - & -- & -. & -. & -- & -- \\
\hline 3 & 5.85 & 7.20 & -- & -. & -- & -- & -- & -- & -- & -- & -- & -- \\
\hline 4 & 6.32 & 5.47 & -- & -- & -- & -- & -- & -- & -- & -- & -- & -. \\
\hline 5 & 6.22 & 5.10 & -- & -- & - & -. & -- & -- & -- & -- & -- & -- \\
\hline 6 & 6.37 & 5.04 & -. & -- & -- & -- & -- & -- & -. & -. & -- & -. \\
\hline 7 & 6.35 & 4.77 & -- & -. & -- & -- & -- & -- & -. & -. & -- & - \\
\hline 8 & 6.71 & 4.53 & -- & -- & -- & - & - & -. & -. & -. & -- & -- \\
\hline 9 & 7.68 & 4.38 & -- & -- & -- & -- & -- & -- & -- & -- & - & -- \\
\hline 10 & 7.92 & 4.60 & -- & -- & -- & -- & -- & -- & -. & -. & -- & -- \\
\hline 11 & 7.95 & 5.67 & -- & -- & -- & -- & -- & -- & -- & -- & -. & -- \\
\hline 12 & 7.94 & 4.38 & -- & -- & -- & -- & -- & -- & -. & .. & -. & -- \\
\hline 13 & 8.33 & 4.53 & -. & -- & -- & -- & - & -. & -. & -. & -- & - \\
\hline 14 & 7.84 & 4.79 & -- & -- & -- & -- & -- & -- & -- & -. & -. & -- \\
\hline 15 & 7.44 & 4.79 & -- & -- & -- & -- & -- & -- & -. & -. & -- & -- \\
\hline 16 & 7.23 & 5.44 & -- & -- & -- & -- & -. & -- & -- & -- & -- & -- \\
\hline 17 & 8.26 & 5.82 & -- & -- & -- & -- & -- & -- & -- & -- & -. & -- \\
\hline 18 & 8.28 & 5.58 & - & -- & -- & -- & -- & -- & -- & -. & -- & -- \\
\hline 19 & 7.08 & 5.60 & -- & -- & -- & -- & -- & -- & -- & -- & -- & -- \\
\hline 20 & 6.96 & 5.83 & -- & -- & -- & -- & -- & -- & -- & -- & -- & -- \\
\hline 21 & 6.73 & 5.20 & -- & -- & -- & -- & -- & -- & -- & -- & -- & -- \\
\hline 22 & 6.99 & 5.18 & -- & -- & -- & -- & -- & -- & -- & -. & -- & -- \\
\hline 23 & 6.49 & 4.90 & -- & -- & -- & -- & -- & -- & -- & -- & -. & -- \\
\hline 24 & 6.21 & 4.81 & -- & -- & -- & -- & -- & -- & -- & -- & -- & -- \\
\hline 25 & 6.04 & 4.90 & -- & -- & -- & -- & -- & -. & -. & -. & -- & -- \\
\hline 26 & 5.85 & 5.07 & -- & - & -- & -- & -- & -- & -- & -- & -- & -- \\
\hline 27 & 5.56 & 5.34 & -- & -- & -- & -- & -- & -. & -- & -- & -- & -- \\
\hline 28 & 5.68 & 4.90 & -. & -- & -- & -- & -- & -. & -- & - & -- & -- \\
\hline 29 & 5.31 & -- & -- & - & -. & -- & -. & -- & -. & -. & -. & -- \\
\hline 30 & 5.24 & -- & -- & -- & -- & -. & -- & -- & -. & -. & -- & -- \\
\hline 31 & 5.53 & -- & -- & -- & -- & -- & -- & -- & -- & -- & -- & -- \\
\hline
\end{tabular}


Table 10b. Mean daily ground-water levels in observation well CRM-2 (map ID \#2), West Well Field, Cedar Rapids, lowa, July 1997 through February 1999

[Water levels measured as feet below land surface; --, value not measured or recorded]

\begin{tabular}{|c|c|c|c|c|c|c|c|c|c|c|c|c|}
\hline Day & Jan & Feb & Mar & Apr & May & Jun & Jul & Aug & Sep & Oct & Nov & Dec \\
\hline & & & & & & 1997 & & & & & & \\
\hline 1 & -- & -- & -- & -- & -- & -- & -- & 5.71 & 6.27 & 5.16 & 3.45 & 8.39 \\
\hline 2 & -. & -- & -- & -- & -- & -- & -- & 5.45 & 6.30 & 5.56 & 2.87 & 8.63 \\
\hline 3 & -- & -- & -- & -- & -- & - & -- & 5.24 & 6.18 & 5.32 & 4.00 & 9.45 \\
\hline 4 & -- & -- & -- & -- & -- & -- & -- & 5.37 & 6.01 & 5.31 & 4.00 & 9.68 \\
\hline 5 & -- & -- & -- & -- & -- & -- & -- & 5.79 & 5.47 & 5.37 & 4.41 & 9.38 \\
\hline 6 & -- & -- & -- & -- & -- & -. & -. & 5.73 & 6.18 & 5.03 & 4.52 & 9.20 \\
\hline 7 & -- & -- & -- & -- & -- & -- & -- & 5.74 & 6.12 & 5.08 & 5.01 & 8.14 \\
\hline 8 & -- & -- & -- & -- & -- & -- & -- & 5.30 & 6.24 & 5.23 & 5.64 & 8.12 \\
\hline 9 & -- & -- & -- & -- & -. & -- & -- & 5.26 & 5.79 & 5.53 & 5.69 & 8.62 \\
\hline 10 & -- & -- & -- & -- & -- & -- & -- & 5.18 & 5.48 & 5.68 & 6.10 & 8.55 \\
\hline 11 & - & -- & - & -- & -- & -- & -- & 5.34 & 5.41 & 5.41 & 6.16 & 8.39 \\
\hline 12 & -- & -- & - & -- & -- & -- & -- & 5.01 & 5.32 & 5.35 & 6.05 & 6.29 \\
\hline 13 & -- & -- & - & -- & -- & -- & -- & 5.21 & 3.62 & 5.18 & 6.50 & 5.07 \\
\hline 14 & -- & -- & -- & -- & -- & -- & -- & 5.20 & 3.38 & 5.92 & -- & 4.79 \\
\hline 15 & -- & -- & -- & -- & -- & -- & 5.34 & 4.74 & 3.18 & 6.69 & -- & 4.89 \\
\hline 16 & -. & -- & -- & -- & -- & -- & 5.34 & 5.58 & 3.38 & 6.81 & -- & 4.90 \\
\hline 17 & -- & -- & - & -- & -- & -- & 5.48 & 5.21 & 3.33 & 7.12 & -- & 4.80 \\
\hline 18 & -. & -. & -- & -- & -- & -- & 5.43 & 5.51 & 3.37 & 6.77 & -- & 5.39 \\
\hline 19 & - & -- & -- & -- & -- & -- & 5.22 & 5.12 & 3.41 & 6.84 & -- & 5.20 \\
\hline 20 & -. & -- & -- & -- & -- & -- & 5.25 & 5.30 & 3.54 & 7.43 & 7.53 & 5.06 \\
\hline 21 & -- & -- & -- & -- & -- & -- & 5.25 & 5.38 & 3.50 & 7.54 & 7.04 & 4.62 \\
\hline 22 & - & -- & -- & -- & -- & -- & 5.27 & 5.61 & 3.32 & 7.45 & 7.05 & 4.89 \\
\hline 23 & -. & -- & -- & -- & -- & -- & 5.29 & 5.59 & 3.92 & 7.70 & 7.08 & 4.52 \\
\hline 24 & -- & -- & -- & -- & -- & - & 5.32 & 5.32 & 5.09 & 7.86 & 7.28 & 4.74 \\
\hline 25 & -- & -- & -- & -- & -- & -- & 5.51 & 5.21 & 5.42 & 7.53 & 7.34 & 5.18 \\
\hline 26 & -- & -- & -- & -- & -- & -- & 5.79 & 5.32 & 5.04 & 6.11 & 7.23 & 4.94 \\
\hline 27 & -- & -- & -- & - & -. & - & 5.44 & 5.26 & 5.35 & 3.68 & 7.26 & 4.86 \\
\hline 28 & -- & -- & -- & -- & -- & -- & 5.41 & 5.35 & 5.29 & 4.04 & 7.55 & 4.47 \\
\hline 29 & -- & -- & -- & -- & -- & -- & 5.86 & 5.35 & 4.62 & 3.89 & 7.57 & 4.75 \\
\hline 30 & -- & -- & -- & - & -- & - & 5.76 & 5.59 & 5.26 & 4.35 & 7.69 & 4.65 \\
\hline 31 & -- & -- & -- & -- & -. & -- & 5.97 & 6.24 & -. & 3.40 & -- & -- \\
\hline
\end{tabular}


Table 10b. Mean daily ground-water levels in observation well CRM-2 (map ID \#2), West Well Field, Cedar Rapids, lowa, July 1997 through February 1999--Continued

\begin{tabular}{|c|c|c|c|c|c|c|c|c|c|c|c|c|}
\hline Day & Jan & Feb & Mar & Apr & May & Jun & Jul & Aug & Sep & Oct & Nov & Dec \\
\hline \multicolumn{13}{|c|}{1998} \\
\hline 1 & -- & 8.77 & 10.61 & 4.36 & 3.70 & 4.89 & -0.55 & 4.07 & 2.47 & 5.43 & 5.11 & -- \\
\hline 2 & 4.94 & 8.64 & 10.56 & 2.67 & 4.54 & 5.04 & -0.92 & 3.94 & 2.75 & 5.17 & 4.83 & -- \\
\hline 3 & 4.61 & 9.07 & 10.65 & 1.64 & 4.68 & 4.20 & -1.68 & 3.50 & 3.57 & 5.11 & 5.08 & -- \\
\hline 4 & 4.36 & 8.81 & 10.13 & 1.99 & 4.71 & 3.60 & -1.44 & 3.55 & 3.05 & 5.30 & 5.53 & -- \\
\hline 5 & 4.32 & 8.67 & 10.20 & 2.00 & 4.87 & 3.11 & -0.28 & 4.04 & 2.97 & 5.44 & 5.51 & -- \\
\hline 6 & 4.12 & 8.75 & 9.98 & 2.45 & 4.73 & 2.82 & 0.59 & 3.90 & 3.12 & 5.37 & 5.76 & -- \\
\hline 7 & 4.21 & 8.73 & 9.67 & 1.48 & 4.29 & 2.84 & 1.67 & 3.66 & 3.13 & 5.44 & 5.71 & -- \\
\hline 8 & 4.52 & 8.78 & 9.79 & 2.13 & 4.38 & 2.50 & 2.22 & 3.91 & 3.29 & 5.87 & 5.70 & -- \\
\hline 9 & 4.54 & 8.88 & 9.94 & 3.93 & 3.85 & 2.73 & 2.38 & 3.85 & 3.53 & 5.23 & 5.35 & -- \\
\hline 10 & 4.47 & 9.27 & 9.82 & 4.04 & 3.77 & 2.50 & 2.58 & 3.77 & 2.90 & 5.02 & 5.03 & -- \\
\hline 11 & 4.46 & 9.24 & 9.95 & 4.21 & 4.07 & 2.10 & 2.84 & 4.05 & 2.23 & 5.02 & 5.68 & -- \\
\hline 12 & 4.59 & 9.15 & 9.78 & 4.45 & 4.41 & 2.23 & 2.86 & 4.31 & 2.24 & 5.24 & 5.87 & -- \\
\hline 13 & 4.83 & 9.35 & 8.74 & 3.93 & 3.81 & 1.91 & 3.10 & 3.76 & 3.31 & 5.11 & 5.50 & -- \\
\hline 14 & 4.45 & 9.47 & 8.47 & 5.11 & 4.55 & -0.20 & 3.76 & 3.22 & 3.71 & 4.87 & 5.70 & -- \\
\hline 15 & 4.53 & 9.36 & 8.72 & 4.37 & 5.43 & -0.75 & 3.92 & 3.08 & 3.31 & 4.88 & 5.46 & 2.93 \\
\hline 16 & 5.43 & 9.63 & 9.09 & 4.82 & 5.36 & 0.69 & 4.16 & 3.09 & 3.56 & 4.98 & 5.51 & 2.48 \\
\hline 17 & 4.87 & 8.96 & 9.11 & 4.61 & 5.42 & 0.99 & 4.11 & 2.94 & 3.34 & 4.96 & 5.37 & 2.58 \\
\hline 18 & 4.85 & 9.78 & 9.40 & 4.69 & 5.17 & 1.65 & 4.27 & 3.28 & 3.39 & 4.60 & 5.73 & 3.12 \\
\hline 19 & 5.18 & 10.15 & 8.92 & 4.31 & 5.07 & 1.75 & 4.57 & 3.54 & 3.91 & 4.88 & 5.51 & 4.18 \\
\hline 20 & 6.72 & 10.05 & 9.03 & 4.06 & 4.98 & 1.38 & 4.50 & 3.55 & 3.93 & 4.60 & 5.63 & 4.51 \\
\hline 21 & 7.28 & 9.84 & 9.22 & 4.05 & 5.03 & 0.82 & 4.41 & 3.46 & 3.29 & 4.52 & 5.65 & 4.03 \\
\hline 22 & 7.28 & 10.07 & 8.95 & 3.28 & 5.32 & -0.04 & 4.11 & 2.95 & 3.11 & 4.30 & 6.03 & 3.29 \\
\hline 23 & 7.46 & 10.39 & 8.96 & 3.61 & 5.50 & 0.07 & 4.25 & 3.49 & 2.83 & 4.55 & 5.89 & 3.52 \\
\hline 24 & 7.38 & 10.21 & 9.04 & 4.55 & 5.24 & 0.29 & 4.59 & 3.55 & 2.60 & 4.78 & 5.90 & 3.53 \\
\hline 25 & 7.71 & 10.40 & 8.97 & 4.52 & 4.92 & -0.84 & 4.87 & 3.11 & 3.06 & 4.52 & 6.04 & 3.39 \\
\hline 26 & 7.49 & 9.42 & 9.10 & 4.59 & 4.99 & -1.53 & 4.64 & 3.04 & 3.17 & 4.63 & 5.31 & 3.26 \\
\hline 27 & 7.62 & 9.54 & 8.33 & 5.25 & 5.04 & -1.23 & 4.24 & 3.00 & 2.68 & 4.72 & 5.00 & 3.32 \\
\hline 28 & 8.35 & 10.52 & 8.09 & 5.33 & 5.13 & -1.63 & 4.33 & 2.83 & 2.58 & 4.87 & -- & 3.20 \\
\hline 29 & 8.38 & -- & 8.25 & 4.94 & 4.57 & -1.03 & 4.21 & 2.86 & 4.19 & 5.42 & -- & 3.39 \\
\hline 30 & 8.17 & -- & 7.55 & 4.63 & 4.70 & -0.41 & 4.31 & 2.98 & 5.00 & 4.24 & -- & 3.27 \\
\hline 31 & 8.66 & -- & 5.70 & -- & 4.99 & -. & 4.79 & 3.10 & -- & 5.10 & -- & -- \\
\hline
\end{tabular}


Table 10b. Mean daily ground-water levels in observation well CRM-2 (map ID \#2), West Well Field, Cedar Rapids, lowa, July 1997 through February 1999--Continued

\begin{tabular}{|c|c|c|c|c|c|c|c|c|c|c|c|c|}
\hline Day & Jan & Feb & Mar & Apr & May & Jun & Jul & Aug & Sep & Oct & Nov & Dec \\
\hline & & & & & & 1999 & & & & & & \\
\hline 1 & -- & 3.08 & -- & -. & -- & -- & -- & -- & -- & -- & -- & -- \\
\hline 2 & 3.47 & 3.29 & -- & - & -. & -- & -. & -- & -- & -- & .. & -. \\
\hline 3 & 3.40 & 2.27 & -- & -- & -- & -- & -- & -- & -- & -- & -- & -- \\
\hline 4 & 3.66 & 3.45 & -- & - & -- & -- & -- & -- & -- & -- & -- & -- \\
\hline 5 & 3.35 & 2.88 & -- & - & -- & -- & -- & -- & -- & -- & -- & -- \\
\hline 6 & 3.52 & 2.43 & -- & - & -- & -- & - & -- & -. & -- & -- & -. \\
\hline 7 & 3.66 & 2.90 & -- & -. & -- & -- & -- & -- & -- & -- & -- & -. \\
\hline 8 & 3.69 & 2.90 & -- & - & -- & -- & -- & -- & -- & -- & -- & -. \\
\hline 9 & 4.15 & 2.34 & -- & -. & -- & -- & -- & .- & -- & -- & -- & -- \\
\hline 10 & 4.10 & 2.31 & -- & - & -- & -- & -- & -- & -- & -- & -- & -- \\
\hline 11 & 4.11 & 1.55 & -- & -- & -- & -- & -- & -- & -- & -- & -- & -- \\
\hline 12 & 4.37 & 2.27 & -. & -- & -- & -. & -- & -- & -- & -- & -- & -- \\
\hline 13 & 4.35 & 2.43 & -- & -- & -- & -- & -- & -- & -- & -- & -- & -- \\
\hline 14 & 4.18 & 2.29 & -- & -- & -- & -- & -- & -- & -- & -- & -- & -. \\
\hline 15 & 4.19 & 2.64 & -- & -- & -- & -- & -- & -- & -- & -- & -- & -- \\
\hline 16 & 4.52 & 2.43 & -. & -- & -- & -. & -- & -. & -- & -- & -- & -. \\
\hline 17 & 4.03 & 2.18 & -- & -- & -- & -- & -- & -- & -- & -- & -- & -- \\
\hline 18 & 4.18 & 2.17 & -- & -- & -- & -- & -- & -- & -- & - & -- & -- \\
\hline 19 & 4.28 & 2.21 & -- & -- & -- & -- & -- & -- & -- & -- & -- & -- \\
\hline 20 & 4.51 & 2.71 & -- & -- & -- & -- & -. & -- & -- & -- & -- & -- \\
\hline 21 & 4.79 & 2.43 & -- & -- & -- & -. & -- & -- & -- & -. & -- & -- \\
\hline 22 & 4.45 & 2.56 & -- & -- & - & -- & -- & -- & -- & -- & -- & -- \\
\hline 23 & 4.59 & 2.33 & -- & -- & -- & -- & -- & -- & -- & -- & -- & -- \\
\hline 24 & 4.23 & 2.28 & -- & -- & -- & -. & -. & -- & -. & -- & -- & -- \\
\hline 25 & 4.48 & 2.32 & -- & -- & -- & - & -- & -- & -- & -- & -- & -- \\
\hline 26 & 4.57 & 2.49 & -- & -- & -- & -- & -- & -. & - & -- & -- & -- \\
\hline 27 & 3.36 & 2.28 & -- & -- & -- & - & -- & -- & -- & -- & -- & -- \\
\hline 28 & 3.39 & 2.61 & -- & -- & -- & -- & -- & -- & -- & - & -- & -- \\
\hline 29 & 3.18 & -- & -- & -- & -- & - & -. & -- & -. & -- & -- & -- \\
\hline 30 & 3.13 & -- & -- & -- & -- & - & -- & -- & -- & -- & -- & -- \\
\hline 31 & 3.32 & -- & -- & -- & -. & -. & -- & -- & -- & -- & -- & -- \\
\hline
\end{tabular}


Table 10c. Mean daily ground-water levels in observation well CRM-3 (map ID \#3), Seminole Well Field, Cedar Rapids, lowa, January 1997 through February 1999

[Water levels measured as feet below land surface; --, value not measured or recorded]

\begin{tabular}{|c|c|c|c|c|c|c|c|c|c|c|c|c|}
\hline$\overline{\text { Day }}$ & Jan & Feb & Mar & Apr & May & Jun & Jul & Aug & Sep & Oct & Nov & Dec \\
\hline \multicolumn{13}{|c|}{1997} \\
\hline 1 & -. & 14.37 & 7.71 & 5.19 & 8.62 & 8.49 & 7.01 & 9.49 & 10.66 & 11.37 & 12.69 & 14.56 \\
\hline 2 & -- & 14.65 & 7.24 & 5.38 & 7.94 & 8.68 & 6.97 & 9.70 & 10.36 & 11.44 & 11.21 & 14.58 \\
\hline 3 & -- & 15.20 & 7.02 & 6.07 & 7.24 & 8.73 & 7.16 & 9.97 & 10.22 & 11.22 & 13.14 & 14.63 \\
\hline 4 & -- & 15.38 & 6.51 & 6.54 & 6.57 & 8.70 & 7.33 & 10.74 & 10.95 & 11.21 & 13.94 & 14.77 \\
\hline 5 & -- & 15.11 & 6.09 & 6.36 & 6.27 & 8.84 & 7.69 & 10.16 & 11.24 & 11.20 & 13.81 & 14.78 \\
\hline 6 & -- & 15.10 & 5.81 & 6.77 & 6.18 & 8.98 & 8.17 & 10.08 & 11.12 & 11.40 & 13.46 & 15.06 \\
\hline 7 & -- & 15.18 & 6.04 & 7.05 & 6.16 & 9.06 & 8.37 & 10.09 & 11.14 & 11.44 & 13.57 & 15.32 \\
\hline 8 & -- & 15.35 & 6.22 & 6.97 & 6.09 & 8.78 & 8.62 & 10.25 & 11.16 & 11.29 & 13.67 & 15.26 \\
\hline 9 & -- & 15.38 & 7.23 & 6.70 & 6.19 & 9.03 & 8.56 & 10.17 & 11.22 & 11.55 & 13.54 & 15.48 \\
\hline 10 & -. & 15.44 & 8.02 & 6.53 & 6.21 & 8.96 & 8.69 & 10.55 & 11.33 & 11.66 & 13.64 & 15.41 \\
\hline 11 & -- & 15.54 & 7.55 & 6.67 & 6.33 & 9.12 & 8.61 & 10.64 & 11.26 & 12.73 & 13.69 & 15.43 \\
\hline 12 & -- & 15.63 & 6.52 & 7.04 & 6.54 & 8.97 & 8.68 & 10.69 & 11.37 & 12.45 & 13.60 & 15.34 \\
\hline 13 & -- & 15.70 & 5.77 & 7.29 & 6.73 & 9.02 & 8.79 & 10.60 & 11.45 & 13.03 & 13.56 & 15.34 \\
\hline 14 & -- & 15.74 & 5.01 & 7.41 & 6.94 & 9.25 & 8.86 & 10.20 & 11.53 & 13.67 & 13.64 & 15.28 \\
\hline 15 & -- & 15.81 & 4.25 & 7.38 & 7.27 & 9.08 & 8.73 & 9.28 & 11.28 & 13.71 & 13.70 & 14.68 \\
\hline 16 & -- & 15.90 & 3.37 & 7.41 & 7.63 & 8.89 & 8.49 & 10.43 & 10.97 & 13.85 & 13.39 & 14.66 \\
\hline 17 & -- & 15.94 & 3.08 & 7.55 & 7.91 & 9.02 & 8.17 & 10.48 & 11.05 & 14.65 & 13.92 & 14.84 \\
\hline 18 & -- & 16.09 & 4.16 & 7.81 & 8.24 & 9.11 & 7.94 & 10.43 & 10.96 & 15.41 & 14.32 & 14.99 \\
\hline 19 & -- & 15.42 & 5.30 & 8.06 & 7.67 & 9.34 & 7.91 & 10.47 & 10.96 & 15.68 & 14.45 & 15.13 \\
\hline 20 & -- & 13.45 & 5.76 & 8.19 & 8.06 & 9.16 & 8.21 & 10.49 & 10.83 & 15.68 & 14.40 & 15.11 \\
\hline 21 & -- & 9.73 & 5.53 & 8.25 & 8.14 & 9.14 & 8.47 & 10.74 & 10.58 & 15.33 & 14.39 & 15.27 \\
\hline 22 & -- & 6.86 & 5.68 & 8.36 & 8.27 & 8.87 & 8.35 & 10.73 & 10.98 & 14.76 & 14.59 & 15.21 \\
\hline 23 & -- & 5.71 & 5.41 & 8.47 & 8.34 & 8.14 & 8.40 & 10.57 & 10.90 & 14.50 & 14.85 & 15.40 \\
\hline 24 & 12.30 & 5.50 & 4.94 & 8.47 & 8.45 & 7.94 & 8.47 & 10.53 & 10.99 & 14.53 & 14.64 & 14.96 \\
\hline 25 & 12.56 & 6.19 & 4.57 & 8.61 & 8.51 & 7.12 & 9.20 & 10.76 & 10.90 & 14.46 & 14.76 & 15.11 \\
\hline 26 & 12.72 & 7.00 & 4.10 & 8.66 & 8.54 & 6.11 & 8.96 & 10.81 & 10.85 & 14.27 & 14.69 & 15.16 \\
\hline 27 & 12.96 & 7.62 & 3.60 & 8.70 & 8.55 & 6.16 & 8.92 & 10.88 & 10.66 & 14.85 & 14.48 & 15.07 \\
\hline 28 & 13.03 & 7.97 & 3.28 & 8.85 & 8.44 & 6.44 & 8.74 & 10.86 & 10.53 & 15.03 & 14.65 & 13.85 \\
\hline 29 & 13.41 & -- & 4.36 & 8.88 & 8.28 & 6.75 & 9.04 & 11.12 & 9.33 & 15.36 & 14.59 & 14.24 \\
\hline 30 & 13.76 & -- & 4.99 & 8.44 & 8.39 & 6.64 & 9.09 & 11.22 & 10.93 & 15.20 & 14.48 & 14.10 \\
\hline 31 & 14.03 & -. & 5.21 & -- & 8.39 & -- & 8.88 & 10.83 & -- & 13.10 & -- & 14.62 \\
\hline
\end{tabular}


Table 10c. Mean daily ground-water levels in observation well CRM-3 (map ID \#3), Seminole Well Field, Cedar Rapids, lowa, January 1997 through February 1999--Continued

\begin{tabular}{|c|c|c|c|c|c|c|c|c|c|c|c|c|}
\hline Day & Jan & Feb & Mar & Apr & May & Jun & Jul & Aug & Sep & Oct & Nov & Dec \\
\hline \multicolumn{13}{|c|}{1998} \\
\hline 1 & 14.83 & 14.74 & 9.46 & 2.57 & 7.37 & 6.27 & -- & -- & 9.58 & 10.22 & 8.30 & 9.62 \\
\hline 2 & 14.99 & 15.01 & 9.07 & 3.46 & 7.46 & 6.55 & -- & -- & 9.65 & 10.48 & 8.37 & 9.36 \\
\hline 3 & 14.99 & 14.86 & 9.04 & 3.35 & 7.44 & 7.14 & -- & -- & 9.49 & 10.41 & 8.48 & -- \\
\hline 4 & 14.80 & 15.16 & 9.16 & 3.62 & 7.49 & 7.22 & -. & -. & 9.77 & 10.31 & 8.73 & -- \\
\hline 5 & 14.78 & 15.26 & 9.25 & 3.83 & 7.59 & 7.48 & -- & -- & 10.01 & 9.52 & 8.95 & 9.33 \\
\hline 6 & 14.91 & 15.33 & 9.36 & 3.85 & 7.70 & 7.66 & -- & -- & 9.76 & 9.19 & 8.80 & 9.39 \\
\hline 7 & 15.02 & 15.27 & 9.40 & 1.80 & 7.47 & 7.69 & - & -- & 9.73 & 9.36 & 8.92 & 9.48 \\
\hline 8 & 15.00 & 15.28 & 9.21 & 2.64 & 7.63 & 7.78 & -- & -- & 10.05 & 9.36 & 9.44 & 9.46 \\
\hline 9 & 15.28 & 15.27 & 9.55 & 5.19 & 7.75 & 7.48 & -- & -- & 10.09 & 9.62 & 9.36 & 9.11 \\
\hline 10 & 15.47 & 15.29 & 8.91 & 5.83 & 7.88 & 6.83 & -- & -- & 10.20 & 9.67 & 6.08 & 8.76 \\
\hline 11 & 15.44 & 15.11 & 8.53 & 5.76 & 7.83 & 5.37 & - & -- & 10.17 & 9.63 & 8.28 & 9.10 \\
\hline 12 & 15.47 & 15.35 & 8.82 & 5.31 & 8.02 & 5.46 & -- & -- & 9.98 & 9.70 & 8.57 & 9.46 \\
\hline 13 & 14.68 & 15.51 & 9.39 & 4.36 & 8.04 & 4.98 & -. & -- & 9.95 & 9.81 & 8.81 & 9.47 \\
\hline 14 & 14.99 & 15.88 & 9.36 & 6.03 & 8.04 & 1.97 & -- & 10.09 & 9.68 & 9.69 & 8.28 & 9.35 \\
\hline 15 & 14.72 & 15.88 & 9.36 & 5.08 & 8.09 & 1.63 & -- & 10.12 & 9.93 & 9.44 & 8.71 & 9.56 \\
\hline 16 & 14.67 & 15.73 & 9.78 & 5.57 & 8.28 & 3.64 & -- & 10.22 & 10.11 & 9.43 & 7.87 & 9.63 \\
\hline 17 & 14.42 & 14.60 & 10.32 & 6.00 & 8.31 & 4.69 & -- & 9.83 & 10.24 & 7.86 & 9.03 & 9.56 \\
\hline 18 & 14.54 & 15.70 & 10.36 & 6.05 & 8.29 & 5.27 & -- & 10.15 & 10.17 & 7.72 & 8.53 & 8.82 \\
\hline 19 & 14.63 & 15.94 & 10.16 & 5.75 & 8.21 & 5.18 & -- & 10.11 & 10.01 & 7.18 & 8.77 & 9.47 \\
\hline 20 & 14.58 & 15.82 & 10.13 & 5.36 & 8.40 & 4.92 & -- & 10.06 & 9.80 & 6.94 & 9.14 & 9.40 \\
\hline 21 & 14.67 & 15.60 & 10.00 & 5.39 & 8.37 & 4.45 & -- & 10.23 & 10.01 & 7.14 & 9.36 & 9.14 \\
\hline 22 & 14.86 & 14.80 & 9.75 & 5.51 & 8.22 & 3.65 & -- & 10.18 & 10.24 & 7.30 & 8.90 & 7.77 \\
\hline 23 & 14.79 & 13.60 & 9.56 & 5.48 & 8.22 & -- & -- & 10.09 & 10.40 & 8.06 & 9.16 & 8.59 \\
\hline 24 & 14.95 & 12.65 & 9.26 & 5.47 & 8.12 & -- & -- & 9.84 & 10.15 & 8.76 & 9.44 & 8.60 \\
\hline 25 & 14.87 & 11.48 & 8.83 & 5.20 & 8.00 & -- & -- & 9.78 & 10.07 & 9.07 & 9.07 & 9.00 \\
\hline 26 & 15.08 & 9.15 & 8.46 & 5.51 & 7.65 & -- & -- & 10.18 & 9.88 & 9.30 & 9.27 & 8.86 \\
\hline 27 & 15.00 & 8.98 & 7.13 & 6.04 & 7.19 & -- & -- & 10.00 & 9.97 & 9.26 & 9.49 & 8.86 \\
\hline 28 & 14.97 & 10.04 & 6.51 & 6.35 & 6.97 & -- & -- & 9.69 & 10.30 & 9.30 & 9.52 & 8.87 \\
\hline 29 & 14.86 & -. & 6.92 & 7.02 & 6.70 & -- & -- & 9.77 & 9.97 & 8.34 & 8.76 & 8.91 \\
\hline 30 & 14.85 & -- & 5.74 & 7.12 & 6.02 & -- & -- & 9.82 & 9.82 & 8.27 & 9.08 & 9.28 \\
\hline 31 & 14.85 & -- & 2.20 & -- & 6.07 & -- & -- & 9.84 & -- & 8.13 & -- & 9.54 \\
\hline
\end{tabular}


Table 10c. Mean daily ground-water levels in observation well CRM-3 (map ID \#3), Seminole Well Field, Cedar Rapids, lowa, January 1997 through February 1999--Continued

\begin{tabular}{|c|c|c|c|c|c|c|c|c|c|c|c|c|}
\hline Day & Jan & Feb & Mar & Apr & May & Jun & Jul & Aug & Sep & Oct & Nov & $\overline{\text { Dec }}$ \\
\hline & & & & & & 1999 & & & & & & \\
\hline 1 & 9.37 & 9.17 & -- & .. & -. & -- & -- & -. & -. & -- & -- & -- \\
\hline 2 & 9.46 & 9.07 & -- & -- & -- & -- & -- & -- & -- & -- & -. & - \\
\hline 3 & 9.50 & 7.14 & -- & -- & - & -- & -- & -- & -- & -. & -- & -- \\
\hline 4 & 9.30 & 8.62 & -. & -- & -- & -- & -- & -- & -- & -. & -. & -- \\
\hline 5 & 9.47 & 8.64 & -- & -- & -- & -- & -- & -- & -- & -- & -. & -- \\
\hline 6 & 9.51 & 8.64 & -- & -- & -- & -- & -- & -- & -- & -- & -- & -- \\
\hline 7 & 9.18 & 8.59 & -- & -- & -- & -- & -- & .. & -- & -- & -- & -- \\
\hline 8 & 9.67 & 8.55 & -- & -- & -- & -- & -- & -- & -- & -- & -- & -- \\
\hline 9 & 9.44 & 8.39 & -- & -- & -- & -- & -- & -- & -- & -- & -- & -- \\
\hline 10 & 9.51 & 8.17 & -- & -- & -- & -- & -. & -- & -- & -- & -- & -- \\
\hline 11 & 9.29 & 6.82 & -- & -- & -- & -- & -- & -- & -- & -- & -- & -- \\
\hline 12 & 9.48 & 7.78 & -- & -- & -- & -- & -- & -- & -- & -- & -- & -- \\
\hline 13 & 9.31 & 7.04 & -- & -- & -- & -- & -- & -- & -- & -- & -- & -- \\
\hline 14 & 9.51 & 7.66 & -. & -- & -- & -- & -. & -- & -- & -- & -- & -- \\
\hline 15 & 9.23 & 7.81 & -- & -- & -- & -. & -. & -- & -- & -- & -- & -- \\
\hline 16 & 9.26 & 7.70 & -- & -- & - & -- & -. & -- & -- & -- & -- & -- \\
\hline 17 & 8.28 & 7.72 & -- & -- & -- & -- & -. & -- & -- & -- & -- & -. \\
\hline 18 & 8.52 & 7.74 & -- & -- & -- & -- & -- & -- & -- & -- & -- & -- \\
\hline 19 & 9.39 & 7.97 & -- & -- & -- & -- & -- & -- & -- & -- & -- & -- \\
\hline 20 & 9.33 & 7.71 & -- & -- & -- & -- & -. & -- & -. & -- & -- & -- \\
\hline 21 & 9.27 & 7.25 & $\ldots$ & -- & -- & -- & -. & -- & -. & -- & -- & -- \\
\hline 22 & 8.83 & 7.97 & -- & -- & -- & -- & -- & -- & -- & -- & -- & -- \\
\hline 23 & 9.09 & 8.42 & -. & -- & -- & -- & -- & -. & -. & -. & -- & -- \\
\hline 24 & 9.35 & 8.51 & -- & -- & -- & -- & -- & -- & -- & -- & -- & -- \\
\hline 25 & 8.74 & 8.70 & -- & -- & - & -- & -- & -- & -- & -- & -- & -- \\
\hline 26 & 9.13 & 8.67 & -- & -- & -- & -- & -- & -- & -. & -- & -- & -- \\
\hline 27 & 9.24 & 8.20 & -- & -- & -- & -- & -- & -- & -- & -- & -- & -- \\
\hline 28 & 9.23 & 8.60 & -- & -- & -- & -- & -- & -- & -- & -- & -- & -- \\
\hline 29 & 8.58 & -- & -- & -- & -- & -- & -. & -. & - & -- & -- & -- \\
\hline 30 & 8.47 & -- & -- & -- & -. & -- & -. & -- & -- & -- & -- & -- \\
\hline 31 & 9.10 & -- & -- & -- & -. & -. & -. & -- & -. & -- & -- & -- \\
\hline
\end{tabular}


Table 10d. Mean daily ground-water levels in observation well CRM-4 (map ID \#4), Seminole Well Field, Cedar Rapids, lowa, January 1997 through June 1998

[Water levels measured as feet below land surface; --, value not measured or recorded]

\begin{tabular}{|c|c|c|c|c|c|c|c|c|c|c|c|c|}
\hline Day & Jan & Feb & Mar & Apr & May & Jun & Jul & Aug & Sep & Oct & Nov & Dec \\
\hline \multicolumn{13}{|c|}{1997} \\
\hline 1 & -- & 14.28 & 7.05 & 4.40 & 7.78 & 7.52 & 5.88 & 7.82 & 10.23 & 10.63 & -- & 13.89 \\
\hline 2 & -- & 14.68 & 6.60 & 4.61 & 7.12 & 7.52 & 5.89 & 8.10 & 9.82 & 10.19 & -- & 13.62 \\
\hline 3 & -- & 15.27 & 6.35 & 5.34 & 6.15 & 7.76 & 5.93 & 8.52 & 9.58 & 7.56 & -- & 13.22 \\
\hline 4 & -- & 15.37 & 5.80 & 5.76 & 5.66 & 7.98 & 6.12 & 9.30 & 10.51 & 9.50 & 13.15 & 13.65 \\
\hline 5 & -- & 14.90 & 5.30 & 6.12 & 5.29 & 8.16 & 6.45 & 8.60 & 10.10 & 9.35 & 13.62 & 12.78 \\
\hline 6 & -- & 14.92 & 4.92 & 6.26 & 5.13 & 8.24 & 6.66 & 8.36 & 9.39 & 9.99 & 13.17 & 13.31 \\
\hline 7 & -- & 15.07 & 5.33 & 6.30 & 5.19 & 8.12 & 7.18 & 8.38 & 9.52 & 10.34 & 13.32 & 14.11 \\
\hline 8 & - & 15.12 & 5.68 & 6.11 & 5.18 & 8.48 & 7.15 & 8.52 & 9.79 & 9.60 & 13.17 & 13.59 \\
\hline 9 & - & 15.24 & 6.75 & 5.86 & 5.22 & 8.86 & 7.56 & 8.65 & 9.80 & 10.83 & 12.62 & 14.03 \\
\hline 10 & - & 15.45 & 7.55 & 5.71 & 5.29 & 8.67 & 7.83 & 8.65 & 9.65 & 11.32 & 13.24 & 13.37 \\
\hline 11 & -- & 15.56 & 6.91 & 5.94 & 5.36 & 8.21 & 7.66 & 8.65 & 9.65 & 11.80 & 13.33 & 14.67 \\
\hline 12 & - & 15.56 & 5.76 & 6.19 & 5.55 & 8.24 & 7.30 & 8.80 & 9.65 & 10.22 & 12.68 & 14.58 \\
\hline 13 & -- & 15.66 & 4.78 & 6.49 & 5.73 & 8.27 & 7.29 & 8.76 & 9.65 & 11.42 & 11.87 & 13.74 \\
\hline 14 & - & 15.76 & 4.11 & 6.60 & 5.97 & 8.15 & 7.27 & 7.82 & 9.65 & 13.57 & 12.43 & 14.28 \\
\hline 15 & -. & 15.84 & 3.17 & 6.57 & 6.31 & 8.09 & 7.18 & 6.67 & 9.65 & 13.52 & 12.77 & 12.76 \\
\hline 16 & -- & 15.85 & 2.36 & 6.65 & 6.66 & 8.00 & 6.69 & 8.42 & 8.22 & 13.58 & 13.01 & 13.02 \\
\hline 17 & -- & 15.98 & 2.22 & 6.76 & 6.89 & 8.14 & 6.37 & 9.35 & 9.27 & 14.60 & 13.57 & 12.98 \\
\hline 18 & -- & 16.11 & 3.33 & 6.99 & 7.64 & 8.20 & 6.28 & 10.16 & 9.16 & 14.75 & 13.59 & 13.45 \\
\hline 19 & -- & 15.42 & 4.55 & 7.22 & 6.66 & 8.41 & 6.22 & 9.46 & 9.07 & 14.74 & 13.65 & 13.53 \\
\hline 20 & -- & 13.16 & 5.00 & 7.43 & 7.13 & 8.29 & 6.54 & 8.81 & 10.02 & 15.46 & 12.94 & 14.60 \\
\hline 21 & - & 8.96 & 4.75 & 7.52 & 7.07 & 8.15 & 6.66 & 9.72 & 9.77 & 15.00 & 12.53 & 14.05 \\
\hline 22 & -- & 6.11 & 4.83 & 7.62 & 7.27 & 7.63 & 6.78 & 10.23 & 10.17 & 14.34 & 12.95 & 13.27 \\
\hline 23 & -- & 5.00 & 4.61 & 7.71 & 7.49 & 6.63 & 6.86 & 9.73 & 10.14 & 13.23 & 14.20 & 13.90 \\
\hline 24 & 11.80 & 4.94 & 4.08 & 7.78 & 7.63 & 6.67 & 7.07 & 8.85 & 9.68 & 13.30 & 14.09 & 11.82 \\
\hline 25 & 12.14 & 5.62 & 3.68 & 7.88 & 7.68 & 5.69 & 8.32 & 9.54 & 9.08 & 13.85 & 12.50 & 13.37 \\
\hline 26 & 12.44 & 6.45 & 3.25 & 7.91 & 7.76 & 4.96 & 8.57 & 9.09 & 8.68 & 12.75 & 12.66 & 13.27 \\
\hline 27 & 12.75 & 7.05 & 2.75 & 7.91 & 7.72 & 4.79 & 8.44 & 9.36 & 7.86 & 14.04 & 11.30 & 13.10 \\
\hline 28 & 12.71 & 7.42 & 2.91 & 7.91 & 7.57 & 4.78 & 8.34 & 9.09 & 6.83 & 13.38 & 12.60 & 10.13 \\
\hline 29 & 13.20 & -- & 3.54 & 7.93 & 7.41 & 5.45 & 8.20 & 9.64 & 9.36 & 14.28 & 13.24 & 9.95 \\
\hline 30 & 13.72 & -- & 4.26 & 7.93 & 7.40 & 5.27 & 8.33 & 9.95 & 8.97 & 13.17 & 12.93 & 11.60 \\
\hline 31 & 14.00 & -- & 4.45 & -- & 7.45 & -- & 7.97 & 10.47 & -- & -. & -- & 14.05 \\
\hline
\end{tabular}


Table 10d. Mean daily ground-water levels in observation well CRM-4 (map ID \#4), Seminole Well Field, Cedar Rapids, lowa, January 1997 through June 1998--Continued

\begin{tabular}{|c|c|c|c|c|c|c|c|c|c|c|c|c|}
\hline Day & Jan & Feb & Mar & Apr & May & Jun & Jul & Aug & Sep & Oct & Nov & Dec \\
\hline \multicolumn{13}{|c|}{1998} \\
\hline 1 & 13.13 & 14.75 & 9.11 & 3.18 & 6.92 & 6.47 & -- & -. & -- & -- & -- & -- \\
\hline 2 & 12.95 & 14.42 & 8.84 & 2.81 & 7.02 & 6.81 & -- & -- & -- & -- & -- & -- \\
\hline 3 & 13.44 & 13.97 & 8.72 & 2.58 & 7.21 & 7.20 & -- & -- & -- & -- & -- & -- \\
\hline 4 & 13.33 & 14.33 & 8.70 & 2.10 & 7.32 & 7.37 & -- & -- & -- & -- & -- & -- \\
\hline 5 & 12.68 & 14.84 & 8.00 & 2.83 & 7.34 & 7.25 & -. & -- & -- & -- & -- & -- \\
\hline 6 & 13.30 & 14.82 & 8.16 & 3.39 & 7.44 & 6.74 & -- & -- & -- & -- & -- & -- \\
\hline 7 & 13.55 & 15.17 & 8.79 & 3.15 & 7.40 & 6.76 & -- & -- & -- & -- & -- & -- \\
\hline 8 & 11.74 & 15.23 & 9.13 & 3.82 & 7.55 & 7.31 & -- & -- & -- & -- & -- & -- \\
\hline 9 & 12.51 & 15.34 & 9.04 & 4.89 & 7.63 & 7.53 & -- & -- & -. & -. & -. & -- \\
\hline 10 & 14.66 & 15.33 & 9.06 & 4.84 & 7.60 & 7.03 & -. & -- & -- & -- & -- & -- \\
\hline 11 & 14.61 & 15.28 & 9.24 & 4.94 & 7.73 & 6.24 & - & -- & -. & -- & -- & -- \\
\hline 12 & 13.95 & 15.45 & 8.93 & 5.12 & 7.63 & 5.44 & -- & -- & -- & -. & -- & -- \\
\hline 13 & 14.02 & 15.68 & 8.95 & 5.16 & 7.72 & 4.10 & -- & -- & -- & -- & -- & -. \\
\hline 14 & 13.81 & 15.48 & 8.92 & 5.49 & 7.91 & 2.81 & - & -- & -- & -- & -- & -- \\
\hline 15 & 12.79 & 16.23 & 9.14 & 5.49 & 7.92 & 2.20 & -- & -- & -- & -. & -- & -- \\
\hline 16 & 13.38 & 16.15 & 9.13 & 5.52 & 7.52 & 3.10 & - & -- & -- & -- & -- & -- \\
\hline 17 & 12.72 & 16.12 & 9.83 & 5.19 & 7.32 & 4.11 & -- & -- & -- & -- & -- & -- \\
\hline 18 & 13.54 & 16.19 & 10.26 & 4.91 & 7.94 & 4.44 & -- & -- & -- & -. & .- & -. \\
\hline 19 & 13.63 & 16.29 & 10.37 & 5.23 & 8.15 & 4.20 & -- & -- & -- & -- & -- & -- \\
\hline 20 & 12.83 & 16.25 & 10.01 & 5.03 & 8.02 & 3.99 & -- & -- & -- & -- & -- & -- \\
\hline 21 & 12.70 & 15.88 & 9.63 & 4.42 & 8.17 & 3.74 & -- & -- & -- & -- & -- & -- \\
\hline 22 & 13.21 & 15.20 & 9.52 & 4.34 & 8.16 & 2.58 & -- & -- & -- & -- & -- & -- \\
\hline 23 & 12.52 & 13.91 & 8.98 & 4.97 & 8.14 & -- & -- & -- & -- & -- & -- & -- \\
\hline 24 & 13.20 & 12.52 & 8.25 & 5.12 & 8.14 & -- & -- & -- & -- & -- & -- & -- \\
\hline 25 & 12.55 & 11.55 & 8.56 & 5.24 & 8.01 & -- & -- & -- & -- & -- & -- & -- \\
\hline 26 & 13.93 & 10.76 & 7.98 & 5.41 & 7.61 & -- & -- & -- & -- & -. & -- & -- \\
\hline 27 & 15.07 & 10.21 & 7.39 & 5.00 & 7.19 & -- & -- & -- & -- & -- & -- & -- \\
\hline 28 & 14.83 & 9.72 & 6.92 & 5.33 & 7.05 & -- & -- & -- & -- & -- & -- & -- \\
\hline 29 & 14.95 & -- & 6.97 & 6.31 & 6.80 & -- & -- & -- & -- & -- & -- & -- \\
\hline 30 & 14.96 & -- & 6.64 & 6.92 & 6.42 & -- & -- & -- & -- & -- & -- & -- \\
\hline 31 & 14.86 & -- & 3.81 & -- & 6.23 & -- & -- & -- & -- & -. & -- & -- \\
\hline
\end{tabular}


Table 10e. Mean daily ground-water levels in observation well CRM-6 (map ID \#5), Seminole Well Field, Cedar Rapids, lowa, January 1997 through February 1999

[Water levels measured as feet below land surface; --, value not measured or recorded]

\begin{tabular}{|c|c|c|c|c|c|c|c|c|c|c|c|c|}
\hline Day & Jan & Feb & Mar & Apr & May & Jun & Jul & Aug & Sep & Oct & Nov & Dec \\
\hline & & & & & & 1997 & & & & & & \\
\hline 1 & -- & 5.97 & 0.47 & -1.89 & -0.37 & -1.06 & -2.00 & 1.09 & 1.20 & 0.04 & 1.85 & 1.81 \\
\hline 2 & -- & 5.93 & 0.24 & -1.84 & -1.07 & -1.05 & -2.02 & 1.16 & 0.78 & 0.27 & 1.29 & 1.78 \\
\hline 3 & -- & 6.29 & 0.03 & -1.89 & -1.23 & -0.70 & -2.09 & 1.33 & 0.61 & 0.14 & 1.96 & 1.69 \\
\hline 4 & -- & 6.55 & -0.62 & -1.66 & -1.33 & -0.56 & -1.93 & 1.98 & 1.00 & 0.46 & 2.25 & 1.72 \\
\hline 5 & -. & 6.33 & -1.26 & -2.61 & -1.51 & -0.56 & -1.61 & 1.98 & 0.87 & 0.56 & 2.38 & 1.89 \\
\hline 6 & -- & 6.15 & -1.47 & -1.92 & -1.51 & -0.46 & -1.42 & 1.83 & 0.93 & 0.64 & 2.24 & 1.94 \\
\hline 7 & - & 6.02 & -1.36 & -0.84 & -1.65 & -0.48 & -1.05 & 1.86 & 0.95 & 0.62 & 2.21 & 1.95 \\
\hline 8 & -. & 6.43 & -1.30 & -1.17 & -1.72 & -0.41 & -1.08 & 1.67 & 0.95 & 0.64 & 1.91 & 2.00 \\
\hline 9 & $-\cdot$ & 6.13 & -0.07 & -1.55 & -1.57 & -0.27 & -0.67 & 1.60 & 0.89 & 0.88 & 1.75 & 1.88 \\
\hline 10 & -- & 6.09 & 0.35 & -1.62 & -1.68 & -0.26 & -0.48 & 2.09 & 0.86 & 0.95 & 1.84 & 1.94 \\
\hline 11 & -- & 6.30 & -0.34 & -1.57 & -1.79 & -0.38 & -0.53 & 2.40 & 0.77 & 1.21 & 1.79 & 2.16 \\
\hline 12 & -- & 6.36 & -1.49 & -1.44 & -1.79 & -0.41 & -0.63 & 1.93 & 0.75 & 0.99 & 1.70 & 2.16 \\
\hline 13 & -- & 6.33 & -1.85 & -1.16 & -1.79 & -0.31 & -0.76 & 1.75 & 0.74 & 1.34 & 1.54 & 1.99 \\
\hline 14 & -- & 6.48 & -2.43 & -0.96 & -1.91 & -0.09 & -0.65 & 1.14 & 0.77 & 1.95 & 1.66 & 1.90 \\
\hline 15 & -- & 6.17 & -2.63 & -1.11 & -1.64 & -0.33 & -0.53 & 0.52 & 0.72 & 2.02 & 1.86 & 1.82 \\
\hline 16 & -- & 6.59 & -2.99 & -0.82 & -1.42 & -0.35 & -0.76 & 0.98 & 0.46 & 2.09 & 1.77 & 1.83 \\
\hline 17 & -- & 6.42 & -2.32 & -0.88 & -1.40 & -0.11 & -0.89 & 1.18 & 0.64 & 2.70 & 1.87 & 1.86 \\
\hline 18 & -- & 6.43 & -2.59 & -1.17 & -1.07 & -0.08 & -0.77 & 1.32 & 0.43 & 2.90 & 1.75 & 1.84 \\
\hline 19 & -- & 5.66 & -2.30 & -1.02 & -0.88 & 0.13 & -0.41 & 1.06 & 0.27 & 3.31 & 1.71 & 1.91 \\
\hline 20 & -- & 4.53 & -1.93 & -0.90 & -0.33 & 0.17 & -0.01 & 1.04 & 0.31 & 3.68 & 1.81 & 2.14 \\
\hline 21 & -- & 1.92 & -2.19 & -0.78 & -0.44 & 0.07 & 0.23 & 0.99 & 0.18 & 3.51 & 1.90 & 1.95 \\
\hline 22 & -- & 0.43 & -2.08 & -0.70 & -0.40 & -0.01 & 0.34 & 1.04 & 0.18 & 3.07 & 1.87 & 1.92 \\
\hline 23 & -. & 0.24 & -2.00 & -0.61 & -0.52 & -0.20 & 0.29 & 1.03 & 0.23 & 2.80 & 1.94 & 1.94 \\
\hline 24 & 4.68 & 0.16 & -2.09 & -0.59 & -0.57 & -0.10 & 0.43 & 1.00 & 0.04 & 2.74 & 2.06 & 1.60 \\
\hline 25 & 4.81 & -0.04 & -2.15 & -0.19 & -0.55 & -0.20 & 0.60 & 0.93 & -0.12 & 2.67 & 1.80 & 1.81 \\
\hline 26 & 4.77 & -0.03 & -2.16 & -0.13 & -0.49 & -1.24 & 0.59 & 0.99 & -0.15 & 2.63 & 1.79 & 1.93 \\
\hline 27 & 4.95 & 0.27 & -2.18 & -0.31 & -0.35 & -1.84 & 0.69 & 0.93 & -0.37 & 2.75 & 1.65 & 1.81 \\
\hline 28 & 5.47 & 0.78 & -2.90 & -0.23 & -0.71 & -2.10 & 1.09 & 0.95 & -0.54 & 3.10 & 1.79 & 1.13 \\
\hline 29 & 5.29 & -. & -2.10 & -0.25 & -0.97 & -2.04 & 1.33 & 1.10 & -0.95 & 3.21 & 1.79 & 1.28 \\
\hline 30 & 5.31 & -- & -2.06 & -1.32 & -0.92 & -2.08 & 1.25 & 1.14 & -0.14 & 3.04 & 1.78 & 1.35 \\
\hline 31 & 5.40 & -- & -1.87 & .. & -0.96 & -- & 1.22 & 1.30 & -- & 2.11 & -- & 1.87 \\
\hline
\end{tabular}


Table 10e. Mean daily ground-water levels in observation well CRM-6 (map ID \#5), Seminole Well Field, Cedar Rapids, lowa, January 1997 through February 1999--Continued

\begin{tabular}{|c|c|c|c|c|c|c|c|c|c|c|c|c|}
\hline Day & Jan & Feb & Mar & Apr & May & Jun & Jul & Aug & Sep & Oct & Nov & Dec \\
\hline & & & & & & 1998 & & & & & & \\
\hline 1 & 1.77 & 1.05 & -0.85 & -2.31 & -1.74 & -1.87 & -2.00 & 1.09 & -2.05 & -2.13 & -2.29 & -1.78 \\
\hline 2 & 1.66 & 1.41 & -0.89 & -1.82 & -1.75 & -1.90 & -2.02 & 1.16 & -2.04 & -2.28 & -2.67 & -1.96 \\
\hline 3 & 1.66 & 1.58 & -1.00 & -1.85 & -1.69 & -1.81 & -2.09 & 1.33 & -2.44 & -2.37 & -2.68 & -- \\
\hline 4 & 1.66 & 1.38 & -1.01 & -1.73 & -1.68 & -1.76 & -1.94 & 1.98 & -2.21 & -2.11 & -2.38 & -- \\
\hline 5 & 1.73 & 1.27 & -0.91 & -1.79 & -1.69 & -1.87 & -1.62 & 1.99 & -2.12 & -2.38 & -2.53 & -2.07 \\
\hline 6 & 1.62 & 1.30 & -0.97 & -1.82 & -1.72 & -1.78 & -1.43 & 1.83 & -2.17 & -2.53 & -2.56 & -2.04 \\
\hline 7 & 1.66 & 1.34 & -1.08 & -2.67 & -1.87 & -1.74 & -1.05 & 1.86 & -2.34 & -2.54 & -2.52 & -2.08 \\
\hline 8 & 1.39 & 1.39 & -1.44 & -2.61 & -1.69 & -1.86 & -1.09 & 1.68 & -2.28 & -2.19 & -2.16 & -1.98 \\
\hline 9 & 1.54 & 1.47 & -1.06 & -1.96 & -1.82 & -1.76 & -0.68 & 1.60 & -2.13 & -2.33 & -2.11 & -2.09 \\
\hline 10 & 1.66 & 1.55 & -1.68 & -1.94 & -1.87 & -1.82 & -0.48 & 2.09 & -2.17 & -2.52 & -4.09 & -2.54 \\
\hline 11 & 1.68 & 1.23 & -1.79 & -1.90 & -1.75 & -2.16 & -0.53 & 2.40 & -2.28 & -2.57 & -2.73 & -2.09 \\
\hline 12 & 1.67 & 1.52 & -1.83 & -1.97 & -1.72 & -1.90 & -0.63 & -- & -2.24 & -2.34 & -2.29 & -1.96 \\
\hline 13 & 1.52 & 1.61 & -1.40 & -2.38 & -1.82 & -1.76 & -0.76 & -- & -2.15 & -2.35 & -2.39 & -1.96 \\
\hline 14 & 1.44 & 1.66 & -1.39 & -1.76 & -1.74 & -2.52 & -0.65 & -1.77 & -2.65 & -2.41 & -2.81 & -1.95 \\
\hline 15 & 1.28 & 1.74 & -1.52 & -2.12 & -1.68 & -2.43 & -0.53 & -1.77 & -2.37 & -2.39 & -2.57 & -1.93 \\
\hline 16 & 1.14 & 1.58 & -1.22 & -1.92 & -1.72 & -1.93 & -0.75 & -1.75 & -2.10 & -2.25 & -2.99 & -2.14 \\
\hline 17 & 1.16 & 1.02 & -1.21 & -1.87 & -1.70 & -1.83 & -0.89 & -1.89 & -2.21 & -2.80 & -2.25 & -2.13 \\
\hline 18 & 1.12 & 1.47 & -1.30 & -1.84 & -1.74 & -1.84 & -0.78 & -1.70 & -2.15 & -2.76 & -2.66 & -2.30 \\
\hline 19 & 1.12 & 1.75 & -1.32 & -1.87 & -1.73 & -1.84 & -0.41 & -1.61 & -2.38 & -2.49 & -2.60 & -2.08 \\
\hline 20 & 1.17 & 1.71 & -1.50 & -1.79 & -1.84 & -1.84 & -0.02 & -1.62 & -2.50 & -2.58 & -2.43 & -2.05 \\
\hline 21 & 1.21 & 1.46 & -1.66 & -1.90 & -1.78 & -1.87 & 0.23 & -1.67 & -2.58 & -2.79 & -2.11 & -2.07 \\
\hline 22 & 1.20 & 1.20 & -1.79 & -1.85 & -1.71 & -1.96 & 0.34 & -1.82 & -2.22 & -3.16 & -2.59 & -2.95 \\
\hline 23 & 1.25 & 0.78 & -1.81 & -1.86 & -1.71 & -- & 0.29 & -1.96 & -2.05 & -2.52 & -2.46 & -2.56 \\
\hline 24 & 1.18 & 0.27 & -1.80 & -1.75 & -1.72 & -- & 0.43 & -2.25 & -2.20 & -2.24 & -2.05 & -2.66 \\
\hline 25 & 1.26 & -0.13 & -1.71 & -1.98 & -1.78 & -0.18 & 0.59 & -2.10 & -2.27 & -2.46 & -2.53 & -2.21 \\
\hline 26 & 1.22 & -1.10 & -1.72 & -1.96 & -1.79 & -1.22 & 0.59 & -2.05 & -2.61 & -2.32 & -2.34 & -2.29 \\
\hline 27 & 1.29 & -1.22 & -2.01 & -1.73 & -1.77 & -1.83 & 0.68 & -2.04 & -2.47 & -2.48 & -2.14 & -2.30 \\
\hline 28 & 1.13 & -0.81 & -2.08 & -1.76 & -1.74 & -2.10 & 1.09 & -2.07 & -2.21 & -2.33 & -1.74 & -2.39 \\
\hline 29 & 1.26 & -- & -1.94 & -1.91 & -1.77 & -2.05 & 1.33 & -2.10 & -2.31 & -2.68 & -2.65 & -2.39 \\
\hline 30 & 1.24 & -- & -2.27 & -1.71 & -1.94 & -2.08 & 1.25 & -2.15 & -2.60 & -2.61 & -2.51 & -2.37 \\
\hline 31 & 1.23 & -- & -2.70 & -- & -1.90 & -- & 1.22 & -1.99 & -- & -2.28 & -- & -2.05 \\
\hline
\end{tabular}


Table 10e. Mean daily ground-water levels in observation well CRM-6 (map ID \#5), Seminole Well Field, Cedar Rapids, lowa, January 1997 through February 1999--Continued

\begin{tabular}{|c|c|c|c|c|c|c|c|c|c|c|c|c|}
\hline Day & Jan & Feb & Mar & Apr & May & Jun & Jul & Aug & Sep & Oct & Nov & Dec \\
\hline & & & & & & 1999 & & & & & & \\
\hline 1 & -2.00 & -1.82 & -- & -- & -- & -- & -- & -- & -- & -- & -- & -- \\
\hline 2 & -1.94 & -1.87 & -- & -- & -- & -- & -- & -- & -- & -- & -- & -- \\
\hline 3 & -1.94 & -2.50 & -- & -- & -. & -- & -- & -. & -- & -- & -- & -. \\
\hline 4 & -1.98 & -1.85 & -- & - & -. & -- & -- & -- & -. & -- & -- & - \\
\hline 5 & -1.91 & -1.89 & -- & -- & -- & -- & -- & -- & -- & -- & -- & -- \\
\hline 6 & -1.90 & -1.89 & -- & -- & -- & -- & -- & -- & -- & -- & -- & -- \\
\hline 7 & -2.13 & -1.80 & -- & -- & -- & -- & -- & -- & -- & -- & -- & -- \\
\hline 8 & -1.99 & -1.81 & -- & -- & -- & -- & -- & -- & -- & -- & -- & - \\
\hline 9 & -1.90 & -1.92 & -- & -- & -- & -- & -. & -- & -- & -- & -- & - \\
\hline 10 & -1.84 & -1.94 & -- & -- & -- & -- & -- & -- & -- & -- & -- & -- \\
\hline 11 & -1.96 & -2.30 & -- & -- & -- & -- & -- & -- & -- & -- & -- & -- \\
\hline 12 & -1.81 & -1.87 & -- & -- & -- & -- & -- & -- & -- & -- & -- & -- \\
\hline 13 & -1.92 & -2.32 & -- & -- & -- & -- & -- & -- & -- & -- & -- & -- \\
\hline 14 & -1.90 & -1.82 & -- & -- & -- & -- & -- & -- & -- & -- & -- & -- \\
\hline 15 & -1.90 & -1.79 & -- & -- & -- & -- & -- & -- & -- & -- & -- & -- \\
\hline 16 & -1.82 & -1.79 & -- & -- & -- & -- & -- & -- & -- & - & -- & -- \\
\hline 17 & -2.23 & -1.84 & -- & -- & -- & -- & -- & -- & -- & -- & -- & -- \\
\hline 18 & -2.12 & -1.83 & -- & -- & -- & -- & - & -- & -- & -- & -- & -- \\
\hline 19 & -1.79 & -1.99 & -- & -- & -- & -- & -- & -- & -- & -- & -- & -- \\
\hline 20 & -1.76 & -1.97 & -- & -. & -. & -. & -- & -- & -- & -- & -. & -. \\
\hline 21 & -1.89 & -2.59 & -- & -- & -- & -- & -- & -- & -- & -- & -- & -- \\
\hline 22 & -2.07 & -2.03 & -- & -- & -- & -- & - & -- & -- & -- & -. & -. \\
\hline 23 & -1.92 & -1.92 & -- & -- & -- & -- & -- & -- & -- & -- & -- & -- \\
\hline 24 & -1.91 & -1.90 & -- & -- & -- & -- & -- & -- & -- & -- & -- & -- \\
\hline 25 & -2.21 & -1.96 & -- & -- & -- & -- & -- & -- & -- & -- & -- & -- \\
\hline 26 & -1.87 & -1.88 & -- & -- & -- & -- & - & -- & -- & -- & -- & -- \\
\hline 27 & -1.86 & -2.13 & -- & -- & -- & -- & -- & -- & -- & -- & -- & -- \\
\hline 28 & -1.88 & -1.96 & -- & -- & -- & -. & -- & -- & -- & -- & -- & -- \\
\hline 29 & -2.37 & -- & -- & -- & -- & -- & -- & -- & -- & -- & -- & -- \\
\hline 30 & -2.48 & -- & -- & -- & -- & -- & -- & -- & -- & -- & -- & -- \\
\hline 31 & -1.89 & -- & -- & -- & -- & -. & -- & -- & -- & -- & -- & -- \\
\hline
\end{tabular}


Table 10f. Mean daily ground-water levels in observation well CRM-7 (map ID \#6), Seminole Well Field, Cedar Rapids, lowa, January 1997 through February 1999

[Water levels measured in feet below land surface; --, value not measured or recorded]

\begin{tabular}{|c|c|c|c|c|c|c|c|c|c|c|c|c|}
\hline Day & Jan & Feb & Mar & Apr & May & Jun & Jul & Aug & Sep & Oct & Nov & Dec \\
\hline \multicolumn{13}{|c|}{1997} \\
\hline 1 & -- & 2.89 & 1.58 & 0.96 & -- & 2.58 & 1.98 & 3.08 & 3.58 & 3.24 & 2.59 & 3.01 \\
\hline 2 & -- & 2.98 & 1.59 & 1.00 & -- & -- & 2.11 & 3.10 & -- & 3.20 & 2.37 & 2.97 \\
\hline 3 & -- & 2.89 & 1.41 & 1.54 & 2.21 & -- & 1.98 & 3.06 & -- & 3.01 & 2.90 & 3.06 \\
\hline 4 & -- & 2.92 & 1.24 & 1.77 & 1.93 & 2.72 & 2.23 & 3.23 & 3.26 & 3.24 & 3.06 & 3.09 \\
\hline 5 & -- & 2.84 & 1.09 & 1.93 & 1.62 & 2.71 & 2.39 & 3.40 & 3.15 & 3.28 & 3.26 & 3.01 \\
\hline 6 & -- & 2.83 & 0.88 & 2.04 & 1.70 & 2.77 & 2.54 & 3.43 & 3.21 & 3.15 & 3.29 & 2.91 \\
\hline 7 & -- & 2.88 & 1.18 & 2.17 & 1.61 & 2.71 & 2.63 & 3.43 & 3.12 & 3.18 & 3.29 & 2.90 \\
\hline 8 & -- & 2.86 & 0.07 & 2.05 & 1.48 & 2.91 & 2.55 & 3.21 & 3.20 & 3.22 & 3.13 & 2.99 \\
\hline 9 & -- & 2.90 & 0.96 & 1.94 & 1.65 & 2.81 & 2.77 & 3.24 & 3.28 & 3.38 & 3.00 & 2.96 \\
\hline 10 & -- & 3.04 & 2.01 & 1.85 & 1.62 & 2.85 & -- & 3.32 & 3.23 & 3.36 & 3.27 & 3.01 \\
\hline 11 & -- & 3.11 & 2.02 & 1.96 & 1.65 & 2.77 & 2.84 & 3.39 & 3.12 & 3.29 & 3.14 & 3.07 \\
\hline 12 & -. & 2.95 & 1.62 & 2.05 & 1.79 & 2.81 & 2.67 & 3.23 & 3.13 & 3.03 & 3.06 & -- \\
\hline 13 & -- & 2.97 & 1.19 & 2.28 & 1.92 & 2.86 & 2.61 & 3.37 & 3.12 & 3.04 & 3.05 & 2.81 \\
\hline 14 & -- & 3.01 & 0.79 & 2.38 & 2.02 & 2.78 & 2.63 & -- & 3.26 & 3.19 & -- & 2.69 \\
\hline 15 & -- & 3.09 & 0.20 & 2.31 & 2.24 & 2.77 & 2.70 & 5.90 & 3.19 & 3.10 & 3.21 & 2.65 \\
\hline 16 & -. & 2.98 & -0.55 & 2.39 & 2.36 & 2.83 & 2.51 & 6.32 & 3.11 & 2.98 & 3.24 & 2.87 \\
\hline 17 & -. & 3.02 & -0.85 & 2.40 & 2.47 & 2.82 & 2.63 & 6.26 & 3.26 & 3.07 & 3.20 & 2.90 \\
\hline 18 & -. & 2.84 & -0.01 & 2.46 & 2.47 & 2.83 & 2.74 & 6.49 & 3.18 & 2.94 & 3.07 & 3.05 \\
\hline 19 & -- & 2.03 & 0.95 & 2.62 & 2.27 & 2.83 & 2.72 & 6.32 & 3.20 & 2.97 & 3.12 & 2.94 \\
\hline 20 & -- & 1.67 & 1.25 & 2.67 & 2.55 & 2.88 & 2.86 & -- & 3.41 & 3.26 & 3.20 & 3.14 \\
\hline 21 & -- & 0.91 & 1.18 & 2.72 & -- & 2.77 & 2.95 & -- & 3.35 & 3.29 & 3.05 & 2.95 \\
\hline 22 & -- & 0.35 & 1.27 & 2.73 & 2.49 & 2.72 & 2.97 & -- & 3.32 & 3.20 & 3.06 & 3.00 \\
\hline 23 & -- & -0.14 & 1.16 & -- & 2.60 & 2.57 & 3.00 & -- & 3.42 & 3.14 & 3.08 & 2.89 \\
\hline 24 & 2.99 & 0.06 & 0.67 & 2.66 & 2.68 & 2.07 & 3.05 & -- & 3.26 & 3.18 & 3.10 & 2.79 \\
\hline 25 & 2.88 & 0.86 & 0.31 & 2.75 & 2.67 & 1.84 & 3.11 & -- & 3.28 & 3.19 & 3.01 & 2.98 \\
\hline 26 & 2.90 & 1.50 & -0.11 & 2.71 & 2.71 & 1.79 & 3.30 & -- & 3.16 & 3.12 & 2.98 & 2.98 \\
\hline 27 & 2.97 & 1.67 & -0.58 & 2.70 & 2.76 & 1.58 & 3.17 & 3.11 & 3.10 & 3.00 & 2.91 & 2.93 \\
\hline 28 & 2.85 & 1.79 & -0.54 & 2.81 & 2.66 & 1.51 & -- & 3.16 & 3.01 & 3.07 & 3.11 & 2.62 \\
\hline 29 & 2.83 & -- & 0.13 & 2.82 & 2.50 & 1.72 & 3.35 & -- & 2.72 & 3.01 & 3.14 & 2.73 \\
\hline 30 & 2.98 & -. & 0.79 & 2.67 & 2.71 & 1.68 & 3.21 & 3.22 & -- & 3.06 & 3.10 & 2.78 \\
\hline 31 & 2.97 & -- & 0.97 & -- & 2.69 & -- & 3.17 & 3.66 & -- & 2.55 & -- & -- \\
\hline
\end{tabular}


Table 10f. Mean daily ground-water levels in observation well CRM-7 (map ID \#6), Seminole Well Field, Cedar Rapids, lowa, January 1997 through February 1999--Continued

\begin{tabular}{|c|c|c|c|c|c|c|c|c|c|c|c|c|}
\hline Day & Jan & Feb & Mar & Apr & May & Jun & Jul & Aug & Sep & Oct & Nov & Dec \\
\hline \multicolumn{13}{|c|}{1998} \\
\hline 1 & -- & 2.66 & 2.51 & -0.47 & 2.14 & 2.05 & -1.44 & 3.10 & 2.55 & 2.89 & 2.47 & 2.94 \\
\hline 2 & 2.96 & 2.79 & 2.56 & -0.88 & 2.20 & 2.18 & -1.68 & 3.07 & -- & 2.87 & 2.35 & 2.84 \\
\hline 3 & 2.88 & 2.96 & 2.59 & -1.18 & 2.36 & 2.35 & -2.29 & 2.90 & 2.50 & 2.95 & 2.37 & -- \\
\hline 4 & 2.85 & 2.84 & -- & -0.79 & 2.42 & 2.41 & -2.08 & 2.92 & 2.51 & 2.95 & 2.58 & 2.93 \\
\hline 5 & -- & 2.70 & 2.74 & -0.66 & 2.46 & 2.49 & -1.08 & 3.09 & 2.54 & 2.81 & 2.52 & 2.46 \\
\hline 6 & -- & 2.72 & 2.73 & -0.58 & 2.48 & 2.72 & 0.02 & 2.89 & 2.56 & 2.91 & 2.55 & 2.33 \\
\hline 7 & -- & 2.66 & 2.60 & -0.82 & 2.27 & 2.75 & 0.78 & 2.74 & 2.59 & 2.74 & 2.75 & 2.62 \\
\hline 8 & -- & 2.67 & 2.46 & -0.28 & 2.57 & 2.56 & 1.00 & 2.88 & 2.76 & 2.73 & 2.96 & 2.57 \\
\hline 9 & 2.91 & 2.72 & 2.76 & 0.68 & 2.40 & 2.52 & 1.21 & 2.83 & 2.88 & 2.67 & 3.22 & 2.59 \\
\hline 10 & 2.85 & 2.82 & 2.66 & 1.00 & 2.40 & 2.27 & 1.55 & -- & 2.81 & 2.68 & 1.38 & 2.72 \\
\hline 11 & 2.91 & 2.58 & 2.37 & 0.99 & 2.46 & 1.65 & 1.92 & 2.84 & 2.66 & 2.68 & -- & 2.80 \\
\hline 12 & 2.85 & 2.59 & 1.93 & 0.85 & 2.48 & 1.31 & 1.98 & 2.98 & 2.63 & 2.71 & 2.67 & 2.70 \\
\hline 13 & 3.04 & 2.70 & 1.62 & 0.67 & 2.73 & 0.74 & 2.05 & 2.87 & 2.69 & 2.70 & 2.70 & 2.71 \\
\hline 14 & 2.93 & 2.80 & 2.69 & -- & 2.76 & -0.74 & 2.15 & -- & 2.60 & 2.72 & 2.20 & 2.82 \\
\hline 15 & 2.79 & 2.74 & 2.75 & 1.09 & 2.61 & -1.45 & 2.29 & 2.60 & -- & 2.82 & 2.57 & 2.90 \\
\hline 16 & 2.89 & 2.76 & 2.83 & 1.33 & 2.93 & -0.44 & 2.50 & 2.67 & 3.03 & 2.84 & 1.98 & 2.84 \\
\hline 17 & 2.82 & 2.39 & 2.65 & 1.34 & 3.07 & 0.46 & -- & 2.55 & 2.92 & 2.50 & 2.67 & 3.26 \\
\hline 18 & 2.71 & 2.67 & 2.64 & 1.40 & 2.62 & 0.75 & 2.50 & 2.77 & 2.91 & 2.40 & 2.27 & 1.99 \\
\hline 19 & 2.68 & 2.86 & 2.49 & 1.05 & 2.94 & 0.70 & 2.61 & 2.91 & 2.99 & 2.35 & 2.46 & 2.74 \\
\hline 20 & 2.69 & 2.75 & 2.50 & 0.86 & 3.86 & 0.52 & 2.57 & 2.91 & 3.05 & 2.16 & 2.53 & -- \\
\hline 21 & 2.78 & 2.55 & 2.53 & 0.90 & 2.85 & 0.20 & 2.58 & 2.83 & 2.86 & 2.08 & 2.86 & -- \\
\hline 22 & 2.74 & 2.50 & 2.47 & 1.06 & 3.51 & -0.73 & 2.49 & 2.49 & 3.13 & 2.42 & 2.43 & 2.23 \\
\hline 23 & 2.78 & -- & -- & 0.93 & 2.82 & -1.35 & 2.61 & 2.62 & 3.03 & 2.59 & 2.47 & 2.57 \\
\hline 24 & 2.71 & 2.54 & 2.55 & 1.10 & 2.67 & -1.60 & -- & 2.57 & 2.93 & 2.61 & 2.88 & 2.58 \\
\hline 25 & 2.81 & 2.49 & 2.30 & 1.07 & 2.51 & -2.44 & 2.96 & 2.57 & 3.08 & 2.53 & 2.44 & 2.44 \\
\hline 26 & -- & 2.11 & 2.21 & 1.33 & 2.46 & -2.71 & 2.86 & 2.62 & 3.02 & 2.61 & 2.67 & 2.33 \\
\hline 27 & 2.75 & 2.10 & 1.93 & 1.87 & 2.34 & -2.48 & 2.62 & 2.60 & 2.95 & 2.63 & 2.78 & 2.32 \\
\hline 28 & 2.80 & 2.39 & 1.95 & 2.08 & 2.36 & -2.43 & 2.62 & 2.48 & -- & 2.81 & 3.05 & 2.28 \\
\hline 29 & 2.82 & -. & 2.01 & -- & 2.12 & -2.13 & 2.62 & 2.49 & 2.94 & 2.05 & 2.10 & 2.38 \\
\hline 30 & 2.65 & -- & 1.63 & 2.17 & 1.98 & -1.60 & 2.74 & 2.59 & 2.93 & 2.31 & 2.33 & 2.51 \\
\hline 31 & 2.79 & -- & 0.21 & -- & 2.04 & -- & 3.00 & 2.67 & .- & 2.41 & - & -- \\
\hline
\end{tabular}


Table 10f. Mean daily ground-water levels in observation well CRM-7 (map ID \#6), Seminole Well Field, Cedar Rapids, lowa, January 1997 through February 1999--Continued

\begin{tabular}{|c|c|c|c|c|c|c|c|c|c|c|c|c|}
\hline Day & Jan & Feb & Mar & Apr & May & Jun & Jul & Aug & Sep & Oct & Nov & Dec \\
\hline & & & & & & 1999 & & & & & & \\
\hline 1 & -- & 2.64 & -- & -- & -- & -- & -- & -- & -- & -. & -- & -- \\
\hline 2 & 2.63 & 2.38 & -- & -- & -- & -- & -. & -- & -- & -- & -- & -- \\
\hline 3 & 2.70 & 1.99 & -- & -- & -- & -- & -- & -. & -- & -- & -- & -- \\
\hline 4 & 2.63 & 2.46 & -- & -- & -- & -- & -- & -- & -. & -- & -- & -- \\
\hline 5 & 2.73 & 2.40 & -- & -- & -- & -- & -- & -- & -- & -- & -- & -- \\
\hline 6 & 2.78 & 2.49 & -- & - & -- & -- & -. & -- & -- & -- & -- & -- \\
\hline 7 & 2.67 & 2.33 & -- & -- & -- & -- & -- & -- & -- & -- & -- & -- \\
\hline 8 & 2.68 & 2.31 & -- & -- & -- & -- & -- & -- & -- & -- & -- & -- \\
\hline 9 & 2.69 & 2.34 & -- & -- & -- & -- & -- & -- & -- & -- & -- & -- \\
\hline 10 & 2.74 & 2.06 & -- & -- & -- & -- & -- & -- & -- & -- & -- & -- \\
\hline 11 & 2.57 & 1.84 & -- & - & -- & -- & -- & -- & -- & -- & -- & -- \\
\hline 12 & 2.78 & 2.49 & -- & - & -- & -- & -- & -- & -- & .- & -- & -- \\
\hline 13 & 2.70 & 2.25 & -- & -- & -- & -- & -- & -- & -- & -- & -- & -- \\
\hline 14 & 2.68 & 2.34 & -- & -- & -- & -- & -- & -- & -- & -- & -- & - \\
\hline 15 & 2.66 & 2.34 & -- & -- & -- & -- & -- & -- & -- & -- & -- & -- \\
\hline 16 & 2.73 & 2.50 & -- & -- & -- & -- & -- & -- & -- & -- & -- & -- \\
\hline 17 & 2.42 & 2.51 & -- & -- & -- & -- & -- & -- & -- & -- & -- & -- \\
\hline 18 & 2.59 & 2.52 & -- & -- & -. & -- & -- & -- & -- & -- & -- & -- \\
\hline 19 & 2.89 & 2.36 & -- & -- & -- & -- & -- & -- & -- & -- & -- & -- \\
\hline 20 & 2.84 & 2.43 & -. & -- & -. & -- & -- & -- & -- & -- & -- & -- \\
\hline 21 & 2.57 & 2.39 & -- & -- & -- & -- & -- & -- & -- & -- & -- & -- \\
\hline 22 & 2.36 & 2.44 & -- & -- & -- & -- & -- & -- & -- & -- & -- & -- \\
\hline 23 & 2.45 & 2.52 & -- & -. & -. & -- & - & -- & -- & -- & -- & -- \\
\hline 24 & 2.56 & 2.58 & -- & -- & -- & -- & -- & -- & -- & -- & -- & -- \\
\hline 25 & 2.57 & 2.56 & -- & -- & -- & -- & -- & -- & -- & -- & -- & -- \\
\hline 26 & 2.62 & 2.51 & -- & -- & -- & -- & -- & -- & -- & -- & -- & -- \\
\hline 27 & 2.63 & 2.27 & -- & -- & -- & -- & -- & -- & -- & -- & -- & -- \\
\hline 28 & 2.62 & 2.50 & -- &.- & -- & -- & -- & -- & -- & -- & -- & -- \\
\hline 29 & 2.56 & -- & -- & -- & -- & -- & -- & -- & -- & -- & -- & -- \\
\hline 30 & 2.55 & -- & -- & -- & -- & -- & -- & -- & -- & -- & -- & -- \\
\hline 31 & 2.59 & -. & .. & -- & -. & -- & -- & -- & -. & -. & -- & -- \\
\hline
\end{tabular}


Table 10g. Mean daily ground-water levels in observation well CRM-9 (map ID \#7), Seminole Well Field, Cedar Rapids, lowa, January 1997 through February 1999

[Water levels measured as feet below land surface; --, value not measured or recorded]

\begin{tabular}{|c|c|c|c|c|c|c|c|c|c|c|c|c|}
\hline Day & Jan & Feb & Mar & Apr & May & Jun & Jul & Aug & Sep & Oct & Nov & Dec \\
\hline \multicolumn{13}{|c|}{1997} \\
\hline 1 & -- & 3.04 & 1.66 & 1.05 & 2.79 & 2.92 & 2.43 & 2.89 & 3.25 & 3.07 & 2.98 & 2.94 \\
\hline 2 & -- & 2.99 & 1.49 & 1.22 & 2.52 & 2.91 & 2.42 & 2.92 & 3.31 & 3.22 & 2.96 & 2.97 \\
\hline 3 & -- & 2.99 & 1.59 & 1.60 & 2.46 & 2.91 & 2.55 & 2.98 & 3.27 & 3.21 & 3.04 & 2.99 \\
\hline 4 & -- & 2.94 & 1.32 & 1.97 & 1.99 & 2.92 & 2.66 & 2.97 & 3.06 & 3.23 & 3.00 & 2.99 \\
\hline 5 & -- & 3.05 & 1.04 & 2.05 & 1.84 & 2.89 & 2.74 & 3.06 & 3.08 & 3.26 & 3.05 & 3.04 \\
\hline 6 & -- & 3.06 & 1.10 & 2.23 & 1.64 & 2.92 & 2.84 & 3.06 & 3.19 & 3.17 & 3.12 & 3.02 \\
\hline 7 & -- & 3.09 & 1.26 & 2.21 & 1.68 & 2.99 & 2.85 & 3.06 & 3.13 & 3.11 & 3.08 & 2.94 \\
\hline 8 & -- & 3.10 & 0.02 & 2.19 & 1.77 & 2.90 & 2.94 & 3.01 & 3.20 & 3.23 & 2.99 & 2.96 \\
\hline 9 & -- & 3.07 & 1.00 & 2.08 & 1.70 & 2.97 & 2.87 & 3.11 & 3.24 & 3.16 & 3.00 & 2.88 \\
\hline 10 & -- & 3.03 & 2.25 & 1.97 & 1.78 & 2.95 & 2.90 & 3.03 & 3.24 & 3.16 & 3.05 & 2.99 \\
\hline 11 & -- & 2.96 & 2.10 & 2.07 & 1.86 & 2.97 & 2.88 & 3.03 & 3.13 & 3.15 & 2.96 & 2.96 \\
\hline 12 & -- & 3.04 & 1.62 & 2.43 & 2.03 & 2.89 & 2.91 & 3.07 & 3.11 & 3.15 & 3.00 & 2.96 \\
\hline 13 & -- & 2.99 & 1.23 & 2.46 & 2.20 & 2.94 & 2.93 & 3.05 & 3.06 & 3.10 & 3.00 & 2.92 \\
\hline 14 & -- & 2.99 & 0.97 & 2.37 & 2.34 & 3.04 & 2.98 & 3.06 & 3.07 & 3.03 & 3.07 & 2.77 \\
\hline 15 & -- & 3.01 & 0.30 & 2.47 & 2.52 & 2.97 & 2.91 & 3.03 & 3.16 & 3.07 & 3.10 & 2.79 \\
\hline 16 & -- & 3.10 & -0.34 & 2.48 & 2.57 & 2.92 & 2.91 & 3.09 & 3.22 & 3.05 & 3.13 & 2.89 \\
\hline 17 & -- & 3.02 & -0.63 & 2.51 & 2.59 & 3.00 & 2.82 & 2.96 & 3.25 & 2.97 & 3.04 & 2.97 \\
\hline 18 & -- & 2.88 & 0.08 & 2.64 & 2.47 & 2.99 & 2.77 & 3.04 & 3.17 & 2.93 & 3.02 & 2.96 \\
\hline 19 & -- & 2.02 & 1.14 & 2.65 & 2.67 & 3.03 & 2.80 & 3.05 & 3.22 & 2.98 & 3.05 & 2.98 \\
\hline 20 & -- & 1.77 & 1.38 & 2.69 & 2.79 & 3.01 & 2.86 & 3.13 & 3.15 & 3.06 & 3.12 & 3.01 \\
\hline 21 & -- & 0.98 & 1.18 & 2.68 & 2.83 & 2.93 & 2.93 & 3.05 & 3.15 & 3.08 & 3.09 & 2.95 \\
\hline 22 & -- & 0.18 & 1.31 & 2.78 & 2.86 & 2.98 & 2.87 & 3.00 & 3.08 & 3.04 & 3.06 & 3.00 \\
\hline 23 & -- & 0.04 & 1.17 & 2.82 & 2.85 & 2.82 & 2.90 & 3.00 & 3.13 & 3.09 & 2.97 & 2.99 \\
\hline 24 & 3.04 & 0.31 & 0.85 & 2.87 & 2.81 & 2.61 & 2.89 & 3.12 & 3.13 & 3.14 & 3.02 & 2.89 \\
\hline 25 & 2.89 & 1.01 & 0.50 & 2.91 & 2.84 & 2.15 & 2.98 & 3.06 & 3.21 & 3.04 & 3.03 & 2.92 \\
\hline 26 & 2.93 & 1.57 & -0.12 & 2.92 & 2.86 & 1.76 & 3.00 & 3.15 & 3.16 & 3.10 & 3.06 & 2.98 \\
\hline 27 & 3.00 & 1.84 & -0.51 & 2.89 & 2.87 & 1.74 & 3.01 & 3.10 & 3.10 & 2.98 & 3.01 & 2.95 \\
\hline 28 & 3.12 & 1.96 & -0.53 & 2.85 & 2.84 & 1.92 & 3.03 & 3.14 & 3.14 & 3.08 & 3.06 & 2.87 \\
\hline 29 & 3.07 & -- & 0.42 & 2.83 & 2.89 & 2.19 & 3.05 & 3.08 & 3.16 & 3.03 & 3.06 & 2.85 \\
\hline 30 & 2.96 & -- & 1.07 & 2.80 & 2.84 & 2.08 & 3.00 & 3.12 & 3.22 & 3.02 & 3.07 & 2.92 \\
\hline 31 & 3.03 & -- & 1.13 & -- & 2.87 & -- & 2.96 & 3.29 & -- & 2.98 & -- & -- \\
\hline
\end{tabular}


Table 10g. Mean daily ground-water levels in observation well CRM-9 (map ID \#7), Seminole Well Field, Cedar Rapids, lowa, January 1997 through February 1999--Continued

\begin{tabular}{|c|c|c|c|c|c|c|c|c|c|c|c|c|}
\hline Day & Jan & Feb & Mar & Apr & May & Jun & Jul & Aug & Sep & Oct & Nov & Dec \\
\hline \multicolumn{13}{|c|}{1998} \\
\hline 1 & -- & 2.75 & 2.91 & -1.02 & 2.53 & 2.16 & -1.23 & 3.27 & 2.92 & 3.01 & 2.30 & 2.75 \\
\hline 2 & 3.15 & 2.92 & 2.86 & -0.71 & 2.62 & 2.29 & -1.51 & 3.03 & 2.91 & 2.99 & 2.57 & 2.87 \\
\hline 3 & 3.08 & 2.95 & 2.96 & -1.03 & 2.67 & 2.60 & -2.12 & 3.14 & 2.98 & 3.05 & 2.54 & 3.01 \\
\hline 4 & 2.99 & 2.97 & 2.82 & -0.96 & 2.67 & 2.58 & -1.95 & 3.12 & 2.93 & 2.95 & 2.54 & 2.92 \\
\hline 5 & 2.90 & 3.08 & 2.73 & -0.59 & 2.79 & 2.75 & -1.00 & 2.97 & 2.97 & 2.96 & 2.56 & 2.83 \\
\hline 6 & 3.00 & 3.06 & 2.76 & -0.42 & 2.81 & 2.67 & 0.12 & 2.92 & 2.87 & 2.83 & 2.81 & 3.00 \\
\hline 7 & 3.04 & 2.98 & 2.81 & -1.86 & 2.38 & 2.65 & 0.82 & 2.92 & 2.96 & 2.88 & 2.91 & 2.77 \\
\hline 8 & 2.88 & 2.96 & 2.63 & -1.16 & 2.71 & 2.69 & 1.31 & 2.87 & 2.99 & 2.92 & 2.71 & 2.75 \\
\hline 9 & 2.98 & 2.89 & 2.95 & 0.71 & 2.68 & 2.71 & 1.53 & 2.85 & 2.97 & 2.80 & 2.56 & 3.03 \\
\hline 10 & 2.96 & 2.95 & 2.98 & 1.10 & 2.71 & 2.53 & 1.72 & 2.93 & 3.00 & 2.89 & 1.84 & 3.11 \\
\hline 11 & 3.01 & 2.62 & 2.63 & 1.10 & 2.68 & 1.52 & 1.89 & 2.86 & 3.07 & 2.89 & 2.66 & 3.02 \\
\hline 12 & 3.02 & 2.85 & 2.21 & 0.90 & 2.75 & 1.38 & 2.08 & 2.91 & 2.94 & 2.80 & 2.63 & 2.75 \\
\hline 13 & 3.28 & 2.86 & 1.79 & 0.06 & -- & 0.96 & 2.19 & 2.92 & 2.90 & 2.83 & 2.56 & 2.89 \\
\hline 14 & 3.07 & 3.01 & 2.80 & 1.66 & 2.78 & -1.60 & 2.41 & 2.92 & 3.04 & 2.83 & 2.66 & 2.98 \\
\hline 15 & 2.92 & 2.93 & 3.03 & 0.69 & 2.74 & -2.03 & 2.62 & 2.86 & 2.97 & 2.69 & 2.61 & 2.80 \\
\hline 16 & 3.15 & 2.80 & 3.02 & 1.22 & 2.92 & -0.30 & 2.73 & 3.01 & 2.93 & 2.77 & 2.34 & 2.97 \\
\hline 17 & 2.97 & 1.84 & 2.87 & 1.48 & 2.94 & 0.53 & 2.75 & 2.87 & 3.03 & 2.71 & 2.68 & 2.97 \\
\hline 18 & 3.04 & 2.71 & 2.94 & 1.48 & 2.79 & 1.08 & 2.66 & 2.82 & 3.03 & 2.48 & 2.63 & 2.39 \\
\hline 19 & 3.05 & 3.05 & 2.78 & 1.36 & 3.10 & 1.04 & 2.78 & 2.91 & 3.17 & 2.35 & 2.70 & 3.02 \\
\hline 20 & 2.92 & 2.89 & 2.89 & 1.03 & 3.61 & 0.80 & 2.78 & 2.93 & 3.20 & 2.35 & 2.69 & 3.04 \\
\hline 21 & 2.93 & 2.90 & 2.88 & 1.08 & 3.04 & 0.32 & 2.77 & 2.92 & 3.05 & 2.31 & 2.62 & 2.60 \\
\hline 22 & 3.00 & 2.76 & 2.86 & 1.09 & 3.27 & -0.50 & 2.80 & 2.78 & 3.00 & 2.45 & 2.83 & 2.61 \\
\hline 23 & 2.86 & 2.73 & 2.76 & 1.15 & 2.93 & -1.25 & 2.90 & 3.02 & 3.03 & 2.61 & 2.76 & 3.00 \\
\hline 24 & 3.00 & 2.90 & 2.57 & 1.29 & 2.77 & -1.42 & 2.79 & 3.04 & 2.94 & 2.54 & 2.68 & 2.99 \\
\hline 25 & 2.88 & 2.67 & 2.66 & 0.98 & 2.71 & -2.21 & 2.86 & 2.93 & 3.15 & 2.63 & 2.87 & 2.56 \\
\hline 26 & 3.03 & 1.23 & 2.63 & 1.17 & 2.66 & -2.53 & 2.92 & 3.04 & 3.25 & 2.66 & 2.72 & 2.47 \\
\hline 27 & 2.93 & 1.37 & 1.91 & 1.76 & 2.48 & -2.16 & 2.90 & 2.98 & 3.11 & 2.75 & 2.77 & 2.55 \\
\hline 28 & 3.03 & 2.71 & 1.80 & 1.92 & 2.49 & -2.45 & 2.77 & 2.85 & 3.04 & 2.74 & 2.75 & 2.50 \\
\hline 29 & 2.94 & -. & 2.11 & 2.11 & 2.24 & -1.91 & 2.94 & 2.93 & 2.93 & 2.82 & 2.44 & 2.49 \\
\hline 30 & 2.89 & -- & 1.15 & 2.38 & 1.97 & -1.42 & 2.95 & 3.05 & 2.96 & 2.25 & 2.71 & 2.67 \\
\hline 31 & 2.97 & .. & -1.08 & -- & 2.17 & -- & 3.05 & 2.98 & -- & 2.42 & -- & -- \\
\hline
\end{tabular}


Table 10g. Mean daily ground-water levels in observation well CRM-9 (map ID \#7), Seminole Well Field, Cedar Rapids, lowa, January 1997 through February 1999--Continued

\begin{tabular}{|c|c|c|c|c|c|c|c|c|c|c|c|c|}
\hline Day & Jan & Feb & Mar & Apr & May & Jun & Jul & Aug & Sep & Oct & Nov & Dec \\
\hline & & & & & & 1999 & & & & & & \\
\hline 1 & -- & 2.84 & -- & -- & -. & -- & -- & -- & - & -- & -- & - \\
\hline 2 & 2.87 & 2.77 & -- & -- & -- & -- & -- & -- & -- & -- & -- & -- \\
\hline 3 & 2.89 & 1.30 & -- & -- & -- & -- & -- & -- & -- & -- & -- & -- \\
\hline 4 & 2.86 & 2.53 & -- & -- & -- & -- & -- & -- & -- & -- & -- & -- \\
\hline 5 & 2.73 & 2.63 & -- & -- & -- & -- & -- & -- & -- & -- & -- & -- \\
\hline 6 & 2.96 & 2.58 & -- & -- & -- & -- & -- & -- & -- & -- & -- & -- \\
\hline 7 & 3.02 & 2.78 & -- & -. & -- & -- & -- & -- & -- & -- & -- & -- \\
\hline 8 & 2.90 & 2.74 & -- & -- & -- & -- & -- & -- & -- & -- & -- & -- \\
\hline 9 & 2.83 & 2.46 & -- & -- & -- & -. & .- & -- & -- & -- & -- & -- \\
\hline 10 & 2.77 & 2.16 & -. & -. & -- & -- & -. & -- & -- & -- & -- & -. \\
\hline 11 & 2.69 & 1.45 & -- & -- & -. & -- & -- & -- & -- & -- & -- & -. \\
\hline 12 & 3.03 & 2.47 & -- & -- & -- & -- & -- & -- & -- & -- & -- & -- \\
\hline 13 & 2.99 & 2.53 & -- & -- & -- & -- & -- & -- & -- & -- & -- & -- \\
\hline 14 & 2.89 & 2.29 & -- & -- & -- & -- & -- & -- & -- & -- & -- & -- \\
\hline 15 & 2.68 & 2.67 & - & - & -- & -. & -- & -- & -- & -- & -- & -- \\
\hline 16 & 2.79 & 2.53 & - & - & -- & -- & -. & -- & -. & -. & -. & -- \\
\hline 17 & 2.02 & 2.45 & -- & - & -- & -- & -- & -- & -- & -- & -. & -. \\
\hline 18 & 2.21 & 2.46 & -- & -. & -- & -. & -- & -- & -. & -- & -. & -- \\
\hline 19 & 2.82 & 2.57 & -. & -- & -. & -- & -- & -- & -. & -- & -- & -- \\
\hline 20 & 2.86 & 2.58 & .. & -- & -- & -- & -- & -- & -. & -- & -- & -- \\
\hline 21 & 2.83 & 2.74 & -- & -- & -- & -- & -- & -- & -. & -. & -. & -. \\
\hline 22 & 2.36 & 2.66 & -- & - & -- & -- & -- & -- & -- & -- & -- & -- \\
\hline 23 & 2.61 & 2.66 & -- & -- & -- & -- & -- & -- & -- & -- & - & -- \\
\hline 24 & 2.76 & 2.66 & -- & -- & -- & -- & -- & -- & -- & -- & -- & -- \\
\hline 25 & 2.93 & 2.77 & -- & -- & -- & -- & -- & -- & -- & -- & -- & -- \\
\hline 26 & 2.84 & 2.77 & -- & -- & -- & -. & -- & -- & -- & -- & -- & -- \\
\hline 27 & 2.79 & 2.25 & -- & -- & -- & -- & -- & -- & -- & -- & -- & -- \\
\hline 28 & 2.81 & 2.69 & -- & -- & -- & -- & -- & -- & -- & -- & -- & -- \\
\hline 29 & 2.99 & - & -- & -- & -- & -- & -- & -- & -- & -- & -- & -- \\
\hline 30 & 2.99 & -- & -- & -- & -- & -- & -- & -- & -- & -- & -- & -- \\
\hline 31 & 2.79 & -- & -- & -- & -- & -- & -- & -- & -- & -- & -- & -- \\
\hline
\end{tabular}


Table 10h. Mean daily ground-water levels in observation well CRM-11 (map ID \#9), West Well Field, Cedar Rapids, lowa, July 1997 through February 1999

[Water levels measured as feet below land surface; --, value not measured or not recorded]

\begin{tabular}{|c|c|c|c|c|c|c|c|c|c|c|c|c|}
\hline Day & Jan & Feb & Mar & Apr & May & Jun & Jul & Aug & Sep & Oct & Nov & Dec \\
\hline & & & & & & 1997 & & & & & & \\
\hline 1 & -- & -- & -- & -- & -- & -- & -- & 4.79 & 4.61 & 4.26 & 4.04 & 7.51 \\
\hline 2 & -- & -- & -- & -- & -- & -- & -- & 4.72 & 4.51 & 4.40 & 3.89 & 7.73 \\
\hline 3 & -- & -- & -- & -- & -- & -- & -- & 4.55 & 4.40 & 4.28 & 4.27 & 7.95 \\
\hline 4 & -- & -- & -- & -- & -- & -- & -- & 4.64 & 4.26 & 4.41 & 4.39 & 8.12 \\
\hline 5 & -- & -. & -. & .. & -- & -- & -- & 4.71 & 4.02 & 4.43 & 4.42 & 8.14 \\
\hline 6 & -- & - & -- & -. & -- & -- & -- & 4.72 & 4.51 & 4.37 & 4.39 & 8.08 \\
\hline 7 & -- & -- & -- & -- & -- & -- & -- & 4.73 & 4.53 & 4.42 & 4.68 & 7.17 \\
\hline 8 & -. & -. & -. & -- & -- & -- & -- & 4.64 & 4.56 & 4.41 & 5.32 & 6.85 \\
\hline 9 & -- & -. & -. & -. & -- & -- & -- & 4.56 & 4.29 & 4.59 & 5.41 & 7.08 \\
\hline 10 & -- & -- & -. & -- & -- & -- & -. & 4.54 & 4.26 & 4.55 & 5.65 & 7.07 \\
\hline 11 & -- & -- & -. & -. & -- & -- & -- & 4.58 & 4.20 & 4.56 & 5.69 & 7.13 \\
\hline 12 & -- & -- & - & -- & -. & -- & $-\cdot$ & 4.43 & 4.26 & 4.44 & 5.64 & 5.86 \\
\hline 13 & -- & -- & -- & -- & -. & -- & -- & 4.51 & 3.19 & 4.48 & 5.70 & 5.02 \\
\hline 14 & -- & -- & -- & -- & -- & - & -- & 4.28 & 2.86 & 4.86 & -- & 4.80 \\
\hline 15 & -- & -- & -- & -- & -- & -- & 4.34 & 4.16 & 2.61 & 5.58 & -- & 4.63 \\
\hline 16 & -- & -- & -- & -- & -- & -. & 4.30 & 4.35 & 2.63 & 5.77 & -- & 4.61 \\
\hline 17 & -- & -- & -- & -- & -- & - & 4.32 & 4.32 & 2.81 & 5.84 & -- & 4.59 \\
\hline 18 & - & -- & -- & -- & -- & -- & 4.27 & 4.37 & 2.83 & 5.82 & -- & 4.69 \\
\hline 19 & -- & -- & -- & -- & -- & -. & 4.32 & 4.23 & 2.93 & 5.93 & -- & 4.85 \\
\hline 20 & .. & -. & -. & -- & -- & -- & 4.34 & 4.53 & 3.06 & 6.13 & 6.67 & 4.78 \\
\hline 21 & .. & .. & -- & -- & -- & -- & 4.34 & 4.58 & 2.94 & 6.22 & 6.61 & 4.61 \\
\hline 22 & -- & -- & -. & -- & -- & -. & 4.40 & 4.74 & 2.92 & 6.31 & 6.66 & 4.61 \\
\hline 23 & -- & -- & -- & -- & -- & -- & 4.41 & 4.67 & 3.38 & 6.43 & 6.78 & 4.60 \\
\hline 24 & -- & - & - & -- & -- & -- & 4.44 & 4.39 & 3.99 & 6.55 & 6.77 & 4.44 \\
\hline 25 & -- & -. & -- & -- & -- & -. & 4.36 & 4.34 & 4.27 & 6.66 & 6.67 & 4.57 \\
\hline 26 & -- & - & -- & -- & -- & -- & 4.45 & 4.30 & 4.31 & 5.64 & 6.75 & 4.52 \\
\hline 27 & -- & -- & $-\cdot$ & -. & -. & -- & 4.41 & 4.23 & 4.13 & 4.08 & 6.62 & 4.40 \\
\hline 28 & -- & -- & -- & -- & -- & -- & 4.55 & 4.21 & 4.10 & 4.39 & 6.73 & 4.18 \\
\hline 29 & -- & -- & -- & -- & -- & -- & 4.63 & 4.20 & 4.00 & 4.35 & 6.72 & 4.21 \\
\hline 30 & -- & -- & -- & -- & -- & -- & 4.64 & 4.37 & 4.29 & 4.32 & 6.85 & 4.16 \\
\hline 31 & -- & -- & -- & -- & -- & -- & 4.78 & 4.65 & -- & 4.03 & -- & -- \\
\hline
\end{tabular}


Table 10h. Mean daily ground-water levels in observation well CRM-11 (map ID \#9), West Well Field, Cedar Rapids, lowa, July 1997 through February 1999--Continued

\begin{tabular}{|c|c|c|c|c|c|c|c|c|c|c|c|c|}
\hline Day & Jan & Feb & Mar & Apr & May & Jun & Jul & Aug & Sep & Oct & Nov & Dec \\
\hline \multicolumn{13}{|c|}{1998} \\
\hline 1 & -- & 7.13 & 8.85 & 3.98 & 2.19 & 2.96 & -1.08 & 2.29 & 2.09 & 4.13 & 3.70 & 3.68 \\
\hline 2 & 3.86 & 7.22 & 8.85 & 1.67 & 2.85 & 3.12 & -1.35 & 2.31 & 2.11 & 4.19 & 3.62 & 3.59 \\
\hline 3 & 3.79 & 7.33 & 8.86 & 0.79 & 2.97 & 2.90 & -1.85 & 2.33 & 2.61 & 4.33 & 3.67 & 3.61 \\
\hline 4 & 3.65 & 7.38 & 8.91 & 0.65 & 3.02 & 2.57 & -1.75 & 2.40 & 2.45 & 4.29 & 3.79 & 3.12 \\
\hline 5 & 3.55 & 7.42 & 8.78 & 0.66 & 3.09 & 2.52 & -0.90 & 2.59 & 2.43 & 4.04 & 3.81 & 2.42 \\
\hline 6 & 3.56 & 7.45 & 8.62 & 0.73 & 3.05 & 2.21 & -0.07 & 2.72 & 2.37 & 4.25 & 3.88 & 2.29 \\
\hline 7 & 3.53 & 7.46 & 8.48 & 1.24 & 3.03 & 2.13 & 0.48 & 2.51 & 2.45 & 4.47 & 3.83 & 2.37 \\
\hline 8 & 3.49 & 7.48 & 8.46 & 1.63 & 3.03 & 2.07 & 1.02 & 2.48 & 2.56 & 4.46 & 3.86 & 2.41 \\
\hline 9 & 3.57 & 7.50 & 8.51 & 1.77 & 3.10 & 2.06 & 1.24 & 2.38 & 2.66 & 4.08 & 3.87 & 2.45 \\
\hline 10 & 3.67 & 7.51 & 8.42 & 1.98 & 3.08 & 2.05 & 1.36 & 2.54 & 2.54 & 4.06 & 4.21 & 2.44 \\
\hline 11 & 3.65 & 7.70 & 8.39 & 1.97 & 2.99 & 2.09 & 1.53 & 2.73 & 2.43 & 4.05 & 4.07 & 2.46 \\
\hline 12 & 3.68 & 7.74 & 8.14 & 1.97 & 3.03 & 1.70 & 1.59 & 2.70 & 2.41 & 4.06 & 4.01 & 2.49 \\
\hline 13 & 3.78 & 7.88 & 7.48 & 2.26 & 3.14 & 1.19 & 1.68 & 2.41 & 2.90 & 4.05 & 3.88 & 2.55 \\
\hline 14 & 3.70 & 8.03 & 7.23 & 2.16 & 3.30 & 0.77 & 2.03 & 2.18 & 2.94 & 4.03 & 3.96 & 2.57 \\
\hline 15 & 3.87 & 8.01 & 7.41 & 2.36 & 3.55 & 0.04 & 2.17 & 2.17 & 3.03 & 3.95 & 3.91 & 2.56 \\
\hline 16 & 4.56 & 8.06 & 7.53 & 2.34 & 3.69 & 0.17 & 2.27 & 2.23 & 3.09 & 4.06 & 4.04 & 2.52 \\
\hline 17 & 4.38 & 8.37 & 7.51 & 2.24 & 3.65 & 0.44 & 2.28 & 2.14 & 3.10 & 3.78 & 3.99 & 2.51 \\
\hline 18 & 4.70 & 8.34 & 7.56 & 2.25 & 3.60 & 0.65 & 2.28 & 2.18 & 3.11 & 3.70 & 3.93 & 3.00 \\
\hline 19 & 4.97 & 8.37 & 7.65 & 2.09 & 3.61 & 0.60 & 2.31 & 2.28 & 3.08 & 3.67 & 3.99 & 3.27 \\
\hline 20 & 5.76 & 8.51 & 7.84 & 1.85 & 3.58 & 0.33 & 2.26 & 2.32 & 3.12 & 3.50 & 4.04 & 3.30 \\
\hline 21 & 6.12 & 8.67 & 7.95 & 1.90 & 3.51 & 0.25 & 2.14 & 2.35 & 3.20 & 3.48 & 4.04 & 3.10 \\
\hline 22 & 6.31 & 8.70 & 7.75 & 1.53 & 3.39 & -0.25 & 2.17 & 2.20 & 2.86 & 3.42 & 4.11 & 2.76 \\
\hline 23 & 6.35 & 8.78 & 7.65 & 1.73 & 3.44 & -0.56 & 2.40 & 2.25 & 2.78 & 3.51 & 4.21 & 2.82 \\
\hline 24 & 6.46 & 8.94 & 7.54 & 2.09 & 3.30 & -0.88 & 2.49 & 2.34 & 2.87 & 3.63 & 4.08 & 2.80 \\
\hline 25 & 6.43 & 8.93 & 7.39 & 2.47 & 3.26 & -1.91 & 2.58 & 2.37 & 2.91 & 3.63 & 4.16 & 2.73 \\
\hline 26 & 6.52 & 9.19 & 7.29 & 2.69 & 3.21 & -2.32 & 2.57 & 2.43 & 2.94 & 3.70 & 3.98 & 2.48 \\
\hline 27 & 6.50 & 9.19 & 7.13 & 2.74 & 3.14 & -2.20 & 2.51 & 2.41 & 2.97 & 3.80 & 3.71 & 2.43 \\
\hline 28 & 6.62 & 8.92 & 7.02 & 2.87 & 3.03 & -2.15 & 2.47 & 2.30 & 2.90 & 3.87 & 3.57 & 2.43 \\
\hline 29 & 6.85 & -- & 6.87 & 3.01 & 2.88 & -1.97 & 2.59 & 2.34 & 3.46 & 3.84 & 3.68 & 2.62 \\
\hline 30 & 6.95 & -- & 6.90 & 2.77 & 2.91 & -1.49 & 2.66 & 2.43 & 3.99 & 3.40 & 3.67 & 2.57 \\
\hline 31 & 6.97 & -- & 6.23 & -- & 2.92 & -- & 2.67 & 2.41 & -- & 3.72 & -- & -- \\
\hline
\end{tabular}


Table 10h. Mean daily ground-water levels in observation well CRM-11 (map ID \#9), West Well Field, Cedar Rapids, lowa, July 1997 through February 1999--Continued

\begin{tabular}{|c|c|c|c|c|c|c|c|c|c|c|c|c|}
\hline Day & Jan & Feb & Mar & Apr & May & Jun & Jul & Aug & Sep & Oct & Nov & $\overline{\text { Dec }}$ \\
\hline & & & & & & 1999 & & & & & & \\
\hline 1 & -- & 2.50 & -- & -- & -- & -- & -- & -- & -. & -. & -. & -- \\
\hline 2 & 2.54 & 2.56 & -- & -- & -- & -- & -- & -- & -- & -- & -- & -- \\
\hline 3 & 2.54 & 2.94 & -- & -- & -- & -- & - & -- & -- & -- & -- & $\ldots$ \\
\hline 4 & 2.53 & 3.20 & -- & -- & -- & -- & -- & -- & -- & -- & -- & -- \\
\hline 5 & 2.40 & 2.71 & -- & -- & -- & -- & -- & -- & -- & -- & -- & -. \\
\hline 6 & 2.48 & 2.60 & -- & -- & -- & -- & - & -- & -- & -. & -- & -- \\
\hline 7 & 2.58 & 2.52 & -- & -- & -. & -- & -- & -- & -- & -. & -- & -- \\
\hline 8 & 2.85 & 2.59 & -- & -- & -- & -- & -- & -. & -- & -. & -- & -- \\
\hline 9 & 2.90 & 2.56 & -- & -. & -- & -- & -- & -- & -- & - & -- & -- \\
\hline 10 & 2.95 & 2.43 & -- & -- & -- & -- & -- & -- & -- & -. & -- & -- \\
\hline 11 & 2.96 & 2.20 & -- & -- & -- & -- & -- & -- & -- & -- & -- & -- \\
\hline 12 & 3.00 & 2.25 & -- & -- & -- & -- & -- & -- & -- & -- & -- & -- \\
\hline 13 & 3.05 & 2.26 & -- & -- & -- & -- & -- & -- & -- & -. & -. & -- \\
\hline 14 & 2.98 & 2.15 & -- & -- & -- & -- & -- & -- & -- & -- & -- & -- \\
\hline 15 & 2.99 & 2.19 & -- & -- & -- & -- & -- & -- & -- & -- & -- & -- \\
\hline 16 & 3.16 & 2.22 & -- & -- & -- & -- & -- & -. & -- & -- & -- & -- \\
\hline 17 & 3.31 & 2.26 & -- & -- & -- & -- & -- & .. & -- & -- & -- & -- \\
\hline 18 & 3.43 & 2.29 & -- & -. & -- & -. & -- & -- & -- & -- & -- & -- \\
\hline 19 & 3.38 & 2.35 & -- & -- & -- & -- & -- & -- & -- & -- & -- & -- \\
\hline 20 & 3.33 & 2.38 & -- & -- & -- & -- & -- & -- & -- & -- & -- & -- \\
\hline 21 & 3.36 & 2.36 & -- & -- & -- & -- & -- & -- & -- & -- & -- & -- \\
\hline 22 & 3.43 & 2.34 & -- & -. & -- & -- & -- & -- & -- & -. & -- & -- \\
\hline 23 & 3.30 & 2.41 & -- & -- & -- & -- & -- & -- & -. & -- & -. & -- \\
\hline 24 & 3.29 & 2.41 & -- & -- & -- & -- & -- & -- & -- & -- & -- & -- \\
\hline 25 & 3.23 & 2.48 & -- & -- & -. & -- & -- & -- & -- & -- & -- & -- \\
\hline 26 & 3.13 & 2.43 & -- & -- & -- & -- & -- & -- & -- & -- & -- & -- \\
\hline 27 & 2.78 & 2.59 & -- & -- & -- & -- & -- & -- & -- & -- & -- & -- \\
\hline 28 & 2.66 & 2.53 & -- & -- & -- & -- & -- & -- & -- & -- & -- & -- \\
\hline 29 & 2.55 & -- & -- & -- & .. & -- & -- & -- & -- & - & -- & -- \\
\hline 30 & 2.53 & -- & -- & -- & -- & -- & .- & -. & -- & -- & -- & -- \\
\hline 31 & 2.54 & -- & -- & -- & -- & -- & -. & -- & -- & .. & -- & -- \\
\hline
\end{tabular}


Table 10i. Mean daily ground-water levels in observation well CRM-12 (map ID \#10), East Well Field, Cedar Rapids, lowa, July 1997 through February 1999

[Water levels measured as feet below land surface; --, value not measured or recorded]

\begin{tabular}{|c|c|c|c|c|c|c|c|c|c|c|c|c|}
\hline Day & Jan & Feb & Mar & Apr & May & Jun & Jul & Aug & Sep & Oct & Nov & Dec \\
\hline & & & & & & 1997 & & & & & & \\
\hline 1 & -- & -- & -- & -- & -- & -- & -- & 11.70 & 10.02 & 7.95 & 19.05 & 23.44 \\
\hline 2 & -- & -- & -- & -- & -- & -. & .- & 11.59 & 9.03 & 7.58 & 19.76 & 22.73 \\
\hline 3 & -- & -- & -- & -- & -- & -- & -- & 11.65 & 7.02 & 7.37 & 20.94 & 21.61 \\
\hline 4 & -- & -- & -- & -- & -- & -- & -- & 11.65 & 5.91 & 7.40 & 21.97 & 21.60 \\
\hline 5 & -- & -- & -- & - & -- & -- & -- & 11.53 & 5.41 & 7.78 & 22.71 & 21.51 \\
\hline 6 & -- & -- & -- & -- & -- & -- & -- & 11.46 & 5.86 & 7.66 & 23.08 & 21.31 \\
\hline 7 & -- & -- & -- & -- & -- & -- & -- & 11.33 & 4.54 & 8.50 & 23.66 & 21.29 \\
\hline 8 & -- & -- & -- & -- & -- & -- & -- & 11.04 & 4.30 & 8.83 & 23.98 & 20.93 \\
\hline 9 & -- & -- & -- & -- & -- & -. & -- & 10.78 & 3.92 & 9.10 & 23.79 & 19.82 \\
\hline 10 & -- & -. & -- & -- & -- & -. & -. & 11.02 & 3.32 & 8.70 & 23.72 & 18.86 \\
\hline 11 & -- & -- & -- & -- & -- & -- & -- & 11.00 & 2.75 & 8.51 & 23.66 & 19.25 \\
\hline 12 & -- & -. & -- & -- & -- & -- & -- & 10.58 & 3.04 & 7.97 & 23.65 & 19.07 \\
\hline 13 & -- & -- & -- & -- & -- & -- & -- & 10.57 & 3.99 & 7.77 & 24.13 & 18.61 \\
\hline 14 & -- & -. & -- & -- & -- & -. & -- & 10.01 & 4.58 & 7.68 & 24.66 & 18.49 \\
\hline 15 & -- & -- & -. & -- & -- & -- & 15.40 & 9.50 & 5.14 & 7.20 & 25.18 & 18.02 \\
\hline 16 & -. & -- & -- & -- & - & -- & 14.80 & 9.43 & 6.31 & 7.01 & 25.34 & 17.71 \\
\hline 17 & -- & -- & -- & - & -- & -. & 14.38 & 9.30 & 6.75 & 7.17 & 25.67 & 17.41 \\
\hline 18 & -- & -- & -- & -. & -- & -- & 13.85 & 9.78 & 6.65 & 7.17 & 25.09 & 17.92 \\
\hline 19 & -- & -- & -- & -- & -- & -- & 13.38 & 11.48 & 7.19 & 7.07 & 25.91 & 18.42 \\
\hline 20 & -. & -- & -. & -- & -- & -- & 12.93 & 12.31 & 7.00 & 7.25 & 25.87 & 17.49 \\
\hline 21 & -- & -- & -- & -- & -- & -- & 12.59 & 11.63 & 6.72 & 7.27 & 25.95 & 16.05 \\
\hline 22 & -- & -- & -- & -- & -- & -- & 12.54 & 10.61 & 6.92 & 7.41 & 26.08 & 15.20 \\
\hline 23 & -- & -. & -- & -- & -- & -- & 12.31 & 9.53 & 7.05 & 7.86 & 25.67 & 14.76 \\
\hline 24 & -- & -- & .- & -- & -- & -- & 12.16 & 8.67 & 6.93 & 9.51 & 24.23 & 14.86 \\
\hline 25 & -- & -- & -. & -- & -- & -- & 11.93 & 8.02 & 6.79 & 10.44 & 23.26 & 14.54 \\
\hline 26 & -- & -. & -- & -- & -. & -- & 12.29 & 7.21 & 7.09 & 10.24 & 23.60 & 13.99 \\
\hline 27 & -- & -- & -- & -- & -. & -- & 12.28 & 6.96 & 7.14 & 12.58 & 23.67 & 14.78 \\
\hline 28 & -- & -- & -- & -- & -. & -- & 12.21 & 7.87 & 6.98 & 14.42 & 23.58 & 15.06 \\
\hline 29 & -- & -- & - & -- & -. & -- & 12.04 & 10.22 & 6.58 & 15.92 & 23.44 & 15.42 \\
\hline 30 & -- & -- & -. & -- & -- & -- & 11.71 & 10.44 & 8.02 & 17.06 & 23.28 & 15.05 \\
\hline 31 & -- & -- & -. & -- & -- & -- & 11.67 & 11.39 & -- & 17.96 & -- & -- \\
\hline
\end{tabular}


Table 10i. Mean daily ground-water levels in observation well CRM-12 (map ID \#10), East Well Field, Cedar Rapids, lowa, July 1997 through February 1999--Continued

\begin{tabular}{|c|c|c|c|c|c|c|c|c|c|c|c|c|}
\hline Day & Jan & Feb & Mar & Apr & May & Jun & Jul & Aug & Sep & Oct & Nov & Dec \\
\hline & & & & & & 1998 & & & & & & \\
\hline 1 & -- & 25.91 & 21.15 & 16.33 & 14.02 & 13.27 & 0.69 & 2.79 & 7.32 & 7.01 & 2.70 & 2.54 \\
\hline 2 & 14.79 & 26.65 & 20.55 & 15.16 & 13.85 & 13.19 & 0.51 & 2.42 & 7.37 & 5.62 & 2.19 & 2.62 \\
\hline 3 & 15.11 & 27.34 & 20.45 & 10.57 & 12.05 & 13.42 & 0.04 & 1.81 & 7.10 & 4.99 & 2.16 & 2.47 \\
\hline 4 & 14.85 & 26.34 & 20.79 & 6.96 & 12.19 & 13.38 & -0.25 & 1.72 & 6.98 & 4.70 & 2.64 & 2.34 \\
\hline 5 & 15.25 & 26.07 & 19.71 & 4.60 & 13.08 & 13.86 & 0.82 & 1.66 & 7.16 & 3.78 & 2.16 & 1.95 \\
\hline 6 & 15.72 & 27.08 & 19.97 & 2.87 & 11.96 & 14.96 & 2.27 & 1.00 & 7.52 & 3.33 & 1.48 & 2.13 \\
\hline 7 & 17.42 & 26.68 & 19.92 & 0.93 & 11.41 & 16.89 & 2.80 & 0.56 & 7.06 & 3.37 & 1.23 & 2.29 \\
\hline 8 & 17.41 & 25.97 & 18.98 & 0.84 & 12.12 & 18.22 & 3.41 & 0.89 & 7.58 & 3.85 & 1.36 & 2.77 \\
\hline 9 & 17.95 & 25.69 & 19.16 & 3.49 & 11.02 & 19.58 & 5.78 & 0.90 & 7.35 & 3.91 & 5.59 & 3.06 \\
\hline 10 & 18.56 & 25.58 & 19.07 & 3.84 & 9.68 & 20.24 & 6.82 & 0.73 & 7.74 & 3.92 & 6.96 & 3.14 \\
\hline 11 & 19.97 & 25.08 & 20.18 & 2.42 & 9.99 & 19.80 & 6.21 & 1.12 & 7.96 & 3.88 & 8.15 & 3.46 \\
\hline 12 & 21.18 & 25.20 & 21.34 & 1.59 & 12.12 & 20.06 & 5.98 & 1.55 & 8.42 & 3.81 & 7.21 & 3.86 \\
\hline 13 & 22.17 & 25.12 & 21.89 & 1.29 & 11.89 & 18.59 & 5.18 & 2.20 & 8.45 & 3.10 & 6.04 & 3.77 \\
\hline 14 & 22.60 & 25.03 & 21.06 & 6.34 & 11.79 & 15.67 & 3.87 & 2.96 & 7.69 & 3.31 & 4.62 & 3.54 \\
\hline 15 & 22.36 & 24.87 & 20.31 & 7.26 & 11.99 & 11.35 & 3.73 & 3.39 & 6.90 & 2.89 & 4.09 & 3.39 \\
\hline 16 & 22.78 & 24.72 & 21.41 & 6.68 & 11.38 & 11.33 & 3.80 & 2.30 & 6.29 & 3.15 & 3.58 & 2.93 \\
\hline 17 & 23.20 & 24.32 & 22.04 & 3.97 & 10.36 & 11.83 & 3.75 & 2.30 & 5.59 & 2.44 & 5.28 & 2.84 \\
\hline 18 & 23.52 & 24.73 & 22.33 & 3.07 & 9.64 & 11.95 & 3.84 & 2.87 & 4.95 & 1.86 & 6.32 & 2.30 \\
\hline 19 & 24.71 & 25.13 & 22.47 & 2.50 & 9.52 & 13.18 & 3.35 & 2.53 & 4.58 & 1.56 & 7.02 & 2.73 \\
\hline 20 & 25.79 & 25.13 & 22.60 & 4.43 & 10.24 & 14.09 & 2.34 & 3.91 & 4.43 & 1.27 & 5.49 & 2.81 \\
\hline 21 & 25.42 & 24.98 & 22.77 & 4.99 & 10.65 & 14.30 & 1.71 & 3.49 & 4.08 & 1.44 & 4.83 & 2.84 \\
\hline 22 & 25.10 & 25.01 & 23.25 & 7.43 & 10.88 & 12.91 & 1.07 & 2.80 & 4.13 & 2.13 & 4.66 & 2.63 \\
\hline 23 & 25.16 & 25.08 & 24.09 & 8.69 & 10.86 & 11.14 & 0.91 & 2.97 & 4.17 & 3.46 & 4.70 & 2.74 \\
\hline 24 & 25.18 & 24.88 & 24.39 & 6.81 & 10.46 & 7.31 & 1.12 & 3.94 & 4.12 & 3.53 & 4.47 & 2.75 \\
\hline 25 & 25.39 & 24.17 & 23.15 & 3.78 & 9.84 & 2.99 & 1.23 & 4.62 & 4.24 & 3.61 & 4.08 & 2.71 \\
\hline 26 & 25.38 & 22.31 & 21.78 & 4.85 & 9.47 & 0.60 & 1.08 & 4.52 & 4.25 & 4.59 & 3.73 & 2.53 \\
\hline 27 & 25.53 & 21.69 & 21.15 & 10.16 & 11.20 & 0.66 & 1.00 & 5.36 & 4.37 & 4.74 & 3.43 & 2.84 \\
\hline 28 & 25.60 & 21.59 & 19.64 & 9.77 & 12.11 & 0.21 & 1.22 & 6.11 & 4.39 & 4.32 & 3.02 & 3.37 \\
\hline 29 & 25.81 & -- & 17.71 & 11.92 & 12.42 & 0.17 & 1.17 & 6.36 & 6.03 & 4.09 & 2.26 & 4.03 \\
\hline 30 & 25.81 & -- & 17.15 & 12.90 & 12.37 & 0.60 & 1.41 & 6.71 & 8.05 & 2.56 & 2.40 & 4.47 \\
\hline 31 & 26.03 & -- & 17.43 & -- & 13.09 & -- & 2.98 & 7.08 & -- & 2.72 & -- & -- \\
\hline
\end{tabular}


Table 10i. Mean daily ground-water levels in observation well CRM-12 (map ID \#10), East Well Field, Cedar Rapids, lowa, July 1997 through February 1999--Continued

\begin{tabular}{|c|c|c|c|c|c|c|c|c|c|c|c|c|}
\hline Day & Jan & Feb & Mar & Apr & May & Jun & Jul & Aug & Sep & Oct & Nov & Dec \\
\hline & & & & & & 1999 & & & & & & \\
\hline 1 & -- & 5.65 & -- & -- & -- & -- & -. & .. & -- & -- & -- & -- \\
\hline 2 & 6.18 & 5.45 & -. & - & -- & -- & -- & -- & -- & -- & -- & -- \\
\hline 3 & 6.32 & 5.63 & -- & - & -- & -- & -- & -- & -- & -- & -- & -- \\
\hline 4 & 6.53 & 5.83 & -- & - & -- & -- & -- & - & -- & -- & -- & -- \\
\hline 5 & 6.58 & 5.61 & -- & - & -- & -- & -- & -- & -- & -- & -- & -- \\
\hline 6 & 6.65 & 5.60 & -- & - & -- & -- & -- & -- & -- & - & -- & -- \\
\hline 7 & 6.62 & 5.28 & -- & -- & -- & -- & -. & -. & -- & - & -- & -- \\
\hline 8 & 7.15 & 5.03 & -- & -. & -- & -- & -- & -- & -- & -- & -- & -- \\
\hline 9 & 7.79 & 4.90 & -- & - & -- & -- & -- & -- & -- & -- & -- & -- \\
\hline 10 & 8.06 & 4.88 & -- & -- & -- & -- & -- & -- & -- & -- & -- & -- \\
\hline 11 & 8.20 & 4.67 & -- & - & -- & -- & -- & -- & -- & -- & -- & -- \\
\hline 12 & 8.45 & 4.86 & -- & - & -- & -- & -- & -- & -- & - & -- & -- \\
\hline 13 & 8.56 & 4.95 & -- & -- & -- & -- & -- & -- & -- & -- & -- & -- \\
\hline 14 & 8.14 & 5.21 & -- & -- & -- & -- & -- & -- & -- & -- & -- & -- \\
\hline 15 & 7.68 & 5.28 & -- & -- & -- & -- & -- & -- & -- & -- & -- & -- \\
\hline 16 & 7.46 & 5.94 & -- & -- & -- & -- & -- & -- & -- & -- & -- & -- \\
\hline 17 & 7.31 & 6.53 & -- & -- & -- & -- & - & -- & -- & -- & -- & -- \\
\hline 18 & 7.69 & 6.32 & -- & -- & -- & -- & -- & -- & -- & -- & -- & -- \\
\hline 19 & 7.67 & 6.26 & -- & - & -- & -- & -- & -- & -- & -. & -- & -- \\
\hline 20 & 7.37 & 6.20 & -- & -- & -- & -- & -- & -- & -- & -- & -- & -- \\
\hline 21 & 7.00 & 5.83 & -- & -- & -- & -- & -- & -- & -- & -- & -- & -- \\
\hline 22 & 6.82 & 5.53 & -- & -- & -. & -. & -- & -- & -- & -- & -- & -- \\
\hline 23 & 6.73 & 5.40 & -- & -- & -- & -- & -- & -- & -- & -- & -- & - \\
\hline 24 & 6.78 & 5.36 & -- & -- & -- & -- & -- & -- & -- & -- & -- & -- \\
\hline 25 & 6.48 & 5.45 & -- & -- & -- & -. & -- & -- & -- & -- & -. & -- \\
\hline 26 & 6.28 & 5.69 & - & -- & -- & -- & -- & -- & -- & -- & -. & -- \\
\hline 27 & 6.10 & 5.24 & -- & -- & -. &.- & -- & -- & -- & -- & -. & -- \\
\hline 28 & 6.05 & 5.09 & -- & -- & -- & -- & -- & -- & -- & -- & -- & -- \\
\hline 29 & 5.75 & -- & -- & -- & -- & -- & -- & -- & -- & -- & -- & -- \\
\hline 30 & 5.77 & -- & -- & -- & -- & -- & -- & -- & -- & -- & -- & -- \\
\hline 31 & 5.86 & -- & -- & -- & -- & -- & -- & -- & -- & -- & -- & -- \\
\hline
\end{tabular}


Table 10j. Mean daily ground-water levels in observation well CRM-15 (map ID \#12), Seminole Well Field, Cedar Rapids, lowa, January 1997 through February 1999

[Water levels measured as feet below land surface; --, value not measured or recorded]

\begin{tabular}{|c|c|c|c|c|c|c|c|c|c|c|c|c|}
\hline Day & Jan & Feb & Mar & $\overline{\text { Apr }}$ & May & Jun & Jul & Aug & Sep & Oct & Nov & Dec \\
\hline \multicolumn{13}{|c|}{1997} \\
\hline 1 & -- & 12.13 & 6.58 & 1.87 & 6.56 & 6.05 & 5.82 & 7.22 & 9.22 & 12.06 & 9.19 & 9.77 \\
\hline 2 & -- & 12.21 & 6.51 & 1.95 & 6.70 & .. & 5.63 & 7.45 & 8.95 & 12.30 & 9.00 & 9.81 \\
\hline 3 & -- & 12.20 & 6.38 & 2.12 & 6.62 & 6.06 & 5.73 & 7.68 & 8.96 & 12.33 & 9.03 & 9.73 \\
\hline 4 & -- & 12.23 & 6.14 & 2.29 & 6.65 & 6.04 & 5.76 & 7.55 & 9.22 & 12.30 & 8.91 & 9.62 \\
\hline 5 & -- & 12.27 & 5.92 & 4.07 & 6.28 & 5.87 & 5.81 & 7.46 & 9.39 & 12.26 & 8.55 & 9.64 \\
\hline 6 & -- & 12.42 & 5.60 & 3.67 & 6.10 & 5.84 & 5.94 & 7.50 & 9.58 & 12.35 & 8.89 & 9.78 \\
\hline 7 & -- & 12.63 & 5.32 & 3.10 & 5.70 & 6.16 & 6.06 & 7.71 & 9.74 & 12.27 & 8.65 & 9.80 \\
\hline 8 & -- & 12.72 & 5.06 & 3.21 & 5.48 & 6.06 & 6.35 & 8.07 & 9.83 & 12.29 & 8.67 & 9.89 \\
\hline 9 & -- & 12.86 & 4.99 & 3.43 & 5.03 & 6.23 & 6.35 & 8.22 & 9.91 & 11.99 & 8.93 & 10.04 \\
\hline 10 & -- & 12.97 & 4.90 & 3.70 & 4.63 & 6.37 & 6.52 & 8.04 & 10.08 & 12.19 & 8.73 & 10.25 \\
\hline 11 & -. & 13.05 & 5.09 & 3.92 & 4.23 & 6.60 & 6.61 & 7.83 & 10.15 & 12.04 & 8.79 & 10.18 \\
\hline 12 & -- & 13.16 & 4.99 & 3.92 & 4.16 & 6.78 & 6.84 & 8.21 & 10.25 & 12.24 & 9.09 & 10.45 \\
\hline 13 & -- & 13.25 & 4.69 & 4.15 & 4.17 & 7.08 & 6.94 & 8.00 & 10.34 & 12.28 & 9.29 & 11.05 \\
\hline 14 & -- & 13.51 & 4.48 & 4.20 & 4.38 & 7.55 & 7.16 & 8.11 & 10.30 & 11.98 & -- & 11.17 \\
\hline 15 & -- & 13.62 & 4.01 & 4.29 & 4.34 & 7.87 & 7.14 & 8.07 & 10.64 & 12.21 & -- & 11.48 \\
\hline 16 & -- & 13.71 & 3.63 & 4.41 & 4.29 & 8.05 & 7.34 & 8.14 & 10.77 & 12.08 & -- & 11.61 \\
\hline 17 & -- & 13.69 & 3.01 & 4.61 & 4.39 & 8.05 & 7.51 & 8.10 & 10.93 & 11.84 & -- & 11.73 \\
\hline 18 & -- & 13.50 & 2.29 & 4.85 & 4.97 & 8.23 & 7.64 & 7.74 & 11.06 & 11.71 & -- & 11.74 \\
\hline 19 & -- & 13.72 & 2.27 & 4.91 & 4.38 & 8.33 & 7.57 & 8.03 & 11.24 & 11.56 & -. & 11.82 \\
\hline 20 & -- & 13.51 & 2.35 & 5.14 & 4.08 & 8.29 & 7.45 & 8.08 & 11.11 & 10.95 & -- & 11.51 \\
\hline 21 & -- & 13.27 & 2.53 & 5.39 & 3.94 & 8.33 & 7.39 & 8.12 & 11.37 & 10.63 & 10.49 & 11.81 \\
\hline 22 & -- & 12.35 & 2.45 & 5.63 & 4.05 & 8.37 & 7.14 & 7.99 & 11.20 & 10.47 & 10.42 & 12.01 \\
\hline 23 & -- & 11.30 & 2.59 & 5.92 & 4.27 & 8.20 & 7.02 & 8.10 & 11.35 & 10.48 & 10.13 & 12.14 \\
\hline 24 & 11.05 & 10.27 & 2.75 & 6.35 & 4.21 & 8.10 & 6.90 & 8.38 & 11.60 & 10.28 & 10.08 & 12.20 \\
\hline 25 & 11.05 & 8.94 & 2.91 & 6.48 & 4.44 & 8.03 & 6.93 & 8.52 & 11.83 & 9.92 & 10.19 & 12.30 \\
\hline 26 & 11.04 & 7.97 & 2.72 & 6.54 & 4.78 & 7.67 & 6.83 & 8.65 & 11.99 & 9.94 & 10.15 & 12.48 \\
\hline 27 & 11.05 & 7.38 & 2.61 & 6.55 & 5.00 & 7.26 & 7.12 & 8.79 & 12.02 & 9.79 & 10.08 & 12.67 \\
\hline 28 & 11.23 & 6.86 & 3.38 & 6.39 & 5.21 & 6.93 & 7.04 & 8.91 & 12.12 & 9.73 & 10.03 & 12.88 \\
\hline 29 & 11.24 & -- & 2.03 & 6.52 & -- & 6.40 & 7.07 & 9.04 & 12.15 & 9.64 & 10.00 & 13.07 \\
\hline 30 & 11.52 & -- & 1.84 & 7.76 & 5.64 & 6.06 & 7.00 & 9.12 & 12.25 & 9.47 & 9.98 & 13.19 \\
\hline 31 & 12.14 & -- & 1.82 & -- & 5.83 & -- & 7.00 & 9.18 & -- & 9.29 & -- & 13.09 \\
\hline
\end{tabular}


Table 10j. Mean daily ground-water levels in observation well CRM-15 (map ID \#12), Seminole Well Field, Cedar Rapids, lowa, January 1997 through February 1999--Continued

\begin{tabular}{|c|c|c|c|c|c|c|c|c|c|c|c|c|}
\hline Day & Jan & Feb & Mar & Apr & May & Jun & Jul & Aug & Sep & Oct & Nov & Dec \\
\hline & & & & & & 1998 & & & & & & \\
\hline 1 & 13.47 & 12.88 & 11.60 & 9.29 & 5.22 & 10.25 & 0.16 & 8.66 & 10.89 & 9.66 & 9.55 & 10.25 \\
\hline 2 & 13.62 & 12.61 & 11.30 & 7.97 & 5.50 & -- & 0.10 & 8.66 & 10.88 & 9.63 & 9.83 & 10.63 \\
\hline 3 & 13.65 & 12.40 & 11.00 & 6.09 & 5.76 & 9.93 & -0.11 & 9.18 & 10.83 & 9.69 & 9.68 & 10.72 \\
\hline 4 & 13.69 & 12.53 & 10.62 & 3.96 & 6.04 & 9.72 & -0.28 & 9.37 & 10.64 & 9.56 & 9.54 & 10.81 \\
\hline 5 & 13.87 & 12.73 & 9.99 & 3.00 & 6.30 & 9.39 & -0.08 & 9.38 & 10.45 & 9.70 & 9.55 & 10.95 \\
\hline 6 & 13.86 & 12.71 & 9.76 & 2.51 & 6.57 & 8.92 & -- & 9.68 & 10.37 & 9.65 & 9.71 & 11.02 \\
\hline 7 & 13.77 & 12.85 & 9.77 & 2.01 & 6.83 & 8.79 & -- & 9.84 & 10.19 & 9.33 & 9.90 & 10.79 \\
\hline 8 & 13.83 & 12.87 & 9.84 & 1.79 & 7.06 & 8.94 & 6.35 & 9.76 & 9.73 & 9.16 & 9.65 & 10.80 \\
\hline 9 & 13.74 & 12.92 & 9.60 & 1.82 & 7.27 & 9.08 & 6.35 & 9.81 & 9.48 & 9.17 & 9.94 & 11.16 \\
\hline 10 & 13.31 & 12.95 & 9.32 & 1.76 & 7.44 & 9.05 & -- & 10.02 & 9.51 & 9.32 & 9.99 & 11.37 \\
\hline 11 & 13.24 & 13.01 & 9.10 & 1.84 & 7.68 & 8.97 & 2.37 & 9.87 & 9.76 & 9.37 & 9.60 & 11.42 \\
\hline 12 & 13.32 & 12.99 & 9.18 & 2.06 & 7.89 & 8.87 & 3.12 & 9.81 & 9.94 & 9.25 & 9.57 & 11.29 \\
\hline 13 & 13.06 & 12.99 & 9.61 & 2.16 & 8.04 & 8.50 & 3.68 & 9.91 & 10.03 & 9.37 & 9.75 & 11.42 \\
\hline 14 & 12.90 & 12.80 & 9.70 & 2.35 & 8.29 & 7.62 & 4.14 & 10.24 & 10.15 & 9.69 & 9.94 & 11.55 \\
\hline 15 & 12.88 & 13.04 & 9.84 & 2.30 & 8.51 & 5.56 & 4.56 & 10.32 & 10.13 & 9.90 & 9.87 & 11.52 \\
\hline 16 & 12.79 & 13.08 & 9.81 & 2.24 & 8.44 & 3.93 & 4.82 & 10.33 & 10.12 & 10.05 & 9.88 & 11.88 \\
\hline 17 & 12.71 & 13.10 & 9.96 & 2.19 & 8.53 & 3.01 & 5.30 & 10.41 & 10.49 & 10.22 & 9.74 & 11.97 \\
\hline 18 & 12.61 & 13.15 & 10.16 & 2.33 & 8.97 & 2.57 & 5.75 & 10.11 & 10.89 & 10.16 & 9.85 & 12.18 \\
\hline 19 & 12.63 & 13.17 & 10.23 & 2.82 & 9.17 & 2.33 & 6.03 & -- & 11.17 & 10.02 & 9.81 & 12.00 \\
\hline 20 & 12.69 & 13.20 & 10.26 & 3.01 & 9.27 & 2.23 & 6.32 & 10.06 & 11.38 & 10.17 & 9.62 & 12.01 \\
\hline 21 & 12.73 & 13.09 & 10.24 & 2.90 & 9.47 & 2.20 & 6.58 & 10.08 & 11.52 & 10.06 & 9.71 & 12.02 \\
\hline 22 & 12.76 & 13.00 & 10.32 & 2.91 & 9.65 & 2.16 & 6.83 & 10.38 & 11.31 & 9.81 & 9.98 & 12.12 \\
\hline 23 & 12.88 & 12.92 & 10.22 & 3.30 & 9.82 & 1.87 & 6.94 & 10.53 & 11.36 & 10.04 & 9.84 & 12.51 \\
\hline 24 & 12.89 & 12.75 & 10.07 & 3.51 & 9.99 & 1.41 & 6.93 & 10.59 & 11.53 & 9.74 & 9.82 & 12.47 \\
\hline 25 & 12.93 & 12.63 & 10.43 & 3.69 & 10.15 & -0.26 & 7.04 & 10.61 & 11.41 & 9.81 & 10.13 & 12.45 \\
\hline 26 & 12.83 & 12.37 & 10.46 & 3.85 & 10.28 & -0.68 & 7.34 & 10.51 & 11.23 & 9.76 & 10.16 & 12.58 \\
\hline 27 & 12.86 & 12.13 & 10.41 & 3.76 & 10.41 & -0.43 & 7.78 & 10.64 & 10.93 & 10.02 & 10.21 & 12.72 \\
\hline 28 & 12.88 & 11.91 & 10.34 & 3.99 & 10.49 & -0.41 & 7.99 & 10.76 & 10.60 & 10.04 & 10.30 & 12.69 \\
\hline 29 & 12.87 & -- & 10.30 & 4.48 & 10.47 & -0.21 & 8.13 & 10.79 & 10.44 & 10.03 & 10.41 & 12.48 \\
\hline 30 & 12.84 & -- & 10.16 & 4.93 & 10.39 & 0.03 & 8.18 & 10.78 & 10.16 & 9.76 & 10.35 & 12.32 \\
\hline 31 & 12.86 & -- & 9.89 & -- & 10.32 & -- & 8.29 & 10.67 & -- & 9.53 & -. & 12.08 \\
\hline
\end{tabular}


Table 10j. Mean daily ground-water levels in observation well CRM-15 (map ID \#12), Seminole Well Field, Cedar Rapids, lowa, January 1997 through February 1999--Continued

\begin{tabular}{|c|c|c|c|c|c|c|c|c|c|c|c|c|}
\hline Day & Jan & Feb & Mar & Apr & May & Jun & Jul & Aug & Sep & Oct & Nov & Dec \\
\hline & & & & & & 1999 & & & & & & \\
\hline 1 & 12.11 & 11.44 & -- & -- & -- & -- & -- & -- & -- & -- & -- & -- \\
\hline 2 & 12.43 & 11.47 & -- & -- & -- & -- & -- & -- & -- & -- & -- & -- \\
\hline 3 & 12.33 & 11.37 & -- & -- & -- & -- & .. & -. & -. & -- & -- & -- \\
\hline 4 & 12.01 & 11.02 & -- & -- & -- & -- & -- & -- & -- & - & -. & -- \\
\hline 5 & 12.09 & 11.17 & -- & -. & -. & -- & -- & -- & -- & -- & -- & -- \\
\hline 6 & 12.20 & 11.14 & .. & -- & -- & -- & -- & -- & -- & -- & -- & -- \\
\hline 7 & 12.14 & 11.16 & -- & -- & -- & -- & -- & -- & -- & -- & -- & -- \\
\hline 8 & 12.08 & 11.12 & -- & -- & -- & -- & -. & -- & -- & -- & -- & -- \\
\hline 9 & 11.85 & 10.91 & -- & -- & -. & -- & -- & .- & -- & -- & -. & -. \\
\hline 10 & 11.82 & 11.01 & -- & -- & -- & -- & -- & -- & -- & -- & -- & -- \\
\hline 11 & 12.00 & 10.94 & -- & -. & -- & -- & -- & -- & -- & -- & -- & -- \\
\hline 12 & 11.96 & 10.77 & -- & -- & -- & -- & -- & -- & -- & -- & -- & -- \\
\hline 13 & 11.63 & 10.70 & -- & -- & -- & -- & -- & -- & -- & -- & -- & -- \\
\hline 14 & 11.55 & 10.59 & -- & -. & -- & -- & -- & -- & -- & -- & -- & -- \\
\hline 15 & 11.81 & 10.66 & -. & -- & -- & -- & -- & -. & -- & -- & -- & -- \\
\hline 16 & 11.78 & 10.48 & -- & -- & -- & -- & -- & -- & -- & -- & -- & -- \\
\hline 17 & 11.72 & 10.35 & - & -- & -- & -- & -- & -- & -- & -- & -- & -- \\
\hline 18 & 11.68 & 10.27 & -- & -- & -- & -- & -- & -- & -- & -- & -- & -- \\
\hline 19 & 11.65 & 10.08 & -- & -- & -- & -- & -- & -- & -- & -- & -- & -- \\
\hline 20 & 11.61 & 9.92 & -- & - & -. & -- & -- & -. & -- & -- & -- & -- \\
\hline 21 & 11.61 & 9.99 & -- & -- & -- & -- & -- & -- & -. & -- & -- & - \\
\hline 22 & 11.60 & 9.92 & -- & -- & -- & -- & -- & -- & -- & -- & - & -- \\
\hline 23 & 11.59 & 9.89 & -- & -- & -- & -- & -- & - & -- & -- & -- & -- \\
\hline 24 & 11.38 & 9.90 & -- & -- & -- & -- & -- & -- & -- & -- & -- & -- \\
\hline 25 & 11.36 & 9.81 & -- & -- & -- & -- & -- & -- & -- & -. & -- & -- \\
\hline 26 & 11.34 & 9.91 & -- & -- & -- & -- & .. & -- & -- & -- & -- & -- \\
\hline 27 & 11.51 & 9.87 & -- & -- & -- & -- & -- & -- & -- & -- & -- & -- \\
\hline 28 & 11.19 & 9.73 & -- & -- & -- & -- & -- & -- & -- & -- & -. & -- \\
\hline 29 & 11.39 & -- & - & -- & -- & -- & -- & -- & -- & -- & -. & -- \\
\hline 30 & 11.33 & -- & -- & -- & -- & -- & -- & -- & -- & -- & -. & -- \\
\hline 31 & 11.16 & .- & -- & .- & -- & -- & -. & -. & -- & .- & .. & .. \\
\hline
\end{tabular}


Table 10k. Mean daily ground-water levels in observation well CRM-16 (map ID \#13), Seminole Well Field, Cedar Rapids, lowa, January 1997 through February 1999

[Water levels measured as feet below land surface; --, value not measured or recorded]

\begin{tabular}{|c|c|c|c|c|c|c|c|c|c|c|c|c|}
\hline Day & $\operatorname{Jan}$ & Feb & Mar & Apr & May & Jun & Jul & Aug & Sep & Oct & Nov & Dec \\
\hline \multicolumn{13}{|c|}{1997} \\
\hline 1 & -- & 18.58 & 7.81 & 4.15 & 8.83 & 8.91 & 6.82 & 9.55 & 10.78 & 14.62 & 9.89 & 11.26 \\
\hline 2 & -- & 18.48 & 7.64 & 4.10 & 9.48 & 8.68 & 6.72 & 10.29 & 11.47 & 14.29 & 9.68 & 11.19 \\
\hline 3 & -- & 18.45 & 7.14 & 4.82 & 9.15 & 8.26 & 7.16 & 10.36 & 11.91 & 14.12 & 10.23 & 11.10 \\
\hline 4 & - & 19.24 & 6.70 & 5.26 & 7.21 & 8.11 & 7.81 & 10.91 & 12.11 & 14.40 & 10.90 & 12.39 \\
\hline 5 & - & 19.52 & 6.16 & 4.84 & 6.11 & 7.85 & 8.33 & 10.91 & 12.24 & 14.36 & 10.98 & 13.58 \\
\hline 6 & -- & 19.60 & 5.74 & 5.40 & 6.15 & 7.85 & 8.84 & 10.97 & 12.37 & 14.48 & 10.59 & 14.18 \\
\hline 7 & -- & 19.68 & 5.84 & 6.28 & 6.12 & 8.34 & 9.42 & 10.33 & 12.54 & 14.56 & 10.99 & 13.82 \\
\hline 8 & -- & 20.30 & 6.12 & 6.92 & 5.92 & 9.28 & 9.68 & 10.37 & 12.62 & 14.40 & 11.81 & 14.38 \\
\hline 9 & -- & 20.11 & 6.84 & 6.75 & 5.26 & 9.61 & 9.33 & 10.15 & 12.70 & 14.40 & 12.19 & 14.86 \\
\hline 10 & -- & 20.41 & 7.17 & 6.14 & 5.90 & 10.04 & 9.34 & 10.36 & 13.27 & 14.23 & 13.14 & 15.16 \\
\hline 11 & -- & 20.76 & 7.10 & 6.64 & 5.58 & 10.25 & 10.34 & 10.35 & 13.58 & 14.49 & 13.64 & 15.53 \\
\hline 12 & -- & 20.59 & 5.99 & 6.90 & 5.36 & 10.32 & 9.93 & 10.29 & 13.79 & 14.26 & 13.92 & 15.58 \\
\hline 13 & -- & 20.73 & 5.34 & 7.51 & 5.49 & 11.67 & 9.35 & 10.39 & 13.98 & 14.36 & 13.72 & 15.75 \\
\hline 14 & -- & 20.70 & 4.70 & 7.78 & 5.77 & 11.41 & 9.37 & 9.99 & 14.18 & 14.14 & 13.91 & 15.91 \\
\hline 15 & -- & 20.77 & 4.14 & 7.82 & 6.61 & 11.54 & 10.06 & 9.69 & 14.18 & 14.31 & 14.28 & 15.57 \\
\hline 16 & -- & 20.60 & 2.67 & 7.75 & 7.46 & 11.36 & 10.63 & 10.10 & 14.01 & 13.40 & 14.48 & 15.38 \\
\hline 17 & -- & 20.26 & 2.37 & 7.88 & 8.04 & 11.44 & 10.42 & 10.27 & 14.21 & 11.93 & -- & 15.33 \\
\hline 18 & -- & 20.11 & 3.49 & 8.06 & 7.20 & 11.08 & 9.97 & 10.26 & 14.13 & 11.42 & -- & 15.41 \\
\hline 19 & -- & 19.70 & 4.63 & 8.31 & 6.46 & 10.67 & 8.99 & 10.33 & 14.14 & 11.20 & -- & 15.64 \\
\hline 20 & -- & 15.69 & 4.60 & 8.68 & 6.79 & 10.41 & 8.64 & 10.42 & 14.11 & 10.74 & -- & 15.48 \\
\hline 21 & -- & 11.86 & 4.12 & 9.02 & 6.92 & 10.10 & 8.54 & 11.35 & 14.16 & 10.68 & 11.65 & 15.69 \\
\hline 22 & -- & 8.47 & 5.19 & 9.41 & 7.13 & 10.00 & 8.74 & 10.96 & 14.51 & 10.90 & 11.22 & 15.68 \\
\hline 23 & -- & 5.31 & 5.53 & 9.30 & 7.54 & 9.84 & 8.81 & 10.98 & 14.39 & 10.74 & 11.15 & 16.07 \\
\hline 24 & 17.12 & 4.10 & 4.97 & 9.47 & 7.49 & 8.37 & 9.00 & 10.96 & 14.61 & 10.98 & 11.29 & 15.76 \\
\hline 25 & 16.93 & 5.06 & 4.58 & 9.55 & 8.17 & 6.35 & 9.42 & 11.49 & 14.63 & 11.32 & 11.62 & 16.08 \\
\hline 26 & 16.92 & 5.50 & 4.15 & 10.03 & 9.19 & 5.16 & 9.98 & 12.11 & 14.61 & 11.31 & 11.96 & 16.40 \\
\hline 27 & 17.86 & 7.23 & 3.07 & 10.36 & 9.60 & 5.48 & 10.29 & 12.41 & 14.47 & 11.16 & 11.82 & 16.69 \\
\hline 28 & 18.26 & 8.24 & 2.00 & 10.46 & 9.97 & 5.89 & 9.94 & 11.50 & 14.48 & 10.59 & 11.41 & 16.49 \\
\hline 29 & 18.69 & -- & 3.04 & 9.51 & 9.94 & 6.33 & 9.40 & 11.48 & 14.21 & 10.59 & 10.94 & 16.65 \\
\hline 30 & 19.09 & -- & 3.97 & 8.28 & 9.35 & 6.30 & 9.19 & 11.30 & 14.61 & 10.26 & 10.99 & 16.73 \\
\hline 31 & 18.65 & -- & 4.04 & -- & 9.08 & -. & 9.33 & 10.82 & -- & 9.85 & -- & 17.03 \\
\hline
\end{tabular}


Table 10k. Mean daily ground-water levels in observation well CRM-16 (map ID \#13), Seminole Well Field, Cedar Rapids, lowa, January 1997 through February 1999--Continued

\begin{tabular}{|c|c|c|c|c|c|c|c|c|c|c|c|c|}
\hline Day & Jan & Feb & Mar & Apr & May & Jun & Jul & Aug & Sep & Oct & Nov & Dec \\
\hline & & & & & & 1998 & & & & & & \\
\hline 1 & 17.24 & 15.37 & 11.83 & 3.96 & 8.55 & 10.48 & 2.04 & 12.11 & 12.63 & 11.06 & 10.72 & 11.90 \\
\hline 2 & 17.35 & 15.46 & 11.48 & 2.25 & 9.03 & 10.16 & 1.58 & 12.18 & 11.82 & 11.25 & 10.97 & 11.97 \\
\hline 3 & 17.19 & 15.29 & 11.28 & 1.97 & 9.53 & 10.21 & 0.89 & 12.59 & 10.99 & 11.33 & 10.94 & 12.06 \\
\hline 4 & 16.54 & 15.46 & 11.58 & 2.16 & 9.96 & 10.11 & 1.17 & 12.79 & 10.81 & 11.32 & 10.90 & 11.94 \\
\hline 5 & 16.11 & 15.63 & 11.30 & 2.52 & 10.40 & 10.26 & 2.36 & 12.71 & 10.75 & 11.24 & 11.10 & 12.02 \\
\hline 6 & 16.15 & 15.64 & 11.56 & 2.38 & 10.91 & 9.97 & 3.62 & 12.70 & 10.46 & 11.28 & 11.10 & 11.99 \\
\hline 7 & 15.96 & 15.58 & 11.68 & 2.46 & 11.47 & 9.80 & 4.42 & 12.50 & 10.50 & 11.47 & 11.04 & 12.28 \\
\hline 8 & 15.69 & 15.67 & 11.64 & 2.96 & 11.74 & 9.87 & 5.22 & 12.29 & 10.61 & 11.55 & 11.00 & 12.28 \\
\hline 9 & 15.64 & 15.65 & 11.75 & 3.47 & 12.28 & 9.64 & 4.93 & 12.24 & 10.72 & 12.05 & 10.46 & 12.42 \\
\hline 10 & 15.66 & 15.57 & 11.94 & 3.87 & 12.70 & 9.50 & 5.64 & 12.31 & 10.98 & 12.28 & 10.48 & 12.61 \\
\hline 11 & 15.39 & 15.64 & 12.22 & 3.76 & 12.84 & 8.85 & 6.37 & 12.09 & 11.19 & 12.47 & 10.56 & 12.55 \\
\hline 12 & 15.11 & 15.66 & 12.80 & 3.74 & 13.14 & 6.36 & 6.80 & 12.02 & 11.09 & 12.50 & 10.45 & 12.48 \\
\hline 13 & 14.74 & 15.68 & 12.96 & 3.91 & 13.53 & 4.76 & 7.11 & 12.23 & 11.54 & 12.83 & 10.35 & 12.53 \\
\hline 14 & 14.66 & 15.94 & 13.15 & 4.07 & 13.56 & 3.56 & 7.52 & 12.29 & 12.02 & 12.94 & 10.24 & 12.57 \\
\hline 15 & 14.72 & 15.87 & 13.49 & 4.19 & 13.49 & 2.13 & 8.04 & 12.28 & 12.57 & 12.52 & 10.27 & 12.63 \\
\hline 16 & 15.46 & 15.82 & 13.71 & 4.15 & 13.77 & 2.50 & 8.59 & 12.26 & 12.82 & 12.51 & 10.11 & 13.57 \\
\hline 17 & 15.87 & 16.26 & 13.82 & 4.99 & 13.88 & 3.06 & 8.53 & 12.37 & 13.18 & 12.26 & 10.47 & 13.98 \\
\hline 18 & 16.42 & 16.95 & 13.83 & 5.22 & 13.97 & 3.41 & 8.23 & 12.42 & 13.24 & 12.06 & 10.41 & 13.97 \\
\hline 19 & 16.49 & 16.93 & 14.01 & 5.13 & 13.95 & 3.31 & 8.40 & 12.32 & 13.21 & 11.41 & 10.67 & 14.02 \\
\hline 20 & 16.40 & 15.99 & 14.13 & 4.81 & 14.23 & 3.65 & 8.65 & 12.35 & 13.35 & 10.84 & 10.88 & 13.49 \\
\hline 21 & 16.55 & 16.01 & 14.11 & 5.24 & 14.24 & 4.28 & 8.85 & 12.40 & 13.65 & 10.28 & 11.01 & 13.29 \\
\hline 22 & 16.88 & 15.44 & 14.08 & 5.34 & 14.15 & 3.21 & 9.27 & 12.39 & 13.19 & 10.26 & 11.05 & 13.26 \\
\hline 23 & 16.61 & 14.53 & 14.01 & 5.42 & 14.31 & 1.98 & 9.52 & 12.64 & 11.60 & 10.30 & 11.32 & 13.10 \\
\hline 24 & 16.27 & 14.17 & 13.62 & 5.45 & 14.31 & 1.34 & 9.48 & 12.82 & 10.64 & 10.20 & 11.23 & 13.14 \\
\hline 25 & 15.81 & 13.70 & 13.16 & 5.81 & 14.26 & 0.22 & 9.55 & 12.98 & 10.19 & 10.38 & 11.03 & 13.05 \\
\hline 26 & 15.99 & 13.38 & 12.74 & 6.15 & 13.84 & 0.02 & 9.94 & 13.31 & 10.04 & 10.42 & 11.09 & 12.87 \\
\hline 27 & 15.69 & 12.94 & 12.50 & 6.47 & 12.99 & 0.38 & 10.48 & 13.01 & 9.98 & 10.52 & 11.29 & 12.62 \\
\hline 28 & 15.50 & 12.34 & 12.33 & 6.99 & 12.27 & 0.36 & 10.95 & 12.76 & 10.47 & 10.43 & 11.39 & 12.45 \\
\hline 29 & 15.39 & -- & 12.13 & 7.70 & 11.89 & 0.82 & 11.67 & 12.85 & 10.69 & 10.21 & 11.72 & 12.31 \\
\hline 30 & 15.46 & -- & 11.60 & 7.95 & 11.50 & 1.46 & 11.99 & 13.00 & 10.95 & 10.70 & 11.81 & 12.48 \\
\hline 31 & 15.32 & -- & 7.03 & -- & 11.16 & -- & 11.89 & 12.99 & -- & 10.70 & -- & 12.44 \\
\hline
\end{tabular}


Table 10k. Mean daily ground-water levels in observation well CRM-16 (map ID \#13), Seminole Well Field, Cedar Rapids, lowa, January 1997 through February 1999--Continued

\begin{tabular}{|c|c|c|c|c|c|c|c|c|c|c|c|c|}
\hline Day & Jan & Feb & Mar & Apr & May & Jun & JuI & Aug & Sep & Oct & Nov & Dec \\
\hline \multicolumn{13}{|c|}{1999} \\
\hline 1 & 12.39 & 12.22 & -- & -- & -- & -- & -- & -- & -- & -- & -- & -- \\
\hline 2 & 12.14 & 11.81 & -- & -- & -- & - & -- & -- & -- & - & -- & -- \\
\hline 3 & 12.29 & 12.28 & -- & -- & -- & -- & -- & -- & -- & -- & -- & -- \\
\hline 4 & 12.38 & 12.25 & -- & -- & -- & -- & -- & -- & -- & -- & -- & -- \\
\hline 5 & 12.34 & 12.20 & -- & -. & -- & -- & -- & -. & -- & -- & -- & -- \\
\hline 6 & 12.45 & 12.53 & -- & -- & -- & -- & -- & -- & -- & -- & -- & -- \\
\hline 7 & 12.63 & 11.82 & -- & -- & -- & -- & -- & -- & -- & -- & -- & -- \\
\hline 8 & 12.57 & 11.62 & -- & -- & -- & -- & -- & -- & -- & -- & -- & -- \\
\hline 9 & 12.47 & 12.19 & -- & -- & -- & -- & -- & -- & -- & -- & -- & -- \\
\hline 10 & 12.46 & 12.54 & -- & -- & -- & -- & -- & -- & -- & -- & -- & -- \\
\hline 11 & 12.43 & 12.84 & -- & -- & -- & -- & -- & -- & -- & -- & -- & -- \\
\hline 12 & 12.54 & 12.72 & -- & -- & -- & -- & -- & -- & -- & -- & -- & - \\
\hline 13 & 12.79 & 12.95 & -- & -- & -- & -. & -- & - & -- & -- & -- & -- \\
\hline 14 & 12.68 & 12.45 & -. & -- & -- & -- & -- & -- & -- & -- & -- & -- \\
\hline 15 & 12.53 & 12.22 & -- & -- & -- & -- & -- & -- & -- & -- & -- & -- \\
\hline 16 & 12.58 & 12.57 & -- & -- & -- & -- & -- & -- & - & -- & -- & -- \\
\hline 17 & 12.55 & 12.53 & -- & -- & -- & -- & -- & -- & -- & -- & -- & -- \\
\hline 18 & 12.46 & 12.55 & -- & -- & -- & -- & -- & -- & -. & -- & -- & -- \\
\hline 19 & 12.50 & 12.76 & -- & -- & -- & -- & -- & -- & -- & -- & -- & -- \\
\hline 20 & 13.07 & 12.12 & -- & -- & -- & -- & -. & -- & -- & -- & -- & -- \\
\hline 21 & 12.80 & 12.03 & -. & -- & -- & -- & -- & -- & -- & -- & -- & -- \\
\hline 22 & 12.56 & 11.72 & -. & -- & -- & -- & -- & -- & - & -- & -- & -- \\
\hline 23 & 12.42 & 11.63 & -- & -- & - & -- & - & -. & -- & -- & -- & -- \\
\hline 24 & 12.70 & 11.67 & -- & - & -- & -- & -- & -. & -- & -- & -- & -- \\
\hline 25 & 12.85 & 11.79 & -- & -- & -- & -. & -- & -- & -- & -- & -- & -- \\
\hline 26 & 12.57 & 11.48 & -- & -- & -- & -- & - & - & -- & -- & -- & -- \\
\hline 27 & 12.42 & 11.29 & -- & -- & -- & -- & -- & -- & -- & -. & - & -- \\
\hline 28 & 12.46 & 11.07 & -- & -- & -- & -- & -- & -- & -- & -- & -- & -- \\
\hline 29 & 12.71 & -- & -- & -- & -- & -- & - & -- & - & -- & - & -- \\
\hline 30 & 12.63 & -- & -- & -- & -- & -- & -- & -- & -- & -- & -- & -- \\
\hline 31 & 12.30 & -- & -- & -- & -- & -- & -- & -- & -- & -- & -. & -- \\
\hline
\end{tabular}


Table 10I. Mean daily ground-water levels in observation well CRM-17 (map ID \#14), Seminole Well Field, Cedar Rapids, lowa, November 1997 through September 1998.

[Water levels measured as feet below land surface; --, value not measured or recorded]

\begin{tabular}{|c|c|c|c|c|c|c|c|c|c|c|c|c|}
\hline Day & Jan & Feb & Mar & Apr & May & Jun & Jul & Aug & Sep & Oct & Nov & Dec \\
\hline & & & & & & 1997 & & & & & & \\
\hline 1 & -- & -- & -- & -- & -- & -- & -- & -- & -- & -- & -- & 11.80 \\
\hline 2 & -- & -- & -- & -- & -- & -. & - & -- & -- & -- & -- & 11.66 \\
\hline 3 & -. & -- & -- & -- & -- & -- & -- & -- & -- & -- & -- & 11.87 \\
\hline 4 & -. & -- & -- & -- & -- & -- & -- & -- & -- & -- & -- & 12.95 \\
\hline 5 & -- & -- & -- & -- & -- & -- & -- & -- & -- & -- & -- & 13.75 \\
\hline 6 & -- & -- & -- & -- & - & -- & -- & -- & -- & -- & -- & 14.37 \\
\hline 7 & - & -- & -- & - & -- & -- & -- & -- & -- & -- & -- & 13.94 \\
\hline 8 & -- & -- & -- & -- & -- & -- & -- & -- & -- & -- & -- & 14.46 \\
\hline 9 & -- & -. & -- & -. & -- & -- & -- & .- & -- & -. & -- & 15.19 \\
\hline 10 & -- & -- & -- & -- & -- & -- & -- & -- & -- & -- & -- & 15.30 \\
\hline 11 & -- & -- & -- & -- & - & -- & -- & -- & -- & -- & -- & 15.57 \\
\hline 12 & -. & -- & -- & -- & -- & -- & -- & -- & -- & -- & -- & 15.51 \\
\hline 13 & -- & -- & -- & -- & -- & -- & -- & -- & -- & -- & -- & 15.61 \\
\hline 14 & -- & -- & -- & -- & -- & -- & -- & -- & -- & -- & -- & 15.74 \\
\hline 15 & -- & -- & -- & -- & -- & -- & -- & -- & -- & -- & -- & 15.44 \\
\hline 16 & -. & -- & -. & -- & -- & -- & -- & -- & -- & -- & -- & 15.31 \\
\hline 17 & -- & -- & -- & -- & -- & -- & -- & -- & -- & -- & -- & 15.26 \\
\hline 18 & -- & -- & -- & -- & -. & -- & -. & -- & -. & -- & -- & 15.33 \\
\hline 19 & -- & -- & -- & -- & -- & -- & -- & -- & -- & -- & -- & 15.56 \\
\hline 20 & -- & -- & -- & - & -- & -- & -- & -- & -- & -- & -- & 15.43 \\
\hline 21 & -- & -- & -- & -- & -- & -- & -- & -- & -- & -- & 12.12 & 15.71 \\
\hline 22 & -- & -- & -- & -- & -- & -. & -- & -- & -- & -- & 11.71 & 15.56 \\
\hline 23 & -- & -- & -- & -- & -- & -- & -- & -- & -- & -- & 11.67 & 15.97 \\
\hline 24 & -- & -- & -- & -- & -- & -- & -- & -- & -- & -- & 11.50 & 16.22 \\
\hline 25 & -- & -- & -- & -- & -- & -- & -- & -- & - & -- & 12.37 & 16.13 \\
\hline 26 & -- & -- & -- & -- & -- & -- & -- & -- & -- & -- & 12.64 & 16.24 \\
\hline 27 & -- & -- & -- & -- & -- & -- & -- & -- & -- & -- & 12.73 & 16.64 \\
\hline 28 & -- & -- & -- & -- & -- & -- & -- & -- & -- & -- & 12.12 & 17.15 \\
\hline 29 & -- & -- & -- & -- & -- & -- & -- & -- & -- & -- & 11.66 & 17.28 \\
\hline 30 & -- & -- & -- & -- & -- & -- & -- & -- & -- & -- & 11.66 & 17.09 \\
\hline 31 & -- & -- & -- & -- & -- & -- & -- & -- & -- & -- & - & 16.64 \\
\hline
\end{tabular}


Table 10l. Mean daily ground-water levels in observation well CRM-17 (map ID \#14), Seminole Well Field, Cedar Rapids, lowa, November 1997 through September 1998--Continued

\begin{tabular}{|c|c|c|c|c|c|c|c|c|c|c|c|c|}
\hline Day & Jan & Feb & Mar & Apr & May & Jun & Jul & Aug & Sep & Oct & Nov & Dec \\
\hline & & & & & & 1998 & & & & & & \\
\hline 1 & 16.93 & 15.71 & 12.25 & 4.44 & 9.48 & 11.40 & 2.56 & 11.07 & 13.29 & -- & -- & -- \\
\hline 2 & 17.27 & 15.66 & 11.71 & 2.72 & 10.05 & 11.03 & 2.25 & 12.83 & 12.69 & -- & -- & -- \\
\hline 3 & 17.09 & 15.28 & 11.60 & 2.48 & 10.24 & 10.86 & 1.55 & 13.06 & 12.25 & -- & -- & -- \\
\hline 4 & 16.45 & 15.69 & 11.88 & 2.96 & 10.71 & 10.82 & 1.93 & 13.28 & 11.63 & -- & -- & -- \\
\hline 5 & 16.11 & 15.44 & 12.00 & 3.15 & 11.23 & 10.81 & 3.14 & 13.61 & 11.55 & -- & -- & -- \\
\hline 6 & 16.06 & 15.57 & 12.25 & 3.17 & 11.76 & 10.86 & 4.22 & 13.52 & 11.37 & -- & -- & - \\
\hline 7 & 15.86 & 15.62 & 12.12 & 2.73 & 12.56 & 10.64 & 5.07 & 13.14 & 11.39 & -- & -. & -- \\
\hline 8 & 16.03 & 15.64 & 12.35 & 3.38 & 12.38 & 10.64 & 5.83 & 13.13 & 11.66 & -- & -. & -- \\
\hline 9 & 15.87 & 15.62 & 11.79 & 4.41 & 12.81 & 10.26 & 5.40 & 13.06 & 11.77 & -- & -- & -- \\
\hline 10 & 15.91 & 15.48 & 7.53 & 4.51 & 13.14 & 10.18 & 6.12 & 12.88 & 12.02 & -- & -- & -- \\
\hline 11 & 15.65 & 15.92 & 8.79 & 4.42 & 13.35 & 9.75 & 6.99 & 13.11 & 11.93 & -- & -- & -- \\
\hline 12 & 15.30 & 15.57 & 9.22 & 4.72 & 13.82 & 7.28 & 7.22 & 12.62 & 11.94 & -- & -. & -- \\
\hline 13 & 13.15 & 15.55 & 13.18 & 4.89 & 13.80 & 5.52 & 7.54 & 13.10 & 12.29 & -- & -. & -. \\
\hline 14 & 14.89 & 15.88 & 10.94 & 5.04 & 13.91 & 4.07 & 7.92 & 12.97 & 12.88 & -- & -- & -- \\
\hline 15 & 14.92 & 15.81 & 10.21 & 5.19 & 14.00 & 2.83 & 8.34 & 13.11 & 13.20 & -- & -- & -- \\
\hline 16 & 15.54 & 15.94 & 11.76 & 5.07 & 14.33 & 3.38 & 9.03 & 13.06 & 13.56 & -- & -- & -- \\
\hline 17 & 15.85 & 16.30 & 13.97 & 5.63 & 14.36 & 3.62 & 8.97 & 13.20 & 13.56 & -- & -- & -- \\
\hline 18 & 16.20 & 16.96 & 14.02 & 5.94 & 14.23 & 4.33 & 8.77 & 13.51 & 13.41 & -- & -- & -- \\
\hline 19 & 16.20 & 16.63 & 14.15 & 5.66 & 14.31 & 4.12 & 8.93 & 13.26 & 13.62 & -- & -- & -- \\
\hline 20 & 16.28 & 15.85 & 14.10 & 5.46 & 14.48 & 4.47 & 9.27 & 13.15 & 13.65 & -- & -- & -- \\
\hline 21 & 16.40 & 15.83 & 14.08 & 5.86 & 14.49 & 4.91 & 9.53 & 13.13 & 13.77 & -- & -- & -- \\
\hline 22 & 16.61 & 15.30 & 14.04 & 6.14 & 14.43 & 3.58 & 9.86 & 13.20 & 13.67 & -- & -- & -- \\
\hline 23 & 16.52 & 14.50 & 14.23 & 5.92 & 14.59 & 2.56 & 10.14 & 13.50 & 11.92 & -- & -- & -- \\
\hline 24 & 16.15 & 14.14 & 14.01 & 6.00 & 14.62 & 2.12 & 10.57 & 13.83 & 11.04 & -- & -- & -- \\
\hline 25 & 15.76 & 13.98 & 13.54 & 6.63 & 14.62 & 0.99 & 10.39 & 13.74 & 10.57 & -- & -- & -- \\
\hline 26 & 15.79 & 13.35 & 13.41 & 6.83 & 14.22 & 0.82 & 10.71 & 13.88 & 10.55 & -- & -- & -- \\
\hline 27 & 15.66 & 13.01 & 13.13 & 6.39 & 13.44 & 1.31 & 11.11 & 13.67 & 10.18 & -- & -- & -- \\
\hline 28 & 15.73 & 12.96 & 12.76 & 6.91 & 12.81 & 1.11 & 11.73 & 13.56 & -- & -- & -- & -- \\
\hline 29 & 15.42 & -- & 12.86 & 7.96 & 12.51 & 1.73 & 12.21 & 13.58 & -- & -- & -- & -- \\
\hline 30 & 15.50 & -- & 12.21 & 8.45 & 12.23 & 2.23 & 12.58 & 13.54 & -- & -- & -- & - \\
\hline 31 & 15.37 & -- & 6.82 & -- & 11.92 & -. & 11.73 & 13.77 & -- & -- & -- & -- \\
\hline
\end{tabular}


Table 10m. Mean daily ground-water levels in observation well CRM-18 (map ID \#15), Seminole Well Field, Cedar Rapids, lowa, January 1997 through February 1999

[Water levels measured as feet below land surface; --, value not measured or recorded]

\begin{tabular}{|c|c|c|c|c|c|c|c|c|c|c|c|c|}
\hline Day & Jan & Feb & Mar & Apr & May & Jun & Jul & Aug & Sep & Oct & Nov & Dec \\
\hline \multicolumn{13}{|c|}{1997} \\
\hline 1 & -- & 17.02 & 5.93 & -0.17 & 5.09 & 7.72 & 4.94 & 6.71 & 6.07 & 7.65 & 6.99 & 7.17 \\
\hline 2 & - & 15.52 & 3.44 & 1.28 & 8.18 & 6.35 & 3.87 & 6.98 & 6.28 & 7.77 & 7.18 & 7.26 \\
\hline 3 & -- & 16.52 & 4.37 & 2.12 & 6.51 & 4.91 & 5.25 & 7.44 & 6.21 & 7.90 & 6.95 & 7.40 \\
\hline 4 & -- & 16.47 & 3.10 & 4.95 & 2.71 & 4.70 & 5.87 & 7.33 & 6.18 & 8.00 & 6.92 & 7.85 \\
\hline 5 & - & 16.14 & 0.27 & 8.55 & 1.64 & 4.35 & 6.12 & 7.59 & 6.64 & 7.99 & 6.82 & 8.49 \\
\hline 6 & -- & 17.18 & 2.57 & 7.37 & 1.63 & 4.22 & 6.60 & 7.71 & 6.89 & 7.99 & 6.67 & 8.95 \\
\hline 7 & -- & 18.10 & 0.93 & 2.52 & 3.38 & 5.07 & 6.88 & 7.64 & 6.92 & 7.94 & 6.91 & 8.76 \\
\hline 8 & -- & 16.63 & -0.55 & 4.87 & 4.18 & 4.75 & 7.57 & 7.75 & 6.97 & 8.02 & 7.26 & 9.08 \\
\hline 9 & - & 17.50 & 1.06 & 4.19 & 3.97 & 5.05 & 7.09 & 8.24 & 6.91 & 7.82 & 7.37 & 9.30 \\
\hline 10 & -- & 15.96 & 2.38 & 3.84 & 4.21 & 5.21 & 7.11 & 7.70 & 7.13 & 7.66 & 7.47 & 9.36 \\
\hline 11 & -- & 15.77 & 0.97 & 5.32 & 3.77 & 5.49 & 7.25 & 7.62 & 7.25 & 7.99 & 7.64 & 9.16 \\
\hline 12 & -- & 17.33 & 0.80 & 5.47 & 4.13 & 5.64 & 7.52 & 7.88 & 7.32 & 8.23 & 8.00 & 9.22 \\
\hline 13 & -- & 17.81 & 0.31 & 6.12 & 4.72 & 5.90 & 7.16 & 7.62 & 7.34 & 8.21 & 8.10 & 9.62 \\
\hline 14 & -- & 17.32 & -0.68 & 6.17 & 5.57 & 6.59 & 7.48 & 8.07 & 7.31 & 7.64 & -- & 9.56 \\
\hline 15 & -- & 16.77 & -1.59 & 6.31 & 5.66 & 8.92 & 7.44 & 8.25 & 7.53 & 7.42 & -- & 9.48 \\
\hline 16 & -- & 17.16 & -2.25 & 5.84 & 5.84 & 6.80 & 7.51 & 8.11 & 7.58 & 7.09 & -- & 9.43 \\
\hline 17 & -- & 17.52 & -2.35 & 6.27 & 6.04 & 6.40 & 7.30 & 7.96 & 7.57 & 6.71 & -- & 9.48 \\
\hline 18 & -- & 18.44 & -1.92 & 6.68 & 6.00 & 7.93 & 7.04 & 7.70 & 7.42 & 6.64 & -- & 9.55 \\
\hline 19 & -- & 15.20 & 1.49 & 6.34 & 4.17 & 7.45 & 6.61 & 8.02 & 7.43 & 6.87 & -- & 9.59 \\
\hline 20 & -- & 13.62 & 3.63 & 6.52 & 5.98 & 8.07 & 6.62 & 8.19 & 7.17 & 6.45 & -- & 9.24 \\
\hline 21 & -- & 9.93 & 4.49 & 6.85 & 6.42 & 6.42 & 6.86 & 8.25 & 7.24 & 6.50 & 7.32 & 9.52 \\
\hline 22 & -- & 2.18 & 2.55 & 6.95 & 6.55 & 6.82 & 6.65 & 8.06 & 7.44 & 6.77 & 7.08 & 9.93 \\
\hline 23 & -- & 2.63 & 0.74 & 4.59 & 6.82 & 7.13 & 6.72 & 8.17 & 7.46 & 6.82 & 6.81 & 10.04 \\
\hline 24 & 15.17 & 3.11 & 2.00 & 4.97 & 6.36 & 6.17 & 6.64 & 8.42 & 7.68 & 7.15 & 6.95 & 10.06 \\
\hline 25 & 14.03 & 2.70 & 2.02 & 5.15 & 6.71 & 4.90 & 7.04 & 8.39 & 7.79 & 7.18 & 7.39 & 10.01 \\
\hline 26 & 13.82 & 5.27 & 1.04 & 7.72 & 7.27 & 2.62 & 6.99 & 8.65 & 7.76 & 7.35 & 7.53 & 10.29 \\
\hline 27 & 14.09 & 5.96 & 2.14 & 8.20 & 7.18 & 3.08 & 7.67 & 8.67 & 7.68 & 6.90 & 7.56 & 10.42 \\
\hline 28 & 16.50 & 5.66 & 3.54 & 7.07 & 7.41 & 4.37 & 7.19 & 8.35 & 7.87 & 6.80 & 7.22 & 10.55 \\
\hline 29 & 16.02 & -- & 2.43 & 5.19 & -- & 4.56 & 7.21 & 7.72 & 8.20 & 6.78 & 6.98 & 10.45 \\
\hline 30 & 15.89 & -- & 1.61 & 8.63 & 7.33 & 4.38 & 6.76 & 6.58 & 7.89 & 6.73 & 7.25 & 10.54 \\
\hline 31 & 18.50 & -- & 0.72 & .. & 7.40 & -. & 6.64 & 6.20 & -- & 6.99 & -- & -- \\
\hline
\end{tabular}


Table 10m. Mean daily ground-water levels in observation well CRM-18 (map ID \#15), Seminole Well Field, Cedar Rapids, lowa, January 1997 through February 1999--Continued

\begin{tabular}{|c|c|c|c|c|c|c|c|c|c|c|c|c|}
\hline Day & Jan & Feb & Mar & Apr & May & Jun & Jul & Aug & Sep & Oct & Nov & Dec \\
\hline \multicolumn{13}{|c|}{1998} \\
\hline 1 & -- & 9.96 & 5.94 & 0.16 & 6.74 & 7.42 & 4.96 & 6.68 & 6.85 & 6.02 & 5.02 & 6.44 \\
\hline 2 & 10.65 & 9.80 & 5.79 & -0.91 & 6.70 & 6.84 & 3.88 & 6.70 & 5.82 & 5.80 & 4.79 & 6.34 \\
\hline 3 & 10.49 & 9.58 & 5.70 & -1.08 & 6.34 & -- & 5.27 & 6.81 & 6.25 & 5.89 & 4.99 & 6.29 \\
\hline 4 & 10.26 & 9.75 & 5.84 & -1.16 & 6.62 & 4.34 & 5.87 & 6.81 & 5.58 & 5.87 & 5.57 & 5.92 \\
\hline 5 & 10.29 & 9.96 & 5.51 & -0.73 & 6.87 & 4.16 & 6.12 & 6.98 & 5.59 & 5.43 & 5.71 & 7.22 \\
\hline 6 & 10.12 & 9.99 & 5.69 & -0.35 & 7.41 & 3.96 & 6.61 & 6.53 & 5.66 & 5.51 & 5.70 & 6.15 \\
\hline 7 & 10.00 & 10.16 & 5.90 & 0.39 & 9.91 & 4.09 & 6.89 & 6.20 & 5.78 & 5.58 & 5.47 & 6.27 \\
\hline 8 & 10.11 & 10.20 & 6.05 & 0.83 & 7.82 & 3.96 & 7.59 & 6.25 & 5.70 & 6.99 & 5.63 & 6.48 \\
\hline 9 & 10.14 & 10.27 & 5.97 & 0.95 & 8.23 & 3.66 & 7.09 & 6.22 & 5.97 & 7.72 & 6.87 & 6.49 \\
\hline 10 & 9.68 & 10.24 & 5.85 & 0.98 & 8.36 & 3.36 & 7.11 & 5.94 & 5.97 & 7.65 & 16.93 & 5.98 \\
\hline 11 & 9.49 & 10.24 & 6.25 & 1.03 & 8.44 & 7.23 & 7.25 & 6.16 & 6.05 & 7.97 & 4.97 & 6.56 \\
\hline 12 & 9.57 & 10.17 & 6.35 & 1.29 & 9.16 & 2.68 & 7.53 & 6.63 & 6.22 & 8.41 & 4.93 & 6.63 \\
\hline 13 & 8.97 & 10.31 & 6.64 & 1.50 & 8.78 & -0.28 & 7.17 & 6.52 & 6.18 & 8.45 & 4.46 & 6.76 \\
\hline 14 & 9.17 & 10.24 & 6.67 & 0.88 & 8.89 & 7.84 & 7.50 & 6.56 & 6.49 & 7.82 & 4.96 & 6.84 \\
\hline 15 & 9.39 & 10.56 & 6.82 & 6.05 & 9.07 & 5.77 & 7.44 & 6.68 & 6.41 & 7.02 & 4.64 & 7.45 \\
\hline 16 & 9.46 & 10.56 & 6.95 & 3.48 & 8.98 & 0.57 & 7.51 & 6.59 & 6.72 & 6.77 & 6.36 & 8.98 \\
\hline 17 & 9.68 & 10.81 & 7.39 & 0.55 & 9.07 & -0.27 & -- & 6.77 & 6.64 & 6.94 & 4.51 & 9.39 \\
\hline 18 & 9.72 & 10.70 & 7.49 & 0.62 & 8.88 & 0.70 & 4.36 & 6.67 & 6.77 & 5.57 & 5.41 & 13.16 \\
\hline 19 & 9.80 & 10.64 & 7.42 & 0.56 & 9.00 & -0.01 & 4.32 & 6.98 & 6.83 & 5.37 & 4.98 & 9.26 \\
\hline 20 & 10.07 & 10.32 & 7.22 & 0.33 & 9.03 & -0.03 & 4.63 & -- & 6.72 & 4.69 & 5.17 & 7.58 \\
\hline 21 & 10.27 & 9.73 & 7.05 & 0.12 & 9.09 & -0.38 & 4.88 & 6.95 & 7.04 & 4.25 & 5.63 & 7.35 \\
\hline 22 & 10.38 & 8.97 & 6.99 & 0.29 & 8.91 & -1.36 & -- & 7.11 & 7.04 & 3.51 & 5.74 & 5.96 \\
\hline 23 & 10.54 & 8.24 & 6.95 & 1.44 & 8.96 & -- & 5.12 & 8.22 & 6.43 & 4.40 & 6.08 & 6.55 \\
\hline 24 & 10.42 & 7.67 & 6.64 & 2.67 & 8.99 & -. & 5.41 & 8.84 & 5.96 & 4.75 & 5.83 & 6.54 \\
\hline 25 & 10.38 & 7.52 & 6.62 & 6.30 & 8.95 & 4.91 & 5.76 & 8.51 & 5.56 & 4.56 & 5.47 & 6.88 \\
\hline 26 & 10.24 & 7.40 & 6.32 & 6.02 & 8.42 & 2.63 & 5.67 & 8.23 & 5.70 & 4.72 & 5.54 & 6.35 \\
\hline 27 & 10.14 & 6.94 & 6.23 & 4.31 & 7.50 & 3.08 & 5.89 & 8.08 & 5.53 & 4.77 & 5.72 & 6.35 \\
\hline 28 & 9.98 & 6.24 & 6.10 & 4.84 & 6.88 & 4.38 & 6.02 & 8.11 & 5.50 & 4.84 & 5.92 & 6.75 \\
\hline 29 & 10.00 & -- & 5.92 & 5.10 & 6.64 & 4.58 & 6.24 & 8.07 & 5.63 & 4.59 & 8.93 & 7.24 \\
\hline 30 & 9.99 & -- & 5.80 & 5.54 & 8.70 & 4.40 & 6.25 & 7.99 & 5.71 & 5.08 & 7.01 & 6.39 \\
\hline 31 & 9.95 & -- & 1.74 & - & 7.60 & -- & 6.67 & 7.91 & -- & 5.30 & -- & -- \\
\hline
\end{tabular}


Table 10m. Mean daily ground-water levels in observation well CRM-18 (map ID \#15), Seminole Well Field, Cedar Rapids, lowa, January 1997 through February 1999--Continued

\begin{tabular}{|c|c|c|c|c|c|c|c|c|c|c|c|c|}
\hline Day & Jan & Feb & Mar & Apr & May & Jun & Jul & Aug & Sep & Oct & Nov & $\overline{\text { Dec }}$ \\
\hline & & & & & & 1999 & & & & & & \\
\hline 1 & -- & 6.36 & -- & -- & -- & -- & -- & -- & -- & -. & -- & -. \\
\hline 2 & 6.98 & 7.12 & -- & -- & -- & -- & -- & -- & -- & -. & -- & -- \\
\hline 3 & 6.60 & 14.83 & - & -- & -. & -- & -- & -- & -- & -. & -- & -- \\
\hline 4 & 6.77 & 8.46 & -- & -- & -- & -- & -- & -- & -- & -. & -- & -- \\
\hline 5 & 6.70 & 8.26 & -- & -- & -- & -- & -- & -- & -- & -- & -- & -- \\
\hline 6 & 6.62 & 8.28 & -- & -- & -- & -- & -- & -- & -- & -- & -- & -- \\
\hline 7 & 6.69 & 6.16 & -- & -- & -- & -- & -- & -- & -- & -- & -- & -- \\
\hline 8 & 6.65 & 5.95 & -- & -- & -- & -- & -- & -- & - & -- & -. & -- \\
\hline 9 & 6.92 & 7.42 & -- &.- & -- & -- & -- & -- & -- & -- & -- & -- \\
\hline 10 & 6.91 & 10.07 & -- & -- & -- & -- & -- & -- & -. & -- & -- & -- \\
\hline 11 & 8.25 & 14.37 & -- & - & -- & -- & -- & -- & -- & -- & -. & -- \\
\hline 12 & 6.78 & 8.76 & -- & -- & -- & -. & -- & -- & -- & -- & -- & -. \\
\hline 13 & 6.99 & 8.35 & -- & -- & -- & -- & -- & -- & -- & -- & -- & - \\
\hline 14 & 6.88 & 8.72 & -- & -- & -. & -. & -. & -. & -- & -- & -- & -- \\
\hline 15 & 8.22 & 8.73 & -- & -- & -- & -- & -- & -- & -- & -- & -- & -- \\
\hline 16 & 7.57 & 8.66 & -- & -- & -- & -. & .. & -. & -- & -- & -- & -- \\
\hline 17 & 11.76 & 8.89 & -- & - & -- & -- & -- & - & -- & -- & -- & -- \\
\hline 18 & 10.64 & 8.95 & -- & -- & -- & -- & -- & -. & -- & -- & -- & -- \\
\hline 19 & 8.02 & 8.79 & -- & -- & -- & -. & -- & -. & -- & -. & -. & -- \\
\hline 20 & 9.03 & 8.89 & -- & -- & -- & -- & -- & - & -- & -. & -- & -- \\
\hline 21 & 9.14 & 8.20 & -- & -- & -- & -- & -- & -- & -- & -. & - & -- \\
\hline 22 & 10.28 & 8.78 & -- & -- & - & -- & -- & -- & -- & - & -. & -- \\
\hline 23 & 8.42 & 8.89 & -- & -- & -- & -- & -- & -- & -- & -- & -- & -- \\
\hline 24 & 6.70 & 9.06 & -- & -- & -- & -- & -- & -- & -- & -. & -- & -- \\
\hline 25 & 6.58 & 9.00 & -- & -- & -- & -- & -- & -- & -- & -- & -- & -- \\
\hline 26 & 6.76 & 9.34 & -- & - & -- & -- & -- & -- & -- & -- & -- & -- \\
\hline 27 & 7.25 & 12.76 & -- & -- & -- & -- & -- & -- & -- & -- & -- & -- \\
\hline 28 & 6.74 & 10.60 & - & -- & -- & -. & -- & -- & -- & -- & -- & -- \\
\hline 29 & 6.23 & -- & -- & -- & -- & -- & -. & -- & -- & - & -- & -- \\
\hline 30 & 6.02 & -- & -- & -- & -- & -- & -- & -- & -- & -- & -- & -- \\
\hline 31 & 6.60 & -- & -. & -- & -. & .. & -- & -. & -- & -. & .. & -. \\
\hline
\end{tabular}


Table 10n. Mean daily ground-water levels in observation well CRM-19 (map ID \#16), Seminole Well Field, Cedar Rapids, lowa, January 1997 through February 1999

[Water levels measured as feet below land surface; --, value not measured or recorded]

\begin{tabular}{|c|c|c|c|c|c|c|c|c|c|c|c|c|}
\hline Day & Jan & Feb & Mar & Apr & May & Jun & Jul & Aug & Sep & Oct & Nov & Dec \\
\hline \multicolumn{13}{|c|}{1997} \\
\hline 1 & -. & 19.87 & 7.28 & 4.18 & 6.30 & 8.42 & 6.10 & 7.60 & 6.99 & 8.21 & 7.78 & 7.94 \\
\hline 2 & -- & 19.80 & 6.39 & 4.63 & 8.12 & 6.86 & 5.14 & 7.80 & 7.07 & 8.54 & 7.86 & 7.95 \\
\hline 3 & -- & 19.65 & 6.12 & 5.26 & 7.12 & 5.28 & 6.48 & 8.07 & 6.81 & 8.55 & 8.00 & 8.57 \\
\hline 4 & -- & 19.62 & 5.29 & 6.23 & 3.40 & 5.28 & 6.51 & 8.26 & 6.91 & 8.75 & 7.82 & 8.92 \\
\hline 5 & -- & 19.39 & 4.28 & 6.59 & 2.96 & 5.70 & 6.81 & 8.74 & 7.14 & 8.81 & 8.19 & 9.20 \\
\hline 6 & -- & 19.60 & 4.52 & 6.77 & 2.56 & 5.76 & 7.31 & 8.85 & 7.45 & 8.34 & 7.98 & 9.29 \\
\hline 7 & -- & 19.88 & 3.35 & 5.33 & 4.79 & 5.66 & 7.76 & 8.86 & 7.31 & 8.30 & 8.28 & 9.08 \\
\hline 8 & -- & 19.56 & 2.75 & 6.14 & 5.51 & 5.44 & 8.29 & 8.64 & 7.43 & 8.64 & 8.19 & 9.74 \\
\hline 9 & -. & 20.12 & 3.56 & 5.60 & 5.31 & 5.80 & 8.06 & 8.91 & 7.43 & 8.67 & 8.08 & 9.87 \\
\hline 10 & -- & 20.55 & 4.38 & 5.12 & 5.43 & 5.81 & 8.19 & 8.70 & 7.37 & 8.47 & 8.45 & 9.81 \\
\hline 11 & - & 20.70 & 4.02 & 6.27 & 5.82 & 6.01 & 8.21 & 8.80 & -- & 8.79 & 8.59 & 9.64 \\
\hline 12 & -- & 20.90 & 3.19 & 7.13 & 5.96 & 6.16 & 8.22 & 8.63 & -- & 8.92 & 8.85 & 9.72 \\
\hline 13 & -- & 21.17 & 2.26 & 7.06 & 6.12 & 6.33 & 8.34 & 8.67 & -- & 8.78 & 9.09 & 9.70 \\
\hline 14 & -- & 21.34 & 1.32 & 7.11 & 6.60 & 6.56 & 8.31 & 8.91 & -- & 8.64 & -- & 9.52 \\
\hline 15 & -- & 21.56 & -0.01 & 7.02 & 6.76 & 8.65 & 9.53 & 8.85 & -- & 7.94 & -- & 9.64 \\
\hline 16 & -- & 21.13 & -0.33 & 6.92 & 7.03 & 6.50 & 7.95 & 9.07 & - & 7.51 & -- & 9.82 \\
\hline 17 & - & 21.60 & -0.40 & 7.36 & 7.68 & 7.35 & 7.75 & 8.85 & 8.22 & 7.68 & - & 9.77 \\
\hline 18 & - & 21.65 & 0.37 & 7.86 & 5.79 & 8.93 & 7.47 & 8.91 & 8.08 & 7.40 & -- & 10.02 \\
\hline 19 & -. & 18.84 & 3.29 & 8.20 & 5.08 & 8.44 & 7.07 & 8.84 & 8.06 & 7.63 & -- & 9.74 \\
\hline 20 & - & 15.77 & 5.43 & 8.37 & 7.31 & 9.02 & 7.30 & 9.14 & 7.94 & 7.82 & -- & 9.94 \\
\hline 21 & - & 11.25 & 5.08 & 8.43 & 7.54 & 7.23 & 7.51 & 8.86 & 7.95 & 7.92 & 8.32 & 9.77 \\
\hline 22 & - & 6.10 & 4.84 & 8.67 & 7.80 & 6.94 & 7.49 & 8.90 & 8.17 & 7.94 & 8.05 & 10.24 \\
\hline 23 & -- & 3.97 & 4.75 & 6.05 & 7.92 & 7.21 & 7.57 & 9.03 & 8.37 & 7.96 & 7.78 & 9.94 \\
\hline 24 & 16.73 & 3.64 & 4.27 & 5.72 & 8.26 & 6.65 & 7.57 & 9.24 & 8.31 & 8.31 & 8.07 & 10.28 \\
\hline 25 & 16.51 & 4.86 & 3.78 & 6.26 & 8.49 & 5.31 & 8.19 & 8.97 & 8.62 & 8.06 & 8.44 & 10.43 \\
\hline 26 & 17.12 & 6.24 & 3.27 & 8.88 & 8.31 & 3.52 & 8.60 & 9.30 & 8.23 & 8.37 & 8.32 & 10.64 \\
\hline 27 & 17.63 & 7.02 & 3.00 & 9.34 & 8.38 & 3.84 & 8.45 & 9.16 & 8.35 & 7.69 & 8.51 & 10.49 \\
\hline 28 & 17.76 & 7.51 & 1.79 & 8.66 & 8.22 & 5.06 & 8.09 & 9.12 & 8.47 & 7.89 & 8.34 & 10.29 \\
\hline 29 & 18.41 & -- & 3.62 & 6.28 & - & 5.80 & 8.36 & 8.35 & 8.53 & 7.67 & 8.14 & 10.25 \\
\hline 30 & 19.32 & -- & 4.07 & 6.15 & 7.93 & 5.76 & 7.45 & 7.20 & 8.52 & 7.94 & 8.34 & 10.41 \\
\hline 31 & 19.52 & -- & 4.38 & $\cdots$ & 8.02 & -- & 7.40 & 7.16 & - & 7.80 & -- & - \\
\hline
\end{tabular}


Table 10n. Mean daily ground-water levels in observation well CRM-19 (map ID \#16), Seminole Well Field, Cedar Rapids, lowa, January 1997 through February 1999--Continued

\begin{tabular}{|c|c|c|c|c|c|c|c|c|c|c|c|c|}
\hline Day & Jan & Feb & Mar & Apr & May & Jun & Jul & Aug & Sep & Oct & Nov & Dec \\
\hline \multicolumn{13}{|c|}{1998} \\
\hline 1 & -- & 9.96 & 6.47 & -0.10 & 7.41 & 7.35 & 5.27 & 6.52 & - & -- & -- & -- \\
\hline 2 & 10.84 & 9.79 & 5.63 & -0.99 & 7.69 & 6.38 & 4.39 & 7.05 & -- & -- & -- & -- \\
\hline 3 & 10.32 & 10.01 & 5.25 & -1.25 & 8.08 & -- & 5.45 & 7.51 & -- & -- & -- & -- \\
\hline 4 & 10.03 & 9.77 & 4.45 & -0.90 & 8.24 & 5.55 & 5.79 & 7.33 & -- & -- & -- & -- \\
\hline 5 & 10.16 & 9.64 & 3.09 & -0.68 & 8.37 & 5.30 & 6.08 & 7.87 & -. & -. & -- & -- \\
\hline 6 & 9.72 & 9.71 & 3.99 & -0.17 & 8.60 & 5.35 & 6.69 & 7.56 & -- & -- & -- & - \\
\hline 7 & 9.84 & 9.83 & 2.39 & 0.08 & 8.62 & 5.51 & 6.84 & 7.79 & -- & -- & -- & -- \\
\hline 8 & 10.26 & 9.90 & 1.76 & 0.65 & 8.95 & 5.41 & 7.48 & 7.78 & -- & -- & -- & -. \\
\hline 9 & 10.26 & 10.08 & 2.72 & 1.24 & 8.66 & 5.57 & 6.96 & 8.15 & -- & -- & -- & -- \\
\hline 10 & 9.63 & 10.38 & 3.45 & 1.08 & 8.74 & 4.71 & 6.91 & 7.75 & -- & -- & - & -- \\
\hline 11 & 9.57 & 10.20 & 2.99 & 1.20 & 9.22 & 4.02 & 7.16 & 7.52 & -- & -- & -- & -- \\
\hline 12 & 9.59 & 9.97 & 2.45 & 1.58 & 9.42 & 2.49 & 7.47 & 8.06 & -- & -- & -- & -- \\
\hline 13 & 9.24 & 10.33 & 1.38 & 1.53 & 9.07 & 1.51 & 7.38 & 7.71 & -- & -- & - & -- \\
\hline 14 & 9.37 & 10.24 & 0.44 & 2.18 & 9.26 & 0.38 & 7.46 & -- & -- & -- & -- & -- \\
\hline 15 & 9.54 & 10.53 & -0.29 & 2.19 & 9.69 & -0.37 & -- & -- & -- & -- & -- & -- \\
\hline 16 & 9.79 & 10.74 & -0.89 & 2.70 & 9.54 & 0.58 & 7.35 & -- & -- & -- & -- & -- \\
\hline 17 & 9.83 & 10.60 & -1.06 & 1.88 & 9.61 & 1.38 & 6.92 & -- & -- & -- & -- & -- \\
\hline 18 & 9.44 & 10.64 & -0.29 & 1.90 & 9.67 & 1.93 & 6.60 & -- & -. & -- & -- & -- \\
\hline 19 & 9.47 & 10.68 & 2.71 & 1.60 & 9.65 & 1.67 & 6.53 & $-\cdot$ & -- &.- & -- & -- \\
\hline 20 & 9.81 & 9.98 & 4.51 & 1.54 & 9.51 & 1.54 & 6.73 & -- & -- & -- & -- & -- \\
\hline 21 & 10.09 & 8.67 & 4.62 & 1.26 & 9.79 & 1.04 & 6.98 & -- & -- & -- & -- & -- \\
\hline 22 & 10.02 & 7.96 & -- & 1.49 & 10.20 & -0.29 & 6.88 & -- & -- & -- & -- & -- \\
\hline 23 & 10.38 & -- & -- & 2.83 & 10.34 & -- & 6.97 & -- & -. & -- & -- & -- \\
\hline 24 & 10.02 & -- & 5.98 & 4.85 & 10.19 & -- & 6.88 & -- & -- & -- & -- & -- \\
\hline 25 & 10.36 & 4.29 & 5.87 & 5.05 & 9.52 & 4.66 & 7.31 & -. & -- & -- & -- & -- \\
\hline 26 & 9.88 & 5.68 & 5.70 & 5.37 & 9.03 & 2.53 & 7.48 & -- & - & -- &.- & -- \\
\hline 27 & 9.92 & 6.42 & 5.34 & 5.84 & 8.42 & 3.06 & 7.57 & -- & - & -- & -- & -- \\
\hline 28 & 10.04 & 6.76 & 5.21 & 6.20 & 8.19 & 4.32 & 7.13 & -- & -. & -- & -- & -. \\
\hline 29 & 10.01 & -. & 5.09 & 6.39 & 7.61 & 4.75 & 8.07 & -- & -- & -- & -- & -. \\
\hline 30 & 9.74 & - & 4.77 & 7.16 & 7.52 & 4.68 & 7.09 & -- & -- & -- & -- & -- \\
\hline 31 & 10.02 & -- & 1.24 & -- & 7.40 & -- & 6.75 & -- & -. & -. & -- & -- \\
\hline
\end{tabular}


Table 10n. Mean daily ground-water levels in observation well CRM-19 (map ID \#16), Seminole Well Field, Cedar Rapids, lowa, January 1997 through February 1999--Continued

\begin{tabular}{|c|c|c|c|c|c|c|c|c|c|c|c|c|}
\hline Day & Jan & Feb & Mar & Apr & May & Jun & Jul & Aug & Sep & Oct & Nov & $\overline{D e c}$ \\
\hline & & & & & & 1999 & & & & & & \\
\hline 1 & 6.68 & 6.49 & -- & -. & -. & -. & -. & -- & -- & -- & -- & -- \\
\hline 2 & 6.84 & 6.51 & - & -- & -- & -- & - & -- & -- & -- & -- & -- \\
\hline 3 & 6.79 & 5.93 & - & -. & -- & -- & -- & -- & -- & -. & -- & -. \\
\hline 4 & 6.78 & 8.57 & -- & -. & -- & -- & -- & -- & -- & -- & -- & -- \\
\hline 5 & 6.43 & 8.51 & - & - & -. & -- & -- & -- & -- & -- & -- & -- \\
\hline 6 & 6.84 & 8.25 & - & - & -- & -- & -- & -- & -- & - & -- & -- \\
\hline 7 & 6.74 & 6.40 & - & - & -- & - & -- & -- & - & -- & -- & -- \\
\hline 8 & 6.64 & 5.97 & -- & -. & -- & -- & - & -- & -- & -- & -- & -- \\
\hline 9 & 6.85 & 7.35 & -- & -- & -. & -- & -- & -- & -- & -- & -- & -- \\
\hline 10 & 6.79 & 8.57 & -- & - & -- & - & - & -- & -- & -- & -- & -- \\
\hline 11 & 6.81 & 7.74 & - & -. & -- & - & - & -- & -- & -- & -- & -- \\
\hline 12 & 7.10 & 8.29 & - & -- & -- & -. & -- & -- & -- & -- & -- & -- \\
\hline 13 & 6.96 & 8.03 & -- & -- & -- & - & -- & -- & -- & -- & -- & -- \\
\hline 14 & 6.86 & 8.34 & - & -- & -- & -. & -- & -- & - & -- & -- & -- \\
\hline 15 & 6.83 & 8.67 & -. & - & - & -. & $\sim$ & -- & -- & -- & -- & -- \\
\hline 16 & 6.89 & 8.48 & - & -- & -- & -- & -. & -- & - & -- & -- & -- \\
\hline 17 & 6.20 & 8.35 & -- & -- & -- & -. & -- & -- & -- & -- & -- & .- \\
\hline 18 & 6.26 & 8.36 & - & -- & -. & -. & - & -- & - & - & -- & - \\
\hline 19 & 8.03 & 8.39 & -- & -- & -- & -- & - & -- & -- & -- & -- & -- \\
\hline 20 & 9.36 & 8.61 & -- & -- & -- & -. & - & -. & -. & -- & -- & -. \\
\hline 21 & 8.06 & 8.13 & -- & - & -. & -. & -. & - & -- & - & - & - \\
\hline 22 & 6.71 & 8.64 & -- & -- & -- & - & -- & -. & -- & -- & -- & -- \\
\hline 23 & 6.79 & 8.64 & -- & -- & -- & - & -- & -- & - & -- & -- & -- \\
\hline 24 & 6.55 & 8.82 & -- & -- & -- & -- & - & -. & -. & $\cdots$ & -- & -- \\
\hline 25 & 6.48 & 8.92 & -- & -- & -- & -- & -- & - & - & - & - & - \\
\hline 26 & 6.74 & 9.17 & - & -- & -- & - & - & - & - & -- & -- & - \\
\hline 27 & 6.69 & 8.88 & -- & -- & -- & -- & -- & -- & - & -- & - & - \\
\hline 28 & 6.61 & 9.21 & -- & -- & -- & -- & -- & -- & -. & -- & - & -- \\
\hline 29 & 6.20 & -- & -- & -- & -- & -- & $-\cdot$ & -. & - & -- & - & - \\
\hline 30 & 6.02 & -- & -- & -. & -- & -- & -- & -- & - & - & - & - \\
\hline 31 & 6.45 & -- & -- & -. & -- & -- & -- & -- & -- & -- & - & -- \\
\hline
\end{tabular}


Table 100. Mean daily ground-water levels in observation well CRM-22 (map ID \#19), Seminole Well Field, Cedar Rapids, lowa, February 1998 through February 1999

[Water levels measured as feet below land surface; --, value not measured or recorded]

\begin{tabular}{|c|c|c|c|c|c|c|c|c|c|c|c|c|}
\hline Day & Jan & Feb & Mar & Apr & May & Jun & Jul & Aug & Sep & $\overline{\text { Oct }}$ & Nov & Dec \\
\hline & & & & & & 1998 & & & & & & \\
\hline 1 & -- & -- & 2.60 & 4.75 & -- & 2.94 & -- & -- & 2.99 & 3.76 & 1.62 & 4.14 \\
\hline 2 & -- & -- & 3.15 & -- & -- & 3.84 & -- & -- & 3.30 & 3.61 & 2.22 & 4.24 \\
\hline 3 & -- & -- & 3.56 & -. & -- & 1.68 & -- & -- & 4.45 & 3.53 & 2.52 & -- \\
\hline 4 & -- & - & 3.57 & -. & -- & 1.03 & -. & -- & 2.32 & 3.33 & 2.97 & -- \\
\hline 5 & -- & -- & 3.73 & -- & 1.48 & 1.14 & -- & -- & 2.56 & 3.32 & 2.96 & 6.08 \\
\hline 6 & -- & -- & 3.70 & -- & 1.86 & 1.19 & -- & -- & 2.39 & 3.15 & 3.10 & 3.86 \\
\hline 7 & -- & -- & 3.84 & -. & 4.44 & 1.30 & -- & -- & 2.62 & 2.34 & 3.30 & 2.29 \\
\hline 8 & -- & -- & 6.78 & -- & 1.23 & 1.31 & -- & -- & 3.11 & 3.45 & 2.41 & 2.29 \\
\hline 9 & -- & -- & 4.17 & -- & 1.49 & 1.11 & -- & -- & 3.36 & 3.46 & 4.21 & 2.33 \\
\hline 10 & -- & - & 4.10 & -. & 1.43 & 0.95 & -- & -- & 3.44 & 3.38 & 10.07 & 2.20 \\
\hline 11 & -- & -- & 3.81 & -- & 1.62 & 5.67 & - & -- & 3.60 & 2.79 & 1.94 & 2.62 \\
\hline 12 & -- & -- & 2.99 & -- & 3.74 & 2.62 & -- & -- & 3.49 & 2.76 & 3.07 & 3.64 \\
\hline 13 & -- & -- & 1.68 & -- & 2.35 & -0.80 & -- & 3.17 & 3.10 & 3.15 & 2.94 & 3.86 \\
\hline 14 & -- & - & 2.79 & -- & 2.57 & 5.98 & -- & 2.97 & 3.93 & 3.42 & 3.83 & 4.00 \\
\hline 15 & -- & -- & 2.34 & -- & 3.18 & 4.53 & -- & 3.33 & 3.06 & 3.39 & 3.59 & 4.00 \\
\hline 16 & -- & - & 2.15 & -- & 2.64 & 0.18 & -- & 3.17 & 3.34 & 3.31 & 5.02 & 4.01 \\
\hline 17 & -- & -- & 2.07 & -- & 2.63 & -1.48 & -- & 2.86 & 3.39 & 3.30 & 3.14 & 4.05 \\
\hline 18 & -- & -- & 2.39 & -- & 2.49 & 0.56 & -- & 2.98 & 3.32 & 1.97 & 4.00 & 8.05 \\
\hline 19 & -- & 1.88 & 2.34 & -- & 2.66 & -0.08 & -. & 3.08 & 3.17 & 1.50 & 2.98 & 4.23 \\
\hline 20 & -- & 2.94 & 2.58 & -- & 2.76 & -0.51 & -- & 3.08 & 3.14 & 1.49 & 3.10 & 4.31 \\
\hline 21 & -- & 4.22 & 2.27 & -- & 2.66 & -1.72 & -- & 3.20 & 3.49 & 1.74 & 3.54 & 3.85 \\
\hline 22 & -- & 3.89 & 2.14 & -- & 1.80 & -2.75 & -- & 3.19 & 3.62 & 2.33 & 3.46 & 3.23 \\
\hline 23 & -- & 2.81 & 2.09 & -- & 1.69 & -- & -- & 3.44 & 3.99 & 2.72 & 3.71 & 3.88 \\
\hline 24 & -- & 2.37 & 2.68 & -- & 1.51 & -- & -- & 4.23 & 3.78 & 2.78 & 3.63 & 3.95 \\
\hline 25 & -- & 4.09 & 4.00 & -- & 1.90 & -- & -. & 3.12 & 3.83 & 2.66 & 3.73 & 3.75 \\
\hline 26 & -- & 10.69 & 5.33 & -- & 1.50 & -- & -- & 3.01 & 4.51 & 2.79 & 3.86 & 3.55 \\
\hline 27 & -- & 9.82 & 7.80 & -- & 1.00 & -- & -- & 3.12 & 3.59 & 2.54 & 3.83 & 4.00 \\
\hline 28 & -- & 4.28 & 7.17 & -- & 0.87 & -- & -- & 3.36 & 3.58 & 2.20 & 3.68 & 4.65 \\
\hline 29 & -- & -- & 5.37 & -- & 1.57 & -- & -- & 3.36 & 3.17 & 1.27 & 7.02 & 5.04 \\
\hline 30 & -- & -- & 8.82 & -. & 3.66 & -- & -- & 2.98 & 3.04 & 1.34 & 4.53 & 3.99 \\
\hline 31 & -. & - & 9.78 & -- & 2.79 & -- & -- & 2.98 & -- & 1.58 & -- & 4.27 \\
\hline
\end{tabular}


Table 10o. Mean daily ground-water levels in observation well CRM-22 (map ID \#19), Seminole Well Field, Cedar Rapids, lowa, February 1998 through February 1999--Continued

\begin{tabular}{|c|c|c|c|c|c|c|c|c|c|c|c|c|}
\hline Day & Jan & Feb & Mar & Apr & May & Jun & Jul & Aug & Sep & Oct & Nov & Dec \\
\hline & & & & & & 1999 & & & & & & \\
\hline 1 & 4.32 & 4.64 & -- & -- & -. & -. & -- & -- & -- & -- & -- & -. \\
\hline 2 & 5.24 & 6.19 & -- & -- & -- & -- & - & -- & -- & -- & -- & - \\
\hline 3 & 4.53 & 11.81 & -- & -- & -- & - & -- & -- & -- & -- & -- & -- \\
\hline 4 & 4.33 & 4.26 & -- & -- & -- & -- & -- & -- & -- & -- & -- & -- \\
\hline 5 & 4.22 & 4.31 & -- & -- & -- & -- & -- & -- & -- & -- & -- & -- \\
\hline 6 & 4.24 & 4.10 & -- & -- & -- & -- & -- & -- & -- & -- & -. & -. \\
\hline 7 & 4.35 & 4.68 & -- & -- & -- & -- & -- & -- & -- & -- & -- & -- \\
\hline 8 & 4.26 & 4.83 & -- & -- & -- & -- & -- & -- & -- & -- & -- & - \\
\hline 9 & 4.49 & 3.92 & -- & -- & -- & -- & - & -- & -- & -- & -- & -- \\
\hline 10 & 4.44 & 5.54 & -- & -- & -- & -- & -- & -- & -- & -- & -- & -- \\
\hline 11 & 6.53 & 8.92 & -- & -- & -- & -- & -- & - & -- & -- & -- & -- \\
\hline 12 & 4.82 & 3.65 & - & - & -- & -. & -. & -. & - & - & -- & -- \\
\hline 13 & 4.54 & 3.58 & -. & -- & -- & -- & -. & -- & -- & -- & -- & -- \\
\hline 14 & 4.43 & 3.59 & -- & -- & -- & -- & -- & -- & -- & - & -- & -- \\
\hline 15 & 6.31 & 4.29 & - & -- & -- & -- & -- & -- & -- & -- & -- & -- \\
\hline 16 & 5.55 & 3.59 & -- & -- & -- & -- & -- & -- & -- & -- & -- & -- \\
\hline 17 & 9.50 & 3.83 & -- & -- & -- & -- & -- & -- & -- & -- & -- & -- \\
\hline 18 & 8.34 & 3.88 & - & -- & -- & -- & -- & -- & -- & -- & -- & -- \\
\hline 19 & 4.69 & 3.72 & - & -- & -- & -- & -- & -- & -- & -- & -- & -- \\
\hline 20 & 4.49 & 3.89 & - & - & -- & -- & -- & -- & -- & -- & -- & -- \\
\hline 21 & 6.66 & 3.89 & -- & -- & -- & -. & -- & -- & -- & -- & -- & -- \\
\hline 22 & 8.70 & 3.98 & - & -- & -- & -- & -- & -- & -- & - & -- & -- \\
\hline 23 & 6.86 & 4.14 & - & -- & -- & -- & -- & -- & -- & -- & -- & -- \\
\hline 24 & 4.39 & 4.28 & - & -- & -- & -- & -- & -- & -- & -- & -- & -- \\
\hline 25 & 4.54 & 4.19 & -. & -- & -- & -- & -- & -- & -- & -- & -- & -- \\
\hline 26 & 4.64 & 5.09 & - & -- & -- & -- & -- & -- & -- & -- & -- & -- \\
\hline 27 & 5.52 & 8.94 & - & -- & -- & - & -- & -- & -- & -- & -- & -- \\
\hline 28 & 4.54 & 6.73 & - & -- & -- & -- & -- & -- & -- & -- & -- & -- \\
\hline 29 & 4.56 & -- & -. & -- & -- & -- & -- & -- & -- & -- & -. & -- \\
\hline 30 & 4.54 & -- & -- & -- & -- & -- & -- & -. & -- & -- & -- & -- \\
\hline 31 & 4.56 & -- & -- & -- & -- & -- & -- & -- & -- & -- & -- & -- \\
\hline
\end{tabular}


Table 10p. Mean daily ground-water levels in observation well CRM-23 (map ID \#20), Seminole Well Field, Cedar Rapids, lowa, February 1998 through February 1999

[Water levels measures as feet below land surface; --, value not measured or recorded]

\begin{tabular}{|c|c|c|c|c|c|c|c|c|c|c|c|c|}
\hline Day & Jan & Feb & Mar & Apr & May & Jun & Jul & Aug & Sep & Oct & Nov & Dec \\
\hline \multicolumn{13}{|c|}{1998} \\
\hline 1 & -- & -- & 1.61 & -2.05 & -- & 0.24 & - & -- & 2.75 & -- & -- & 3.52 \\
\hline 2 & -- & -- & 3.25 & -- & -. & 0.62 & -- & -. & -- & -. & -. & 3.79 \\
\hline 3 & -- & -- & 3.63 & -- & -- & 0.22 & -. & -- & -- & -. & -. & 4.06 \\
\hline 4 & -- & -- & 3.56 & -- & -- & -0.74 & -. & -- & -- & -. & -. & 3.91 \\
\hline 5 & -- & -- & 3.74 & -- & -0.51 & -0.51 & -- & -- & -- & -- & -- & 4.35 \\
\hline 6 & -- & -- & 3.72 & -- & -0.38 & -0.34 & -- & -- & -- & -- & -- & 3.09 \\
\hline 7 & -. & -. & 3.83 & -- & -0.01 & -0.24 & -- & -- & -- & -- & -- & 1.35 \\
\hline 8 & -. & -. & 4.39 & -- & -0.31 & -0.20 & -- & -- & -- & - & -- & 1.21 \\
\hline 9 & -- & -- & 4.13 & -- & -0.28 & -0.27 & -- & -- & -- & -- & -- & 1.06 \\
\hline 10 & -- & -- & 4.03 & - & -0.20 & -0.56 & -- & -- & -- & -- & -- & 0.89 \\
\hline 11 & -- & -- & 3.74 & -- & 0.01 & 0.10 & -- & -- & -- & -- & - & 1.45 \\
\hline 12 & -- & -- & 3.19 & -- & 1.86 & -0.66 & -. & -- & -- & -- & -- & 3.11 \\
\hline 13 & -- & -- & 1.49 & -- & 1.74 & -1.87 & -- & -- & -- & -- & 2.46 & 3.35 \\
\hline 14 & -- & -- & 1.60 & -- & 1.90 & -2.73 & -- & -- & -- & -- & 2.81 & 3.51 \\
\hline 15 & -- & -- & 0.99 & -- & 2.21 & -3.33 & -- & -- & - & -- & 2.70 & 3.66 \\
\hline 16 & -- & -- & 0.80 & -- & 2.25 & -2.47 & -- & -- & -. & - & 2.88 & 3.76 \\
\hline 17 & -- & -- & 1.04 & -- & 2.35 & -2.16 & -- & -- & -- & -- & 2.74 & 3.85 \\
\hline 18 & -- & -- & 1.25 & -- & -- & -1.36 & -- & -- & -- & -- & 2.96 & 4.19 \\
\hline 19 & -- & -. & 1.15 & -- & -- & -1.53 & -- & 2.71 & -- & -- & 2.82 & 3.94 \\
\hline 20 & -- & -- & 1.78 & -- & -- & -2.54 & -- & 2.75 & -- & -- & 2.94 & 3.97 \\
\hline 21 & -- & 3.82 & 1.66 & -- & -- & -3.51 & -- & 2.73 & -- & -- & 2.95 & 3.72 \\
\hline 22 & -- & 3.82 & 1.41 & -- & - & -4.46 & -- & 2.60 & -- & -- & 3.19 & 3.10 \\
\hline 23 & -- & 2.91 & 1.37 & -- & -- & -- & -- & 2.94 & -- & -- & 3.23 & 3.57 \\
\hline 24 & -- & 1.82 & 2.27 & -- & -- & -- & -- & 3.16 & -- & -- & 3.21 & 3.69 \\
\hline 25 & -- & 1.98 & 3.18 & -- & -- & -- & -- & 2.86 & - & -- & 3.49 & 3.66 \\
\hline 26 & -- & 1.93 & 3.50 & -- & -- & -- & -- & 2.95 & -- & - & 3.40 & 3.58 \\
\hline 27 & -- & 1.81 & 3.26 & -- & -- & -- & -- & 2.90 & -- & -- & 3.50 & 3.69 \\
\hline 28 & -- & 1.88 & 2.56 & -- & -- & -- & -- & 2.73 & -- & $\cdots$ & 3.38 & 3.77 \\
\hline 29 & -- & -. & 1.11 & -- & -- & -- & - & 2.82 & -- & -. & 3.87 & 3.87 \\
\hline 30 & -- & -- & 1.11 & -- & -- & -- & -- & 2.95 & -- & -. & 3.65 & 3.87 \\
\hline 31 & -- & -- & -1.33 & -- & 0.15 & -- & - & 2.96 & -- & -- & -- & 4.08 \\
\hline
\end{tabular}


Table 10p. Mean daily ground-water levels in observation well CRM-23 (map ID \#20), Seminole Well Field, Cedar Rapids, lowa, February 1998 through February 1999--Continued

\begin{tabular}{|c|c|c|c|c|c|c|c|c|c|c|c|c|}
\hline Day & Jan & Feb & Mar & Apr & May & Jun & Jul & Aug & Sep & Oct & Nov & Dec \\
\hline & & & & & & 1999 & & & & & & \\
\hline 1 & 4.10 & 4.56 & -- & -- & -- & -- & -- & -- & -- & -- & -- & -- \\
\hline 2 & 4.31 & 4.82 & -- & -- & -- & -- & -- & -- & -- & -- & -- & -- \\
\hline 3 & 4.21 & 4.55 & -- & -- & -- & -- & -- & -- & -- & -- & -- & -- \\
\hline 4 & 4.13 & 4.21 & -- & -- & -- & -- & -- & -- & -- & -- & -- & -- \\
\hline 5 & 4.12 & 4.23 & -- & -- & -- & -- & -- & -- & -- & -- & -- & -- \\
\hline 6 & 4.15 & 4.13 & -- & -- & -- & -- & -. & -. & -- & -- & -- & -- \\
\hline 7 & 4.07 & 4.40 & -- & -- & -- & -- & -- & -- & -- & -- & -- & -- \\
\hline 8 & 4.30 & 4.38 & -- & -- & -. & -- & -. & -- & -- & -- & -- & - \\
\hline 9 & 4.41 & 4.01 & -- & -- & -- & -- & -- & -- & -- & -- & -- & -- \\
\hline 10 & 4.41 & 4.01 & -- & -- & -- & -- & -- & -- & -- & -- & -- & -. \\
\hline 11 & 4.69 & 3.68 & -- & -- & -- & -- & -. & -- & -- & -- & -- & -- \\
\hline 12 & 4.57 & 3.60 & -- & -- & -- & -- & -- & -- & -- & -- & -- & -- \\
\hline 13 & 4.47 & 3.49 & -- & -- & -- & -- & -- & -- & -- & -- & -- & -- \\
\hline 14 & 4.45 & 3.69 & -- & -- & -- & -- & -. & -- & -- & -- & -- & -- \\
\hline 15 & 4.59 & 3.94 & -- & -- & -- & -- & -- & -- & -- & -- & -- & -- \\
\hline 16 & 4.55 & 3.70 & -- & -- & -- & -- & -- & -- & -- & -- & -- & -- \\
\hline 17 & 4.63 & 3.68 & -- & -- & -- & -- & -- & -- & -- & -- & -- & -- \\
\hline 18 & 4.57 & 3.70 & -- & -- & -- & -- & -- & -- & -- & -- & -- & - \\
\hline 19 & 4.37 & 3.84 & -- & -- & -- & -- & -- & -- & -- & -- & -- & -- \\
\hline 20 & 4.44 & 3.86 & -- & -- & -- & -- & -- & -- & -- & -- & -- & -- \\
\hline 21 & 4.87 & 3.84 & -- & -- & -- & -- & -- & -- & -- & -- & -- & -- \\
\hline 22 & 4.85 & 3.98 & -- & -- & -- & -- & -- & -- & -- & -- & -- & -- \\
\hline 23 & 4.76 & 4.11 & -- & -- & -- & -- & -. & -- & -. & -- & -- & -- \\
\hline 24 & 4.43 & 4.17 & -- & -- & -- & -- & -- & -- & -- & -- & -- & -- \\
\hline 25 & 4.40 & 4.32 & -- & -- & -- & -- & -- & -- & -- & -- & -- & -- \\
\hline 26 & 4.49 & 4.62 & -- & -- & -- & -- & -- & -- & -- & -- & -- & - \\
\hline 27 & 4.65 & 4.92 & -- & -- & -- & -- & -- & -- & -- & - & -- & - \\
\hline 28 & 4.53 & 4.88 & -- & -- & -- & -- & -- & -- & -- & -- & -- & -- \\
\hline 29 & 4.43 & -- & -- & -- & -- & -- & -. & -- & -- & -- & -- & -- \\
\hline 30 & 4.43 & -. & -- & -- & -- & -- & -- & -- & -- & -- & -- & -- \\
\hline 31 & 4.53 & -- & -- & -- & -- & -- & -. & -- & -- & -- & -- & -- \\
\hline
\end{tabular}


Table 10q. Mean daily ground-water levels in observation well CRM-24 (map ID \#21), Seminole Well Field, Cedar Rapids, lowa, February 1998 through February 1999

[Water levels measured as feet below land surface; --, value not measured or recorded]

\begin{tabular}{|c|c|c|c|c|c|c|c|c|c|c|c|c|}
\hline Day & Jan & Feb & Mar & Apr & May & Jun & Jul & Aug & Sep & Oct & Nov & Dec \\
\hline \multicolumn{13}{|c|}{1998} \\
\hline 1 & -- & -- & 1.36 & 0.92 & -- & 2.00 & -. & - & 3.24 & 3.85 & 0.39 & 4.63 \\
\hline 2 & -- & -- & 1.43 & -0.99 & -- & 2.47 & -- & -- & 3.35 & 3.64 & 0.25 & -- \\
\hline 3 & -- & -- & 3.81 & -- & -- & 1.99 & -- & -- & 3.44 & 3.65 & 2.24 & -- \\
\hline 4 & -- & -- & 4.30 & -- & -- & 0.32 & -- & -. & 3.44 & 3.57 & 3.02 & -- \\
\hline 5 & -- & -- & 4.39 & -- & -0.02 & 0.25 & -- & -- & 1.31 & 3.22 & 3.16 & 4.41 \\
\hline 6 & -- & -- & 4.31 & -- & 0.03 & 0.37 & -- & -- & 1.41 & 3.69 & 3.40 & 2.91 \\
\hline 7 & -- & -- & 4.15 & - & 0.33 & 0.52 & -- & -- & 1.41 & 3.61 & 3.41 & 1.16 \\
\hline 8 & -- & -- & 4.14 & -- & 0.37 & 0.48 & -- & -- & 1.79 & 1.78 & 3.22 & 1.17 \\
\hline 9 & -- & -- & 4.93 & -- & 0.43 & 0.46 & -- & -- & 3.49 & 3.51 & 1.03 & 1.10 \\
\hline 10 & -- & -- & 4.75 & -- & 0.36 & 0.13 & - & -- & 3.42 & 3.51 & 2.63 & 0.76 \\
\hline 11 & -- & -- & 4.44 & -- & 0.79 & 1.77 & -- & 3.11 & 3.52 & 3.60 & 0.70 & 1.82 \\
\hline 12 & -- & -- & 4.14 & -- & 2.82 & 0.48 & -- & 3.35 & 3.76 & 2.49 & 1.54 & 3.96 \\
\hline 13 & -- & -- & 3.24 & -- & 3.02 & -0.47 & -- & 3.24 & 3.55 & 1.90 & 3.08 & 4.21 \\
\hline 14 & -- & -- & 2.49 & -- & 3.28 & -0.20 & -- & 3.31 & 3.17 & 2.92 & 3.10 & 4.38 \\
\hline 15 & -- & -- & 2.91 & -- & 3.35 & -1.94 & -- & 3.48 & 3.26 & 3.56 & 3.11 & 4.41 \\
\hline 16 & -- & -- & 1.69 & -- & 3.40 & -1.69 & -- & 3.41 & 3.33 & 3.61 & 3.49 & 4.30 \\
\hline 17 & -- & -. & 0.77 & -- & 3.44 & -0.47 & -- & 3.24 & 3.20 & 3.20 & 3.30 & 4.39 \\
\hline 18 & -- & -- & 1.10 & -- & 3.47 & -0.21 & -- & 2.87 & 3.35 & 2.62 & 3.19 & 4.91 \\
\hline 19 & -- & -. & 1.63 & -- & 3.62 & -0.43 & -- & 3.33 & 3.05 & 2.41 & 3.56 & 4.66 \\
\hline 20 & -- & -- & 1.39 & -- & 3.57 & -1.97 & -- & 3.36 & 1.44 & 1.45 & 3.85 & 4.78 \\
\hline 21 & -- & -- & 2.03 & -- & 3.74 & -2.69 & -- & 3.28 & 2.85 & 1.28 & 3.52 & 4.39 \\
\hline 22 & -- & 4.52 & 1.67 & - & 1.97 & -3.82 & -- & 3.25 & 3.76 & 1.26 & 3.62 & 3.41 \\
\hline 23 & -- & 4.48 & 1.43 & -- & 1.08 & -- & -- & 3.17 & 3.92 & 2.11 & 3.90 & 4.19 \\
\hline 24 & -. & 3.23 & 1.47 & -- & 0.93 & -- & -- & 3.12 & 4.12 & 2.54 & 4.07 & 4.25 \\
\hline 25 & -- & 1.59 & 2.50 & -- & 0.99 & -- & -- & 3.48 & 3.99 & 2.51 & 3.74 & 4.25 \\
\hline 26 & -- & 2.45 & 3.63 & -- & 0.79 & -- & -- & 3.28 & 3.88 & 2.70 & 4.11 & 4.07 \\
\hline 27 & .- & 2.64 & 4.11 & -- & 0.54 & -- & -- & 3.41 & 4.03 & 2.63 & 4.28 & 4.14 \\
\hline 28 & -- & 1.69 & 4.10 & -- & 0.93 & -- & -- & 3.52 & 3.67 & 1.87 & 3.70 & 4.23 \\
\hline 29 & -- & -- & 3.21 & -- & 2.40 & -- & - & 3.49 & 3.86 & 0.77 & 3.89 & 4.51 \\
\hline 30 & -- & -- & 2.13 & -- & 2.12 & -- & -- & 3.39 & 3.80 & 0.40 & 4.38 & 4.49 \\
\hline 31 & -- & -- & 3.61 & -- & 1.82 & -. & -- & 3.35 & -- & 0.57 & -- & 4.83 \\
\hline
\end{tabular}


Table 10q. Mean daily ground-water levels in observation well CRM-24 (map ID \#21), Seminole Well Field, Cedar Rapids, lowa, February 1998 through February 1999--Continued

\begin{tabular}{|c|c|c|c|c|c|c|c|c|c|c|c|c|}
\hline Day & Jan & Feb & Mar & $\overline{\text { Apr }}$ & May & Jun & Jul & Aug & Sep & Oct & Nov & $\overline{\text { Dec }}$ \\
\hline & & & & & & 1999 & & & & & & \\
\hline 1 & 4.92 & 5.59 & -. & -. & -- & -- & -- & -- & -. & -- & -- & -- \\
\hline 2 & 4.80 & 5.48 & -. & -- & -- & -- & -- & -- & -. & -- & -- & -- \\
\hline 3 & 4.82 & 6.36 & -- & -- & -- & -- & -- & -- & -- & -- & -- & -- \\
\hline 4 & 4.95 & 5.37 & -- & -- & -- & -- & -- & -- & -- & -- & -- & -- \\
\hline 5 & 5.01 & 5.16 & -- & -- & -- & -- & -- & -- & -- & -- & -- & -- \\
\hline 6 & 4.94 & 5.27 & -- & -- & -- & -- & - & -- & -- & -- & -- & -- \\
\hline 7 & 5.00 & 5.14 & -- & -- & -- & -- & -- & -- & -- & -- & -- & -- \\
\hline 8 & 4.99 & 5.14 & -. & -. & -- & -- & -. & -. & -- & -. & -- & -. \\
\hline 9 & 5.37 & 5.01 & -- & -- & -. & -- & -- & -- & -- & -- & -- & -- \\
\hline 10 & 5.42 & 5.01 & -- & -- & -- & -- & -- & -- & -- & -- & -- & -- \\
\hline 11 & 5.44 & 5.17 & -- & -- & -- & -- & -- & -- & -- & -- & -- & -- \\
\hline 12 & 5.27 & 4.88 & -- & -- & -- & -- & -- & -- & -- & -- & -- & -- \\
\hline 13 & 5.46 & 4.62 & -- & -- & -- & -- & -- & -- & -- & -- & -- & -- \\
\hline 14 & 5.33 & 4.98 & -- & -- & -- & -- & - & -- & -- & -- & -- & -- \\
\hline 15 & 5.48 & 4.88 & -- & -- & - & -- & -- & -- & -- & -- & -- & -- \\
\hline 16 & 5.43 & 4.99 & -- & -- & -- & -- & -- & - & -- & -- & - & -- \\
\hline 17 & 5.93 & 5.08 & -- & -- & -- & -- & -- & -- & -- & -- & -- & -- \\
\hline 18 & 5.89 & 5.09 & -- & - & -- & -- & -- & -- & -- & -- & -- & -- \\
\hline 19 & 5.61 & 4.84 & -- & -- & -- & -- & -- & -- & -- & -- & -- & -- \\
\hline 20 & 5.57 & 5.11 & -- & -- & -- & -- & -- & -- & -- & -- & -- & -- \\
\hline 21 & 5.51 & 4.88 & -- & -- & -- & -- & -- & -- & -- & -- & -- & -- \\
\hline 22 & 5.70 & 5.18 & -- & -- & -- & -- & -- & -- & -- & -- & -- & -- \\
\hline 23 & 5.57 & 5.29 & -- & -- & -- & -- & -- & -- & -- & -. & -- & -- \\
\hline 24 & 5.37 & 5.44 & -- & -- & -- & -- & -- & -- & -- & -- & -- & -- \\
\hline 25 & 5.45 & 5.44 & -- & -- & -- & -- & -- & -- & -- & -- & -- & -- \\
\hline 26 & 5.57 & 5.75 & -- & -- & -- & -- & -- & -- & -- & -. & -- & -- \\
\hline 27 & 5.62 & 6.07 & -- & -- & -- & -- & -- & -- & -- & -- & -- & -- \\
\hline 28 & 5.63 & 5.90 & -- & -- & -- & -- & -- & -- & -- & -- & -. & -- \\
\hline 29 & 5.43 & -- & -- & -- & -- & -- & -- & -- & -- & -- & -- & -- \\
\hline 30 & 5.38 & -. & -- & -- & -- & -- & -- & -- & -- & -- & -- & -- \\
\hline 31 & 5.66 & -- & -- & -- & -- & -- & -- & -- & -- & -- & -- & -- \\
\hline
\end{tabular}


Table 10r. Mean daily ground-water levels in observation well CRM-25 (map ID \#22), Seminole Well Field, Cedar Rapids, lowa, February 1998 through February 1999

[Water levels measured as feet below land surface; --, value not measured or recorded]

\begin{tabular}{|c|c|c|c|c|c|c|c|c|c|c|c|c|}
\hline Day & Jan & Feb & Mar & Apr & May & Jun & Jul & Aug & Sep & Oct & Nov & Dec \\
\hline \multicolumn{13}{|c|}{1998} \\
\hline 1 & -- & -- & 5.85 & 0.35 & 2.93 & 3.04 & -0.64 & 4.61 & 4.76 & 5.36 & 4.11 & 5.04 \\
\hline 2 & -- & -- & 5.71 & -0.18 & 2.99 & 3.16 & -1.05 & 4.66 & 4.81 & 5.35 & 4.04 & 5.17 \\
\hline 3 & -- & -- & 5.72 & -0.39 & 2.98 & 3.31 & -1.44 & 4.74 & 5.00 & 5.35 & 4.05 & 5.25 \\
\hline 4 & -. & -- & 5.79 & -0.18 & 3.12 & 3.44 & -1.35 & 4.75 & 5.00 & 5.30 & 4.18 & 5.29 \\
\hline 5 & -- & -- & 5.79 & 0.11 & 3.26 & 3.56 & -0.39 & 4.71 & 5.06 & 4.89 & 4.25 & 5.49 \\
\hline 6 & -- & -- & 5.83 & 0.35 & 3.31 & 3.66 & 0.70 & 4.50 & 5.12 & 4.85 & 4.37 & 5.42 \\
\hline 7 & - & -- & 5.89 & 0.08 & 3.47 & 3.78 & 1.32 & 4.28 & 5.16 & 4.92 & 4.47 & 5.24 \\
\hline 8 & -- & -- & 6.01 & 0.76 & 3.49 & 3.87 & 1.69 & 4.44 & 5.14 & 4.96 & 4.53 & 5.11 \\
\hline 9 & -- & -- & 5.96 & 1.73 & 3.65 & 3.76 & 1.87 & 4.53 & 5.18 & 4.89 & 4.46 & 5.10 \\
\hline 10 & -- & -- & 6.01 & 1.87 & 3.74 & 3.75 & 2.04 & 4.59 & 5.25 & 4.93 & 3.58 & 5.01 \\
\hline 11 & -- & -. & 5.85 & 1.87 & 3.81 & 2.87 & 2.21 & 4.67 & 5.37 & 5.02 & 4.28 & 4.90 \\
\hline 12 & - & -- & 5.86 & 1.89 & 3.98 & 2.36 & 2.35 & 4.76 & 5.48 & 5.09 & 4.18 & 4.89 \\
\hline 13 & -- & -- & 4.77 & 1.77 & 4.07 & 1.56 & 2.44 & 4.79 & 5.53 & 5.12 & 4.13 & 5.02 \\
\hline 14 & -- & 5.36 & 4.28 & 2.13 & 4.21 & 0.15 & 2.53 & 4.85 & 5.38 & 5.17 & 4.27 & 5.16 \\
\hline 15 & -- & 5.47 & 4.41 & 1.97 & 4.29 & -0.38 & 2.66 & 4.90 & 5.20 & 5.04 & 4.24 & 5.26 \\
\hline 16 & -- & 5.51 & 4.54 & 2.05 & 4.36 & 0.46 & 2.81 & 4.95 & 5.24 & 5.11 & 4.26 & 5.39 \\
\hline 17 & -- & 5.37 & 4.61 & 2.08 & 4.43 & 1.12 & 3.10 & 4.75 & 5.31 & 4.98 & 4.26 & 5.47 \\
\hline 18 & -- & 5.57 & 4.70 & 2.07 & 4.46 & 1.51 & 3.40 & 4.65 & 5.40 & 4.60 & 4.31 & 5.54 \\
\hline 19 & -- & 5.50 & 4.82 & 1.98 & 4.46 & 1.41 & 3.55 & 4.76 & 5.47 & 4.03 & 4.32 & 5.65 \\
\hline 20 & -- & 5.65 & 4.95 & 1.75 & 4.43 & 1.24 & 3.73 & 4.83 & 5.48 & 3.28 & 4.30 & 5.71 \\
\hline 21 & -- & 5.82 & 5.06 & 1.61 & 4.43 & 0.88 & 3.87 & 4.83 & 5.50 & 3.01 & 4.34 & 5.73 \\
\hline 22 & -- & 5.96 & 5.19 & 1.72 & 4.39 & 0.08 & 3.97 & 4.85 & 5.47 & 3.08 & 4.38 & 5.49 \\
\hline 23 & -- & 6.07 & 5.22 & 1.82 & 4.44 & -0.61 & 4.04 & 4.96 & 5.49 & 3.26 & 4.45 & 5.53 \\
\hline 24 & -- & 6.15 & 5.21 & 1.85 & 4.40 & -0.97 & 4.14 & 5.10 & 5.51 & 3.48 & 4.48 & 5.60 \\
\hline 25 & -- & 6.18 & 5.36 & 1.98 & 4.47 & -1.85 & 4.25 & 5.06 & 5.49 & 3.74 & 4.59 & 5.69 \\
\hline 26 & -- & 5.69 & 5.47 & 2.11 & 4.52 & -2.01 & 4.35 & 4.99 & 5.57 & 3.97 & 4.71 & 5.69 \\
\hline 27 & -- & 5.70 & 5.24 & 2.32 & 4.50 & -1.69 & 4.46 & 4.95 & 5.54 & 4.11 & 4.79 & 5.78 \\
\hline 28 & -- & 6.05 & 4.52 & 2.43 & 4.23 & -1.65 & 4.45 & 4.71 & 5.50 & 4.25 & 4.84 & 5.81 \\
\hline 29 & -- & -- & 4.09 & 2.53 & 3.81 & -1.34 & 4.43 & 4.68 & 5.43 & 4.25 & 5.04 & 5.81 \\
\hline 30 & -- & -. & 3.36 & 2.68 & 3.20 & -0.89 & 4.41 & 4.71 & 5.40 & 4.18 & 5.05 & 5.81 \\
\hline 31 & -- & -. & 0.89 & -- & 3.03 & -. & 4.51 & 4.72 & -- & 4.12 & -- & 5.85 \\
\hline
\end{tabular}


Table 10r. Mean daily ground-water levels in observation well CRM-25 (map ID \#22), Seminole Well Field, Cedar Rapids, lowa, February 1998 through February 1999--Continued

\begin{tabular}{|c|c|c|c|c|c|c|c|c|c|c|c|c|}
\hline Day & Jan & Feb & Mar & Apr & May & Jun & Jul & Aug & Sep & Oct & Nov & Dec \\
\hline & & & & & & 1999 & & & & & & \\
\hline 1 & 5.93 & 5.97 & -- & -- & -- & -- & -- & -- & -- & -- & -- & -- \\
\hline 2 & 6.06 & 5.96 & -- & -- & -- & -- & -- & -- & -- & -- & -- & -- \\
\hline 3 & 6.04 & 5.52 & -- & -- & -. & -- & -- & -- & -- & .- & -. & .- \\
\hline 4 & 6.05 & 5.73 & -- & -- & -- & -- & -- & - & -- & -- & -- & -- \\
\hline 5 & 6.07 & 5.71 & -- & -- & -- & -- & -- & -- & -- & -- & -- & -- \\
\hline 6 & 6.15 & 5.73 & -- & -- & -- & -- & -- & -- & -- & -- & -- & -- \\
\hline 7 & 6.11 & 5.70 & -- & -- & -- & -- & -- & -- & -- & -- & -- & -- \\
\hline 8 & 6.07 & 5.72 & -- & -- & -- & -- & - & -- & -- & -- & -- & -- \\
\hline 9 & 6.06 & 5.65 & -- & -- & -- & -- & -- & -- & -- & -- & -- & -- \\
\hline 10 & 6.07 & 5.67 & -- & -- & -- & -- & -- & -- & -- & -- & -- & -- \\
\hline 11 & 6.11 & 5.19 & -- & -- & -- & -- & -- & -- & -- & - & -- & -- \\
\hline 12 & 6.08 & 5.05 & -- & -- & -- & -- & -- & -- & -- & -- & -- & -- \\
\hline 13 & 6.08 & 4.97 & -- & -- & -- & -- & -- & -- & -- & -- & -- & -- \\
\hline 14 & 6.03 & 4.97 & -- & -- & -- & -- & -- & -- & -- & -- & -- & -- \\
\hline 15 & 6.08 & 5.03 & -- & -- & -- & -- & -- & -- & -- & -- & -- & -- \\
\hline 16 & 6.09 & 5.02 & -- & -- & -. & -- & -- & -- & -- & -- & -- & -- \\
\hline 17 & 5.86 & 5.08 & -- & -- & -- & -- & -- & -- & -- & -- & -- & -- \\
\hline 18 & 5.91 & 5.12 & -- & -- & -- & -- & -- & -- & -- & -- & -- & -- \\
\hline 19 & 6.10 & 5.10 & -- & -- & -- & -- & -- & -- & -- & -- & -- & -- \\
\hline 20 & 6.06 & 5.12 & -- & -- & -- & -- & -- & -- & -- & -- & -- & -- \\
\hline 21 & 6.11 & 5.14 & -- & -- & -- & -- & -- & -- & -- & -- & -- & -- \\
\hline 22 & 6.08 & 5.16 & -- & -- & -- & -- & -- & -- & -- & -- & -- & -- \\
\hline 23 & 6.05 & 5.24 & -- & -- & -- & -. & -- & -- & -- & -- & -- & -- \\
\hline 24 & 5.95 & 5.30 & -- & -- & -- & -- & -- & -- & -- & -- & -- & -- \\
\hline 25 & 5.95 & 5.33 & -- & -- & -- & -- & -- & -- & -- & -- & -- & -- \\
\hline 26 & 5.93 & 5.36 & - & -- & -- & -- & -- & -- & -- & -- & -- & -- \\
\hline 27 & 6.01 & 5.40 & -- & -- & -- & -- & -- & -- & -- & -- & -- & -- \\
\hline 28 & 5.96 & 5.43 & -- & -- & -- & -- & -- & -- & -- & -- & -- & -- \\
\hline 29 & 5.99 & -- & -- & -- & -- & -- & -- & -- & -- & - & -- & -- \\
\hline 30 & 6.00 & -- & -- & -- & -- & -- & -- & -- & -- & -- & -- & -- \\
\hline 31 & 5.97 & -- & -- & -- & -- & -- & -- & -- & -- & -- & -- & -- \\
\hline
\end{tabular}


Table 10s. Mean daily ground-water levels in observation well CRM-27 (map ID \#24), Seminole Well Field, Cedar Rapids, lowa, February 1998 through February 1999

[Water levels measured as feet below land surface; --, value not measured or recorded]

\begin{tabular}{|c|c|c|c|c|c|c|c|c|c|c|c|c|}
\hline Day & Jan & Feb & Mar & Apr & May & Jun & Jul & Aug & Sep & Oct & Nov & Dec \\
\hline \multicolumn{13}{|c|}{1998} \\
\hline 1 & -- & -- & 3.32 & -1.23 & 1.47 & 1.10 & -3.11 & 3.57 & 3.29 & 3.04 & 2.03 & 3.50 \\
\hline 2 & -- & -- & 4.20 & -2.57 & 1.55 & 1.47 & -3.46 & 3.74 & 3.63 & 3.10 & 2.02 & 3.84 \\
\hline 3 & -- & -- & 4.42 & -2.75 & 1.47 & 1.36 & -4.06 & 3.75 & 3.91 & 3.11 & 2.24 & 4.04 \\
\hline 4 & -- & -- & 4.19 & -2.48 & 1.68 & 2.36 & -3.87 & 3.76 & 3.74 & 3.09 & 2.38 & 4.08 \\
\hline 5 & -- & -- & 4.32 & -2.29 & 1.95 & 2.56 & -2.92 & 3.78 & 3.62 & 2.94 & 2.58 & 4.21 \\
\hline 6 & -- & -- & 4.29 & -1.70 & 2.06 & 2.73 & -1.50 & 3.42 & 3.69 & 2.91 & 2.67 & 3.63 \\
\hline 7 & -- & -- & 4.38 & -0.98 & 2.92 & 2.92 & -0.55 & 3.31 & 3.81 & 3.64 & 2.69 & 2.43 \\
\hline 8 & -- & -- & 5.02 & -0.38 & 2.83 & 2.89 & -0.16 & 3.40 & 3.79 & 2.52 & 2.82 & 2.61 \\
\hline 9 & -- & -- & 4.73 & -0.02 & 2.70 & 2.97 & 0.18 & 3.43 & 3.79 & 2.73 & 1.98 & 2.19 \\
\hline 10 & -- & -- & 3.95 & -0.16 & 2.90 & 2.50 & 0.59 & 3.43 & 3.88 & 3.64 & 2.38 & 1.90 \\
\hline 11 & -- & -- & 3.61 & -0.18 & 2.88 & 1.81 & 0.86 & 3.45 & 3.94 & 3.78 & 1.44 & 2.22 \\
\hline 12 & -- & -- & 3.47 & 0.29 & 3.54 & 0.71 & 1.00 & 3.64 & 3.99 & 3.74 & 1.27 & 3.46 \\
\hline 13 & -- & -- & 2.40 & 0.71 & 3.18 & -0.59 & 1.05 & 3.67 & 3.97 & 3.74 & 1.68 & 3.77 \\
\hline 14 & -- & 2.45 & 2.55 & 0.80 & 3.25 & -1.22 & 1.29 & 3.65 & 3.91 & 3.62 & 1.87 & 3.83 \\
\hline 15 & -- & 2.44 & 2.71 & 1.02 & 3.63 & -1.99 & 1.69 & 3.61 & 3.72 & 3.50 & 1.76 & 3.98 \\
\hline 16 & -- & 2.94 & 3.38 & 0.90 & 3.46 & -1.39 & 2.14 & 3.74 & 3.69 & 3.79 & 1.94 & 4.15 \\
\hline 17 & -- & 3.20 & 3.85 & 0.21 & 3.59 & -0.94 & 2.46 & 3.47 & 3.76 & 3.66 & 1.76 & 4.28 \\
\hline 18 & -- & 3.03 & 4.11 & 0.25 & 2.79 & -0.13 & 2.69 & 3.55 & 3.88 & 2.68 & 1.99 & 4.59 \\
\hline 19 & -- & 3.20 & 3.80 & -0.11 & 2.58 & -0.34 & 3.04 & 3.69 & 4.08 & 1.83 & 1.91 & 4.27 \\
\hline 20 & -- & 4.28 & 3.95 & -0.60 & 2.60 & -0.74 & 3.09 & 3.73 & 4.10 & 0.99 & 1.92 & 4.29 \\
\hline 21 & -- & 4.66 & 4.06 & -0.51 & 2.63 & -1.55 & 3.14 & 3.66 & 3.89 & 0.80 & 1.95 & 4.11 \\
\hline 22 & -- & 4.58 & 4.03 & -0.37 & 3.36 & -2.57 & 3.08 & 3.52 & 3.67 & 0.99 & 2.27 & 3.33 \\
\hline 23 & - & 4.57 & 3.94 & -0.32 & 3.43 & -3.20 & 3.25 & 4.00 & 3.16 & 1.57 & 2.55 & 3.77 \\
\hline 24 & -- & 4.39 & 3.89 & 0.00 & 2.75 & -3.32 & 3.32 & 4.15 & 3.15 & 2.09 & 3.00 & 3.89 \\
\hline 25 & - & 4.70 & 4.00 & 0.67 & 3.37 & -4.11 & 3.54 & 3.24 & 3.45 & 2.40 & 3.30 & 4.00 \\
\hline 26 & -- & 4.99 & 4.29 & 0.71 & 3.41 & -4.30 & 3.56 & 3.00 & 3.46 & 2.60 & 3.18 & 4.03 \\
\hline 27 & -- & 4.85 & 4.01 & 0.80 & 3.11 & -3.88 & 3.17 & 2.89 & 3.25 & 2.69 & 3.29 & 4.15 \\
\hline 28 & - & 4.50 & 3.10 & 1.04 & 2.62 & -4.24 & 2.51 & 2.60 & 3.13 & 2.77 & 3.48 & 4.22 \\
\hline 29 & -- & -- & 2.27 & 1.25 & 1.26 & -3.68 & 2.48 & 2.63 & 3.20 & 2.93 & 3.74 & 4.30 \\
\hline 30 & -- & -- & 2.26 & 0.96 & 1.15 & -3.22 & 2.93 & 2.80 & 3.19 & 2.35 & 3.63 & 4.32 \\
\hline 31 & -- & - & -0.20 & -- & 1.08 & - & 3.50 & 2.77 & -- & 2.21 & -- & 4.49 \\
\hline
\end{tabular}


Table 10s. Mean daily ground-water levels in observation well CRM-27 (map ID \#24), Seminole Well Field, Cedar Rapids, lowa, February 1998 through February 1999--Continued

\begin{tabular}{|c|c|c|c|c|c|c|c|c|c|c|c|c|}
\hline Day & Jan & Feb & Mar & Apr & May & Jun & Jul & Aug & Sep & Oct & Nov & Dec \\
\hline & & & & & & 1999 & & & & & & \\
\hline 1 & 4.49 & 3.56 & -- & -- & -- & -- & -- & -- & -- & -- & -- & -- \\
\hline 2 & 4.78 & 3.62 & -- & -- & -- & -- & -- & -- & -- & -- & -- & -- \\
\hline 3 & 4.76 & 3.70 & -- & -- & - & -- & -- & -- & -- & -- & -- & -- \\
\hline 4 & 4.53 & 3.08 & -- & -- & -- & -- & -- & -- & -- & -- & -- & -- \\
\hline 5 & 4.69 & 3.17 & -- & -. & -- & - & -- & -- & -- & -- & -- & -- \\
\hline 6 & 4.38 & 3.11 & -- & -- & -- & -- & -- & -- & -- & -- & -- & -- \\
\hline 7 & 3.34 & 3.29 & -- & -- & -- & -- & -- & -- & -- & -- & -- & -- \\
\hline 8 & 3.79 & 3.24 & -- & -- & -- & -- & -- & -- & -. & -- & -- & -- \\
\hline 9 & 3.58 & 2.92 & -. & -- & -- & -- & -- & -- &.- & -- & -- & -- \\
\hline 10 & 3.53 & 2.95 & -- & -- & -- & -- & -- & -- & -- & -- & -- & -- \\
\hline 11 & 3.76 & 2.82 & -- & -- & -- & -- & -- & -- & -- & -- & -- & -- \\
\hline 12 & 3.73 & 2.41 & -. & -- & -- & -- & -- & -. & -- & -- & -- & -- \\
\hline 13 & 3.42 & 2.05 & -- & -- & -- & -- & -- & -- & -. & - & - & -- \\
\hline 14 & 3.45 & 2.41 & -- & -- & -- & -- & -- & -- & -- & -- & -- & -- \\
\hline 15 & 3.70 & 2.74 & -- & -- & -- & -- & -- & -- & -- & -- & -- & -- \\
\hline 16 & 3.74 & 2.69 & -- & -- & -- & -- & -- & - & -- & - & -- & -- \\
\hline 17 & 3.85 & 2.58 & -- & -- & -- & -- & -- & -- & -- & - & -- & -- \\
\hline 18 & 3.81 & 2.60 & - & -- & -- & -- & -- & -- & -- & -- & -- & -- \\
\hline 19 & 3.50 & 2.63 & -- & -- & -- & -- & -- & -- & -- & -- & -- & -- \\
\hline 20 & 3.60 & 2.54 & -- & -- & -- & -- & -- & -- & -- & -- & -- & -- \\
\hline 21 & 3.78 & 2.31 & -- & -- & -- & -- & -- & -- & -- & -- & -- & -- \\
\hline 22 & 3.75 & 2.64 & -- & -- & -- & - & -- & -- & -- & -- & -- & -- \\
\hline 23 & 3.67 & 2.83 & -- & -- & -- & -- & -- & -- & -- & -- & -- & -- \\
\hline 24 & 3.37 & 2.88 & -- & -- & -- & -- & -- & -- & -- & -- & -- & -- \\
\hline 25 & 3.16 & 2.90 & -- & -- & -- & -- & -- & -- & -- & -- & -- & -- \\
\hline 26 & 3.40 & 2.70 & -- & -- & -- & -- & -- & -- & -- & -- & -- & -- \\
\hline 27 & 3.62 & 3.27 & -- & -- & -- & -- & -- & -- & -- & -- & -- & -- \\
\hline 28 & 3.37 & 3.37 & -- & -- & -- & -- & -- & -- & -- & -- & -- & -- \\
\hline 29 & 3.15 & -- & -- & -- & -- & -- & -- & -- & -- & -- & -- & -- \\
\hline 30 & 3.13 & -- & - & -- & -- & -- & -- & -- & -- & -- & -- & -- \\
\hline 31 & 3.32 & -- & -- & -- & -- & -- & -- & -- & -. & -- & -- & -- \\
\hline
\end{tabular}


Table 11a. Mean daily specific conductance in ground water at site CRM-1 (map ID \#1), East Well Field, Cedar Rapids, lowa, April 1996 through June 1997

[All values in microsiemens per centimeter at 25 degrees Centigrade; --, value not measured, not recorded, or rejected after data review]

\begin{tabular}{|c|c|c|c|c|c|c|c|c|c|c|c|c|}
\hline Day & Jan & Feb & Mar & Apr & May & Jun & Jul & Aug & Sep & Oct & Nov & Dec \\
\hline \multicolumn{13}{|c|}{1996} \\
\hline 1 & -- & -- & -- & 643 & 517 & -- & 582 & 604 & -- & -- & -- & -- \\
\hline 2 & -- & -- & -- & 641 & 518 & -- & 583 & 603 & -- & -- & -- & -- \\
\hline 3 & -. & -- & -- & 630 & 519 & -- & 584 & 605 & -- & -- & -- & -- \\
\hline 4 & -- & -- & -- & 596 & 521 & -- & 585 & 609 & -- & -- & -- & 611 \\
\hline 5 & -- & -- & -- & 569 & 521 & -- & 586 & 612 & -- & -- & -- & 611 \\
\hline 6 & -- & -- & -- & 547 & 521 & -- & 587 & 603 & -- & -- & -- & 611 \\
\hline 7 & -- & -- & -- & 533 & 521 & -- & 588 & 594 & -- & -- & -- & 611 \\
\hline 8 & -- & - & -- & 529 & 522 & -- & 589 & 597 & -- & -- & -- & 611 \\
\hline 9 & -- & -- & -- & 530 & -- & -- & 588 & 606 & -- & -- & -- & 611 \\
\hline 10 & -- & -- & -- & 527 & -- & -- & 589 & 611 & -- & -- & -- & 612 \\
\hline 11 & -- & -- & -- & 517 & -- & -- & 592 & 615 & -- & -- & -- & 563 \\
\hline 12 & -- & -. & -- & 515 & -- & -- & 594 & 616 & -- & -- & -- & 619 \\
\hline 13 & -- & -- & -- & 515 & -- & -- & 591 & 617 & -- & -- & -- & 618 \\
\hline 14 & -- & -- & -. & 514 & -- & -- & 592 & 615 & 607 & -- & -- & 618 \\
\hline 15 & -- & -- & -- & 514 & -- & -- & 591 & 589 & 603 & -- & -- & 619 \\
\hline 16 & -- & -- & -- & 514 & -- & -- & 585 & 614 & 592 & -- & -- & 620 \\
\hline 17 & -- & -- & -- & 514 & -- & -- & 584 & 614 & 592 & -- & -- & 570 \\
\hline 18 & -- & -- & -- & 514 & -- & .. & 593 & 618 & 592 & -- & -- & 560 \\
\hline 19 & -- & -- & -- & 515 & -- & -- & 595 & 659 & 593 & -- & -- & 565 \\
\hline 20 & -- & -- & -- & 515 & -- & -- & 593 & 629 & 593 & -- & -- & 570 \\
\hline 21 & -. & -- & -- & 514 & -- & -- & 593 & 631 & 595 & -- & -- & 581 \\
\hline 22 & -- & -- & -- & 513 & -- & -- & 594 & 635 & 597 & -- & -- & 590 \\
\hline 23 & -- & -- & -- & 514 & -- & -- & 594 & 607 & 598 & -- & -- & 595 \\
\hline 24 & - & -- & -- & 515 & -- & -- & 595 & 631 & 598 & -- & -- & 599 \\
\hline 25 & -- & -- & -- & 515 & -- & -- & 596 & 631 & 598 & -- & -- & 612 \\
\hline 26 & -- & -- & -- & 515 & -- & -- & 598 & -- & 597 & -- & -- & 615 \\
\hline 27 & - & -- & -- & 515 & -- & -- & 636 & -- & 597 & -- & -- & 621 \\
\hline 28 & -- & -- & -- & 514 & -- & 577 & 602 & -- & -- & -- & -- & 640 \\
\hline 29 & -. & -- & -- & 515 & -- & 580 & 603 & -- & -- & -- & -- & 654 \\
\hline 30 & -- & -- & -- & 516 & -. & 582 & 601 & -- & -- & -- & -- & 651 \\
\hline 31 & -- & -- & -- & -- & -- & -- & 603 & -- & -- & -- & -- & 633 \\
\hline
\end{tabular}


Table 11a. Mean daily specific conductance in ground water at site CRM-1 (map ID \#1), East Well Field, Cedar Rapids, lowa, April 1996 through June 1997--Continued

\begin{tabular}{|c|c|c|c|c|c|c|c|c|c|c|c|c|}
\hline Day & Jan & Feb & Mar & Apr & May & Jun & Jul & Aug & Sep & Oct & Nov & Dec \\
\hline & & & & & & 1997 & & & & & & \\
\hline 1 & 664 & 786 & 622 & -- & -- & 604 & -- & -- & -- & -- & -- & -- \\
\hline 2 & 682 & 695 & 623 & -- & -- & 589 & -- & -- & -- & -- & -- & -- \\
\hline 3 & 659 & 621 & 624 & -- & -- & 573 & -- & -- & -- & -- & -- & -- \\
\hline 4 & 670 & 618 & 626 & -- & -- & 568 & -- & -. & -- & -- & -- & -- \\
\hline 5 & 680 & 618 & 629 & -- & -- & 552 & -- & -- & -- & -- & -- & -- \\
\hline 6 & 688 & 618 & 631 & -- & -- & 539 & -- & -- & -- & -- & -- & -- \\
\hline 7 & 698 & 656 & 633 & -- & -- & 536 & -- & -- & -- & -- & -- & -- \\
\hline 8 & 701 & 740 & 635 & -- & -- & 525 & -- & -- & -- & -- & -- & -- \\
\hline 9 & 706 & 754 & 638 & -- & -- & 513 & -- & -- & -- & -. & -- & -- \\
\hline 10 & 688 & 761 & 642 & -- & -- & 517 & -- & -- & -- & -- & -- & -- \\
\hline 11 & 670 & 722 & 641 & -. & -- & 520 & -- & -- & -- & -- & -- & -- \\
\hline 12 & 660 & 720 & 641 & -- & -- & 521 & -- & -- & -- & -- & -- & -- \\
\hline 13 & 666 & 717 & 641 & -- & -- & -- & -- & -- & -- & -- & -- & -- \\
\hline 14 & 689 & 714 & 642 & -- & -- & -- & -- & -- & -- & -- & -- & -- \\
\hline 15 & 749 & 712 & 642 & -- & -- & -- & -- & -- & -- & -- & -- & -- \\
\hline 16 & 811 & 707 & 643 & -- & -- & -- & -- & - & -- & -- & -- & -- \\
\hline 17 & 811 & 668 & 647 & -- & -- & -- & -- & - & -- & -- & -- & -- \\
\hline 18 & 759 & 646 & 649 & -- & -- & -- & -- & -- & -- & -- & -- & -- \\
\hline 19 & 629 & 644 & 648 & -. & -- & -- & -- & -- & -- & -- & -- & -- \\
\hline 20 & 705 & 645 & 645 & -. & -- & -- & -- & -- & -- & -- & -- & -- \\
\hline 21 & 828 & 644 & 645 & -- & -- & -- & -- & -- & -- & -- & -- & -- \\
\hline 22 & 850 & 639 & 651 & - & -- & -- & -- & -- & -- & -- & -- & -- \\
\hline 23 & 798 & 628 & 648 & -- & 610 & -- & -- & -- & -- & -- & -- & -- \\
\hline 24 & 626 & 619 & 649 & -- & 610 & -- & -- & -. & -- & -- & -- & -- \\
\hline 25 & 697 & 617 & 647 & -- & 610 & -- & -- & -- & -- & -- & -- & -- \\
\hline 26 & 821 & 617 & 645 & -- & 608 & -- & -- & -- & -- & -- & -- & -- \\
\hline 27 & 833 & 619 & 644 & -- & 614 & -- & -- & -- & -- & -- & -- & -- \\
\hline 28 & 814 & 621 & 643 & -- & 633 & -- & -- & -- & -- & -- & -- & -- \\
\hline 29 & 812 & -- & 641 & -- & 626 & -- & -- & -- & -- & -- & -- & -- \\
\hline 30 & 813 & -- & -- & -- & 619 & -- & -- & -- & -- & -- & -- & -- \\
\hline 31 & 831 & -- & -- & -- & 611 & -- & -- & -- & -- & -- & -- & -- \\
\hline
\end{tabular}


Table 11b. Mean daily specific conductance in ground water at site CRM-2 (map ID \#2), West Well Field, Cedar Rapids, lowa, April 1996 through June 1997

[All values listed in microsiemens per centimeter at 25 degrees Centigrade; --, value not measured, not recorded, or rejected after data review]

\begin{tabular}{|c|c|c|c|c|c|c|c|c|c|c|c|c|}
\hline Day & Jan & Feb & Mar & Apr & May & Jun & Jul & Aug & Sep & Oct & Nov & Dec \\
\hline & & & & & & 1996 & & & & & & \\
\hline 1 & -- & -- & -- & 503 & 505 & -- & -- & 563 & 498 & 470 & 469 & 493 \\
\hline 2 & -- & -- & -- & 502 & 505 & -- & .- & 564 & 494 & 471 & 469 & 492 \\
\hline 3 & -- & -- & -- & 500 & 503 & -- & -- & 565 & 493 & 471 & 469 & 493 \\
\hline 4 & -- & -- & -- & 500 & 503 & -- & -- & 566 & 493 & 472 & 470 & 500 \\
\hline 5 & -- & -- & -- & 500 & 502 & -- & -- & 566 & 494 & 473 & 472 & 502 \\
\hline 6 & -- & -- & -- & 501 & 502 & -- & -- & 566 & 495 & 473 & 474 & 500 \\
\hline 7 & -- & -- & -- & 501 & 501 & -- & -. & 564 & 496 & 474 & 475 & 501 \\
\hline 8 & -- & -- & -- & 502 & 501 & -- & -- & 565 & 497 & 474 & 476 & 504 \\
\hline 9 & -- & -- & -- & 501 & -- & -- & -- & 565 & 497 & 473 & 474 & 509 \\
\hline 10 & -- & -- & -- & 500 & -- & -- & -- & 565 & 498 & 474 & 473 & 513 \\
\hline 11 & -- & -- & -- & 502 & -- & -- & -- & 565 & 498 & 475 & 475 & 494 \\
\hline 12 & -- & -- & -- & 502 & -- & -- & -- & 565 & 499 & 474 & 477 & 516 \\
\hline 13 & -- & -- & -- & 502 & -- & -- & -. & 565 & 496 & 475 & 478 & 515 \\
\hline 14 & -- & -- & -- & 502 & -- & -- & -- & 564 & 488 & 475 & 479 & 516 \\
\hline 15 & - & -- & -- & 502 & -- & -- & -- & 564 & 486 & 475 & 480 & 518 \\
\hline 16 & -- & -- & -- & 502 & -- & -- & -- & 564 & 484 & 476 & 478 & 521 \\
\hline 17 & -- & -- & -- & 503 & -- & -- & -- & 564 & 482 & 476 & 477 & 522 \\
\hline 18 & -- & -- & -- & 502 & -- & -- & -- & 564 & 480 & 474 & 478 & 524 \\
\hline 19 & -- & -- & -- & 502 & -- & -- & -- & 564 & 474 & 469 & 481 & 525 \\
\hline 20 & -- & -- & -- & 503 & -- & -- & -- & 563 & 471 & 470 & 481 & 526 \\
\hline 21 & -- & -- & -- & 504 & -- & -- & -- & 563 & 471 & 471 & 481 & 527 \\
\hline 22 & -- & -- & -- & 496 & -- & -- & -- & 563 & 470 & 469 & 482 & 527 \\
\hline 23 & -- & -- & -- & -- & -- & -- & -- & 563 & 467 & 468 & 482 & 538 \\
\hline 24 & -- & -- & -- & 506 & -- & -- & -- & 563 & 467 & 467 & 483 & 540 \\
\hline 25 & -- & -. & -- & 517 & -- & -- & -- & 564 & 466 & 467 & 484 & 542 \\
\hline 26 & -- & -- & -- & 512 & -- & -- & -- & 564 & 465 & 466 & 490 & 545 \\
\hline 27 & -- & -- & -- & 506 & -- & -- & -- & 564 & 465 & 465 & 489 & 542 \\
\hline 28 & -- & -- & -- & 505 & -- & -- & -- & 564 & 464 & 467 & 488 & 524 \\
\hline 29 & -- & - & -- & 505 & -- & -- & -- & 540 & 465 & 468 & 490 & 480 \\
\hline 30 & -- & -- & -- & 505 & -- & -- & -- & 496 & 469 & 469 & 492 & 502 \\
\hline 31 & -- & -- & -- & -- & -- & -- & 563 & 498 & -- & 466 & -- & 479 \\
\hline
\end{tabular}


Table 11b. Mean daily specific conductance in ground water at site CRM-2 (map ID \#2), West Well Field, Cedar Rapids, lowa, April 1996 through July 1997--Continued

\begin{tabular}{|c|c|c|c|c|c|c|c|c|c|c|c|c|}
\hline Day & $\operatorname{Jan}$ & Feb & Mar & Apr & May & Jun & Jul & Aug & Sep & Oct & Nov & Dec \\
\hline & & & & & & 1997 & & & & & & \\
\hline 1 & 477 & 545 & 508 & -- & 600 & 594 & -- & -- & -- & -- & -- & -- \\
\hline 2 & 477 & 547 & 508 & -- & 604 & 593 & -- & -- & -- & -- & -- & -- \\
\hline 3 & 477 & 548 & 509 & -- & 606 & 593 & -- & -- & -- & -- & -- & -- \\
\hline 4 & 497 & 549 & 509 & -- & 610 & 591 & -- & -- & -- & -- & -- & -- \\
\hline 5 & 506 & 550 & 508 & 552 & 613 & 588 & -- & -- & -- & -- & -- & -- \\
\hline 6 & 509 & 552 & 509 & 552 & 616 & 586 & -- & -- & -- & -- & -- & -- \\
\hline 7 & 517 & 544 & 509 & 554 & 618 & 584 & -- & -- & -- & -- & -- & - \\
\hline 8 & 528 & 530 & 509 & 556 & 620 & 582 & -- & -- & -- & -- & -- & -- \\
\hline 9 & 522 & 490 & 533 & 560 & 622 & 580 & -- & -- & -- & -- & -- & -- \\
\hline 10 & 484 & 490 & 513 & 559 & -- & 576 & -- & -- & -- & -- & -- & -- \\
\hline 11 & 479 & 490 & 514 & 562 & -. & -- & -- & -- & -- & -- & -- & -- \\
\hline 12 & 480 & 490 & 514 & 563 & - & -- & -- & - & -- & -- & -- & -- \\
\hline 13 & 481 & 490 & 514 & 566 & -- & -- & -- & -- & -- & -- & -- & -- \\
\hline 14 & 482 & 490 & 515 & 566 & -- & -- & -- & -- & -- & -- & -- & -- \\
\hline 15 & 484 & 489 & 516 & 567 & -- & -- & -- & -- & -- & -- & -- & -- \\
\hline 16 & 482 & 490 & 517 & 568 & -. & -- & - & -- & -- & -- & -- & -- \\
\hline 17 & 483 & 490 & 531 & 569 & -- & -- & -- & -- & -- & -- & -- & -- \\
\hline 18 & 485 & 490 & 540 & 572 & -- & -- & -- & -- & -- & -- & -- & -- \\
\hline 19 & 485 & 504 & 623 & 574 & -- & -- & -- & -- & -- & -- & -- & -- \\
\hline 20 & 484 & 527 & 546 & 579 & -. & -. & -. & -- & -- & - & -- & -- \\
\hline 21 & 487 & 533 & 586 & 581 & -. & -- & -- & -- & -- & -- & -- & -- \\
\hline 22 & 486 & 543 & 650 & 582 & -- & -- & -- & -- & -- & -- & -- & -- \\
\hline 23 & 486 & 552 & 673 & 583 & 649 & -- & -- & - & -- & -- & -- & -- \\
\hline 24 & 488 & 553 & 681 & 584 & 649 & -- & -- & -- & -- & -- & -- & -- \\
\hline 25 & 493 & 514 & 689 & 585 & 652 & -- & -- & -- & -- & -- & -- & -- \\
\hline 26 & 491 & 509 & 687 & 586 & 653 & -- & -- & -- & -- & -- & - & -- \\
\hline 27 & 536 & 508 & 673 & 588 & 654 & -- & -- & -- & -- & -- & -- & -- \\
\hline 28 & 538 & 509 & 661 & 590 & 656 & -- & -- & -- & -- & -- & -- & -- \\
\hline 29 & 539 & -- & 580 & 592 & 657 & -- & -- & -- & -- & -- & -- & -- \\
\hline 30 & 541 & -- & -- & 597 & 661 & -- & -- & -- & -- & -- & -- & -- \\
\hline 31 & 543 & -- & -- & -- & 673 & -- & -- & -- & -- & -- & -- & -- \\
\hline
\end{tabular}


Table 11c. Mean daily specific conductance in ground water at site CRM-11 (map ID \#9), West Well Field, Cedar Rapids, lowa, April 1996 through June 1997

[All values listed in microsiemens per centimeter at 25 degress Centigrade; --, value not measured, not recorded, or rejected after data review]

\begin{tabular}{|c|c|c|c|c|c|c|c|c|c|c|c|c|}
\hline Day & Jan & Feb & Mar & Apr & May & Jun & Jul & Aug & Sep & Oct & Nov & Dec \\
\hline \multicolumn{13}{|c|}{1996} \\
\hline 1 & -- & -- & -- & 324 & 321 & -- & 349 & 359 & 372 & 358 & 357 & 360 \\
\hline 2 & -- & -- & -- & 327 & 323 & -- & 350 & 361 & 372 & 359 & 357 & 362 \\
\hline 3 & -. & -- & -- & 326 & 324 & -- & 351 & 363 & 372 & 359 & 359 & 364 \\
\hline 4 & -- & -. & -- & 325 & 323 & -- & 352 & 365 & 372 & 359 & 358 & 354 \\
\hline 5 & -- & -. & -- & 322 & 324 & -- & 352 & 367 & 371 & 359 & 360 & 374 \\
\hline 6 & -- & -- & -- & 325 & 325 & -- & 352 & 369 & 371 & 359 & 358 & 376 \\
\hline 7 & -- & -- & -- & 322 & 326 & -- & 352 & 373 & 371 & 359 & 357 & 378 \\
\hline 8 & -- & .- & -- & 321 & 326 & -- & 351 & 374 & 371 & 359 & 357 & 379 \\
\hline 9 & -- & -- & -- & 329 & -- & -- & 350 & 373 & 371 & 358 & 357 & 381 \\
\hline 10 & -- & -- & -- & 328 & -- & -- & 350 & 373 & 371 & 358 & 357 & 381 \\
\hline 11 & -- & -. & -- & 329 & -- & -- & 350 & 372 & 371 & 359 & 357 & 382 \\
\hline 12 & -- & -. & -- & 323 & -- & -- & 350 & 371 & 371 & 359 & 357 & 383 \\
\hline 13 & -- & -- & -- & 327 & -- & -- & 350 & 370 & 365 & 359 & 356 & 384 \\
\hline 14 & -- & -. & -- & 320 & -- & -- & 349 & 370 & 357 & 359 & 357 & 384 \\
\hline 15 & -- & -. & -- & 321 & -- & -- & 349 & 370 & 357 & 359 & 356 & 385 \\
\hline 16 & -. & -- & -- & 330 & -- & -- & 352 & 370 & 357 & 359 & 355 & 385 \\
\hline 17 & -- & -- & - & 331 & -- & - & 350 & 370 & 357 & 359 & 355 & 386 \\
\hline 18 & -- & -- & -- & 331 & -- & -- & 349 & 370 & 357 & 359 & 355 & 386 \\
\hline 19 & -- & -- & -- & 330 & -- & -- & 349 & 370 & 357 & 358 & 355 & 387 \\
\hline 20 & -- & -. & -- & 331 & -- & -- & 350 & 370 & 357 & 358 & 355 & 387 \\
\hline 21 & -- & -- & -- & 329 & -- & -- & 351 & 370 & 357 & 358 & 354 & 388 \\
\hline 22 & -- & -- & -- & 327 & -- & -- & 351 & 370 & 358 & 358 & 355 & 388 \\
\hline 23 & -- & -- & -- & 310 & -- & -- & 351 & 370 & 358 & 358 & 355 & 389 \\
\hline 24 & -- & -. & -- & 316 & -- & -- & 351 & 370 & 358 & 358 & 355 & 394 \\
\hline 25 & -. & -. & -- & 308 & -- & -- & 351 & 370 & 358 & 358 & 355 & 399 \\
\hline 26 & -. & -- & - & 310 & -- & -- & 351 & 354 & 358 & 358 & 355 & 404 \\
\hline 27 & -. & -. & -- & 314 & -- & -- & 350 & 370 & 358 & 358 & 355 & 408 \\
\hline 28 & -- & -. & -- & 316 & -- & 349 & 350 & 372 & 358 & 358 & 355 & 410 \\
\hline 29 & -- & -- & -- & 319 & -- & 349 & 351 & 372 & 358 & 358 & 356 & 411 \\
\hline 30 & -- & -- & -- & 320 & -- & 349 & 355 & 372 & 358 & 358 & 358 & 413 \\
\hline 31 & -- & -- & -- & -- & -- & -- & 359 & 372 & -- & 357 & -- & 396 \\
\hline
\end{tabular}


Table 11c. Mean daily specific conductance in ground water at site CRM-11 (map ID \#9), West Well Field, Cedar Rapids, lowa, April 1996 through June 1997--Continued

\begin{tabular}{|c|c|c|c|c|c|c|c|c|c|c|c|c|}
\hline Day & Jan & Feb & Mar & Apr & May & Jun & Jul & Aug & Sep & Oct & Nov & Dec \\
\hline & & & & & & 1997 & & & & & & \\
\hline 1 & 411 & 466 & 438 & -- & 409 & 438 & -- & -- & -. & -- & -- & -- \\
\hline 2 & 409 & 472 & 438 & -- & 410 & 435 & -- & -- & -- & -- & -- & -- \\
\hline 3 & 404 & 477 & 437 & -- & 411 & 429 & -- & -- & -- & -- & -- & -- \\
\hline 4 & 401 & 480 & 437 & -- & 412 & 428 & -- & -- & -- & -- & -- & -- \\
\hline 5 & 404 & 484 & 435 & 406 & 413 & 427 & -- & -- & -- & -- & -- & -- \\
\hline 6 & 409 & 485 & 430 & 404 & 414 & 423 & -- & -- & -- & -- & - & -- \\
\hline 7 & 415 & 476 & 425 & 414 & 416 & 419 & -- & -- & -- & -- & -- & -- \\
\hline 8 & 419 & 470 & 422 & 403 & 416 & 417 & - & -- & -- & -- & -- & -. \\
\hline 9 & 425 & 461 & 418 & 402 & 418 & 413 & -- & -- & -- & -- & -- & -- \\
\hline 10 & 427 & 459 & 416 & 402 & -- & 409 & -- & -- & -. & -- & -- & -- \\
\hline 11 & 427 & 456 & 416 & 402 & -- & 408 & -- & -- & -- & -- & -- & -- \\
\hline 12 & 423 & 451 & 415 & 402 & -- & 408 & -- & -. & -- & -- & -- & -- \\
\hline 13 & 421 & 449 & 414 & 401 & -- & -- & -- & -- & -- & -- & -- & -- \\
\hline 14 & 421 & 448 & 415 & 401 & -- & -- & -- & -- & $-\cdot$ & -- & -- & -- \\
\hline 15 & 430 & 447 & 415 & 401 & -- & -- & $-\cdot$ & -- & -- & -- & -- & -- \\
\hline 16 & 414 & 448 & 416 & 401 & -- & -- & -- & -- & -- & -- & -- & -- \\
\hline 17 & 419 & 450 & 417 & 401 & -- & -- & -- & -- & -- & -- & -- & -- \\
\hline 18 & 419 & 450 & 417 & 401 & -- & -- & -- & -- & -- & -- & -- & -- \\
\hline 19 & 416 & 453 & 415 & 402 & -- & -- & -- & -- & -- & -- & -- & -- \\
\hline 20 & 414 & 462 & 414 & 403 & -- & -- & -- & -- & -- & -- & -- & -- \\
\hline 21 & 416 & 463 & 412 & 403 & -- & -- & -- & -- & -- & -- & -- & -- \\
\hline 22 & 415 & 464 & 412 & 404 & -- & -- & -- & -- & -- & -- & -- & -- \\
\hline 23 & 414 & 461 & 413 & 404 & 432 & -- & -- & - & -- & -- & -- & -- \\
\hline 24 & 416 & 453 & 414 & 404 & 436 & -- & -- & -- & -- & -- & -- & -- \\
\hline 25 & 416 & 445 & 417 & 405 & 438 & -- & -- & -- & -- & -- & -- & -- \\
\hline 26 & 425 & 440 & 416 & 405 & 438 & -- & -- & -- & -- & -- & -- & -- \\
\hline 27 & 452 & 438 & 413 & 406 & 438 & -- & -- & -- & -- & -- & -- & -- \\
\hline 28 & 456 & 438 & 412 & 406 & 438 & -- & -- & -- & -- & -- & -- & -- \\
\hline 29 & 457 & -- & 410 & 407 & 438 & -- & -- & -- & -- & -- & -- & -- \\
\hline 30 & 459 & -- & - & 408 & 437 & -- & -- & -- & -- & -- & -- & -- \\
\hline 31 & 462 & -- & -- & -. & 438 & -- & -- & .- & -- & -- & -- & -- \\
\hline
\end{tabular}


Table 11d. Mean daily specific conductance in ground water at site CRM-12 (map ID \#10), East Well Field, Cedar Rapids lowa, April 1996 through July 1997

[All values in microsiemens per centimeter at 25 degrees Centigrade; --, value not measured, not recorded, or rejected after data review]

\begin{tabular}{|c|c|c|c|c|c|c|c|c|c|c|c|c|}
\hline Day & Jan & Feb & Mar & Apr & May & Jun & Jul & Aug & Sep & Oct & Nov & Dec \\
\hline \multicolumn{13}{|c|}{1996} \\
\hline 1 & -- & -- & -- & 665 & 660 & -- & 645 & 631 & 627 & 617 & 631 & 659 \\
\hline 2 & -- & -- & -- & 664 & 659 & -- & 644 & 631 & 627 & 617 & 632 & 657 \\
\hline 3 & -- & -- & -- & 658 & 657 & -- & 641 & 631 & 627 & 617 & 632 & 645 \\
\hline 4 & .. & -- & -- & 655 & 655 & -- & 639 & 630 & 627 & 618 & 632 & 654 \\
\hline 5 & -- & -- & -- & 656 & 657 & -- & 638 & 630 & 627 & 622 & 632 & 678 \\
\hline 6 & -- & -- & -- & 655 & 660 & -- & 635 & 630 & 627 & 619 & 632 & 666 \\
\hline 7 & -. & -- & -- & 654 & 656 & -- & 632 & 631 & 628 & 618 & 632 & 668 \\
\hline 8 & -- & -- & -- & 653 & 654 & - & 629 & 632 & 628 & 619 & 633 & 669 \\
\hline 9 & -- & -- & -- & 651 & -- & -- & 627 & 633 & 629 & 623 & 633 & 668 \\
\hline 10 & -- & -- & -- & 650 & -- & -- & 626 & 632 & 629 & 632 & 634 & 683 \\
\hline 11 & -- & -- & -- & 652 & -- & -- & 627 & 631 & 629 & 627 & 636 & 648 \\
\hline 12 & -- & -- & -- & 655 & -- & -- & 628 & 631 & 630 & 626 & 635 & 701 \\
\hline 13 & -- & -- & -- & 657 & -- & -- & 630 & 630 & 598 & 626 & 634 & 698 \\
\hline 14 & -- & -- & -- & 654 & -- & -- & 631 & 630 & 614 & 629 & 634 & 698 \\
\hline 15 & -- & -- & -- & 661 & -- & -- & 631 & 630 & 613 & 631 & 634 & 701 \\
\hline 16 & -- & -- & -- & 665 & -- & -- & 633 & 630 & 613 & 633 & 634 & 703 \\
\hline 17 & -- & -- & -- & 667 & -- & - & 635 & 630 & 613 & 634 & 635 & 705 \\
\hline 18 & -- & -- & -- & 668 & -- & -- & 634 & 630 & 613 & 632 & 635 & 706 \\
\hline 19 & -- & -- & -- & 668 & -- & -- & 632 & 629 & 613 & 630 & 643 & 705 \\
\hline 20 & -- & -- & -- & 667 & -- & -- & 632 & 627 & 613 & 624 & 637 & 705 \\
\hline 21 & -- & -- & -- & 664 & -- & -- & 632 & 626 & 614 & 623 & 669 & 706 \\
\hline 22 & -- & -- & -- & 661 & -- & -- & 631 & 625 & 615 & 622 & 662 & 706 \\
\hline 23 & -- & -- & -- & 634 & -- & -- & 631 & 624 & 615 & 619 & 661 & 706 \\
\hline 24 & -- & -- & -- & 662 & -- & -- & 631 & 624 & 615 & 617 & 662 & 706 \\
\hline 25 & -- & -- & -- & 662 & -- & -- & 630 & 624 & 615 & 616 & 651 & 701 \\
\hline 26 & -- & -- & -- & 662 & -- & -- & 630 & 621 & 616 & 616 & 694 & 701 \\
\hline 27 & -- & -- & -- & 662 & -- & -- & 629 & 625 & 616 & 616 & 695 & 731 \\
\hline 28 & -- & -- & -- & 661 & -- & 649 & 628 & 625 & 616 & 616 & 695 & 761 \\
\hline 29 & -- & -- & -- & 661 & -- & 648 & 627 & 626 & 617 & 616 & 692 & 711 \\
\hline 30 & -- & -- & -- & 660 & -- & 647 & 629 & 627 & 617 & 621 & 673 & 786 \\
\hline 31 & -- & -- & -- & -. & -- & -- & 632 & 627 & -- & 630 & -- & 778 \\
\hline
\end{tabular}


Table 11d. Mean daily specific conductance in ground water at site CRM-12 (map ID \#10), East Well Field, Cedar Rapids, lowa, April 1996 through July 1997--Continued

\begin{tabular}{|c|c|c|c|c|c|c|c|c|c|c|c|c|}
\hline Day & Jan & Feb & Mar & Apr & May & Jun & Jul & Aug & Sep & Oct & Nov & Dec \\
\hline & & & & & & 1997 & & & & & & \\
\hline 1 & 774 & 740 & 673 & -- & 712 & 670 & 673 & -- & -- & -- & -- & -- \\
\hline 2 & 791 & 730 & 673 & -- & 710 & 632 & 677 & -- & -- & -- & -- & -- \\
\hline 3 & 788 & 724 & 673 & -- & 709 & 656 & 678 & -. & -- & -- & -- & -- \\
\hline 4 & 783 & 713 & 673 & -- & 707 & 678 & 676 & -- & -- & -- & -- & -- \\
\hline 5 & 783 & 709 & 672 & 685 & 708 & 675 & 676 & -- & -- & -- & -- & -- \\
\hline 6 & 780 & 699 & 669 & 688 & 709 & 670 & 677 & -- & -- & -. & -- & -- \\
\hline 7 & 769 & 696 & 667 & 691 & 710 & 655 & 678 & -- & -- & -- & -- & -- \\
\hline 8 & 754 & 688 & 665 & 693 & 711 & 654 & 678 & -- & -- & -- & -- & -- \\
\hline 9 & 753 & 682 & 664 & 696 & 713 & 667 & 677 & -- & -- & -- & -- & -- \\
\hline 10 & 751 & 679 & 658 & 698 & -- & 636 & 676 & -- & -- & -- & -- & -- \\
\hline 11 & 720 & 679 & 660 & 700 & -- & 646 & -- & -- & -- & -- & -- & -- \\
\hline 12 & 694 & 683 & 661 & 703 & -- & 666 & -- & -- & -- & -- & -- & -- \\
\hline 13 & 675 & 688 & 661 & 704 & -- & 665 & -- & -- & -- & -- & -- & -- \\
\hline 14 & 664 & 691 & 662 & 704 & -- & 661 & -- & -- & -- & -- & -- & -. \\
\hline 15 & 658 & 695 & 661 & 702 & -- & 662 & -- & -- & -- & -- & -- & -- \\
\hline 16 & 654 & 697 & 661 & 702 & -- & 663 & -- & -- & -- & -- & -- & -- \\
\hline 17 & 652 & 697 & 661 & 703 & -- & 659 & -- & -- & -- & -- & -- & -- \\
\hline 18 & 648 & 698 & 662 & 703 & -- & 659 & -- & -- & -- & -- & -- & -- \\
\hline 19 & 645 & 695 & 662 & 703 & -- & 662 & -- & -- & -- & -- & -- & -. \\
\hline 20 & 643 & 691 & 665 & 703 & -- & 663 & -- & -- & -- & -- & -- & -- \\
\hline 21 & 651 & 686 & 669 & 711 & -- & 665 & -- & -- & -- & -- & -- & -- \\
\hline 22 & 658 & 678 & 672 & 713 & -- & 664 & -- & -- & -- & -- & -- & -- \\
\hline 23 & 664 & 674 & 673 & 712 & 631 & 669 & -- & -- & -- & -- & -- & -- \\
\hline 24 & 655 & 669 & 675 & 713 & 630 & 675 & -- & -- & -- & -- & - & -- \\
\hline 25 & 698 & 666 & 678 & 713 & 630 & 650 & -- & -- & -- & -- & -- & - \\
\hline 26 & 788 & 663 & 681 & 713 & 631 & 678 & -- & -- & -- & -- & -- & -- \\
\hline 27 & 802 & 667 & 685 & 713 & 630 & 676 & -- & -- & -- & -- & -- & -- \\
\hline 28 & 794 & 672 & 688 & 713 & 630 & 673 & -- & -- & -- & -- & -- & -- \\
\hline 29 & 789 & -- & 687 & 713 & 630 & 671 & -- & -- & -- & -- & -- & -- \\
\hline 30 & 775 & -- & -. & 712 & 624 & 671 & -- & -- & -- & -- & -- & -- \\
\hline 31 & 754 & -- & $\ldots$ & -- & 682 & -- & -- & -- & - & -- & -- & -- \\
\hline
\end{tabular}


Table 11e. Mean daily specific conductance in ground water at site CRM-20 (map ID \#17), Northwest Well Field, Cedar Rapids, lowa, February 1998 through March 1999

[All values in microsiemens per centimeter at 25 degrees Centigrade; --, value not measured, not recorded, or rejected after data review]

\begin{tabular}{|c|c|c|c|c|c|c|c|c|c|c|c|c|}
\hline Day & Jan & Feb & Mar & Apr & May & Jun & Jul & $\overline{\text { Aug }}$ & Sep & Oct & Nov & Dec \\
\hline & & & & & & 1998 & & & & & & \\
\hline 1 & -- & -- & 495 & 550 & 478 & 491 & 399 & 426 & -- & 420 & 505 & 526 \\
\hline 2 & -. & -- & 493 & 634 & 483 & 490 & 401 & 425 & -- & 420 & 503 & 528 \\
\hline 3 & -- & -- & 490 & 552 & 490 & 478 & 405 & 423 & -. & 419 & 499 & 531 \\
\hline 4 & -- & -- & 486 & 565 & 533 & 481 & 414 & 420 & -. & 418 & -- & 533 \\
\hline 5 & -- & -- & 483 & 508 & 542 & 481 & 430 & 417 & -- & 417 & 493 & 536 \\
\hline 6 & -- & -- & 479 & 480 & 550 & 480 & 434 & 413 & -- & 417 & 490 & 539 \\
\hline 7 & -- & - & 477 & 479 & 560 & 483 & 424 & 410 & -- & 421 & 479 & 541 \\
\hline 8 & -- & -- & 475 & 479 & 562 & 488 & 416 & 408 & -- & 424 & 473 & 543 \\
\hline 9 & -- & -- & 474 & 475 & 556 & 493 & 416 & 403 & -- & 426 & 481 & 545 \\
\hline 10 & -- & 519 & 462 & 475 & 551 & 497 & 427 & 401 & -- & 424 & 493 & 546 \\
\hline 11 & -- & 516 & 446 & 481 & 548 & 498 & 443 & 395 & -- & 420 & $498^{-}$ & 546 \\
\hline 12 & -- & 514 & 443 & 488 & 548 & 503 & 458 & 392 & -- & 420 & 504 & 545 \\
\hline 13 & -- & 511 & 441 & 493 & 549 & 521 & 461 & 389 & -- & 423 & 506 & 543 \\
\hline 14 & -- & 508 & 439 & 488 & 550 & 529 & 451 & 388 & -- & 428 & 508 & 542 \\
\hline 15 & -- & 504 & 437 & 483 & 549 & 547 & 444 & 388 & -- & 431 & 510 & 540 \\
\hline 16 & -- & 500 & 435 & 485 & 550 & 565 & 437 & 383 & -- & 432 & 512 & 539 \\
\hline 17 & -- & 495 & 431 & 490 & 551 & 516 & 439 & -- & -- & 437 & 515 & 540 \\
\hline 18 & -- & 490 & 425 & 492 & 550 & 445 & 465 & -- & -- & 446 & 518 & 542 \\
\hline 19 & -- & 485 & 422 & 490 & 548 & 452 & 491 & -- & -- & 456 & 520 & 540 \\
\hline 20 & -- & 481 & 424 & 484 & 547 & 477 & 496 & -- & - & 463 & 523 & 539 \\
\hline 21 & -- & 478 & 432 & 474 & 546 & 489 & 496 & -- & -- & 460 & 527 & 538 \\
\hline 22 & -- & 475 & 442 & 473 & 545 & 477 & 495 & -- & -- & 454 & 530 & 537 \\
\hline 23 & -- & 490 & 470 & 474 & 545 & 463 & 492 & -- & 429 & 460 & 537 & 536 \\
\hline 24 & -- & 502 & 490 & 474 & 543 & 462 & 477 & -- & 426 & 484 & 539 & 535 \\
\hline 25 & -- & 503 & 492 & 474 & 540 & 453 & 459 & -- & 429 & 490 & 538 & 536 \\
\hline 26 & -- & 501 & 493 & 474 & 524 & 433 & 442 & -. & 429 & 499 & 535 & 536 \\
\hline 27 & -- & 498 & 494 & 475 & 510 & 421 & 427 & -- & 426 & 398 & 531 & 536 \\
\hline 28 & -- & 496 & 497 & 478 & 501 & 413 & 426 & -- & 422 & 400 & 528 & 536 \\
\hline 29 & -- & -- & 504 & 481 & 492 & 407 & 431 & -- & 419 & 510 & 525 & 535 \\
\hline 30 & -- & -- & 516 & 481 & 489 & 399 & 430 & -- & 419 & 510 & 525 & 534 \\
\hline 31 & -- & -- & 516 & -- & 490 & -- & 428 & -- & -- & 504 & -- & 532 \\
\hline
\end{tabular}


Table 11e. Mean daily specific conductance in ground water at site CRM-20 (map ID \#17), Northwest Well Field, Cedar Rapids, lowa, February 1998 through March 1999--Continued

\begin{tabular}{|c|c|c|c|c|c|c|c|c|c|c|c|c|}
\hline Day & Jan & Feb & Mar & Apr & May & Jun & Jul & Aug & Sep & Oct & Nov & Dec \\
\hline \multicolumn{13}{|c|}{1999} \\
\hline 1 & 531 & 586 & 477 & -- & -- & -. & -- & -- & -- & -- & -- & -- \\
\hline 2 & 530 & 589 & 460 & -- & -- & -- & -- & -- & -- & -- & -- & -- \\
\hline 3 & 530 & 589 & 462 & -- & -- & -- & -- & -- & -- & -- & -- & -- \\
\hline 4 & 532 & 587 & 470 & -- & -- & -- & -- & -- & -- & -- & -- & -- \\
\hline 5 & 536 & 584 & 479 & -- & -- & -- & -- & -- & -. & -- & -- & -- \\
\hline 6 & 550 & 582 & 485 & -- & -- & -. & .. & -- & -- & -- & -- & -- \\
\hline 7 & 556 & 582 & 489 & - & -- & -- & -- & -- & - & -- & - & -- \\
\hline 8 & 566 & 578 & 494 & -- & -- & -- & -- & -- & -- & -- & -- & -- \\
\hline 9 & 577 & 574 & 498 & -- & -- & -- & -- & -- & -- & -- & -- & -- \\
\hline 10 & 578 & 569 & 502 & -- & -- & -- & -- & -. & -- & -- & -- & -- \\
\hline 11 & 577 & 566 & 506 & -- & -- & -- & -- & -- & -- & -- & -- & -- \\
\hline 12 & 578 & 566 & 511 & -- & -- & -- & -- & -- & -- & -- & -- & -- \\
\hline 13 & 582 & 565 & 516 & -- & -- & -- & -- & -- & -- & -- & -- & -- \\
\hline 14 & 586 & 563 & 519 & -- & -- & -- & -- & -- & -- & -- & -- & -- \\
\hline 15 & 588 & 557 & 522 & -- & -- & -- & -. & -- & -. & -- & -- & -- \\
\hline 16 & 589 & 549 & 517 & -- & -- & -- & -- & -- & -- & -- & -- & - \\
\hline 17 & 587 & 539 & 503 & -- & -- & -- & -- & -- & -- & -- & -- & -- \\
\hline 18 & 585 & 529 & 492 & -- & -- & -- & -- & .- & -- & -. & -- & -- \\
\hline 19 & 583 & 523 & 491 & -- & -- & -- & -- & -- & -- & -- & -- & -- \\
\hline 20 & 581 & 519 & 498 & -- & -- & -- & -- & -- & -- & -- & -. & -- \\
\hline 21 & 581 & 517 & 505 & -- & -- & -- & - & -- & -- & -- & -- & -- \\
\hline 22 & 568 & 515 & 509 & -- & -- & -- & -- & -- & -. & -- & -- & -- \\
\hline 23 & 551 & 513 & 515 & -- & -- & -- & -- & -- & -- & -- & -- & -- \\
\hline 24 & 550 & 511 & 520 & -- & -- & -- & -- & -- & -- & -- & -. & -- \\
\hline 25 & 547 & 507 & 520 & -- & -- & -- & -- & -- & -- & -- & -- & -- \\
\hline 26 & 543 & 504 & 535 & -- & -- & -- & -- & -- & -- & -- & -- & -- \\
\hline 27 & 539 & 499 & 538 & -- & -- & -- & -- & -- & -- & -- & -- & -- \\
\hline 28 & 537 & 494 & 540 & -- & -- & -- & -- & -- & -- & -- & -- & -- \\
\hline 29 & 561 & -- & 539 & -- & -- & -. & -- & -- & -- & -- & -- & -- \\
\hline 30 & 584 & -- & 537 & -- & -- & -- & -- & -- & -- & -- & -- & -- \\
\hline 31 & 584 & -- & 537 & -- & -- & -- & -- & -- & -- & -- & -- & -- \\
\hline
\end{tabular}


Table 11f. Mean daily specific conductance in ground water at site CRM-21 (map ID \#18), Northwest Well Field, Cedar Rapids, lowa, February 1998 through March 1999

[All values in microsiemens per centimeter at 25 degrees Centigrade; --, value not measured, not recorded, or rejected after data review]

\begin{tabular}{|c|c|c|c|c|c|c|c|c|c|c|c|c|}
\hline Day & Jan & Feb & Mar & Apr & May & Jun & Jul & Aug & Sep & Oct & Nov & Dec \\
\hline \multicolumn{13}{|c|}{1998} \\
\hline 1 & -- & -. & 612 & 369 & 541 & 574 & 508 & 758 & -- & 706 & 725 & 655 \\
\hline 2 & -- & -- & 606 & 395 & 546 & 562 & 510 & 760 & -- & 704 & 741 & 669 \\
\hline 3 & -- & -- & 585 & 419 & 578 & 579 & 379 & 759 & -- & 696 & 776 & 681 \\
\hline 4 & -- & -- & 569 & 451 & 606 & 612 & 401 & 757 & -- & 695 & 779 & 692 \\
\hline 5 & -- & -- & 562 & 480 & 593 & 620 & 413 & 746 & -- & 704 & 792 & 700 \\
\hline 6 & -- & -- & 574 & 499 & 577 & 637 & 414 & 736 & -- & 715 & 798 & 704 \\
\hline 7 & -- & -- & 585 & 510 & 569 & 647 & 416 & 724 & -- & 726 & 728 & 702 \\
\hline 8 & -- & -- & 583 & 520 & 569 & 647 & 422 & 722 & -- & 739 & 658 & 698 \\
\hline 9 & -- & -- & 579 & 528 & 573 & 645 & 439 & 720 & -- & 767 & 619 & 692 \\
\hline 10 & -- & 577 & 594 & 536 & 575 & 642 & 446 & 702 & -- & 777 & 596 & 687 \\
\hline 11 & -- & 577 & 609 & 542 & 575 & 638 & 442 & 693 & -- & 788 & 596 & 683 \\
\hline 12 & -. & 578 & 592 & 544 & 574 & 645 & 437 & 692 & -- & 787 & 631 & 679 \\
\hline 13 & -- & 578 & 590 & 551 & 577 & 689 & 432 & 695 & -- & 780 & 688 & 672 \\
\hline 14 & -- & 579 & 597 & 519 & 579 & 678 & 419 & 693 & -- & 771 & 688 & 664 \\
\hline 15 & -- & 580 & 596 & 526 & 576 & 664 & 470 & 689 & -- & 760 & 688 & 659 \\
\hline 16 & -- & 581 & 594 & 525 & 574 & 653 & 544 & 684 & -- & 741 & 726 & 659 \\
\hline 17 & -- & 581 & 593 & 526 & 574 & 643 & 515 & -- & -- & 722 & 690 & 666 \\
\hline 18 & -- & 582 & 595 & 527 & 576 & 632 & 516 & -- & -- & 706 & 607 & 678 \\
\hline 19 & -- & 583 & 599 & 529 & 575 & 622 & 467 & -- & -- & 693 & 573 & 693 \\
\hline 20 & -- & 583 & 602 & 531 & 571 & 614 & 467 & -- & -- & 707 & 561 & 685 \\
\hline 21 & -. & 590 & 604 & 532 & 569 & 602 & 472 & -- & -- & 743 & 557 & 676 \\
\hline 22 & -. & 600 & 602 & 532 & 570 & 597 & 474 & -- & -- & 783 & 555 & 674 \\
\hline 23 & -- & 600 & 603 & 530 & 573 & 579 & 474 & -- & 682 & 756 & 581 & 670 \\
\hline 24 & -- & 591 & 602 & 525 & 575 & 527 & 567 & -- & 690 & 712 & 600 & 669 \\
\hline 25 & -- & 593 & 588 & 517 & 576 & 342 & 700 & -- & 695 & 703 & 595 & 666 \\
\hline 26 & -- & 600 & 582 & 507 & 597 & 364 & 726 & -- & 695 & 689 & 591 & 665 \\
\hline 27 & -- & 606 & 582 & 499 & 691 & 365 & 733 & -- & 698 & 686 & 591 & 665 \\
\hline 28 & -- & 611 & 571 & 495 & 739 & 386 & 732 & -- & 703 & 715 & 602 & 668 \\
\hline 29 & -- & -- & 568 & 499 & 755 & 426 & 736 & -- & 699 & 733 & 621 & 669 \\
\hline 30 & -- & -- & 575 & 514 & 685 & 444 & 746 & -- & 702 & 729 & 640 & 668 \\
\hline 31 & -- & -- & 492 & -. & 604 & -- & 754 & -- &.- & 744 & -. & 665 \\
\hline
\end{tabular}


Table 11f. Mean daily specific conductance in ground water at site CRM-21 (map ID \#18), Northwest Well Field, Cedar Rapids, lowa, February 1998 through March 1999--Continued

\begin{tabular}{|c|c|c|c|c|c|c|c|c|c|c|c|c|}
\hline Day & Jan & Feb & Mar & Apr & May & Jun & Jul & Aug & Sep & Oct & Nov & Dec \\
\hline & & & & & & 1999 & & & & & & \\
\hline 1 & 664 & 680 & 480 & -- & -- & -- & -- & -- & -- & -- & -- & -- \\
\hline 2 & 664 & 679 & 469 & -- & -- & -- & -- & - & - & -- & -. & -- \\
\hline 3 & 664 & 679 & 472 & -- & -. & -- & -- & -- & -- & -. & -- & -- \\
\hline 4 & 666 & 679 & 467 & -- & -- & -- & -- & -- & -- & -- & -- & -- \\
\hline 5 & 672 & 680 & 469 & -- & -- & -- & -- & -- & -- & -- & -- & -- \\
\hline 6 & 683 & 680 & 477 & -- & -- & -- & -- & -- & -- & -- & -- & -- \\
\hline 7 & 686 & 681 & 506 & -- & -- & -- & -- & -- & -- & -- & -- & -- \\
\hline 8 & 686 & 681 & 560 & -- & -- & -- & -- & -- & -- & -- & -- & -- \\
\hline 9 & 687 & 682 & 614 & -- & -- & -- & -- & -- & -- & -- & -- & -- \\
\hline 10 & 690 & 682 & 642 & -- & -- & -- & -- & -- & -- & -- & -- & -- \\
\hline 11 & 696 & 683 & 652 & -- & -- & -- & -- & -. & -- & -- & -- & -. \\
\hline 12 & 704 & 683 & 650 & -- & -- & -- & -- & -- & -- & -- & -- & -- \\
\hline 13 & 710 & 680 & 638 & -- & -- & -- & -- & -- & -- & -- & -- & -- \\
\hline 14 & 714 & 676 & 629 & -- & -- & -- & -- & -- & -- & -- & -- & -- \\
\hline 15 & 719 & 682 & 629 & -- & -- & -- & -- & -- & -- & -- & -- & -- \\
\hline 16 & 722 & 690 & 635 & -- & -- & -. & -- & -- & -- & -- & -- & -- \\
\hline 17 & 723 & 696 & 643 & -- & -- & -- & -- & -- & -- & -- & -- & -- \\
\hline 18 & 725 & 710 & 654 & -- & -- & -- & -- & -. & -- & - & -. & -- \\
\hline 19 & 726 & 706 & 667 & -- & -- & -- & -- & -- & -- & -- & -- & -- \\
\hline 20 & 726 & 660 & 677 & -- & -- & -- & -- & -- & -- & -- & -- & -- \\
\hline 21 & 722 & 618 & 679 & -- & -- & - & -- & -- & -- & -- & -- & -- \\
\hline 22 & 694 & 576 & 678 & -- & -- & -- & -- & -- & -- & -- & -- & -- \\
\hline 23 & 657 & 543 & 677 & -- & -- & -- & -- & -- & -- & -- & -- & -- \\
\hline 24 & 650 & 523 & 677 & -- & -- & -- & -- & -- & -- & -- & -- & -- \\
\hline 25 & 643 & 506 & 676 & -- & -- & -- & -- & -- & -- & - & -- & -- \\
\hline 26 & 638 & 494 & 691 & -- & -- & -- & -- & -- & -- & -- & -- & -- \\
\hline 27 & 634 & 488 & 712 & -- & -- & -- & -- & -- & -- & -- & -- & -- \\
\hline 28 & 631 & 485 & 713 & -- & -- & -- & -- & -- & -- & -- & -- & -- \\
\hline 29 & 659 & -- & 711 & -- & -- & -- & -- & -- & -- & -- & -- & -- \\
\hline 30 & 681 & -- & 710 & -- & -- & -- & -- & -- & -- & -- & -- & -- \\
\hline 31 & 680 & -- & 708 & -- & -- & .- & -- & -- & -- & -- & -- & -- \\
\hline
\end{tabular}


Table 11g. Mean daily specific conductance in ground water at site CRM-22 (map ID \#19), Seminole Well Field, Cedar Rapids, lowa, February 1998 through March 1999

[All values in microsiemens per centimeter at 25 degrees Centigrade; --, value not measured, not recorded, or rejected after data review]

\begin{tabular}{|c|c|c|c|c|c|c|c|c|c|c|c|c|}
\hline Day & Jan & Feb & Mar & $\overline{\text { Apr }}$ & May & Jun & Jul & Aug & Sep & Oct & Nov & $\overline{\text { Dec }}$ \\
\hline & & & & & & 1998 & & & & & & \\
\hline 1 & -- & -. & 612 & 369 & 563 & 476 & -- & -- & -- & 454 & 548 & 588 \\
\hline 2 & -- & -- & 606 & 395 & 564 & 477 & -- & -- & -- & 463 & 529 & 588 \\
\hline 3 & -- & -- & 585 & 419 & 564 & -- & -- & -. & -- & 473 & 528 & 588 \\
\hline 4 & -- & -. & 569 & 451 & 561 & -- & -. & -- & -- & 475 & 540 & 586 \\
\hline 5 & -- & -- & 562 & 480 & 544 & -- & -- & -- & -- & 471 & 556 & 583 \\
\hline 6 & -- & -- & 574 & 499 & 545 & -- & -. & -- & -- & 463 & 563 & 576 \\
\hline 7 & -. & -- & 585 & 510 & 547 & -- & -. & -- & -- & 456 & 569 & 573 \\
\hline 8 & -- & -- & 583 & 520 & 548 & -- & -. & -- & -- & 456 & 571 & 572 \\
\hline 9 & -- & -- & 579 & 528 & 547 & -- & -- & -- & -- & 451 & 571 & 572 \\
\hline 10 & -- & 577 & 594 & 536 & 545 & -- & - & -- & -- & 456 & 570 & 571 \\
\hline 11 & -- & 577 & 609 & 542 & 543 & -- & -- & -- & -- & 464 & 570 & 570 \\
\hline 12 & .. & 578 & 592 & 544 & 538 & -- & -- & -- & -- & 475 & 571 & 560 \\
\hline 13 & -- & 578 & 590 & 551 & 537 & -- & -- & -- & -- & 477 & 572 & 545 \\
\hline 14 & -- & 579 & 597 & 523 & 538 & -- & -- & -- & -- & 482 & 579 & 533 \\
\hline 15 & -- & 580 & 596 & 562 & 528 & -- & -- & -- & -- & 489 & 590 & 527 \\
\hline 16 & -- & 581 & 594 & 561 & 531 & - & -- & -- & -- & 485 & 600 & 525 \\
\hline 17 & -. & 581 & 593 & 563 & 520 & -. & -- & -- & -- & 487 & 612 & 526 \\
\hline 18 & -- & 582 & 595 & 565 & 506 & -- & -- & -- & -- & 498 & 619 & 529 \\
\hline 19 & -- & 583 & 599 & 564 & 498 & -- & -- & -- & -. & 513 & 620 & 536 \\
\hline 20 & -- & 583 & 602 & 560 & 489 & -. & -. & -- & -- & 528 & 614 & 538 \\
\hline 21 & -- & 590 & 604 & 561 & 484 & -- & - & -- & -- & 543 & 599 & 545 \\
\hline 22 & -- & 600 & 602 & 562 & 483 & -. & -- & -- & -- & 557 & 584 & 547 \\
\hline 23 & -- & 600 & 603 & 561 & 485 & -- & -- & -- & 476 & 563 & 545 & 551 \\
\hline 24 & -- & 591 & 602 & 546 & 487 & -. & -- & -- & 468 & 565 & 583 & 557 \\
\hline 25 & -- & 593 & 588 & 550 & 487 & -- & -- & -- & 460 & 567 & 582 & 566 \\
\hline 26 & -- & 600 & 582 & 553 & 489 & -- & -- & -- & 457 & 564 & 584 & 576 \\
\hline 27 & -- & 606 & 582 & 557 & 486 & -- & -- & -- & 465 & 561 & 585 & 582 \\
\hline 28 & -- & 611 & 571 & 559 & 484 & -- & -- & -- & 463 & 558 & 586 & 583 \\
\hline 29 & -- & -- & 568 & 560 & 473 & -- & -- & -- & 459 & 555 & 587 & 584 \\
\hline 30 & -. & -. & 575 & 562 & 473 & -- & -- & -- & 454 & 554 & 587 & 583 \\
\hline 31 & -- & -- & 492 & -- & 470 & -- & -- & -- & -- & 553 & -- & 582 \\
\hline
\end{tabular}


Table 11g. Mean daily specific conductance in ground water at site CRM-22 (map ID \#19), Seminole Well Field, Cedar Rapids, lowa, February 1998 through March 1999--Continued

\begin{tabular}{|c|c|c|c|c|c|c|c|c|c|c|c|c|}
\hline Day & Jan & Feb & Mar & Apr & May & Jun & Jul & Aug & Sep & Oct & Nov & Dec \\
\hline \multicolumn{13}{|c|}{1999} \\
\hline 1 & 581 & 601 & 518 & -- & -- & -- & -- & -- & -. & -- & -- & -- \\
\hline 2 & 579 & 605 & 517 & -- & -- & -- & -- & -- & -- & -- & -- & -- \\
\hline 3 & 578 & 606 & 526 & -- & -- & -- & -- & -- & -- & -- & -. & -- \\
\hline 4 & 580 & 603 & 533 & -- & -- & -- & -- & -- & -- & -- & -- & -- \\
\hline 5 & 587 & 599 & 537 & -- & -- & -- & -- & -- & -- & -- & -- & -- \\
\hline 6 & 596 & 596 & 540 & -- & -- & -- & -- & -- & -- & -- & -- & -- \\
\hline 7 & 604 & 597 & 543 & -. & -- & -- & -. & -. & .- & -- & -- & -- \\
\hline 8 & 613 & 599 & 546 & -- & -- & -- & -- & -- & -- & -- & -- & -- \\
\hline 9 & 620 & 593 & 549 & -. & -- & -- & -. & -- & -- & -- & -- & -- \\
\hline 10 & 624 & 587 & 553 & -- & -- & -- & -- & -- & -- & -- & -- & -- \\
\hline 11 & 625 & 584 & 558 & -- & -- & -- & -- & -- & -- & -- & -- & -- \\
\hline 12 & 627 & 581 & 563 & -- & -- & -- & -- & -- & -- & -- & -- & -- \\
\hline 13 & 629 & 581 & 566 & -- & -- & -- & -- & -- & -- & -- & -- & -- \\
\hline 14 & 634 & 581 & 570 & -- & -- & -- & -- & -- & -- & -- & -- & -- \\
\hline 15 & 638 & 577 & 565 & -- & -- & -- & -- & -- & -- & -- & -- & -- \\
\hline 16 & 640 & 572 & 545 & -- & -- & -- & -- & -- & -- & -- & -- & - \\
\hline 17 & 639 & 564 & 530 & -- & -- & -- & -- & -- & -- & -- & -- & -. \\
\hline 18 & 636 & 554 & 531 & -- & -- & -- & -- & -- & - & -- & -- & - \\
\hline 19 & 633 & 544 & 536 & -- & -- & -- & -- & -- & -- & -- & -- & -- \\
\hline 20 & 630 & 535 & 545 & -- & -- & -. & -- & -- & -- & -- & -- & -- \\
\hline 21 & 627 & 531 & 542 & -- & -- & -- & -- & -- & -- & -- & -- & -- \\
\hline 22 & 627 & 532 & 541 & -- & -- & -- & -- & -- & -- & - & -- & -- \\
\hline 23 & 627 & 529 & 541 & -- & -- & -- & -- & -- & -- & -- & -- & -- \\
\hline 24 & 626 & 522 & 543 & -- & -- & -- & -- & -- & -- & -- & -- & -- \\
\hline 25 & 622 & 514 & 545 & -- & -- & -- & $-\cdot$ & -- & -- & -- & -- & -- \\
\hline 26 & 617 & 505 & 546 & -- & -- & -- & -- & -- & -- & -- & -- & -- \\
\hline 27 & 611 & 499 & 559 & -- & -- & -- & -- & -- & -- & -- & -- & -- \\
\hline 28 & 607 & 499 & 567 & -- & -- & -- & -- & -- & -- & -- & -- & -- \\
\hline 29 & 605 & -- & 574 & -- & -- & -- & -- & -- & -- & -- & -- & -- \\
\hline 30 & 603 & -- & 574 & -- & -- & .. & -- & -- & -- & -- & -- & -- \\
\hline 31 & 601 & -- & 573 & -- & -- & -- & -- & -- & -- & -- & .. & -- \\
\hline
\end{tabular}


Table 11h. Mean daily specific conductance in ground water at site CRM-23 (map ID \#20), Seminole Well Field, Cedar Rapids, lowa, February 1998 through March 1999

[All values in microsiemens per centimeter at 25 degrees Centigrade; --, value not measured, not recorded, or rejected after data review]

\begin{tabular}{|c|c|c|c|c|c|c|c|c|c|c|c|c|}
\hline Day & Jan & Feb & Mar & Apr & May & Jun & Jul & Aug & Sep & Oct & Nov & Dec \\
\hline & & & & & & 1998 & & & & & & \\
\hline 1 & -- & -- & 504 & 516 & 500 & 494 & 476 & 573 & -- & 509 & 536 & 506 \\
\hline 2 & -- & -- & 493 & 522 & 500 & 495 & 432 & 570 & -- & 509 & 538 & 514 \\
\hline 3 & -- & -- & 493 & 526 & 501 & 508 & 418 & 565 & -- & 512 & 538 & 524 \\
\hline 4 & -- & -- & 496 & 522 & 505 & 513 & 418 & 561 & -- & 516 & 544 & 533 \\
\hline 5 & -. & -- & 500 & 516 & 516 & 509 & 426 & 557 & -. & 520 & 550 & 540 \\
\hline 6 & -- & -- & 502 & 508 & 519 & 506 & 423 & 552 & -- & 512 & 555 & 546 \\
\hline 7 & -- & -- & 500 & 497 & 519 & 508 & 420 & 547 & -- & 511 & 561 & 549 \\
\hline 8 & - & -. & 494 & 487 & 516 & 510 & 421 & 544 & -- & 525 & 560 & 551 \\
\hline 9 & -. & -. & 483 & 482 & 515 & 510 & 428 & 545 & -- & 515 & 559 & 551 \\
\hline 10 & -- & 453 & 487 & 475 & 517 & 521 & 427 & 540 & -- & 521 & 557 & 550 \\
\hline 11 & -- & 453 & 494 & 478 & 519 & 500 & 427 & 532 & -- & 534 & 554 & 550 \\
\hline 12 & -- & 451 & 494 & 485 & 501 & 506 & 430 & 535 & -- & 547 & 549 & 538 \\
\hline 13 & - & 449 & 495 & 491 & 504 & 505 & 437 & 543 & -- & 552 & 513 & 533 \\
\hline 14 & $\ldots$ & 447 & 504 & 489 & 507 & 500 & 443 & 550 & -. & 555 & 485 & 543 \\
\hline 15 & -- & 446 & 511 & 484 & 509 & 494 & 448 & 551 & -- & 567 & 469 & 557 \\
\hline 16 & -- & 445 & 512 & 484 & 505 & 492 & 447 & 545 & -- & 574 & 463 & 573 \\
\hline 17 & -- & 444 & 511 & 484 & 503 & 489 & 445 & -- & -- & 580 & 457 & 587 \\
\hline 18 & -- & 448 & 510 & 487 & 504 & 489 & 444 & -- & - & 579 & 458 & 603 \\
\hline 19 & - & 454 & 510 & 491 & 505 & 489 & 443 & -- & -- & 581 & 457 & 614 \\
\hline 20 & -- & 446 & -- & 494 & 504 & 497 & 462 & -- & -- & 533 & 455 & 617 \\
\hline 21 & -- & 442 & - & 501 & 504 & 507 & 450 & -- & -- & 518 & 454 & 626 \\
\hline 22 & -. & 446 & -- & 504 & 512 & 513 & 452 & -- & -- & 521 & 455 & 640 \\
\hline 23 & - & 479 & -- & 500 & 514 & 518 & 454 & -- & 524 & 518 & 462 & 654 \\
\hline 24 & -. & 505 & 515 & 496 & 524 & 520 & 462 & -- & 524 & 515 & 471 & 667 \\
\hline 25 & -- & 506 & 514 & 497 & 525 & 438 & 482 & -- & 523 & 517 & 477 & 680 \\
\hline 26 & - & 504 & 509 & 496 & 527 & 403 & 501 & -- & 524 & 522 & 485 & 689 \\
\hline 27 & -- & 503 & 499 & 497 & 531 & 406 & 516 & -- & 522 & 525 & 491 & 694 \\
\hline 28 & -- & 504 & 492 & 500 & 532 & 424 & 530 & -- & 518 & 529 & 493 & 693 \\
\hline 29 & -- & -- & 492 & 499 & 516 & 468 & 541 & -- & 514 & 530 & 494 & 693 \\
\hline 30 & -- & -- & 492 & 500 & 502 & 479 & 554 & - & 511 & 534 & 498 & 689 \\
\hline 31 & -- & -- & 501 & -- & 496 & -- & 567 & -- & -- & 534 & -- & 682 \\
\hline
\end{tabular}


Table 11h. Mean daily specific conductance in ground water at site CRM-23 (map ID \#20), Seminole Well Field, Cedar Rapids, lowa, February 1998 through March 1999--Continued

\begin{tabular}{|c|c|c|c|c|c|c|c|c|c|c|c|c|}
\hline Day & Jan & Feb & Mar & Apr & May & Jun & Jul & Aug & Sep & Oct & Nov & Dec \\
\hline & & & & & & 1999 & & & & & & \\
\hline 1 & 675 & 520 & 459 & -- & -- & -- & -- & -- & -- & -- & -- & -- \\
\hline 2 & 669 & 518 & 455 & -- & -- & -- & -- & -- & -- & -- & -- & -- \\
\hline 3 & 663 & 516 & 450 & -- & -- & -- & -- & -- & -- & -- & -- & -- \\
\hline 4 & 658 & 515 & 447 & -- & -- & -- & -- & -- & -- & -- & -- & -- \\
\hline 5 & 654 & 514 & 445 & -- & -- & -- & -- & -- & -- & -- & -- & -- \\
\hline 6 & 654 & 514 & 443 & -- & -- & -- & -- & -- & -- & -- & -- & -- \\
\hline 7 & 669 & 513 & 443 & -- & -- & -- & -- & -- & -- & -- & -- & -- \\
\hline 8 & 703 & 513 & 442 & -- & -- & -- & -. & -. & -- & -- & -- & -- \\
\hline 9 & 726 & 512 & 439 & -- & -- & -- & -- & -- & -- & -- & -- & -- \\
\hline 10 & 746 & 511 & 434 & -- & -- & -- & -- & -- & -- & -- & -- & .- \\
\hline 11 & 750 & 509 & 429 & -- & -- & -- & -- & -- & -- & -- & -- & -- \\
\hline 12 & 732 & 507 & 425 & -- & -- & -- & -- & -- & -- & -- & -- & -- \\
\hline 13 & 702 & 505 & 422 & -- & -- & -- & -- & -- & -- & -- & -- & -- \\
\hline 14 & 676 & 504 & 420 & -- & -- & -- & -- & -- & -- & -- & -- & -- \\
\hline 15 & 653 & 505 & 419 & -- & -- & -- & -- & -- & -- & -- & -- & -- \\
\hline 16 & 633 & 506 & 419 & -- & -- & -- & -- & -- & -- & -- & -- & -- \\
\hline 17 & 616 & 505 & 420 & -- & -- & -- & -- & -- & -- & -- & -- & -- \\
\hline 18 & 604 & 505 & 420 & -- & -- & -- & -- & -- & -- & -- & -- & -- \\
\hline 19 & 596 & 503 & 420 & -- & -- & -- & -- & -- & -- & -- & -- & -- \\
\hline 20 & 590 & 501 & 426 & -- & -- & -- & - & -- & -- & -- & -- & -- \\
\hline 21 & 584 & 499 & 442 & -- & -- & -- & -- & -- & -- & -- & -- & -- \\
\hline 22 & 576 & 495 & 462 & -- & -- & -- & -- & -- & -- & -- & -- & -- \\
\hline 23 & 567 & 492 & 470 & -- & -- & -. & -- & -- & -- & -- & -- & -- \\
\hline 24 & 561 & 489 & 470 & -- & -- & -. & -- & -- & -- & -- & -- & -- \\
\hline 25 & 554 & 484 & 469 & -- & -- & -- & -- & -- & -- & -- & -- & -- \\
\hline 26 & 547 & 478 & 469 & -- & -- & -- & -- & -- & -- & -- & -- & -- \\
\hline 27 & 541 & 471 & 462 & -- & -- & -- & -- & -- & -- & -- & -- & -- \\
\hline 28 & 535 & 464 & 459 & -- & -- & -- & -- & -- & -- & -- & -- & -- \\
\hline 29 & 531 & -- & 459 & -- & -- & -- & - & -- & -- & -. & -- & -- \\
\hline 30 & 528 & -- & 459 & -- & -- & -- & -- & -- & -- & -- & -- & -- \\
\hline 31 & 523 & -- & 466 & -- & -- & -- & -- & -- & -- & -- & -- & -- \\
\hline
\end{tabular}


Table 11i. Mean daily specific conductance in ground water at site CRM-25 (map ID \#22), Seminole Well Field, Cedar Rapids, lowa, February 1998 through February 1999

[All values in microsiemens per centimeter at 25 degrees Centigrade; --, value not measured, not recorded, or rejected after data review]

\begin{tabular}{|c|c|c|c|c|c|c|c|c|c|c|c|c|}
\hline Day & Jan & Feb & Mar & Apr & May & Jun & Jul & Aug & Sep & Oct & Nov & Dec \\
\hline & & & & & & 1998 & & & & & & \\
\hline 1 & -- & -- & 521 & 176 & 470 & 443 & 513 & 533 & 470 & 505 & 499 & 488 \\
\hline 2 & -- & -- & 522 & 149 & 471 & 447 & 514 & 533 & 473 & 509 & 492 & 488 \\
\hline 3 & -- & -- & 521 & 167 & 472 & 449 & 508 & 533 & 476 & 506 & 493 & 483 \\
\hline 4 & -- & -- & 527 & 302 & 473 & 452 & 515 & 532 & 482 & 505 & 495 & 480 \\
\hline 5 & -- & -- & 532 & 363 & 472 & 454 & 510 & 530 & 484 & 497 & 496 & 477 \\
\hline 6 & -- & -- & 539 & 361 & 469 & 458 & 523 & 525 & 485 & 497 & 498 & 481 \\
\hline 7 & -- & -- & 543 & 407 & 468 & 464 & 535 & 517 & 490 & 505 & 498 & 471 \\
\hline 8 & -- & -- & 547 & 422 & 466 & 467 & 538 & 512 & 496 & 499 & 497 & 465 \\
\hline 9 & -- & -- & 534 & 443 & 465 & 461 & 540 & 508 & 500 & 493 & 492 & 462 \\
\hline 10 & -- & -- & 531 & 449 & 463 & 463 & 542 & 504 & 501 & 497 & 493 & 462 \\
\hline 11 & -- & -- & 529 & 454 & 461 & 457 & 537 & 503 & 503 & 501 & 486 & 461 \\
\hline 12 & -- & - & 541 & 457 & 458 & 453 & 535 & 503 & 504 & 503 & 483 & 469 \\
\hline 13 & -- & -- & 455 & 457 & 456 & 378 & 536 & 503 & 503 & 502 & 482 & 476 \\
\hline 14 & -- & 361 & 315 & 456 & 455 & 312 & 537 & 504 & 502 & 501 & 483 & 481 \\
\hline 15 & -- & 361 & 272 & 456 & 453 & 304 & 536 & 503 & 495 & 497 & 484 & 482 \\
\hline 16 & -- & 362 & 262 & 457 & 453 & 389 & 534 & 506 & 486 & 497 & 483 & 483 \\
\hline 17 & -- & 361 & 260 & 455 & 454 & 448 & 533 & 503 & 482 & 493 & 461 & 484 \\
\hline 18 & -- & 359 & 268 & 457 & 454 & 483 & 531 & 497 & 479 & 488 & 483 & 482 \\
\hline 19 & -- & 358 & 275 & 460 & 454 & 503 & 529 & 496 & 478 & 481 & 484 & 478 \\
\hline 20 & -- & 352 & 286 & 459 & 455 & 511 & 528 & 491 & 477 & 475 & 485 & 485 \\
\hline 21 & - & 342 & 291 & 460 & 457 & 520 & 527 & 488 & 479 & 479 & 486 & 483 \\
\hline 22 & -- & 332 & 378 & 462 & 458 & 497 & 527 & 488 & 481 & 479 & 485 & 480 \\
\hline 23 & -- & 324 & 491 & 462 & 460 & 474 & 527 & 489 & 481 & 484 & 487 & 477 \\
\hline 24 & -- & 329 & 468 & 463 & 460 & 476 & 528 & 488 & 480 & 493 & 490 & 479 \\
\hline 25 & -- & 412 & 463 & 465 & 460 & 464 & 529 & 489 & 484 & 497 & 490 & 477 \\
\hline 26 & -- & 501 & 461 & 466 & 460 & 469 & 529 & 485 & 489 & 499 & 491 & 476 \\
\hline 27 & -- & 506 & 460 & 469 & 459 & 494 & 530 & 484 & 494 & 496 & 490 & 471 \\
\hline 28 & -- & 506 & 460 & 468 & 459 & 500 & 530 & 491 & 497 & 497 & 489 & 468 \\
\hline 29 & -- & -- & 454 & 467 & 448 & 509 & 530 & 479 & 492 & 496 & 488 & 467 \\
\hline 30 & -- & -- & 450 & 468 & 439 & 512 & 531 & 474 & 496 & 497 & 488 & 470 \\
\hline 31 & .- & -- & 321 & -- & 442 & -- & 532 & 471 & -- & 502 & -- & 471 \\
\hline
\end{tabular}


Table 11i. Mean daily specific conductance in ground water at site CRM-25 (map ID \#22), Seminole Well Field, Cedar Rapids, lowa, February 1998 through February 1999--Continued

\begin{tabular}{|c|c|c|c|c|c|c|c|c|c|c|c|c|}
\hline Day & Jan & Feb & Mar & Apr & May & Jun & Jul & Aug & Sep & Oct & Nov & $\overline{\mathrm{Dec}}$ \\
\hline & & & & & & 1999 & & & & & & \\
\hline 1 & 469 & 326 & -- & -- & -- & -- & -- & -- & -- & -- & -- & -- \\
\hline 2 & 465 & 337 & -- & -- & -- & -- & -- & -- & -- & -- & -- & -. \\
\hline 3 & 464 & 339 & -- & -- & -- & -- & -- & -- & -- & -- & -- & -. \\
\hline 4 & 464 & 309 & -- & -- & -- & -- & -- & -- & -- & -- & -- & -- \\
\hline 5 & 464 & 346 & -- & -- & -- & -- & -- & -- & -- & -- & -- & -- \\
\hline 6 & 462 & 321 & -- & -- & -- & -- & -- & -- & -- & -- & -- & -- \\
\hline 7 & 453 & 334 & -- & -- & -- & -- & -- & -- & -- & -- & -- & -- \\
\hline 8 & 442 & 336 & -- & -- & -- & -- & -- & -- & -- & -- & -- & -. \\
\hline 9 & 444 & 332 & -- & -- & -- & -- & -- & -- & -- & -- & -- & -- \\
\hline 10 & 437 & 357 & -- & -- & -- & -- & -- & -- & -- & -- & -- & -- \\
\hline 11 & 431 & 396 & -- & -- & -- & -- & -- & -- & -- & -- & -- & -- \\
\hline 12 & 378 & 410 & -- & -- & -- & -- & -- & -- & -- & -- & -- & -- \\
\hline 13 & 330 & 383 & -- & -- & -- & -- & -- & -- & -- & -- & -- & -- \\
\hline 14 & 347 & 363 & -- & -- & -. & -- & -- & -- & -- & -- & -- & -- \\
\hline 15 & 351 & 345 & -- & -- & -- & -- & -- & -- & -- & -- & -- & -- \\
\hline 16 & 325 & 327 & -- & -- & -- & -- & - & -- & -- & -- & -- & -- \\
\hline 17 & 347 & 322 & -- & -- & -- & -- & -- & -- & -- & -- & -- & -- \\
\hline 18 & 319 & 322 & -- & -- & -- & -- & -- & -- & -- & -- & -- & -- \\
\hline 19 & 315 & 319 & -- & -- & -- & -- & -- & -- & -- & -- & -- & -- \\
\hline 20 & 329 & 311 & -- & -- & -- & -- & -- & -- & -- & -- & -- & -- \\
\hline 21 & 328 & 319 & -- & -- & -- & -- & -- & -- & -- & -- & -- & -- \\
\hline 22 & 341 & 330 & -- & -- & -- & -- & -- & -- & -- & -- & -- & -- \\
\hline 23 & 350 & 319 & -- & -- & -- & -- & -- & -- & -- & -- & -- & -- \\
\hline 24 & 329 & 387 & -- & -- & -- & -- & -- & -- & -- & -- & -- & -- \\
\hline 25 & 310 & 431 & -- & -- & -- & -- & - & $-\cdot$ & -- & -- & -- & -- \\
\hline 26 & 326 & 430 & -- & -- & -- & -- & -- & -- & -- & -- & -- & -- \\
\hline 27 & 316 & 425 & -- & -- & -- & -- & -- & -- & -- & -- & -- & -- \\
\hline 28 & 289 & 428 & -- & -- & -- & -- & -- & -- & -- & -- & -- & -- \\
\hline 29 & 339 & - & -- & -- & -- & -- & -- & -- & -- & -- & -- & -- \\
\hline 30 & 313 & -- & -- & -- & -- & -- & -- & -- & -- & -- & -- & -- \\
\hline 31 & 320 & -- & -- & -- & -- & -- & -- & -- & -- & -- & -- & -- \\
\hline
\end{tabular}


Table 11j. Mean daily specific conductance in ground water at site CRM-27 (map ID \#24), Seminole Well Field, Cedar Rapids, lowa, February 1998 through March 1999

[All values in microsiemens per centimeter at 25 degrees Centigrade; --, value not measured, not recorded, or rejected after data review]

\begin{tabular}{|c|c|c|c|c|c|c|c|c|c|c|c|c|}
\hline Day & Jan & Feb & Mar & Apr & May & Jun & Jul & Aug & Sep & Oct & Nov & Dec \\
\hline & & & & & & 1998 & & & & & & \\
\hline 1 & -- & -- & 424 & 292 & 315 & 366 & 396 & 470 & 529 & 513 & 422 & 407 \\
\hline 2 & -- & -- & 416 & 274 & 315 & 368 & 383 & 479 & 520 & 504 & 424 & 408 \\
\hline 3 & -- & -- & 413 & 293 & 315 & 371 & 385 & 485 & 518 & 495 & 441 & 409 \\
\hline 4 & -- & -- & 410 & 308 & 314 & 383 & 390 & 488 & 502 & 493 & 444 & 411 \\
\hline 5 & -- & -- & 407 & 310 & 314 & 396 & 366 & 489 & 493 & 485 & 437 & 412 \\
\hline 6 & -- & -- & 406 & 321 & 314 & 395 & 370 & 490 & 486 & 488 & 431 & 455 \\
\hline 7 & -- & -- & 405 & 332 & 317 & 394 & 371 & 486 & 482 & 470 & 425 & 524 \\
\hline 8 & -- & -- & 402 & 331 & 319 & 401 & 377 & 478 & 481 & 509 & 420 & 495 \\
\hline 9 & -- & -- & 399 & 325 & 318 & 408 & 398 & 483 & 479 & 500 & 447 & 514 \\
\hline 10 & -- & -- & 399 & 323 & 322 & 423 & 418 & 486 & 475 & 478 & 468 & 523 \\
\hline 11 & - & -- & 400 & 323 & 328 & 425 & 428 & 489 & 471 & 452 & 471 & 514 \\
\hline 12 & -- & -- & 405 & 323 & 331 & 433 & 434 & 490 & 470 & 447 & 481 & 470 \\
\hline 13 & -- & -- & 385 & 323 & 333 & 433 & 435 & 492 & 467 & 442 & 483 & 458 \\
\hline 14 & -- & 421 & 372 & 322 & 337 & 411 & 421 & 492 & 473 & 450 & 481 & 451 \\
\hline 15 & -- & 422 & 365 & 320 & 340 & 349 & 409 & 479 & 472 & 439 & 484 & 429 \\
\hline 16 & -- & 423 & 361 & 319 & 345 & 389 & 404 & 474 & 471 & 432 & 474 & 415 \\
\hline 17 & -- & 424 & 353 & 318 & 349 & 373 & 403 & 475 & 471 & 425 & 488 & 410 \\
\hline 18 & -- & 425 & 344 & 318 & 344 & 380 & 405 & 475 & 470 & 501 & 486 & 408 \\
\hline 19 & -- & 424 & 337 & 317 & 340 & 413 & 408 & 472 & 469 & 429 & 470 & 406 \\
\hline 20 & -- & 423 & 331 & 318 & 339 & 436 & 412 & 471 & 468 & 426 & 458 & 406 \\
\hline 21 & -- & 421 & 326 & 318 & 339 & 438 & 418 & 471 & 465 & 424 & 449 & 433 \\
\hline 22 & -- & 418 & 320 & 316 & 346 & 438 & 423 & 471 & 489 & 426 & 444 & 474 \\
\hline 23 & -- & 419 & 312 & 315 & 353 & 438 & 429 & 471 & 544 & 422 & 441 & 425 \\
\hline 24 & -- & 419 & 303 & 314 & 351 & 438 & 436 & 471 & 564 & 415 & 452 & 419 \\
\hline 25 & -. & 419 & 304 & 315 & 358 & 445 & 443 & 507 & 552 & 414 & 458 & 424 \\
\hline 26 & -- & 418 & 305 & 314 & 361 & 364 & 447 & 554 & 562 & 418 & 450 & 431 \\
\hline 27 & -. & 415 & 316 & 314 & 362 & 304 & 452 & 563 & 558 & 423 & 427 & 440 \\
\hline 28 & -- & 416 & 313 & 314 & 363 & 307 & 458 & 558 & 582 & 429 & 411 & 444 \\
\hline 29 & -- & -- & 309 & 314 & 356 & 318 & 464 & 572 & 544 & 432 & 406 & 447 \\
\hline 30 & -. & -- & 302 & 314 & 362 & 335 & 467 & 562 & 526 & 429 & 405 & 453 \\
\hline 31 & -- & -- & 302 & -. & 364 & -- & 467 & 562 & -- & 426 & -- & 462 \\
\hline
\end{tabular}


Table 11j. Mean daily specific conductance in ground water at site CRM-27 (map ID \#24), Seminole Well Field, Cedar Rapids, lowa, February 1998 through March 1999--Continued

\begin{tabular}{|c|c|c|c|c|c|c|c|c|c|c|c|c|}
\hline Day & Jan & Feb & Mar & Apr & May & Jun & Jul & Aug & Sep & Oct & Nov & $\overline{\mathrm{Dec}}$ \\
\hline & & & & & & 1999 & & & & & & \\
\hline 1 & 474 & 477 & 435 & -- & -- & -- & -. & -- & -- & -- & -- & -- \\
\hline 2 & 482 & 475 & -- & -- & -- & -- & -- & -- & -- & -- & -- & -- \\
\hline 3 & 488 & 473 & -- & -- & -- & -- & -- & -- & -- & -- & -- & -. \\
\hline 4 & 493 & 471 & -- & -- & -- & - & -. & - & -- & -- & -- & -- \\
\hline 5 & 493 & 474 & -- & -- & -- & -- & -- & - & -- & -- & -- & -- \\
\hline 6 & 494 & 475 & -- & -- & -- & -- & -- & -- & -- & -- & -- & -- \\
\hline 7 & 496 & 472 & -- & -- & -- & -- & -- & -- & -- & -- & -- & -- \\
\hline 8 & 495 & 468 & -- & -- & -- & -- & -- & -- & -- & -- & -- & -- \\
\hline 9 & 491 & 464 & -- & -. & -- & -- & -- & - & -. & - & -- & -. \\
\hline 10 & 490 & 469 & -- & -. & -- & -- & -- & - & -- & -- & -- & -- \\
\hline 11 & 489 & 462 & -- & -- & -- & -- & -- & - & -- & -- & -- & -- \\
\hline 12 & 489 & 437 & -- & -- & -- & -- & -- & -- & -- & -- & -- & -- \\
\hline 13 & 490 & 440 & -- & -. & -- & -- & -- & -. & -- & -- & -- & -- \\
\hline 14 & 491 & 445 & -- & -- & -- & -- & -- & - & -- & -- & -- & -- \\
\hline 15 & 492 & 444 & -- & -- & -. & -- & -- & -- & -- & -- & -- & -- \\
\hline 16 & 493 & 442 & -- & -- & -- & -- & -- & -- & -- & -- & -- & -- \\
\hline 17 & 494 & 441 & -- & -. & -- & -- & -- & -- & -- & -- & -- & -. \\
\hline 18 & 495 & 441 & -- & -- & -- & -- & -- & - & -- & -- & -- & -- \\
\hline 19 & 494 & 441 & -- & -- & -- & -- & -- & -. & -- & -- & -- & -- \\
\hline 20 & 494 & 441 & -- & -- & -- & -- & -- & -- & -- & -- & -- & -- \\
\hline 21 & 494 & 447 & -- & -- & -- & -- & -- & - & -- & -- & -- & -- \\
\hline 22 & 493 & 447 & -- & -- & -- & -- & -- & -- & -- & -- & -- & -- \\
\hline 23 & 491 & 445 & -- & -- & -- & -- & -- & -- & -- & -- & -- & -- \\
\hline 24 & 491 & 442 & -- & -- & -- & -- & -- & - & -- & -- & -- & -- \\
\hline 25 & 490 & 441 & -- & -- & -- & -- & -- & -- & -- & -- & -- & -- \\
\hline 26 & 489 & 460 & -- & -- & -- & -- & -- & -- & -- & -- & -- & -- \\
\hline 27 & 486 & 464 & -- & -- & - & -- & -- & -- & -- & -- & -- & -- \\
\hline 28 & 484 & 450 & -- & -- & -- & -- & -- & -- & -- & -- & -- & -- \\
\hline 29 & 483 & - & -- & -- & -- & -- & -- & -. & -- & -- & -- & -- \\
\hline 30 & 481 & -- & -- & -- & -- & -- & -- & -- & -- & -- & -- & -- \\
\hline 31 & 479 & -- & -- & -- & - & -- & -- & -- & -- & -- & -. & -- \\
\hline
\end{tabular}


Table 11k. Mean daily specific conducatnce in the Cedar River at site SW-2 (map ID \#32), Seminole Well Field, Cedar Rapids, lowa, April 1996 through March 1999

[All values in microsiemens per centimeter at 25 degrees Centigrade; --, value not measured, not recorded, or rejected after data review]

\begin{tabular}{|c|c|c|c|c|c|c|c|c|c|c|c|c|}
\hline Day & Jan & Feb & Mar & Apr & May & Jun & Jul & Aug & Sep & $\overline{\text { Oct }}$ & Nov & Dec \\
\hline \multicolumn{13}{|c|}{1996} \\
\hline 1 & -- & -- & -- & 394 & 425 & - & 558 & 364 & 370 & 432 & 479 & 496 \\
\hline 2 & -- & -- & -- & 367 & 410 & -- & 579 & 362 & 369 & 453 & 483 & 499 \\
\hline 3 & -- & -- & -- & 347 & 413 & -- & 579 & 363 & 368 & 479 & 490 & -- \\
\hline 4 & -- & -- & -- & 363 & 418 & -- & 582 & 374 & 358 & 510 & 501 & 579 \\
\hline 5 & -- & -- & -. & 392 & 409 & -- & 572 & 386 & 348 & 525 & 507 & 570 \\
\hline 6 & -- & -- & -- & 419 & 406 & -- & 544 & 367 & 343 & 549 & 512 & 560 \\
\hline 7 & -- & -- & -- & 440 & -- & -- & 530 & 347 & 346 & 560 & 503 & 549 \\
\hline 8 & -. & -- & -- & 458 & -- & - & 512 & 339 & 352 & 572 & 493 & 549 \\
\hline 9 & -- & -- & -- & 472 & -. & -- & 494 & 343 & 362 & 583 & 492 & 553 \\
\hline 10 & -- & -- & -- & 484 & -- & -- & 472 & 343 & 373 & 594 & 493 & 545 \\
\hline 11 & $\cdots$ & -- & -- & 494 & -- & -- & 440 & 355 & 387 & 621 & $49 \overline{6}$ & 534 \\
\hline 12 & -- & -- & -- & 505 & -- & -- & 441 & 359 & 379 & 637 & 497 & 526 \\
\hline 13 & -- & -- & -- & 508 & -- & -- & 458 & 351 & 382 & 645 & 499 & 519 \\
\hline 14 & -- & -- & -- & 507 & -- & -- & 428 & 338 & 390 & 638 & 499 & 513 \\
\hline 15 & - & -- & -- & 503 & -- & -. & 411 & 339 & 381 & 625 & 501 & 497 \\
\hline 16 & -- & - & -- & 498 & -- & - & 393 & 344 & 361 & 619 & 509 & 493 \\
\hline 17 & -- & -- & -- & 471 & -- & -- & 407 & 350 & 350 & 599 & 506 & 500 \\
\hline 18 & -- & -- & - & 458 & -- & - & 404 & 362 & 357 & 584 & 511 & 512 \\
\hline 19 & -- & -- & -- & 458 & -- & -- & 424 & 374 & 352 & 549 & 498 & 525 \\
\hline 20 & -- & -- & -- & 464 & -- & -- & 407 & 376 & 356 & 542 & 487 & 540 \\
\hline 21 & - & -- & -- & 462 & -- & -- & 414 & 375 & 364 & 550 & 478 & 544 \\
\hline 22 & -- & -- & -- & 432 & -- & -- & 421 & 356 & 365 & 561 & 477 & 547 \\
\hline 23 & - & -- & -- & 382 & -- & -- & 420 & 364 & 361 & 541 & 488 & 560 \\
\hline 24 & - & -- & -- & 377 & -- & -- & 425 & 371 & 367 & 533 & 491 & 564 \\
\hline 25 & -- & -- & -- & 420 & -- & -- & 391 & 353 & 367 & 564 & 487 & 568 \\
\hline 26 & -- & -- & -- & 443 & -- & -- & 388 & 384 & 378 & 581 & 493 & 573 \\
\hline 27 & -- & -- & -- & 439 & -- & -- & 412 & 404 & 392 & 604 & 505 & 584 \\
\hline 28 & -- & -- & -- & 426 & -- & 588 & 402 & 408 & 414 & 617 & 511 & 586 \\
\hline 29 & -- & -- & -. & 423 & -- & 590 & 365 & 397 & 408 & 622 & 508 & 577 \\
\hline 30 & -- & -- & -- & 439 & -- & 551 & 367 & 380 & 420 & -- & 501 & 573 \\
\hline 31 & -- & -- & -- & -- & -- & -- & 365 & 372 & -- & 503 & -- & 564 \\
\hline
\end{tabular}


Table 11k. Mean daily specific conducatnce in the Cedar River at site SW-2 (map ID \#32), Seminole Well Field, Cedar Rapids, lowa, April 1996 through March 1999--Continued

\begin{tabular}{|c|c|c|c|c|c|c|c|c|c|c|c|c|}
\hline Day & Jan & Feb & Mar & Apr & May & Jun & Jul & Aug & Sep & Oct & Nov & Dec \\
\hline & & & & & & 1997 & & & & & & \\
\hline 1 & 560 & 519 & 325 & -- & 404 & 493 & 375 & 445 & 294 & 296 & 516 & 469 \\
\hline 2 & 549 & 499 & 327 & -- & 408 & 487 & 366 & 465 & 336 & 351 & 512 & 470 \\
\hline 3 & 533 & 505 & -- & -- & 417 & 466 & 350 & 473 & 394 & 347 & 507 & 474 \\
\hline 4 & 507 & 492 & -- & -- & 437 & 447 & 368 & 464 & 385 & 348 & 505 & 481 \\
\hline 5 & 498 & 472 & -- & 471 & 455 & 436 & 384 & 429 & 357 & 346 & 502 & 479 \\
\hline 6 & 477 & 465 & - & 471 & 470 & 428 & 390 & 398 & 352 & 345 & 502 & 492 \\
\hline 7 & 447 & 465 & -- & 451 & 484 & 424 & 386 & 371 & 353 & 355 & 504 & 474 \\
\hline 8 & 400 & 466 & -- & 476 & 484 & 416 & 382 & 374 & 381 & 385 & 505 & 473 \\
\hline 9 & 384 & 478 & -- & 478 & 486 & 415 & 374 & 381 & 397 & 391 & 505 & 472 \\
\hline 10 & 421 & 488 & -- & 485 & 490 & 422 & 365 & 383 & 380 & 398 & 499 & 470 \\
\hline 11 & 458 & 494 & - & 486 & 496 & 416 & 465 & 389 & 317 & 394 & 490 & 480 \\
\hline 12 & 489 & 497 & -- & 489 & 502 & 418 & 512 & 409 & 304 & 395 & 509 & 490 \\
\hline 13 & 514 & 503 & -- & 498 & 506 & 385 & 510 & 414 & 282 & 400 & 514 & 490 \\
\hline 14 & 530 & 512 & -- & 509 & 504 & 371 & 512 & 403 & 305 & 410 & 499 & 481 \\
\hline 15 & 539 & 524 & -- & 507 & 501 & 341 & 515 & 391 & 294 & 421 & 488 & 479 \\
\hline 16 & 545 & 530 & -- & 511 & 495 & 315 & 511 & 371 & 288 & 448 & 478 & -- \\
\hline 17 & 552 & 531 & -- & 513 & 483 & 343 & 459 & 353 & 285 & 451 & 484 & 497 \\
\hline 18 & 558 & 485 & -- & 514 & 471 & 366 & 483 & 359 & 280 & 461 & 487 & 496 \\
\hline 19 & 562 & 235 & -- & 515 & 442 & 346 & 500 & 369 & 334 & 475 & 472 & 495 \\
\hline 20 & 560 & 270 & -- & 514 & 456 & 344 & 514 & 371 & 346 & 487 & 475 & 497 \\
\hline 21 & 557 & 233 & -- & 508 & 436 & 335 & 522 & 366 & 351 & 492 & 474 & 495 \\
\hline 22 & 523 & 208 & -- & 502 & 458 & 341 & 524 & 356 & 344 & 500 & 473 & 491 \\
\hline 23 & 473 & 202 & -- & 486 & 460 & 340 & 530 & 344 & 352 & 507 & 465 & 492 \\
\hline 24 & 501 & 220 & -- & 471 & 454 & 290 & 522 & 342 & 364 & 511 & 462 & 494 \\
\hline 25 & 504 & 259 & -- & 452 & 454 & 314 & 517 & 339 & 358 & 510 & 465 & 510 \\
\hline 26 & 484 & 297 & -- & 432 & 459 & 301 & 511 & 340 & 332 & 504 & 467 & 503 \\
\hline 27 & 488 & 324 & -- & 420 & 466 & 334 & 504 & 351 & 285 & 495 & 470 & 500 \\
\hline 28 & 500 & 343 & -- & 405 & 475 & 353 & 500 & 340 & 261 & 512 & 473 & 503 \\
\hline 29 & 499 & -- & -- & 391 & 492 & 371 & 477 & 320 & 274 & 532 & 478 & 500 \\
\hline 30 & 507 & -- & -- & 388 & 511 & 336 & 464 & 329 & 257 & 516 & 475 & 499 \\
\hline 31 & 519 & -- & -- & -- & 510 & -- & 431 & 323 & -- & 515 & -- & 504 \\
\hline
\end{tabular}


Table 11k. Mean daily specific conducatnce in the Cedar River at site SW-2 (map ID \#32), Seminole Well Field, Cedar Rapids, lowa, April 1996 through March 1999--Continued

\begin{tabular}{|c|c|c|c|c|c|c|c|c|c|c|c|c|}
\hline Day & Jan & Feb & Mar & Apr & May & Jun & Jul & Aug & Sep & Oct & Nov & Dec \\
\hline & & & & & & 1998 & & & & & & \\
\hline 1 & 515 & 475 & 475 & 371 & 436 & 533 & 400 & 315 & -- & 429 & 570 & 580 \\
\hline 2 & 514 & 468 & 487 & 392 & 436 & 552 & 378 & 319 & -. & 442 & 576 & 583 \\
\hline 3 & 500 & 455 & 492 & 417 & 440 & 558 & 371 & 328 & -- & 463 & 570 & 583 \\
\hline 4 & 478 & 456 & 495 & 437 & 484 & 566 & 388 & 327 & -- & 494 & 574 & 583 \\
\hline 5 & 476 & 459 & 503 & 449 & 515 & 542 & 420 & 320 & -- & 477 & 576 & 584 \\
\hline 6 & 464 & 461 & 504 & 458 & 522 & 543 & 435 & 332 & -- & 452 & 577 & 579 \\
\hline 7 & 486 & 452 & 505 & 465 & 519 & 545 & 486 & 330 & -- & 453 & 575 & 571 \\
\hline 8 & 483 & 449 & 497 & 472 & 516 & 538 & 510 & 400 & - & 478 & 575 & 567 \\
\hline 9 & 478 & 449 & 488 & 476 & 513 & 508 & 539 & 424 & -- & 507 & 577 & 564 \\
\hline 10 & 495 & -- & 489 & 476 & 505 & 470 & 537 & 432 & -- & 522 & 569 & 565 \\
\hline 11 & 514 & -- & 490 & 476 & 484 & 482 & 556 & 457 & -- & 534 & 554 & 565 \\
\hline 12 & 525 & -- & 498 & 482 & 458 & 445 & 557 & 464 & -- & 543 & 547 & 568 \\
\hline 13 & 530 & -- & 502 & 487 & 453 & 373 & 564 & 478 & -- & 548 & 550 & 566 \\
\hline 14 & 541 & -- & 498 & 468 & 443 & 355 & 549 & 475 & -- & 546 & 559 & 570 \\
\hline 15 & 543 & 443 & 506 & 439 & 443 & 380 & 526 & 466 & -- & 521 & 565 & 566 \\
\hline 16 & 541 & 460 & 510 & 424 & 442 & 416 & 525 & 512 & -- & 523 & 570 & 566 \\
\hline 17 & 541 & 467 & 509 & 412 & 438 & 414 & 521 & - & -- & 508 & 573 & 566 \\
\hline 18 & 544 & 442 & 501 & 419 & 432 & 442 & 522 & -. & -- & 436 & 579 & 572 \\
\hline 19 & 537 & 396 & 483 & 418 & 429 & 404 & 502 & -- & -- & 466 & 581 & 576 \\
\hline 20 & 528 & 408 & 480 & 415 & 432 & 411 & 465 & -. & -- & 475 & 578 & 572 \\
\hline 21 & 525 & 408 & 481 & 412 & 437 & 374 & 455 & -- & -- & 455 & 577 & 580 \\
\hline 22 & 520 & 382 & 480 & 416 & 429 & 361 & 449 & - & -- & 500 & 582 & 606 \\
\hline 23 & 515 & 398 & 501 & 417 & 436 & 380 & 457 & -- & 420 & 540 & 586 & 628 \\
\hline 24 & 514 & 417 & 518 & 422 & 442 & 369 & 415 & -. & 425 & 574 & 586 & 635 \\
\hline 25 & 510 & 428 & 508 & 427 & 454 & 373 & 387 & -- & 440 & 584 & 587 & 637 \\
\hline 26 & 512 & 448 & 504 & 429 & 478 & 386 & 391 & -- & 447 & 588 & 584 & 641 \\
\hline 27 & 511 & 454 & 496 & 428 & 525 & 405 & 391 & -- & 453 & 589 & 584 & 633 \\
\hline 28 & 502 & 463 & 484 & 429 & 530 & 405 & 330 & -- & 432 & 587 & 583 & 630 \\
\hline 29 & 491 & -- & 480 & 432 & 527 & 423 & 314 & -- & 416 & 581 & 584 & 629 \\
\hline 30 & 486 & -- & 475 & 435 & 535 & 417 & 318 & -- & 416 & 561 & 586 & 645 \\
\hline 31 & 481 & -- & 378 & -. & 532 & -- & 314 & -- & -- & 564 & -- & 649 \\
\hline
\end{tabular}


Table 11k. Mean daily specific conducatnce in the Cedar River at site SW-2 (map ID \#32), Seminole Well Field, Cedar Rapids, lowa, April 1996 through March 1999--Continued

\begin{tabular}{|c|c|c|c|c|c|c|c|c|c|c|c|c|}
\hline Day & Jan & Feb & Mar & Apr & May & Jun & Jul & Aug & Sep & Oct & Nov & $\overline{D e c}$ \\
\hline & & & & & & 1999 & & & & & & \\
\hline 1 & 651 & 557 & -- & -- & -- & -- & -- & -- & -- & -- & -- & -- \\
\hline 2 & 642 & 544 & 579 & -- & -- & -- & -- & -- & -- & -- & -- & -- \\
\hline 3 & 634 & 516 & 571 & -- & -- & -- & -- & -- & -- & -- & -- & .- \\
\hline 4 & 628 & 501 & 517 & -- & -- & -- & -- & -- & -- & -- & -- & -- \\
\hline 5 & 626 & 504 & 517 & -- & -- & -- & -- & -- & -- & -- & -- & -- \\
\hline 6 & 622 & 511 & 536 & -- & -- & -- & -- & -- & -- & -- & -- & -- \\
\hline 7 & 618 & 508 & 555 & -- & -- & -- & -- & -- & -. & -- & -- & -- \\
\hline 8 & 616 & 504 & 558 & -- & -- & -- & -- & -- & -- & -- & -- & -- \\
\hline 9 & 614 & 507 & 561 & -- & -- & -- & -- & -- & -- & -. & -- & -- \\
\hline 10 & 614 & 496 & 563 & -- & -- & -- & -- & -- & -- & -- & -- & -- \\
\hline 11 & 608 & 494 & 572 & -- & -- & -- & -- & -- & -- & -- & -- & -- \\
\hline 12 & 600 & 477 & 583 & -- & -- & -- & -- & -- & -- & -- & -- & -- \\
\hline 13 & 593 & 485 & 575 & -- & -- & -- & -- & -- & -- & -- & -- & -- \\
\hline 14 & 586 & 497 & 574 & -- & -- & -- & -- & -- & -. & -- & -- & -- \\
\hline 15 & 586 & 511 & 579 & -- & -. & -- & -- & -- & -- & -. & -. & -- \\
\hline 16 & 586 & 523 & 578 & -- & -- & -- & -- & -- & -- & -- & -- & -- \\
\hline 17 & 584 & 528 & 579 & -- & -- & -- & -- & -- & -- & -- & -- & -- \\
\hline 18 & 583 & 535 & 578 & -- & -- & -- & -- & -- & -- & -- & -- & -. \\
\hline 19 & 588 & 540 & 580 & -- & -- & -- & -- & -- & -- & -- & -- & -- \\
\hline 20 & 594 & 536 & 576 & -- & -- & -- & -- & -- & -- & -- & -- & -- \\
\hline 21 & 588 & 536 & 535 & -- & -- & -- & -- & -- & -. & -- & -- & -- \\
\hline 22 & 577 & 540 & 521 & -- & -- & -- & -- & -- & -- & -- & -- & -- \\
\hline 23 & 574 & 542 & 524 & -- & -- & -- & -- & -- & -- & -- & -- & -- \\
\hline 24 & 571 & 543 & 534 & -- & -. & -- & -- & -- & -- & -. & -- & -- \\
\hline 25 & 586 & 549 & 541 & -- & -- & -- & -- & -- & -- & -- & -- & -- \\
\hline 26 & 573 & 556 & 543 & -- & -- & -- & -- & -- & -- & -- & -- & -- \\
\hline 27 & 564 & 563 & 549 & -- & -- & -- & -- & -- & -- & -- & -- & -- \\
\hline 28 & 562 & 568 & 554 & -- & -- & -- & -- & -- & -- & -- & -- & -- \\
\hline 29 & 560 & -- & 555 & -. & -- & -- & -- & -- & -- & -- & -- & -- \\
\hline 30 & 566 & -- & 558 & -- & -- & -- & -- & -- & -- & -- & -- & -- \\
\hline 31 & 564 & -- & 556 & -- & -- & -- & -- & -- & -- & -- & -- & -- \\
\hline
\end{tabular}


Table 12a. Mean daily pH in ground water at site CRM-1 (map ID \#1), East Well Field, Cedar Rapids, lowa, April 1996 through June 1997

[All values in standard units; --, value not measured, not recorded, or rejected after data review]

\begin{tabular}{|c|c|c|c|c|c|c|c|c|c|c|c|c|}
\hline Day & Jan & Feb & Mar & Apr & May & Jun & Jul & Aug & Sep & Oct & Nov & $\overline{D e c}$ \\
\hline \multicolumn{13}{|c|}{1996} \\
\hline 1 & -. & -- & -- & 7.8 & 7.6 & -. & 7.6 & 7.6 & -- & -- & -- & -- \\
\hline 2 & -- & - & -- & 7.8 & 7.6 & -. & 7.6 & 7.6 & - & -- & -- & -. \\
\hline 3 & -- & -- & -. & 7.8 & 7.7 & -- & 7.6 & 7.6 & -- & -- & -- & -- \\
\hline 4 & -- & -- & -- & 7.9 & 7.7 & -- & 7.6 & 7.6 & -- & -- & -- & 7.2 \\
\hline 5 & -- & -- & -- & 7.9 & 7.7 & -- & 7.6 & 7.6 & -- & -- & -- & 7.2 \\
\hline 6 & -. & -- & -- & 7.9 & 7.7 & -- & 7.7 & 7.6 & -- & -- & -- & 7.2 \\
\hline 7 & -- & -- & -- & 7.9 & 7.7 & -- & 7.7 & 7.6 & -- & -- & -- & 7.2 \\
\hline 8 & -- & -- & -- & 7.9 & 7.7 & -- & 7.7 & 7.6 & -- & -- & -- & 7.2 \\
\hline 9 & -- & -- & -- & 7.9 & -- & -- & 7.7 & 7.6 & -- & -- & -- & 7.2 \\
\hline 10 & -- & -- & -. & 7.9 & -- & -- & 7.7 & 7.7 & -- & -- & -- & 7.2 \\
\hline 11 & -- & -- & -- & 7.9 & -- & -. & 7.7 & 7.7 & -- & -- & -- & 7.2 \\
\hline 12 & -- & -. & -- & 7.9 & -- & -- & 7.6 & 7.7 & -- & -- & -- & 7.3 \\
\hline 13 & -- & -- & -- & 7.9 & -- & -- & 7.6 & 7.7 & -- & -- & -- & 7.3 \\
\hline 14 & -- & -- & -- & 7.8 & -- & -- & 7.6 & 7.7 & 7.5 & -- & -- & 7.3 \\
\hline 15 & - & -- & -- & 7.8 & -- & -- & 7.7 & 7.8 & 7.5 & -- & -- & 7.3 \\
\hline 16 & -- & -- & -- & 7.8 & - & - & 7.6 & 7.8 & 7.5 & -- & -- & 7.3 \\
\hline 17 & - & -- & -- & 7.8 & -- & -- & 7.6 & 7.8 & 7.6 & -- & -- & 7.3 \\
\hline 18 & -- & -- & -- & 7.8 & - & -- & 7.7 & 7.8 & 7.6 & -- & -- & 7.3 \\
\hline 19 & -- & -- & -- & 7.8 & -- & -- & 7.7 & 7.7 & 7.6 & -- & -- & 7.4 \\
\hline 20 & -- & - & -. & 7.7 & -- & -- & 7.7 & 7.7 & 7.6 & -- & -- & 7.3 \\
\hline 21 & -- & -. & -. & 7.7 & -- & -- & 7.7 & 7.8 & 7.6 & -- & -- & 7.3 \\
\hline 22 & -. & -- & -- & 7.7 & -- & -- & 7.7 & 7.8 & 7.6 & -- & -- & 7.2 \\
\hline 23 & -. & -- & -- & 7.6 & -- & -- & 7.8 & 7.7 & 7.6 & -- & -- & 7.2 \\
\hline 24 & -. & -- & -- & 7.6 & -- & -- & 7.8 & 7.7 & 7.6 & -- & -- & 7.2 \\
\hline 25 & -- & -- & -- & 7.6 & -. & -- & 7.8 & 7.7 & 7.7 & -- & -- & 7.2 \\
\hline 26 & -- & -- & -- & 7.6 & - & -- & 7.8 & -- & 7.7 & -- & -- & 7.3 \\
\hline 27 & -- & -- & -- & 7.6 & -- & -- & 7.7 & -- & 7.7 & -- & -- & 7.3 \\
\hline 28 & -- & -- & -- & 7.6 & -- & 7.5 & 7.7 & -- & -- & -- & -- & 7.5 \\
\hline 29 & -- & -- & -- & 7.6 & -- & 7.5 & 7.7 & -- & -- & -- & -- & 7.4 \\
\hline 30 & -- & -- & -- & 7.6 & -- & 7.6 & 7.6 & -- & -- & -- & -- & 7.4 \\
\hline 31 & -- & -- & -- & -- & -- & -- & 7.5 & -- & -- & -. & -- & 7.2 \\
\hline
\end{tabular}


Table 12a. Mean daily pH in ground water at site CRM-1 (map ID \#1), East Well Field, Cedar Rapids, lowa, April 1996 through June 1997--.Continued

\begin{tabular}{|c|c|c|c|c|c|c|c|c|c|c|c|c|}
\hline Day & Jan & Feb & Mar & Apr & May & Jun & Jul & Aug & Sep & Oct & Nov & Dec \\
\hline \multicolumn{13}{|c|}{1997} \\
\hline 1 & 7.0 & 7.5 & 7.2 & -- & -- & 7.3 & -- & -- & -- & -- & -- & -- \\
\hline 2 & 7.0 & 7.4 & 7.2 & -- & -. & 7.3 & -- & -- & -- & -. & -- & -- \\
\hline 3 & 7.1 & 7.3 & 7.2 & -- & -- & 7.4 & -- & -. & -. & -- & -. & -- \\
\hline 4 & 7.1 & 7.3 & 7.3 & -- & -- & 7.4 & -- & -- & -- & -- & -- & -- \\
\hline 5 & 7.2 & 7.3 & 7.3 & -- & -- & 7.5 & -- & -- & -- & -- & -- & -- \\
\hline 6 & 7.2 & 7.3 & 7.3 & -- & -. & 7.5 & -- & -- & -- & -- & -- & -- \\
\hline 7 & 7.2 & 7.0 & 7.3 & -- & -- & 7.5 & -- & -- & -- & -- & -- & -- \\
\hline 8 & 7.3 & 7.1 & 7.3 & -. & -. & 7.5 & -- & -. & -- & -- & -- & -- \\
\hline 9 & 7.2 & 7.1 & 7.4 & -- & -- & 7.5 & -- & -- & -- & -- & -- & -- \\
\hline 10 & 7.2 & 7.2 & 7.4 & -- & -- & 7.5 & -- & -- & -- & -- & -- & -- \\
\hline 11 & 7.2 & 7.2 & 7.4 & -- & -- & 7.5 & -- & -- & -- & -- & -- & -- \\
\hline 12 & 7.2 & 7.3 & 7.4 & -- & -- & 7.5 & -- & -- & -- & -- & -- & -- \\
\hline 13 & 7.2 & 7.3 & 7.4 & -- & .. & -- & -- & -- & -- & -- & -- & -- \\
\hline 14 & 7.2 & 7.3 & 7.5 & -- & -- & -- & -- & -- & -- & -- & -- & -- \\
\hline 15 & 7.3 & 7.3 & 7.5 & -- & -- & -- & -- & -- & -- & -- & -- & -- \\
\hline 16 & 7.4 & 7.3 & 7.5 & -- & -- & -- & -- & -- & -- & -- & -- & -- \\
\hline 17 & 7.4 & 7.1 & 7.5 & -- & -- & -- & -- & -- & -- & -- & -- & -- \\
\hline 18 & 7.3 & 7.0 & 7.5 & -- & -- & -- & -- & -- & -- & -- & -- & -- \\
\hline 19 & 7.2 & 7.0 & 7.5 & -- & -- & -- & -- & -- & -- & -- & -- & -- \\
\hline 20 & 7.3 & 7.0 & 7.6 & -. & -- & -- & -. & -- & -- & -- & -- & -- \\
\hline 21 & 7.4 & 7.0 & 7.6 & -- & -- & -- & -- & -- & -- & -- & -- & -- \\
\hline 22 & 7.4 & 7.1 & 7.6 & -- & -- & -- & -- & -- & -- & -- & -- & -- \\
\hline 23 & 7.4 & 7.1 & 7.6 & -- & 6.7 & -- & -- & -- & -- & -- & -- & -- \\
\hline 24 & 7.2 & 7.1 & 7.6 & -- & 6.7 & -- & -- & -- & -- & -- & -- & -- \\
\hline 25 & 7.3 & 7.1 & 7.6 & -. & 6.8 & -- & -- & -- & -- & -- & -- & -- \\
\hline 26 & 7.5 & 7.1 & 7.5 & -- & 6.8 & -- & -- & -- & -- & -- & -- & -- \\
\hline 27 & 7.5 & 7.1 & 7.5 & -- & 6.9 & -- & -- & -- & -- & -- & -- & -- \\
\hline 28 & 7.5 & 7.2 & 7.5 & -- & 7.2 & -- & -- & -- & -- & -- & -- & -- \\
\hline 29 & 7.5 & -- & 7.5 & -- & 7.1 & -- & -- & -- & -- & -- & -- & -- \\
\hline 30 & 7.5 & -- & -. & -- & 7.2 & -- & -- & -. & -- & -- & -- & -- \\
\hline 31 & 7.5 & -- & -- & -- & 7.3 & -- & -- & -- & -- & -- & -- & -- \\
\hline
\end{tabular}


Table 12b. Mean daily pH in ground water at site CRM-2 (map ID \#2), West Well Field, Cedar Rapids, lowa, April 1996 through July 1997

[All values in standard units; --, value not measured, not recorded, or rejected after data review]

\begin{tabular}{|c|c|c|c|c|c|c|c|c|c|c|c|c|}
\hline Day & Jan & Feb & Mar & Apr & May & Jun & Jul & Aug & Sep & Oct & Nov & Dec \\
\hline \multicolumn{13}{|c|}{1996} \\
\hline 1 & .- & -- & -- & 6.5 & 6.7 & -- & -- & 7.4 & 7.7 & 7.2 & 7.6 & 7.8 \\
\hline 2 & -- & -- & -- & 6.5 & 6.7 & -- & -- & 7.4 & 7.7 & 7.2 & 7.6 & 7.9 \\
\hline 3 & - & -- & -- & 6.6 & 6.7 & -- & -- & 7.4 & 7.7 & 7.2 & 7.6 & 7.9 \\
\hline 4 & -- & -- & -- & 6.6 & 6.7 & -- & -- & 7.4 & 7.7 & 7.3 & 7.6 & 7.6 \\
\hline 5 & -- & -- & -- & 6.6 & 6.7 & -- & -- & 7.4 & 7.7 & 7.3 & 7.7 & 7.4 \\
\hline 6 & -. & -- & -- & 6.6 & 6.7 & -- & -- & 7.4 & 7.7 & 7.3 & 7.7 & 7.4 \\
\hline 7 & -- & -- & -- & 6.6 & 6.7 & - & -- & 7.4 & 7.7 & 7.3 & 7.7 & 7.4 \\
\hline 8 & -- & -- & -- & 6.6 & 6.7 & -- & -- & 7.4 & 7.7 & 7.3 & 7.7 & 7.4 \\
\hline 9 & -- & -- & -- & 6.6 & -- & -- & -- & 7.4 & 7.7 & 7.3 & 7.7 & 7.4 \\
\hline 10 & -- & -- & -- & 6.6 & -. & -- & -- & 7.4 & 7.7 & 7.3 & 7.7 & 7.5 \\
\hline 11 & -- & -- & -- & 6.6 & - & -- & -- & 7.4 & 7.7 & 7.3 & 7.7 & 7.4 \\
\hline 12 & -- & -. & -- & 6.6 & -- & -- & -- & 7.4 & 7.7 & 7.3 & 7.7 & 7.4 \\
\hline 13 & -- & -- & -- & 6.7 & -- & -- & -- & 7.5 & 7.5 & 7.3 & 7.7 & 7.4 \\
\hline 14 & -- & -- & -- & 6.7 & -- & -- & - & 7.5 & 7.1 & 7.3 & 7.7 & 7.5 \\
\hline 15 & -- & -- & -- & 6.7 & -- & -- & -- & 7.5 & 7.2 & 7.3 & 7.7 & 7.5 \\
\hline 16 & -- & -- & -- & 6.7 & -- & -- & -- & 7.5 & 7.2 & 7.3 & 7.8 & 7.5 \\
\hline 17 & -- & -- & -- & 6.7 & -- & -- & -- & 7.5 & 7.2 & 7.3 & 7.8 & 7.5 \\
\hline 18 & -- & -- & -- & 6.7 & -. & -- & -- & 7.5 & 7.2 & 7.3 & 7.8 & 7.5 \\
\hline 19 & -- & -- & -- & 6.7 & -- & -- & -- & 7.5 & 7.2 & 7.3 & 7.8 & 7.5 \\
\hline 20 & -- & -- & -- & 6.7 & -- & -- & -- & 7.5 & 7.2 & 7.3 & 7.8 & 7.5 \\
\hline 21 & -- & -- & -- & 6.7 & -- & -- & -- & 7.5 & 7.2 & 7.3 & 7.8 & 7.5 \\
\hline 22 & -- & -- & -- & 6.7 & -- & - & -- & 7.5 & 7.2 & 7.3 & 7.8 & 7.5 \\
\hline 23 & -- & -- & -- & 6.7 & -- & -- & -- & 7.5 & 7.2 & 7.4 & 7.8 & 7.5 \\
\hline 24 & -- & -- & -- & 6.7 & -. & -- & -- & 7.5 & 7.2 & 7.4 & 7.8 & 7.5 \\
\hline 25 & -- & -- & -- & 6.6 & -- & -- & -- & 7.5 & 7.2 & 7.4 & 7.8 & 7.5 \\
\hline 26 & -- & -- & -- & 6.6 & -- & - & -- & 7.5 & 7.2 & 7.4 & 7.8 & 7.6 \\
\hline 27 & -- & -- & -- & 6.6 & -- & -- & -- & 7.5 & 7.2 & 7.4 & 7.8 & 7.6 \\
\hline 28 & -- & -- & -- & 6,6 & -- & -- & -- & 7.6 & 7.2 & 7.4 & 7.8 & 7.6 \\
\hline 29 & -. & -- & -- & 6.7 & -- & -- & -- & 7.6 & 7.2 & 7.4 & 7.8 & 7.6 \\
\hline 30 & -- & -- & -- & 6.7 & -- & -- & -- & 7.7 & 7.2 & 7.5 & 7.8 & 7.6 \\
\hline 31 & $\ldots$ & -- & -- & -- & -- & -- & 7.3 & 7.7 & -- & 7.6 & -- & 7.4 \\
\hline
\end{tabular}


Table 12b. Mean daily pH in ground water at site CRM-2 (map ID \#2), West Well Field, Cedar Rapids, lowa, April 1996 through July 1997--Continued

\begin{tabular}{|c|c|c|c|c|c|c|c|c|c|c|c|c|}
\hline Day & Jan & Feb & Mar & Apr & May & Jun & Jul & Aug & Sep & Oct & Nov & Dec \\
\hline \multicolumn{13}{|c|}{1997} \\
\hline 1 & 7.3 & 7.6 & 7.6 & -. & 7.4 & 8.1 & 7.1 & -- & -- & -- & -- & -- \\
\hline 2 & 7.3 & 7.6 & 7.6 & -- & 7.4 & 8.2 & 7.1 & -- & -- & -. & -. & -- \\
\hline 3 & 7.4 & 7.6 & 7.6 & -- & 7.4 & 8.2 & 7.1 & -- & -- & -- & -- & -- \\
\hline 4 & 7.4 & 7.6 & 7.6 & -- & 7.4 & 8.3 & 7.1 & -- & -- & -- & -- & -- \\
\hline 5 & 7.4 & 7.6 & 7.6 & 7.3 & 7.4 & 8.3 & 7.1 & -- & -- & -- & -- & -- \\
\hline 6 & 7.4 & 7.6 & 7.6 & 7.3 & 7.4 & 8.3 & 7.1 & -- & -. & -- & -. & -. \\
\hline 7 & 7.4 & 7.5 & 7.7 & 7.3 & 7.4 & 8.4 & 7.1 & -- & -- & -- & -- & -- \\
\hline 8 & 7.4 & 7.4 & 7.7 & 7.3 & 7.4 & 8.4 & 7.2 & -- & -. & -- & - & -- \\
\hline 9 & 7.4 & 7.5 & 7.6 & 7.3 & 7.4 & 8.4 & 7.2 & -- & -- & -- & -- & -- \\
\hline 10 & 7.5 & 7.5 & 7.6 & 7.3 & -- & 8.5 & 7.2 & -- & -- & -- & -- & -- \\
\hline 11 & 7.5 & 7.5 & 7.6 & 7.3 & -- & 8.5 & -- & -- & -- & - & -- & -- \\
\hline 12 & 7.5 & 7.5 & 7.6 & 7.3 & -- & 8.5 & -- & -- & -- & -- & -- & -- \\
\hline 13 & 7.5 & 7.5 & 7.6 & 7.3 & -- & - & -- & -- & -- & -- & -- & -- \\
\hline 14 & 7.5 & 7.5 & 7.7 & 7.4 & -- & 6.9 & -- & -- & -- & -- & -- & -- \\
\hline 15 & 7.5 & 7.5 & 7.7 & 7.4 & -- & 6.9 & -- & -- & -- & -- & -- & -- \\
\hline 16 & 7.5 & 7.6 & 7.7 & 7.4 & -- & 7.0 & -- & -- & -- & -- & - & -- \\
\hline 17 & 7.5 & 7.6 & 7.7 & 7.4 & -- & 7.0 & -- & -- & -- & -- & -- & -- \\
\hline 18 & 7.6 & 7.6 & 7.7 & 7.4 & -- & 7.0 & -- & -- & -- & -- & -- & -- \\
\hline 19 & 7.6 & 7.6 & 7.6 & 7.4 & -- & 7.0 & -- & -- & -- & -- & -- & -- \\
\hline 20 & 7.6 & 7.6 & 7.7 & 7.4 & -- & 7.0 & -- & -- & -- & -- & -- & -- \\
\hline 21 & 7.6 & 7.6 & 7.5 & 7.4 & -- & 7.0 & -- & -- & -- & -- & -- & -- \\
\hline 22 & 7.6 & 7.5 & 7.5 & 7.4 & -- & 7.0 & -- & -- & -- & -- & -- & -- \\
\hline 23 & 7.6 & 7.5 & 7.5 & 7.4 & 7.5 & 7.0 & -- & -- & -- & -- & -- & -- \\
\hline 24 & 7.6 & 7.5 & 7.5 & 7.4 & 7.5 & 7.0 & -- & -- & -- & -- & -- & -- \\
\hline 25 & 7.6 & 7.6 & 7.6 & 7.4 & 7.5 & 7.1 & -- & -- & -- & -- & -- & -- \\
\hline 26 & 7.6 & 7.6 & 7.6 & 7.4 & 7.5 & 7.1 & -- & -- & -- & -- & -- & -- \\
\hline 27 & 7.6 & 7.6 & 7.6 & 7.4 & 7.5 & 7.1 & -- & -- & -- & -- & -- & -- \\
\hline 28 & 7.6 & 7.6 & 7.6 & 7.4 & 7.5 & 7.1 & -- & -- & -- & -- & -- & -- \\
\hline 29 & 7.6 & -- & 7.7 & 7.4 & 7.5 & 7.1 & -- & -- & -- & -- & -- & -- \\
\hline 30 & 7.6 & -- & -- & 7.4 & 7.7 & 7.1 & -- & -- & -- & -- & - & -- \\
\hline 31 & 7.6 & -- & -- & -- & 8.0 & -- & -- & -- & - & -- & -- & -- \\
\hline
\end{tabular}


Table 12c. Mean daily pH in ground water at site CRM-11 (map ID \#9), West Well Field, Cedar Rapids, lowa, April 1996 through June 1997

[All values in standard units; --, value not measured, not recorded, or rejected after data review]

\begin{tabular}{|c|c|c|c|c|c|c|c|c|c|c|c|c|}
\hline Day & Jan & Feb & Mar & Apr & May & Jun & Jul & Aug & Sep & Oct & Nov & Dec \\
\hline & & & & & & 1996 & & & & & & \\
\hline 1 & -- & -- & -- & 7.8 & 7.8 & -- & 7.9 & 7.3 & 7.5 & 7.4 & 7.4 & 7.6 \\
\hline 2 & -. & -- & -- & 7.8 & 7.8 & -- & 8.0 & 7.3 & 7.5 & 7.4 & 7.4 & 7.6 \\
\hline 3 & -- & -- & -- & 7.8 & 7.8 & -- & 8.0 & 7.3 & 7.5 & 7.4 & 7.4 & 7.6 \\
\hline 4 & -- & -- & -- & 7.8 & 7.8 & -- & 8.0 & 7.3 & 7.5 & 7.4 & 7.5 & 7.5 \\
\hline 5 & -. & - & -- & 7.7 & 7.8 & - & 8.0 & 7.3 & 7.5 & 7.4 & 7.5 & 7.4 \\
\hline 6 & -- & -- & -- & 7.8 & 7.8 & -. & 8.0 & 7.3 & 7.5 & 7.4 & 7.5 & 7.4 \\
\hline 7 & -. & -- & -- & 7.7 & 7.8 & -- & 8.0 & 7.3 & 7.5 & 7.4 & 7.5 & 7.4 \\
\hline 8 & -- & .- & -. & 7.7 & 7.8 & -- & 8.1 & 7.3 & 7.5 & 7.4 & 7.5 & 7.4 \\
\hline 9 & -. & -- & - & 7.8 & -- & -- & 8.1 & 7.3 & 7.5 & 7.4 & 7.5 & 7.5 \\
\hline 10 & -. & -- & -- & 7.8 & -- & -- & 8.1 & 7.3 & 7.5 & 7.5 & 7.5 & 7.5 \\
\hline 11 & -. & -- & -. & 7.8 & -- & -- & 8.1 & 7.3 & 7.5 & 7.5 & 7.5 & 7.5 \\
\hline 12 & -- & -- & -- & 7.8 & -- & -- & 8.1 & 7.3 & 7.5 & 7.5 & 7.5 & 7.5 \\
\hline 13 & -- & -- & -- & 7.8 & -- & -- & 8.1 & 7.3 & 7.4 & 7.5 & 7.5 & 7.5 \\
\hline 14 & -. & -- & -- & 7.8 & -- & -- & 8.1 & 7.3 & 7.3 & 7.5 & 7.5 & 7.5 \\
\hline 15 & -. & -- & -- & 7.8 & -. & -- & 8.2 & 7.3 & 7.3 & 7.5 & 7.5 & 7.5 \\
\hline 16 & -- & -- & -- & 7.8 & -- & -- & 8.2 & 7.3 & 7.3 & 7.5 & 7.5 & 7.5 \\
\hline 17 & -- & -- & -- & 7.8 & - & -- & 8.2 & 7.4 & 7.3 & 7.5 & 7.5 & 7.5 \\
\hline 18 & -. & -- & - & 7.8 & -- & -- & 8.2 & 7.4 & 7.3 & 7.5 & 7.5 & 7.5 \\
\hline 19 & -- & -- & -- & 7.8 & -. & -- & 8.2 & 7.4 & 7.3 & 7.5 & 7.5 & 7.5 \\
\hline 20 & -- & -- & -- & 7.8 & -- & -- & 8.2 & 7.4 & 7.3 & 7.5 & 7.6 & 7.5 \\
\hline 21 & -- & -- & -- & 7.8 & -- & -- & 8.2 & 7.4 & 7.3 & 7.5 & 7.6 & 7.5 \\
\hline 22 & -- & -- & -- & 7.8 & -. & -- & 8.3 & 7.4 & 7.4 & 7.5 & 7.6 & 7.5 \\
\hline 23 & -- & -- & -- & -- & -. & -- & 8.3 & 7.4 & 7.4 & 7.5 & 7.6 & 7.5 \\
\hline 24 & -- & -- & -- & 7.7 & -. & -- & 8.3 & 7.4 & 7.4 & 7.5 & 7.6 & 7.5 \\
\hline 25 & -- & -- & -- & 7.7 & -- & -- & 8.3 & 7.4 & 7.4 & 7.5 & 7.6 & 7.5 \\
\hline 26 & -- & -- & -- & 7.7 & -. & -- & 8.3 & 7.4 & 7.4 & 7.5 & 7.6 & 7.5 \\
\hline 27 & -- & -- & -- & 7.7 & - & -- & 8.3 & 7.4 & 7.4 & 7.5 & 7.6 & 7.5 \\
\hline 28 & -. & -- & -. & 7.7 & -- & 7.9 & 8.3 & 7.4 & 7.4 & 7.5 & 7.6 & 7.5 \\
\hline 29 & -- & -- & -- & 7.8 & - & 7.9 & 8.3 & 7.4 & 7.4 & 7.6 & 7.6 & 7.6 \\
\hline 30 & -. & -- & -. & 7.7 & -. & 7.9 & 7.9 & 7.5 & 7.4 & 7.5 & 7.6 & 7.6 \\
\hline 31 & -- & .. & -. & -- & -. & -- & 7.3 & 7.5 & -. & 7.4 & -- & 7.5 \\
\hline
\end{tabular}


Table 12c. Mean daily pH in ground water at site CRM-11 (map ID \#9), West Well Field, Cedar Rapids, lowa, April 1996 through June 1997---Continued

\begin{tabular}{|c|c|c|c|c|c|c|c|c|c|c|c|c|}
\hline Day & Jan & Feb & Mar & Apr & May & Jun & Jul & Aug & Sep & Oct & Nov & Dec \\
\hline \multicolumn{13}{|c|}{1997} \\
\hline 1 & 7.5 & 7.6 & 7.4 & -- & 7.6 & 7.4 & -- & -- & -- & -- & -- & -- \\
\hline 2 & 7.5 & 7.7 & 7.4 & -- & 7.6 & 7.6 & -- & -- & -- & -- & -- & -- \\
\hline 3 & 7.5 & 7.7 & 7.4 & -- & 7.6 & 7.8 & -- & -- & -- & -- & -- & -- \\
\hline 4 & 7.5 & 7.7 & 7.4 & -- & 7.6 & 7.6 & -- & -- & -- & -- & -- & -- \\
\hline 5 & 7.5 & 7.7 & 7.4 & 7.4 & 7.6 & 7.5 & - & -- & -- & -- & -- & -- \\
\hline 6 & 7.5 & 7.7 & 7.4 & 7.4 & 7.6 & 7.5 & - & -- & -- & -- & -- & -- \\
\hline 7 & 7.5 & 7.4 & 7.4 & 7.4 & 7.6 & 7.5 & -- & -- & -- & -- & -- & -- \\
\hline 8 & 7.5 & 7.2 & 7.4 & 7.4 & 7.6 & 7.5 & -- & -- & -- & -- & -- & -- \\
\hline 9 & 7.5 & 7.3 & 7.4 & 7.4 & 7.7 & 7.5 & -- & -- & -- & -- & -- & -- \\
\hline 10 & 7.6 & 7.3 & 7.4 & 7.4 & -- & 7.5 & - & -- & -- & -- & -- & -- \\
\hline 11 & 7.6 & 7.4 & 7.4 & 7.4 & -- & 7.5 & -- & -- & -. & -- & -- & -- \\
\hline 12 & 7.6 & 7.4 & 7.4 & 7.4 & -- & 7.5 & -- & -- & -- & -. & -- & -- \\
\hline 13 & 7.6 & 7.4 & 7.4 & 7.4 & -. & -- & -- & -. & -. & -- & -- & -- \\
\hline 14 & 7.6 & 7.4 & 7.4 & 7.5 & -- & -- & -- & -- & -- & -- & -- & -- \\
\hline 15 & 7.6 & 7.4 & 7.4 & 7.5 & -- & -- & -- & -. & -- & -- & -- & -. \\
\hline 16 & 7.7 & 7.4 & 7.5 & 7.5 & -- & -- & -- & -- & -- & -- & -- & -- \\
\hline 17 & 7.6 & 7.4 & 7.5 & 7.5 & -- & -- & -- & -- & -- & -- & -- & -- \\
\hline 18 & 7.7 & 7.4 & 7.5 & 7.5 & -- & -- & -- & -- & -- & -- & -- & -- \\
\hline 19 & 7.7 & 7.4 & 7.5 & 7.5 & -- & -- & -. & -- & -- & -- & -- & -- \\
\hline 20 & 7.7 & 7.3 & 7.5 & 7.5 & -. & -- & -- & -- & -- & -- & -. & -- \\
\hline 21 & 7.7 & 7.3 & 7.5 & 7.5 & -- & -- & -. & -- & -- & -- & -- & -- \\
\hline 22 & 7.7 & 7.3 & 7.5 & 7.5 & -- & -- & -- & -- & -- & -- & -- & -- \\
\hline 23 & 7.7 & 7.3 & 7.5 & 7.5 & 7.4 & -- & -- & -- & -- & -- & -- & -- \\
\hline 24 & 7.7 & 7.3 & 7.5 & 7.5 & 7.4 & -- & -- & -- & -- & -- & -- & -- \\
\hline 25 & 7.7 & 7.3 & 7.5 & 7.5 & 7.4 & -- & -- & -- & -- & -- & -- & -- \\
\hline 26 & 7.7 & 7.3 & 7.5 & 7.5 & 7.4 & -- & -. & -- & -. & -- & -. & -- \\
\hline 27 & 7.6 & 7.3 & 7.5 & 7.5 & 7.4 & -- & -- & -- & -- & -- & -- & -- \\
\hline 28 & 7.6 & 7.3 & 7.5 & 7.5 & 7.4 & -- & -- & -- & -- & -- & -- & -- \\
\hline 29 & 7.6 & -- & 7.5 & 7.5 & 7.4 & -- & -- & -- & -- & -- & -- & -- \\
\hline 30 & 7.7 & -- & -- & 7.6 & 7.4 & -- & -. & -- & -- & -- & -- & -- \\
\hline 31 & 7.6 & -- & -- & -- & 7.4 & -- & -- & -. & -- & -- & -- & -- \\
\hline
\end{tabular}


Table 12d. Mean daily pH in ground water at site CRM-12 (map ID \#10), East Well Field, Cedar Rapids, lowa, April 1996 through July 1997

[All values in standard units; --, value not measured, not recorded, or rejected after data review]

\begin{tabular}{|c|c|c|c|c|c|c|c|c|c|c|c|c|}
\hline Day & Jan & Feb & Mar & Apr & May & Jun & Jul & Aug & Sep & Oct & Nov & Dec \\
\hline & & & & & & 1996 & & & & & & \\
\hline 1 & -- & -- & -- & 7.5 & 7.8 & -- & 7.1 & 7.2 & 7.5 & 7.2 & 7.1 & 7.3 \\
\hline 2 & -- & -- & -- & 7.5 & 7.8 & -- & 7.2 & 7.2 & 7.5 & 7.3 & 7.1 & 7.4 \\
\hline 3 & -- & .. & .. & 7.6 & 7.8 & -. & 7.2 & 7.3 & 7.5 & 7.3 & 7.2 & 7.1 \\
\hline 4 & -- & -- & -- & 7.6 & 7.8 & -- & 7.2 & 7.3 & 7.5 & 7.3 & 7.2 & 7.0 \\
\hline 5 & -. & -- & -- & 7.6 & 7.8 & -. & 7.2 & 7.3 & 7.5 & 7.3 & 7.2 & 7.3 \\
\hline 6 & -- & -. & -- & 7.6 & 7.8 & -- & 7.2 & 7.3 & 7.5 & 7.3 & 7.2 & 7.4 \\
\hline 7 & -. & -- & -- & 7.6 & 7.8 & -- & 7.2 & 7.3 & 7.5 & 7.3 & 7.2 & 7.4 \\
\hline 8 & -- & -- & -. & 7.6 & 7.8 & -- & 7.3 & 7.3 & 7.5 & 7.3 & 7.2 & 7.5 \\
\hline 9 & -- & -- & -- & 7.6 & -- & -- & 7.3 & 7.3 & 7.5 & 7.3 & 7.2 & 7.6 \\
\hline 10 & -- & -. & -- & 7.6 & -- & -- & 7.3 & 7.3 & 7.5 & 7.3 & 7.2 & 7.6 \\
\hline 11 & -- & -- & -- & 7.6 & -. & -- & 7.3 & 7.3 & 7.5 & 7.3 & 7.2 & 7.3 \\
\hline 12 & -. & -- & -- & 7.6 & -- & -- & 7.3 & 7.4 & 7.5 & 7.3 & 7.3 & 7.4 \\
\hline 13 & -- & -- & -- & 7.7 & -- & -- & 7.3 & 7.4 & 7.3 & 7.3 & 7.3 & 7.4 \\
\hline 14 & -- & -- & -- & 7.7 & -- & -- & 7.3 & 7.4 & 7.0 & 7.3 & 7.3 & 7.5 \\
\hline 15 & -- & -- & -- & 7.7 & -. & -. & 7.3 & 7.4 & 7.1 & 7.3 & 7.3 & 7.5 \\
\hline 16 & -- & -- & -- & 7.7 & -- & -- & 7.3 & 7.4 & 7.1 & 7.3 & 7.3 & 7.5 \\
\hline 17 & -- & -. & -. & 7.7 & .. & -. & 7.3 & 7.4 & 7.1 & 7.3 & 7.3 & 7.5 \\
\hline 18 & -- & -- & -- & 7.7 & -- & -- & 7.3 & 7.4 & 7.1 & 7.3 & 7.3 & 7.6 \\
\hline 19 & -. & -- & -- & 7.7 & - & -- & 7.3 & 7.4 & 7.1 & 7.3 & 7.4 & 7.6 \\
\hline 20 & -- & -- & -. & 7.7 & -- & -- & 7.3 & 7.4 & 7.1 & 7.4 & 7.4 & 7.6 \\
\hline 21 & -- & -- & -. & 7.7 & -. & -- & 7.3 & 7.4 & 7.1 & 7.4 & 7.3 & 7.6 \\
\hline 22 & -- & -- & -- & 7.7 & -- & -- & 7.3 & 7.4 & 7.2 & 7.3 & 7.3 & 7.7 \\
\hline 23 & -- & -- & -. & 7.7 & -. & -. & 7.3 & 7.4 & 7.2 & 7.3 & 7.3 & 7.7 \\
\hline 24 & -- & -- & -- & 7.7 & -- & -- & 7.4 & 7.4 & 7.2 & 7.3 & 7.3 & 7.7 \\
\hline 25 & -- & -- & -- & 7.7 & -- & -. & 7.4 & 7.5 & 7.2 & 7.4 & 7.5 & 7.7 \\
\hline 26 & -- & -- & -- & 7.7 & -. & -. & 7.4 & 7.5 & 7.2 & 7.4 & 7.3 & 7.7 \\
\hline 27 & -- & -- & -- & 7.7 & -- & -- & 7.4 & 7.5 & 7.2 & 7.4 & 7.3 & 7.7 \\
\hline 28 & -- & -- & -- & 7.7 & -- & 7.1 & 7.4 & 7.5 & 7.2 & 7.4 & 7.3 & 7.6 \\
\hline 29 & -- & -- & -- & 7.8 & -- & 7.1 & 7.4 & 7.5 & 7.2 & 7.4 & 7.3 & 7.7 \\
\hline 30 & - & -- & -- & 7.8 & -- & 7.1 & 7.3 & 7.5 & 7.2 & 7.3 & 7.3 & 7.5 \\
\hline 31 & .. & -. & .- & -. & -- & -- & 7.2 & 7.5 & -- & 7.1 & -- & 7.4 \\
\hline
\end{tabular}


Table 12d. Mean daily pH in ground water at site CRM-12 (map ID \#10), East Well Field, Cedar Rapids, lowa, April 1996 through July 1997--Continued

\begin{tabular}{|c|c|c|c|c|c|c|c|c|c|c|c|c|}
\hline Day & Jan & Feb & Mar & Apr & May & Jun & Jul & Aug & Sep & Oct & Nov & Dec \\
\hline \multicolumn{13}{|c|}{1997} \\
\hline 1 & 7.3 & 7.6 & 7.7 & -. & 6.7 & 6.7 & 6.8 & -- & - & -. & -- & -- \\
\hline 2 & 7.3 & 7.7 & 7.7 & -. & 6.7 & 6.8 & 6.9 & -. & -- & -- & -- & -- \\
\hline 3 & 7.3 & 7.7 & 7.7 & -- & 6.7 & 6.8 & 6.9 & -- & -- & -- & -- & -- \\
\hline 4 & 7.4 & 7.7 & 7.7 & -- & 6.7 & 6.8 & 6.9 & -- & -- & -- & -- & -- \\
\hline 5 & 7.4 & 7.7 & 7.7 & 6.7 & 6.7 & 6.8 & 6.9 & -- & -- & -- & -- & -- \\
\hline 6 & 7.4 & 7.7 & 7.7 & 6.7 & 6.7 & 6.8 & 6.9 & -- & -- & -- & -- & -- \\
\hline 7 & 7.4 & 7.4 & 7.7 & 6.6 & 6.7 & 6.8 & 6.9 & -- & -- & -- & -- & -. \\
\hline 8 & 7.4 & 7.2 & 7.7 & 6.6 & 6.7 & 6.8 & 6.9 & -- & -- & -. & -- & -- \\
\hline 9 & 7.4 & 7.3 & 7.7 & 6.6 & 6.7 & 6.8 & 6.9 & -- & -- & -- & -- & -- \\
\hline 10 & 7.5 & 7.3 & 7.7 & 6.6 & -- & 6.8 & 6.9 & -- & -. & -- & -- & -- \\
\hline 11 & 7.5 & 7.4 & 7.7 & 6.6 & -- & 6.8 & -- & -- & -- & -- & -- & -- \\
\hline 12 & 7.5 & 7.4 & 7.7 & 6.6 & -- & 6.8 & -- & -- & -- & -- & -- & -- \\
\hline 13 & 7.5 & 7.4 & 7.8 & 6.6 & - & 6.8 & -- & -- & -- & -- & -- & -- \\
\hline 14 & 7.5 & 7.4 & 7.8 & 6.6 & -- & 6.8 & -- & -- & -- & -- & -- & -- \\
\hline 15 & 7.5 & 7.5 & 7.8 & 6.8 & -- & 6.8 & -- & - & -- & -- & -- & -- \\
\hline 16 & 7.5 & 7.5 & 7.8 & 6.8 & -- & 6.8 & -. & -- & -- & -- & -- & -- \\
\hline 17 & 7.5 & 7.5 & 7.8 & 6.8 & -- & 6.8 & - & -- & -- & -- & -- & -- \\
\hline 18 & 7.6 & 7.5 & 7.8 & 6.7 & -- & 6.8 & -- & -- & -- & -- & -- & -- \\
\hline 19 & 7.6 & 7.5 & 7.8 & 6.8 & -- & 6.8 & -- & -- & -- & -- & -- & -- \\
\hline 20 & 7.6 & 7.5 & 7.8 & 6.8 & -- & 6.8 & -. & -- & -- & -- & -- & -. \\
\hline 21 & 7.6 & 7.6 & 7.8 & 6.6 & - & 6.8 & -. & -- & -- & -- & -- & -- \\
\hline 22 & 7.7 & 7.6 & 7.8 & 6.6 & -- & 6.8 & -- & -- & - & -- & -- & -- \\
\hline 23 & 7.7 & 7.6 & 7.8 & 6.6 & 6.8 & 6.8 & -- & -- & -- & -- & -- & -- \\
\hline 24 & 7.7 & 7.6 & 7.8 & 6.6 & 6.8 & 6.8 & -- & -- & -- & -- & -- & -- \\
\hline 25 & 7.6 & 7.6 & 7.8 & 6.6 & 6.8 & 6.8 & - & -- & -- & -- & -- & -- \\
\hline 26 & 7.6 & 7.6 & 7.8 & 6.6 & 6.8 & 6.8 & -. & -- & -- & -- & -- & -- \\
\hline 27 & 7.6 & 7.6 & 7.8 & 6.6 & 6.8 & 6.8 & -- & -. & -- & -. & -- & -- \\
\hline 28 & 7.6 & 7.6 & 7.8 & 6.6 & 6.8 & 6.8 & -- & -- & -- & -- & -- & -- \\
\hline 29 & 7.6 & -- & 7.8 & 6.7 & 6.8 & 6.8 & -- & -- & -- & -- & -- & -- \\
\hline 30 & 7.6 & - & -- & 6.7 & 6.8 & 6.8 & - & -- & - & -- & -- & -- \\
\hline 31 & 7.6 & -. & - & -- & 6.7 & -- & -. & -- & -- & -- & - & -- \\
\hline
\end{tabular}


Table 12e. Mean daily pH in ground water at site CRM-20 (map ID \#17), Northwest Well Field, Cedar Rapids, lowa, February 1998 through March 1999

[All values in standard units; --, value not measured, not recorded, or rejected after data review]

\begin{tabular}{|c|c|c|c|c|c|c|c|c|c|c|c|c|}
\hline Day & Jan & Feb & Mar & Apr & May & Jun & Jul & Aug & Sep & Oct & Nov & Dec \\
\hline \multicolumn{13}{|c|}{1998} \\
\hline 1 & -. & -- & 7.4 & 7.3 & 7.2 & 7.1 & 7.3 & 7.2 & -- & 7.4 & 7.5 & 7.4 \\
\hline 2 & -- & -. & 7.4 & 7.3 & 7.2 & 7.1 & 7.3 & 7.2 & -- & 7.4 & 7.5 & 7.4 \\
\hline 3 & -- & -- & 7.4 & 7.3 & 7.2 & 7.1 & 7.2 & 7.2 & -- & 7.4 & 7.5 & 7.4 \\
\hline 4 & -- & -- & 7.4 & 7.2 & 7.3 & 7.1 & 7.2 & 7.2 & -. & 7.4 & 7.5 & 7.4 \\
\hline 5 & -- & -- & 7.4 & 7.2 & 7.3 & 7.1 & 7.2 & 7.2 & -- & 7.4 & 7.5 & 7.4 \\
\hline 6 & -- & -- & 7.4 & 7.2 & 7.4 & 7.1 & 7.2 & 7.2 & -- & 7.4 & 7.5 & 7.4 \\
\hline 7 & -- & -- & 7.4 & 7.1 & 7.4 & 7.1 & 7.2 & 7.2 & -- & 7.4 & 7.5 & 7.4 \\
\hline 8 & -. & -- & 7.4 & 7.1 & 7.4 & 7.1 & 7.2 & 7.2 & -. & 7.4 & 7.5 & 7.4 \\
\hline 9 & -- & -- & 7.4 & 7.1 & 7.4 & 7.1 & 7.2 & 7.3 & - & 7.4 & 7.5 & 7.5 \\
\hline 10 & -. & 7.7 & 7.5 & 7.1 & 7.4 & 7.1 & 7.2 & 7.3 & -- & 7.4 & 7.5 & 7.4 \\
\hline 11 & -- & 7.7 & 7.6 & 7.1 & 7.4 & 7.1 & 7.2 & 7.3 & -- & 7.4 & 7.5 & 7.4 \\
\hline 12 & -- & 7.7 & 7.6 & 7.1 & 7.4 & 7.1 & 7.2 & 7.3 & -. & 7.5 & 7.5 & 7.4 \\
\hline 13 & -- & 7.7 & 7.6 & 7.1 & 7.4 & 7.1 & 7.2 & 7.3 & -- & 7.5 & 7.5 & 7.4 \\
\hline 14 & -- & 7.7 & 7.6 & 7.2 & 7.4 & 7.1 & 7.2 & 7.3 & -- & 7.4 & 7.5 & 7.4 \\
\hline 15 & -- & 7.7 & 7.5 & 7.3 & 7.3 & 7.1 & 7.2 & 7.3 & -- & 7.4 & 7.5 & 7.4 \\
\hline 16 & -- & 7.7 & 7.5 & 7.3 & 7.3 & 7.1 & 7.3 & 7.3 & -- & 7.4 & 7.5 & 7.3 \\
\hline 17 & -. & 7.7 & 7.5 & 7.2 & 7.3 & 7.1 & 7.3 & -- & -- & 7.4 & 7.5 & 7.3 \\
\hline 18 & -- & 7.7 & 7.6 & 7.2 & 7.3 & 7.2 & 7.2 & -- & - & 7.4 & 7.5 & 7.3 \\
\hline 19 & -. & 7.7 & 7.6 & 7.2 & 7.3 & 7.2 & 7.2 & -. & -- & 7.4 & 7.5 & 7.3 \\
\hline 20 & .. & 7.7 & 7.5 & 7.2 & 7.3 & 7.2 & 7.2 & -- & -- & 7.4 & 7.5 & 7.2 \\
\hline 21 & -- & 7.7 & 7.5 & 7.2 & 7.3 & 7.2 & 7.2 & -- & -- & 7.4 & 7.5 & 7.2 \\
\hline 22 & -. & 7.7 & 7.5 & 7.2 & 7.3 & 7.2 & 7.2 & -- & -- & 7.4 & 7.5 & 7.2 \\
\hline 23 & -- & 7.6 & 7.5 & 7.2 & 7.3 & 7.2 & 7.2 & -- & 7.3 & 7.4 & 7.4 & 7.2 \\
\hline 24 & -- & 7.5 & 7.4 & 7.2 & 7.3 & 7.3 & 7.2 & -- & 7.3 & 7.4 & 7.3 & 7.1 \\
\hline 25 & -- & 7.5 & 7.4 & 7.2 & 7.3 & 7.3 & 7.2 & -- & 7.3 & 7.4 & 7.3 & 7.1 \\
\hline 26 & -- & 7.5 & 7.4 & 7.2 & 7.2 & 7.3 & 7.2 & -- & 7.3 & 7.4 & 7.3 & 7.1 \\
\hline 27 & -- & 7.5 & 7.4 & 7.2 & 7.2 & 7.3 & 7.2 & -- & 7.3 & 7.4 & 7.3 & 7.1 \\
\hline 28 & -- & 7.4 & 7.4 & 7.2 & 7.1 & 7.3 & 7.1 & -- & 7.3 & 7.4 & 7.3 & 7.1 \\
\hline 29 & - & -- & 7.3 & 7.2 & 7.1 & 7.3 & 7.1 & -- & 7.3 & 7.4 & 7.3 & 7.1 \\
\hline 30 & -- & -. & 7.3 & 7.2 & 7.1 & 7.3 & 7.1 & -- & 7.4 & 7.5 & 7.3 & 7.1 \\
\hline 31 & -. & -. & 7.3 & -- & 7.1 & -- & 7.1 & -- & -- & 7.5 & -- & 7.1 \\
\hline
\end{tabular}


Table 12e. Mean daily pH in ground water at site CRM-20 (map ID \#17), Northwest Well Field, Cedar Rapids, lowa, February 1998 through March 1999--Continued

\begin{tabular}{|c|c|c|c|c|c|c|c|c|c|c|c|c|}
\hline Day & Jan & Feb & Mar & $\overline{A p r}$ & May & Jun & Jul & Aug & Sep & Oct & Nov & Dec \\
\hline & & & & & & 1999 & & & & & & \\
\hline 1 & 7.1 & 7.4 & 7.5 & -- & -- & -- & -- & -- & -- & -- & -- & -. \\
\hline 2 & 7.1 & 7.4 & 7.5 & -- & -- & -- & - & -- & -- & -- & -. & -. \\
\hline 3 & 7.2 & 7.4 & 7.5 & -- & -- & -- & -- & -- & -- & -. & -- & -- \\
\hline 4 & 7.2 & 7.4 & 7.5 & -- & -- & -- & -- & -- & -- & -- & -- & -. \\
\hline 5 & 7.2 & $7 . \dot{4}$ & 7.5 & -- & -- & -. & -- & -- & -- & -- & -- & -- \\
\hline 6 & 7.2 & 7.4 & 7.4 & -- & -- & -- & -- & -- & -- & -- & -- & -- \\
\hline 7 & 7.2 & 7.4 & 7.4 & -- & -- & -- & -- & -- & -- & -- & -- & -- \\
\hline 8 & 7.2 & 7.4 & 7.4 & -- & -- & -- & -- & -- & -- & -- & -- & -- \\
\hline 9 & 7.2 & 7.4 & 7.4 & -- & -- & -- & -- & -- & -- & -- & -. & -. \\
\hline 10 & 7.2 & 7.4 & 7.4 & - & -- & -- & -- & -- & -- & -- & -- & -- \\
\hline 11 & 7.2 & 7.4 & 7.4 & -- & -- & -- & -- & -- & -- & -- & -- & -- \\
\hline 12 & 7.2 & 7.4 & 7.4 & -. & -. & -. & -- & -- & -- & -- & .- & -- \\
\hline 13 & 7.2 & 7.4 & 7.4 & -- & -- & -- & -- & -- & -- & -- & -- & -- \\
\hline 14 & 7.3 & 7.4 & 7.4 & -- & -- & -- & -- & -- & -- & -- & -- & -- \\
\hline 15 & 7.3 & 7.4 & 7.4 & -- & -- & -- & -- & -- & -- & -- & -. & -- \\
\hline 16 & 7.3 & 7.4 & 7.4 & -- & -- & -- & -- & -- & -- & -- & -- & -- \\
\hline 17 & 7.3 & 7.5 & 7.4 & -- & -. & -- & -- & -. & -- & -- & -- & -- \\
\hline 18 & 7.3 & 7.5 & 7.4 & -. & -- & -- & -- & -- & -. & -- & -- & -- \\
\hline 19 & 7.3 & 7.5 & 7.4 & -- & -- & -- & -- & -- & -- & -- & -- & -- \\
\hline 20 & 7.3 & 7.5 & 7.4 & -- & -- & -- & -- & -- & -- & -- & -- & -- \\
\hline 21 & 7.3 & 7.5 & 7.4 & -. & -. & -- & -- & -- & -- & -- & -- & -- \\
\hline 22 & 7.4 & 7.5 & 7.4 & -- & -. & -- & -- & -- & -- & -- & -- & -- \\
\hline 23 & 7.4 & 7.5 & 7.4 & -- & -- & -- & -- & -- & -- & -- & -- & -- \\
\hline 24 & 7.4 & 7.5 & 7.4 & -- & -- & -- & -- & -- & -- & -- & -- & -- \\
\hline 25 & 7.4 & 7.5 & 7.4 & -- & -- & -- & -- & -- & -- & -- & -- & -- \\
\hline 26 & 7.4 & 7.5 & 7.6 & -- & -- & -- & -- & -- & -- & -- & -- & -. \\
\hline 27 & 7.4 & 7.5 & 7.7 & -- & -- & -- & -- & -- & -- & -- & -- & -- \\
\hline 28 & 7.4 & 7.5 & 7.7 & -- & -- & -- & -- & -- & -- & -- & -- & -- \\
\hline 29 & 7.4 & -- & 7.7 & -- & -- & -- & -- & -- & -- & -- & -- & -- \\
\hline 30 & 7.4 & -- & 7.7 & -- & -- & -- & -- & -- & -- & -- & -- & -- \\
\hline 31 & 7.4 & -- & 7.7 & -- & -- & -- & -- & -- & -. & -. & -- & -- \\
\hline
\end{tabular}


Table 12f. Mean daily pH in ground water at site CRM-21 (map ID \#18), Northwest Well Field, Cedar Rapids, lowa, February 1998 through March 1999

[All values in standard units; --, value not measured, not recorded, or rejected after data review]

\begin{tabular}{|c|c|c|c|c|c|c|c|c|c|c|c|c|}
\hline Day & Jan & Feb & Mar & Apr & May & Jun & Jul & Aug & Sep & Oct & Nov & Dec \\
\hline & & & & & & 1998 & & & & & & \\
\hline 1 & -- & -- & 7.7 & 7.7 & 7.2 & 7.2 & 7.5 & 6.9 & -- & 7.0 & 7.0 & 6.9 \\
\hline 2 & -- & -- & 7.7 & 7.7 & 7.2 & 7.2 & 7.5 & 6.9 & -. & 7.0 & 7.0 & 6.9 \\
\hline 3 & -- & -- & 7.7 & 7.7 & 7.2 & 7.1 & 7.6 & 6.9 & -- & 7.0 & 6.9 & 6.9 \\
\hline 4 & -- & -- & 7.7 & 7.8 & 7.2 & 7.1 & 7.6 & 6.9 & -- & 7.0 & 6.9 & 6.9 \\
\hline 5 & -- & -- & 7.7 & 7.8 & 7.2 & 7.1 & 7.6 & 7.0 & -- & 7.0 & 6.9 & 6.9 \\
\hline 6 & -- & -- & 7.7 & 7.9 & 7.2 & 7.1 & 7.5 & 7.0 & -- & 7.0 & 6.9 & 6.9 \\
\hline 7 & -- & -- & 7.7 & 7.9 & 7.2 & 7.1 & 7.4 & 7.0 & -- & 7.0 & 6.9 & 6.9 \\
\hline 8 & -- & -- & 7.7 & 7.9 & 7.2 & 7.1 & 7.4 & 7.0 & -- & 7.0 & 6.9 & 6.9 \\
\hline 9 & -- & -- & 7.6 & 7.9 & 7.2 & 7.1 & 7.4 & 7.0 & -- & 6.9 & 6.9 & 6.9 \\
\hline 10 & -- & 7.8 & 7.6 & 7.9 & 7.2 & 7.1 & 7.4 & 7.0 & -. & 6.9 & 6.9 & 6.9 \\
\hline 11 & -- & 7.8 & 7.7 & 8.0 & 7.2 & 7.1 & 7.4 & 7.0 & -- & 6.9 & 7.0 & 6.9 \\
\hline 12 & -- & 7.8 & 7.7 & 8.0 & 7.2 & 7.1 & 7.4 & 7.0 & -- & 6.9 & 6.9 & 6.9 \\
\hline 13 & -. & 7.8 & 7.7 & 8.0 & 7.2 & 7.0 & 7.4 & 7.0 & -- & 6.9 & 6.9 & 6.9 \\
\hline 14 & -- & 7.8 & 7.7 & 7.6 & 7.2 & 7.1 & 7.4 & 7.0 & - & 7.0 & 6.9 & 7.0 \\
\hline 15 & -- & 7.8 & 7.7 & 7.2 & 7.2 & 7.1 & 7.3 & 7.0 & -- & 7.0 & 6.9 & 7.0 \\
\hline 16 & -- & 7.8 & 7.7 & 7.2 & 7.2 & 7.1 & 7.2 & 7.0 & -- & 7.0 & 6.9 & 7.0 \\
\hline 17 & -- & 7.8 & 7.7 & 7.2 & 7.2 & 7.1 & 7.2 & -- & - & 7.0 & 6.9 & 7.0 \\
\hline 18 & -- & 7.8 & 7.7 & 7.2 & 7.2 & 7.1 & 7.2 & -- & -- & 7.0 & 7.0 & 6.9 \\
\hline 19 & -- & 7.8 & 7.6 & 7.2 & 7.2 & 7.1 & 7.3 & -- & -- & 7.0 & 7.0 & 6.9 \\
\hline 20 & -- & 7.8 & 7.6 & 7.2 & 7.2 & 7.1 & 7.3 & -- & -. & 7.0 & 7.0 & 6.9 \\
\hline 21 & -- & 7.8 & 7.6 & 7.2 & 7.2 & 7.1 & 7.2 & -- & -. & 7.0 & 7.0 & 6.9 \\
\hline 22 & -- & 7.8 & 7.6 & 7.2 & 7.2 & 7.1 & 7.2 & -- & -- & 7.0 & 7.0 & 6.9 \\
\hline 23 & -- & 7.8 & 7.6 & 7.2 & 7.2 & 7.1 & 7.2 & -- & 7.0 & 7.0 & 7.0 & 6.9 \\
\hline 24 & -- & 7.7 & 7.6 & 7.2 & 7.2 & 7.2 & 7.1 & -- & 6.9 & 7.0 & 6.9 & 6.9 \\
\hline 25 & -- & 7.7 & 7.6 & 7.2 & 7.2 & 7.6 & 7.0 & -- & 6.9 & 7.0 & 6.9 & 6.9 \\
\hline 26 & -- & 7.7 & 7.6 & 7.2 & 7.2 & 7.6 & 6.9 & -- & 6.9 & 7.0 & 6.9 & 6.9 \\
\hline 27 & - & 7.7 & 7.6 & 7.2 & 7.1 & 7.6 & 6.9 & -- & 6.9 & 7.0 & 6.9 & 6.9 \\
\hline 28 & -- & 7.7 & 7.6 & 7.2 & 7.1 & 7.6 & 6.9 & -- & 6.9 & 7.0 & 6.9 & 6.9 \\
\hline 29 & -- & -- & 7.6 & 7.2 & 7.1 & 7.7 & 6.9 & -- & 7.0 & 7.0 & 6.9 & 6.9 \\
\hline 30 & -- & -- & 7.6 & 7.2 & 7.1 & 7.6 & 6.9 & -- & 7.0 & 7.0 & 6.9 & 6.9 \\
\hline 31 & -. & .- & 7.6 & -- & 7.1 & .. & 6.9 & -- & .. & 7.0 & .- & 6.9 \\
\hline
\end{tabular}


Table 12f. Mean daily pH in ground water at site CRM-21 (map ID \#18), Northwest Well Field, Cedar Rapids, lowa, February 1998 through March 1999--Continued

\begin{tabular}{|c|c|c|c|c|c|c|c|c|c|c|c|c|}
\hline Day & Jan & Feb & Mar & Apr & May & Jun & Jul & Aug & Sep & Oct & Nov & Dec \\
\hline \multicolumn{13}{|c|}{1999} \\
\hline 1 & 6.9 & 7.0 & 7.2 & -- & -- & -- & -- & -- & -- & -- & -- & -- \\
\hline 2 & 6.9 & 7.0 & 6.9 & -- & -- & -- & -- & -- & -- & -- & -- & -- \\
\hline 3 & 6.9 & 7.0 & 7.1 & -- & -- & -- & -- & --- & -- & -- & -- & -- \\
\hline 4 & 6.9 & 7.0 & 7.4 & -- & -- & -- & -- & -- & -- & -- & -- & -- \\
\hline 5 & 6.9 & 7.0 & 7.4 & -- & -- & -- & -- & -- & -- & -- & -. & -- \\
\hline 6 & 6.9 & 7.0 & 7.4 & -- & -- & -. & -. & -- & -- & -- & -- & -- \\
\hline 7 & 6.9 & 7.0 & 7.4 & -- & -- & -- & -- & -- & -- & -- & -- & -- \\
\hline 8 & 6.9 & 7.0 & 7.4 & -- & -- & -- & -- & -- & -- & -- & -- & -. \\
\hline 9 & 6.9 & 7.0 & 7.3 & -- & -- & -- & - & -- & -- & -- & -- & -- \\
\hline 10 & 6.9 & 7.0 & 7.3 & -- & -- & -- & -- & -- & -- & -- & -- & -- \\
\hline 11 & 6.9 & 7.0 & 7.3 & -- & -. & -- & -- & -- & -- & -- & -- & -- \\
\hline 12 & 6.9 & 7.0 & 7.3 & -- & -- & -- & -- & -- & -- & -- & -- & -- \\
\hline 13 & 6.9 & 7.0 & 7.3 & -. & -- & -- & -- & -- & -- & -- & -- & -- \\
\hline 14 & 6.9 & 7.0 & 7.3 & -- & -- & -- & - & -- & -- & -- & -. & -- \\
\hline 15 & 6.9 & 7.0 & 7.3 & -- & -- & -- & -- & -- & -- & -- & -- & -- \\
\hline 16 & 6.9 & 7.0 & 7.3 & -- & -- & -- & -- & -- & -- & - & -- & -- \\
\hline 17 & 6.9 & 7.0 & 7.3 & -- & -- & -- & -- & -- & -- & -- & -- & -- \\
\hline 18 & 6.9 & 7.0 & 7.3 & -- & -- & -- & -- & -- & -- & -- & -- & -- \\
\hline 19 & 6.9 & 7.0 & 7.3 & - & -- & -- & -- & -- & -- & -- & -- & -- \\
\hline 20 & 6.9 & 7.0 & 7.3 & -- & -- & -- & -- & -- & -- & -- & -- & -- \\
\hline 21 & 6.9 & 7.1 & 7.3 & -- & - & -- & -- & -- & -- & -- & -- & -- \\
\hline 22 & 7.0 & 7.1 & 7.3 & -- & -- & -- & -- & -- & -- & -- & -- & -. \\
\hline 23 & 7.0 & 7.1 & 7.3 & -- & -- & -- & -- & -- & -- & -- & -- & -- \\
\hline 24 & 7.0 & 7.2 & 7.3 & -- & -- & -- & -- & -- & -- & -- & -- & -- \\
\hline 25 & 7.0 & 7.2 & 7.3 & -- & -- & -- & -- & -- & -- & -- & - & -- \\
\hline 26 & 7.0 & 7.2 & 7.2 & -- & -- & -- & -- & -- & -- & -- & -- & -- \\
\hline 27 & 7.0 & 7.3 & 7.2 & -- & -- & -- & -- & -- & -- & -- & -- & -- \\
\hline 28 & 7.0 & 7.3 & 7.2 & -- & -- & -. & -- & -- & -- & -- & -- & -- \\
\hline 29 & 7.0 & -- & 7.2 & -- & -- & -- & -- & -- & -- & -- & -- & -- \\
\hline 30 & 7.0 & -. & 7.2 & -- & -- & -- & -- & -- & -- & -- & -- & -- \\
\hline 31 & 7.0 & -- & 7.2 & -- & -- & -. & -- & -- & -. & -- & -- & -- \\
\hline
\end{tabular}


Table 12g. Mean daily $\mathrm{pH}$ in ground water at site CRM-22 (map ID \#19), Seminole Well Field, Cedar Rapids, lowa, February 1998 through March 1999

[All values in standard units; --, value not measured, not recorded, or rejected after data review]

\begin{tabular}{|c|c|c|c|c|c|c|c|c|c|c|c|c|}
\hline Day & Jan & Feb & Mar & Apr & May & Jun & Jul & Aug & Sep & Oct & Nov & Dec \\
\hline & & & & & & 1998 & & & & & & \\
\hline 1 & -- & -- & 7.7 & 7.7 & 7.6 & 7.3 & -- & -- & -- & 7.4 & 7.6 & 7.6 \\
\hline 2 & -- & -- & 7.7 & 7.7 & 7.6 & 7.3 & -- & -- & -- & 7.4 & 7.6 & 7.6 \\
\hline 3 & -- & -- & 7.7 & 7.7 & 7.6 & -- & -- & -. & -- & 7.4 & 7.6 & 7.6 \\
\hline 4 & -- & -- & 7.7 & 7.8 & 7.5 & -- & -- & -- & -- & 7.4 & 7.6 & 7.6 \\
\hline 5 & -- & -- & 7.7 & 7.8 & 7.5 & -- & -- & -- & - & 7.4 & 7.6 & 7.6 \\
\hline 6 & -. & -- & 7.7 & 7.9 & 7.5 & -- & -- & -- & -- & 7.4 & 7.6 & 7.6 \\
\hline 7 & -- & -. & 7.7 & 7.9 & 7.5 & -- & -- & -- & -- & 7.4 & 7.6 & 7.6 \\
\hline 8 & -- & -- & 7.7 & 7.9 & 7.5 & -- & -- & -- & -- & 7.4 & 7.6 & 7.6 \\
\hline 9 & -- & -- & 7.6 & 7.9 & 7.5 & -- & -- & -- & -- & 7.4 & 7.6 & 7.6 \\
\hline 10 & -- & 7.8 & 7.6 & 7.9 & 7.4 & -- & -- & -- & -- & 7.4 & 7.6 & 7.6 \\
\hline 11 & -- & 7.8 & 7.7 & 8.0 & 7.4 & -- & -- & -- & -- & 7.4 & 7.6- & 7.6 \\
\hline 12 & -- & 7.8 & 7.7 & 8.0 & 7.4 & -- & -- & -- & -- & 7.4 & 7.6 & 7.6 \\
\hline 13 & -- & 7.8 & 7.7 & 8.0 & 7.4 & -- & -- & -- & -- & 7.4 & 7.6 & 7.6 \\
\hline 14 & -- & 7.8 & 7.7 & 7.9 & 7.4 & -- & -- & -- & -- & 7.4 & 7.6 & 7.6 \\
\hline 15 & -. & 7.8 & 7.7 & 7.7 & 7.4 & -- & -- & -- & -- & 7.4 & 7.6 & 7.6 \\
\hline 16 & -- & 7.8 & 7.7 & 7.5 & 7.4 & -- & -- & -- & -. & 7.4 & 7.6 & 7.6 \\
\hline 17 & -- & 7.8 & 7.7 & 7.4 & 7.4 & -- & -- & -- & -- & 7.4 & 7.6 & 7.6 \\
\hline 18 & -- & 7.8 & 7.7 & 7.4 & 7.3 & -- & -- & -- & -- & 7.4 & 7.6 & 7.6 \\
\hline 19 & -- & 7.8 & 7.6 & 7.4 & 7.4 & -- & -- & -- & -- & 7.4 & 7.6 & 7.6 \\
\hline 20 & -- & 7.8 & 7.6 & 7.4 & 7.4 & -- & -- & -- & - & 7.4 & 7.6 & 7.6 \\
\hline 21 & -- & 7.8 & 7.6 & 7.4 & 7.4 & -- & -- & -- & -- & 7.4 & 7.6 & 7.6 \\
\hline 22 & -- & 7.8 & 7.6 & 7.4 & 7.4 & -- & -- & -- & -- & 7.4 & 7.6 & 7.6 \\
\hline 23 & -- & 7.8 & 7.6 & 7.4 & 7.4 & -- & -- & -- & 7.3 & 7.5 & 7.6 & 7.6 \\
\hline 24 & -- & 7.7 & 7.6 & 8.1 & 7.4 & -- & -- & -- & 7.3 & 7.6 & 7.7 & 7.6 \\
\hline 25 & -- & 7.7 & 7.6 & 8.1 & 7.4 & -- & -- & -- & 7.3 & 7.6 & 7.7 & 7.6 \\
\hline 26 & -- & 7.7 & 7.6 & 8.0 & 7.3 & -- & -- & -- & 7.3 & 7.6 & 7.7 & 7.6 \\
\hline 27 & -- & 7.7 & 7.6 & 7.9 & 7.3 & -- & -- & -- & 7.3 & 7.6 & 7.7 & 7.6 \\
\hline 28 & -- & 7.7 & 7.6 & 7.8 & 7.3 & -- & -- & -- & 7.3 & 7.6 & 7.7 & 7.5 \\
\hline 29 & -- & -- & 7.6 & 7.7 & 7.3 & -- & -- & -- & 7.3 & 7.6 & 7.6 & 7.5 \\
\hline 30 & -- & -- & 7.6 & 7.6 & 7.3 & -- & -- & -- & 7.3 & 7.6 & 7.6 & 7.5 \\
\hline 31 & -- & -- & 7.6 & -- & 7.3 & -- & -- & -. & -- & 7.6 & -- & 7.4 \\
\hline
\end{tabular}


Table 12g. Mean daily pH in ground water at site CRM-22 (map ID \#19), Seminole Well Field, Cedar Rapids, lowa, February 1998 through March 1999--Continued

\begin{tabular}{|c|c|c|c|c|c|c|c|c|c|c|c|c|}
\hline Day & Jan & Feb & Mar & Apr & May & Jun & Jul & Aug & Sep & Oct & Nov & Dec \\
\hline & & & & & & 1999 & & & & & & \\
\hline 1 & 7.4 & 7.7 & 8.0 & -- & -- & -- & -- & -- & -- & -- & -- & -- \\
\hline 2 & 7.4 & 7.7 & 8.1 & -- & -- & -- & -- & -- & -- & -- & -- & -- \\
\hline 3 & 7.4 & 7.7 & 8.1 & -- & -- & -- & -- & -- & - & -- & -- & -- \\
\hline 4 & 7.4 & 7.7 & 8.1 & -- & -- & -- & -- & -- & -. & -- & -- & -- \\
\hline 5 & 7.4 & 7.7 & 8.1 & -- & -- & -- & -- & -- & -- & -- & -- & -- \\
\hline 6 & 7.4 & 7.7 & 8.1 & -- & -- & -- & -- & - & -- & -- & -- & - \\
\hline 7 & 7.4 & 7.7 & 8.1 & -- & -- & -- & -- & -- & -- & -- & -- & -. \\
\hline 8 & 7.3 & 7.7 & 8.1 & -- & -- & -- & -- & -- & -- & -- & -- & -- \\
\hline 9 & 7.3 & 7.7 & 8.1 & -- & -- & -- & -- & -- & -- & -- & -- & -- \\
\hline 10 & 7.3 & 7.7 & 8.1 & -- & -- & -- & -- & -- & -- & -- & -- & -- \\
\hline 11 & 7.3 & 7.7 & 8.1 & -- & -- & -- & -- & -- & -- & -- & -- & -- \\
\hline 12 & 7.4 & 7.7 & 8.1 & -- & -- & -- & -- & -- & -- & -- & -- & -- \\
\hline 13 & 7.4 & 7.7 & 8.1 & -- & -- & -- & -- & -- & -- & -- & -- & -- \\
\hline 14 & 7.4 & 7.8 & 8.1 & -- & -- & -- & -- & -- & -- & -- & -- & - \\
\hline 15 & 7.4 & 7.8 & 8.1 & -- & -- & -- & -- & -- & -. & -- & -- & -- \\
\hline 16 & 7.4 & 7.8 & 8.1 & -- & -- & -- & -- & -- & -- & -- & -- & -- \\
\hline 17 & 7.5 & 7.8 & 8.1 & -- & -. & -- & -- & -- & -- & -- & - & - \\
\hline 18 & 7.5 & 7.8 & 8.1 & -- & -- & -- & -- & -- & -- & -- & -- & -- \\
\hline 19 & 7.5 & 7.8 & 8.1 & -- & -- & -- & -- & -- & -- & -- & -- & -- \\
\hline 20 & 7.5 & 7.8 & 8.1 & -- & -- & -- & -- & -- & -- & -- & -- & -- \\
\hline 21 & 7.5 & 7.8 & 8.1 & -- & -- & -- & -- & -- & -- & -- & -- & - \\
\hline 22 & 7.6 & 7.8 & 8.1 & -- & -- & -- & -- & -- & -- & -- & -- & -- \\
\hline 23 & 7.6 & 7.8 & 8.1 & -- & -- & -- & -- & -- & -- & -- & -- & -- \\
\hline 24 & 7.6 & 7.8 & 8.1 & -- & -- & -- & -- & -. & -- & -- & -- & -- \\
\hline 25 & 7.6 & 7.8 & 8.1 & -- & -- & -- & -- & -- & -- & -- & -. & -- \\
\hline 26 & 7.6 & 7.8 & 8.0 & -- & -- & -- & -- & -- & -. & -- & -- & -- \\
\hline 27 & 7.6 & 7.8 & 7.9 & -- & -- & -- & -- & -- & -. & -- & -- & -- \\
\hline 28 & 7.7 & 7.8 & 7.9 & -- & -- & -- & -- & -- & -- & -- & -- & -- \\
\hline 29 & 7.7 & -- & 7.9 & -- & -- & -- & -- & -. & -- & -- & -- & -- \\
\hline 30 & 7.7 & -- & 7.9 & -- & -- & -- & -- & -- & -- & -- & -- & -- \\
\hline 31 & 7.7 & -- & 7.9 & -- & -- & -- & -- & -- & -- & -- & -- & -- \\
\hline
\end{tabular}


Table 12h. Mean daily $\mathrm{pH}$ in ground water at site CRM-23 (map ID \#20), Seminole Well Field, Cedar Rapids, lowa, February 1998 through March 1999

[All values in standard units; --, value not measured, not recorded, or rejected after data review]

\begin{tabular}{|c|c|c|c|c|c|c|c|c|c|c|c|c|}
\hline Day & $\operatorname{Jan}$ & Feb & Mar & Apr & May & Jun & Jul & Aug & Sep & Oct & Nov & Dec \\
\hline & & & & & & 1998 & & & & & & \\
\hline 1 & -- & -- & 7.1 & 7.0 & 7.1 & 7.0 & 7.6 & 7.0 & -. & 6.7 & 6.8 & 6.8 \\
\hline 2 & -- & -- & 7.1 & 7.0 & 7.1 & 7.0 & 7.7 & 7.0 & - & 6.7 & 6.8 & 6.8 \\
\hline 3 & -- & -- & 7.1 & 7.0 & 7.1 & 7.0 & 7.7 & 7.0 & -- & 6.7 & 6.8 & 6.8 \\
\hline 4 & -- & -- & 7.1 & 7.0 & 7.1 & 7.0 & 7.6 & 7.0 & -- & 6.7 & 6.8 & 6.8 \\
\hline 5 & -- & -- & 7.1 & 7.0 & 7.0 & 7.0 & 7.6 & 7.0 & -- & 6.7 & 6.8 & 6.8 \\
\hline 6 & -- & -- & 7.1 & 7.0 & 7.0 & 7.0 & 7.5 & 7.0 & -- & 6.7 & 6.8 & 6.8 \\
\hline 7 & -- & -- & 7.1 & 7.0 & 7.0 & 7.0 & 7.5 & 7.0 & -- & 6.7 & 6.8 & 6.8 \\
\hline 8 & -- & -- & 7.1 & 7.0 & 7.1 & 7.0 & 7.5 & 7.0 & -- & 6.7 & 6.8 & 6.8 \\
\hline 9 & -- & -- & 7.1 & 7.0 & 7.1 & 7.0 & 7.5 & 7.0 & -. & 6.7 & 6.8 & 6.8 \\
\hline 10 & -- & 7.2 & 7.1 & 7.0 & 7.1 & 7.0 & 7.5 & 7.0 & -- & 6.6 & 6.8 & 6.9 \\
\hline 11 & -- & 7.2 & 7.1 & 7.0 & 7.0 & 7.0 & 7.5 & 7.0 & - & 6.6 & 6.8 & 6.9 \\
\hline 12 & -- & 7.2 & 7.1 & 7.0 & 7.0 & 7.0 & 7.5 & 7.0 & -. & 6.7 & 6.8 & 6.9 \\
\hline 13 & -- & 7.2 & 7.1 & 7.0 & 7.0 & 7.0 & 7.5 & 7.0 & - & 6.7 & 6.8 & 6.9 \\
\hline 14 & -- & 7.2 & 7.1 & 7.1 & 7.0 & 7.0 & 7.5 & 6.9 & .- & 6.6 & 6.9 & 6.8 \\
\hline 15 & - & 7.2 & 7.1 & 7.1 & 7.0 & 7.0 & 7.4 & 6.9 & - & 6.6 & 6.9 & 6.8 \\
\hline 16 & -- & 7.2 & 7.1 & 7.1 & 6.9 & 7.0 & 7.4 & 6.9 & - & 6.6 & 6.9 & 6.8 \\
\hline 17 & -. & 7.2 & 7.1 & 7.1 & 6.9 & 7.0 & 7.4 & -- & -- & 6.6 & 6.9 & 6.8 \\
\hline 18 & -- & 7.2 & 7.1 & 7.1 & 6.9 & 7.0 & 7.4 & -- & - & 6.7 & 6.9 & 6.8 \\
\hline 19 & -. & 7.2 & 7.1 & 7.1 & 6.9 & 6.9 & 7.4 & -- & -- & 6.7 & 6.9 & 6.8 \\
\hline 20 & -. & 7.2 & -. & 7.1 & 6.9 & 6.9 & 7.4 & -- & -- & 6.7 & 6.9 & 6.8 \\
\hline 21 & - & 7.2 & -- & 7.1 & 6.9 & 6.9 & 7.4 & -- & -- & 6.7 & 6.9 & 6.8 \\
\hline 22 & .. & 7.2 & -- & 7.1 & 6.9 & 6.9 & 7.4 & -- & -- & 6.7 & 6.9 & 6.7 \\
\hline 23 & -. & 7.1 & -- & 7.1 & 7.0 & 6.9 & 7.4 & -- & 6.7 & 6.8 & 6.9 & 6.7 \\
\hline 24 & - & 7.1 & 7.1 & 7.1 & 7.0 & 6.9 & 7.4 & -- & 6.7 & 6.8 & 6.9 & 6.7 \\
\hline 25 & - & 7.1 & 7.0 & 7.1 & 7.0 & 7.3 & 7.2 & -- & 6.7 & 6.8 & 6.9 & 6.7 \\
\hline 26 & -. & 7.1 & 7.0 & 7.1 & 7.0 & 7.6 & 7.2 & .. & $6: 7$ & 6.8 & 6.8 & 6.7 \\
\hline 27 & -- & 7.1 & 7.0 & 7.1 & 7.0 & 7.6 & 7.1 & -- & 6.7 & 6.8 & 6.8 & 6.7 \\
\hline 28 & -. & 7.1 & 7.0 & 7.1 & 7.0 & 7.7 & 7.1 & -- & 6.7 & 6.8 & 6.8 & 6.7 \\
\hline 29 & -. & -. & 7.0 & 7.1 & 6.9 & 7.7 & 7.0 & -- & 6.7 & 6.8 & 6.8 & 6.7 \\
\hline 30 & -- & -- & 7.0 & 7.1 & 7.0 & 7.7 & 7.0 & -. & 6.7 & 6.8 & 6.8 & 6.7 \\
\hline 31 & -- & -. & 7.0 & -- & 7.0 & -- & 7.0 & -- & -- & 6.8 & -- & 6.7 \\
\hline
\end{tabular}


Table 12h. Mean daily pH in ground water at site CRM-23 (map ID \#20), Seminole Well Field, Cedar Rapids, lowa, February 1998 through March 1999--Continued

\begin{tabular}{|c|c|c|c|c|c|c|c|c|c|c|c|c|}
\hline Day & Jan & Feb & Mar & Apr & May & Jun & Jul & Aug & Sep & Oct & Nov & $\overline{D e c}$ \\
\hline \multicolumn{13}{|c|}{1999} \\
\hline 1 & 6.7 & 6.8 & 6.9 & -- & -. & -. & -- & - & -- & -- & -- & -- \\
\hline 2 & 6.7 & 6.8 & 6.9 & -- & -- & -- & -- & -- & -- & -- & -- & -- \\
\hline 3 & 6.7 & 6.8 & 6.9 & -- & -- & -- & -- & - & -- & -- & -- & -- \\
\hline 4 & 6.8 & 6.8 & 6.9 & -- & -- & -- & -- & -- & -- & -- & -- & -- \\
\hline 5 & 6.8 & 6.8 & 6.8 & -- & -- & -- & -- & -- & -- & -- & -- & -. \\
\hline 6 & 6.8 & 6.8 & 6.8 & -- & -- & -. & -- & -- & -- & -- & -- & -. \\
\hline 7 & 6.8 & 6.8 & 6.8 & -- & -- & -- & -- & -- & -- & -- & -- & -- \\
\hline 8 & 6.7 & 6.8 & 6.8 & -- & -- & -- & -- & -- & -- & -- & -- & -- \\
\hline 9 & 6.7 & 6.8 & 6.8 & -- & -- & -- & -- & -- & -- & -- & -- & -- \\
\hline 10 & 6.7 & 6.8 & 6.8 & -- & -- & -- & -- & -- & -- & -- & -- & -- \\
\hline 11 & 6.7 & 6.8 & 6.8 & -- & -- & -- & -- & -- & -- & -- & - & -- \\
\hline 12 & 6.8 & 6.8 & 6.8 & -- & -- & -- & -- & -- & -- & -- & -- & -- \\
\hline 13 & 6.8 & 6.8 & 6.8 & -- & -- & -- & -- & -- & -- & -- & -- & -- \\
\hline 14 & 6.8 & 6.8 & 6.8 & -- & -- & -- & -- & -- & -- & -- & -- & -- \\
\hline 15 & 6.8 & 6.8 & 6.8 & -- & -- & -- & -- & -- & -- & -- & -- & -- \\
\hline 16 & 6.8 & 6.8 & 6.8 & -- & -- & -- & -- & -- & -- & -- & -- & -. \\
\hline 17 & 6.8 & 6.8 & 6.8 & -- & -- & -- & -- & -- & -- & -- & -- & -- \\
\hline 18 & 6.8 & 6.8 & 6.8 & -- & -- & -. & -. & -- & -- & -- & -- & -- \\
\hline 19 & 6.8 & 6.8 & 6.8 & -- & -- & -- & -- & -- & -- & -. & -- & -- \\
\hline 20 & 6.8 & 6.8 & 6.8 & -- & -- & -- & -- & -- & -- & -- & -- & -- \\
\hline 21 & 6.8 & 6.8 & 6.8 & -- & -- & -- & -- & -- & -- & -- & -- & -. \\
\hline 22 & 6.8 & 6.8 & 6.8 & -- & -- & -- & -- & -- & -- & -- & -- & -- \\
\hline 23 & 6.8 & 6.8 & 6.8 & -- & -- & -- & -- & -- & -- & -- & -- & -- \\
\hline 24 & 6.8 & 6.9 & 6.8 & -- & -- & -- & -- & -- & -- & -- & -- & -- \\
\hline 25 & 6.8 & 6.9 & 6.8 & -- & -- & -- & -- & -- & -- & -. & -- & -. \\
\hline 26 & 6.8 & 6.9 & 6.9 & -- & -. & -- & -- & -- & -- & -- & -- & -- \\
\hline 27 & 6.8 & 6.9 & 6.9 & -. & -- & -- & -- & -- & -- & -- & -- & -- \\
\hline 28 & 6.8 & 6.9 & 6.9 & -- & -- & -- & -- & -- & -- & -- & -- & -- \\
\hline 29 & 6.8 & -- & 6.9 & -- & -- & -- & -- & -- & -- & -- & -- & -- \\
\hline 30 & 6.8 & -- & 6.9 & -- & -- & -- & -- & -- & -- & -- & -- & -- \\
\hline 31 & 6.8 & -- & 6.9 & -- & -- & -- & -. & -- & -- & -- & -- & -- \\
\hline
\end{tabular}


Table 12i. Mean daily pH in the Cedar River at site SW-2 (map ID \#32), Seminole Well Field, Cedar Rapids, lowa, October 1996 through March 1999

[All values in standard units; --, value not measured, not recorded, or rejected after data review]

\begin{tabular}{|c|c|c|c|c|c|c|c|c|c|c|c|c|}
\hline Day & Jan & Feb & Mar & Apr & May & Jun & Jul & Aug & Sep & $\overline{\text { Oct }}$ & Nov & Dec \\
\hline & & & & & & 1996 & & & & & & \\
\hline 1 & -. & -- & -. & -- & -- & -. & -- & -- & -- & 8.5 & 8.1 & -- \\
\hline 2 & -- & -- & -- & -- & -- & -- & -- & -- & -- & 8.6 & 8.0 & -- \\
\hline 3 & -- & -- & -. & -- & -- & -- & -- & -- & -- & 8.7 & 8.1 & -- \\
\hline 4 & -- & -- & -- & -- & -- & -- & -- & -- & -- & 8.7 & -. & 8.1 \\
\hline 5 & -- & -- & -- & -- & -- & -- & -- & -. & -- & 8.7 & -- & 8.1 \\
\hline 6 & -- & -- & -- & -- & -. & -- & -- & -- & -- & 8.6 & -- & 8.1 \\
\hline 7 & -- & -- & - & -- & -- & -. & -- & -- & -- & 8.4 & -- & 8.1 \\
\hline 8 & -- & -- & -- & -- & -- & -- & -- & -- & -- & 8.4 & -- & 8.1 \\
\hline 9 & -. & -- & -- & -. & -. & -- & -- & - & -- & 8.3 & -- & 8.1 \\
\hline 10 & -- & -- & -- & -- & -- & -- & -- & -- & -- & 8.3 & -- & 8.2 \\
\hline 11 & -- & -- & -- & -- & -- & -- & -- & -- & -- & 8.3 & -- & 8.2 \\
\hline 12 & -- & -- & -- & -- & -- & -- & -- & - & -- & 8.3 & -- & 8.2 \\
\hline 13 & -- & -- & -- & -- & -- & -- & -- & - & -. & 8.2 & -- & 8.2 \\
\hline 14 & -- & -- & -- & -- & -- & -- & -- & - & -- & 8.2 & -- & 8.2 \\
\hline 15 & -- & -- & -. & -- & -- & -- & -- & - & -- & 8.2 & -- & 8.2 \\
\hline 16 & -- & -- & -. & -- & -- & -- & -- & -- & -- & 8.1 & -- & 8.2 \\
\hline 17 & -- & -- & -- & - & -- & -- & -- & - & -- & 8.0 & -- & 8.2 \\
\hline 18 & -- & -- & -- & -- & -- & -- & -- & -- & -- & 8.1 & -- & 8.2 \\
\hline 19 & -- & -- & -. & -- & -- & -- & -- & - & -- & 8.2 & -- & 8.1 \\
\hline 20 & -- & -- & -. & -- & -- & -- & -- & -- & -- & 8.1 & -- & 8.1 \\
\hline 21 & -. & -- & -- & -- & -- & -- & -- & -- & -- & 8.0 & -- & 8.1 \\
\hline 22 & -- & -- & -- & -- & - & -- & -- & -- & -- & 7.9 & -- & 8.0 \\
\hline 23 & -- & -- & -- & -- & -- & -- & -- & -- & -- & 7.9 & -- & 8.1 \\
\hline 24 & -- & -- & -- & -- & -- & -- & -- & -- & -- & 8.0 & -- & 8.1 \\
\hline 25 & -- & -- & -- & -- & -- & -- & -- & -- & -- & 8.0 & -- & 8.1 \\
\hline 26 & -- & -- & -. & -- & -- & -. & -- & -- & -- & 7.9 & -- & 8.0 \\
\hline 27 & -- & -- & -- & -- & -- & -- & -- & -- & -- & 8.0 & -- & 8.0 \\
\hline 28 & -- & -- & -- & -. & -- & -- & -- & -- & -- & 8.0 & -- & 8.0 \\
\hline 29 & -- & -- & -- & -. & -. & -- & -- & -- & -- & 8.0 & -- & 8.0 \\
\hline 30 & -- & -- & -- & -- & -. & -- & -- & -- & -- & 8.0 & -- & 8.0 \\
\hline 31 & -- & -- & -- & -- & -. & -- & -- & -- & -- & 8.0 & -- & 8.0 \\
\hline
\end{tabular}


Table 12i. Mean daily pH in the Cedar River at site SW-2 (map ID \#32), Seminole Well Field, Cedar Rapids, lowa, April 1996 through March 1999--Continued

\begin{tabular}{|c|c|c|c|c|c|c|c|c|c|c|c|c|}
\hline Day & Jan & Feb & Mar & Apr & May & Jun & Jul & Aug & Sep & Oct & Nov & Dec \\
\hline & & & & & & 1997 & & & & & & \\
\hline 1 & 8.0 & 8.2 & 8.8 & -- & -- & 8.7 & 8.7 & 8.5 & 8.5 & 9.0 & 8.9 & 8.7 \\
\hline 2 & 8.0 & 8.2 & 8.8 & -- & -- & 8.8 & 8.7 & 8.6 & 8.5 & 9.0 & 9.0 & 8.6 \\
\hline 3 & 8.0 & 8.2 & -- & -- & -. & 8.7 & 8.8 & 8.8 & 8.7 & 9.0 & 9.0 & 8.6 \\
\hline 4 & 8.0 & 8.3 & -- & -- & -- & 8.6 & 8.7 & 8.9 & 8.9 & 9.1 & 9.1 & 8.5 \\
\hline 5 & 8.0 & 8.3 & -- & 8.7 & -- & 8.8 & 8.8 & 8.7 & 9.0 & 9.1 & 9.1 & 8.3 \\
\hline 6 & 8.0 & 8.3 & -- & 8.9 & -- & 8.7 & 8.9 & 8.6 & 9.1 & 9.3 & 9.1 & 8.2 \\
\hline 7 & 8.0 & 8.3 & -- & 9.0 & -. & 8.5 & 9.0 & 8.7 & 9.0 & 9.2 & 9.2 & 8.2 \\
\hline 8 & 7.9 & 8.3 & -- & 8.9 & -- & 8.4 & 9.1 & 8.6 & 8.8 & 9.1 & 9.2 & 8.1 \\
\hline 9 & 7.9 & 8.4 & -- & 8.8 & -- & 8.4 & 9.1 & 8.5 & 8.9 & 9.2 & 9.1 & 8.2 \\
\hline 10 & 7.9 & 8.4 & -. & 9.3 & -- & 8.3 & 9.1 & 8.4 & 8.9 & 9.3 & 9.0 & 8.1 \\
\hline 11 & 7.9 & 8.4 & -- & -- & -- & 8.3 & 8.8 & 8.4 & 9.0 & 9.3 & 8.9 & 8.2 \\
\hline 12 & 7.9 & 8.5 & -- & -- & -- & 9.1 & 8.5 & 8.4 & 9.1 & 9.1 & 8.8 & 8.1 \\
\hline 13 & 8.0 & 8.5 & -- & -- & -- & 8.9 & 8.3 & 8.4 & 9.1 & 9.2 & 8.8 & 8.2 \\
\hline 14 & 8.0 & 8.5 & -- & -- & -- & 8.8 & 8.4 & 8.4 & 9.1 & 9.0 & 8.7 & 8.1 \\
\hline 15 & 8.0 & 8.6 & -. & -- & -. & 8.9 & 8.5 & 8.5 & 9.0 & 8.9 & 8.6 & 8.1 \\
\hline 16 & 8.0 & 8.6 & - & -- & .- & 8.6 & 8.4 & 8.6 & 9.0 & 9.0 & 8.7 & 8.2 \\
\hline 17 & 8.0 & 8.6 & -- & -- & -- & 8.8 & 8.1 & 8.5 & 9.1 & 9.0 & 8.7 & 8.3 \\
\hline 18 & 8.0 & 8.6 & -- & -- & -- & 9.0 & 8.1 & 8.6 & 9.0 & 9.1 & 8.5 & 8.3 \\
\hline 19 & 8.0 & 8.7 & -- & -- & -- & 8.9 & 8.2 & 8.5 & 8.9 & 9.2 & 8.5 & 8.2 \\
\hline 20 & 8.0 & 8.6 & -- & -- & -- & 9.0 & 8.2 & 8.5 & 9.0 & 9.1 & 8.5 & 8.3 \\
\hline 21 & 8.0 & 8.6 & -- & -- & -- & 9.0 & 8.3 & 8.6 & 9.0 & 9.0 & 8.6 & 8.3 \\
\hline 22 & 8.1 & 8.6 & -- & -- & -- & 9.1 & 8.4 & 8.7 & 8.8 & 8.9 & 8.5 & 8.2 \\
\hline 23 & 8.1 & 8.7 & -- & -- & -- & 8.9 & 8.4 & 8.6 & 8.9 & 8.8 & 8.6 & 8.3 \\
\hline 24 & 8.1 & 8.6 & -- & -- & -- & 8.8 & 8.4 & 8.7 & 8.8 & 8.8 & 8.6 & 8.3 \\
\hline 25 & 8.1 & 8.6 & -- & -- & 8.8 & 8.8 & 8.5 & 8.6 & 8.7 & 8.7 & 8.7 & 8.2 \\
\hline 26 & 8.1 & 8.6 & -- & -- & 8.8 & 8.7 & 8.6 & 8.5 & 8.8 & 8.6 & 8.7 & 8.2 \\
\hline 27 & 8.1 & 8.7 & -- & -- & 8.8 & 8.8 & 8.7 & 8.5 & 8.9 & 8.5 & 8.0 & 8.3 \\
\hline 28 & 8.1 & 8.7 & -- & -- & 8.7 & 8.6 & 8.7 & 8.4 & 9.0 & 8.5 & 8.8 & 8.2 \\
\hline 29 & 8.1 & -- & -- & -- & 8.8 & 8.6 & 8.8 & 8.4 & 9.0 & 8.5 & 8.8 & 8.2 \\
\hline 30 & 8.1 & -- & -- & -- & 8.7 & 8.7 & 8.7 & 8.4 & 8.9 & 8.6 & 8.8 & 8.2 \\
\hline 31 & 8.2 & -- & -- & -- & 8.8 & -- & 8.5 & 8.6 & -- & 8.8 & -- & 8.2 \\
\hline
\end{tabular}


Table 12i. Mean daily pH in the Cedar River at site SW-2 (map ID \#32), Seminole Well Field, Cedar Rapids, lowa, April 1996 through March 1999--Continued

\begin{tabular}{|c|c|c|c|c|c|c|c|c|c|c|c|c|}
\hline Day & Jan & Feb & Mar & Apr & May & Jun & Jul & Aug & Sep & Oct & Nov & $\overline{\text { Dec }}$ \\
\hline \multicolumn{13}{|c|}{1998} \\
\hline 1 & 8.2 & 7.9 & 8.2 & 7.7 & 8.3 & 7.8 & 7.8 & 8.3 & -- & 7.9 & 8.1 & 8.2 \\
\hline 2 & 8.1 & -- & 8.3 & 7.7 & 8.3 & 7.9 & 7.8 & 8.3 & -- & 7.9 & 8.1 & 8.3 \\
\hline 3 & 8.2 & 8.0 & 8.3 & 7.8 & 8.3 & 8.0 & 7.8 & 8.3 & -- & 8.0 & 8.1 & 8.3 \\
\hline 4 & 8.2 & -- & 8.3 & 7.9 & 8.4 & 8.1 & 7.8 & 8.2 & -- & 8.1 & 8.1 & 8.3 \\
\hline 5 & -- & - & 8.3 & 7.9 & 8.4 & 8.2 & 7.9 & 8.0 & -- & 7.9 & 8.2 & 8.3 \\
\hline 6 & -- & -- & 8.4 & 7.9 & 8.4 & 8.2 & 7.9 & 8.0 & -- & 7.9 & 8.2 & 8.3 \\
\hline 7 & -- & -- & 8.4 & 7.9 & 8.3 & 8.3 & 8.0 & 7.9 & -- & 8.0 & 8.2 & 8.3 \\
\hline 8 & -- & -- & 8.4 & 7.9 & 8.4 & 8.3 & 8.0 & 8.1 & -- & 8.1 & 8.2 & 8.4 \\
\hline 9 & 8.1 & -- & 8.4 & 8.0 & 8.4 & 8.2 & 8.0 & 8.2 & -- & 8.2 & 8.1 & 8.4 \\
\hline 10 & 8.1 & -- & 8.4 & 8.0 & 8.4 & 8.0 & 8.1 & 8.2 & -- & 8.2 & 8.1 & 8.4 \\
\hline 11 & - & - & 8.4 & 8.0 & 8.3 & 7.9 & 8.1 & 8.3 & -- & 8.2 & 8.1 & 8.4 \\
\hline 12 & 8.0 & -- & 8.4 & 8.0 & 8.3 & 7.7 & 8.2 & 8.4 & -- & 8.3 & 8.1 & 8.4 \\
\hline 13 & -- & -- & 8.4 & 8.0 & 8.2 & 7.5 & 8.3 & 8.5 & -- & 8.3 & 8.1 & 8.3 \\
\hline 14 & -- & -- & 8.4 & 8.1 & 8.2 & 7.5 & 8.3 & 8.3 & -- & 8.3 & 8.1 & 8.3 \\
\hline 15 & -- & -. & 8.4 & 8.1 & 8.2 & 7.4 & 8.4 & 8.2 & -- & 8.3 & 8.1 & 8.3 \\
\hline 16 & -- & -- & 8.4 & 8.1 & 8.3 & 7.5 & 8.5 & 8.2 & -- & 8.4 & 8.1 & 8.3 \\
\hline 17 & 7.9 & -- & 8.4 & 8.1 & 8.2 & 7.6 & 8.6 & -- & -- & 8.3 & 8.2 & 8.3 \\
\hline 18 & -- & 7.7 & 8.4 & 8.1 & 8.2 & 7.7 & 8.6 & -- & -- & 8.0 & 8.2 & 8.3 \\
\hline 19 & 7.8 & -- & 8.3 & 8.1 & 8.1 & 7.6 & 8.4 & -- & .. & 8.0 & 8.2 & 8.3 \\
\hline 20 & 7.8 & -- & 8.3 & 8.1 & 8.1 & 7.4 & 8.4 & -. & -- & 8.0 & 8.2 & 8.3 \\
\hline 21 & -- & -- & 8.3 & 8.1 & 8.2 & 7.3 & 8.3 & -- & -- & 8.0 & 8.2 & 8.3 \\
\hline 22 & -- & -- & 8.3 & 8.1 & 8.2 & 7.5 & 8.3 & -- & - & 8.1 & 8.2 & 8.3 \\
\hline 23 & -- & -- & 8.2 & 8.1 & 8.3 & 7.7 & 8.4 & -- & 8.5 & 8.2 & 8.2 & 8.2 \\
\hline 24 & 7.8 & 8.0 & 8.2 & 8.1 & 8.2 & 7.7 & 8.3 & -- & 8.4 & 8.2 & 8.2 & 8.2 \\
\hline 25 & -- & 8.1 & 8.1 & 8.2 & 8.3 & 7.7 & 8.2 & -- & 8.3 & 8.1 & 8.2 & 8.1 \\
\hline 26 & 7.8 & 8.1 & 8.1 & 8.2 & 8.3 & 7.7 & 8.3 & -- & 8.3 & 8.1 & 8.2 & 8.2 \\
\hline 27 & -- & 8.1 & 8.1 & 8.2 & 8.0 & 7.7 & 8.3 & -- & 8.2 & 8.1 & 8.2 & 8.1 \\
\hline 28 & -- & 8.2 & 8.1 & 8.3 & 7.9 & 7.7 & 8.3 & -- & 8.1 & 8.1 & 8.2 & 8.1 \\
\hline 29 & 7.9 & -- & 8.1 & 8.3 & 7.9 & 7.8 & 8.3 & -- & 8.0 & 8.1 & 8.2 & 8.1 \\
\hline 30 & 7.8 & -- & 8.1 & 8.3 & 7.8 & 7.8 & 8.3 & -- & 7.9 & 8.1 & 8.2 & 8.1 \\
\hline 31 & 7.9 & -- & 7.8 & -- & 7.8 & -- & 8.3 & -- & -. & 8.1 & .- & 8.1 \\
\hline
\end{tabular}


Table 12i. Mean daily pH in the Cedar River at site SW-2 (map ID \#32), Seminole Well Field, Cedar Rapids, lowa, April 1996 through March 1999--Continued

\begin{tabular}{|c|c|c|c|c|c|c|c|c|c|c|c|c|}
\hline Day & Jan & $\mathrm{Feb}$ & Mar & Apr & May & Jun & Jul & Aug & Sep & Oct & Nov & Dec \\
\hline & & & & & & 1999 & & & & & & \\
\hline 1 & 8.0 & 8.0 & -- & -- & -- & -- & -- & -- & -- & -- & -- & -- \\
\hline 2 & 8.0 & 8.0 & 8.1 & -- & -- & -- & -- & -- & -- & -- & -- & -- \\
\hline 3 & 8.0 & 8.0 & 8.1 & -- & -- & -- & -- & -- & -- & -- & -- & -- \\
\hline 4 & 7.9 & 8.0 & 8.0 & -- & -- & -- & -- & -- & -- & -- & -- & -- \\
\hline 5 & 7.9 & 8.0 & 8.0 & -- & - & -- & -- & -- & -- & -- & -- & -- \\
\hline 6 & 7.9 & 8.0 & 8.0 & -- & -- & -- & -- & -- & -- & -- & -- & -- \\
\hline 7 & 7.9 & 8.1 & 8.1 & -- & -- & -- & -- & -- & -- & -- & -- & -- \\
\hline 8 & 7.8 & 8.1 & 8.1 & -- & -- & -- & -- & -- & - & -- & - & -- \\
\hline 9 & 7.8 & 8.1 & 8.1 & -- & -- & -- & -- & -- & -- & -- & -- & -- \\
\hline 10 & 7.8 & 8.1 & 8.1 & -- & -- & -- & -- & -- & -- & -- & -- & -- \\
\hline 11 & 7.7 & 8.1 & 8.1 & -- & -- & -- & -- & -- & -- & -- & -- & -- \\
\hline 12 & 7.7 & 8.1 & 8.1 & -- & -- & -- & -- & -- & -- & -- & -- & -- \\
\hline 13 & 7.7 & 8.1 & 8.1 & -- & -- & -- & -- & -- & -- & -- & -- & -- \\
\hline 14 & 7.7 & 8.1 & 8.1 & -- & -- & -- & -- & -- & -- & -- & -- & -- \\
\hline 15 & 7.7 & 8.1 & 8.1 & - & -- & -- & -- & -- & -- & -- & -- & -- \\
\hline 16 & 7.7 & 8.1 & 8.1 & -- & -- & -- & -- & -- & -- & -- & -- & -- \\
\hline 17 & 7.7 & 8.1 & 8.1 & -- & -- & -- & -- & -- & -- & -- & -- & -- \\
\hline 18 & 7.7 & 8.2 & 8.2 & -- & -- & -- & -- & -- & -- & -- & -- & -- \\
\hline 19 & 7.8 & 8.2 & 8.2 & -- & -- & -- & -- & -- & -- & -- & -- & -- \\
\hline 20 & 7.8 & 8.2 & 8.1 & -- & -. & -- & -- & -- & -- & -- & -- & -- \\
\hline 21 & 7.8 & 8.2 & 8.0 & -- & -- & -- & -- & - & -- & -- & -- & -- \\
\hline 22 & 7.8 & 8.2 & 8.1 & -- & -- & -- & -- & -- & -- & -- & -- & -- \\
\hline 23 & 7.8 & 8.2 & 8.1 & -- & -- & -- & -- & -- & -- & -. & -- & -- \\
\hline 24 & 7.8 & 8.2 & 8.1 & -- & -- & -- & -- & -- & -- & -. & -. & -- \\
\hline 25 & 7.8 & 8.2 & 8.2 & -- & -- & -- & -- & -- & -- & -- & - & -- \\
\hline 26 & 7.9 & 8.2 & 8.3 & -- & -- & -- & -- & -- & -- & -- & -- & -- \\
\hline 27 & 7.9 & 8.2 & 8.5 & -- & -- & -- & -- & -- & -- & -- & -- & -- \\
\hline 28 & 7.9 & 8.2 & 8.5 & -- & -- & -- & -- & -- & -- & - & -- & -- \\
\hline 29 & 7.9 & -- & 8.5 & -- & -- & -- & -- & -- & -- & -- & -- & -- \\
\hline 30 & 8.0 & -- & 8.5 & -- & -- & -- & -- & -- & -- & -- & -- & -- \\
\hline 31 & 8.0 & -- & 8.5 & -- & -- & -- & -- & -- & -- & -- & -- & -- \\
\hline
\end{tabular}


Table 13a. Mean daily dissolved-oxygen concentration in ground water at site CRM-2 (map ID \#2), West Well Field, Cedar Rapids, lowa, April 1996 through June 1997

[Dissoved oxygen in milligrams per liter; --, value not measured, not recorded, or rejected after data review]

\begin{tabular}{|c|c|c|c|c|c|c|c|c|c|c|c|c|}
\hline Day & Jan & Feb & Mar & Apr & May & Jun & Jul & Aug & Sep & Oct & Nov & Dec \\
\hline \multicolumn{13}{|c|}{1996} \\
\hline 1 & -- & -- & -- & 8.4 & 8.1 & -- & -- & -- & -. & -- & -- & -- \\
\hline 2 & -- & -- & -- & 8.3 & 8.0 & -. & -- & -- & -- & -- & -- & -- \\
\hline 3 & -. & -- & -- & 8.3 & 8.0 & -- & -- & -- & -- & -- & -- & -- \\
\hline 4 & -- & -. & -- & 8.3 & 7.7 & -- & -- & -. & -- & -- & -- & -- \\
\hline 5 & -- & -- & -- & 8.2 & 7.7 & -- & -- & -- & -- & -- & -- & 3.9 \\
\hline 6 & -- & -- & -- & 8.2 & 7.8 & -- & -- & -- & -- & -- & -- & 4.7 \\
\hline 7 & -- & -- & -- & 8.2 & 8.0 & -- & -- & -- & -- & -- & -- & 4.7 \\
\hline 8 & -- & -- & -- & 8.1 & 8.1 & -- & -- & -- & -- & -- & -- & 4.7 \\
\hline 9 & -- & -- & -- & 8.1 & -- & -. & -- & -- & -. & -- & -. & 4.7 \\
\hline 10 & -- & -- & -- & 8.1 & -- & -- & -- & -- & -- & -- & -. & 4.7 \\
\hline 11 & -. & -- & -- & 8.1 & -- & -- & - & -- & -- & -- & -- & 4.1 \\
\hline 12 & -- & -- & -- & 8.1 & -- & -- & .. & $-\cdot$ & -- & - & -- & 3.0 \\
\hline 13 & - & -- & -- & 8.1 & -- & -- & - & -- & -- & - & -- & 4.5 \\
\hline 14 & -- & -- & -- & 8.1 & -- & -- & - & -- & -- & -- & -- & 4.6 \\
\hline 15 & -- & -- & -- & 8.2 & -- & -- & -- & -- & - & -- & -- & 4.8 \\
\hline 16 & -- & -- & -- & 8.2 & -- & - & - & -- & -- & -- & - & 4.8 \\
\hline 17 & -- & -- & -- & 8.3 & -- & -- & -- & -- & - & -- & -- & 4.8 \\
\hline 18 & -- & -- & -- & 8.3 & -- & -- & -- & -- & -- & -- & -- & 4.7 \\
\hline 19 & -- & -. & -- & 8.3 & -- & -- & - & -- & -- & -- & -- & 4.7 \\
\hline 20 & -- & -- & -- & 8.3 & -- & - & -- & -- & -- & - & -- & 4.7 \\
\hline 21 & - & -- & -- & 8.7 & -- & -- & -- & -- & -- & -- & -- & 4.7 \\
\hline 22 & - & -. & -- & 9.3 & -- & -- & -- & -- & -- & -- & -- & 4.8 \\
\hline 23 & -- & -. & -- & -- & -- & -- & -- & -- & -- & -- & -- & 4.8 \\
\hline 24 & -- & - & -- & 8.2 & -- & -- & -- & -- & -- & -- & -- & 4.8 \\
\hline 25 & -- & -- & -- & 7.5 & -- & -- & -- & -- & -- & -- & -- & 4.8 \\
\hline 26 & -. & -- & -- & 7.5 & -- & -- & -- & -- & -- & -- & -- & 4.8 \\
\hline 27 & -- & -- & -- & 8.8 & -- & -- & -. & -- & -- & -- & -- & 4.7 \\
\hline 28 & -- & -- & -- & 9.3 & -- & -- & -- & -. & -- & -- & -. & 4.8 \\
\hline 29 & -. & -- & -- & 7.9 & -- & -- & -- & -- & -- & -- & -- & 4.7 \\
\hline 30 & - & -- & -- & 8.1 & -- & -- & -. & -- & -- & -- & -- & 4.1 \\
\hline 31 & -- & -- & -- & -- & -. & -- & -- & -- & -- & -- & -- & 2.2 \\
\hline
\end{tabular}


Table 13a. Mean daily dissolved-oxygen concentration in ground water at site CRM-2 (map ID \#2), West Well Field, Cedar Rapids, Iowa, April 1996 through June 1997--Continued

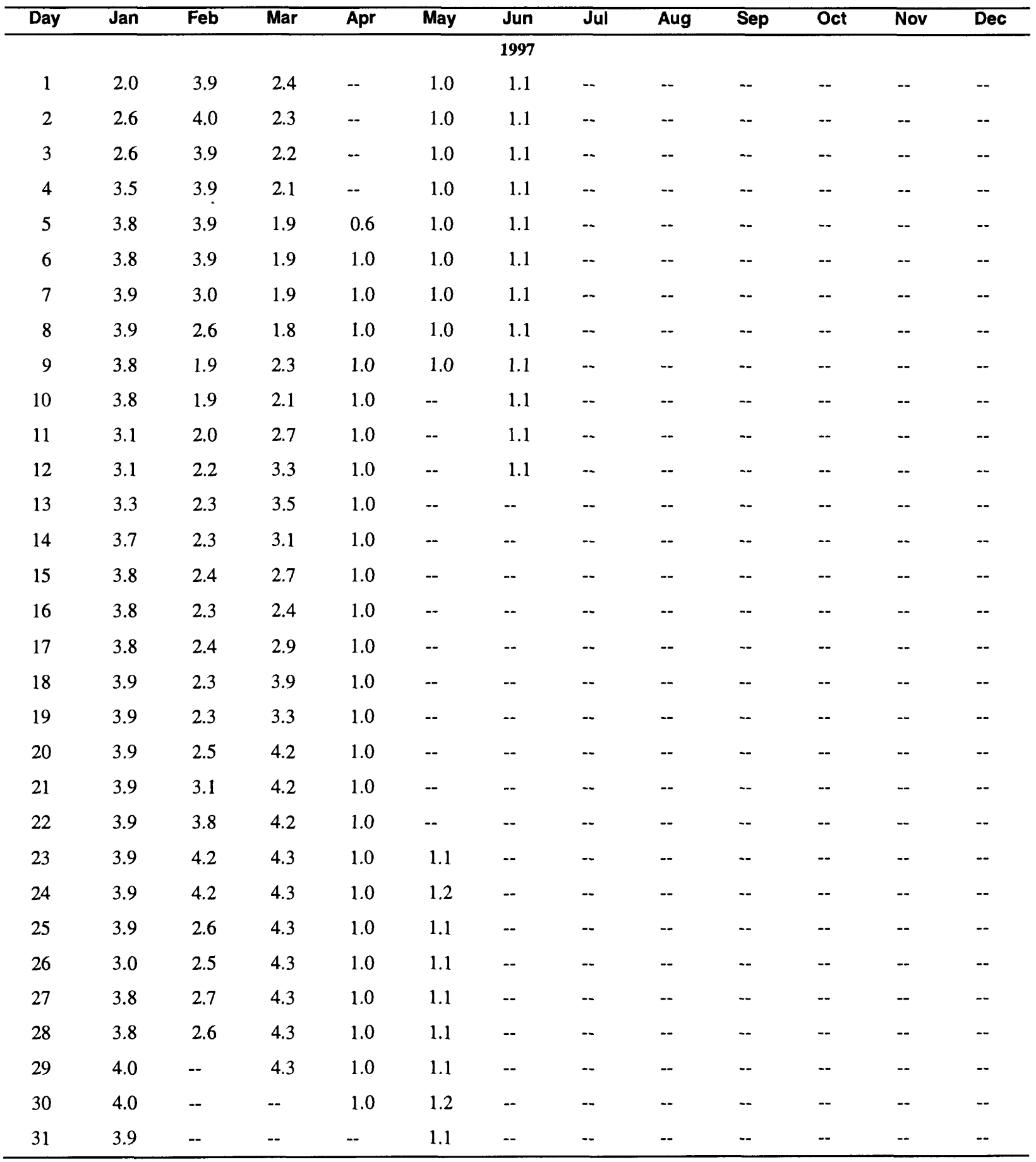

TABLES

165 
Table 13b. Mean daily dissolved-oxygen concentration in ground water at site CRM-12 (map ID \#10), East Well Field, Cedar Rapids, lowa, April 1996 through July 1997

[Dissolved oxygen in milligrams per liter; --, value not measured, not recorded, or rejected after data review]

\begin{tabular}{|c|c|c|c|c|c|c|c|c|c|c|c|c|}
\hline Day & Jan & Feb & Mar & Apr & May & Jun & Jul & Aug & Sep & Oct & Nov & $\overline{D e c}$ \\
\hline & & & & & & 1996 & & & & & & \\
\hline 1 & -- & - & -. & 2.7 & 2.8 & -. & 4.1 & 3.6 & 3.7 & 3.2 & 1.5 & 3.4 \\
\hline 2 & -- & -- & -- & 2.7 & 2.8 & -. & 4.2 & 4.0 & 3.6 & 3.2 & 2.0 & 3.3 \\
\hline 3 & -- & -- & -- & 2.7 & 2.8 & -- & 4.2 & 4.0 & 3.6 & 3.2 & 2.8 & 3.0 \\
\hline 4 & -- & -- & -. & 2.7 & 2.8 & -. & 4.2 & 4.0 & 3.5 & 3.2 & 2.9 & 2.1 \\
\hline 5 & -- & -- & -. & 2.7 & 2.9 & -- & 4.2 & 4.0 & 3.5 & 3.2 & 3.0 & 1.8 \\
\hline 6 & -- & -- & -- & 2.7 & 2.9 & -. & 4.2 & 4.0 & 3.4 & 3.2 & 3.0 & 2.2 \\
\hline 7 & -- & -. & -- & 2.7 & 2.9 & -- & 4.2 & 3.9 & 3.3 & 3.1 & 3.1 & 3.1 \\
\hline 8 & -. & -- & -- & 2.7 & 3.0 & -- & 4.2 & 3.9 & 3.3 & 3.1 & 3.1 & 3.4 \\
\hline 9 & -- & -. & -. & 2.7 & -- & -. & 4.1 & 3.9 & 3.3 & 3.1 & 3.2 & 3.5 \\
\hline 10 & -- & -- & - & 2.7 & -- & -- & 4.1 & 3.9 & 3.3 & 3.1 & 3.2 & 3.6 \\
\hline 11 & -- & -- & -- & 2.7 & -. & -- & 4.0 & 3.8 & 3.2 & 3.1 & 3.2 & 3.8 \\
\hline 12 & -- & -- & -. & 2.7 & -- & -- & 4.0 & 3.7 & 3.2 & 3.1 & 3.1 & 2.2 \\
\hline 13 & -- & -- & -- & 2.8 & -- & -. & 4.0 & 3.7 & 3.8 & 3.1 & 3.1 & 3.2 \\
\hline 14 & -- & -- & -- & 2.8 & -- & -- & 3.9 & 3.6 & 1.5 & 3.1 & 3.1 & 3.4 \\
\hline 15 & -- & -- & -- & 2.8 & -- & -- & 3.9 & 3.5 & 1.6 & 3.1 & 3.1 & 3.4 \\
\hline 16 & -- & -- & -- & 2.8 & -- & -- & 3.9 & 3.5 & 2.5 & 3.1 & 3.3 & 3.4 \\
\hline 17 & -- & -- & -- & 2.8 & -. & -. & 3.9 & 3.5 & 3.1 & 3.1 & 3.3 & 3.4 \\
\hline 18 & -- & -- & -- & 2.8 & -- & -- & 3.9 & 3.4 & 3.3 & 3.1 & 3.3 & 3.4 \\
\hline 19 & -- & -- & -- & 2.8 & -- & -- & 3.9 & 3.4 & 3.3 & 3.2 & 3.4 & 3.4 \\
\hline 20 & -- & .. & -- & 2.8 & -- & -. & 3.9 & 3.4 & 3.3 & 3.2 & 3.4 & 3.4 \\
\hline 21 & -- & -- & -- & 2.8 & -- & -- & 3.8 & 3.4 & 3.3 & 3.2 & 3.5 & 3.4 \\
\hline 22 & -- & -- & -- & 3.0 & -- & -- & 3.8 & 3.5 & 3.3 & 3.2 & 3.4 & 3.4 \\
\hline 23 & -- & -- & -- & 2.7 & - & -. & 3.7 & 3.5 & 3.3 & 3.2 & 3.4 & 3.4 \\
\hline 24 & -- & -- & -- & 2.4 & -- & -- & 3.7 & 3.5 & 3.3 & 3.3 & 3.4 & 3.4 \\
\hline 25 & -- & -- & -- & 2.7 & -- & -- & 3.7 & 3.5 & 3.3 & 3.3 & 3.3 & 3.4 \\
\hline 26 & -- & -- & -- & 2.8 & -- & -- & 3.7 & 3.3 & 3.2 & 3.3 & 3.4 & 3.4 \\
\hline 27 & -- & -- & -- & 2.8 & -. & -- & 3.6 & 3.5 & 3.2 & 3.3 & 3.4 & 3.5 \\
\hline 28 & -- & -. & -- & 2.7 & -- & 1.9 & 3.7 & 4.3 & 3.2 & 3.3 & 3.3 & 3.5 \\
\hline 29 & -- & -- & -- & 2.7 & -- & 3.2 & 3.7 & 4.2 & 3.2 & 3.3 & 3.3 & 3.4 \\
\hline 30 & -- & -- & -- & 2.8 & -- & 4.0 & 2.8 & 4.0 & 3.2 & 3.3 & 3.4 & 3.7 \\
\hline 31 & -- & -- & -- & -- & -- & -- & 1.9 & 3.9 & -- & 1.4 & -- & 3.1 \\
\hline
\end{tabular}


Table 13b. Mean daily dissolved-oxygen concentration in ground water at site CRM-12 (map ID \#10), East Well Field, Cedar Rapids, lowa, April 1996 through July 1997--Continued

\begin{tabular}{|c|c|c|c|c|c|c|c|c|c|c|c|c|}
\hline Day & Jan & Feb & Mar & Apr & May & Jun & Jul & Aug & Sep & Oct & Nov & $\overline{\text { Dec }}$ \\
\hline & & & & & & 1997 & & & & & & \\
\hline 1 & 1.2 & 3.8 & 4.1 & -- & 4.5 & 4.0 & 4.6 & - & -- & -- & -. & -- \\
\hline 2 & 2.6 & 3.8 & 4.1 & -- & 4.5 & 3.9 & 4.6 & -- & -- & -- & -- & -- \\
\hline 3 & 3.5 & 3.8 & 4.1 & -- & 4.5 & 3.9 & 4.6 & -- & -- & -- & -- & -- \\
\hline 4 & 3.5 & 3.7 & 4.1 & -- & 4.4 & 3.9 & 4.6 & -- & -- & -- & -- & -- \\
\hline 5 & 3.6 & 3.8 & 4.1 & 3.3 & 4.4 & 3.9 & 4.8 & -- & -- & -- & -- & -- \\
\hline 6 & 3.9 & 3.8 & 4.0 & 3.9 & 4.4 & 4.1 & 4.7 & - & -- & -- & -- & -- \\
\hline 7 & 3.9 & 4.8 & 4.1 & 4.0 & 4.5 & 4.3 & 4.7 & -- & - & -- & -- & -- \\
\hline 8 & 3.8 & 2.6 & 4.1 & 4.1 & 4.5 & 4.5 & 4.7 & -- & -- & -- & -- & -- \\
\hline 9 & 3.7 & 3.8 & 4.0 & 4.1 & 4.6 & 4.6 & 4.8 & -. & -- & -- & -- & -- \\
\hline 10 & 3.7 & 3.8 & 4.0 & 4.2 & -- & 4.6 & 4.8 & -- & -- & -- & -- & -- \\
\hline 11 & 3.6 & 3.8 & 4.0 & 4.1 & -- & 4.6 & -- & -- & -- & -- & -- & -- \\
\hline 12 & 3.6 & 3.7 & 4.0 & 4.1 & - & 4.7 & -- & -. & -- & -- & -- & -- \\
\hline 13 & 3.6 & 3.7 & 4.0 & 4.1 & -- & 5.3 & -- & -- & - & -- & -- & -- \\
\hline 14 & 3.7 & 3.8 & 3.9 & 4.1 & -- & 3.9 & -- & -- & -- & - & -- & -- \\
\hline 15 & 3.6 & 4.1 & 3.9 & 4.2 & -- & 4.7 & -- & -- & -- & -- & -- & -- \\
\hline 16 & 3.7 & 4.1 & 3.9 & 4.2 & -- & 4.7 & -- & -- & -- & - & -- & -- \\
\hline 17 & 3.7 & 4.1 & 4.0 & 4.4 & -- & 4.7 & -- & - & -- & - & -- & -- \\
\hline 18 & 3.7 & 4.1 & 4.2 & 4.4 & -- & 4.7 & -- & -- & -- & -- & -- & - \\
\hline 19 & 3.7 & 4.1 & 4.2 & 4.5 & -- & 4.7 & - & - & -- & -. & -- & - \\
\hline 20 & 3.7 & 4.1 & 4.2 & 4.5 & -- & 4.8 & -- & -- & -- & -- & - & -- \\
\hline 21 & 3.6 & 4.1 & 4.2 & 4.6 & -- & 4.8 & -- & -- & -- & -- & -- & -- \\
\hline 22 & 3.7 & 4.1 & 4.2 & 4.5 & - & 4.8 & -- & -- & -- & -- & -- & -- \\
\hline 23 & 3.7 & 4.0 & 4.2 & 4.6 & 3.2 & 4.9 & -- & - & -- & -- & -- & -- \\
\hline 24 & 3.6 & 4.0 & 4.3 & 4.5 & 3.9 & 5.0 & -- & -- & -- & -- & -- & -- \\
\hline 25 & 3.7 & 4.0 & 4.2 & 4.5 & 4.0 & 4.7 & -- & -- & -- & -- & -- & -- \\
\hline 26 & 3.9 & 4.0 & 4.3 & 4.5 & 4.1 & 4.3 & -- & -- & -- & -- & -- & -- \\
\hline 27 & 3.9 & 4.0 & 4.3 & 4.5 & 4.0 & 4.6 & -- & $\cdots$ & -- & -- & -- & -- \\
\hline 28 & 3.9 & 4.1 & 4.3 & 4.5 & 4.0 & 4.6 & -- & $-\cdot$ & -- & -- & -- & - \\
\hline 29 & 3.8 & -- & 4.2 & 4.5 & 4.0 & 4.6 & -- & -- & -- & -- & -- & - \\
\hline 30 & 3.8 & -- & -- & 4.5 & 3.7 & 4.6 & -- & - & -- & - & -- & - \\
\hline 31 & 3.8 & -- & -- & -- & 3.8 & -- & -- & -- & -- & -- & - & - \\
\hline
\end{tabular}


Table 13c. Mean daily dissolved-oxygen concentration in ground water at site CRM-20 (map ID \#17), Northwest Well Field, Cedar Rapids, lowa, February 1998 through March 1999

[Dissolved oxygen in milligrams per liter, --, value not measured, not recorded, or rejected after data review]

\begin{tabular}{|c|c|c|c|c|c|c|c|c|c|c|c|c|}
\hline Day & Jan & Feb & Mar & Apr & May & Jun & Jul & Aug & Sep & Oct & Nov & Dec \\
\hline & & & & & & 1998 & & & & & & \\
\hline 1 & -- & -- & 0.1 & 0.1 & 0.1 & 0.1 & 0.1 & 0.1 & -- & 0.1 & 0.1 & 0.1 \\
\hline 2 & -- & -- & 0.1 & 0.1 & 0.1 & 0.1 & 0.1 & 0.1 & -- & 0.1 & 0.1 & 0.1 \\
\hline 3 & -- & -- & 0.1 & 0.1 & 0.1 & 0.1 & 0.1 & 0.1 & -- & 0.1 & 0.1 & 0.1 \\
\hline 4 & -- & -- & 0.1 & 0.1 & 0.1 & 0.1 & 0.1 & 0.1 & -- & 0.1 & 0.1 & 0.1 \\
\hline 5 & -. & -. & 0.1 & 0.1 & 0.1 & 0.1 & 0.1 & 0.1 & -- & 0.1 & 0.1 & 0.1 \\
\hline 6 & - & -- & 0.1 & 0.1 & 0.1 & 0.1 & 0.1 & 0.1 & -- & 0.1 & 0.1 & 0.1 \\
\hline 7 & -- & -- & 0.1 & 0.1 & 0.1 & 0.1 & 0.1 & 0.1 & -- & 0.1 & 0.1 & 0.1 \\
\hline 8 & -. & -- & 0.1 & 0.1 & 0.1 & 0.1 & 0.1 & 0.1 & -- & 0.1 & 0.1 & 0.1 \\
\hline 9 & -- & -- & 0.1 & 0.1 & 0.1 & 0.1 & 0.1 & 0.1 & -- & 0.1 & 0.1 & 0.1 \\
\hline 10 & -- & 0.2 & 0.1 & 0.1 & 0.1 & 0.1 & 0.1 & 0.1 & -- & 0.1 & 0.1 & 0.1 \\
\hline 11 & - & 0.2 & 0.1 & 0.1 & 0.1 & 0.1 & 0.1 & 0.1 & -- & 0.1 & 0.1 & 0.1 \\
\hline 12 & -- & 0.2 & 0.1 & 0.1 & 0.1 & 0.1 & 0.1 & 0.1 & -- & 0.1 & 0.1 & 0.1 \\
\hline 13 & -- & 0.2 & 0.1 & 0.1 & 0.1 & 0.1 & 0.1 & 0.1 & -- & 0.1 & 0.1 & 0.1 \\
\hline 14 & -- & 0.2 & 0.1 & 0.1 & 0.1 & 0.1 & 0.1 & 0.1 & -- & 0.1 & 0.1 & 0.1 \\
\hline 15 & -- & 0.2 & 0.1 & 0.1 & 0.1 & 0.1 & 0.1 & 0.1 & -- & 0.1 & 0.1 & 0.1 \\
\hline 16 & -. & 0.2 & 0.1 & 0.1 & 0.1 & 0.1 & 0.1 & 0.1 & -- & 0.1 & 0.1 & 0.2 \\
\hline 17 & -- & 0.2 & 0.1 & 0.1 & 0.1 & 0.1 & 0.1 & -- & -- & 0.1 & 0.1 & 0.2 \\
\hline 18 & -- & 0.1 & 0.1 & 0.1 & 0.1 & 0.1 & 0.1 & -. & -- & 0.1 & 0.1 & 0.1 \\
\hline 19 & -- & 0.1 & 0.1 & 0.1 & 0.1 & 0.1 & 0.1 & -- & -- & 0.1 & 0.1 & 0.1 \\
\hline 20 & -- & 0.1 & 0.1 & 0.1 & 0.1 & 0.1 & 0.1 & -- & -- & 0.1 & 0.1 & 0.1 \\
\hline 21 & -- & 0.1 & 0.1 & 0.1 & 0.1 & 0.1 & 0.1 & -- & -- & 0.1 & 0.1 & 0.1 \\
\hline 22 & -- & 0.1 & 0.1 & 0.1 & 0.1 & 0.1 & 0.1 & -- & -- & 0.1 & 0.1 & 0.1 \\
\hline 23 & -- & 0.2 & 0.1 & 0.1 & 0.1 & 0.1 & 0.1 & -- & 0.1 & 0.1 & 0.1 & 0.1 \\
\hline 24 & -- & 0.2 & 0.1 & 0.1 & 0.1 & 0.1 & 0.1 & -. & 0.1 & 0.1 & 0.1 & 0.1 \\
\hline 25 & -- & 0.2 & 0.1 & 0.1 & 0.1 & 0.1 & 0.1 & -. & 0.1 & 0.1 & 0.1 & 0.1 \\
\hline 26 & -- & 0.2 & 0.1 & 0.1 & 0.1 & 0.1 & 0.1 & -- & 0.1 & 0.1 & 0.1 & 0.2 \\
\hline 27 & -- & 0.2 & 0.1 & 0.1 & 0.1 & 0.1 & 0.1 & -. & 0.1 & 0.1 & 0.1 & 0.2 \\
\hline 28 & -- & 0.1 & 0.1 & 0.1 & 0.1 & 0.1 & 0.1 & -. & 0.1 & 0.1 & 0.1 & 0.3 \\
\hline 29 & -- & -- & 0.1 & 0.1 & 0.1 & 0.1 & 0.1 & -- & 0.1 & 0.1 & 0.1 & 0.5 \\
\hline 30 & -- & -- & 0.1 & 0.1 & 0.1 & 0.1 & 0.1 & -- & 0.1 & 0.1 & 0.1 & 0.7 \\
\hline 31 & -- & -- & 0.1 & -- & 0.1 & -- & 0.1 & -- & -- & 0.1 & -- & 0.9 \\
\hline
\end{tabular}


Table 13c. Mean daily dissolved-oxygen concentration in ground water at site CRM-20 (map ID \#17), Northwest Well Field, Cedar Rapids, lowa, February 1998 through March 1999--Continued

\begin{tabular}{|c|c|c|c|c|c|c|c|c|c|c|c|c|}
\hline Day & Jan & Feb & Mar & Apr & May & Jun & Jul & Aug & Sep & Oct & Nov & Dec \\
\hline & & & & & & 1999 & & & & & & \\
\hline 1 & 1.3 & 6.4 & 6.8 & -. & -- & -. & -- & -- & -- & -- & -- & -- \\
\hline 2 & 2.0 & 6.4 & 7.2 & -- & -- & -. & -- & -- & -- & -- & -. & -- \\
\hline 3 & 2.8 & 6.5 & 7.5 & -- & -- & -- & -- & -- & -. & -- & -- & -- \\
\hline 4 & 3.5 & 6.6 & 7.7 & -- & -- & -. & -. & -- & -- & -. & -- & -. \\
\hline 5 & 3.9 & 6.6 & 7.9 & -- & -- & -- & -- & -- & -- & -- & -- & -- \\
\hline 6 & 5.2 & 6.6 & 8.1 & - & -- & -- & -- & -- & -- & -- & -. & -- \\
\hline 7 & 6.3 & 6.6 & 8.3 & -. & -- & -- & -- & -- & -- & -- & .. & -- \\
\hline 8 & 7.0 & 6.7 & 8.5 & -. & -- & -- & -. & -- & -- & - & -- & -- \\
\hline 9 & 7.3 & 6.9 & 8.6 & -- & -- & -- & -- & -- & -- & -- & -- & -- \\
\hline 10 & 7.3 & 7.2 & 8.6 & -- & -- & -- & .. & -- & -- & -. & -- & .. \\
\hline 11 & 7.4 & 7.6 & 8.3 & -- & -- & -- & -- & -- & -- & -- & -- & -- \\
\hline 12 & 7.4 & 7.9 & 8.0 & -- & -. & -. & -. & -- & -- & -- & -. & -- \\
\hline 13 & 7.3 & 8.0 & 7.9 & - & -- & -- & -- & -- & -- & -- & -- & -- \\
\hline 14 & 6.8 & 7.9 & 7.7 & - & -- & -- & -- & -- & -. & -- & -- & -- \\
\hline 15 & 7.1 & 7.8 & 7.6 & -- & -- & -- & -- & -- & -- & -- & -- & -- \\
\hline 16 & 7.3 & 7.8 & 7.6 & -. & -- & -- & -- & -- & -- & -- & -- & -- \\
\hline 17 & 7.3 & 7.9 & 7.5 & -. & -- & -- & -. & -- & -- & -- & -- & -- \\
\hline 18 & 7.1 & 8.0 & 7.7 & -- & -- & -- & -- & -. & -- & - & -- & -- \\
\hline 19 & 7.3 & 8.0 & 8.0 & - & -- & -- & -. & -- & -- & -- & -- & -- \\
\hline 20 & 7.1 & 7.7 & 8.3 & -- & -- & -- & -- & -- & -- & -- & -- & -- \\
\hline 21 & 6.8 & 7.5 & 8.8 & -- & -- & -. & -- & -- & -- & -- & -- & -- \\
\hline 22 & 6.2 & 7.4 & 8.8 & -- & -. & -. & -- & -. & -- & -- & -- & -. \\
\hline 23 & 5.9 & 7.2 & 8.3 & -- & -- & -- & -- & -- & - & -- & -- & -. \\
\hline 24 & 5.8 & 7.1 & 7.8 & -- & -- & -. & -- & -- & -. & -- & -- & -. \\
\hline 25 & 5.8 & 7.0 & 7.3 & -- & -- & -- & -- & -- & -- & -. & -- & -. \\
\hline 26 & 6.0 & 7.0 & 6.9 & -. & -. & -- & -- & -- & -- & -. & -- & -- \\
\hline 27 & 6.1 & 6.8 & 6.3 & -- & -- & -- & -- & -- & -- & -. & -- & -- \\
\hline 28 & 6.0 & 6.7 & 5.8 & -- & -- & .. & - & -- & -- & -. & -. & -- \\
\hline 29 & 6.2 & .. & 5.1 & - & -- & -- & -- & -- & -- & -- & -- & -- \\
\hline 30 & 6.4 & -- & 4.9 & -- & -- & -- & -- & -- & - & -- & -- & -- \\
\hline 31 & 6.4 & -. & 4.8 & -- & -- & -- & -- & -- & -- & -- & -- & -- \\
\hline
\end{tabular}


Table 13d. Mean daily dissolved-oxygen concentration in ground water at site CRM-21 (map ID \#18), Northwest Well Field, Cedar Rapids, lowa, February 1998 through March 1999

[Dissolved oxygen in milligrams per liter; --, value not measured, not recorded, or rejected after data review]

\begin{tabular}{|c|c|c|c|c|c|c|c|c|c|c|c|c|}
\hline Day & Jan & Feb & Mar & Apr & May & Jun & Jul & Aug & Sep & Oct & Nov & Dec \\
\hline & & & & & & 1998 & & & & & & \\
\hline 1 & -- & -- & 0.1 & 7.9 & 4.1 & 0.1 & 0.6 & 0.1 & -- & 0.0 & 0.1 & 0.2 \\
\hline 2 & - & -- & 0.1 & 8.3 & 4.8 & 0.1 & 1.2 & 0.1 & -- & 0.0 & 0.1 & 0.2 \\
\hline 3 & -- & -- & 0.1 & 8.6 & 3.7 & 0.2 & 6.0 & 0.1 & -- & 0.0 & 0.1 & 0.1 \\
\hline 4 & -- & -- & 0.3 & 9.0 & 1.7 & 0.1 & 5.8 & 0.1 & -- & 0.0 & 0.1 & 0.1 \\
\hline 5 & -. & -- & 0.2 & 9.3 & 3.1 & 0.1 & 4.1 & 0.1 & -- & 0.0 & 0.1 & 0.1 \\
\hline 6 & -. & -- & 0.1 & 9.3 & 4.4 & 0.1 & 2.1 & 0.1 & -- & 0.0 & 0.1 & 0.1 \\
\hline 7 & -- & -- & 0.1 & 9.0 & 4.6 & 0.1 & 0.7 & 0.1 & -- & 0.1 & 0.1 & 0.1 \\
\hline 8 & -- & -- & 0.1 & 8.7 & 4.9 & 0.1 & 0.1 & 0.1 & - & 0.0 & 0.0 & 0.4 \\
\hline 9 & -- & -- & 0.1 & 8.4 & 5.3 & 0.1 & 0.0 & 0.1 & -- & 0.1 & 0.0 & 0.8 \\
\hline 10 & -- & 0.8 & 0.2 & 8.6 & 5.5 & 0.1 & 0.0 & 0.1 & -- & 0.1 & 0.0 & 1.2 \\
\hline 11 & -- & 0.7 & 0.1 & 9.1 & 5.5 & 0.1 & 0.0 & 0.1 & -. & 0.1 & 0.0 & 1.7 \\
\hline 12 & -- & 0.6 & 0.2 & 9.2 & 5.5 & 0.1 & 0.0 & 0.1 & -- & 0.1 & 0.0 & 2.2 \\
\hline 13 & -- & 0.5 & 0.2 & 8.8 & 5.2 & 0.0 & 0.1 & 0.1 & -- & 0.1 & 0.0 & 2.6 \\
\hline 14 & -- & 0.6 & 0.2 & 9.8 & 4.5 & 0.0 & 0.1 & 0.1 & -- & 0.1 & 0.0 & 2.9 \\
\hline 15 & -- & 0.5 & 0.1 & 11.5 & 3.4 & 0.0 & 0.0 & 0.1 & -- & 0.1 & 0.0 & 3.0 \\
\hline 16 & -- & 0.5 & 0.1 & 10.9 & 2.5 & 0.0 & 0.0 & 0.1 & -- & 0.0 & 0.0 & 2.8 \\
\hline 17 & -- & 0.5 & 0.1 & 10.0 & 2.0 & 0.0 & 0.0 & -- & -- & 0.0 & 0.0 & 2.3 \\
\hline 18 & - & 0.5 & 0.1 & 8.8 & 1.9 & 0.0 & 0.0 & -- & -- & 0.0 & 0.1 & 1.8 \\
\hline 19 & -- & 0.4 & 0.1 & 7.4 & 1.7 & 0.0 & 0.0 & -- & -- & 0.0 & 0.1 & 1.5 \\
\hline 20 & -- & 0.3 & 0.1 & 6.0 & 1.5 & 0.0 & 0.0 & -- & -- & 0.0 & 0.1 & 1.4 \\
\hline 21 & -- & 0.2 & 0.1 & 4.4 & 1.1 & 0.0 & 0.0 & - & -. & 0.0 & 0.1 & 1.3 \\
\hline 22 & -- & 0.2 & 0.1 & 2.7 & 0.7 & 0.0 & 0.0 & - & -- & 0.1 & 0.1 & 1.3 \\
\hline 23 & - & 0.1 & 0.2 & 1.3 & 0.4 & 0.0 & 0.0 & -- & 0.1 & 0.3 & 0.1 & 1.2 \\
\hline 24 & -- & 0.1 & 0.3 & 0.5 & 0.2 & 1.0 & 0.1 & -- & 0.1 & 0.6 & 0.1 & 1.1 \\
\hline 25 & -- & 0.1 & 0.2 & 0.2 & 0.2 & 6.0 & 0.3 & -- & 0.1 & 0.8 & 0.3 & 1.0 \\
\hline 26 & -- & 0.1 & 0.2 & 0.1 & 0.4 & 5.7 & 0.6 & -- & 0.1 & 0.3 & 0.4 & 0.9 \\
\hline 27 & - & 0.1 & 0.2 & 0.1 & 1.4 & 5.5 & 0.7 & -- & 0.1 & 0.0 & 0.4 & 0.8 \\
\hline 28 & -- & 0.1 & 0.1 & 0.1 & 2.2 & 5.5 & 0.7 & -- & 0.1 & 0.0 & 0.3 & 0.7 \\
\hline 29 & -- & -- & 0.1 & 0.1 & 2.1 & 5.5 & 0.9 & -- & 0.1 & 0.1 & 0.2 & 0.6 \\
\hline 30 & - & -- & 0.1 & 0.8 & 1.1 & 3.7 & 0.7 & -- & 0.1 & 0.1 & 0.2 & 0.5 \\
\hline 31 & -- & -- & 3.5 & -- & 0.2 & -- & 0.5 & -- & -- & 0.1 & -- & 0.5 \\
\hline
\end{tabular}


Table 13d. Mean daily dissolved-oxygen concentration in ground water at site CRM-21 (map ID \#18), Northwest Well Field, Cedar Rapids, lowa, February 1998 through March 1999--Continued

\begin{tabular}{|c|c|c|c|c|c|c|c|c|c|c|c|c|}
\hline Day & Jan & Feb & Mar & Apr & May & Jun & Jul & Aug & Sep & Oct & Nov & Dec \\
\hline & & & & & & 1999 & & & & & & \\
\hline 1 & 0.5 & 0.2 & 12.1 & -- & -- & -- & -- & -- & -- & -- & -- & -- \\
\hline 2 & 0.5 & 0.2 & 11.9 & -- & -- & -- & -- & - & -- & -- & -- & -- \\
\hline 3 & 0.4 & 0.2 & 11.5 & -. & -- & -- & -- & -- & -- & -. & -- & -- \\
\hline 4 & 0.4 & 0.2 & 11.0 & -- & - & -- & -- & -- & -- & -- & -- & -- \\
\hline 5 & 0.4 & 0.2 & 9.5 & -- & -- & -- & -- & -- & -- & -- & -- & -- \\
\hline 6 & 0.7 & 0.2 & 7.3 & -- & -- & -. & -- & -. & -- & -- & -- & -- \\
\hline 7 & 1.1 & 0.2 & 4.3 & -- & -- & -- & -- & -. & -- & -- & -- & -- \\
\hline 8 & 1.3 & 0.2 & 2.7 & -- & -. & -- & -. & -- & -- & -- & -. & -- \\
\hline 9 & 1.4 & 0.2 & 2.4 & -- & -- & -- & -- & -- & -- & -- & -- & -- \\
\hline 10 & 1.4 & 0.1 & 2.1 & -- & -- & -- & -- & -- & -- & -- & -- & -- \\
\hline 11 & 1.4 & 0.1 & 2.0 & -- & -- & -- & -- & -- & -- & -- & -- & -- \\
\hline 12 & 1.4 & 0.1 & 2.5 & -- & -- & -- & -- & -- & -- & -- & -- & -- \\
\hline 13 & 1.3 & 0.1 & 2.8 & -- & -- & -. & -- & -. & -- & -- & -- & -. \\
\hline 14 & 1.3 & 0.1 & 3.0 & -- & -- & -- & -- & -. & -- & -- & -- & - \\
\hline 15 & 1.6 & 0.1 & 2.9 & -- & - & -. & -- & -. & -- & -- & - & -- \\
\hline 16 & 1.8 & 0.1 & 2.7 & -- & -- & -- & -- & -- & -- & -- & -- & -- \\
\hline 17 & 1.9 & 0.1 & 2.4 & -. & -- & -. & -- & - & -- & -- & -- & -- \\
\hline 18 & 2.1 & 0.1 & 1.9 & -- & -. & -- & -- & -- & -- & -- & -- & -- \\
\hline 19 & 2.3 & 1.7 & 1.5 & -- & -- & -- & -- & -- & -- & -- & -- & -- \\
\hline 20 & 2.1 & 4.8 & 1.2 & -- & -- & -- & -- & -- & -- & -- & -- & -- \\
\hline 21 & 1.7 & 7.0 & 1.0 & -- & -- & -- & -- & -- & -- & -- & -- & -- \\
\hline 22 & 1.2 & 8.8 & 0.9 & -- & -- & -- & - & -- & -- & -- & -- & -- \\
\hline 23 & 0.9 & 10.2 & 0.8 & -. & -- & -- & -- & -- & -- & -. & - & -- \\
\hline 24 & 0.7 & 11.2 & 0.8 & -. & - & -- & - & - & -- & -. & -- & -. \\
\hline 25 & 0.5 & 11.9 & 0.8 & -- & - & -- & -- & -- & -- & -- & -- & -- \\
\hline 26 & 0.4 & 12.4 & 0.8 & .. & -- & -- & -. & -- & -- & -. & -- & -- \\
\hline 27 & 0.3 & 12.6 & 0.6 & -- & -- & -- & -- & -- & -- & -- & -- & -- \\
\hline 28 & 0.3 & 12.5 & 0.6 & -- & -- & -- & -- & -- & -- & -- & -- & -- \\
\hline 29 & 0.3 & -- & 0.6 & -- & -- & -- & -- & -- & -- & -- & -- & -- \\
\hline 30 & 0.2 & -- & 0.6 & -- & -- & -- & -- & -- & -- & -- & -- & -. \\
\hline 31 & 0.2 & -- & 0.5 & -- & -- & -- & -- & -- & -- & -- & -- & -- \\
\hline
\end{tabular}


Table 13e. Mean daily dissolved-oxygen concentration in ground water at site CRM-22 (map ID \#19), Seminole Well Field, Cedar Rapids, lowa, February 1998 through March 1999

[Dissolved oxygen in milligrams per liter; -- , value not measured, not recorded, or rejected after data review]

\begin{tabular}{|c|c|c|c|c|c|c|c|c|c|c|c|c|}
\hline Day & Jan & Feb & Mar & Apr & May & Jun & Jul & Aug & Sep & Oct & Nov & Dec \\
\hline \multicolumn{13}{|c|}{1998} \\
\hline 1 & -. & -- & 0.1 & 7.9 & 0.4 & 0.0 & - & -- & -- & 0.0 & 0.0 & 0.1 \\
\hline 2 & -- & -- & 0.1 & 8.3 & 0.1 & 0.1 & -. & -- & -- & 0.0 & 0.1 & 0.1 \\
\hline 3 & -- & -- & 0.1 & 8.6 & 0.1 & 0.0 & -- & -- & -- & 0.0 & 0.1 & 0.1 \\
\hline 4 & -- & -- & 0.3 & 9.0 & 0.3 & 0.0 & -- & -- & -- & 0.0 & 0.0 & 0.1 \\
\hline 5 & -- & -- & 0.2 & 9.3 & 0.4 & 0.0 & -- & -- & -- & 0.0 & 0.0 & 0.1 \\
\hline 6 &.- & -- & 0.1 & 9.3 & 0.2 & 0.0 & -- & -- & -- & 0.0 & 0.0 & 0.0 \\
\hline 7 & -- & -- & 0.1 & 9.0 & 0.1 & 0.0 & -. & -- & - & 0.0 & 0.0 & 0.0 \\
\hline 8 & -. & -- & 0.1 & 8.7 & 0.1 & 0.0 & -- & -- & -- & 0.0 & 0.0 & 0.1 \\
\hline 9 & -- & -- & 0.1 & 8.4 & 0.1 & 0.0 & -- & -- & -- & 0.0 & 0.0 & 0.1 \\
\hline 10 & -- & 0.8 & 0.2 & 8.6 & 0.1 & 0.0 & -- & -- & -- & 0.1 & 0.1 & 0.1 \\
\hline 11 & -- & 0.7 & 0.1 & 9.1 & 0.1 & 0.0 & -- & -- & -- & 0.0 & 0.1 & 0.1 \\
\hline 12 & -- & 0.6 & 0.2 & 9.2 & 0.1 & 0.0 & -- & -- & -- & 0.0 & 0.1 & 0.1 \\
\hline 13 & -- & 0.5 & 0.2 & 8.8 & 0.1 & 0.0 & -- & -- & -- & 0.0 & 0.1 & 0.1 \\
\hline 14 & -- & 0.6 & 0.2 & 6.8 & 0.1 & 0.0 & -- & -- & -- & 0.0 & 0.0 & 0.1 \\
\hline 15 & -- & 0.5 & 0.1 & 3.2 & 0.1 & 0.0 & -- & -- & -- & 0.0 & 0.0 & 0.1 \\
\hline 16 & -- & 0.5 & 0.1 & 2.3 & 0.1 & 0.0 & -- & -- & -- & 0.0 & 0.0 & 0.1 \\
\hline 17 & -- & 0.5 & 0.1 & 1.5 & 0.2 & 0.0 & -- & -- & -- & 0.0 & 0.0 & 0.1 \\
\hline 18 & -- & 0.5 & 0.1 & 1.0 & 0.7 & 0.0 & -- & -- & -- & 0.0 & 0.0 & 0.1 \\
\hline 19 & -- & 0.4 & 0.1 & 1.0 & 0.5 & 0.0 & -- & -- & -- & 0.0 & 0.0 & 0.1 \\
\hline 20 & -- & 0.3 & 0.1 & 1.2 & 0.1 & 0.0 & -- & -- & -- & 0.0 & 0.0 & 0.1 \\
\hline 21 & -- & 0.2 & 0.1 & 0.5 & 0.1 & 0.0 & -- & - & -- & 0.0 & 0.0 & 0.1 \\
\hline 22 & -- & 0.2 & 0.1 & 0.4 & 0.1 & 0.0 & -- & -- & -- & 0.0 & 0.1 & 0.1 \\
\hline 23 & -- & 0.1 & 0.2 & 0.5 & 0.0 & -- & -- & -- & 0.1 & 0.1 & 0.8 & 0.1 \\
\hline 24 & - & 0.1 & 0.3 & 9.5 & 0.0 & -- & -- & -- & 0.1 & 0.0 & 0.1 & 0.1 \\
\hline 25 & -. & 0.1 & 0.2 & 9.1 & 0.0 & -. & -- & -- & 0.0 & 0,0 & 0.1 & 0.0 \\
\hline 26 & -- & 0.1 & 0.2 & 7.0 & 0.1 & -- & -- & -- & 0.0 & 0.0 & 0.1 & 0.0 \\
\hline 27 & -- & 0.1 & 0.2 & 4.9 & 0.1 & -- & -- & -- & 0.0 & 0.0 & 0.1 & 0.1 \\
\hline 28 & -- & 0.1 & 0.1 & 3.6 & 0.1 & -- & -. & -- & 0.0 & 0.0 & 0.1 & 0.5 \\
\hline 29 & -- & -- & 0.1 & 2.4 & 0.1 & -- & -- & -- & 0.0 & 0.0 & 0.1 & 1.5 \\
\hline 30 & -- & -- & 0.1 & 1.2 & 0.1 & -- & - & -- & 0.0 & 0.0 & 0.1 & 1.6 \\
\hline 31 & -- & -. & 3.5 & -- & 0.0 & -- & -. & -- & -. & 0.0 & -- & 2.2 \\
\hline
\end{tabular}


Table 13e. Mean daily dissolved-oxygen concentration in ground water at site CRM-22 (map ID \#19), Seminole Well Field, Cedar Rapids, lowa, February 1998 through February 1999--Continued

\begin{tabular}{|c|c|c|c|c|c|c|c|c|c|c|c|c|}
\hline Day & Jan & Feb & Mar & Apr & May & Jun & Jul & Aug & Sep & Oct & Nov & Dec \\
\hline & & & & & & 1999 & & & & & & \\
\hline 1 & 2.8 & 6.6 & 8.6 & -- & -- & -- & -- & .- & -- & -- & -- & -- \\
\hline 2 & 3.3 & 6.6 & 8.0 & -- & -- & -- & -- & -- & -- & -- & -. & -- \\
\hline 3 & 3.7 & 6.7 & 8.3 & -- & -- & -. & -- & -- & -- & -- & -- & -- \\
\hline 4 & 4.0 & 6.7 & 8.5 & -- & - & -. & - & -- & -- & -. & -- & -- \\
\hline 5 & 4.3 & 6.8 & 8.6 & .- & -. & -- & -. & -- & -- & -. & -. & -- \\
\hline 6 & 4.6 & 6.8 & 8.7 & -- & -. & -- & -- & -- & -- & -- & -- & -- \\
\hline 7 & 4.7 & 6.8 & 8.8 & -- & -- & -. & - & -- & -- & -- & -- & - \\
\hline 8 & 5.3 & 6.8 & 8.9 & -- & -- & .- & -- & -- & -- & -- & -- & - \\
\hline 9 & 6.0 & 6.9 & 8.9 & -- & -- & -- & -- & -- & -- & -- & -- & -- \\
\hline 10 & 6.6 & 7.0 & 8.9 & -- & -- & -- & -- & -- & -- & -- & -- & -. \\
\hline 11 & 7.3 & 7.2 & 8.7 & -- & - & -- & -- & -- & -- & -- & -- & -- \\
\hline 12 & 7.2 & 7.3 & 8.4 & -- & -- & -- & -- & -- & -- & -- & -- & -- \\
\hline 13 & 7.2 & 7.5 & 8.2 & -- & -- & -- & -- & -- & -- & -- & -- & -- \\
\hline 14 & 7.4 & 7.5 & 8.1 & -- & -- & -- & -- & -- & -- & -- & -- & -- \\
\hline 15 & 7.3 & 7.5 & 7.9 & -- & - & -- & -- & -- & -- & -- & -- & -- \\
\hline 16 & 6.9 & 7.4 & 7.7 & -- & -- & -. & -- & -- & -- & -. & -- & -- \\
\hline 17 & 6.5 & 6.9 & 7.7 & -- & -- & -- & -- & -- & -- & -- & -- & -- \\
\hline 18 & 6.4 & 6.7 & 7.9 & -- & -- & -- & -- & -- & -- & -- & -- & -- \\
\hline 19 & 6.3 & 6.4 & 8.0 & -- & -- & -- & -- & -- & -- & -- & -- & -- \\
\hline 20 & 6.3 & 6.3 & 7.6 & -- & - & -- & -- & -- & -- & -- & -- & -- \\
\hline 21 & 6.3 & 6.1 & 7.5 & -- & -- & -- & -- & - & -- & -- & -- & -- \\
\hline 22 & 6.3 & 5.8 & 7.5 & -- & -- & -- & -- & -- & -- & -- & -- & -- \\
\hline 23 & 6.2 & 5.8 & 7.4 & -- & -- & -- & -- & - & -- & -- & -- & -. \\
\hline 24 & 6.2 & 6.0 & 7.4 & -- & -- & -- & -- & -- & -- & -- & -- & -- \\
\hline 25 & 6.2 & 6.4 & 7.2 & -- & -. & -- & -- & -- & -- & -- & -- & -- \\
\hline 26 & 6.3 & 6.9 & 7.2 & -. & -. & -- & - & -- & -- & -. & -- & -- \\
\hline 27 & 6.4 & 7.3 & 7.5 & -- & -- & -. & -. & -- & -- & -- & -- & -- \\
\hline 28 & 6.3 & 8.0 & 7.4 & -- & -. & -. & -- & -- & -. & -- & -- & -- \\
\hline 29 & 6.5 & -- & 6.7 & -. & -- & -- & -- & -- & -. & -. & -- & -. \\
\hline 30 & 6.5 & -- & 6.5 & -- & -- & -- & -- & -- & -- & -- & -- & -- \\
\hline 31 & 6.5 & -- & 6.4 & -- & -- & -. & -- & -. & -- & -. & -- & -- \\
\hline
\end{tabular}


Table 13f. Mean daily dissolved-oxygen concentration in ground water at site CRM-23 (map ID \#20), Seminole Well Field, Cedar Rapids, lowa, February 1998 through March 1999

[Dissolved oxygen in milligrams per liter; --, value not measured, not recorded, or rejected after data review]

\begin{tabular}{|c|c|c|c|c|c|c|c|c|c|c|c|c|}
\hline Day & Jan & Feb & Mar & Apr & May & Jun & Jul & Aug & Sep & Oct & Nov & Dec \\
\hline \multicolumn{13}{|c|}{1998} \\
\hline 1 & -- & -- & 0.1 & 0.1 & 0.1 & 0.1 & 1.8 & 0.1 & -- & 0.1 & 0.1 & 0.2 \\
\hline 2 & -- & -- & 0.1 & 0.1 & 0.1 & 0.1 & 5.0 & 0.1 & -. & 0.1 & 0.1 & 0.2 \\
\hline 3 & -- & -- & 0.1 & 0.1 & 0.1 & 0.1 & 5.8 & 0.1 & -- & 0.1 & 0.1 & 0.2 \\
\hline 4 & -. & -- & 0.1 & 0.1 & 0.1 & 0.1 & 3.2 & 0.1 & -- & 0.1 & 0.2 & 0.2 \\
\hline 5 & -- & -- & 0.1 & 0.1 & 0.1 & 0.1 & 0.2 & 0.1 & -- & 0.1 & 0.2 & 0.2 \\
\hline 6 & -- & -- & 0.1 & 0.1 & 0.1 & 0.1 & 0.1 & 0.1 & -- & 0.1 & 0.2 & 0.2 \\
\hline 7 & -- & -- & 0.1 & 0.1 & 0.1 & 0.1 & 0.1 & 0.1 & -- & 0.1 & 0.1 & 0.2 \\
\hline 8 & -- & -- & 0.1 & 0.1 & 0.1 & 0.1 & 0.1 & 0.1 & -- & 0.1 & 0.1 & 0.2 \\
\hline 9 & -- & -- & 0.1 & 0.1 & 0.1 & 0.1 & 0.1 & 0.1 & -. & 0.1 & 0.1 & 0.2 \\
\hline 10 & -- & 0.4 & 0.1 & 0.1 & 0.1 & 0.1 & 0.1 & 0.1 & -- & 0.1 & 0.1 & 0.2 \\
\hline 11 & -- & 0.2 & 0.1 & 0.1 & 0.1 & 0.1 & 0.1 & 0.1 & -- & 0.1 & 0.1 & 0.2 \\
\hline 12 & -- & 0.4 & 0.0 & 0.1 & 0.1 & 0.1 & 0.1 & 0.1 & -. & 0.1 & 0.1 & 0.4 \\
\hline 13 & -- & 0.6 & 0.1 & 0.1 & 0.1 & 0.1 & 0.1 & 0.1 & -. & 0.1 & 0.3 & 0.3 \\
\hline 14 & -- & 0.5 & 0.1 & 0.1 & 0.1 & 0.1 & 0.1 & 0.1 & -- & 0.1 & 0.2 & 0.2 \\
\hline 15 & -. & 0.4 & 0.1 & 0.1 & 0.1 & 0.1 & 0.1 & 0.1 & -- & 0.1 & 0.1 & 0.2 \\
\hline 16 & -- & 0.3 & 0.1 & 0.1 & 0.1 & 0.1 & 0.1 & 0.1 & -- & 0.1 & 0.1 & 0.2 \\
\hline 17 & -- & 0.3 & 0.1 & 0.1 & 0.1 & 0.1 & 0.1 & -- & -- & 0.1 & 0.1 & 0.2 \\
\hline 18 & -- & 0.3 & 0.1 & 0.1 & 0.1 & 0.1 & 0.1 & -. & -- & 0.1 & 0.1 & 0.2 \\
\hline 19 & -- & 0.2 & 0.2 & 0.1 & 0.1 & 0.1 & 0.1 & -- & -. & 0.1 & 0.1 & 0.2 \\
\hline 20 & -- & 0.2 & -- & 0.1 & 0.1 & 0.1 & 0.1 & -- & -- & 0.1 & 0.1 & 0.2 \\
\hline 21 & $\ldots$ & 0.2 & -- & 0.1 & 0.1 & 0.1 & 0.1 & -- & -- & 0.1 & 0.1 & 0.2 \\
\hline 22 & - & 0.1 & -- & 0.1 & 0.1 & 0.1 & 0.1 & -- & -- & 0.1 & 0.1 & 0.2 \\
\hline 23 & -- & 0.1 & -- & 0.1 & 0.1 & 0.1 & 0.1 & -- & 0.1 & 0.1 & 0.1 & 0.3 \\
\hline 24 & -. & 0.1 & 0.2 & 0.1 & 0.1 & 0.1 & 0.1 & -. & 0.1 & 0.1 & 0.1 & 0.3 \\
\hline 25 & -- & 0.1 & 0.2 & 0.1 & 0.1 & 3.7 & 0.1 & -- & 0.1 & 0.1 & 0.1 & 0.2 \\
\hline 26 & -- & 0.1 & 0.1 & 0.1 & 0.1 & 6.0 & 0.1 & -- & 0.1 & 0.1 & 0.1 & 0.2 \\
\hline 27 & -- & 0.1 & 0.1 & 0.1 & 0.1 & 5.8 & 0.1 & -- & 0.1 & 0.1 & 0.2 & 0.2 \\
\hline 28 & -- & 0.1 & 0.1 & 0.1 & 0.1 & 5.7 & 0.1 & -- & 0.1 & 0.1 & 0.2 & 0.2 \\
\hline 29 & -- & -- & 0.1 & 0.1 & 0.1 & 5.9 & 0.1 & -- & 0.1 & 0.1 & 0.2 & 0.2 \\
\hline 30 & - & .- & 0.1 & 0.1 & 0.1 & 4.7 & 0.1 & -- & 0.1 & 0.1 & 0.2 & 0.2 \\
\hline 31 & -. & -- & 0.1 & -- & 0.1 & .. & 0.1 & -- & -. & 0.1 & -- & 0.3 \\
\hline
\end{tabular}




\begin{tabular}{|c|c|c|c|c|c|c|c|c|c|c|c|c|}
\hline Day & Jan & Feb & Mar & Apr & May & Jun & Jul & Aug & Sep & Oct & Nov & Dec \\
\hline & & & & & & 1999 & & & & & & \\
\hline 1 & 0.4 & 0.2 & 0.2 & - & -- & -- & -- & -- & -- & -- & -- & - \\
\hline 2 & 0.3 & 0.2 & 0.4 & -. & -. & -- & -- & -- & - & -- & -- & -- \\
\hline 3 & 0.2 & 0.2 & 0.2 & -- & -- & -- & - & -- & -- & -- & -- & -- \\
\hline 4 & 0.2 & 0.2 & 0.1 & -- & -- & -- & -- & -- & -- & -- & -- & -- \\
\hline 5 & 0.2 & 0.2 & 0.1 & -- & -- & -- & -- & -- & -- & -- & -- & -- \\
\hline 6 & 0.2 & 0.2 & 0.1 & -- & -- & -- & -- & -- & -- & -- & -. & -- \\
\hline 7 & 0.2 & 0.2 & 0.1 & -- & -- & -. & -- & -- & -- & -- & -- & -- \\
\hline 8 & 0.2 & 0.2 & 0.1 & - & - & -- & -. & -. & -- & -. & -- & -- \\
\hline 9 & 0.4 & 0.2 & 0.1 & -- & -- & -- & -. & -- & -- & -- & -- & -- \\
\hline 10 & 0.3 & 0.2 & 0.1 & -- & -- & -- & -- & -- & -- & -- & -- & -- \\
\hline 11 & 0.2 & 0.2 & 0.1 & - & -- & -. & -- & -- & -- & -- & -- & -- \\
\hline 12 & 0.2 & 0.2 & 0.1 & -- & - & -. & -. & -- & -. & -- & -- & -- \\
\hline 13 & 0.2 & 0.2 & 0.1 & -- & -- & -- & -- & -- & -- & -- & -- & -- \\
\hline 14 & 0.2 & 0.2 & 0.1 & - & -- & -- & -- & -- & -- & -- & -- & -- \\
\hline 15 & 0.2 & 0.2 & 0.1 & -- & -- & -- & -- & - & -- & -- & -- & -- \\
\hline 16 & 0.2 & 0.2 & 0.1 & -- & -- & -- & -- & -- & -- & -- & -- & -- \\
\hline 17 & 0.1 & 0.2 & 0.1 & -. & -- & -- & -- & -- & -- & - & -- & -- \\
\hline 18 & 0.2 & 0.2 & 0.1 & -- & -- & - & - & -- & -. & -- & - & -- \\
\hline 19 & 0.2 & 0.2 & 0.1 & -- & -- & -- & -- & -- & -- & -- & -- & -- \\
\hline 20 & 0.2 & 0.2 & 0.1 & -- & -- & -- & -- & -- & -- & -- & -- & -- \\
\hline 21 & 0.2 & 0.2 & 0.1 & - & -- & -- & -- & - & -- & -- & -- & -- \\
\hline 22 & 0.2 & 0.2 & 0.1 & -- & -- & -- & - & -- & - & -- & -- & -- \\
\hline 23 & 0.2 & 0.2 & 0.1 & - & -- & - & -- & -. & -. & -- & -- & -- \\
\hline 24 & 0.2 & 0.2 & 0.1 & - & - & - & -- & -. & -. & -- & -- & -- \\
\hline 25 & 0.2 & 0.2 & 0.1 & -- & -- & - & -- & -- & -- & -- & -- & -- \\
\hline 26 & 0.2 & 0.2 & 0.1 & -- & -- & -- & -- & -- & -- & -- & -- & -- \\
\hline 27 & 0.2 & 0.2 & 0.1 & - & - & - & -- & -- & -- & -- & - & -- \\
\hline 28 & 0.2 & 0.2 & 0.1 & - & - & - & -- & -- & -- & -- & - & - \\
\hline 29 & 0.2 & -- & 0.1 & -- & -- & -- & -- & -- & -- & -- & - & -- \\
\hline 30 & 0.2 & -- & 0.1 & - & - & -. &.- & -- & -- & -- & -- & -- \\
\hline 31 & 0.2 & -- & 0.1 & -- & - & -. & -- & -- & -- & -- & -- & -- \\
\hline
\end{tabular}


Table 13g. Mean daily dissolved-oxygen concentration in the Cedar River at site SW-2 (map ID \#32), Seminole Well Field, Cedar Rapids, lowa, April 1996 through March 1999

[Dissolved oxygen in milligrams per liter; --, value not measured, not recorded, or rejected after data review]

\begin{tabular}{|c|c|c|c|c|c|c|c|c|c|c|c|c|}
\hline Day & Jan & Feb & Mar & Apr & May & Jun & Jul & Aug & Sep & Oct & Nov & Dec \\
\hline \multicolumn{13}{|c|}{1996} \\
\hline 1 & -. & -- & -- & 10.9 & 7.6 & -- & 7.0 & - & 10.5 & 8.7 & 13.2 & 14.2 \\
\hline 2 & -- & - & -- & 10.7 & 5.6 & -- & 7.3 & -- & 11.1 & 7.2 & 13.7 & 14.3 \\
\hline 3 & -- & -- & -- & 10.2 & 3.8 & -- & 7.3 & -- & -- & 7.3 & 13.6 & 15.5 \\
\hline 4 & -- & -- & -- & 10.0 & 2.4 & -- & 7.5 & 9.8 & - & 7.3 & 12.8 & 17.4 \\
\hline 5 & -- & -- & -- & 10.3 & 2.1 & -- & 8.3 & 11.4 & 9.4 & 7.7 & 12.1 & 17.1 \\
\hline 6 & -- & -- & -- & 10.2 & 1.7 & -- & 8.9 & -- & 7.1 & 7.5 & 11.5 & 17.0 \\
\hline 7 & - & -- & -- & 10.4 & -- & -- & 9.3 & -- & 6.2 & 6.7 & 11.4 & 16.8 \\
\hline 8 & -- & -- & -- & 10.4 & -- & -- & 9.2 & -- & 5.4 & 8.2 & 11.7 & 16.7 \\
\hline 9 & -- & -- & - & 10.3 & -- & -- & 9.3 & 10.7 & 5.8 & 7.8 & 12.3 & 16.7 \\
\hline 10 & -- & -- & -- & 9.9 & -- & - & 10.2 & 9.7 & 6.7 & 8.5 & 13.1 & 16.8 \\
\hline 11 & -- & -- & -- & 9.7 & -- & -- & 10.9 & 9.8 & 8.4 & 9.4 & 14.0 & 16.7 \\
\hline 12 & -- & -- & -- & 9.1 & -- & -- & 10.3 & 10.7 & 11.3 & 9.1 & 14.6 & 16.3 \\
\hline 13 & -- & -- & -- & 9.7 & -- & -- & 10.6 & 10.8 & -- & 8.3 & 14.7 & 16.1 \\
\hline 14 & -- & -- & -- & 10.1 & -- & -- & 10.2 & 10.8 & -- & 7.1 & 14.7 & 16.1 \\
\hline 15 & -- & - & -- & 11.4 & -- & -- & 10.4 & 10.5 & -- & 7.0 & 14.8 & 15.9 \\
\hline 16 & -- & -- & -- & 12.3 & -- & -- & 9.9 & 10.8 & -- & 7.1 & 13.1 & 16.1 \\
\hline 17 & - & -- & -- & 12.3 & -- & -- & 7.7 & 10.9 & -- & 5.6 & 11.5 & 16.5 \\
\hline 18 & -. & -. & -- & 10.8 & -- & -- & 8.9 & 8.9 & 12.7 & 6.5 & 12.4 & 16.5 \\
\hline 19 & -. & -- & -- & 10.6 & -. & -- & 8.7 & 8.4 & 11.7 & 7.5 & 13.0 & 16.4 \\
\hline 20 & -- & -- & -- & 10.2 & -- & -- & 7.5 & 10.0 & 10.4 & 9.3 & 13.1 & 16.2 \\
\hline 21 & -- & -- & -- & 11.8 & -. & -- & 7.8 & -- & 10.0 & 9.6 & 13.7 & 15.6 \\
\hline 22 & -- & -- & - & 12.0 & -- & -- & 8.6 & 9.9 & 10.5 & 9.3 & 14.0 & 15.3 \\
\hline 23 & -- & -- & -- & 12.3 & -- & -- & 10.0 & -- & 8.1 & 10.7 & 13.9 & 15.4 \\
\hline 24 & -- & -. & -. & 11.2 & -. & -- & 10.7 & - & 8.5 & 8.7 & 13.8 & 15.6 \\
\hline 25 & -- & -- & -- & 8.9 & -- & -- & 10.6 & -- & 7.1 & 8.5 & 14.5 & 15.5 \\
\hline 26 & -- & -- & -- & 8.9 & -- & -- & 10.9 & - & 6.3 & 8.8 & 14.6 & 15.3 \\
\hline 27 & -- & -. & -- & 9.8 & -- & -- & 9.8 & - & 8.1 & 9.0 & 15.1 & 15.2 \\
\hline 28 & -- & -. & -- & 8.7 & -- & 7.7 & 10.1 & -- & 9.2 & 10.0 & 14.8 & 14.8 \\
\hline 29 & -- & -. & - & 7.3 & -- & 7.6 & 11.2 & -- & 8.9 & 12.0 & 14.5 & 14.6 \\
\hline 30 & -- & -- & -- & 8.5 & -- & 6.8 & 11.8 & -- & 9.7 & 12.0 & 14.3 & 14.4 \\
\hline 31 & -- & -- & -- & -- & -- & -- & 12.6 & 11.3 & -- & 12.2 & -- & 14.4 \\
\hline
\end{tabular}


Table 13g. Mean daily dissolved-oxygen concentration in the Cedar River at site SW-2 (map ID \#32), Seminole Well Field, Cedar Rapids, lowa, April 1996 through March 1999--Continued

\begin{tabular}{|c|c|c|c|c|c|c|c|c|c|c|c|c|}
\hline Day & Jan & Feb & Mar & Apr & May & Jun & Jul & Aug & Sep & Oct & Nov & Dec \\
\hline & & & & & & 1997 & & & & & & \\
\hline 1 & 14.4 & 12.0 & 13.2 & -- & 9.7 & 10.4 & 6.4 & 7.4 & 7.6 & 8.4 & 9.6 & 13.4 \\
\hline 2 & 14.2 & 12.3 & 13.5 & -- & 8.6 & 11.1 & 6.1 & 7.9 & 7.4 & 8.2 & 10.0 & 13.4 \\
\hline 3 & 14.3 & 12.3 & 13.6 & -- & 8.7 & 10.9 & 6.5 & 7.7 & 9.7 & 7.1 & 10.7 & 13.4 \\
\hline 4 & 14.1 & 12.5 & -- & -- & 9.0 & 11.6 & 7.1 & 7.7 & 10.9 & 7.0 & 11.1 & 13.1 \\
\hline 5 & 14.2 & 12.7 & -- & 9.8 & 8.9 & 10.5 & 7.5 & 8.6 & 10.7 & 8.3 & 11.2 & 13.6 \\
\hline 6 & 14.3 & 12.5 & -- & 10.1 & 8.8 & 10.2 & 7.7 & 8.2 & 11.2 & 9.0 & 11.0 & 14.1 \\
\hline 7 & 14.4 & 12.2 & -- & 11.3 & 8.3 & 11.2 & 7.9 & 9.4 & 9.2 & 9.5 & 10.7 & 14.0 \\
\hline 8 & 13.9 & 12.3 & -- & 11.8 & 8.1 & 11.3 & 7.9 & 10.5 & 6.9 & 8.6 & 10.3 & 14.0 \\
\hline 9 & 13.5 & 12.5 & -- & 12.2 & 8.3 & 11.5 & 7.7 & 10.1 & 7.8 & 9.3 & 10.2 & 13.8 \\
\hline 10 & 13.3 & 12.5 & -- & 12.4 & 8.7 & 11.6 & 7.9 & 9.6 & 9.1 & 9.3 & 10.7 & 13.5 \\
\hline 11 & 13.3 & 12.7 & -- & 12.7 & 8.7 & 11.0 & 8.5 & 8.1 & 9.8 & 8.9 & 11.4 & 13.2 \\
\hline 12 & 13.5 & 12.7 & -- & 13.0 & 9.0 & 10.4 & 8.2 & 7.3 & 9.8 & 6.5 & 13.3 & 13.4 \\
\hline 13 & 13.3 & 12.8 & -- & 12.7 & 9.3 & 11.8 & 7.2 & 8.5 & 7.9 & 6.4 & 14.6 & 13.7 \\
\hline 14 & 13.2 & 12.9 & -- & 12.1 & 9.5 & 12.5 & 7.3 & 8.3 & 9.2 & 7.7 & 14.6 & 13.7 \\
\hline 15 & 13.2 & 12.9 & -- & 11.6 & 10.3 & 11.7 & 8.0 & 9.7 & 10.3 & 7.7 & 14.9 & 13.7 \\
\hline 16 & 13.1 & 12.8 & -- & 11.5 & 10.4 & 8.2 & 7.0 & 9.9 & 9.2 & 8.0 & 15.7 & -- \\
\hline 17 & 13.1 & 12.9 & -- & 11.6 & 10.2 & 7.8 & 5.8 & 8.1 & 9.4 & 7.8 & 15.9 & 14.4 \\
\hline 18 & 13.0 & 13.2 & -- & 11.5 & 9.8 & 7.9 & 5.9 & 8.2 & 9.3 & 7.8 & 15.7 & 14.1 \\
\hline 19 & 12.8 & 14.1 & -- & 11.5 & 9.1 & 7.9 & 6.2 & 8.2 & 7.4 & 8.1 & 15.5 & 13.8 \\
\hline 20 & 12.8 & 14.1 & -- & 11.2 & 9.5 & 7.7 & 6.3 & 9.0 & 7.8 & 8.5 & 15.0 & 13.6 \\
\hline 21 & 12.8 & 14.1 & -- & 11.1 & 10.0 & 6.4 & 6.4 & 10.1 & 8.9 & 8.8 & 14.6 & 14.0 \\
\hline 22 & 12.8 & 13.5 & -- & 11.2 & 11.0 & 6.3 & 6.2 & 10.3 & 8.0 & 9.2 & 14.7 & 14.2 \\
\hline 23 & 13.2 & 12.7 & - & 11.0 & 12.2 & 5.2 & 6.4 & 9.5 & 8.9 & 8.8 & 15.4 & 14.0 \\
\hline 24 & 13.0 & 11.9 & - & 11.1 & 11.1 & 5.4 & 7.0 & 9.0 & 9.1 & 8.1 & 15.9 & 13.8 \\
\hline 25 & 12.8 & 11.4 & -- & 11.5 & 9.9 & 5.8 & 7.6 & 7.8 & 9.9 & 8.2 & 15.4 & 14.1 \\
\hline 26 & 12.8 & 11.4 & -- & 11.2 & 9.5 & 5.9 & 7.5 & 7.7 & 8.8 & 9.4 & 14.4 & 14.0 \\
\hline 27 & 12.5 & 12.1 & -- & 11.2 & 10.2 & 6.1 & 6.9 & 7.6 & 8.4 & 10.7 & 14.2 & 13.7 \\
\hline 28 & 12.3 & 12.6 & -- & 11.3 & 9.5 & 6.1 & 7.5 & 8.7 & 7.7 & 11.2 & 13.8 & 13.2 \\
\hline 29 & 12.1 & -- & -- & 11.5 & 9.6 & 6.3 & 8.2 & 8.0 & 8.0 & 11.1 & 13.0 & 12.5 \\
\hline 30 & 12.1 & -- & -- & 9.1 & 9.9 & 6.2 & 7.9 & 7.5 & 8.9 & 10.4 & 12.8 & 12.0 \\
\hline 31 & 12.0 & -- & -- & -- & 10.0 & -- & 7.2 & 7.9 & -- & 9.9 & -- & 12.7 \\
\hline
\end{tabular}


Table 13g. Mean daily dissolved-oxygen concentration in the Cedar River at site SW-2 (map ID \#32), Seminole Well Field, Cedar Rapids, lowa, April 1996 through March 1999--Continued

\begin{tabular}{|c|c|c|c|c|c|c|c|c|c|c|c|c|}
\hline Day & Jan & Feb & Mar & Apr & May & Jun & Jul & Aug & Sep & Oct & Nov & Dec \\
\hline \multicolumn{13}{|c|}{1998} \\
\hline 1 & 13.4 & 12.6 & 3.5 & 8.0 & 4.7 & 5.1 & 9.2 & 3.3 & -- & 3.2 & 4.5 & 3.9 \\
\hline 2 & 13.3 & 12.8 & 3.5 & 9.0 & 4.6 & 4.7 & 9.7 & 3.1 & -- & 3.0 & 4.3 & 3.6 \\
\hline 3 & 12.9 & 12.8 & 3.3 & 9.2 & 4.6 & 4.7 & 10.1 & 2.9 & -- & 2.8 & 4.1 & 3.5 \\
\hline 4 & 12.7 & 12.9 & 3.5 & 9.0 & 4.5 & 4.5 & 10.0 & 3.0 & -- & 2.9 & 3.9 & 3.6 \\
\hline 5 & 12.8 & 13.1 & 3.6 & 8.7 & 4.4 & 4.4 & 8.9 & 3.0 & -- & 2.8 & 3.7 & 3.4 \\
\hline 6 & 12.7 & 13.2 & 3.5 & 8.2 & 4.3 & 4.5 & 7.4 & 3.2 & -- & 3.1 & 3.6 & 3.4 \\
\hline 7 & 12.9 & 13.3 & 3.3 & 7.4 & 4.2 & 4.4 & 6.5 & 3.4 & -- & 3.3 & 3.6 & 3.6 \\
\hline 8 & 13.1 & 13.3 & 2.9 & 6.8 & 4.4 & 4.2 & 6.2 & 3.5 & -- & 3.5 & 3.3 & 3.6 \\
\hline 9 & 13.4 & 13.2 & 3.1 & 6.5 & 4.5 & 4.1 & 6.0 & -- & -- & 3.5 & 3.0 & 3.7 \\
\hline 10 & 13.6 & -- & 3.3 & 6.7 & 4.5 & 5.0 & 5.7 & -- & -- & 3.3 & 2.3 & 3.8 \\
\hline 11 & 13.8 & -- & 3.5 & 6.6 & 4.3 & 5.2 & 5.4 & -- & -- & 3.3 & 3.5 & 3.6 \\
\hline 12 & 13.6 & -- & 3.9 & 6.3 & 4.1 & 6.3 & 5.1 & -- & -- & 3.3 & 3.9 & 3.5 \\
\hline 13 & 13.4 & -- & 3.7 & 5.9 & 4.4 & 7.4 & 4.9 & -- & -- & 3.2 & 3.7 & 3.4 \\
\hline 14 & 13.5 & -- & 3.1 & 6.0 & 4.3 & 8.0 & 4.7 & -- & -- & 3.1 & 3.5 & 3.4 \\
\hline 15 & 13.1 & 12.1 & 3.1 & 5.8 & 4.1 & 8.7 & 4.4 & -. & -- & 3.0 & 3.8 & 3.4 \\
\hline 16 & 12.9 & 11.4 & 3.1 & 5.9 & 4.3 & 8.2 & 4.3 & -- & -- & 3.1 & 3.5 & 3.2 \\
\hline 17 & 12.7 & 11.3 & 2.7 & 6.4 & 4.3 & 7.5 & 4.0 & -- & -- & 3.2 & 3.8 & 3.2 \\
\hline 18 & 12.5 & 11.4 & 2.6 & 6.5 & 4.2 & 6.9 & 3.8 & -- & -- & 4.5 & 3.5 & 3.1 \\
\hline 19 & 12.4 & 11.3 & 3.0 & 6.7 & 4.2 & 7.2 & 3.6 & -- & -- & 4.8 & 3.5 & 4.0 \\
\hline 20 & 12.2 & 11.4 & 3.2 & 6.9 & 4.2 & 7.4 & 3.6 & -- & -- & 5.3 & 3.5 & 4.1 \\
\hline 21 & 12.2 & 11.5 & 3.4 & 7.1 & 4.0 & 8.0 & 3.6 & -- & -- & 5.3 & 3.4 & 4.4 \\
\hline 22 & 11.9 & 11.7 & 3.5 & 7.0 & 3.8 & 9.1 & 3.6 & - & -- & 4.0 & 3.0 & 5.3 \\
\hline 23 & 11.7 & 7.3 & 3.8 & 6.9 & 3.7 & 9.4 & 3.6 & -- & 3.1 & 4.5 & 3.5 & 4.7 \\
\hline 24 & 11.4 & 3.6 & 4.4 & 6.6 & 3.8 & 9.4 & 3.3 & -- & 2.9 & 4.0 & 3.9 & 4.6 \\
\hline 25 & 11.3 & 3.3 & 4.2 & 6.2 & 4.0 & 10.3 & 3.4 & -- & 2.8 & 3.8 & 3.6 & 4.7 \\
\hline 26 & 11.5 & 2.9 & 4.3 & 6.1 & 4.4 & 10.5 & 3.3 & -- & 2.8 & 3.7 & 3.7 & 4.8 \\
\hline 27 & 11.7 & 3.1 & 4.5 & 6.2 & 4.9 & 10.2 & 3.2 & -- & 3.1 & 3.6 & 3.7 & 4.6 \\
\hline 28 & 11.9 & 3.5 & 4.7 & 5.9 & 4.9 & 10.3 & 3.2 & -- & 3.2 & 3.5 & 3.6 & 4.5 \\
\hline 29 & 12.1 & -- & 4.7 & 5.4 & 5.3 & 9.9 & 3.2 & -- & 3.0 & 3.4 & 3.4 & 4.3 \\
\hline 30 & 12.4 & -- & 4.8 & 5.0 & 5.2 & 9.3 & 3.3 & -- & 3.1 & 4.1 & 3.7 & 4.3 \\
\hline 31 & 12.5 & -- & 7.0 & -- & 5.3 & -- & 3.4 & -- & -- & 4.4 & -- & 4.0 \\
\hline
\end{tabular}


Table 13g. Mean daily dissolved-oxygen concentration in the Cedar River at site SW-2 (map ID \#32), Seminole Well Field, Cedar Rapids, lowa, April 1996 through March 1999--Continued

\begin{tabular}{|c|c|c|c|c|c|c|c|c|c|c|c|c|}
\hline Day & Jan & Feb & Mar & Apr & May & Jun & Jul & Aug & Sep & Oct & Nov & $\overline{D e c}$ \\
\hline & & & & & & 1999 & & & & & & \\
\hline 1 & 4.2 & 4.1 & 4.0 & -- & -- & -- & -- & -- & -- & - & -- & -- \\
\hline 2 & 3.8 & 4.1 & 4.1 & -- & -- & -- & -- & -- & -- & -- & -- & -- \\
\hline 3 & 3.9 & 4.1 & 4.4 & -- & -- & -- & -- & -- & -- & -- & -- & -- \\
\hline 4 & 4.2 & 4.9 & 4.3 & -- & -- & -- & -- & -- & -- & -- & -- & -- \\
\hline 5 & 4.0 & 4.7 & 4.2 & -. & -- & -- & -- & -- & -- & -- & -- & -- \\
\hline 6 & 4.0 & 4.7 & 4.5 & -- & -- & -- & -. & -- & -- & -- & -- & -- \\
\hline 7 & 4.3 & 4.6 & 4.7 & -- & -- & -- & -- & -. & -- & -- & - & -- \\
\hline 8 & 4.0 & 4.6 & 4.2 & -- & -- & -- & -. & -- & -- & -- & -- & -- \\
\hline 9 & 4.2 & 5.0 & 4.0 & -- & -- & -. & -- & -. & -- & -- & -- & -- \\
\hline 10 & 4.0 & 4.7 & 4.4 & -- & -- & -- & -- & -- & -- & -- & -- & -- \\
\hline 11 & 3.8 & 4.7 & 4.3 & -- & - & -- & -- & -- & -- & -- & -- & -- \\
\hline 12 & 3.8 & 5.2 & 4.4 & -- & -- & -- & -- & -- & -- & -- & -- & -- \\
\hline 13 & 4.2 & 5.6 & 4.3 & -- & -- & -- & -- & -- & -- & -- & -- & -- \\
\hline 14 & 4.2 & 5.1 & 4.3 & -- & -- & -- & -- & -- & -- & -- & -- & -- \\
\hline 15 & 3.8 & 4.7 & 4.2 & -- & -- & -- & -- & -- & -. & -- & -- & -- \\
\hline 16 & 3.8 & 4.9 & 3.9 & -- & -- & -- & -- & -- & -- & -- & -- & -- \\
\hline 17 & 3.6 & 5.0 & 4.1 & -- & -- & -- & -- & -- & -- & -- & -- & -- \\
\hline 18 & 3.7 & 5.0 & 4.6 & -- & -. & -- & -- & -- & -- & -- & -- & -- \\
\hline 19 & 4.0 & 5.0 & 4.9 & -- & -- & -- & -- & -- & -- & -- & -- & -- \\
\hline 20 & 4.0 & 5.1 & 5.0 & -. & -- & -- & -- & -- & -- & -. & -- & -- \\
\hline 21 & 3.8 & 5.2 & 5.0 & -- & -- & -- & -- & -- & -- & -- & -- & -- \\
\hline 22 & 3.7 & 4.9 & 4.9 & -- & -- & -- & -- & -- & -- & -- & -- & -- \\
\hline 23 & 3.9 & 4.6 & 4.7 & -- & -- & -- & -- & - & -- & -- & -- & -- \\
\hline 24 & 4.3 & 4.5 & 4.8 & -- & -- & -- & -- & -- & -- & -- & -- & -- \\
\hline 25 & 4.6 & 4.4 & 4.9 & -- & -- & - & -- & -- & -- & -- & -- & -- \\
\hline 26 & 4.3 & 4.2 & 4.6 & -- & -- & -- & -- & -- & -- & -- & -- & -- \\
\hline 27 & 4.0 & 3.9 & 4.6 & -- & -- & -- & -. & -- & -- & -- & -- & -- \\
\hline 28 & 4.4 & 4.0 & 4.4 & -- & -- & -- & -- & -- & -- & -- & -- & -- \\
\hline 29 & 4.7 & -- & 4.7 & -- & -- & -- & -- & -- & -- & -- & -- & -- \\
\hline 30 & 4.7 & -- & 4.7 & - & -- & -- & -- & -- & -- & -- & -- & -- \\
\hline 31 & 4.5 & -- & 4.3 & -. & -- & -- & -- & -- & -- & -- & -- & -- \\
\hline
\end{tabular}


Table 14a. Mean daily ground-water temperature at site CRM-1 (map ID \#1), East Well Field, Cedar Rapids, lowa, April 1996 through February 1999

[Temperature in degrees Centigrade; --, value not measured or not recorded]

\begin{tabular}{|c|c|c|c|c|c|c|c|c|c|c|c|c|}
\hline Day & Jan & Feb & Mar & Apr & May & Jun & Jul & Aug & Sep & Oct & Nov & Dec \\
\hline \multicolumn{13}{|c|}{1996} \\
\hline 1 & -- & -- & -- & 4.6 & 2.8 & -- & 3.9 & 5.2 & -- & -- & -- & -- \\
\hline 2 & -- & -- & -- & 4.4 & 2.8 & -- & 4.0 & 5.3 & -- & -- & -- & -- \\
\hline 3 & -- & -- & -- & 4.2 & 2.9 & -. & 4.0 & 5.4 & -. & -- & -- & -- \\
\hline 4 & -- & -- & -- & 4.0 & 3.0 & -- & 4.1 & 5.4 & -. & -. & -- & 13.2 \\
\hline 5 & -- & -- & -- & 3.8 & 3.1 & -- & 4.1 & 5.5 & -- & -- & -- & 13.2 \\
\hline 6 & -- & -- & -- & 3.7 & 3.0 & -. & 4.2 & 5.6 & -. & -- & -- & 13.2 \\
\hline 7 & -- & -- & -- & 3.5 & 3.0 & -- & 4.3 & 5.5 & -- & -- & -- & 13.3 \\
\hline 8 & -- & -- & -- & 3.3 & 3.1 & -. & 4.3 & 5.4 & -. & -- & -- & 13.4 \\
\hline 9 & -- & -- & - & 3.2 & -- & - & 4.4 & 5.3 & -- & -- & -- & 13.6 \\
\hline 10 & -- & -- & -- & 3.1 & -- & -- & 4.5 & 5.2 & -- & -- & -- & 13.7 \\
\hline 11 & -- & -- & -- & 3.0 & -- & -- & 4.6 & 5.2 & -- & -- & -- & 13.2 \\
\hline 12 & -- & -- & -- & 2.9 & -- & -- & 4.7 & 5.2 & -- & -- & -- & 13.8 \\
\hline 13 & -- & -- & -- & 2.8 & -- & -- & 4.8 & 5.2 & -- & -- & -- & 13.9 \\
\hline 14 & -- & -- & -- & 2.7 & -- & -- & 4.9 & 5.2 & 6.6 & -- & -- & 13.9 \\
\hline 15 & -- & -- & -- & 2.7 & -- & -. & 4.8 & 5.2 & 6.7 & -- & -- & 14.0 \\
\hline 16 & -- & -- & -- & 2.6 & -- & -- & 4.6 & 5.3 & 6.7 & -- & -- & 14.1 \\
\hline 17 & -- & -- & -- & 2.7 & -- & -- & 4.5 & 5.3 & 6.8 & -- & -- & 14.4 \\
\hline 18 & -- & -- & -- & 2.7 & -- & -- & 4.5 & 5.4 & 6.9 & -- & -- & 14.6 \\
\hline 19 & -- & -- & -- & 2.7 & -- & -- & 4.5 & 5.4 & 7.0 & -- & -- & 14.6 \\
\hline 20 & -- & -- & -- & 2.8 & -- & -- & 4.6 & 5.4 & 7.0 & -- & -- & 14.7 \\
\hline 21 & -- & -- & -- & 2.8 & -- & -- & 4.6 & 5.5 & 7.1 & -- & -. & 14.7 \\
\hline 22 & -- & -- & -- & 2.7 & -. & -- & 4.7 & 5.5 & 7.1 & -- & -- & 14.8 \\
\hline 23 & -- & -- & -- & 3.4 & -- & -- & 4.7 & 5.5 & 7.2 & -- & -- & 14.8 \\
\hline 24 & -- & -- & -- & 2.8 & -- & -- & 4.8 & 5.6 & 7.2 & -- & - & 14.8 \\
\hline 25 & -- & -- & -- & 2.8 & -- & -- & 4.8 & 5.6 & 7.3 & -- & -- & 14.8 \\
\hline 26 & -- & -- & -- & 2.7 & -- & -- & 4.8 & -- & 7.3 & -- & -- & 14.8 \\
\hline 27 & -- & -- & -- & 2.7 & -- & -- & 4.8 & -- & 7.4 & -- & -- & 14.7 \\
\hline 28 & -- & -- & -- & 2.7 & -- & 3.8 & 4.9 & -- & -- & -- & -- & 14.7 \\
\hline 29 & -. & -. & -- & 2.6 & -- & 3.8 & 4.9 & -- & -- & -- & -- & 14.7 \\
\hline 30 & -- & - & -. & 2.7 & -. & 3.9 & 5.2 & - & -- & -- & -- & 14.7 \\
\hline 31 & -- & - & -. & -- & -. & .. & 5.1 & -- & -- & -- & -- & 14.9 \\
\hline
\end{tabular}


Table 14a. Mean daily ground-water temperature at site CRM-1 (map ID \#1), East Well Field, Cedar Rapids, lowa, April 1996 through February 1999--Continued

\begin{tabular}{|c|c|c|c|c|c|c|c|c|c|c|c|c|}
\hline Day & Jan & Feb & Mar & Apr & May & Jun & Jul & Aug & Sep & Oct & Nov & Dec \\
\hline & & & & & & 1997 & & & & & & \\
\hline 1 & 15.1 & 14.0 & 13.9 & -- & - & 7.8 & -- & 8.2 & 8.5 & 8.7 & 10.7 & 11.0 \\
\hline 2 & 15.1 & 14.0 & 13.9 & -- & -- & 7.5 & -- & 8.2 & 8.5 & 8.7 & 10.8 & 11.0 \\
\hline 3 & 15.0 & 14.0 & 13.8 & -- & -. & 7.2 & -- & 8.2 & 8.4 & 8.7 & 10.9 & 11.0 \\
\hline 4 & 15.0 & 13.9 & 13.8 & -- & -- & 7.0 & -- & 8.2 & 8.4 & 8.7 & 10.9 & 11.0 \\
\hline 5 & 15.0 & 13.8 & 13.7 & -- & -- & 6.9 & -- & 8.2 & 8.5 & 8.7 & 10.9 & 11.0 \\
\hline 6 & 15.0 & 13.8 & 13.7 & -. & -. & 6.7 & -- & 8.1 & 8.5 & 8.7 & 10.9 & 11.0 \\
\hline 7 & 14.9 & 13.5 & 13.7 & - & -. & 6.6 & - & 8.1 & 8.5 & 8.7 & 10.9 & 11.1 \\
\hline 8 & 14.9 & 13.3 & 13.6 & -. & -- & 6.5 & -- & 8.1 & 8.5 & 8.7 & 10.9 & 11.1 \\
\hline 9 & 14.8 & 12.9 & 13.6 & -- & -- & 6.5 & -- & 8.1 & 8.5 & 8.7 & 10.9 & 11.1 \\
\hline 10 & 14.8 & 12.5 & 13.6 & -- & -- & 6.6 & -- & 8.1 & 8.6 & 8.7 & 10.9 & 11.1 \\
\hline 11 & 14.8 & 12.4 & 13.5 & -- & -- & 6.8 & -- & 8.1 & 8.6 & 8.7 & 10.9 & 11.1 \\
\hline 12 & 14.8 & 12.7 & 13.5 & -- & -- & 6.9 & -- & 8.1 & 8.6 & 8.6 & 10.9 & 11.2 \\
\hline 13 & 14.7 & 12.9 & 13.5 & -- & -- & -- & -- & 8.0 & 8.7 & 8.6 & 10.9 & 11.2 \\
\hline 14 & 14.7 & 13.1 & 13.4 & -- & -- & -- & -- & 8.0 & 8.7 & 8.6 & 10.9 & 11.3 \\
\hline 15 & 14.6 & 13.3 & 13.4 & -- & -- & -- & -- & 8.1 & 8.8 & 8.6 & 10.9 & 11.3 \\
\hline 16 & 14.6 & 13.5 & 13.3 & -- & -- & -- & -- & 8.4 & 8.8 & 8.6 & 10.9 & 11.4 \\
\hline 17 & 14.5 & 13.5 & 13.3 & -- & -- & -- & -- & 8.4 & 8.8 & 8.6 & 10.9 & 11.5 \\
\hline 18 & 14.4 & 13.6 & 13.2 & -- & -- & -- & -- & 8.4 & 8.7 & 8.6 & 10.9 & 11.6 \\
\hline 19 & 14.3 & 13.8 & 13.2 & -- & -- & -- & -- & 8.7 & 8.8 & 8.6 & 10.9 & 11.8 \\
\hline 20 & 14.2 & 13.9 & 13.1 & -- & -- & -- & -- & 8.7 & 8.7 & 8.6 & 10.9 & 11.9 \\
\hline 21 & 14.1 & 13.9 & 12.9 & -- & -- & -- & -- & 8.6 & 8.7 & 8.7 & 10.9 & 11.9 \\
\hline 22 & 14.1 & 14.0 & 12.7 & -- & -- & -- & -- & 8.4 & 8.7 & 8.7 & 10.9 & 11.9 \\
\hline 23 & 14.1 & 14.0 & 12.5 & -- & 9.9 & -- & -- & 8.4 & 8.7 & 8.7 & 10.9 & 11.9 \\
\hline 24 & 14.0 & 14.0 & 12.4 & - & 9.7 & -- & 8.2 & 8.3 & 8.7 & 8.9 & 10.9 & 11.9 \\
\hline 25 & 13.9 & 14.0 & 12.3 & -- & 9.6 & -- & 8.2 & 8.2 & 8.7 & 9.0 & 11.0 & 11.9 \\
\hline 26 & 13.9 & 14.0 & 12.2 & -- & 9.4 & -- & 8.3 & 8.2 & 8.7 & 9.0 & 11.0 & 11.9 \\
\hline 27 & 14.0 & 14.0 & 12.1 & -- & 9.3 & -- & 8.3 & 8.2 & 8.7 & 9.4 & 11.0 & 11.9 \\
\hline 28 & 14.1 & 13.9 & 12.0 & -- & 9.1 & -- & 8.2 & 8.3 & 8.6 & 9.6 & 11.0 & 11.9 \\
\hline 29 & 14.1 & -- & 11.9 & -- & 8.8 & -- & 8.2 & 8.5 & 8.6 & 10.0 & 11.0 & 12.0 \\
\hline 30 & 14.1 & -- & -- & -- & 8.6 & -. & 8.2 & 8.4 & 8.7 & 10.2 & 11.0 & 12.1 \\
\hline 31 & 14.1 & -. & -- & -- & 8.2 & -- & 8.2 & 8.5 & -- & 10.5 & -- & 12.1 \\
\hline
\end{tabular}


Table 14a. Mean daily ground-water temperature at site CRM-1 (map ID \#1), East Well Field, Cedar Rapids, lowa, April 1996 through February 1999--Continued

\begin{tabular}{|c|c|c|c|c|c|c|c|c|c|c|c|c|}
\hline Day & Jan & Feb & Mar & Apr & May & Jun & Jul & Aug & Sep & Oct & Nov & $\overline{D e c}$ \\
\hline \multicolumn{13}{|c|}{1998} \\
\hline 1 & 12.2 & 11.6 & 10.6 & 8.6 & 8.1 & 6.8 & 7.0 & 7.3 & 8.1 & 8.2 & 9.2 & 9.4 \\
\hline 2 & 12.2 & 11.6 & 10.7 & 8.6 & 8.0 & 6.7 & 7.0 & 7.3 & 8.1 & 8.2 & 9.2 & 9.4 \\
\hline 3 & 12.3 & 11.4 & 10.9 & 8.5 & 7.9 & 6.7 & 7.1 & 7.3 & 8.1 & 8.1 & 9.2 & 9.4 \\
\hline 4 & 12.4 & 11.2 & 10.9 & 8.4 & 7.8 & 6.6 & 7.1 & 7.3 & 8.2 & 8.1 & 9.2 & 9.4 \\
\hline 5 & 12.4 & 11.2 & 10.8 & 8.6 & 7.7 & 6.6 & 7.2 & 7.3 & 8.2 & 8.1 & 9.2 & 9.4 \\
\hline 6 & 12.5 & 11.1 & 10.9 & 8.7 & 7.6 & 6.5 & 7.2 & 7.3 & 8.3 & 8.2 & 9.3 & 9.5 \\
\hline 7 & 12.6 & 11.0 & 10.9 & 8.7 & 7.5 & 6.5 & 7.1 & 7.3 & 8.3 & 8.2 & 9.3 & 9.5 \\
\hline 8 & 12.6 & 10.9 & 10.8 & 8.7 & 7.5 & 6.4 & 7.0 & 7.3 & 8.3 & 8.2 & 9.3 & 9.5 \\
\hline 9 & 12.7 & 10.9 & 10.8 & 8.6 & 7.5 & 6.4 & 7.0 & 7.4 & 8.4 & 8.2 & 9.4 & 9.5 \\
\hline 10 & 12.8 & 10.8 & 10.7 & 8.6 & 7.4 & 6.3 & 6.9 & 7.4 & 8.4 & 8.3 & 9.4 & 9.6 \\
\hline 11 & 12.7 & 10.7 & 10.5 & 8.6 & 7.4 & 6.2 & 6.8 & 7.4 & 8.5 & 8.3 & 9.4 & 9.6 \\
\hline 12 & 12.3 & 10.6 & 10.1 & 8.6 & 7.4 & 6.2 & 6.8 & 7.4 & 8.5 & 8.3 & 9.4 & 9.6 \\
\hline 13 & 12.3 & 10.6 & 9.7 & 8.5 & 7.4 & 6.2 & 6.8 & 7.5 & 8.6 & 8.3 & 9.5 & 9.6 \\
\hline 14 & 12.3 & 10.5 & 9.5 & 8.5 & 7.4 & 6.2 & 6.8 & 7.5 & 8.6 & 8.4 & 9.5 & 9.6 \\
\hline 15 & 12.3 & 10.5 & 9.3 & 8.6 & 7.3 & 6.2 & 6.8 & 7.6 & 8.6 & 8.4 & 9.5 & 9.6 \\
\hline 16 & 12.3 & 10.4 & 9.2 & 8.5 & 7.3 & 6.3 & 6.9 & 7.5 & 8.5 & 8.4 & 9.6 & 9.6 \\
\hline 17 & 12.3 & 10.4 & 8.9 & 8.5 & 7.3 & 6.3 & 6.9 & 7.6 & 8.5 & 8.5 & 9.6 & 9.7 \\
\hline 18 & 12.3 & 10.4 & 8.7 & 8.5 & 7.3 & 6.4 & 7.0 & 7.6 & 8.5 & 8.5 & 9.5 & 9.7 \\
\hline 19 & 12.3 & 10.4 & 8.6 & 8.5 & 7.3 & 6.4 & 7.0 & 7.7 & 8.4 & 8.5 & 9.5 & 9.7 \\
\hline 20 & 12.3 & 10.4 & 8.7 & 8.5 & 7.3 & 6.5 & 7.0 & 7.8 & 8.4 & 8.6 & 9.5 & 9.7 \\
\hline 21 & 12.3 & 10.3 & 8.7 & 8.5 & 7.3 & 6.4 & 7.0 & 7.8 & 8.4 & 8.6 & 9.4 & 9.7 \\
\hline 22 & 12.3 & 10.3 & 8.7 & 8.4 & 7.2 & 6.4 & 7.1 & 7.8 & 8.4 & 8.7 & 9.4 & 9.7 \\
\hline 23 & 12.3 & 10.2 & 8.7 & 8.4 & 7.2 & 6.4 & 7.1 & 7.8 & 8.4 & 8.8 & 9.4 & 9.7 \\
\hline 24 & 12.3 & 10.2 & 8.7 & 8.4 & 7.1 & 6.4 & 7.1 & 7.8 & 8.4 & 8.8 & 9.4 & 9.7 \\
\hline 25 & 12.3 & 10.1 & 8.7 & 8.4 & 7.1 & 6.6 & 7.1 & 7.8 & 8.4 & 8.9 & 9.4 & 9.7 \\
\hline 26 & 12.1 & 10.1 & 8.7 & 8.3 & 7.0 & 6.8 & 7.1 & 7.8 & 8.4 & 8.9 & 9.4 & 9.7 \\
\hline 27 & 11.9 & 10.2 & 8.7 & 8.3 & 7.0 & 6.8 & 7.1 & 7.8 & 8.4 & 9.0 & 9.4 & 9.7 \\
\hline 28 & 11.8 & 10.4 & 8.7 & 8.3 & 6.9 & 6.9 & 7.2 & 7.8 & 8.4 & 9.0 & 9.4 & 9.8 \\
\hline 29 & 11.8 & -- & 8.7 & 8.2 & 6.9 & 7.0 & 7.2 & 7.9 & 8.4 & 9.0 & 9.4 & 9.8 \\
\hline 30 & 11.7 & -- & 8.7 & 8.1 & 6.9 & 7.0 & 7.2 & 7.9 & 8.4 & 9.1 & 9.4 & 9.8 \\
\hline 31 & 11.7 & -. & 8.7 & -. & 6.8 & -- & 7.2 & 8.0 & -- & 9.2 & -. & 9.8 \\
\hline
\end{tabular}


Table 14a. Mean daily ground-water temperature at site CRM-1 (map ID \#1), East Well Field, Cedar Rapids, lowa, April 1996 through February 1999--Continued

\begin{tabular}{|c|c|c|c|c|c|c|c|c|c|c|c|c|}
\hline Day & Jan & Feb & Mar & Apr & May & Jun & Jul & Aug & Sep & Oct & Nov & Dec \\
\hline & & & & & & 1999 & & & & & & \\
\hline 1 & 9.9 & 9.4 & -- & -- & -- & -- & -- & -- & -- & -- & -- & -- \\
\hline 2 & 9.9 & 9.4 & -- & -- & -- & -- & - & -- & -- & -- & -- & -- \\
\hline 3 & 9.9 & 9.4 & -- & -- & -- & -- & -- & -- & -- & -- & -- & -- \\
\hline 4 & 9.9 & 9.4 & -- & -- & -- & -- & -- & -- & -- & -- & -- & -- \\
\hline 5 & 9.8 & 9.4 & -- & -- & -- & -- & -- & -- & -- & -- & -- & -- \\
\hline 6 & 9.8 & 9.4 & -- & -- & -- & -- & -- & -- & -- & -- & -- & -- \\
\hline 7 & 9.8 & 9.4 & -- & -- & -- & -- & -- & -- & -- & -- & -- & - \\
\hline 8 & 9.8 & 9.4 & -- & -- & -- & -- & -. & -- & -- & -- & - & -- \\
\hline 9 & 9.8 & 9.4 & -- & -- & -- & -- & -- & -- & -- & -- & -- & -- \\
\hline 10 & 9.8 & 9.4 & -- & -- & -- & -- & -- & -- & -- & -- & -- & -- \\
\hline 11 & 9.7 & 9.4 & -- & -- & -- & -- & -- & -- & -- & -- & -- & -- \\
\hline 12 & 9.7 & 9.4 & -- & -- & -- & -- & -- & -- & -- & -- & -- & -- \\
\hline 13 & 9.7 & 9.4 & -- & -. & -. & - & -- & -- & -- & -. & -- & -- \\
\hline 14 & 9.7 & 9.4 & -- & -- & -- & -- & -- & -- & -- & -- & -- & -- \\
\hline 15 & 9.7 & 9.4 & -- & -- & -- & -- & -- & -- & -. & -- & -- & -- \\
\hline 16 & 9.7 & 9.4 & -- & -- & -- & -- & -- & -- & -- & -- & -- & -- \\
\hline 17 & 9.6 & 9.4 & -- & -- & -- & -- & -- & -- & -- & - & -- & -- \\
\hline 18 & 9.6 & 9.4 & -- & -- & -- & -- & -- & -- & -- & -- & -- & -- \\
\hline 19 & 9.6 & 9.4 & -- & -- & -- & -. & -- & -- & -- & -- & - & -- \\
\hline 20 & 9.6 & 9.4 & -- & -- & -- & -- & -- & -- & - & -- & -- & -- \\
\hline 21 & 9.5 & 9.3 & -- & -- & -- & -- & -- & -- & -- & -- & -- & -- \\
\hline 22 & 9.5 & 9.3 & -- & -- & -- & -- & -- & -- & -- & -- & -- & -- \\
\hline 23 & 9.5 & 9.3 & -- & -- & -. & -- & -- & - & -- & -- & -- & -- \\
\hline 24 & 9.5 & 9.3 & -- & -- & -- & -- & -- & -- & -- & -- & -- & -- \\
\hline 25 & 9.5 & 9.3 & -- & -- & -- & -- & -- & -- & -- & -- & -- & -- \\
\hline 26 & 9.4 & 9.3 & -- & -- & -- & -- & -- & -- & -- & -- & -- & -- \\
\hline 27 & 9.4 & 9.3 & -- & -- & -- & -- & -- & -- & -- & -- & -- & -- \\
\hline 28 & 9.4 & 9.3 & -- & - & -- & -- & -- & - & -- & -- & -- & -- \\
\hline 29 & 9.4 & -- & -- & -- & - & -- & -- & -- & -- & -- & -- & -. \\
\hline 30 & 9.4 & -- & -- & -- & -- & -- & -- & -- & -- & -- & -- & -- \\
\hline 31 & 9.4 & .. & -- & -- & -- & -- & -- & -- & -- & -- & -- & -- \\
\hline
\end{tabular}


Table 14b. Mean daily ground-water temperature at site CRM-2 (map ID \#2), West Well Field, Cedar Rapids, lowa, April 1996 through February 1999

[Temperature in degrees Centigrade; --, value not measured or not recorded]

\begin{tabular}{|c|c|c|c|c|c|c|c|c|c|c|c|c|}
\hline Day & Jan & Feb & Mar & Apr & May & Jun & Jul & Aug & Sep & Oct & Nov & Dec \\
\hline \multicolumn{13}{|c|}{1996} \\
\hline 1 & -- & -- & -- & 15.1 & 16.7 & -- & -- & 14.1 & 11.8 & 11.3 & 12.1 & 12.9 \\
\hline 2 & -- & -- & -- & 15.2 & 16.7 & -- & -- & 14.0 & 11.7 & 11.4 & 12.2 & 13.0 \\
\hline 3 & -- & -- & -- & 15.3 & 16.7 & -- & -- & 14.0 & 11.6 & 11.5 & 12.2 & 12.9 \\
\hline 4 & -- & -- & -- & 15.3 & 16.7 & -- & -- & 13.9 & 11.6 & 11.6 & 12.2 & 13.0 \\
\hline 5 & -- & - & -- & 15.4 & 16.6 & -- & -- & 13.9 & 11.5 & 11.5 & 12.3 & 12.9 \\
\hline 6 & -- & -- & -- & 15.5 & 16.6 & -. & -- & 13.8 & 11.5 & 11.5 & 12.3 & 12.9 \\
\hline 7 & -- & -- & -- & 15.6 & 16.5 & -- & -- & 13.8 & 11.4 & 11.5 & 12.3 & 12.9 \\
\hline 8 & -- & -. & -. & 15.6 & 16.5 & -- & -- & 13.7 & 11.4 & 11.5 & 12.3 & 12.9 \\
\hline 9 & -- & -- & -. & 15.6 & -- & -- & -- & 13.6 & 11.3 & 11.6 & 12.4 & 12.9 \\
\hline 10 & -- & -- & -- & 15.6 & -- & -- & -- & 13.5 & 11.3 & 11.7 & 12.4 & 12.9 \\
\hline 11 & -- & -- & -- & 15.6 & -- & -- & -- & 13.4 & 11.2 & 11.7 & 12.5 & 12.8 \\
\hline 12 & -- & -- & -. & 15.6 & -. & -- & -- & 13.3 & 11.2 & 11.7 & 12.5 & 12.9 \\
\hline 13 & -- & - & -- & 15.6 & -- & -- & -- & 13.2 & 11.2 & 11.8 & 12.5 & 12.9 \\
\hline 14 & -- & -- & -- & 15.7 & -- & -. & -- & 13.2 & 11.1 & 11.8 & 12.5 & 12.9 \\
\hline 15 & -- & - & -- & 15.7 & -- & -- & -- & 13.2 & 11.1 & 11.8 & 12.5 & 12.9 \\
\hline 16 & -- & - & -- & 15.8 & -- & -- & -- & 13.1 & 11.1 & 11.7 & 12.6 & 13.0 \\
\hline 17 & -- & .- & -- & 15.8 & -- & -. & -- & 13.1 & 11.0 & 11.7 & 12.7 & 13.0 \\
\hline 18 & -- & -- & -- & 15.8 & -- & -- & -- & 13.1 & 11.0 & 11.7 & 12.7 & 13.0 \\
\hline 19 & -- & -. & -- & 15.9 & -- & -- & -- & 13.1 & 11.0 & 11.7 & 12.7 & 13.0 \\
\hline 20 & -- & - & -- & 15.9 & -- & -- & -- & 13.1 & 11.0 & 11.7 & 12.7 & 13.0 \\
\hline 21 & -- & -- & -- & 15.9 & -- & -. & -- & 13.1 & 10.9 & 11.7 & 12.8 & 13.0 \\
\hline 22 & -- & -- & -- & 15.9 & -- & -- & -- & 13.0 & 11.0 & 11.7 & 12.8 & 13.0 \\
\hline 23 & -- & -- & -- & -- & -- & -- & -- & 13.0 & 11.1 & 11.8 & 12.9 & 13.0 \\
\hline 24 & -- & -. & -. & 16.1 & -- & -. & -- & 13.0 & 11.1 & 11.8 & 12.9 & 13.0 \\
\hline 25 & -- & -- & -- & 16.2 & -- & -- & -- & 13.0 & 11.1 & 11.8 & 13.0 & 13.0 \\
\hline 26 & -- & -- & -- & 16.3 & -- & -- & -- & 13.0 & 11.1 & 11.9 & 12.9 & 13.1 \\
\hline 27 & -- & .- & -- & 16.3 & -- & -. & -- & 13.0 & 11.1 & 11.9 & 12.9 & 13.1 \\
\hline 28 & -- & -- & -- & 16.4 & -- & -. & -- & 12.9 & 11.1 & 12.0 & 12.9 & 13.2 \\
\hline 29 & -- & .- & -- & 16.5 & -- & -. & -- & 12.8 & 11.2 & 12.0 & 12.9 & 13.2 \\
\hline 30 & -- & -- & -- & 16.6 & -- & -- & -. & 12.1 & 11.2 & 12.0 & 12.9 & 13.2 \\
\hline 31 & -. & -- & -- & -- & -- & -. & 14.1 & 11.9 & -- & 12.1 & -- & 13.3 \\
\hline
\end{tabular}


Table 14b. Mean daily ground-water temperature at site CRM-2 (map ID \#2), West Well Field, Cedar Rapids, lowa, April 1996 through February 1999--Continued

\begin{tabular}{|c|c|c|c|c|c|c|c|c|c|c|c|c|}
\hline Day & Jan & Feb & Mar & Apr & May & Jun & Jul & Aug & Sep & Oct & Nov & Dec \\
\hline & & & & & & 1997 & & & & & & \\
\hline 1 & 13.4 & 13.7 & 13.0 & -- & 12.2 & 11.9 & -- & 10.7 & 19.5 & 21.0 & 22.0 & 22.8 \\
\hline 2 & 13.5 & 13.7 & 13.0 & -- & 12.2 & 11.8 & -- & 10.6 & 19.7 & 21.1 & 22.1 & 22.7 \\
\hline 3 & 13.5 & 13.7 & 13.0 & -- & 12.2 & 11.8 & -- & 10.6 & 19.8 & 21.1 & 22.1 & 22.7 \\
\hline 4 & 13.6 & 13.6 & 13.0 & -- & 12.2 & 11.8 & -- & 10.5 & 19.9 & 21.1 & 22.1 & 22.7 \\
\hline 5 & 13.5 & 13.6 & 12.9 & 12.4 & 12.2 & 11.9 & -- & 10.5 & 19.9 & 21.2 & 22.1 & 22.8 \\
\hline 6 & 13.5 & 13.6 & 12.9 & 12.4 & 12.2 & 11.9 & -- & 10.5 & 20.0 & 21.2 & 21.7 & 22.8 \\
\hline 7 & 13.5 & 13.6 & 12.9 & 12.5 & 12.2 & 11.9 & -- & 10.5 & 20.1 & 21.2 & 22.1 & 22.8 \\
\hline 8 & 13.5 & 13.6 & 12.9 & 12.5 & 12.1 & 11.9 & -- & 10.5 & 20.2 & 21.2 & 22.3 & 22.8 \\
\hline 9 & 13.5 & 13.5 & 12.8 & 12.4 & 12.1 & 11.9 & -- & 10.5 & 20.2 & 21.2 & 22.5 & 22.8 \\
\hline 10 & 13.6 & 13.5 & 12.6 & 12.4 & -- & 11.9 & -- & 10.6 & 20.2 & 21.2 & 22.5 & 22.8 \\
\hline 11 & 13.6 & 13.5 & 12.7 & 12.4 & -- & 11.9 & -- & 10.8 & 20.3 & 21.2 & 22.6 & 22.8 \\
\hline 12 & 13.6 & 13.5 & 12.6 & 12.4 & -- & 11.9 & -- & 11.0 & 20.3 & 21.2 & 22.7 & 22.8 \\
\hline 13 & 13.6 & 13.4 & 12.5 & 12.4 & -- & - & -- & 11.3 & 20.3 & 21.2 & 22.8 & 22.7 \\
\hline 14 & 13.6 & 13.4 & 12.5 & 12.4 & -- & -- & -- & 11.6 & 20.4 & 21.2 & -- & 22.6 \\
\hline 15 & 13.6 & 13.4 & 12.4 & 12.3 & -- & -- & 11.0 & 12.0 & 20.5 & 21.2 & -- & 22.6 \\
\hline 16 & 13.6 & 13.3 & 12.2 & 12.3 & -- & -- & 11.0 & 12.5 & 20.5 & 21.2 & -- & 22.6 \\
\hline 17 & 13.6 & 13.3 & 12.0 & 12.3 & -- & -- & 10.9 & 13.0 & 20.6 & 21.2 & -- & 22.6 \\
\hline 18 & 13.6 & 13.3 & 12.1 & 12.3 & -- & -- & 10.9 & 13.5 & 20.6 & 21.2 & -- & 22.5 \\
\hline 19 & 13.6 & 13.3 & 12.3 & 12.3 & -- & -- & 10.9 & 14.0 & 20.7 & 21.2 & -- & 22.5 \\
\hline 20 & 13.6 & 13.3 & 12.2 & 12.3 & -- & -- & 10.9 & 14.2 & 20.7 & 21.3 & 22.9 & 22.4 \\
\hline 21 & 13.6 & 13.2 & 12.6 & 12.3 & -- & -- & 10.9 & 15.0 & 20.8 & 21.3 & 22.9 & 22.4 \\
\hline 22 & 13.6 & 13.2 & 12.7 & 12.3 & -- & -- & 10.9 & 15.6 & 20.9 & 21.4 & 22.9 & 22.4 \\
\hline 23 & 13.6 & 13.2 & 12.7 & 12.3 & 11.9 & -- & 10.9 & 16.3 & 20.9 & 21.5 & 22.9 & 22.4 \\
\hline 24 & 13.6 & 13.2 & 12.6 & 12.3 & 11.9 & -- & 10.9 & 16.9 & 20.9 & 21.5 & 22.9 & 22.4 \\
\hline 25 & 13.6 & 13.0 & 12.6 & 12.3 & 11.9 & -- & 10.9 & 17.4 & 20.9 & 21.6 & 22.9 & 22.3 \\
\hline 26 & 13.6 & 13.0 & 12.7 & 12.3 & 11.9 & -- & 10.9 & 17.8 & 20.8 & 21.6 & 22.9 & 22.3 \\
\hline 27 & 13.6 & 13.0 & 12.7 & 12.3 & 11.9 & -- & 10.9 & 18.2 & 20.9 & 21.7 & 22.8 & 22.3 \\
\hline 28 & 13.6 & 13.0 & 12.7 & 12.3 & 11.9 & -- & 10.8 & 18.6 & 20.9 & 21.8 & 22.8 & 22.2 \\
\hline 29 & 13.7 & -- & 12.3 & 12.2 & 11.9 & -- & 10.8 & 18.9 & 20.9 & 21.9 & 22.8 & 22.2 \\
\hline 30 & 13.7 & -- & -- & 12.2 & 11.9 & -- & 10.8 & 19.1 & 21.0 & 21.9 & 22.8 & 22.2 \\
\hline 31 & 13.7 & -- & -- & -- & 11.9 & -- & 10.7 & 19.3 & - & 22.0 & -- & 22.2 \\
\hline
\end{tabular}


Table 14b. Mean daily ground-water temperature at site CRM-2 (map ID \#2), West Well Field, Cedar Rapids, lowa, April 1996 through February 1999--Continued

\begin{tabular}{|c|c|c|c|c|c|c|c|c|c|c|c|c|}
\hline Day & Jan & Feb & Mar & Apr & May & Jun & Jul & Aug & Sep & Oct & Nov & Dec \\
\hline \multicolumn{13}{|c|}{1998} \\
\hline 1 & 22.1 & 21.3 & 20.3 & 19.2 & 14.9 & 5.0 & 4.3 & 10.3 & 18.0 & 21.9 & 24.3 & -- \\
\hline 2 & 22.1 & 21.3 & 20.3 & 19.0 & 14.6 & 4.8 & 4.2 & 10.6 & 18.1 & 22.1 & 24.3 & -- \\
\hline 3 & 22.1 & 21.3 & 20.3 & 18.7 & 14.2 & 4.7 & 4.3 & 10.8 & 18.2 & 22.4 & 24.3 & -- \\
\hline 4 & 22.1 & 21.3 & 20.2 & 18.3 & 13.8 & 4.5 & 4.3 & 11.0 & 18.4 & 22.6 & 24.3 & -- \\
\hline 5 & 22.0 & 21.3 & 20.2 & 17.9 & 13.4 & 4.5 & 4.4 & 11.3 & 18.5 & 22.9 & 24.3 & -- \\
\hline 6 & 22.0 & 21.2 & 20.1 & 17.5 & 13.0 & 4.4 & 4.5 & 11.5 & 18.7 & 23.1 & 24.3 & -- \\
\hline 7 & 22.0 & 21.2 & 20.1 & 17.4 & 12.6 & 4.3 & 4.5 & 11.8 & 18.8 & 23.3 & 24.3 & -- \\
\hline 8 & 21.9 & 21.2 & 20.0 & 17.3 & 12.3 & 4.2 & 4.5 & 12.1 & 19.0 & 23.5 & 24.3 & -- \\
\hline 9 & 21.9 & 21.2 & 20.0 & 17.2 & 12.0 & 4.2 & 4.6 & 12.5 & 19.1 & 23.6 & 24.3 & -- \\
\hline 10 & 21.9 & 21.1 & 20.0 & 17.2 & 11.7 & 4.1 & 4.6 & 12.8 & 19.2 & 23.7 & 24.3 & -- \\
\hline 11 & 21.8 & 21.1 & 19.9 & 17.1 & 11.3 & 4.1 & 4.7 & 13.2 & 19.2 & 23.8 & 24.3 & -- \\
\hline 12 & 21.8 & 21.1 & 19.9 & 17.0 & 11.0 & 4.1 & 4.8 & 13.6 & 19.3 & 23.9 & 24.2 & -- \\
\hline 13 & 21.8 & 21.0 & 19.8 & 17.0 & 10.6 & 4.0 & 4.9 & 13.9 & 19.4 & 24.0 & 24.2 & -- \\
\hline 14 & 21.8 & 21.0 & 19.8 & 17.0 & 10.2 & 4.0 & 5.0 & 14.3 & 19.5 & 24.1 & 24.2 & -- \\
\hline 15 & 21.7 & 20.9 & 19.8 & 17.0 & 9.9 & 4.0 & 5.1 & 14.6 & 19.7 & 24.1 & 24.1 & 21.8 \\
\hline 16 & 21.7 & 20.9 & 19.7 & 17.0 & 9.6 & 4.0 & 5.3 & 14.9 & 19.9 & 24.2 & 24.1 & 21.8 \\
\hline 17 & 21.7 & 20.9 & 19.7 & 17.0 & 9.2 & 3.9 & 5.6 & 15.1 & 20.0 & 24.2 & 24.1 & 21.7 \\
\hline 18 & 21.6 & 20.8 & 19.6 & 17.0 & 8.8 & 3.9 & 5.9 & 15.3 & 20.2 & 24.2 & 24.0 & 21.6 \\
\hline 19 & 21.6 & 20.8 & 19.6 & 16.9 & 8.4 & 3.9 & 6.2 & 15.4 & 20.3 & 24.2 & 24.0 & 21.6 \\
\hline 20 & 21.6 & 20.7 & 19.6 & 16.9 & 8.1 & 3.9 & 6.4 & 15.7 & 20.4 & 24.3 & 23.9 & 21.6 \\
\hline 21 & 21.6 & 20.7 & 19.5 & 16.8 & 7.7 & 3.9 & 6.7 & 15.9 & 20.6 & 24.3 & 23.9 & 21.5 \\
\hline 22 & 21.6 & 20.7 & 19.5 & 16.8 & 7.4 & 4.0 & 7.0 & 16.1 & 20.7 & 24.3 & 23.8 & 21.4 \\
\hline 23 & 21.6 & 20.6 & 19.5 & 16.7 & 7.1 & 4.0 & 7.3 & 16.4 & 20.7 & 24.4 & 23.8 & 21.3 \\
\hline 24 & 21.6 & 20.6 & 19.4 & 16.6 & 6.8 & 4.0 & 7.6 & 16.6 & 20.8 & 24.4 & 23.7 & 21.2 \\
\hline 25 & 21.5 & 20.5 & 19.4 & 16.5 & 6.5 & 4.0 & 7.9 & 16.9 & 20.9 & 24.4 & 23.6 & 21.1 \\
\hline 26 & 21.5 & 20.5 & 19.4 & 16.3 & 6.3 & 4.1 & 8.3 & 17.1 & 21.0 & 24.4 & 23.6 & 20.9 \\
\hline 27 & 21.5 & 20.4 & 19.3 & 16.1 & 6.0 & 4.1 & 8.7 & 17.3 & 21.1 & 24.4 & 23.5 & 20.8 \\
\hline 28 & 21.4 & 20.4 & 19.3 & 15.8 & 5.8 & 4.1 & 9.0 & 17.5 & 21.3 & 24.4 & -- & 20.7 \\
\hline 29 & 21.4 & -- & 19.3 & 15.6 & 5.6 & 4.2 & 9.4 & 17.7 & 21.5 & 24.4 & -- & 20.6 \\
\hline 30 & 21.4 & -- & 19.3 & 15.3 & 5.4 & 4.2 & 9.7 & 17.8 & 21.6 & 24.4 & -- & 20.5 \\
\hline 31 & 21.4 & -- & 19.3 & -- & 5.2 & -- & 10.1 & 18.0 & -. & 24.3 & -- & 20.3 \\
\hline
\end{tabular}


Table 14b. Mean daily ground-water temperature at site CRM-2 (map ID \#2), West Well Field, Cedar Rapids, lowa, April 1996 through February 1999--Continued

\begin{tabular}{|c|c|c|c|c|c|c|c|c|c|c|c|c|}
\hline Day & $\operatorname{Jan}$ & Feb & Mar & Apr & May & Jun & Jul & Aug & Sep & Oct & Nov & Dec \\
\hline & & & & & & 1999 & & & & & & \\
\hline 1 & 20.2 & 15.3 & -- & -- & -- & -- & -- & -- & -- & -- & -- & -- \\
\hline 2 & 20.1 & 15.3 & -- & -- & -- & -- & -- & -- & -- & -- & -- & -- \\
\hline 3 & 20.0 & 15.2 & -- & -- & -- & -- & -- & -- & -- & -- & -- & -- \\
\hline 4 & 19.8 & 15.1 & -- & -- & -- & -- & -- & -- & -- & -- & -- & -- \\
\hline 5 & 19.7 & 15.0 & -- & -- & - & -- & -. & -- & -- & -- & -- & -- \\
\hline 6 & 19.6 & 15.0 & -- & -- & -- & -- & -- & -- & -- & -- & -- & -- \\
\hline 7 & 19.4 & 14.9 & -- & -- & -- & -- & -- & -- & -- & -- & -- & -- \\
\hline 8 & 19.3 & 14.8 & -- & -- & -- & -- & -- & -- & -- & -- & -- & -- \\
\hline 9 & 19.1 & 14.8 & -- & -- & -- & -- & $\cdots$ & -- & -. & -- & -- & -- \\
\hline 10 & 18.9 & 14.7 & -- & -- & -- & -- & -- & -- & -- & -- & -- & -- \\
\hline 11 & 18.7 & 14.6 & -- & -- & -- & -- & -- & -- & -- & -- & -- & -- \\
\hline 12 & 18.5 & 14.6 & -- & -- & -- & -- & -- & -- & -- & -- & -- & -- \\
\hline 13 & 18.2 & 14.6 & -- & -- & -. & -- & -- & -- & -- & -- & -- & -- \\
\hline 14 & 18.0 & 14.5 & -- & -- & -- & -. & -- & -- & -- & -. & -- & -- \\
\hline 15 & 17.8 & 14.5 & -- & -- & -- & -- & -- & -- & -- & -- & -- & -- \\
\hline 16 & 17.6 & 14.4 & -- & -- & -- & -- & -- & -- & -- & -- & -- & -- \\
\hline 17 & 17.4 & 14.4 & -- & -- & -- & -- & -- & -- & -- & -- & -- & -- \\
\hline 18 & 17.1 & 14.3 & -- & $-\cdot$ & -- & $\cdots$ & -- & -- & -- & -- & -- & -. \\
\hline 19 & 17.0 & 14.3 & -. & -- & -- & -- & -- & -- & -- & -- & -- & -- \\
\hline 20 & 16.7 & 14.2 & -- & -- & -- & -- & -- & -- & -- & -. & -- & -- \\
\hline 21 & 16.6 & 14.2 & -- & -- & -- & -- & -. & -- & -- & -- & -- & -- \\
\hline 22 & 16.4 & 14.1 & -- & -. & -- & -- & -- & -- & -- & -- & -- & -- \\
\hline 23 & 16.2 & 14.1 & -- & -- & -- & -- & -- & -- & -- & -- & -- & -- \\
\hline 24 & 16.1 & 14.1 & -- & -- & -- & -- & -- & -- & -- & -- & -- & -- \\
\hline 25 & 16.0 & 14.0 & -- & -- & -- & -- & -- & -- & -- & -- & -- & -- \\
\hline 26 & 15.8 & 13.9 & -- & -- & -- & -- & -- & -- & -- & -- & -- & -- \\
\hline 27 & 15.7 & 13.9 & -- & -- & -. & -- & -- & -- & -- & -. & -- & -- \\
\hline 28 & 15.6 & 13.8 & -- & -- & -- & -- & -- & -- & -- & -- & -- & -- \\
\hline 29 & 15.6 & -- & -- & -- & -- & -- & -- & -- & -- & -- & -- & -- \\
\hline 30 & 15.5 & -- & -- & -- & -- & -- & -- & -- & -- & -- & -- & -- \\
\hline 31 & 15.4 & -- & -- & -- & -- & -- & -- & -- & -- & -- & -- & -. \\
\hline
\end{tabular}


Table 14c. Mean daily ground-water temperature at site CRM-3 (map ID \#3), Seminole Well Field, Cedar Rapids, lowa, January 1997 through February 1999

[Temperature in degrees Centigrade; --, value not measured or not recorded]

\begin{tabular}{|c|c|c|c|c|c|c|c|c|c|c|c|c|}
\hline Day & Jan & Feb & Mar & Apr & May & Jun & Jul & Aug & Sep & Oct & Nov & Dec \\
\hline & & & & & & 1997 & & & & & & \\
\hline 1 & -- & 10.8 & 10.8 & 10.3 & 9.9 & 9.5 & 9.2 & 8.9 & 8.8 & 9.2 & 9.8 & 10.2 \\
\hline 2 & -. & 10.9 & 10.8 & 10.3 & 9.9 & 9.5 & 9.2 & 8.8 & 8.8 & 9.2 & 9.8 & 10.2 \\
\hline 3 & - & 10.9 & 10.8 & 10.3 & 9.9 & 9.5 & 9.2 & 8.8 & 8.8 & 9.2 & 9.8 & 10.2 \\
\hline 4 & -- & 10.9 & 10.8 & 10.2 & 9.9 & 9.5 & 9.2 & 8.8 & 8.8 & 9.2 & 9.8 & 10.2 \\
\hline 5 & -. & 10.9 & 10.7 & 10.2 & 9.9 & 9.5 & 9.2 & 8.8 & 8.8 & 9.2 & 9.8 & 10.2 \\
\hline 6 & -- & 10.9 & 10.7 & 10.2 & 9.9 & 9.5 & 9.2 & 8.8 & 8.8 & 9.2 & 9.8 & 10.2 \\
\hline 7 & -- & 10.9 & 10.7 & 10.2 & 9.9 & 9.5 & 9.2 & 8.8 & 8.8 & 9.2 & 9.8 & 10.2 \\
\hline 8 & -- & 10.9 & 10.7 & 10.2 & 9.9 & 9.5 & 9.1 & 8.8 & 8.8 & 9.2 & 9.8 & 10.3 \\
\hline 9 & -- & 10.9 & 10.7 & 10.2 & 9.9 & 9.4 & 9.1 & 8.8 & 8.9 & 9.3 & 9.8 & 10.3 \\
\hline 10 & -- & 10.9 & 10.7 & 10.2 & 9.9 & 9.4 & 9.1 & 8.8 & 8.9 & 9.3 & 9.8 & 10.3 \\
\hline 11 & -- & 10.9 & 10.7 & 10.1 & 9.8 & 9.4 & 9.1 & 8.7 & 8.9 & 9.3 & 9.8 & 10.3 \\
\hline 12 & -- & 10.9 & 10.6 & 10.1 & 9.8 & 9.4 & 9.1 & 8.7 & 8.9 & 9.3 & 9.8 & 10.3 \\
\hline 13 & -- & 10.9 & 10.6 & 10.1 & 9.8 & 9.4 & 9.1 & 8.7 & 8.9 & 9.4 & 9.8 & 10.3 \\
\hline 14 & -- & 10.9 & 10.6 & 10.1 & 9.8 & 9.4 & 9.1 & 8.7 & 8.9 & 9.4 & 9.8 & 10.3 \\
\hline 15 & -- & 10.9 & 10.6 & 10.1 & 9.8 & 9.4 & 9.1 & 8.6 & 8.9 & 9.4 & 9.8 & 10.3 \\
\hline 16 & -- & 10.9 & 10.6 & 10.1 & 9.7 & 9.4 & 9.0 & 8.6 & 8.9 & 9.5 & 9.9 & 10.4 \\
\hline 17 & -- & 10.8 & 10.6 & 10.1 & 9.7 & 9.4 & 9.0 & 8.6 & 9.0 & 9.5 & 9.9 & 10.4 \\
\hline 18 & -- & 10.8 & 10.6 & 10.0 & 9.7 & 9.4 & 9.0 & 8.7 & 9.0 & 9.5 & 9.9 & 10.4 \\
\hline 19 & -- & 10.8 & 10.6 & 10.0 & 9.7 & 9.4 & 9.0 & 8.7 & 9.0 & 9.6 & 9.9 & 10.4 \\
\hline 20 & -- & 10.8 & 10.5 & 10.0 & 9.7 & 9.4 & 9.0 & 8.7 & 9.0 & 9.6 & 9.9 & 10.4 \\
\hline 21 & -- & 11.0 & 10.5 & 10.0 & 9.7 & 9.4 & 9.0 & 8.7 & 9.0 & 9.6 & 10.0 & 10.4 \\
\hline 22 & -- & 11.0 & 10.5 & 10.0 & 9.6 & 9.3 & 9.0 & 8.7 & 9.0 & 9.7 & 10.0 & 10.4 \\
\hline 23 & -. & 11.0 & 10.5 & 10.0 & 9.6 & 9.4 & 9.0 & 8.7 & 9.0 & 9.7 & 10.0 & 10.5 \\
\hline 24 & 10.8 & 10.8 & 10.5 & 9.9 & 9.6 & 9.4 & 8.9 & 8.7 & 9.0 & 9.7 & 10.0 & 10.5 \\
\hline 25 & 10.8 & 10.8 & 10.5 & 9.9 & 9.6 & 9.3 & 8.9 & 8.7 & 9.1 & 9.7 & 10.1 & 10.5 \\
\hline 26 & 10.8 & 10.8 & 10.5 & 9.9 & 9.6 & 9.3 & 8.9 & 8.7 & 9.1 & 9.7 & 10.1 & 10.5 \\
\hline 27 & 10.8 & 10.8 & 10.5 & 9.9 & 9.6 & 9.3 & 8.9 & 8.8 & 9.1 & 9.8 & 10.1 & 10.5 \\
\hline 28 & 10.8 & 10.8 & 10.4 & 9.9 & 9.6 & 9.2 & 8.9 & 8.7 & 9.1 & 9.8 & 10.1 & 10.5 \\
\hline 29 & 10.8 & -- & 10.4 & 9.9 & 9.5 & 9.2 & 8.9 & 8.7 & 9.1 & 9.8 & 10.1 & 10.5 \\
\hline 30 & 10.8 & -- & 10.4 & 9.9 & 9.5 & 9.2 & 8.9 & 8.7 & 9.2 & 9.8 & 10.1 & 10.5 \\
\hline 31 & 10.8 & -- & 10.4 & -- & 9.5 & -- & 8.9 & 8.8 & -- & 9.8 & -- & 10.5 \\
\hline
\end{tabular}


Table 14c. Mean daily ground-water temperature at site CRM-3 (map ID \#3), Seminole Well Field, Cedar Rapids, lowa, January 1997 through February 1999--Continued

\begin{tabular}{|c|c|c|c|c|c|c|c|c|c|c|c|c|}
\hline Day & Jan & Feb & Mar & Apr & May & Jun & Jul & Aug & Sep & Oct & Nov & Dec \\
\hline & & & & & & 1998 & & & & & & \\
\hline 1 & 10.5 & 10.2 & 10.1 & 9.6 & 9.5 & 10.1 & -- & -- & 20.0 & 22.1 & 23.2 & 23.2 \\
\hline 2 & 10.5 & 10.2 & 10.1 & 9.7 & 9.5 & 10.1 & -- & -- & 20.2 & 22.3 & 23.3 & 23.2 \\
\hline 3 & 10.5 & 10.2 & 10.0 & 9.7 & 9.6 & 10.1 & -- & -- & 20.2 & 22.6 & 23.3 & -- \\
\hline 4 & 10.5 & 10.2 & 10.0 & 9.6 & 9.5 & 10.1 & -- & -- & 20.3 & 22.7 & 23.4 & -- \\
\hline 5 & 10.5 & 10.2 & 10.0 & 9.6 & 9.7 & 10.1 & -- & -- & 20.3 & 22.8 & 23.4 & 23.1 \\
\hline 6 & 10.5 & 10.2 & 9.9 & 9.6 & 9.9 & 10.1 & -- & -- & 20.5 & 22.8 & 23.4 & 23.0 \\
\hline 7 & 10.4 & 10.2 & 9.9 & 9.6 & 10.0 & 10.0 & -- & -- & 20.5 & 22.8 & 23.5 & 23.0 \\
\hline 8 & 10.4 & 10.2 & 9.9 & 9.6 & 10.0 & 10.0 & -- & -- & 20.5 & 22.8 & 23.5 & 22.9 \\
\hline 9 & 10.4 & 10.1 & 9.9 & 9.6 & 10.0 & 10.0 & -- & -- & 20.7 & 22.8 & 23.5 & 22.9 \\
\hline 10 & 10.4 & 10.1 & 9.9 & 9.6 & 10.0 & 9.9 & -- & -- & 20.7 & 22.8 & 23.5 & 22.8 \\
\hline 11 & 10.4 & 10.1 & 9.9 & 9.6 & 10.0 & 9.9 & -- & -- & 20.8 & 22.7 & 23.5 & 22.8 \\
\hline 12 & 10.4 & 10.1 & 9.9 & 9.5 & 10.0 & 9.6 & -- & -- & 20.8 & 22.7 & 23.5 & 22.8 \\
\hline 13 & 10.4 & 10.1 & 9.9 & 9.5 & 9.9 & 9.3 & -- & -- & 20.9 & 22.7 & 23.6 & 22.7 \\
\hline 14 & 10.4 & 10.1 & 9.9 & 9.5 & 10.0 & 9.3 & -- & 16.0 & 20.9 & 22.7 & 23.6 & 22.7 \\
\hline 15 & 10.4 & 10.1 & 9.8 & 9.5 & 10.1 & 9.3 & -- & 16.2 & 20.9 & 22.7 & 23.7 & 22.6 \\
\hline 16 & 10.4 & 10.1 & 9.8 & 9.5 & 10.0 & 9.3 & -- & 16.5 & 21.0 & 22.7 & 23.7 & 22.6 \\
\hline 17 & 10.4 & 10.1 & 9.8 & 9.5 & 10.1 & 9.3 & -- & 16.9 & 20.9 & 22.8 & 23.7 & 22.6 \\
\hline 18 & 10.3 & 10.1 & 9.8 & 9.5 & 10.1 & 9.3 & -- & 17.3 & 21.0 & 22.9 & 23.7 & 22.5 \\
\hline 19 & 10.4 & 10.0 & 9.8 & 9.5 & 10.1 & 9.3 & -- & 17.5 & 21.0 & 22.9 & 23.7 & 22.5 \\
\hline 20 & 10.3 & 10.0 & 9.7 & 9.5 & 10.1 & 9.2 & -- & 17.9 & 21.0 & 22.9 & 23.7 & 22.5 \\
\hline 21 & 10.3 & 10.0 & 9.7 & 9.5 & 10.1 & 9.2 & -- & 18.1 & 21.1 & 22.9 & 23.7 & 22.4 \\
\hline 22 & 10.3 & 10.0 & 9.7 & 9.5 & 10.1 & 9.2 & -- & 18.4 & 21.2 & 22.8 & 23.6 & 22.4 \\
\hline 23 & 10.3 & 10.0 & 9.7 & 9.5 & 10.1 & -- & -- & 18.8 & 21.2 & 22.8 & 23.6 & 22.3 \\
\hline 24 & 10.3 & 10.0 & 9.6 & 9.5 & 10.2 & -- & -- & 19.1 & 21.2 & 23.0 & 23.6 & 22.3 \\
\hline 25 & 10.3 & 10.1 & 9.6 & 9.5 & 10.2 & -- & -- & 19.4 & 21.2 & 22.7 & 23.6 & 22.2 \\
\hline 26 & 10.3 & 10.1 & 9.6 & 9.5 & 10.2 & -- & -- & 19.7 & 21.1 & 22.7 & 23.5 & 22.2 \\
\hline 27 & 10.3 & 10.1 & 9.6 & 9.5 & 10.1 & -- & -- & 19.7 & 21.2 & 22.8 & 23.5 & 22.1 \\
\hline 28 & 10.3 & 10.1 & 9.6 & 9.5 & 10.1 & -- & -- & 19.8 & 21.4 & 22.9 & 23.4 & 22.1 \\
\hline 29 & 10.2 & -- & 9.6 & 9.5 & 10.0 & -- & -- & 19.9 & 21.7 & 23.0 & 23.3 & 22.1 \\
\hline 30 & 10.2 & -- & 9.6 & 9.5 & 10.0 & -- & -- & 20.2 & 21.9 & 23.1 & 23.3 & 22.0 \\
\hline 31 & 10.2 & -- & 9.6 & -- & 10.0 & -- & -- & 19.8 & -- & 23.2 & -- & 22.0 \\
\hline
\end{tabular}


Table 14c. Mean daily ground-water temperature at site CRM-3 (map ID \#3), Seminole Well Field, Cedar Rapids, lowa, January 1997 through February 1999--Continued

\begin{tabular}{|c|c|c|c|c|c|c|c|c|c|c|c|c|}
\hline Day & Jan & Feb & Mar & Apr & May & Jun & Jul & Aug & Sep & Oct & Nov & Dec \\
\hline \multicolumn{13}{|c|}{1999} \\
\hline 1 & 22.0 & 21.3 & -- & -- & -- & -- & -- & -- & -- & -- & -- & -. \\
\hline 2 & 21.9 & 21.3 & -- & -- & -. & -- & -- & -- & -- & -- & -- & -- \\
\hline 3 & 21.9 & 21.3 & -- & -- & -- & -- & -- & -- & -- & -- & -- & -. \\
\hline 4 & 21.9 & 21.2 & -- & -- & -. & -- & -- & -- & -- & -- & -- & -- \\
\hline 5 & 21.8 & 21.2 & -- & -- & -- & -- & -- & -- & -- & -- & -. & -- \\
\hline 6 & 21.8 & 21.2 & -- & -- & -- & -- & -- & -- & -- & -- & -- & -- \\
\hline 7 & 21.8 & 21.2 & -- & -- & -- & -- & -- & -- & -- & -- & -- & -- \\
\hline 8 & 21.7 & 21.2 & -- & -- & -- & -- & -- & -- & -- & -- & -- & -- \\
\hline 9 & 21.7 & 21.1 & -- & -- & -. & -- & -- & -- & -- & -- & -. & -- \\
\hline 10 & 21.7 & 21.1 & -- & -- & -- & -. & -- & -- & -- & -- & -- & -- \\
\hline 11 & 21.7 & 21.0 & -- & -- & -- & -- & -- & -. & -. & -- & -- & -- \\
\hline 12 & 21.7 & 21.0 & -. & -- & -- & -- & -- & -- & -. & -- & -- & -- \\
\hline 13 & 21.7 & 21.0 & -- & -- & -- & -- & -- & -- & -- & -- & -- & -- \\
\hline 14 & 21.6 & 20.9 & -- & -- & -- & -- & -- & -- & -- & -- & -- & -- \\
\hline 15 & 21.6 & 20.9 & -- & -- & -- & -- & -- & -- & -- & -- & -- & -- \\
\hline 16 & 21.6 & 20.9 & -- & -- & -- & -. & -- & -- & -- & -- & -- & -- \\
\hline 17 & 21.6 & 20.8 & - & -- & -- & - & -- & -- & -- & -- & -- & -- \\
\hline 18 & 21.6 & 20.8 & -. & -- & -- & -- & -- & -- & -- & .- & -- & -- \\
\hline 19 & 21.6 & 20.8 & -- & -- & -- & -- & -- & -- & -. & -- & -- & -- \\
\hline 20 & 21.5 & 20.7 & -- & -- & -- & -- & -- & -- & -- & -- & -- & -- \\
\hline 21 & 21.5 & 20.7 & -- & -- & -- & -. & -- & -- & -- & -- & -- & -- \\
\hline 22 & 21.5 & 20.7 & -- & -- & -- & -- & -- & -- & -- & -- & -- & -- \\
\hline 23 & 21.5 & 20.6 & -- & -- & -- & -- & -- & -- & -- & -- & -- & -- \\
\hline 24 & 21.5 & 20.6 & -- & -- & -- & -- & -- & -- & -- & -- & -- & -- \\
\hline 25 & 21.4 & 20.6 & -- & -- & -- & -. & -- & -- & -- & -- & -- & -- \\
\hline 26 & 21.4 & 20.6 & -- & -- & -- & -- & -- & -- & -- & -- & -- & -- \\
\hline 27 & 21.5 & 20.5 & -- & -- & -- & -- & -- & -- & -- & -- & -- & -- \\
\hline 28 & 21.4 & 20.5 & -- & -- & -- & -- & -- & -- & -- & -- & -- & -- \\
\hline 29 & 21.4 & -- & -- & -- & -- & -- & -- & -- & -- & -- & -- & -- \\
\hline 30 & 21.4 & -- & -- & -- & -- & -- & -- & - & -- & -- & -- & -- \\
\hline 31 & 21.3 & -- & -- & -- & -- & -. & -- & -- & -- & -. & -. & -. \\
\hline
\end{tabular}


Table 14d. Mean daily ground-water temperature at site CRM-4 (map ID \#4), Seminole Well Field, Cedar Rapids, lowa, January 1997 through June 1998

[Temperature in degrees Centigrade; --, value not measured or not recorded]

\begin{tabular}{|c|c|c|c|c|c|c|c|c|c|c|c|c|}
\hline Day & Jan & Feb & Mar & Apr & May & Jun & Jul & Aug & Sep & Oct & Nov & Dec \\
\hline \multicolumn{13}{|c|}{1997} \\
\hline 1 & -- & 10.0 & 9.9 & 8.5 & 8.0 & 6.8 & 8.9 & 22.8 & 24.8 & 23.1 & 10.0 & 10.4 \\
\hline 2 & - & 10.0 & 9.9 & 8.5 & 7.9 & 6.8 & 9.2 & 22.9 & 24.8 & 23.0 & 10.0 & 10.4 \\
\hline 3 & -- & 10.1 & 9.9 & 8.5 & 7.8 & 6.8 & 9.5 & 23.1 & 24.8 & 22.8 & 10.0 & 10.4 \\
\hline 4 & -- & 10.1 & 9.9 & 8.5 & 7.8 & 6.7 & 9.9 & 24.0 & 24.8 & 22.7 & 10.0 & 10.4 \\
\hline 5 & -- & 10.0 & 9.9 & 8.4 & 7.7 & 6.7 & 10.2 & 24.7 & 24.8 & 22.6 & 10.0 & 10.4 \\
\hline 6 & -- & 10.1 & 9.9 & 8.4 & 7.7 & 6.6 & 10.4 & 24.7 & 24.7 & 22.6 & 10.0 & 10.5 \\
\hline 7 & -- & 10.0 & 9.8 & 8.4 & 7.6 & 6.6 & 10.7 & 24.8 & 24.7 & 22.5 & 10.0 & 10.5 \\
\hline 8 & -- & 10.1 & 9.7 & 8.4 & 7.6 & 6.6 & 11.0 & 24.8 & 24.6 & 22.4 & 10.0 & 10.5 \\
\hline 9 & -- & 10.0 & 9.8 & 8.4 & 7.5 & 6.5 & 11.3 & 24.9 & 24.5 & 22.3 & 10.0 & 10.5 \\
\hline 10 & -- & 10.0 & 9.7 & 8.3 & 7.5 & 6.5 & 11.6 & 24.9 & 24.4 & 22.2 & 10.1 & 10.6 \\
\hline 11 & -- & 10.1 & 9.7 & 8.3 & 7.5 & 6.5 & 12.0 & 25.1 & 24.3 & 21.9 & 10.1 & 10.6 \\
\hline 12 & -- & 10.1 & 9.7 & 8.3 & 7.5 & 6.4 & 12.4 & 25.2 & 24.2 & 21.7 & 10.1 & 10.6 \\
\hline 13 & -- & 10.1 & 9.6 & 8.3 & 7.4 & 6.4 & 12.8 & 25.2 & 24.1 & 21.4 & 10.1 & 10.6 \\
\hline 14 & -- & 10.1 & 9.5 & 8.3 & 7.4 & 6.4 & 13.3 & 25.2 & 23.9 & 21.1 & 10.1 & 10.6 \\
\hline 15 & -- & 10.1 & 9.5 & 8.3 & 7.4 & 6.3 & 13.8 & 25.2 & 23.7 & 20.7 & 10.1 & 10.7 \\
\hline 16 & -- & 10.1 & 9.4 & 8.3 & 7.4 & 6.3 & 14.3 & 25.2 & 23.6 & 20.1 & 10.1 & 10.7 \\
\hline 17 & -- & 10.1 & 9.3 & 8.3 & 7.5 & 6.3 & 14.9 & 25.1 & 23.4 & 19.3 & 10.1 & 10.7 \\
\hline 18 & -- & 10.1 & 9.2 & 8.2 & 7.2 & 6.3 & 15.8 & 25.0 & 23.3 & 18.4 & 10.1 & 10.7 \\
\hline 19 & -- & 10.1 & 9.2 & 8.2 & 6.9 & 6.2 & 16.6 & 25.0 & 23.3 & 17.3 & 10.1 & 10.7 \\
\hline 20 & -- & 10.0 & 9.1 & 8.2 & 7.0 & 6.1 & 16.9 & 24.9 & 23.3 & 16.1 & 10.1 & 10.7 \\
\hline 21 & -- & 9.9 & 9.1 & 8.2 & 7.0 & 6.1 & 17.2 & 24.8 & 23.2 & 15.0 & 10.2 & 10.7 \\
\hline 22 & -- & 9.9 & 9.1 & 8.2 & 7.0 & 6.1 & 17.4 & 24.8 & 23.2 & 14.2 & 10.2 & 10.7 \\
\hline 23 & -- & 9.9 & 9.0 & 8.2 & 7.0 & 6.0 & 17.7 & 24.7 & 23.2 & 13.4 & 10.2 & 10.7 \\
\hline 24 & 9.4 & 9.9 & 9.0 & 8.1 & 7.0 & 6.0 & 17.9 & 24.7 & 23.2 & 12.7 & 10.2 & 10.7 \\
\hline 25 & 9.6 & 9.9 & 8.9 & 8.1 & 7.0 & 6.5 & 18.7 & 24.7 & 23.2 & 12.2 & 10.2 & 10.7 \\
\hline 26 & 9.8 & 9.9 & 8.9 & 8.1 & 7.0 & 7.1 & 19.7 & 24.7 & 23.2 & 11.8 & 10.2 & 10.7 \\
\hline 27 & 9.9 & 9.9 & 8.8 & 8.0 & 7.0 & 7.6 & 19.9 & 24.7 & 23.2 & 11.4 & 10.3 & 10.7 \\
\hline 28 & 9.9 & 9.9 & 8.8 & 8.0 & 7.0 & 8.0 & 20.1 & 24.8 & 23.2 & 10.9 & 10.3 & 10.7 \\
\hline 29 & 9.9 & -- & 8.7 & 8.0 & 6.9 & 8.3 & 20.3 & 24.7 & 23.2 & 10.5 & 10.3 & 10.7 \\
\hline 30 & 10.0 & -- & 8.6 & 8.0 & 6.9 & 8.6 & 20.9 & 24.8 & 23.2 & 10.2 & 10.3 & 10.6 \\
\hline 31 & 10.0 & -- & 8.6 & -- & 6.9 & -- & 22.5 & 24.8 & -- & 10.1 & -- & 10.6 \\
\hline
\end{tabular}


Table 14d. Mean daily ground-water temperature at site CRM-4 (map ID \#4), Seminole Well Field, Cedar Rapids, lowa, January 1997 through June 1998--Continued

\begin{tabular}{|c|c|c|c|c|c|c|c|c|c|c|c|c|}
\hline Day & Jan & Feb & Mar & Apr & May & Jun & Jul & Aug & Sep & Oct & Nov & Dec \\
\hline \multicolumn{13}{|c|}{1998} \\
\hline 1 & 10.5 & 10.1 & 9.6 & 9.3 & 8.8 & 8.0 & - & - & -- & - & -- & -. \\
\hline 2 & 10.4 & 10.1 & 9.6 & 9.3 & 8.8 & 8.1 & -- & -- & -- & -- & -- & - \\
\hline 3 & 10.4 & 10.1 & 9.6 & 9.2 & 8.7 & 8.1 & -. & -- & -. & -- & -. & -. \\
\hline 4 & 10.4 & 10.0 & 9.6 & 9.2 & 8.7 & 8.1 & -- & -- & -- & - & -- & - \\
\hline 5 & 10.4 & 10.0 & 9.6 & 9.2 & 8.7 & 8.2 & -- & -- & -- & -- & -- & - \\
\hline 6 & 10.4 & 10.0 & 9.6 & 9.2 & 8.7 & 8.2 & -- & -- & -- & - & -- & - \\
\hline 7 & 10.4 & 10.0 & 9.6 & 9.2 & 8.6 & 8.2 & -- & -- & -- & -- & -- & -- \\
\hline 8 & 10.4 & 10.0 & 9.6 & 9.2 & 8.6 & 8.3 & -- & -- & -- & -- & -- & -. \\
\hline 9 & 10.4 & 10.0 & 9.6 & 9.1 & 8.6 & 8.3 & -- & -- & -- & -- & -- & -- \\
\hline 10 & 10.4 & 10.0 & 9.6 & 9.1 & 8.5 & 8.4 & -- & -- & -- & -- & -- & -- \\
\hline 11 & 10.3 & 10.0 & 9.5 & 9.1 & 8.5 & 8.5 & -- & -- & -- & -- & -- & - \\
\hline 12 & 10.3 & 10.0 & 9.5 & 9.1 & 8.4 & 8.6 & -- & -- & -- & -- & -- & -- \\
\hline 13 & 10.3 & 10.0 & 9.4 & 9.1 & 8.4 & 8.7 & -- & -- & -- & -- & -- & -- \\
\hline 14 & 10.3 & 9.9 & 9.4 & 9.1 & 8.3 & 8.9 & - & -- & -- & -- & -- & -- \\
\hline 15 & 10.3 & 9.9 & 9.4 & 9.1 & 8.3 & 9.1 & -- & -- & -- & -- & -- & -- \\
\hline 16 & 10.3 & 9.9 & 9.4 & 9.1 & 8.3 & 9.2 & -- & -- & -- & -- & -- & - \\
\hline 17 & 10.3 & 9.9 & 9.4 & 9.1 & 8.2 & 9.4 & -- & -- & -- & -- & -- & -- \\
\hline 18 & 10.3 & 9.9 & 9.4 & 9.0 & 8.2 & 9.5 & -- & -- & -- & -- & -- & -- \\
\hline 19 & 10.2 & 9.8 & 9.4 & 9.0 & 8.1 & 9.8 & -- & -- & -- & -- & -- & -- \\
\hline 20 & 10.2 & 9.8 & 9.4 & 9.0 & 8.1 & 10.0 & -- & -- & -- & - & -- & -- \\
\hline 21 & 10.2 & 9.8 & 9.3 & 9.0 & 8.1 & 10.3 & -- & -- & -. & .- & -- & -- \\
\hline 22 & 10.2 & 9.7 & 9.3 & 9.0 & 8.0 & 10.6 & -. & -- & -. & -. & -- & -. \\
\hline 23 & 10.2 & 9.7 & 9.3 & 8.9 & 8.0 & -- & -- & -- & -- & -- & -- & -- \\
\hline 24 & 10.2 & 9.6 & 9.3 & 8.9 & 8.0 & -- & -- & -. & -- & -- & -- & -- \\
\hline 25 & 10.2 & 9.6 & 9.3 & 8.9 & 8.0 & -- & -- & -. & -- & - & - & -. \\
\hline 26 & 10.2 & 9.7 & 9.3 & 8.9 & 8.0 & -- & -- & - & -- & - & -- & -- \\
\hline 27 & 10.2 & 9.6 & 9.3 & 8.9 & 8.0 & -- & -- & -- & -- & -- & -- & -- \\
\hline 28 & 10.1 & 9.6 & 9.3 & 8.9 & 8.0 & -- & -- & -- & -- & -- & -- & -- \\
\hline 29 & 10.1 & -- & 9.2 & 8.8 & 8.0 & -- & -- & -- & -- & -- & -- & -- \\
\hline 30 & 10.1 & -- & 9.2 & 8.8 & 8.0 & -- & -. & -- & -- & - & -- & -- \\
\hline 31 & 10.1 & -- & 9.3 & -- & 8.0 & -- & -- & -- & -- & -- & -- & -- \\
\hline
\end{tabular}


Table 14e. Mean daily ground-water temperature at site CRM-6 (map ID \#5), Seminole Well Field, Cedar Rapids, lowa, January 1997 through February 1999

[Temperature in degrees Centigrade; --, value not measured or not recorded]

\begin{tabular}{|c|c|c|c|c|c|c|c|c|c|c|c|c|}
\hline Day & Jan & Feb & Mar & Apr & May & Jun & Jul & Aug & Sep & Oct & Nov & Dec \\
\hline & & & & & & 1997 & & & & & & \\
\hline 1 & -- & 10.4 & 10.5 & 10.1 & 9.3 & 8.1 & 11.8 & 24.0 & 25.9 & 23.5 & 9.7 & 10.1 \\
\hline 2 & -- & 10.4 & 10.5 & 10.1 & 9.3 & 8.1 & 12.0 & 24.3 & 25.8 & 23.5 & 9.7 & 10.2 \\
\hline 3 & -- & 10.4 & 10.5 & 10.0 & 9.2 & 8.0 & 12.3 & 24.5 & 25.7 & 23.4 & 9.7 & 10.2 \\
\hline 4 & -- & 10.4 & 10.5 & 9.9 & 9.2 & 7.9 & 12.5 & 25.1 & -- & 23.3 & 9.7 & 10.2 \\
\hline 5 & -- & 10.4 & 10.5 & 9.8 & 9.3 & 7.8 & 12.6 & 26.0 & 25.3 & 23.2 & 9.7 & 10.2 \\
\hline 6 & -- & 10.4 & 10.5 & 9.8 & 9.3 & 7.8 & 12.8 & 26.3 & 25.1 & 23.1 & 9.7 & 10.2 \\
\hline 7 & -- & 10.4 & 10.5 & 9.8 & 9.3 & 7.8 & 12.8 & 26.3 & 25.0 & 23.1 & 9.7 & 10.2 \\
\hline 8 & -. & 10.4 & 10.5 & 9.7 & 9.4 & 7.8 & 12.9 & 26.4 & 24.9 & 23.0 & 9.7 & 10.2 \\
\hline 9 & - & 10.4 & 10.4 & 9.7 & 9.4 & 7.8 & 13.0 & 26.4 & 24.7 & 22.9 & 9.7 & 10.2 \\
\hline 10 & -- & 10.4 & 10.4 & 9.7 & 9.3 & 7.8 & 13.2 & 26.4 & 24.6 & 22.8 & 9.8 & 10.2 \\
\hline 11 & -- & 10.4 & 10.4 & 9.7 & 9.4 & 7.8 & 13.4 & 26.5 & 24.4 & 22.5 & 9.8 & 10.3 \\
\hline 12 & -- & 10.4 & 10.4 & 9.6 & 9.3 & 7.7 & 13.6 & 26.5 & 24.3 & 22.1 & 9.8 & 10.3 \\
\hline 13 & -- & 10.4 & 10.3 & 9.6 & 9.3 & 7.7 & 13.8 & 26.6 & 24.1 & 21.6 & 9.8 & 10.3 \\
\hline 14 & - & 10.4 & 10.3 & 9.6 & 9.2 & 7.7 & 14.1 & 26.6 & 24.0 & 21.0 & 9.8 & 10.3 \\
\hline 15 & -- & 10.4 & 10.3 & 9.6 & 9.0 & 7.7 & 14.3 & 26.6 & 23.8 & 20.2 & 9.9 & 10.3 \\
\hline 16 & - & 10.4 & 10.3 & 9.6 & 8.9 & 7.7 & 14.6 & 26.6 & 23.6 & 19.3 & 9.9 & 10.3 \\
\hline 17 & -- & 10.4 & 10.4 & 9.5 & 8.9 & 7.7 & 15.0 & 26.6 & 23.5 & 18.4 & 9.9 & 10.2 \\
\hline 18 & -- & 10.4 & 10.3 & 9.5 & 8.7 & 7.7 & 15.7 & 26.6 & 23.5 & 17.2 & 9.9 & 10.2 \\
\hline 19 & -- & 10.4 & 10.2 & 9.5 & 8.4 & 7.6 & 16.4 & 26.6 & 23.5 & 16.0 & 9.9 & 10.2 \\
\hline 20 & -- & 10.4 & 10.2 & 9.5 & 8.3 & 7.6 & 16.6 & 26.5 & 23.6 & 14.7 & 9.9 & 10.2 \\
\hline 21 & -- & 10.5 & 10.2 & 9.5 & 8.3 & 7.6 & 16.7 & 26.5 & 23.6 & 13.7 & 10.0 & 10.2 \\
\hline 22 & -- & 10.5 & 10.1 & 9.5 & 8.2 & 7.6 & 16.9 & 26.4 & 23.6 & 12.9 & 10.0 & 10.2 \\
\hline 23 & -- & 10.5 & 10.1 & 9.5 & 8.2 & 7.7 & 17.0 & 26.4 & 23.7 & 12.3 & 10.0 & 10.2 \\
\hline 24 & 10.2 & 10.5 & 10.2 & 9.4 & 8.2 & 7.8 & 17.1 & 26.3 & 23.6 & 11.7 & 10.0 & 10.2 \\
\hline 25 & 10.3 & 10.5 & 10.2 & 9.4 & 8.2 & 8.6 & 17.8 & 26.3 & 23.6 & 11.3 & 10.0 & 10.2 \\
\hline 26 & 10.3 & 10.5 & 10.3 & 9.4 & 8.2 & 9.8 & 19.8 & 26.2 & 23.6 & 11.0 & 10.1 & $10: 2$ \\
\hline 27 & 10.3 & 10.5 & 10.2 & 9.3 & 8.2 & 10.5 & 20.4 & 26.2 & 23.6 & 10.7 & 10.1 & 10.2 \\
\hline 28 & 10.4 & 10.5 & 10.2 & 9.3 & 8.2 & 11.1 & 20.8 & 26.1 & 23.5 & 10.4 & 10.1 & 10.1 \\
\hline 29 & 10.4 & -- & 10.2 & 9.3 & 8.2 & 11.4 & 21.0 & 26.1 & 23.5 & 10.0 & 10.1 & 10.1 \\
\hline 30 & 10.4 & -- & 10.2 & 9.3 & 8.2 & 11.6 & 21.4 & 26.0 & 23.5 & 9.8 & 10.1 & 10.1 \\
\hline 31 & 10.4 & -- & 10.1 & -- & 8.1 & -- & 23.0 & 26.0 & -. & 9.7 & -- & 10.1 \\
\hline
\end{tabular}


Table 14e. Mean daily ground-water temperature at site CRM-6 (map ID \#5), Seminole Well Field, Cedar Rapids, lowa, January 1997 through February 1999--Continued

\begin{tabular}{|c|c|c|c|c|c|c|c|c|c|c|c|c|}
\hline Day & Jan & $\mathrm{Feb}$ & Mar & Apr & May & Jun & Jul & Aug & Sep & Oct & Nov & Dec \\
\hline \multicolumn{13}{|c|}{1998} \\
\hline 1 & 10.1 & 9.9 & 9.9 & 10.0 & 9.9 & 9.6 & 11.8 & 24.0 & 22.4 & 24.6 & 19.4 & 15.4 \\
\hline 2 & 10.1 & 9.9 & 9.9 & 10.0 & 9.9 & 9.6 & 12.0 & 24.3 & 22.3 & 24.6 & 19.4 & 15.3 \\
\hline 3 & 10.1 & 9.9 & 9.9 & 10.0 & 9.9 & 9.6 & 12.3 & 24.5 & 22.5 & 24.6 & 19.3 & -. \\
\hline 4 & 10.1 & 9.9 & 9.9 & 10.0 & 9.9 & 9.6 & 12.5 & 25.1 & 22.7 & 24.4 & 19.3 & -- \\
\hline 5 & 10.1 & 9.9 & 9.9 & 10.0 & 9.9 & 9.6 & 12.6 & 26.0 & 22.7 & 24.2 & 19.3 & 15.1 \\
\hline 6 & 10.1 & 9.9 & 9.9 & 10.0 & 9.9 & 9.6 & 12.8 & 26.3 & 22.7 & 24.1 & 19.3 & 15.0 \\
\hline 7 & 10.1 & 9.9 & 9.9 & 10.0 & 9.8 & 9.6 & 12.8 & 26.3 & 23.0 & 24.0 & 19.2 & 14.9 \\
\hline 8 & 10.1 & 9.9 & 9.9 & 10.0 & 9.8 & 9.6 & 12.9 & 26.4 & 23.2 & 23.9 & 19.0 & 14.9 \\
\hline 9 & 10.0 & 9.9 & 9.9 & 10.0 & 9.8 & 9.7 & 13.0 & 26.4 & 23.3 & 23.7 & 18.6 & 14.8 \\
\hline 10 & 10.0 & 9.9 & 9.8 & 10.0 & 9.8 & 9.7 & 13.2 & 26.4 & 23.4 & 23.7 & 18.7 & 14.7 \\
\hline 11 & 10.0 & 9.9 & 9.8 & 10.0 & 9.8 & 9.8 & 13.4 & 26.5 & 23.4 & 23.6 & 18.7 & 14.6 \\
\hline 12 & 10.0 & 9.9 & 9.8 & 10.0 & 9.7 & 9.8 & 13.6 & -- & 23.7 & 23.5 & 18.5 & 14.4 \\
\hline 13 & 10.0 & 9.8 & 9.8 & 10.0 & 9.7 & 9.9 & 13.8 & -- & 23.8 & 23.3 & 18.2 & 14.3 \\
\hline 14 & 10.0 & 9.8 & 9.8 & 10.0 & 9.7 & 10.0 & 14.1 & 22.3 & 24.0 & 22.9 & 18.1 & 14.2 \\
\hline 15 & 10.0 & 9.8 & 9.8 & 10.0 & 9.7 & 10.0 & 14.3 & 22.7 & 23.9 & 22.7 & 17.9 & 14.1 \\
\hline 16 & 10.0 & 9.8 & 9.8 & 10.0 & 9.6 & 10.0 & 14.6 & 22.8 & 23.7 & 22.3 & 17.7 & 13.9 \\
\hline 17 & 10.0 & 9.8 & 9.8 & 10.0 & 9.6 & 10.1 & 15.0 & 22.9 & 23.5 & 21.8 & 17.4 & 13.8 \\
\hline 18 & 10.0 & 9.8 & 9.8 & 10.0 & 9.6 & 10.1 & 15.7 & 23.0 & 23.2 & 21.6 & 17.2 & 13.7 \\
\hline 19 & 10.0 & 9.8 & 9.8 & 10.0 & 9.6 & 10.1 & 16.4 & 23.1 & 23.3 & 21.1 & 17.1 & 13.5 \\
\hline 20 & 10.0 & 9.8 & 9.8 & 10.0 & 9.5 & 10.2 & 16.6 & 23.2 & 23.9 & 20.7 & 17.0 & 13.4 \\
\hline 21 & 10.0 & 9.8 & 9.8 & 10.0 & 9.5 & 10.3 & 16.7 & 23.0 & 24.2 & 20.7 & 16.8 & 13.3 \\
\hline 22 & 10.0 & 9.8 & 9.8 & 10.0 & 9.5 & 10.3 & 16.9 & 22.8 & 24.4 & 20.5 & 16.7 & 13.0 \\
\hline 23 & 10.0 & 9.8 & 9.8 & 10.0 & 9.5 & -- & 17.0 & 22.8 & 24.3 & 20.4 & 16.6 & 12.8 \\
\hline 24 & 10.0 & 9.8 & 9.8 & 10.0 & 9.5 & -- & 17.1 & 23.1 & 24.3 & 20.3 & 16.4 & 12.7 \\
\hline 25 & 10.0 & 9.8 & 9.9 & 10.0 & 9.5 & 8.5 & 17.8 & 23.3 & 24.3 & 20.4 & 16.2 & 12.6 \\
\hline 26 & 9.9 & 9.8 & 9.9 & 10.0 & 9.5 & 9.8 & 19.8 & 23.4 & 24.4 & 20.3 & 16.1 & 12.4 \\
\hline 27 & 9.9 & 9.8 & 9.9 & 9.9 & 9.5 & 10.5 & 20.4 & 23.2 & 24.5 & 20.2 & 16.0 & 12.2 \\
\hline 28 & 9.9 & 9.8 & 9.9 & 9.9 & 9.5 & 11.1 & 20.8 & 23.0 & 24.4 & 20.0 & 15.8 & 12.1 \\
\hline 29 & 9.9 & -- & 9.9 & 9.9 & 9.5 & 11.4 & 21.0 & 22.9 & 24.4 & 19.8 & 15.7 & 11.9 \\
\hline 30 & 9.9 & -- & 9.9 & 9.9 & 9.5 & 11.6 & 21.4 & 23.0 & 24.6 & 19.8 & 15.6 & 11.7 \\
\hline 31 & 9.9 & -- & 10.0 & -- & 9.5 & -- & 23.0 & 22.7 & - & 19.6 & -- & 11.5 \\
\hline
\end{tabular}


Table 14e. Mean daily ground-water temperature at site CRM-6 (map ID \#5), Seminole Well Field, Cedar Rapids, lowa, January 1997 through February 1999--Continued

\begin{tabular}{|c|c|c|c|c|c|c|c|c|c|c|c|c|}
\hline Day & Jan & Feb & $\overline{\text { Mar }}$ & Apr & May & Jun & Jul & Aug & Sep & Oct & Nov & Dec \\
\hline & & & & & & 1999 & & & & & & \\
\hline 1 & 11.4 & 8.8 & -- & -- & -- & -- & -- & -- & -- & - & -- & -- \\
\hline 2 & 11.3 & 8.8 & -- & -- & -- & -- & -- & -- & -- & -- & -- & -- \\
\hline 3 & 11.1 & 8.8 & -- & -- & -- & -. & -- & -- & -- & -- & -- & -- \\
\hline 4 & 10.9 & 8.6 & -- & -- & -- & -- & -- & -- & -- & -- & -- & -- \\
\hline 5 & 10.8 & 8.6 & -- & -- & -- & -- & -- & -- & -- & -- & -- & -- \\
\hline 6 & 10.7 & 8.5 & -- & -- & -- & -- & -- & -- & -- & -- & -- & -- \\
\hline 7 & 10.4 & 8.5 & -- & -- & - & -- & -- & -- & -- & -- & -- & -- \\
\hline 8 & 10.3 & 8.4 & -- & -- & -- & -- & -- & -- & -- & -. & -- & -- \\
\hline 9 & 10.2 & 8.3 & -- & -- & -- & -. & -- & - & -- & -- & -- & -- \\
\hline 10 & 10.1 & 8.3 & -- & -- & -. & -- & -- & -- & -- & -- & -- & -- \\
\hline 11 & 10.0 & 8.3 & -. & -- & -- & -- & -- & -. & -- & - & -- & -- \\
\hline 12 & 10.0 & 8.2 & -- & -- & -- & -- & -- & -. & -- & -- & -- & -- \\
\hline 13 & 9.9 & 8.1 & -- & -- & -- & -- & -- & -- & -- & -- & -- & -- \\
\hline 14 & 9.8 & 8.1 & -- & -- & -- & -- & -- & -- & -- & -- & -- & -. \\
\hline 15 & 9.8 & 8.2 & -- & -- & -- & -- & -- & -- & -- & -- & -- & -- \\
\hline 16 & 9.7 & 8.1 & -- & -- & -- & -- & -- & -- & -- & -- & -- & -- \\
\hline 17 & 9.7 & 8.1 & -- & -- & - & -- & -- & -- & -- & -- & -- & -- \\
\hline 18 & 9.6 & 8.0 & -- & -- & -. & -- & -- & -. & -- & -- & -- & -- \\
\hline 19 & 9.4 & 7.9 & -- & -- & -- & - & -- & -- & -- & -- & -- & -- \\
\hline 20 & 9.4 & 7.9 & -- & -- & -- & -- & -- & -- & - & -- & -- & -- \\
\hline 21 & 9.4 & 7.7 & -- & -- & -- & -- & -- & -- & -- & -- & -- & -- \\
\hline 22 & 9.3 & 7.7 & -- & -- & -- & -- & -- & -- & -. & -- & -- & -- \\
\hline 23 & 9.3 & 7.7 & -- & -- & - & -- & -- & -- & - & -- & -- & -- \\
\hline 24 & 9.2 & 7.6 & -- & -- & -- & -- & -- & -- & -- & -- & -- & -- \\
\hline 25 & 9.0 & 7.6 & -- & -- & -- & -- & -- & -- & -- & -- & -- & -- \\
\hline 26 & 9.0 & 7.5 & -- & -- & -. & -- & -- & -- & -. & -- & -- & -- \\
\hline 27 & 9.0 & 7.5 & -- & -- & -- & -- & -- & -- & -- & -- & -- & -- \\
\hline 28 & 8.9 & 7.5 & -. & -- & -- & -- & -- & -- & -- & -- & -- & -- \\
\hline 29 & 8.8 & -- & -- & -- & -- & -- & -- & -- & -- & -- & -- & -- \\
\hline 30 & 8.8 & -- & -- & -. & -- & -- & -- & -- & -- & -- & -- & -- \\
\hline 31 & 8.7 & -- & -- & -- & -- & -- & -- & -- & -- & -- & -- & -- \\
\hline
\end{tabular}


Table 14f. Mean daily ground-water temperature at site CRM-7 (map ID \#6), Seminole Well Field, Cedar Rapids, lowa, January 1997 through February 1999

[Temperature in degrees Centigrade; --, value not measured or not recorded]

\begin{tabular}{|c|c|c|c|c|c|c|c|c|c|c|c|c|}
\hline Day & Jan & Feb & Mar & $\overline{\text { Apr }}$ & May & Jun & Jul & Aug & Sep & Oct & Nov & Dec \\
\hline & & & & & & 1997 & & & & & & \\
\hline 1 & -- & 10.9 & 10.9 & 10.7 & 10.5 & 10.3 & 10.2 & 10.2 & 10.2 & 10.3 & 10.4 & 10.5 \\
\hline 2 & -- & 10.9 & 10.9 & 10.7 & 10.5 & 10.3 & 10.2 & 10.2 & 10.2 & 10.3 & 10.4 & 10.5 \\
\hline 3 & -- & 10.9 & 10.9 & 10.7 & 10.5 & 10.3 & 10.2 & 10.1 & 10.2 & 10.3 & 10.4 & 10.5 \\
\hline 4 & -- & 10.9 & 10.9 & 10.7 & 10.5 & 10.3 & 10.2 & 10.2 & 10.2 & 10.3 & 10.4 & 10.5 \\
\hline 5 & -- & 10.9 & 10.8 & 10.7 & 10.5 & 10.3 & 10.2 & 10.2 & 10.2 & 10.3 & 10.4 & 10.5 \\
\hline 6 & -- & 10.9 & 10.8 & 10.7 & 10.5 & 10.3 & 10.2 & 10.2 & 10.2 & 10.3 & 10.4 & 10.5 \\
\hline 7 & -- & 10.9 & 10.8 & 10.7 & 10.5 & 10.3 & 10.2 & 10.2 & 10.2 & 10.3 & 10.4 & 10.5 \\
\hline 8 & -- & 10.9 & 10.8 & 10.7 & 10.4 & 10.3 & 10.2 & 10.2 & 10.2 & 10.3 & 10.4 & 10.5 \\
\hline 9 & -- & 10.9 & 10.8 & 10.7 & 10.4 & 10.3 & 10.2 & 10.2 & 10.2 & 10.3 & 10.4 & 10.5 \\
\hline 10 & -- & 10.9 & 10.8 & 10.6 & 10.4 & 10.3 & 10.2 & 10.2 & 10.2 & 10.3 & 10.4 & 10.5 \\
\hline 11 & -- & 10.9 & 10.8 & 10.6 & 10.4 & 10.3 & 10.2 & 10.2 & 10.2 & 10.3 & 10.4 & 10.5 \\
\hline 12 & -. & 10.9 & 10.8 & 10.6 & 10.4 & 10.3 & 10.2 & 10.2 & 10.2 & 10.3 & 10.4 & 10.5 \\
\hline 13 & -- & 11.0 & 10.8 & 10.6 & 10.4 & 10.3 & 10.2 & 10.2 & 10.2 & 10.3 & 10.4 & 10.5 \\
\hline 14 & -- & 11.0 & 10.8 & 10.6 & 10.4 & 10.3 & 10.2 & 10.2 & 10.2 & 10.3 & 10.4 & 10.5 \\
\hline 15 & -- & 10.9 & 10.8 & 10.6 & 10.4 & 10.3 & 10.2 & 10.2 & 10.2 & 10.3 & 10.4 & 10.5 \\
\hline 16 & -- & 10.9 & 10.8 & 10.6 & 10.4 & 10.3 & 10.2 & 10.2 & 10.2 & 10.3 & 10.4 & 10.5 \\
\hline 17 & -- & 10.9 & 10.8 & 10.6 & 10.4 & 10.3 & 10.2 & 10.2 & 10.2 & 10.3 & 10.4 & 10.5 \\
\hline 18 & -- & 11.0 & 10.8 & 10.6 & 10.4 & 10.2 & 10.2 & 10.2 & 10.2 & 10.3 & 10.4 & 10.5 \\
\hline 19 & -- & 10.9 & 10.8 & 10.6 & 10.4 & 10.2 & 10.2 & 10.2 & 10.2 & 10.3 & 10.4 & 10.5 \\
\hline 20 & -- & 10.9 & 10.8 & 10.6 & 10.4 & 10.2 & 10.1 & -- & 10.2 & 10.3 & 10.4 & 10.5 \\
\hline 21 & -- & 10.9 & 10.8 & 10.6 & 10.4 & 10.2 & 10.2 & -- & 10.2 & 10.3 & 10.4 & 10.5 \\
\hline 22 & -- & 10.9 & 10.8 & 10.6 & 10.3 & 10.2 & 10.1 & -- & 10.2 & 10.3 & 10.4 & 10.5 \\
\hline 23 & -- & 10.9 & 10.7 & 10.6 & 10.3 & 10.2 & 10.1 & -- & 10.2 & 10.3 & 10.4 & 10.5 \\
\hline 24 & 10.9 & 10.9 & 10.7 & 10.5 & 10.3 & 10.2 & 10.1 & -- & 10.2 & 10.3 & 10.4 & 10.5 \\
\hline 25 & 10.9 & 10.9 & 10.7 & 10.5 & 10.3 & 10.2 & 10.2 & -- & 10.2 & 10.4 & 10.4 & 10.5 \\
\hline 26 & 10.9 & 10.9 & 10.7 & 10.5 & 10.3 & 10.2 & 10.2 & -- & 10.2 & 10.3 & 10.4 & 10.5 \\
\hline 27 & 10.9 & 10.9 & 10.7 & 10.5 & 10.3 & 10.2 & 10.1 & 10.2 & 10.2 & 10.4 & 10.4 & 10.5 \\
\hline 28 & 10.9 & 10.9 & 10.7 & 10.5 & 10.3 & 10.2 & 10.2 & 10.2 & 10.3 & 10.4 & 10.4 & 10.5 \\
\hline 29 & 10.9 & -- & 10.7 & 10.5 & 10.3 & 10.2 & 10.1 & 10.2 & 10.3 & 10.4 & 10.4 & 10.5 \\
\hline 30 & 10.9 & -. & 10.7 & 10.5 & 10.3 & 10.2 & 10.2 & 10.2 & 10.3 & 10.4 & 10.4 & 10.6 \\
\hline 31 & 10.9 & -- & 10.7 & -- & 10.3 & -. & 10.2 & 10.2 & -- & 10.4 & -- & 10.6 \\
\hline
\end{tabular}


Table 14f. Mean daily ground-water temperature at site CRM-7 (map ID \#6), Seminole Well Field, Cedar Rapids, lowa, January 1997 through February 1999--Continued

\begin{tabular}{|c|c|c|c|c|c|c|c|c|c|c|c|c|}
\hline Day & Jan & Feb & Mar & Apr & May & Jun & Jul & Aug & Sep & Oct & Nov & Dec \\
\hline & & & & & & 1998 & & & & & & \\
\hline 1 & 10.6 & 10.7 & 10.7 & 10.7 & 10.6 & 10.5 & 10.4 & 10.4 & 10.4 & 10.4 & 10.5 & 10.6 \\
\hline 2 & 10.6 & 10.7 & 10.7 & 10.7 & 10.6 & 10.5 & 10.4 & 10.4 & 10.4 & 10.4 & 10.5 & 10.6 \\
\hline 3 & 10.6 & 10.7 & 10.7 & 10.7 & 10.6 & 10.5 & 10.4 & 10.4 & 10.4 & 10.4 & 10.5 & 10.6 \\
\hline 4 & 10.6 & $10: 7$ & 10.7 & 10.7 & 10.6 & 10.5 & 10.4 & 10.4 & 10.4 & 10.4 & 10.5 & 10.6 \\
\hline 5 & -- & 10.7 & 10.7 & 10.7 & 10.6 & 10.5 & 10.4 & 10.4 & 10.4 & 10.4 & 10.5 & 10.6 \\
\hline 6 & -- & 10.7 & 10.7 & 10.7 & 10.6 & 10.5 & 10.4 & 10.4 & 10.4 & 10.4 & 10.5 & 10.6 \\
\hline 7 & -- & 10.7 & 10.7 & 10.7 & 10.6 & 10.5 & 10.4 & 10.4 & 10.4 & 10.4 & 10.5 & 10.6 \\
\hline 8 & 10.6 & 10.7 & 10.7 & 10.7 & 10.5 & 10.5 & 10.4 & 10.4 & 10.4 & 10.4 & 10.5 & 10.6 \\
\hline 9 & 10.6 & 10.7 & 10.7 & 10.7 & 10.6 & 10.5 & 10.4 & 10.4 & 10.4 & 10.4 & 10.5 & 10.6 \\
\hline 10 & 10.6 & 10.7 & 10.7 & 10.6 & 10.5 & 10.5 & 10.4 & 10.4 & 10.4 & 10.4 & 10.5 & 10.6 \\
\hline 11 & 10.6 & 10.7 & 10.7 & 10.6 & 10.5 & 10.5 & 10.4 & 10.4 & 10.4 & 10.4 & 10.5 & 10.6 \\
\hline 12 & 10.6 & 10.7 & 10.7 & 10.6 & 10.5 & 10.5 & 10.4 & 10.4 & 10.4 & 10.4 & 10.5 & 10.6 \\
\hline 13 & 10.6 & 10.7 & 10.7 & 10.6 & 10.5 & 10.5 & 10.4 & 10.4 & 10.4 & 10.4 & 10.5 & 10.6 \\
\hline 14 & 10.6 & 10.7 & 10.7 & 10.6 & 10.5 & 10.5 & 10.4 & 10.4 & 10.4 & 10.4 & 10.5 & 10.6 \\
\hline 15 & 10.6 & 10.7 & 10.7 & 10.6 & 10.5 & 10.5 & 10.4 & 10.4 & 10.4 & 10.4 & 10.5 & 10.6 \\
\hline 16 & 10.6 & 10.7 & 10.7 & 10.6 & 10.5 & 10.5 & 10.4 & 10.4 & 10.4 & 10.4 & 10.5 & 10.6 \\
\hline 17 & 10.6 & 10.7 & 10.7 & 10.6 & 10.5 & 10.5 & 10.4 & 10.4 & 10.4 & 10.4 & 10.5 & 10.6 \\
\hline 18 & 10.6 & 10.7 & 10.7 & 10.6 & 10.5 & 10.4 & 10.4 & 10.4 & 10.4 & 10.4 & 10.5 & 10.6 \\
\hline 19 & 10.6 & 10.7 & 10.7 & 10.6 & 10.5 & 10.4 & 10.4 & 10.4 & 10.4 & 10.4 & 10.5 & 10.6 \\
\hline 20 & 10.6 & 10.7 & 10.7 & 10.6 & 10.5 & 10.4 & 10.4 & 10.4 & 10.4 & 10.4 & 10.5 & -- \\
\hline 21 & 10.6 & 10.7 & 10.7 & 10.6 & 10.5 & 10.4 & 10.4 & 10.4 & 10.4 & 10.4 & 10.5 & -- \\
\hline 22 & 10.6 & 10.7 & 10.7 & 10.6 & 10.5 & 10.4 & 10.4 & 10.4 & 10.4 & 10.4 & 10.5 & 10.6 \\
\hline 23 & 10.6 & 10.7 & 10.7 & 10.6 & 10.5 & 10.4 & 10.4 & 10.4 & 10.4 & 10.4 & 10.5 & 10.6 \\
\hline 24 & 10.6 & 10.7 & 10.7 & 10.6 & 10.5 & 10.4 & 10.4 & 10.4 & 10.4 & 10.4 & 10.5 & 10.6 \\
\hline 25 & 10.6 & 10.7 & 10.7 & 10.6 & 10.5 & 10.4 & 10.4 & 10.4 & 10.4 & 10.4 & 10.5 & 10.6 \\
\hline 26 & 10.6 & 10.7 & 10.7 & 10.6 & 10.5 & 10.4 & 10.4 & 10.4 & 10.4 & 10.4 & 10.5 & 10.7 \\
\hline 27 & 10.6 & 10.7 & 10.7 & 10.6 & 10.5 & 10.4 & 10.4 & 10.4 & 10.4 & 10.4 & 10.5 & 10.6 \\
\hline 28 & 10.6 & 10.7 & 10.7 & 10.6 & 10.5 & 10.4 & 10.4 & 10.4 & 10.4 & 10.4 & 10.5 & 10.7 \\
\hline 29 & 10.6 & -- & 10.7 & 10.6 & 10.5 & 10.4 & 10.4 & 10.4 & 10.4 & 10.5 & 10.6 & 10.7 \\
\hline 30 & 10.7 & -- & 10.7 & 10.6 & 10.5 & 10.4 & 10.4 & 10.4 & 10.4 & 10.5 & 10.6 & 10.7 \\
\hline 31 & 10.7 & -. & 10.7 & -- & 10.5 & -- & 10.4 & 10.4 & -- & 10.5 & -- & 10.7 \\
\hline
\end{tabular}


Table 14f. Mean daily ground-water temperature at site CRM-7 (map ID \#6), Seminole Well Field, Cedar Rapids, lowa, January 1997 through February 1999--Continued

\begin{tabular}{|c|c|c|c|c|c|c|c|c|c|c|c|c|}
\hline Day & Jan & Feb & Mar & Apr & May & Jun & Jul & Aug & Sep & Oct & Nov & Dec \\
\hline & & & & & & 1999 & & & & & & \\
\hline 1 & 10.7 & 10.8 & & -- & -- & -- & -- & -- & -- & -- & -- & -- \\
\hline 2 & 10.7 & 10.8 & & -- & -- & -- & -- & -- & - & -- & -- & -- \\
\hline 3 & 10.7 & 10.8 & & -- & -- & -- & -- & -- & -- & -- & - & -- \\
\hline 4 & 10.7 & 10.8 & & -- & -- & -- & -- & -- & -- & -. & -- & -- \\
\hline 5 & 10.7 & 10.8 & & -- & -- & -- & -- & -- & -- & -- & -- & -- \\
\hline 6 & 10.7 & 10.8 & & -- & -- & -- & -- & -- & -- & -- & -. & -- \\
\hline 7 & 10.7 & 10.8 & & -- & -- & -- & -- & -- & -- & -. & -- & -- \\
\hline 8 & 10.7 & 10.8 & & -- & -- & -- & -- & -- & -- & -- & -- & -- \\
\hline 9 & 10.7 & 10.8 & & -- & -- & -- & -- & - & -- & -- & -- & -- \\
\hline 10 & 10.7 & 10.8 & & -- & -- & -- & -. & -- & -- & -- & -- & -- \\
\hline 11 & 10.7 & 10.8 & & -- & -- & -- & -- & -- & -- & -- & -- & -- \\
\hline 12 & 10.7 & 10.8 & & -- & -- & -- & -- & -- & -- & -- & -- & - \\
\hline 13 & 10.7 & 10.8 & & -- & -- & -- & -- & -- & -- & -- & -- & -- \\
\hline 14 & 10.7 & 10.8 & & -- & -- & -- & -- & -- & -- & -- & -- & -- \\
\hline 15 & 10.7 & 10.8 & & -- & -- & -- & -- & -- & -- & -- & -. & -- \\
\hline 16 & 10.7 & 10.8 & & -- & -. & -- & -- & -- & -- & -- & -. & -- \\
\hline 17 & 10.7 & 10.8 & & -- & -- & -- & -- & -- & -- & -- & -- & -- \\
\hline 18 & 10.7 & 10.8 & & -- & -- & -- & -- & -- & -- & -- & -- & -- \\
\hline 19 & 10.7 & 10.8 & & -- & -- & -- & -- & -- & -- & -- & -- & -- \\
\hline 20 & 10.7 & 10.8 & & -- & -- & -- & -- & -- & -- & -- & -- & -- \\
\hline 21 & 10.7 & 10.8 & & -- & -- & -- & -- & -- & -- & -- & -- & -- \\
\hline 22 & 10.7 & 10.8 & & -- & -- & -. & -- & -- & -- & -- & -- & -- \\
\hline 23 & 10.7 & 10.8 & & -- & -- & -- & -- & -- & -- & -- & -- & -- \\
\hline 24 & 10.7 & 10.8 & & -- & -- & -- & -- & -- & -- & -- & -- & -- \\
\hline 25 & 10.8 & 10.8 & & -- & -- & -- & -- & -- & -- & -- & -- & -- \\
\hline 26 & 10.8 & 10.8 & & -- & -- & -- & -- & -- & - & -- & -- & -- \\
\hline 27 & 10.8 & 10.8 & & -- & -- & -- & -- & -- & -- & -- & -- & -- \\
\hline 28 & 10.8 & 10.8 & & -- & -- & -- & -- & -- & -- & -- & -- & -- \\
\hline 29 & 10.8 & -- & & -- & -. & -- & -- & -- & -- & -- & -- & -- \\
\hline 30 & 10.8 & -- & & -- & -- & -- & -- & -- & -- & -- & -- & -- \\
\hline 31 & 10.8 & -. & & -- & -- & -. & -- & -- & .- & -- & -- & -- \\
\hline
\end{tabular}


Table 14g. Mean daily ground-water temperature at site CRM-9 (map ID \#7), Seminole Well Field, Cedar Rapids, lowa, January 1997 through February 1999

[Temperature in degrees Centigrade; --, value not measured or not recorded]

\begin{tabular}{|c|c|c|c|c|c|c|c|c|c|c|c|c|}
\hline Day & Jan & Feb & Mar & Apr & May & Jun & Jul & Aug & Sep & Oct & Nov & Dec \\
\hline \multicolumn{13}{|c|}{1997} \\
\hline 1 & -- & 10.2 & 9.9 & 9.6 & 9.4 & 9.3 & 9.3 & 9.4 & 9.5 & 9.7 & 9.9 & 10.1 \\
\hline 2 & - & 10.2 & 9.9 & 9.6 & 9.4 & 9.3 & 9.3 & 9.4 & 9.5 & 9.7 & 9.9 & 10.1 \\
\hline 3 & -- & 10.2 & 9.9 & 9.6 & 9.4 & 9.3 & 9.3 & 9.4 & 9.5 & 9.7 & 9.9 & 10.1 \\
\hline 4 & -- & 10.2 & 9.8 & 9.6 & 9.4 & 9.4 & 9.3 & 9.4 & 9.6 & 9.7 & 9.9 & 10.1 \\
\hline 5 & -- & 10.1 & 9.8 & 9.6 & 9.4 & 9.4 & 9.3 & 9.4 & 9.6 & 9.7 & 9.9 & 10.1 \\
\hline 6 & - & 10.1 & 9.8 & 9.6 & 9.4 & 9.4 & 9.3 & 9.4 & 9.6 & 9.7 & 9.9 & 10.1 \\
\hline 7 & -- & 10.1 & 9.8 & 9.6 & 9.4 & 9.4 & 9.3 & 9.4 & 9.6 & 9.7 & 9.9 & 10.1 \\
\hline 8 & -- & 10.1 & 9.8 & 9.6 & 9.4 & 9.4 & 9.3 & 9.4 & 9.6 & 9.7 & 9.9 & 10.1 \\
\hline 9 & -- & 10.1 & 9.8 & 9.6 & 9.3 & 9.3 & 9.3 & 9.4 & 9.6 & 9.7 & 9.9 & 10.1 \\
\hline 10 & -- & 10.1 & 9.8 & 9.5 & 9.3 & 9.3 & 9.3 & 9.4 & 9.6 & 9.7 & 9.9 & 10.1 \\
\hline 11 & -- & 10.1 & 9.8 & 9.5 & 9.3 & 9.3 & 9.3 & 9.4 & 9.6 & 9.7 & 9.9 & 10.1 \\
\hline 12 & -- & 10.0 & 9.8 & 9.5 & 9.3 & 9.3 & 9.3 & 9.4 & 9.6 & 9.7 & 9.9 & 10.2 \\
\hline 13 & -- & 10.0 & 9.8 & 9.5 & 9.3 & 9.3 & 9.3 & 9.4 & 9.6 & 9.7 & 10.0 & 10.2 \\
\hline 14 & -- & 10.0 & 9.8 & 9.5 & 9.3 & 9.3 & 9.3 & 9.4 & 9.6 & 9.8 & 10.0 & 12.2 \\
\hline 15 & -- & 10.0 & 9.8 & 9.5 & 9.3 & 9.3 & 9.3 & 9.4 & 9.6 & 9.8 & 10.0 & 10.2 \\
\hline 16 & -- & 10.0 & 9.7 & 9.5 & 9.3 & 9.3 & 9.3 & 9.5 & 9.6 & 9.8 & 10.0 & 10.2 \\
\hline 17 & -- & 10.0 & 9.7 & 9.5 & 9.3 & 9.3 & 9.3 & 9.4 & 9.6 & 9.8 & 10.0 & 10.2 \\
\hline 18 & -- & 10.0 & 9.7 & 9.5 & 9.3 & 9.3 & 9.3 & 9.5 & 9.6 & 9.8 & 10.0 & 10.2 \\
\hline 19 & -- & 10.0 & 9.7 & 9.5 & 9.3 & 9.3 & 9.3 & 9.5 & 9.6 & 9.8 & 10.0 & 10.2 \\
\hline 20 & -- & 10.0 & 9.7 & 9.5 & 9.3 & 9.3 & 9.3 & 9.5 & 9.6 & 9.8 & 10.0 & 10.2 \\
\hline 21 & -- & 10.0 & 9.7 & 9.4 & 9.3 & 9.3 & 9.3 & 9.5 & 9.6 & 9.8 & 10.0 & 10.2 \\
\hline 22 & -- & 9.9 & 9.7 & 9.4 & 9.3 & 9.3 & 9.4 & 9.5 & 9.6 & 9.8 & 10.0 & 10.2 \\
\hline 23 & -- & 9.9 & 9.7 & 9.4 & 9.3 & 9.3 & 9.3 & 9.5 & 9.6 & 9.8 & 10.0 & 10.2 \\
\hline 24 & 10.2 & 9.9 & 9.7 & 9.4 & 9.3 & 9.3 & 9.3 & 9.5 & 9.6 & 9.8 & 10.0 & 10.2 \\
\hline 25 & 10.2 & 9.9 & 9.7 & 9.4 & 9.3 & 9.3 & 9.4 & 9.5 & 9.7 & 9.8 & 10.0 & 10.2 \\
\hline 26 & 10.2 & 9.9 & 9.6 & 9.4 & 9.3 & 9.3 & 9.4 & 9.5 & 9.7 & 9.8 & 10.0 & 10.3 \\
\hline 27 & 10.2 & 9.9 & 9.6 & 9.4 & 9.3 & 9.3 & 9.4 & 9.5 & 9.7 & 9.8 & 10.0 & 10.3 \\
\hline 28 & 10.2 & 9.9 & 9.6 & 9.4 & 9.3 & 9.3 & 9.4 & 9.5 & 9.7 & 9.8 & 10.1 & 10.3 \\
\hline 29 & 10.2 & -- & 9.6 & 9.4 & 9.3 & 9.3 & 9.4 & 9.5 & 9.7 & 9.9 & 10.1 & 10.3 \\
\hline 30 & 10.2 & -- & 9.6 & 9.4 & 9.3 & 9.3 & 9.4 & 9.5 & 9.7 & 9.9 & 10.1 & 10.3 \\
\hline 31 & 10.2 & .- & 9.6 & -- & 9.3 & -- & 9.4 & 9.5 & -- & 9.9 & -- & 10.3 \\
\hline
\end{tabular}


Table 14g. Mean daily ground-water temperature at site CRM-9 (map ID \#7), Seminole Well Field, Cedar Rapids, lowa, January 1997 through February 1999--Continued

\begin{tabular}{|c|c|c|c|c|c|c|c|c|c|c|c|c|}
\hline Day & Jan & Feb & Mar & Apr & May & Jun & Jul & Aug & Sep & Oct & Nov & Dec \\
\hline \multicolumn{13}{|c|}{1998} \\
\hline 1 & 10.3 & 10.4 & 10.4 & 10.3 & 10.2 & 9.9 & 9.8 & 9.8 & 9.8 & 10.0 & 10.2 & 10.4 \\
\hline 2 & 10.3 & 10.4 & 10.4 & 10.2 & 10.2 & 9.9 & 9.8 & 9.8 & 9.8 & 10.0 & 10.2 & 10.4 \\
\hline 3 & 10.3 & 10.4 & 10.4 & 10.2 & 10.2 & 9.9 & 9.7 & 9.8 & 9.8 & 10.0 & 10.2 & 10.4 \\
\hline 4 & 10.3 & 10.4 & 10.4 & 10.2 & 10.2 & 9.9 & 9.7 & 9.8 & 9.9 & 10.0 & 10.2 & 10.4 \\
\hline 5 & 10.3 & 10.4 & 10.4 & 10.2 & 10.1 & 9.9 & 9.8 & 9.8 & 9.8 & 10.0 & 10.2 & 10.4 \\
\hline 6 & 10.3 & 10.4 & 10.4 & 10.2 & 10.1 & 9.9 & 9.8 & 9.8 & 9.9 & 10.0 & 10.2 & 10.4 \\
\hline 7 & 10.3 & 10.4 & 10.4 & 10.2 & 10.1 & 9.9 & 9.8 & 9.8 & 9.9 & 10.0 & 10.2 & 10.4 \\
\hline 8 & 10.3 & 10.4 & 10.4 & 10.2 & 10.1 & 9.9 & 9.8 & 9.8 & 9.9 & 10.0 & 10.2 & 10.4 \\
\hline 9 & 10.3 & 10.4 & 10.4 & 10.2 & 10.1 & 9.9 & 9.8 & 9.8 & 9.9 & 10.0 & 10.2 & 10.4 \\
\hline 10 & 10.3 & 10.4 & 10.4 & 10.2 & 10.1 & 9.9 & 9.8 & 9.8 & 9.9 & 10.0 & 10.2 & 10.4 \\
\hline 11 & 10.3 & 10.4 & 10.4 & 10.2 & 10.1 & 9.9 & 9.8 & 9.8 & 9.9 & 10.0 & 10.2 & 10.4 \\
\hline 12 & 10.3 & 10.4 & 10.4 & 10.3 & 10.1 & 9.9 & 9.8 & 9.8 & 9.9 & 10.0 & 10.2 & 10.4 \\
\hline 13 & 10.3 & 10.4 & 10.4 & 10.3 & 10.1 & 9.9 & 9.8 & 9.8 & 9.9 & 10.0 & 10.2 & 10.4 \\
\hline 14 & 10.4 & 10.4 & 10.4 & 10.3 & 10.0 & 9.9 & 9.8 & 9.8 & 9.9 & 10.0 & 10.2 & 10.5 \\
\hline 15 & 10.4 & 10.4 & 10.4 & 10.3 & 10.0 & 9.8 & 9.8 & 9.8 & 9.9 & 10.1 & 10.3 & 10.5 \\
\hline 16 & 10.4 & 10.4 & 10.4 & 10.3 & 10.0 & 9.8 & 9.8 & 9.8 & 9.9 & 10.1 & 10.3 & 10.5 \\
\hline 17 & 10.4 & 10.4 & 10.4 & 10.2 & 10.0 & 9.8 & 9.8 & 9.8 & 9.9 & 10.1 & 10.3 & 10.5 \\
\hline 18 & 10.4 & 10.4 & 10.4 & 10.2 & 10.0 & 9.8 & 9.8 & 9.8 & 9.9 & 10.1 & 10.3 & 10.5 \\
\hline 19 & 10.4 & 10.4 & 10.3 & 10.2 & 10.0 & 9.8 & 9.8 & 9.8 & 9.9 & 10.1 & 10.3 & 10.5 \\
\hline 20 & 10.4 & 10.4 & 10.3 & 10.2 & 9.9 & 9.9 & 9.8 & 9.8 & 9.9 & 10.1 & 10.3 & 10.5 \\
\hline 21 & 10.4 & 10.4 & 10.4 & 10.2 & 9.9 & 9.9 & 9.8 & 9.8 & 9.9 & 10.1 & 10.3 & 10.5 \\
\hline 22 & 10.4 & 10.4 & 10.3 & 10.2 & 9.9 & 9.9 & 9.8 & 9.8 & 9.9 & 10.1 & 10.3 & 10.5 \\
\hline 23 & 10.4 & 10.4 & 10.3 & 10.2 & 10.0 & 9.9 & 9.8 & 9.8 & 9.9 & 10.1 & 10.3 & 10.5 \\
\hline 24 & 10.4 & 10.4 & 10.3 & 10.2 & 9.9 & 9.8 & 9.8 & 9.8 & 9.9 & 10.1 & 10.3 & 10.5 \\
\hline 25 & 10.4 & 10.4 & 10.3 & 10.2 & 9.9 & 9.8 & 9.8 & 9.8 & 9.9 & 10.1 & 10.3 & 10.5 \\
\hline 26 & 10.4 & 10.4 & 10.3 & 10.2 & 9.9 & 9.8 & 9.8 & 9.8 & 9.9 & 10.1 & 10.3 & 10.5 \\
\hline 27 & 10.4 & 10.4 & 10.3 & 10.2 & 9.9 & 9.8 & 9.8 & 9.8 & 10.0 & 10.1 & 10.3 & 10.5 \\
\hline 28 & 10.4 & 10.4 & 10.3 & 10.2 & 9.9 & 9.8 & 9.8 & 9.8 & 10.0 & 10.1 & 10.3 & 10.5 \\
\hline 29 & 10.4 & -- & 10.3 & 10.2 & 9.9 & 9.7 & 9.8 & 9.8 & 10.0 & 10.1 & 10.3 & 10.5 \\
\hline 30 & 10.4 & -- & 10.3 & 10.2 & 9.9 & 9.8 & 9.8 & 9.8 & 10.0 & 10.1 & 10.4 & 10.5 \\
\hline 31 & 10.4 & -- & 10.3 & -- & 9.9 & -- & 9.8 & 9.8 & -- & 10.1 & -- & 10.6 \\
\hline
\end{tabular}


Table 14g. Mean daily ground-water temperature at site CRM-9 (map ID \#7), Seminole Well Field, Cedar Rapids, lowa, January 1997 through February 1999--Continued

\begin{tabular}{|c|c|c|c|c|c|c|c|c|c|c|c|c|}
\hline Day & Jan & Feb & Mar & Apr & May & Jun & Jul & Aug & Sep & Oct & Nov & Dec \\
\hline & & & & & & 1999 & & & & & & \\
\hline 1 & 10.6 & 10.7 & -- & -- & -- & -- & -- & -- & -- & -- & -- & -- \\
\hline 2 & 10.6 & 10.7 & -. & -- & -- & -- & -- & -- & -- & -- & -- & -- \\
\hline 3 & 10.6 & 10.7 & -- & -- & -- & .. & - & -. & -- & -- & -- & -- \\
\hline 4 & 10.6 & 10.7 & -- & -- & -. & -- & -- & -- & -- & -- & -- & -- \\
\hline 5 & 10.6 & 10.7 & -- & -- & -- & -- & -- & -- & -- & -- & -- & -- \\
\hline 6 & 10.6 & 10.7 & -- & -- & -- & -- & -- & -- & -- & -- & -- & -- \\
\hline 7 & 10.6 & 10.7 & -- & -- & -- & -- & -- & -- & -- & -- & -- & -- \\
\hline 8 & 10.6 & 10.7 & -. & -- & -- & -- & -- & -- & -- & -- & -- & -- \\
\hline 9 & 10.6 & 10.7 & -- & -- & -- & -- & -. & -- & -- & -- & -- & -- \\
\hline 10 & 10.6 & 10.7 & -. & -- & -- & -- & -- & -- & -- & -- & -- & -- \\
\hline 11 & 10.6 & 10.7 & -. & -- & -- & -- & -- & -- & -- & -- & -- & -- \\
\hline 12 & 10.6 & 10.7 & -- & -- & -- & -- & -- & -- & -- & -- & -- & -- \\
\hline 13 & 10.6 & 10.7 & -- & -- & -- & -- & -- & -- & -- & -- & -- & -- \\
\hline 14 & 10.6 & 10.7 & -- & -- & -- & -- & -- & -- & -- & $-\cdot$ & -- & -- \\
\hline 15 & 10.6 & 10.7 & -- & -- & -- & -- & -- & -- & -- & -- & -- & -- \\
\hline 16 & 10.6 & 10.7 & -- & -. & -- & -- & - & -- & -. & -- & -- & -- \\
\hline 17 & 10.6 & 10.7 & - & -- & -- & -- & -- & -- & -. & -- & -- & -- \\
\hline 18 & 10.6 & 10.7 & -- & -- & -- & -- & -- & -- & -. & -- & -- & -- \\
\hline 19 & 10.6 & 10.7 & - & -- & - & -- & -- & -- & -- & -- & -- & -- \\
\hline 20 & 10.6 & 10.7 & -- & -- & -- & -- & -- & -- & -- & -- & -- & -- \\
\hline 21 & 10.6 & 10.7 & -- & -- & -- & -- & -- & -- & -- & -- & -- & -- \\
\hline 22 & 10.6 & 10.7 & -- & -- & -- & -- & -- & -- & -- & -- & -- & -- \\
\hline 23 & 10.6 & 10.7 & -- & -- & -- & -- & -- & -- & -- & -- & -- & -- \\
\hline 24 & 10.6 & 10.7 & -- & -- & -- & -- & -- & -- & -- & -- & -- & -- \\
\hline 25 & 10.6 & 10.7 & -- & -- & -- & -- & -- & -- & -- & -- & -- & -- \\
\hline 26 & 10.7 & 10.7 & -- & -- & -- & -- & -- & -- & -- & -- & -- & -- \\
\hline 27 & 10.7 & 10.7 & -- & -- & -- & -- & -- & -. & -- & -- & -- & -- \\
\hline 28 & 10.7 & 10.7 & -- & -- & -- & -- & -- & -- & -- & -- & -- & -- \\
\hline 29 & 10.7 & -- & -- & -- & -- & -. & -- & -. & -- & -- & -. & -- \\
\hline 30 & 10.7 & -- & -- & -- & -- & -- & -- & -- & -. & -- & -- & -- \\
\hline 31 & 10.7 & -- & -- & -- & -. & -- & -- & -- & -- & -- & -- & -- \\
\hline
\end{tabular}


[Temperature in degrees Centigrade; --, value not measured or not recorded]

\begin{tabular}{|c|c|c|c|c|c|c|c|c|c|c|c|c|}
\hline Day & Jan & Feb & Mar & Apr & May & Jun & Jul & Aug & Sep & Oct & Nov & Dec \\
\hline & & & & & & 1996 & & & & & & \\
\hline 1 & -- & -- & -- & 16.3 & 15.3 & -- & 11.1 & 11.3 & 11.2 & 10.8 & 10.9 & 9.0 \\
\hline 2 & -- & -- & - & 16.3 & 15.1 & -- & 11.1 & 11.3 & 11.1 & 10.8 & 10.9 & 8.9 \\
\hline 3 & -- & -- & -- & 16.3 & 15.0 & -- & 11.1 & 11.4 & 11.1 & 10.9 & 10.9 & 8.8 \\
\hline 4 & -- & -- & -- & 16.3 & 15.0 & -- & 11.1 & 11.4 & 11.0 & 10.9 & 10.8 & 8.9 \\
\hline 5 & -- & -- & -- & 16.3 & 14.9 & -- & 11.1 & 11.4 & 11.0 & 10.9 & 10.8 & 8.8 \\
\hline 6 & -- & -- & -- & 16.3 & 14.9 & -- & 11.1 & 11.4 & 11.0 & 10.9 & 10.8 & 8.7 \\
\hline 7 & -- & -- & -- & 16.3 & 15.0 & -- & 11.1 & 11.4 & 10.9 & 10.9 & 10.7 & 8.7 \\
\hline 8 & -. & -- & -- & 16.3 & 15.0 & -- & 11.1 & 11.4 & 10.9 & 10.9 & 10.6 & 8.7 \\
\hline 9 & -. & -- & -. & 16.3 & -. & -- & 11.1 & 11.4 & 10.9 & 11.0 & 10.6 & 8.6 \\
\hline 10 & -- & -- & -- & 16.3 & -- & -- & 11.2 & 11.4 & 10.8 & 11.0 & 10.4 & 8.6 \\
\hline 11 & -. & -- & -- & 16.2 & -- & -- & 11.2 & 11.4 & 10.8 & 11.1 & 10.3 & 8.6 \\
\hline 12 & -- & -- & -- & 16.2 & -- & -- & 11.2 & 11.4 & 10.8 & 11.1 & 10.2 & 8.6 \\
\hline 13 & -- & -- & -- & 16.2 & -- & - & 11.2 & 11.4 & 10.7 & 11.1 & 10.1 & 8.6 \\
\hline 14 & -- & -- & -- & 16.2 & -- & -- & 11.2 & 11.4 & 10.7 & 11.1 & 10.0 & 8.6 \\
\hline 15 & -- & -- & -- & 16.2 & -- & -- & 11.2 & 11.4 & 10.7 & 11.1 & 9.9 & 8.6 \\
\hline 16 & -- & -- & -- & 16.2 & -- & -- & 11.2 & 11.4 & 10.6 & 11.1 & 9.8 & 8.5 \\
\hline 17 & -- & -- & -- & 16.2 & -- & -- & 11.2 & 11.4 & 10.6 & 11.1 & 9.8 & 8.5 \\
\hline 18 & -- & -- & -- & 16.1 & -- & -- & 11.2 & 11.4 & 10.6 & 11.1 & 9.8 & 8.5 \\
\hline 19 & -- & -- & -- & 16.1 & -- & -- & 11.2 & 11.4 & 10.6 & 11.1 & 9.8 & 8.5 \\
\hline 20 & -- & -- & -- & 16.1 & -- & -- & 11.2 & 11.4 & 10.6 & 11.1 & 9.8 & 8.5 \\
\hline 21 & -- & - & -- & 16.1 & -- & -- & 11.2 & 11.4 & 10.6 & 11.0 & 9.7 & 8.5 \\
\hline 22 & -- & -- & -- & 16.1 & -- & -- & 11.2 & 11.4 & 10.6 & 11.0 & 9.7 & 8.5 \\
\hline 23 & -- & -- & -- & 15.9 & -- & -- & 11.2 & 11.4 & 10.7 & 11.0 & 9.6 & 8.6 \\
\hline 24 & -- & -- & -- & 16.0 & -- & -- & 11.3 & 11.4 & 10.7 & 11.0 & 9.6 & 8.5 \\
\hline 25 & -- & -- & -- & 16.0 & -- & -- & 11.3 & 11.3 & 10.7 & 11.0 & 9.6 & 8.5 \\
\hline 26 & -- & -- & -- & 15.9 & -- & -- & 11.3 & 11.3 & 10.7 & 11.0 & 9.5 & 8.5 \\
\hline 27 & -- & -- & -- & 15.9 & -- & -- & 11.3 & 11.3 & 10.7 & 11.0 & 9.4 & 8.5 \\
\hline 28 & -- & -- & -- & 15.7 & -- & 11.1 & 11.3 & 11.3 & 10.7 & 11.0 & 9.3 & 8.5 \\
\hline 29 & -- & -- & -- & 15.6 & -- & 11.1 & 11.3 & 11.2 & 10.7 & 11.0 & 9.1 & 8.6 \\
\hline 30 & -- & -- & -- & 15.5 & -- & 11.1 & 11.4 & 11.2 & 10.7 & 11.0 & 9.0 & 8.6 \\
\hline 31 & -- & -- & -- & -- & .- & -- & 11.3 & 11.2 & -- & 11.0 & -- & 8.8 \\
\hline
\end{tabular}


Table 14h. Mean daily ground-water temperature at site CRM-11 (map ID \#9), West Well Field, Cedar Rapids, lowa, April 1996 through February 1999--Continued

\begin{tabular}{|c|c|c|c|c|c|c|c|c|c|c|c|c|}
\hline Day & Jan & Feb & Mar & Apr & May & Jun & Jul & Aug & Sep & Oct & Nov & Dec \\
\hline & & & & & & 1997 & & & & & & \\
\hline 1 & 8.6 & 9.1 & 9.5 & -- & 10.9 & 11.3 & -- & 10.9 & 16.7 & 19.1 & 20.4 & 21.8 \\
\hline 2 & 8.6 & 9.1 & 9.5 & -- & 10.9 & 11.3 & -- & 10.8 & 16.8 & 19.2 & 20.5 & 21.9 \\
\hline 3 & 8.6 & 9.1 & 9.5 & -- & 10.9 & 11.3 & -- & 10.8 & 17.1 & 19.1 & 20.5 & 22.0 \\
\hline 4 & 8.7 & 9.1 & 9.6 & -- & 10.9 & 11.3 & -. & 10.7 & 17.3 & 19.2 & 20.6 & 22.0 \\
\hline 5 & 8.7 & 9.1 & 9.6 & 10.3 & 11.0 & 11.3 & -- & 10.7 & 17.5 & 19.6 & 20.6 & 22.1 \\
\hline 6 & 8.7 & 9.1 & 9.6 & 10.3 & 11.0 & 11.4 & -- & 10.6 & 17.6 & 19.2 & 20.7 & 22.1 \\
\hline 7 & 8.7 & 9.2 & 9.6 & 10.4 & 11.0 & 11.4 & -- & 10.7 & 17.8 & 19.3 & 20.8 & 22.1 \\
\hline 8 & 8.7 & 9.1 & 9.6 & 10.4 & 11.0 & 11.4 & -- & 10.6 & 17.8 & 19.6 & 20.9 & 22.1 \\
\hline 9 & 8.7 & 9.1 & 9.6 & 10.4 & 11.0 & 11.5 & -- & 10.6 & 17.9 & 19.4 & 20.9 & 22.1 \\
\hline 10 & 8.8 & 9.2 & 9.7 & 10.5 & -- & 11.5 & -- & 10.5 & 18.0 & 19.4 & 21.0 & 22.1 \\
\hline 11 & 8.8 & 9.2 & 9.7 & 10.5 & -. & 11.5 & -- & 10.6 & 18.0 & 19.4 & 21.1 & 22.1 \\
\hline 12 & 8.8 & 9.2 & 9.7 & 10.5 & -- & 11.5 & -. & 10.7 & 18.0 & 19.4 & 21.1 & 22.0 \\
\hline 13 & 8.8 & 9.2 & 9.7 & 10.6 & -- & -- & -- & 10.8 & 18.0 & 19.4 & 21.2 & 22.1 \\
\hline 14 & 8.8 & 9.2 & 9.8 & 10.6 & -- & -- & -- & 10.9 & 18.1 & 19.4 & -- & 22.1 \\
\hline 15 & 8.8 & 9.3 & 9.8 & 10.6 & -- & -- & 11.4 & 11.2 & 18.2 & 19.5 & -- & 22.2 \\
\hline 16 & 8.8 & 9.3 & 9.8 & 10.6 & -- & -- & 11.6 & 11.5 & 18.3 & 19.4 & -- & 22.2 \\
\hline 17 & 8.8 & 9.3 & 9.8 & 10.6 & -- & -- & 11.5 & 11.6 & 18.4 & 19.5 & -- & 22.2 \\
\hline 18 & 8.8 & 9.3 & 9.8 & 10.7 & -- & -- & 11.5 & 12.0 & 18.7 & 19.5 & -. & 22.2 \\
\hline 19 & 8.9 & 9.3 & 9.8 & 10.7 & -- & -- & 11.5 & 12.3 & 18.5 & 19.6 & -- & 22.2 \\
\hline 20 & 8.9 & 9.3 & 9.9 & 10.7 & -- & -- & 11.5 & 12.7 & 18.7 & 19.7 & 21.7 & 22.2 \\
\hline 21 & 8.9 & 9.4 & 9.9 & 10.7 & -- & -- & 11.6 & 13.0 & 18.7 & 19.7 & 21.7 & 22.2 \\
\hline 22 & 8.9 & 9.4 & 9.9 & 10.7 & -- & -. & 11.6 & 13.4 & 18.8 & 19.8 & 21.7 & 22.2 \\
\hline 23 & 8.9 & 9.4 & 9.9 & 10.8 & 11.2 & -- & 11.3 & 14.0 & 18.9 & 19.9 & 21.7 & 22.3 \\
\hline 24 & 8.9 & 9.4 & 9.9 & 10.8 & 11.2 & -- & 11.3 & 14.2 & 19.0 & 19.9 & 21.7 & 22.3 \\
\hline 25 & 9.0 & 9.4 & 10.0 & 10.8 & 11.2 & -- & 11.3 & 14.7 & 19.1 & 20.0 & 21.7 & 22.3 \\
\hline 26 & 9.0 & 9.4 & 10.0 & 10.8 & 11.2 & -- & 11.2 & 14.9 & 19.1 & 20.0 & 21.7 & 22.3 \\
\hline 27 & 9.0 & 9.5 & 10.0 & 10.8 & 11.2 & -. & 11.1 & 15.2 & 19.0 & 20.1 & 21.7 & 22.3 \\
\hline 28 & 9.0 & 9.5 & 10.1 & 10.8 & 11.2 & -- & 11.2 & 15.5 & 19.1 & 20.2 & 21.8 & 22.3 \\
\hline 29 & 9.0 & -- & 10.1 & 10.9 & 11.2 & -- & 11.2 & 15.9 & 19.1 & 20.2 & 21.8 & 22.3 \\
\hline 30 & 9.1 & -- & -- & 10.9 & 11.2 & -- & 11.1 & 16.2 & 19.1 & 20.3 & 21.8 & 22.3 \\
\hline 31 & 9.1 & -- & -- & -- & 11.2 & -- & 10.9 & 16.6 & -- & 20.4 & -- & 22.3 \\
\hline
\end{tabular}


Table 14h. Mean daily ground-water temperature at site CRM-11 (map ID \#9), West Well Field, Cedar Rapids, lowa, April 1996 through February 1999--Continued

\begin{tabular}{|c|c|c|c|c|c|c|c|c|c|c|c|c|}
\hline Day & Jan & Feb & Mar & Apr & May & Jun & Jul & Aug & Sep & Oct & Nov & Dec \\
\hline & & & & & & 1998 & & & & & & \\
\hline 1 & 22.3 & 21.5 & 20.9 & 19.9 & 16.5 & 5.4 & 4.1 & 8.4 & 15.1 & 19.7 & 24.0 & 23.5 \\
\hline 2 & 22.3 & 21.5 & 20.9 & 19.9 & 16.1 & 5.2 & 4.1 & 8.6 & 15.3 & 20.0 & 24.0 & 23.4 \\
\hline 3 & 22.2 & 21.5 & 20.8 & 19.6 & 15.7 & 5.0 & 4.1 & 8.8 & 15.5 & 20.3 & 24.1 & 23.3 \\
\hline 4 & 22.2 & 21.5 & 20.8 & 19.2 & 15.3 & 4.8 & 4.1 & 9.0 & 15.6 & 20.4 & 24.1 & 23.2 \\
\hline 5 & 22.2 & 21.5 & 20.8 & 18.9 & 14.8 & 4.6 & 4.1 & 9.3 & 15.8 & 20.7 & 24.2 & 23.1 \\
\hline 6 & 22.2 & 21.5 & 20.7 & 18.6 & 14.4 & 4.5 & 4.1 & 9.5 & 15.9 & 20.9 & 24.2 & 23.0 \\
\hline 7 & 22.1 & 21.5 & 20.7 & 18.4 & 14.0 & 4.5 & 4.1 & 9.7 & 16.1 & 21.2 & 24.3 & 22.9 \\
\hline 8 & 22.1 & 21.5 & 20.7 & 18.3 & 13.7 & 4.4 & 4.1 & 9.9 & 16.2 & 21.3 & 24.3 & 22.9 \\
\hline 9 & 22.1 & 21.5 & 20.6 & 18.3 & 13.4 & 4.4 & 4.1 & 10.1 & 16.4 & 21.5 & 24.3 & 22.8 \\
\hline 10 & 22.1 & 21.4 & 20.6 & 18.2 & 13.0 & 4.3 & 4.2 & 10.4 & 16.5 & 21.6 & 24.4 & 22.7 \\
\hline 11 & 22.1 & 21.4 & 20.5 & 18.1 & 12.6 & 4.3 & 4.2 & 10.7 & 16.6 & 21.8 & 24.4 & 22.7 \\
\hline 12 & 22.0 & 21.4 & 20.5 & 18.1 & 12.1 & 4.3 & 4.3 & 11.0 & 16.7 & 21.9 & 24.4 & 22.6 \\
\hline 13 & 22.0 & 21.4 & 20.5 & 18.2 & 11.7 & 4.2 & 4.3 & 11.3 & 16.9 & 22.1 & 24.4 & 22.5 \\
\hline 14 & 22.0 & 21.3 & 20.5 & 18.2 & 11.2 & 4.2 & 4.4 & 11.6 & 17.1 & 22.2 & 24.3 & 22.5 \\
\hline 15 & 22.0 & 21.3 & 20.4 & 18.2 & 10.8 & 4.1 & 4.5 & 11.9 & 17.3 & 22.3 & 24.3 & 22.4 \\
\hline 16 & 22.0 & 21.3 & 20.4 & 18.2 & 10.4 & 4.1 & 4.6 & 12.1 & 17.3 & 22.6 & 24.3 & 22.3 \\
\hline 17 & 21.9 & 21.3 & 20.3 & 18.2 & 10.0 & 4.1 & 4.7 & 12.3 & 17.6 & 22.5 & 24.3 & 22.2 \\
\hline 18 & 21.9 & 21.2 & 20.3 & 18.2 & 9.5 & 4.0 & 4.8 & 12.5 & 17.7 & 22.7 & 24.3 & 22.2 \\
\hline 19 & 21.9 & 21.2 & 20.3 & 18.2 & 9.2 & 4.1 & 5.0 & 12.7 & 17.8 & 22.8 & 24.2 & 22.1 \\
\hline 20 & 21.8 & 21.2 & 20.2 & 18.2 & 8.8 & 4.1 & 5.2 & 12.9 & 17.9 & 22.9 & 24.2 & 22.0 \\
\hline 21 & 21.8 & 21.2 & 20.2 & 18.2 & 8.4 & 4.0 & 5.4 & 13.1 & 18.1 & 23.0 & 24.2 & 21.9 \\
\hline 22 & 21.7 & 21.1 & 20.2 & 18.1 & 8.1 & 4.0 & 5.6 & 13.3 & 18.2 & 23.1 & 24.1 & 21.8 \\
\hline 23 & 21.7 & 21.1 & 20.1 & 18.0 & 7.7 & 4.0 & 5.8 & 13.6 & 18.3 & 23.3 & 24.1 & 21.7 \\
\hline 24 & 21.7 & 21.1 & 20.1 & 17.9 & 7.4 & 3.9 & 6.0 & 13.8 & 18.4 & 23.4 & 24.0 & 21.5 \\
\hline 25 & 21.7 & 21.0 & 20.1 & 17.7 & 7.1 & 4.0 & 6.3 & 14.0 & 18.6 & 23.4 & 24.0 & 21.4 \\
\hline 26 & 21.7 & 21.0 & 20.0 & 17.7 & 6.8 & 4.0 & 6.6 & 14.2 & 18.7 & 23.5 & 23.9 & 21.3 \\
\hline 27 & 21.7 & 21.0 & 20.0 & 17.5 & 6.5 & 4.0 & 6.9 & 14.3 & 18.9 & 23.6 & 23.8 & 21.2 \\
\hline 28 & 21.6 & 20.9 & 20.0 & 17.2 & 6.2 & 4.1 & 7.2 & 14.6 & 19.0 & 23.7 & 23.8 & 21.0 \\
\hline 29 & 21.6 & -- & 19.9 & 17.0 & 6.0 & 4.1 & 7.5 & 14.7 & 19.3 & 23.8 & 23.7 & 20.9 \\
\hline 30 & 21.6 & -- & 19.9 & 16.7 & 5.8 & 4.1 & 7.8 & 14.8 & 19.5 & 23.8 & 23.6 & 20.8 \\
\hline 31 & 21.6 & -- & 19.9 & -- & 5.6 & -- & 8.1 & 15.0 & -- & 23.9 & -- & 20.6 \\
\hline
\end{tabular}


Table 14h. Mean daily ground-water temperature at site CRM-11 (map ID \#9), West Well Field, Cedar Rapids, lowa, April 1996 through February 1999--Continued

\begin{tabular}{|c|c|c|c|c|c|c|c|c|c|c|c|c|}
\hline Day & Jan & Feb & Mar & Apr & May & Jun & Jul & Aug & Sep & Oct & Nov & Dec \\
\hline & & & & & & 1999 & & & & & & \\
\hline 1 & 20.5 & 15.9 & -- & -- & -- & -- & -- & -- & -- & -- & -. & -- \\
\hline 2 & 20.4 & 15.8 & -- & -. & -- & -- & -- & -- & -- & -- & -- & -- \\
\hline 3 & 20.3 & 15.8 & -- & -- & -- & -- & -- & -- & -. & -- & -- & -- \\
\hline 4 & 20.1 & 15.7 & -- & -- & -- & -- & -- & -- & -- & -- & -. & -- \\
\hline 5 & 20.0 & 15.6 & -- & -- & -- & -- & -- & -- & -- & -- & -- & -- \\
\hline 6 & 19.9 & 15.5 & -- & -- & -. & -- & -- & -- & -- & -- & -- & -- \\
\hline 7 & 19.7 & 15.5 & -- & -- & -- & -- & -. & -- & -- & -- & -- & -- \\
\hline 8 & 19.6 & 15.4 & -- & -- & -- & -- & -- & -. & -- & - & -- & -- \\
\hline 9 & 19.4 & 15.3 & -- & -- & -- & -- & -- & -. & -- & -- & -- & -- \\
\hline 10 & 19.2 & 15.3 & -- & -- & -- & -- & -- & -- & -- & -- & -- & -- \\
\hline 11 & 19.0 & 15.2 & -- & -- & -- & -- & -- & -- & -- & -- & -- & -- \\
\hline 12 & 18.8 & 15.2 & -- & -- & -. & -- & -- & -. & -- & -- & -- & -- \\
\hline 13 & 18.6 & 15.1 & -- & -- & -- & -- & -- & -- & -- & -- & -- & -- \\
\hline 14 & 18.4 & 15.1 & -- & -. & -- & -- & -- & -- & -- & -- & -- & -- \\
\hline 15 & 18.2 & 15.0 & - & -- & -- & -- & -- & -- & -- & -- & -- & -- \\
\hline 16 & 18.1 & 15.0 & -- & -- & -- & -- & -- & -- & -- & -- & -- & -- \\
\hline 17 & 17.9 & 14.9 & -- & -- & -- & -- & -- & -- & -- & -- & -- & -- \\
\hline 18 & 17.7 & 14.9 & -- & - & -- & -. & -- & -- & -- & -- & -- & -- \\
\hline 19 & 17.5 & 14.8 & -- & -- & -- & -- & -- & -- & -- & -- & -- & -- \\
\hline 20 & 17.3 & 14.8 & -- & - & -- & -- & -- & -- & -- & -- & -- & -- \\
\hline 21 & 17.2 & 14.7 & -- & -- & -- & -- & -- & -- & -- & -- & -- & $\cdots$ \\
\hline 22 & 17.0 & 14.7 & -- & -- & -- & -- & -- & -- & -- & - & -- & -- \\
\hline 23 & 16.9 & 14.6 & - & -- & -- & -- & -- & -- & -- & -- & -- & - \\
\hline 24 & 16.7 & 14.6 & -. & -- & -- & -- & -- & -- & -- & -- & -- & -- \\
\hline 25 & 16.6 & 14.5 & -- & -- & -- & -- & -- & -. & -- & -- & -- & -- \\
\hline 26 & 16.5 & 14.5 & -- & -- & -- & -- & -- & -- & -- & -- & -- & -- \\
\hline 27 & 16.4 & 14.4 & -. & -- & -- & -- & -- & -- & -- & -- & -- & -- \\
\hline 28 & 16.3 & 14.4 & -- & -- & -- & -- & - & -- & -- & -- & - & -- \\
\hline 29 & 16.2 & -- & -- & -- & -- & -- & -- & -- & -- & -- & -- & -- \\
\hline 30 & 16.1 & - & -- & -- & -- & -- & -- & -- & -- & -- & -- & -- \\
\hline 31 & 16.0 & -- & -- & -- & -- & -- & -- & -- & -- & -- & -- & - \\
\hline
\end{tabular}


Table 14i. Mean daily ground-water temperature at site CRM-12 (map ID \#10), East Well Field, Cedar Rapids, lowa, April 1996 through February 1999

[Temperature in degrees Centigrade; --, value not measured or not recorded]

\begin{tabular}{|c|c|c|c|c|c|c|c|c|c|c|c|c|}
\hline Day & Jan & Feb & Mar & Apr & May & Jun & Jul & Aug & Sep & Oct & Nov & Dec \\
\hline & & & & & & 1996 & & & & & & \\
\hline 1 & .. & -. & -- & 11.2 & 10.1 & -. & 8.7 & 8.5 & 8.8 & 8.6 & 8.8 & 9.3 \\
\hline 2 & -- & -- & -. & 11.1 & 10.1 & -- & 8.5 & 8.5 & 8.8 & 8.6 & 8.8 & 9.3 \\
\hline 3 & -- & -- & -- & 11.1 & 10.1 & -- & 8.5 & 8.5 & 8.8 & 8.6 & 8.8 & 9.3 \\
\hline 4 & -. & -- & -- & 11.0 & 9.8 & -- & 8.5 & 8.5 & 8.8 & 8.6 & 8.8 & 9.3 \\
\hline 5 & -- & -- & -. & 11.0 & 9.7 & -. & 8.4 & 8.5 & 8.8 & 8.7 & 8.9 & 9.4 \\
\hline 6 & -- & -- & -- & 11.0 & 9.7 & -- & 8.4 & 8.6 & 8.7 & 8.6 & 8.9 & 9.4 \\
\hline 7 & -- & - & -- & 10.9 & 9.6 & -- & 8.4 & 8.7 & 8.7 & 8.6 & 8.9 & 9.4 \\
\hline 8 & -- & -- & -- & 10.9 & 9.5 & -- & 8.4 & 8.6 & 8.6 & 8.6 & 8.9 & 9.4 \\
\hline 9 & -- & -- & -. & 10.8 & -- & -- & 8.4 & 8.7 & 8.6 & 8.7 & 8.9 & 9.4 \\
\hline 10 & -- & -- & -- & 10.7 & -- & -- & 8.4 & 8.7 & 8.6 & 8.7 & 9.0 & 9.4 \\
\hline 11 & -- & -- & -- & 10.7 & -- & -- & 8.5 & 8.7 & 8.6 & 8.7 & 9.0 & 9.3 \\
\hline 12 & -- & -- & -- & 10.6 & -- & -- & 8.5 & 8.7 & 8.6 & 8.7 & 9.0 & 9.5 \\
\hline 13 & -- & -- & -. & 10.6 & -- & -- & 8.5 & 8.6 & 8.7 & 8.7 & 9.0 & 9.5 \\
\hline 14 & -- & -- & -- & 10.6 & -- & -- & 8.6 & 8.6 & 8.6 & 8.7 & 9.0 & 9.5 \\
\hline 15 & -- & -- & -- & 10.5 & -- & -- & 8.6 & 8.6 & 8.6 & 8.7 & 9.0 & 9.5 \\
\hline 16 & - & -- & -- & 10.5 & -- & -- & 8.6 & 8.5 & 8.6 & 8.8 & 9.0 & 9.5 \\
\hline 17 & -- & -- & -- & 10.5 & -- & -- & 8.7 & 8.5 & 8.6 & 8.8 & 9.0 & 9.5 \\
\hline 18 & -- & -- & -- & 10.4 & -- & -- & 8.6 & 8.5 & 8.6 & 8.8 & 9.0 & 9.6 \\
\hline 19 & -- & -- & -- & 10.4 & -- & -- & 8.5 & 8.6 & 8.5 & 8.8 & 9.0 & 9.6 \\
\hline 20 & -. & -- & - & 10.4 & -- & -- & 8.5 & 8.5 & 8.5 & 8.7 & 9.1 & 9.6 \\
\hline 21 & -. & -- & -- & 10.4 & -- & -- & 8.5 & 8.5 & 8.6 & 8.7 & 9.1 & 9.6 \\
\hline 22 & -- & -- & -- & 10.3 & -- & -- & 8.5 & 8.6 & 8.6 & 8.7 & 9.1 & 9.6 \\
\hline 23 & -- & -- & -- & 10.1 & -- & -- & 8.5 & 8.6 & 8.6 & 8.7 & 9.1 & 9.6 \\
\hline 24 & -- & -- & -- & 10.3 & -- & -- & 8.5 & 8.6 & 8.6 & 8.7 & 9.1 & 9.6 \\
\hline 25 & -- & -- & -- & 10.2 & -- & -. & 8.5 & 8.6 & 8.5 & 8.7 & 9.1 & 9.6 \\
\hline 26 & -- & -- & -- & 10.2 & -- & -- & 8.5 & 8.6 & 8.5 & 8.7 & 9.2 & 9.7 \\
\hline 27 & -- & -- & - & 10.2 & -- & -- & 8.5 & 8.7 & 8.6 & 8.7 & 9.2 & 9.7 \\
\hline 28 & -- & -- & -- & 10.2 & -- & 8.7 & 8.5 & 8.7 & 8.6 & 8.7 & 9.2 & 9.7 \\
\hline 29 & -- & -- & -- & 10.1 & -- & 8.7 & 8.5 & 8.8 & 8.6 & 8.7 & 9.2 & 9.7 \\
\hline 30 & -- & -- & -- & 10.2 & - & 8.7 & 8.5 & 8.8 & 8.6 & 8.7 & 9.2 & 9.7 \\
\hline 31 & -. & -- & -- & -- & -- & -. & 8.5 & 8.8 & -. & 8.8 & -- & 9.8 \\
\hline
\end{tabular}


Table 14i. Mean daily ground-water temperature at site CRM-12 (map ID \#10), East Well Field, Cedar Rapids, lowa, April 1996 through February 1999

\begin{tabular}{|c|c|c|c|c|c|c|c|c|c|c|c|c|}
\hline Day & Jan & Feb & Mar & Apr & May & Jun & Jul & Aug & Sep & Oct & Nov & Dec \\
\hline & & & & & & 1997 & & & & & & \\
\hline 1 & 9.9 & 11.0 & 11.1 & 11.5 & 11.3 & 11.0 & 10.1 & 7.0 & 7.0 & 7.0 & 8.1 & 9.8 \\
\hline 2 & 9.9 & 11.0 & 11.1 & 11.6 & 11.3 & 10.9 & 10.1 & 6.9 & 7.1 & 7.0 & 8.2 & 9.9 \\
\hline 3 & 10.0 & 11.0 & 11.1 & 11.5 & 11.3 & 10.9 & 10.1 & 6.9 & 7.1 & 7.1 & 8.3 & 9.9 \\
\hline 4 & 10.0 & 11.0 & 11.2 & 11.5 & 11.2 & 10.9 & 10.0 & 6.8 & 7.2 & 7.1 & 8.3 & 10.0 \\
\hline 5 & 10.1 & 11.1 & 11.2 & 11.5 & 11.2 & 10.9 & 10.0 & 6.8 & 7.2 & 7.0 & 8.4 & 10.0 \\
\hline 6 & 10.1 & 11.1 & 11.2 & 11.4 & 11.2 & 10.8 & 10.0 & 6.7 & 7.3 & 7.0 & 8.4 & 10.1 \\
\hline 7 & 10.1 & 11.1 & 11.2 & 11.4 & 11.2 & 10.8 & 10.0 & 6.7 & 7.4 & 7.0 & 8.5 & 10.2 \\
\hline 8 & 10.1 & 11.0 & 11.2 & 11.4 & 11.2 & 10.8 & 10.0 & 6.6 & 7.4 & 7.0 & 8.5 & 10.3 \\
\hline 9 & 10.0 & 11.1 & 11.2 & 11.4 & 11.2 & 10.8 & 10.0 & 6.6 & 7.4 & 7.0 & 8.6 & 10.3 \\
\hline 10 & 10.0 & 11.1 & 11.3 & 11.4 & -- & 10.6 & 10.0 & 6.6 & 7.4 & 7.0 & 8.6 & 10.3 \\
\hline 11 & 9.9 & 11.1 & 11.3 & 11.3 & -- & 10.6 & -. & 6.5 & 7.5 & 7.1 & 8.6 & 10.3 \\
\hline 12 & 9.9 & 11.1 & 11.4 & 11.3 & -- & 10.7 & -- & 6.5 & 7.5 & 7.1 & 8.6 & 10.3 \\
\hline 13 & 9.9 & 11.1 & 11.4 & 11.3 & -- & 10.7 & -- & 6.5 & 7.4 & 7.1 & 8.6 & 10.4 \\
\hline 14 & 9.9 & 11.0 & 11.5 & 11.3 & -- & 10.7 & -- & 6.5 & 7.4 & 7.1 & 8.6 & 10.5 \\
\hline 15 & 9.9 & 11.0 & 11.5 & 11.3 & -- & 10.7 & 7.6 & 6.5 & 7.3 & 7.1 & 8.7 & 10.7 \\
\hline 16 & 10.0 & 11.0 & 11.5 & 11.3 & -- & 10.7 & 7.7 & 6.4 & 7.3 & 7.1 & 8.7 & 10.9 \\
\hline 17 & 10.0 & 11.0 & 11.5 & 11.3 & -- & 10.8 & 7.8 & 6.4 & 7.2 & 7.1 & 8.8 & 11.2 \\
\hline 18 & 10.0 & 11.0 & 11.6 & 11.3 & -- & 10.8 & 7.8 & 6.4 & 7.1 & 7.2 & 8.9 & 11.4 \\
\hline 19 & 10.0 & 11.0 & 11.6 & 11.3 & -- & 10.7 & 7.8 & 6.4 & 7.1 & 7.2 & 9.0 & 11.7 \\
\hline 20 & 10.0 & 11.0 & 11.6 & 11.3 & -- & 10.5 & 7.8 & 6.7 & 7.1 & 7.2 & 9.2 & 11.9 \\
\hline 21 & 10.1 & 11.0 & 11.6 & 11.3 & -- & 10.4 & 7.8 & 6.7 & 7.1 & 7.2 & 9.5 & 11.9 \\
\hline 22 & 10.2 & 11.0 & 11.6 & 11.3 & -- & 10.4 & 7.7 & 6.7 & 7.1 & 7.2 & 9.7 & 11.8 \\
\hline 23 & 10.3 & 11.0 & 11.6 & 11.3 & 11.0 & 10.3 & 7.6 & 6.7 & 7.1 & 7.2 & 9.8 & 11.6 \\
\hline 24 & 10.4 & 11.0 & 11.5 & 11.3 & 11.0 & 10.2 & 7.6 & 6.7 & 7.0 & 7.3 & 9.7 & 11.6 \\
\hline 25 & 10.5 & 11.1 & 11.5 & 11.3 & 11.0 & 10.4 & 7.5 & 6.7 & 7.0 & 7.3 & 9.6 & 11.6 \\
\hline 26 & 10.7 & 11.1 & 11.5 & 11.3 & 11.0 & 10.2 & 7.4 & 6.7 & 7.0 & 7.4 & 9.5 & 11.5 \\
\hline 27 & 10.8 & 11.1 & 11.5 & 11.3 & 11.0 & 10.2 & 7.4 & 6.7 & 7.0 & 7.5 & 9.5 & 11.4 \\
\hline 28 & 10.9 & 11.1 & 11.5 & 11.3 & 11.0 & 10.2 & 7.3 & 6.7 & 7.0 & 7.7 & 9.5 & 11.5 \\
\hline 29 & 10.9 & -- & 11.5 & 11.3 & 11.0 & 10.2 & 7.2 & 6.7 & 7.0 & 7.8 & 9.5 & 11.7 \\
\hline 30 & 11.0 & -- & 11.5 & 11.3 & 11.0 & 10.1 & 7.2 & 6.8 & 7.0 & 7.9 & 9.6 & 11.9 \\
\hline 31 & 11.0 & -- & 11.5 & -- & 11.0 & -- & 7.1 & 6.9 & -- & 8.0 & -- & 12.1 \\
\hline
\end{tabular}


Table 14i. Mean daily ground-water temperature at site CRM-12 (map ID \#10), East Well Field, Cedar Rapids, lowa, April 1996 through February 1999

\begin{tabular}{|c|c|c|c|c|c|c|c|c|c|c|c|c|}
\hline Day & Jan & Feb & Mar & Apr & May & Jun & Jul & Aug & Sep & Oct & Nov & Dec \\
\hline \multicolumn{13}{|c|}{1998} \\
\hline 1 & 12.2 & 11.9 & 10.7 & 8.4 & 7.7 & 6.7 & 6.7 & 7.0 & 7.7 & 8.1 & 8.9 & 9.1 \\
\hline 2 & 12.4 & 11.9 & 10.8 & 8.3 & 7.7 & 6.7 & 6.7 & 7.0 & 7.7 & 8.0 & 9.0 & 9.1 \\
\hline 3 & 12.4 & 11.6 & 10.9 & 8.1 & 7.7 & 6.6 & 6.8 & 7.0 & 7.7 & 8.0 & 9.0 & 9.1 \\
\hline 4 & 12.6 & 11.4 & 10.9 & 8.0 & 7.6 & 6.6 & 6.8 & 7.0 & 7.8 & 8.0 & 9.0 & 9.1 \\
\hline 5 & 12.6 & 11.4 & 10.9 & 8.0 & 7.6 & 6.5 & 6.8 & 7.0 & 7.8 & 8.0 & 9.0 & 9.1 \\
\hline 6 & 12.7 & 11.3 & 10.9 & 8.1 & 7.5 & 6.5 & 6.8 & 7.1 & 7.9 & 8.0 & 9.0 & 9.1 \\
\hline 7 & 12.9 & 11.1 & 10.9 & 8.1 & 7.5 & 6.5 & 6.7 & 7.1 & 7.9 & 8.0 & 9.0 & 9.1 \\
\hline 8 & 13.0 & 10.9 & 10.8 & 8.2 & 7.4 & 6.2 & 6.7 & 7.1 & 8.0 & 8.1 & 9.1 & 9.2 \\
\hline 9 & 13.1 & 10.9 & 10.8 & 8.3 & 7.4 & 6.2 & 6.7 & 7.1 & 8.0 & 8.1 & 9.2 & 9.2 \\
\hline 10 & 13.1 & 10.8 & 10.7 & 8.3 & 7.4 & 6.1 & 6.7 & 7.1 & 8.0 & 8.1 & 9.1 & 9.2 \\
\hline 11 & 13.1 & 10.8 & 10.6 & 8.3 & 7.4 & 6.1 & 6.8 & 7.1 & 8.1 & 8.1 & 9.1 & 9.2 \\
\hline 12 & 13.2 & 10.8 & 10.3 & 8.3 & 7.4 & 6.3 & 6.8 & 7.1 & 8.2 & 8.2 & 9.1 & 9.2 \\
\hline 13 & 13.4 & 10.7 & 9.9 & 8.2 & 7.4 & 6.3 & 6.8 & 7.1 & 8.2 & 8.2 & 9.1 & 9.3 \\
\hline 14 & 13.5 & 10.7 & 9.6 & 8.2 & 7.4 & 6.3 & 6.8 & 7.1 & 8.2 & 8.2 & 9.1 & 9.3 \\
\hline 15 & 13.5 & 10.7 & 9.4 & 8.2 & 7.4 & 6.3 & 6.9 & 7.1 & 8.2 & 8.2 & 9.1 & 9.3 \\
\hline 16 & 13.4 & 10.7 & 9.1 & 8.1 & 7.3 & 6.3 & 6.9 & 7.1 & 8.2 & 8.3 & 9.1 & 9.3 \\
\hline 17 & 13.4 & 10.8 & 8.8 & 8.1 & 7.4 & 6.4 & 6.9 & 7.2 & 8.2 & 8.3 & 9.2 & 9.3 \\
\hline 18 & 13.2 & 10.8 & 8.6 & 8.2 & 7.4 & 6.4 & 6.9 & 7.2 & 8.2 & 8.3 & 9.2 & 9.3 \\
\hline 19 & 13.1 & 10.8 & 8.4 & 8.2 & 7.4 & 6.4 & 6.9 & 7.2 & 8.2 & 8.4 & 9.1 & 9.3 \\
\hline 20 & 12.9 & 10.7 & 8.4 & 8.2 & 7.4 & 6.4 & 6.9 & 7.2 & 8.2 & 8.4 & 9.1 & 9.3 \\
\hline 21 & 12.8 & 10.7 & 8.4 & 8.1 & 7.4 & 6.4 & 7.0 & 7.2 & 8.2 & 8.4 & 9.1 & 9.4 \\
\hline 22 & 12.6 & 10.6 & 8.4 & 8.2 & 7.3 & 6.4 & 7.0 & 7.3 & 8.2 & 8.5 & 9.1 & 9.4 \\
\hline 23 & 12.4 & 10.6 & 8.5 & 8.2 & 7.3 & 6.4 & 7.0 & 7.3 & 8.2 & 8.6 & 9.1 & 9.4 \\
\hline 24 & 12.3 & 10.5 & 8.5 & 8.2 & 7.2 & 6.5 & 7.0 & 7.3 & 8.2 & 8.6 & 9.1 & 9.4 \\
\hline 25 & 12.1 & 10.4 & 8.5 & 8.2 & 7.1 & 6.5 & 7.0 & 7.3 & 8.2 & 8.7 & 9.1 & 9.4 \\
\hline 26 & 12.1 & 10.4 & 8.5 & 8.2 & 7.1 & 6.6 & 7.0 & 7.3 & 8.2 & 8.7 & 9.0 & 9.4 \\
\hline 27 & 12.1 & 10.5 & 8.5 & 8.1 & 7.0 & 6.6 & 7.0 & 7.4 & 8.2 & 8.8 & 9.0 & 9.4 \\
\hline 28 & 12.1 & 10.6 & 8.5 & 8.0 & 6.9 & 6.7 & 7.0 & 7.4 & 8.2 & 8.8 & 9.0 & 9.4 \\
\hline 29 & 12.0 & -- & 8.5 & 7.8 & 6.9 & 6.7 & 7.0 & 7.5 & 8.3 & 8.8 & 9.1 & 9.4 \\
\hline 30 & 12.0 & -- & 8.4 & 7.7 & 6.8 & 6.7 & 7.1 & 7.6 & 8.2 & 8.8 & 9.0 & 9.4 \\
\hline 31 & 12.0 & -. & 8.4 & -- & 6.8 & -- & 7.0 & 7.6 & -- & 8.9 & -- & 9.5 \\
\hline
\end{tabular}


Table 14i. Mean daily ground-water temperature at site CRM-12 (map ID \#10), East Well Field, Cedar Rapids, lowa, April 1996 through February 1999

\begin{tabular}{|c|c|c|c|c|c|c|c|c|c|c|c|c|}
\hline Day & $\operatorname{Jan}$ & Feb & Mar & Apr & May & Jun & Jul & Aug & Sep & Oct & Nov & Dec \\
\hline & & & & & & 1999 & & & & & & \\
\hline 1 & 9.5 & 9.2 & -- & -- & -- & -- & -- & -- & -- & -- & -- & -- \\
\hline 2 & 9.5 & 9.2 & -- & - & -- & -- & -- & -- & -- & -- & -- & -- \\
\hline 3 & 9.5 & 9.2 & -. & -- & -- & -- & -- & -. & -. & -- & -- & -- \\
\hline 4 & 9.5 & 9.2 & -- & -- & -- & -- & $-\cdot$ & -- & -- & -- & -- & -- \\
\hline 5 & 9.5 & 9.2 & -- & -- & -- & -- & -- & -- & -- & -- & -- & -- \\
\hline 6 & 9.4 & 9.2 & -- & -- & -- & -- & -- & -- & -- & -- & -- & -- \\
\hline 7 & 9.4 & 9.2 & -- & -- & -- & -- & -- & -- & -- & -- & -- & -- \\
\hline 8 & 9.4 & 9.2 & -- & -- & -- & -- & -- & -- & -- & -- & -. & -- \\
\hline 9 & 9.4 & 9.2 & -- & -- & -- & -- & -- & -- & -- & -- & -- & -. \\
\hline 10 & 9.4 & 9.2 & -- & -- & - & -- & -- & -- & -- & -- & -- & -- \\
\hline 11 & 9.4 & 9.2 & -- & -- & - & -- & -- & -- & -- & -- & -- & -- \\
\hline 12 & 9.4 & 9.2 & -- & -- & -. & -- & -- & -- & -- & -- & -- & -- \\
\hline 13 & 9.4 & 9.2 & -- & -- & -. & -- & -- & -- & -- & -- & -- & -- \\
\hline 14 & 9.3 & 9.2 & -- & -- & -- & -- & -- & -- & -- & -- & -- & -- \\
\hline 15 & 9.3 & 9.2 & -- & -- & -- & -- & -- & -- & -- & -- & -- & -- \\
\hline 16 & 9.3 & 9.2 & -- & -- & -- & -- & -- & -- & -- & -- & -- & -- \\
\hline 17 & 9.3 & 9.2 & -- & -- & - & -- & -- & -- & -- & -- & -- & -. \\
\hline 18 & 9.3 & 9.2 & -- & -- & -- & -- & -- & - & -- & -- & -- & -- \\
\hline 19 & 9.3 & 9.2 & -- & -- & - & -- & -- & -- & -- & -- & -- & -- \\
\hline 20 & 9.3 & 9.2 & -- & -- & - & -- & -- & -- & -- & -- & -- & -- \\
\hline 21 & 9.3 & 9.2 & -- & -- & - & -- & -- & -- & -- & -- & -- & -- \\
\hline 22 & 9.2 & 9.1 & -- & -- & -- & -- & -- & -- & -- & -- & -- & -. \\
\hline 23 & 9.2 & 9.1 & -- & -- & -- & -- & -- & -- & - & -- & -- & -- \\
\hline 24 & 9.2 & 9.1 & -- & -- & -- & - & -- & -- & -- & -- & -- & -- \\
\hline 25 & 9.2 & 9.1 & -- & -- & -- & -- & -- & -- & -- & -- & -- & -- \\
\hline 26 & 9.2 & 9.1 & -- & -- & -- & -- & -- & -- & -- & -- & -- & -- \\
\hline 27 & 9.2 & 9.1 & -- & -- & -- & -- & -- & -- & -- & -- & -- & -- \\
\hline 28 & 9.2 & 9.1 & -- & -- & -- & -- & -- & -- & -- & -- & -- & -- \\
\hline 29 & 9.2 & -- & -- & -- & -- & - & -- & -- & -- & -- & -- & -- \\
\hline 30 & 9.2 & -- & -- & -- & -- & -- & -- & -- & -- & -- & -- & -- \\
\hline 31 & 9.2 & -- & -- & -- & -. & -- & -- & -- & -- & -- & -- & -- \\
\hline
\end{tabular}


Table 14j. Mean daily ground-water temperature at site CRM-15 (map ID \#12), Seminole Well Field, Cedar Rapids, lowa, January 1997 through February 1999

[Temperature in degrees Centigrade; --, value not measured or not recorded]

\begin{tabular}{|c|c|c|c|c|c|c|c|c|c|c|c|c|}
\hline Day & Jan & Feb & Mar & Apr & May & Jun & Jul & Aug & Sep & Oct & Nov & Dec \\
\hline \multicolumn{13}{|c|}{1997} \\
\hline 1 & -- & 9.0 & 7.0 & 6.6 & 6.3 & 7.7 & 8.7 & 9.9 & 11.2 & 11.9 & 12.0 & 11.7 \\
\hline 2 & -- & 9.0 & 7.0 & 6.5 & 6.3 & 7.8 & 8.8 & 10.0 & 11.2 & 11.9 & 12.0 & 11.6 \\
\hline 3 & -- & 9.0 & 6.9 & 6.5 & 6.4 & 7.8 & 8.8 & 10.0 & 11.2 & 11.9 & 12.0 & 11.6 \\
\hline 4 & -- & 9.0 & 6.8 & 6.5 & 6.4 & 7.8 & 8.8 & 10.1 & 11.3 & 12.0 & 12.0 & 11.6 \\
\hline 5 & -- & 9.0 & 6.8 & 6.5 & 6.4 & 7.9 & 8.9 & 10.1 & 11.3 & 12.0 & 12.0 & 11.6 \\
\hline 6 & -- & 8.9 & 6.8 & 6.6 & 6.4 & 7.9 & 8.9 & 10.1 & 11.4 & 12.0 & 12.0 & 11.5 \\
\hline 7 & -- & 8.9 & 6.7 & 6.5 & 6.4 & 8.0 & 9.0 & 10.2 & 11.4 & 12.0 & 12.0 & 11.5 \\
\hline 8 & -. & 8.9 & 6.7 & 6.5 & 6.5 & 8.0 & 9.0 & 10.2 & 11.4 & 12.0 & 12.0 & 11.4 \\
\hline 9 & -- & 8.9 & 6.6 & 6.5 & 6.5 & 8.0 & 9.0 & 10.3 & 11.4 & 12.0 & 12.0 & 11.4 \\
\hline 10 & -- & 8.9 & 6.6 & 6.6 & 6.6 & 8.1 & 9.1 & 10.3 & 11.5 & 12.0 & 12.0 & 11.3 \\
\hline 11 & -- & 8.8 & 6.5 & 6.6 & 6.6 & 8.1 & 9.1 & 10.4 & 11.5 & 12.0 & 12.1 & 11.3 \\
\hline 12 & -- & 8.8 & 6.5 & 6.6 & 6.6 & 8.2 & 9.2 & 10.4 & 11.5 & 12.0 & 12.1 & 11.2 \\
\hline 13 & -- & 8.7 & 6.5 & 6.5 & 6.7 & 8.2 & 9.2 & 10.4 & 11.6 & 12.0 & 12.0 & 11.2 \\
\hline 14 & -- & 8.7 & 6.5 & 6.5 & 6.7 & 8.3 & 9.3 & 10.5 & 11.6 & 12.0 & -- & 11.1 \\
\hline 15 & -- & 8.7 & 6.5 & 6.5 & 6.7 & 8.3 & 9.4 & 10.5 & 11.6 & 12.0 & -- & 11.1 \\
\hline 16 & -- & 8.7 & 6.5 & 6.5 & 6.8 & 8.4 & 9.4 & 10.5 & 11.7 & 12.0 & -- & 11.0 \\
\hline 17 & -- & 8.6 & 6.5 & 6.5 & 6.8 & 8.4 & 9.4 & 10.6 & 11.7 & 12.0 & -- & 11.0 \\
\hline 18 & -- & 7.6 & 6.5 & 6.4 & 6.9 & 8.5 & 9.4 & 10.6 & 11.7 & 12.0 & -- & 10.9 \\
\hline 19 & -- & 6.3 & 6.4 & 6.4 & 6.9 & 8.5 & 9.5 & 10.7 & 11.8 & 11.9 & -- & 10.9 \\
\hline 20 & -- & 6.9 & 6.4 & 6.4 & 6.9 & 8.5 & 9.5 & 10.7 & 11.8 & 11.9 & -- & 10.9 \\
\hline 21 & -- & 7.6 & 6.4 & 6.4 & 7.0 & 8.6 & 9.5 & 10.7 & 11.8 & 11.9 & 11.9 & 10.9 \\
\hline 22 & -- & 7.7 & 6.4 & 6.3 & 7.0 & 8.6 & 9.6 & 10.8 & 11.8 & 11.9 & 11.9 & 10.8 \\
\hline 23 & -- & 7.7 & 6.4 & 6.3 & 7.1 & 8.6 & 9.6 & 10.8 & 11.8 & 11.9 & 11.9 & 10.8 \\
\hline 24 & 9.4 & 7.9 & 6.4 & 6.3 & 7.1 & 8.6 & 9.6 & 10.8 & 11.8 & 12.0 & 11.9 & 10.7 \\
\hline 25 & 9.4 & 7.8 & 6.4 & 6.3 & 7.2 & 8.7 & 9.7 & 10.9 & 11.9 & 12.0 & 11.8 & 10.7 \\
\hline 26 & 9.4 & 7.5 & 6.5 & 6.3 & 7.3 & 8.7 & 9.7 & 10.9 & 11.9 & 12.0 & 11.8 & 10.7 \\
\hline 27 & 9.4 & 7.3 & 6.5 & 6.3 & 7.3 & 8.7 & 9.7 & 11.0 & 11.9 & 12.0 & 11.8 & 10.6 \\
\hline 28 & 9.3 & 7.2 & 6.5 & 6.3 & 7.4 & 8.7 & 9.8 & 11.0 & 11.9 & 12.0 & 11.7 & 10.6 \\
\hline 29 & 9.2 & -- & 6.6 & 6.4 & 7.5 & 8.7 & 9.8 & 11.1 & 11.9 & 12.0 & 11.7 & 10.6 \\
\hline 30 & 9.1 & -- & 6.6 & 6.3 & 7.5 & 8.7 & 9.9 & 11.1 & 11.9 & 12.0 & 11.7 & 10.5 \\
\hline 31 & 9.1 & -- & 6.6 & -- & 7.6 & -- & 9.9 & 11.1 & -- & 12.0 & -- & 10.5 \\
\hline
\end{tabular}


Table 14j. Mean daily ground-water temperature at site CRM-15 (map ID \#12), Seminole Well Field, Cedar Rapids, lowa, January 1997 through February 1999--Continued

\begin{tabular}{|c|c|c|c|c|c|c|c|c|c|c|c|c|}
\hline Day & Jan & Feb & Mar & Apr & May & Jun & Jul & Aug & Sep & Oct & Nov & Dec \\
\hline \multicolumn{13}{|c|}{1998} \\
\hline 1 & 10.5 & 9.4 & 8.6 & 7.7 & 7.5 & 8.9 & 9.9 & 12.1 & 12.6 & 13.0 & 12.9 & 12.0 \\
\hline 2 & 10.4 & 9.4 & 8.6 & 7.6 & 7.6 & 8.9 & 10.0 & 12.1 & 12.6 & 13.1 & 12.9 & 12.0 \\
\hline 3 & 10.4 & 9.4 & 8.6 & 7.6 & 7.6 & 8.9 & 10.0 & 12.2 & 12.6 & 13.1 & 12.9 & 11.9 \\
\hline 4 & 10.4 & 9.3 & 8.6 & 7.7 & 7.6 & 8.9 & 10.1 & 12.2 & 12.6 & 13.1 & 12.9 & 11.9 \\
\hline 5 & 10.4 & 9.3 & 8.6 & 7.7 & 7.7 & 9.0 & 10.1 & 12.2 & 12.6 & 13.1 & 12.8 & 11.8 \\
\hline 6 & 10.3 & 9.3 & 8.6 & 7.7 & 7.7 & 9.0 & -- & 12.3 & 12.7 & 13.2 & 12.8 & 11.8 \\
\hline 7 & 10.3 & 9.3 & 8.5 & 7.7 & 7.8 & 9.0 & -- & 12.3 & 12.7 & 13.2 & 12.8 & 11.8 \\
\hline 8 & 10.3 & 9.2 & 8.4 & 7.7 & 7.8 & 9.1 & 9.0 & 12.3 & 12.7 & 13.2 & 12.8 & 11.7 \\
\hline 9 & 10.3 & 9.2 & 8.4 & 7.7 & 7.9 & 9.1 & 9.0 & 12.3 & 12.7 & 13.2 & 12.7 & 11.6 \\
\hline 10 & 10.3 & 9.1 & 8.4 & 7.7 & 7.9 & 9.1 & -- & 12.3 & 12.7 & 13.2 & 12.7 & 11.6 \\
\hline 11 & 10.2 & 9.1 & 8.3 & 7.7 & 7.9 & 9.2 & 10.7 & 12.3 & 12.8 & 13.2 & 12.7 & 11.6 \\
\hline 12 & 10.2 & 9.1 & 8.3 & 7.7 & 8.0 & 9.2 & 10.8 & 12.3 & 12.8 & 13.2 & 12.7 & 11.5 \\
\hline 13 & 10.1 & 9.1 & 8.2 & 7.7 & 8.1 & 9.3 & 10.8 & 12.3 & 12.8 & 13.3 & 12.6 & 11.5 \\
\hline 14 & 10.1 & 9.0 & 8.2 & 7.7 & 8.1 & 9.3 & 10.9 & 12.4 & 12.9 & 13.2 & 12.6 & 11.5 \\
\hline 15 & 10.1 & 9.0 & 8.1 & 7.7 & 8.2 & 9.3 & 11.0 & 12.4 & 12.9 & 13.2 & 12.6 & 11.5 \\
\hline 16 & 10.0 & 8.9 & 8.2 & 7.6 & 8.2 & 9.3 & 11.1 & 12.4 & 13.0 & 13.3 & 12.6 & 11.4 \\
\hline 17 & 10.0 & 8.9 & 8.1 & 7.6 & 8.3 & 9.4 & 11.2 & 12.4 & 13.0 & 13.2 & 12.5 & 11.4 \\
\hline 18 & 9.9 & 8.9 & 8.1 & 7.5 & 8.3 & 9.4 & 11.3 & 12.4 & 13.0 & 13.2 & 12.5 & 11.4 \\
\hline 19 & 9.9 & 8.9 & 8.1 & 7.5 & 8.3 & 9.4 & 11.4 & 12.5 & 13.0 & 13.2 & 12.5 & 11.4 \\
\hline 20 & 9.9 & 8.8 & 8.0 & 7.5 & 8.4 & 9.4 & 11.5 & 12.5 & 13.0 & 13.1 & 12.5 & 11.3 \\
\hline 21 & 9.8 & 8.8 & 8.0 & 7.4 & 8.4 & 9.5 & 11.5 & 12.5 & 13.0 & 13.1 & 12.4 & 11.3 \\
\hline 22 & 9.8 & 8.8 & 8.0 & 7.4 & 8.5 & 9.5 & 11.7 & 12.5 & 13.0 & 13.1 & 12.4 & 11.3 \\
\hline 23 & 9.8 & 8.7 & 7.9 & 7.4 & 8.5 & 9.6 & 11.7 & 12.5 & 13.0 & 13.0 & 12.3 & 11.3 \\
\hline 24 & 9.7 & 8.7 & 7.9 & 7.4 & 8.6 & 9.6 & 11.8 & 12.5 & 13.0 & 13.0 & 12.3 & 11.3 \\
\hline 25 & 9.7 & 8.7 & 7.9 & 7.4 & 8.6 & 9.6 & 11.8 & 12.5 & 13.0 & 13.0 & 12.3 & 11.2 \\
\hline 26 & 9.7 & 8.7 & 7.9 & 7.5 & 8.7 & 9.7 & 11.9 & 12.5 & 13.0 & 13.0 & 12.2 & 11.2 \\
\hline 27 & 9.6 & 8.7 & 7.9 & 7.5 & 8.7 & 9.7 & 11.9 & 12.5 & 13.0 & 13.0 & 12.2 & 11.2 \\
\hline 28 & 9.6 & 8.6 & 7.9 & 7.5 & 8.7 & 9.7 & 12.0 & 12.6 & 13.0 & 13.0 & 12.2 & 11.2 \\
\hline 29 & 9.5 & -- & 7.8 & 7.5 & 8.8 & 9.7 & 12.0 & 12.6 & 13.0 & 12.9 & 12.1 & 11.2 \\
\hline 30 & 9.5 & -- & 7.8 & 7.5 & 8.8 & 9.8 & 12.0 & 12.6 & 13.0 & 12.9 & 12.1 & 11.1 \\
\hline 31 & 9.5 & -- & 7.8 & -- & 8.8 & -- & 12.0 & 12.6 & -- & 12.9 & -- & 11.1 \\
\hline
\end{tabular}


Table 14j. Mean daily ground-water temperature at site CRM-15 (map ID \#12), Seminole Well Field, Cedar Rapids, lowa, January 1997 through February 1999--Continued

\begin{tabular}{|c|c|c|c|c|c|c|c|c|c|c|c|c|}
\hline Day & Jan & Feb & Mar & $\overline{\text { Apr }}$ & May & Jun & Jul & Aug & Sep & Oct & Nov & $\overline{\mathrm{Dec}}$ \\
\hline & & & & & & 1999 & & & & & & \\
\hline 1 & 11.1 & 9.8 & -- & -- & -- & -- & -- & -- & -- & -- & .. & -- \\
\hline 2 & 11.1 & 9.8 & -. & -- & -- & -- & -. & -- & -- & -- & -- & -- \\
\hline 3 & 11.0 & 9.7 & -- & -- & -- & -- & -. & -- & -- & -- & -- & -- \\
\hline 4 & 11.0 & 9.7 & -- & -- & -. & -- & -- & -- & -- & -- & -- & -- \\
\hline 5 & 11.0 & 9.6 & -- & -- & -- & -. & -- & -- & -- & -- & -- & -- \\
\hline 6 & 11.0 & 9.6 & -- & -- & -- & -- & -- & -- & -- & -- & -- & -- \\
\hline 7 & 10.9 & 9.5 & -- & -- & -- & -- & -- & -- & -- & -- & -- & -- \\
\hline 8 & 10.9 & 9.4 & -- & -- & -. & -. & -- & -. & -- & -- & -. & -- \\
\hline 9 & 10.9 & 9.4 & -- & -- & -- & -- & -- & -- & -- & -- & -- & -- \\
\hline 10 & 10.9 & 9.3 & -- & -- & -. & -- & -- & -. & -- & -- & -- & -- \\
\hline 11 & 10.8 & 9.3 & -- & -- & -- & -- & -- & -- & -- & -- & -- & -- \\
\hline 12 & 10.8 & 9.2 & -- & -- & -- & -- & -- & -- & -- & -- & -- & -- \\
\hline 13 & 10.7 & 9.2 & -- & -- & -- & -- & -- & -- & -- & -- & -- & -- \\
\hline 14 & 10.7 & 9.1 & -. & -- & -- & -. & -- & -- & -- & -- & -- & -- \\
\hline 15 & 10.6 & 9.1 & -- & -- & -- & -- & -- & -- & -- & -- & -- & -- \\
\hline 16 & 10.6 & 9.0 & -. & -- & -- & -. & .- & -- & -- & -- & -- & -- \\
\hline 17 & 10.5 & 8.9 & -- & -- & -- & -- & -- & -. & -. & -- & .. & -- \\
\hline 18 & 10.5 & 8.8 & -- & -- & -- & -- & -- & -- & -- & -- & -- & -- \\
\hline 19 & 10.4 & 8.8 & -- & -- & -- & -- & -- & -- & -- & -- & -- & -- \\
\hline 20 & 10.4 & 8.8 & -- & -- & -- & -- & -- & -- & -- & -- & -- & -- \\
\hline 21 & 10.3 & 8.7 & -- & -- & -- & -- & -- & -- & -- & -- & -- & -- \\
\hline 22 & 10.3 & 8.7 & -- & -- & -- & -- & -- & -- & -- & -- & -- & -- \\
\hline 23 & 10.2 & 8.7 & -- & -- & -- & -- & -- & -- & -- & -- & -- & -- \\
\hline 24 & 10.2 & 8.6 & -- & -- & -- & -- & -- & -- & -- & -- & -- & - \\
\hline 25 & 10.1 & 8.6 & -- & -- & -- & -- & -- & -- & -- & -- & -- & -- \\
\hline 26 & 10.1 & 8.5 & -- & -- & -- & -- & -- & -- & -- & -- & -- & -- \\
\hline 27 & 10.0 & 8.5 & -- & -- & -- & -- & -- & -- & -- & -- & -- & -- \\
\hline 28 & 10.0 & 8.4 & -. & -. & -- & -- & -. & -- & -- & -- & -- & -- \\
\hline 29 & 9.9 & -- & -. & -- & -- & -- & -- & -- & -- & -- & -- & -- \\
\hline 30 & 9.9 & -- & -- & -- & -- & -- & -- & -- & -- & -- & -- & -- \\
\hline 31 & 9.9 & -- & -. & -- & -- & -- & -- & .- & .. & -- & -- & -- \\
\hline
\end{tabular}


Table 14k. Mean daily ground-water temperature at site CRM-16 (map ID \#13), Seminole Well Field, Cedar Rapids, lowa, January 1997 through February 1999

[Temperature in degrees Centigrade; --, value not measured or not recorded]

\begin{tabular}{|c|c|c|c|c|c|c|c|c|c|c|c|c|}
\hline Day & Jan & Feb & Mar & Apr & May & Jun & Jul & Aug & Sep & Oct & Nov & Dec \\
\hline & & & & & & 1997 & & & & & & \\
\hline 1 & -- & 9.3 & 4.0 & 1.5 & 3.1 & 3.2 & 3.8 & 5.2 & 6.7 & 9.4 & 11.6 & 13.3 \\
\hline 2 & -- & 9.5 & 3.7 & 1.6 & 3.2 & 3.3 & 3.8 & 5.3 & 6.7 & 9.3 & 11.6 & 13.2 \\
\hline 3 & -- & 9.6 & 3.4 & 1.6 & 3.1 & 3.3 & 3.8 & 5.3 & 6.6 & 9.3 & 11.6 & 13.2 \\
\hline 4 & -- & 9.7 & 3.1 & 1.7 & 3.0 & 3.4 & 3.8 & 5.3 & 6.6 & 9.2 & 11.6 & 13.4 \\
\hline 5 & -- & 9.7 & 3.0 & 1.7 & 2.9 & 3.4 & 3.8 & 5.3 & 6.5 & 9.1 & 11.6 & 13.5 \\
\hline 6 & -- & 9.8 & 2.9 & 1.7 & 2.8 & 3.5 & 3.8 & 5.3 & 6.5 & 9.1 & 11.7 & 13.5 \\
\hline 7 & -- & 9.9 & 2.8 & 1.7 & 2.7 & 3.5 & 3.9 & 5.4 & 6.4 & 9.1 & 11.7 & 13.4 \\
\hline 8 & -. & 9.9 & 2.7 & 1.7 & 2.7 & 3.5 & 3.9 & 5.4 & 6.3 & 9.2 & 11.6 & 13.6 \\
\hline 9 & -- & 9.9 & 2.6 & 1.8 & 2.8 & 3.5 & 4.0 & 5.5 & 6.2 & 9.2 & 11.9 & 13.8 \\
\hline 10 & -- & 9.8 & 2.5 & 2.0 & 2.8 & 3.5 & 4.1 & 5.6 & 6.1 & 9.3 & 12.1 & 14.0 \\
\hline 11 & -. & 9.8 & 2.4 & 2.2 & 2.8 & 3.5 & 4.2 & 5.6 & 6.1 & 9.5 & 12.2 & 14.1 \\
\hline 12 & -- & 9.7 & 2.3 & 2.4 & 2.8 & 3.6 & 4.2 & 5.7 & 6.1 & 9.7 & 12.3 & 14.2 \\
\hline 13 & -- & 9.6 & 2.1 & 2.4 & 2.9 & 3.6 & 4.3 & 5.8 & 6.1 & 9.9 & 12.4 & 14.4 \\
\hline 14 & -- & 9.6 & 1.9 & 2.5 & 2.9 & 3.7 & 4.4 & 5.9 & 6.1 & 10.2 & 12.5 & 14.4 \\
\hline 15 & -- & 9.5 & 1.8 & 2.5 & 3.0 & 3.7 & 4.5 & 5.9 & 6.2 & 10.5 & 12.7 & 14.4 \\
\hline 16 & - & 9.4 & 1.7 & 2.5 & 3.0 & 3.7 & 4.6 & 6.0 & 6.2 & 10.5 & 13.0 & 14.5 \\
\hline 17 & -- & 9.3 & 1.7 & 2.5 & 3.0 & 3.7 & 4.6 & 6.1 & 6.3 & 10.5 & -- & 14.5 \\
\hline 18 & -- & 9.2 & 1.6 & 2.5 & 3.0 & 3.8 & 4.6 & 6.2 & 6.5 & 10.7 & -- & 14.6 \\
\hline 19 & -- & 9.3 & 1.6 & 2.6 & 3.0 & 3.8 & 4.6 & 6.2 & 6.7 & 10.7 & -- & 14.7 \\
\hline 20 & -- & 9.0 & 1.6 & 2.6 & 3.1 & 3.9 & 4.6 & 6.3 & 7.0 & 10.8 & -- & 14.8 \\
\hline 21 & -- & 7.8 & 1.6 & 2.7 & 3.2 & 4.0 & 4.7 & 6.4 & 7.4 & 10.9 & 13.2 & 14.9 \\
\hline 22 & -- & 6.5 & 1.5 & 2.7 & 3.2 & 4.0 & 4.7 & 6.4 & 7.8 & 11.0 & 13.2 & 15.0 \\
\hline 23 & -- & 5.8 & 1.4 & 2.8 & 3.2 & 4.0 & 4.8 & 6.5 & 8.1 & 11.2 & 13.3 & 15.1 \\
\hline 24 & 8.3 & 5.5 & 1.3 & 2.9 & 3.3 & 4.0 & 4.9 & 6.6 & 8.5 & 11.2 & 13.3 & 15.3 \\
\hline 25 & 8.5 & 5.4 & 1.3 & 2.9 & 3.2 & 3.9 & 4.9 & 6.6 & 8.7 & 11.3 & 13.3 & 15.5 \\
\hline 26 & 8.7 & 5.3 & 1.3 & 3.0 & 3.2 & 3.9 & 5.0 & 6.7 & 9.0 & 11.4 & 13.3 & 15.7 \\
\hline 27 & 8.6 & 4.9 & 1.3 & 3.0 & 3.1 & 3.8 & 5.0 & 6.7 & 9.2 & 11.4 & 13.3 & 15.8 \\
\hline 28 & 8.7 & 4.4 & 1.3 & 3.0 & 3.1 & 3.8 & 5.0 & 6.7 & 9.3 & 11.5 & 13.3 & 15.9 \\
\hline 29 & 8.8 & -- & 1.4 & 3.0 & 3.1 & 3.8 & 5.1 & 6.7 & 9.4 & 11.5 & 13.3 & 16.0 \\
\hline 30 & 8.9 & -. & 1.4 & 3.1 & 3.2 & 3.8 & 5.1 & 6.7 & 9.4 & 11.5 & 13.3 & 16.0 \\
\hline 31 & 9.2 & -- & 1.5 & -- & 3.2 & -- & 5.2 & 6.7 & -. & 11.5 & -- & 16.1 \\
\hline
\end{tabular}


Table 14k. Mean daily ground-water temperature at site CRM-16 (map ID \#13), Seminole Well Field, Cedar Rapids, lowa, January 1997 through February 1999--Continued

\begin{tabular}{|c|c|c|c|c|c|c|c|c|c|c|c|c|}
\hline Day & Jan & Feb & Mar & Apr & May & Jun & Jul & Aug & Sep & Oct & Nov & Dec \\
\hline \multicolumn{13}{|c|}{1998} \\
\hline 1 & 16.2 & 13.3 & 13.3 & 8.0 & 7.5 & 6.6 & 7.2 & 7.3 & 9.5 & 9.1 & 13.5 & 17.9 \\
\hline 2 & 16.3 & 13.2 & 13.3 & 7.9 & 7.5 & 6.6 & 7.2 & 7.3 & 9.3 & 9.1 & 13.6 & 18.1 \\
\hline 3 & 16.3 & 13.2 & 13.3 & 7.9 & 7.5 & 6.6 & 7.3 & 7.2 & 9.3 & 9.1 & 13.8 & 18.2 \\
\hline 4 & 16.1 & 13.1 & 13.3 & 7.9 & 7.5 & 6.6 & 7.3 & 7.2 & 9.6 & 9.2 & 14.0 & 18.4 \\
\hline 5 & 16.0 & 13.1 & 13.2 & 7.9 & 7.4 & 6.6 & 7.4 & 7.2 & 10.1 & 9.3 & 14.2 & 18.5 \\
\hline 6 & 16.1 & 13.1 & 13.1 & 7.9 & 7.4 & 6.6 & 7.4 & 7.2 & 10.2 & 9.4 & 14.3 & 18.6 \\
\hline 7 & 16.0 & 13.1 & 13.0 & 8.0 & 7.4 & 6.6 & 7.3 & 7.1 & 10.4 & 9.5 & 14.5 & 18.7 \\
\hline 8 & 16.0 & 13.1 & 12.9 & 8.1 & 7.4 & 6.7 & 7.3 & 7.1 & 10.7 & 9.5 & 14.6 & 18.9 \\
\hline 9 & 15.8 & 13.2 & 12.8 & 8.2 & 7.4 & 6.7 & 7.2 & 7.1 & 10.9 & 9.6 & 14.6 & 19.0 \\
\hline 10 & 15.7 & 13.2 & 12.7 & 8.3 & 7.4 & 6.7 & 7.3 & 7.1 & 11.0 & 9.6 & 14.7 & 19.1 \\
\hline 11 & 15.5 & 13.3 & 12.5 & 8.3 & 7.4 & 6.8 & 7.2 & 7.1 & 11.2 & 9.6 & 14.9 & 19.2 \\
\hline 12 & 15.2 & 13.3 & 12.4 & 8.4 & 7.4 & 6.7 & 7.2 & 7.1 & 11.3 & 9.7 & 15.1 & 19.3 \\
\hline 13 & 15.0 & 13.4 & 12.2 & 8.4 & 7.4 & 6.7 & 7.1 & 7.1 & 11.5 & 9.7 & 15.2 & 19.4 \\
\hline 14 & 14.8 & 13.5 & 12.1 & 8.5 & 7.3 & 6.6 & 7.1 & 7.1 & 11.8 & 9.8 & 15.4 & 19.4 \\
\hline 15 & 14.7 & 13.5 & 12.0 & 8.6 & 7.3 & 6.7 & 7.1 & 7.2 & 9.8 & 9.9 & 15.6 & 19.5 \\
\hline 16 & 14.8 & 13.6 & 11.8 & 8.6 & 7.3 & 6.7 & 7.1 & 7.4 & 8.6 & 10.0 & 15.7 & 19.5 \\
\hline 17 & 14.9 & 13.7 & 11.7 & 8.6 & 7.3 & 6.8 & 7.1 & 8.2 & 8.7 & 10.1 & 15.9 & 19.6 \\
\hline 18 & 14.9 & 13.9 & 11.5 & 8.6 & 7.2 & 6.8 & 7.2 & 8.3 & 8.8 & 10.2 & 16.0 & 19.6 \\
\hline 19 & 14.9 & 13.8 & 11.3 & 8.5 & 7.2 & 6.8 & 7.2 & 8.3 & 8.8 & 10.4 & 16.2 & 19.6 \\
\hline 20 & 14.9 & 13.7 & 11.2 & 8.5 & 7.2 & 6.8 & 7.2 & 8.3 & 8.9 & 10.6 & 16.3 & 19.6 \\
\hline 21 & 15.0 & 13.6 & 11.0 & 8.3 & 7.2 & 6.8 & 7.2 & 8.3 & 8.9 & 11.0 & 16.4 & 19.6 \\
\hline 22 & 15.0 & 13.5 & 10.8 & 8.2 & 7.1 & 6.8 & 7.3 & 8.4 & 8.9 & 11.5 & 16.5 & 19.6 \\
\hline 23 & 14.9 & 13.4 & 10.6 & 8.1 & 7.1 & 6.8 & 7.3 & 8.5 & 8.8 & 11.9 & 16.7 & 19.6 \\
\hline 24 & 14.7 & 13.3 & 10.4 & 8.0 & 7.1 & 6.9 & 7.3 & 8.6 & 8.9 & 12.1 & 16.8 & 19.6 \\
\hline 25 & 14.5 & 13.3 & 10.2 & 7.9 & 7.0 & 6.9 & 7.3 & 8.7 & 8.9 & 12.3 & 17.0 & 19.6 \\
\hline 26 & 14.4 & 13.3 & 10.0 & 7.8 & 7.0 & 7.0 & 7.3 & 8.8 & 8.9 & 12.5 & 17.2 & 19.6 \\
\hline 27 & 14.2 & 13.3 & 9.8 & 7.7 & 6.9 & 7.0 & 7.3 & 8.9 & 9.0 & 12.7 & 17.3 & 19.6 \\
\hline 28 & 14.1 & 13.3 & 9.5 & 7.7 & 6.9 & 7.1 & 7.3 & 9.0 & 9.0 & 12.8 & 17.4 & 19.6 \\
\hline 29 & 13.8 & -- & 9.3 & 7.6 & 6.8 & 7.1 & 7.3 & 9.1 & 9.0 & 13.0 & 17.6 & 19.7 \\
\hline 30 & 13.6 & -- & 9.1 & 7.6 & 6.8 & 7.2 & 7.3 & 9.2 & 9.1 & 13.1 & 17.7 & 19.7 \\
\hline 31 & 13.5 & -- & 8.5 & -- & 6.7 & -- & 7.3 & 9.3 & -- & 13.3 & -- & 19.7 \\
\hline
\end{tabular}


Table 14k. Mean daily ground-water temperature at site CRM-16 (map ID \#13), Seminole Well Field, Cedar Rapids, lowa, January 1997 through February 1999--Continued

\begin{tabular}{|c|c|c|c|c|c|c|c|c|c|c|c|c|}
\hline Day & Jan & Feb & Mar & Apr & May & Jun & Jul & Aug & Sep & Oct & Nov & $\overline{D e c}$ \\
\hline & & & & & & 1999 & & & & & & \\
\hline 1 & 19.7 & 19.6 & -- & -- & -- & -- & -- & -- & -- & -- & -. & -- \\
\hline 2 & 19.8 & 19.6 & -- & -- & - & -- & -- & -- & -- & -- & -- & -- \\
\hline 3 & 19.8 & 19.6 & -- & -- & -- & -- & - & - & -- & -- & -- & -- \\
\hline 4 & 19.8 & 19.5 & .- & -- & -- & -- & -- & -- & - & -- & -. & -- \\
\hline 5 & 19.8 & 19.5 & -- & -- & -- & -- & -- & - & $\cdots$ & -- & -- & -- \\
\hline 6 & 19.9 & 19.4 & -- & -. & - & -- & - & -- & -- & -- & -- & - \\
\hline 7 & 19.9 & 19.4 & -- & -- & - & -- & -- & -- & -- & -- & -- & -- \\
\hline 8 & 19.9 & 19.4 & -- & -- & - & -- & -- & -- & -- & -- & -- & - \\
\hline 9 & 19.9 & 19.3 & -- & -- & -- & -- & -- & -- & -- & -- & -. & -- \\
\hline 10 & 19.9 & 19.3 & -- & -- & -- & -- & -- & -- & -- & -- & -- & -- \\
\hline 11 & 19.9 & 19.3 & - & -- & -- & -- & -- & -- & -- & - & - & - \\
\hline 12 & 19.9 & 19.3 & -- & -- & -- & -- & -- & -- & -- & -- & -- & -- \\
\hline 13 & 19.9 & 19.3 & - & -- & -- & -- & -- & -- & -- & -- & -- & -- \\
\hline 14 & 19.9 & 19.3 & -- & -- & -- & -- & -- & -- & -- & -- & -- & -- \\
\hline 15 & 19.9 & 19.4 & -. & -- & -- & -- & -- & -- & -- & -- & -- & -. \\
\hline 16 & 19.9 & 19.4 & -- & -- & -- & -- & -- & -- & -- & -- & -- & -- \\
\hline 17 & 19.9 & 19.4 & -- & -- & -- & -- & -- & -- & -- & -- & -- & -- \\
\hline 18 & 19.9 & 19.4 & -- & -- & -- & -- & -- & -- & -- & -- & -. & - \\
\hline 19 & 19.9 & 19.4 & -- & -- & -- & -- & -- & -- & -- & -- & -- & -- \\
\hline 20 & 19.9 & 19.3 & -- & -- & -- & -- & -- & -- & -- & -- & -- & -- \\
\hline 21 & 19.9 & 19.2 & -- & -- & -. & -- & -- & - & -- & -- & -- & -- \\
\hline 22 & 19.9 & 19.1 & -- & -- & -. & -- & -- & -- & - & -- & -- & -- \\
\hline 23 & 19.8 & 19.0 & -- & -- & -- & -- & -- & -- & -- & -- & -- & -- \\
\hline 24 & 19.8 & 18.9 & -- & -. & - & - & -- & -- & -- & -- & -- & -- \\
\hline 25 & 19.8 & 18.8 & -- & -- & -. & -. & -- & -- & - & -- & -- & - \\
\hline 26 & 19.8 & 18.7 & -- & -- & -- & -- & -- & -- & -- & -- & -- & - \\
\hline 27 & 19.8 & 18.5 & - & -- & -- & -. & -- & -- & -- & -- & -. & -- \\
\hline 28 & 19.7 & 18.4 & -- & -- & -- & -- & -- & - & -- & -- & - & -- \\
\hline 29 & 19.7 & -- & - & -- & - & - & - & -- & -- & -- & -- & -- \\
\hline 30 & 19.7 & -- & -- & -- & - & - & -- & -- & - & -- & -- & -- \\
\hline 31 & 19.7 & -- & -- & -- & -- & -- & -- & -- & -- & -- & - & -- \\
\hline
\end{tabular}


Table 14I. Mean daily ground-water temperature at site CRM-17 (map ID \#14), Seminole Well Field, Cedar Rapids, lowa, November 1997 through September 1998

[Temperature in degrees Centigrade; --, value not measured or not recorded]

\begin{tabular}{|c|c|c|c|c|c|c|c|c|c|c|c|c|}
\hline Day & Jan & Feb & Mar & $\overline{\text { Apr }}$ & May & Jun & Jul & Aug & Sep & Oct & Nov & Dec \\
\hline & & & & & & 1997 & & & & & & \\
\hline 1 & -- & -- & -- & -- & -- & -- & -- & -- & -- & -- & -- & 15.9 \\
\hline 2 & -- & -- & -- & -- & -- & -- & -- & -. & -- & -- & -- & 15.9 \\
\hline 3 & -- & -- & -- & -- & -. & -- & -- & - & -- & -- & -- & 15.8 \\
\hline 4 & -- & -- & -- & -- & -- & -- & -- & -- & -- & -- & -- & 15.7 \\
\hline 5 & -- & -- & -- & -- & -- & -- & -- & -- & -- & -- & -- & 15.7 \\
\hline 6 & -- & -- & -. & -- & -- & -- & -- & -. & -- & -- & -- & 15.5 \\
\hline 7 & -- & -- & -- & -- & -- & -- & -- & -- & -- & -- & -- & 15.4 \\
\hline 8 & -. & -- & -- & -- & -- & -- & -- & - & -- & -- & -- & 15.1 \\
\hline 9 & -- & -- & -- & -- & -- & -. & -- & -- & -- & -- & -- & 14.8 \\
\hline 10 & -. & -- & -- & -. & -- & -- & -- & -. & -- & -- & -- & 14.5 \\
\hline 11 & -- & -. & -- & -- & -- & -- & -. & -. & -- & -- & -- & 14.3 \\
\hline 12 & -- & -- & -- & -- & -- & -- & -- & -- & -- & -- & -- & 14.1 \\
\hline 13 & -- & -- & -- & -- & -- & -- & -- & -. & -- & -- & -. & 14.1 \\
\hline 14 & -- & -. & -- & -- & -- & -- & -- & -- & -- & -- & -- & 14.2 \\
\hline 15 & -. & -- & -- & -- & -. & -- & -- & -. & -- & -- & -- & 14.4 \\
\hline 16 & -- & -- & -- & -- & -- & -- & -- & -- & -- & -. & -- & 14.6 \\
\hline 17 & -- & -- & -- & -- & -- & - & -- & -. & -- & -- & -- & 14.8 \\
\hline 18 & -. & -. & -- & -- & -- & -- & -- & -- & -- & -- & .- & 14.9 \\
\hline 19 & -- & -- & -- & -- & -- & -- & -- & -- & -- & -- & -- & 15.0 \\
\hline 20 & -- & -- & -- & -- & -. & -- & -- & -- & -- & -- & -- & 15.1 \\
\hline 21 & -- & -- & -- & -- & -- & -- & -- & -- & -- & -- & 16.0 & 15.1 \\
\hline 22 & -- & -- & -- & -- & -- & -- & -- & -- & -- & -- & 16.0 & 15.0 \\
\hline 23 & -- & -- & -- & -- & -- & -- & -- & -- & -- & -- & 16.1 & 14.9 \\
\hline 24 & -- & -- & -- & -- & -- & -- & -- & -- & -- & -- & 16.1 & 14.9 \\
\hline 25 & -- & -- &.- & -- & -- & -- & -- & -- & -- & -- & 16.0 & 14.7 \\
\hline 26 & -- & -- & -- & -- & -- & -- & -- & -- & -- & -- & 16.0 & 14.7 \\
\hline 27 & -- & -- & -- & -- & -- & -- & -- & -- & -- & -- & 16.0 & 14.7 \\
\hline 28 & -- & & -- & -- & -- & -- & -- & -- & -- & -- & 16.0 & 14.7 \\
\hline 29 & -- & -- & - & -- & -- & -- & -- & -. & -- & -- & 16.0 & 14.7 \\
\hline 30 & -- & -- & -- & -- & -- & -- & -- & -- & -- & -- & 15.9 & 14.7 \\
\hline 31 & -. & -- & -- & -- & -. & -- & -- & -- & -- & -- & -- & 14.6 \\
\hline
\end{tabular}


Table 14I. Mean daily ground-water temperature at site CRM-17 (map ID \#14), Seminole Well Field, Cedar Rapids, lowa, November 1997 through September 1998--Continued

\begin{tabular}{|c|c|c|c|c|c|c|c|c|c|c|c|c|}
\hline Day & Jan & Feb & Mar & Apr & May & Jun & Jul & Aug & Sep & Oct & Nov & Dec \\
\hline & & & & & & 1998 & & & & & & \\
\hline 1 & 14.5 & 13.3 & 11.4 & 11.0 & 13.9 & 8.6 & 19.8 & 15.5 & 14.8 & -. & -- & -- \\
\hline 2 & 14.5 & 13.2 & 11.6 & 10.9 & 13.3 & 8.9 & 20.1 & 15.4 & 14.9 & -- & -- & -- \\
\hline 3 & 14.5 & 13.0 & 11.7 & 11.1 & 12.9 & 10.7 & 20.3 & 15.2 & 14.5 & -. & -- & -- \\
\hline 4 & 14.6 & 12.8 & 11.7 & 11.3 & 12.6 & 14.7 & 20.4 & 15.0 & 14.5 & -- & -- & -- \\
\hline 5 & 14.6 & 12.7 & 11.7 & 11.4 & 12.6 & 17.4 & 20.7 & 14.7 & 14.4 & -. & -. & -. \\
\hline 6 & 14.6 & 12.5 & 11.5 & 11.4 & 12.8 & 18.6 & 21.1 & 14.4 & 14.4 & -- & -. & -- \\
\hline 7 & 14.7 & 12.4 & 11.2 & 11.3 & 13.0 & 19.0 & 21.8 & 14.0 & 14.5 & -- & -- & -- \\
\hline 8 & 14.7 & 12.2 & 10.9 & 11.2 & 13.2 & 18.7 & 22.6 & 13.7 & 14.5 & -. & -- & -. \\
\hline 9 & 14.7 & 12.1 & 10.4 & 11.2 & 13.3 & 17.9 & 23.0 & 13.5 & 14.6 & - & -- & -- \\
\hline 10 & 14.7 & 11.9 & 10.1 & 11.1 & 13.4 & 16.8 & 23.2 & 13.2 & 14.7 & -- & -- & -- \\
\hline 11 & 14.7 & 11.7 & 9.8 & 11.1 & 13.6 & 15.5 & 23.3 & 13.0 & 14.8 & -- & -- & -- \\
\hline 12 & 14.7 & 11.6 & 9.6 & 11.0 & 13.8 & 14.7 & 23.0 & 12.8 & 14.9 & -- & -- & -- \\
\hline 13 & 14.7 & 11.5 & 9.5 & 11.0 & 13.8 & 17.5 & 22.6 & 12.7 & 15.3 & -- & -- & -- \\
\hline 14 & 14.6 & 11.4 & 9.5 & 11.0 & 13.6 & 18.8 & 22.2 & 12.6 & 15.6 & -- & -- & -- \\
\hline 15 & 14.5 & 11.2 & 9.5 & 10.9 & 13.4 & 18.5 & 22.0 & 12.5 & 15.8 & -- & -- & -- \\
\hline 16 & 14.3 & 11.1 & 9.5 & 10.9 & 13.2 & 18.3 & 21.7 & 12.5 & 16.0 & -- & -- & -- \\
\hline 17 & 14.2 & 11.0 & 9.5 & 10.9 & 12.9 & 18.2 & 21.2 & 12.6 & 16.1 & -- &.- & -- \\
\hline 18 & 13.8 & 10.9 & 9.6 & 10.9 & 12.5 & 18.1 & 20.4 & 12.5 & 16.2 & -- & -- & -- \\
\hline 19 & 13.6 & 10.9 & 9.6 & 10.6 & 12.1 & 18.0 & 19.8 & 12.5 & 16.3 & -- & -. & -- \\
\hline 20 & 13.5 & 11.0 & 9.6 & 9.9 & 11.8 & 17.9 & 19.2 & 12.6 & 16.4 & -. & -- & -- \\
\hline 21 & 13.3 & 10.9 & 9.6 & 9.3 & 11.5 & 18.1 & 18.6 & 12.7 & 16.4 & -- & -- & -- \\
\hline 22 & 13.3 & 11.1 & 9.6 & 10.0 & 11.2 & 18.6 & 18.1 & 12.8 & 16.3 & -- & -- & -- \\
\hline 23 & 13.3 & 11.2 & 9.6 & 10.9 & 11.1 & 18.8 & 17.6 & 13.0 & 15.8 & -- & -- & -- \\
\hline 24 & 13.5 & 11.3 & 9.5 & 11.2 & 11.0 & 18.9 & 17.2 & 13.1 & 15.6 & -- & -- & -- \\
\hline 25 & 13.6 & 11.2 & 9.5 & 11.2 & 10.9 & 19.0 & 16.8 & 13.3 & 15.6 & -. & -- & - \\
\hline 26 & 13.7 & 11.1 & 9.4 & 11.6 & 10.8 & 19.0 & 16.5 & 13.5 & 15.6 & -- & -- & -- \\
\hline 27 & 13.8 & 11.1 & 9.4 & 12.3 & 10.6 & 19.1 & 16.3 & 13.7 & 15.7 & -. & -- & -- \\
\hline 28 & 13.7 & 11.2 & 9.3 & 13.3 & 10.2 & 19.2 & 16.2 & 13.9 & -- & - & -- & -- \\
\hline 29 & 13.7 & -- & 9.1 & 14.1 & 9.8 & 19.3 & 15.9 & 14.2 & .- & -- & -- & -. \\
\hline 30 & 13.6 & -- & 9.3 & 14.4 & 9.3 & 19.5 & 15.7 & 14.4 & -- & -- & -- & -- \\
\hline 31 & 13.5 & -. & 10.1 & -- & 8.9 & -- & 15.6 & 14.6 & -- & -- & -- & -- \\
\hline
\end{tabular}


Table 14m. Mean daily ground-water temperature at site CRM-18 (map ID \#15), Seminole Well Field, Cedar Rapids, lowa, January 1997 through February 1999

[Temperature in degrees Centigrade; --, value not measured or not recorded]

\begin{tabular}{|c|c|c|c|c|c|c|c|c|c|c|c|c|}
\hline Day & Jan & Feb & Mar & Apr & May & Jun & Jul & Aug & Sep & Oct & Nov & Dec \\
\hline & & & & & & 1997 & & & & & & \\
\hline 1 & -- & 9.9 & 8.7 & 1.7 & 4.6 & 6.2 & 6.2 & 8.5 & 20.7 & 22.8 & 20.9 & 19.2 \\
\hline 2 & -- & 9.8 & 7.7 & 1.9 & 4.7 & 6.1 & 6.3 & 9.0 & 21.4 & 22.8 & 20.9 & 19.1 \\
\hline 3 & -- & 9.7 & 6.9 & 2.0 & 4.7 & 6.2 & 6.9 & 9.5 & 22.4 & 22.8 & 20.8 & 19.0 \\
\hline 4 & -- & 9.9 & 6.4 & 2.1 & 4.9 & 6.3 & 7.7 & 10.1 & 23.1 & 22.9 & 20.8 & 18.8 \\
\hline 5 & -- & 10.3 & 6.4 & 2.2 & 4.9 & 6.3 & 9.0 & 10.7 & 23.6 & 22.9 & 20.8 & 18.5 \\
\hline 6 & -- & 10.5 & 6.3 & 2.4 & 4.9 & 6.3 & 10.0 & 11.6 & 23.9 & 23.0 & 20.7 & 18.1 \\
\hline 7 & -- & 10.7 & 6.2 & 2.7 & 4.9 & 6.3 & 10.3 & 12.3 & 24.3 & 23.0 & 20.7 & 17.7 \\
\hline 8 & -- & 10.8 & 6.5 & 3.0 & 5.0 & 6.3 & 10.1 & 12.7 & 24.4 & 23.0 & 20.6 & 17.2 \\
\hline 9 & -- & 10.8 & 6.8 & 3.5 & 4.9 & 6.3 & 9.6 & 13.1 & 24.3 & 23.1 & 20.6 & 16.6 \\
\hline 10 & -- & 10.8 & 7.1 & 4.1 & 4.8 & 6.2 & 9.1 & 13.5 & 24.0 & 23.1 & 20.5 & 16.1 \\
\hline 11 & -- & 10.8 & 7.5 & 4.4 & 4.8 & 5.9 & 8.5 & 13.8 & 23.8 & 23.1 & 20.4 & 15.8 \\
\hline 12 & -- & 10.7 & 7.9 & 4.6 & 4.8 & 5.5 & 7.9 & 13.9 & 23.7 & 23.1 & 20.3 & 15.4 \\
\hline 13 & -- & 10.7 & 8.0 & 4.7 & 5.1 & 5.0 & 7.7 & 14.0 & 23.7 & 23.0 & 20.2 & 15.1 \\
\hline 14 & -- & 10.8 & 8.0 & 4.8 & 5.9 & 4.3 & 7.7 & 14.1 & 23.6 & 23.0 & -- & 14.9 \\
\hline 15 & -- & 10.8 & 7.8 & 4.8 & 7.2 & 3.7 & 7.6 & 14.2 & 23.6 & 23.0 & -- & 14.8 \\
\hline 16 & -- & 10.6 & 7.6 & 4.9 & 7.8 & 3.5 & 7.0 & 14.3 & 23.6 & 22.9 & -- & 14.5 \\
\hline 17 & -- & 10.4 & 7.4 & 4.9 & 7.9 & 3.1 & 6.1 & 14.3 & 23.6 & 22.9 & -- & 14.2 \\
\hline 18 & -- & 10.1 & 7.2 & 4.9 & 7.7 & 3.1 & 5.5 & 14.4 & 23.6 & 22.8 & -- & 13.9 \\
\hline 19 & -- & 10.5 & 7.2 & 4.8 & 7.6 & 3.1 & 5.3 & 14.4 & 23.6 & 22.7 & -- & 13.5 \\
\hline 20 & -- & 11.4 & 7.4 & 4.8 & 7.4 & 3.2 & 5.3 & 14.4 & 23.5 & 22.4 & -- & 13.1 \\
\hline 21 & -- & 11.2 & 7.2 & 4.8 & 6.9 & 3.2 & 5.3 & 14.7 & 23.4 & 22.2 & 20.0 & 12.7 \\
\hline 22 & -- & 9.6 & 6.3 & 4.8 & 6.7 & 3.1 & 5.4 & 15.3 & 23.4 & 22.0 & 19.9 & 12.1 \\
\hline 23 & -- & 9.4 & 5.4 & 4.8 & 6.6 & 3.0 & 5.5 & 15.8 & 23.3 & 21.8 & 19.9 & 11.5 \\
\hline 24 & 9.3 & 10.9 & 4.7 & 4.8 & 6.3 & 3.0 & 5.6 & 16.4 & 23.2 & 21.7 & 19.9 & 10.9 \\
\hline 25 & 10.5 & 11.6 & 4.3 & 4.8 & 6.1 & 3.0 & 5.7 & 16.9 & 23.1 & 21.5 & 19.9 & 10.5 \\
\hline 26 & 10.8 & 11.4 & 3.7 & 4.7 & 5.9 & 3.5 & 5.8 & 17.6 & 23.0 & 21.4 & 19.8 & 10.0 \\
\hline 27 & 10.9 & 10.5 & 2.4 & 4.7 & 5.9 & 3.7 & 6.1 & 18.2 & 22.9 & 21.3 & 19.7 & 9.4 \\
\hline 28 & 10.8 & 9.6 & 2.3 & 4.8 & 5.9 & 4.4 & 6.6 & 18.6 & 22.9 & 21.2 & 19.6 & 8.8 \\
\hline 29 & 10.5 & -- & 1.9 & 4.7 & 6.2 & 5.2 & 7.0 & 18.6 & 22.9 & 21.1 & 19.5 & 8.2 \\
\hline 30 & 10.2 & -. & 1.7 & 4.6 & 6.2 & 5.7 & 7.4 & 19.1 & 22.8 & 21.0 & 19.4 & 7.7 \\
\hline 31 & 10.0 & -- & 1.6 & -- & 6.2 & -- & 7.9 & 19.9 & -- & 21.0 & -- & 7.2 \\
\hline
\end{tabular}


Table 14m. Mean daily ground-water temperature at site CRM-18 (map ID \#15), Seminole Well Field, Cedar Rapids, lowa, January 1997 through February 1999--Continued

\begin{tabular}{|c|c|c|c|c|c|c|c|c|c|c|c|c|}
\hline Day & Jan & Feb & Mar & Apr & May & Jun & Jul & Aug & Sep & Oct & Nov & Dec \\
\hline \multicolumn{13}{|c|}{1998} \\
\hline 1 & 6.7 & 1.3 & 0.8 & 0.9 & 5.8 & 7.0 & 6.2 & 15.7 & 22.4 & 24.7 & 22.8 & 17.0 \\
\hline 2 & 6.3 & 1.2 & 0.8 & 1.0 & 6.4 & 7.2 & 6.3 & 15.7 & 22.8 & 24.7 & 22.7 & 16.9 \\
\hline 3 & 6.0 & 1.2 & 0.9 & 1.1 & 6.2 & 7.8 & 6.9 & 15.7 & 23.1 & 24.7 & 22.6 & 16.8 \\
\hline 4 & 5.6 & 1.1 & 0.9 & 1.1 & 5.6 & 9.0 & 7.7 & 15.8 & 23.1 & 24.6 & 22.4 & 16.7 \\
\hline 5 & 5.3 & 1.0 & 0.9 & 1.1 & 4.8 & 10.1 & 9.0 & 15.8 & 23.1 & 24.6 & 22.3 & 16.6 \\
\hline 6 & 5.0 & 1.0 & 0.9 & 1.1 & 4.3 & 10.8 & 10.0 & 15.8 & 23.0 & 24.6 & 22.1 & 16.6 \\
\hline 7 & 4.7 & 1.0 & 0.9 & 1.1 & 4.0 & 11.3 & 10.3 & 15.9 & 23.0 & 24.5 & 21.8 & 16.5 \\
\hline 8 & 4.5 & 1.0 & 0.9 & 1.2 & 3.7 & 11.8 & 10.1 & 16.0 & 23.1 & 24.4 & 21.6 & 16.4 \\
\hline 9 & 4.3 & 0.9 & 0.9 & 1.2 & 3.5 & 12.2 & 9.6 & 16.2 & 23.2 & 24.2 & 21.4 & 16.3 \\
\hline 10 & 4.2 & 0.9 & 0.8 & 1.2 & 3.1 & 12.8 & 9.1 & 16.4 & 23.3 & 24.0 & 21.2 & 16.1 \\
\hline 11 & 4.1 & 0.9 & 0.8 & 1.2 & 2.7 & 13.4 & 8.5 & 16.6 & 23.4 & 24.0 & 21.0 & 16.0 \\
\hline 12 & 4.0 & 0.9 & 0.8 & 1.2 & 2.3 & 13.9 & 7.9 & 16.8 & 23.5 & 24.1 & 20.8 & 15.9 \\
\hline 13 & 3.9 & 0.9 & 0.8 & 1.2 & 2.2 & 13.9 & 7.7 & 16.9 & 23.6 & 24.1 & 20.6 & 15.7 \\
\hline 14 & 3.8 & 1.0 & 0.8 & 1.2 & 2.0 & 13.9 & 7.7 & 17.1 & 23.7 & 24.1 & 20.4 & 15.6 \\
\hline 15 & 3.6 & 1.0 & 0.8 & 1.3 & 1.9 & 13.9 & 7.6 & 17.3 & 23.7 & 24.0 & 20.1 & 15.5 \\
\hline 16 & 3.1 & 0.9 & 0.9 & 1.3 & 1.9 & 13.9 & 7.0 & 17.5 & 23.8 & 24.0 & 19.9 & 15.6 \\
\hline 17 & 2.6 & 0.9 & 0.9 & 1.3 & 1.9 & 13.8 & -- & 17.7 & 23.9 & 24.0 & 19.7 & 16.1 \\
\hline 18 & 2.4 & 0.9 & 0.9 & 1.3 & 2.0 & 13.8 & 14.9 & 17.8 & 24.1 & 23.9 & 19.4 & 16.6 \\
\hline 19 & 2.2 & 0.9 & 1.1 & 1.3 & 2.1 & 13.7 & 15.0 & 17.9 & 24.3 & 23.8 & 19.1 & 16.8 \\
\hline 20 & 2.1 & 0.9 & 1.2 & 1.4 & 2.3 & 13.6 & 15.1 & 17.8 & 24.5 & 23.8 & 18.8 & 16.8 \\
\hline 21 & 2.1 & 0.8 & 1.2 & 1.4 & 2.5 & 13.6 & 15.2 & 18.1 & 24.6 & 23.7 & 18.6 & 16.6 \\
\hline 22 & 2.0 & 0.8 & 1.2 & 1.4 & 2.7 & 13.6 & 15.1 & 18.2 & 24.7 & 23.6 & 18.5 & 16.4 \\
\hline 23 & 2.0 & 0.8 & 1.3 & 1.5 & 2.9 & -- & 15.4 & 18.1 & 24.8 & 23.5 & 18.3 & 16.2 \\
\hline 24 & 1.9 & 0.8 & 1.2 & 1.6 & 3.3 & -. & 15.5 & 18.5 & 24.9 & 23.5 & 18.1 & 15.9 \\
\hline 25 & 1.8 & 0.7 & 1.2 & 1.5 & 3.7 & 3.0 & 15.5 & 19.2 & 24.9 & 23.4 & 17.9 & 15.6 \\
\hline 26 & 1.8 & 0.7 & 1.1 & 1.4 & 4.1 & 3.5 & 15.6 & 20.0 & 24.9 & 23.3 & 17.7 & 15.2 \\
\hline 27 & 1.7 & 0.7 & 1.0 & 1.6 & 4.6 & 3.7 & 15.6 & 20.9 & 24.8 & 23.2 & 17.6 & 14.7 \\
\hline 28 & 1.6 & 0.7 & 1.0 & 1.9 & 5.2 & 4.4 & 15.7 & 21.7 & 24.8 & 23.1 & 17.5 & 14.0 \\
\hline 29 & 1.6 & -- & 1.0 & 2.7 & 5.7 & 5.2 & 15.7 & 22.1 & 24.8 & 23.1 & 17.3 & 13.3 \\
\hline 30 & 1.5 & -- & 1.0 & 4.0 & 6.2 & 5.7 & 15.7 & 22.2 & 24.8 & 23.0 & 17.1 & 12.5 \\
\hline 31 & 1.4 & -- & 0.8 & -- & 6.6 & -- & 15.7 & 22.3 & -- & 22.9 & -- & 11.6 \\
\hline
\end{tabular}


Table 14m. Mean daily ground-water temperature at site CRM-18 (map ID \#15), Seminole Well Field, Cedar Rapids, lowa, January 1997 through February 1999--Continued

\begin{tabular}{|c|c|c|c|c|c|c|c|c|c|c|c|c|}
\hline Day & Jan & Feb & Mar & Apr & May & Jun & Jul & Aug & Sep & Oct & Nov & Dec \\
\hline & & & & & & 1999 & & & & & & \\
\hline 1 & 10.8 & 8.7 & -- & -- & -- & -- & -- & -- & -- & -- & -- & -- \\
\hline 2 & 10.2 & 8.5 & -- & -- & -- & -- & -- & -- & -- & -- & -- & -- \\
\hline 3 & 9.6 & 8.2 & -- & -- & -- & -- & -- & -- & -- & -- & -- & -. \\
\hline 4 & 9.2 & 8.4 & -- & -- & -- & -- & -- & -- & -- & -- & -- & -- \\
\hline 5 & 8.8 & 8.8 & -- & -- & -- & -. & -- & -- & -- & -- & -- & -. \\
\hline 6 & 8.6 & 8.8 & -- & -- & -- & -- & -- & -- & -- & -- & -- & -- \\
\hline 7 & 8.5 & 8.8 & -- & -- & -- & -- & -- & -- & -- & -- & -- & -- \\
\hline 8 & 8.4 & 8.7 & -- & -- & -- & -- & -- & -- & -. & -- & -- & -- \\
\hline 9 & 8.4 & 8.5 & -- & -- & -- & -- & -- & -- & -- & -- & -- & -- \\
\hline 10 & 8.4 & 8.3 & -- & -- & -- & -- & -- & -- & -- & -. & -- & -- \\
\hline 11 & 8.4 & 7.6 & -. & -- & -- & -- & -- & -- & -. & -- & .. & -. \\
\hline 12 & 8.5 & 6.9 & -- & -- & -- & -- & -- & -- & -- & -- & -- & -- \\
\hline 13 & 8.6 & 6.4 & -- & -- & -- & -- & -- & -- & -- & -- & -- & -- \\
\hline 14 & 8.6 & 6.0 & -- & -- & -. & -. & -. & -- & -- & -- & -. & -- \\
\hline 15 & 8.7 & 5.8 & -- & -- & -- & -- & -. & -- & -- & -- & -- & -- \\
\hline 16 & 8.8 & 5.7 & -- & -- & -- & -- & -- & -- & -. & -- & -- & -- \\
\hline 17 & 8.8 & 5.5 & -- & -- & -- & -- & -- & -- & -- & -- & -- & -- \\
\hline 18 & 8.8 & 5.3 & -- & -- & -- & -- & -- & -- & -- & -- & -- & -- \\
\hline 19 & 8.8 & 5.1 & -- & -- & -- & -- & -- & -- & -- & -- & -. & -- \\
\hline 20 & 8.8 & 4.9 & -- & -- & -- & -- & -- & -- & -- & -- & -- & -- \\
\hline 21 & 8.9 & 4.6 & -- & -- & -- & -- & -- & -- & -- & -- & -- & -. \\
\hline 22 & 9.0 & 4.5 & -- & -- & -- & -- & -. & -- & -- & -- & -- & -- \\
\hline 23 & 9.0 & 4.4 & -- & -- & -- & -- & -- & -- & -- & -- & -- & -- \\
\hline 24 & 9.0 & 4.4 & -- & -. & -- & -- & -- & -- & -- & -- & -- & -- \\
\hline 25 & 8.9 & 4.4 & -- & -- & -. & -- & -- & -- & -- & -- & -- & -- \\
\hline 26 & 8.9 & 4.4 & -- & -- & -- & -- & -- & -- & -- & -- & -- & -- \\
\hline 27 & 8.9 & 4.4 & -- & -- & -- & -- & -- & -- & -- & -- & -- & -- \\
\hline 28 & 8.9 & 4.4 & -- & -- & -- & -- & -- & -- & -- & -- & -- & -- \\
\hline 29 & 8.9 & -- & -- & -- & -- & -- & -- & -- & -- & -- & -- & -- \\
\hline 30 & 8.9 & -- & -- & -- & -- & -- & -- & -- & -- & -- & -- & -- \\
\hline 31 & 8.8 & -- & -- & -- & -- & -- & .- & -- & -- & -- & -- & -- \\
\hline
\end{tabular}


Table 14n. Mean daily ground-water temperature at site CRM-19 (map ID \#16), Seminole Well Field, Cedar Rapids, lowa, January 1997 through February 1999

[Temperature in degrees Centigrade; --, value not measured or not recorded]

\begin{tabular}{|c|c|c|c|c|c|c|c|c|c|c|c|c|}
\hline Day & Jan & Feb & Mar & Apr & May & Jun & Jul & Aug & Sep & Oct & Nov & Dec \\
\hline & & & & & & 1997 & & & & & & \\
\hline 1 & -- & -0.1 & 0.3 & 0.3 & 4.3 & 10.2 & 14.7 & 23.5 & 22.8 & 22.6 & 19.8 & 14.3 \\
\hline 2 & -- & -0.1 & 0.3 & 0.4 & 4.2 & 10.4 & 15.3 & 23.7 & 22.7 & 22.6 & 19.7 & 14.1 \\
\hline 3 & -- & -0.1 & 0.3 & 0.5 & 4.2 & 10.5 & 15.3 & 23.9 & 22.6 & 22.6 & 19.6 & 13.7 \\
\hline 4 & -- & -0.1 & 0.2 & 0.5 & 4.2 & 10.5 & 16.0 & 24.1 & 22.5 & 22.5 & 19.6 & 13.4 \\
\hline 5 & -- & -0.1 & 0.2 & 0.5 & 4.2 & 10.5 & 16.6 & 24.2 & 22.4 & 22.4 & 19.5 & 13.1 \\
\hline 6 & -- & 0.0 & 0.2 & 0.7 & 4.3 & 10.4 & 17.2 & 24.4 & 22.3 & 22.3 & 19.5 & 12.7 \\
\hline 7 & -- & 0.0 & 0.2 & 0.8 & 4.3 & 10.4 & 17.8 & 24.6 & 22.2 & 22.2 & 19.4 & 12.2 \\
\hline 8 & -- & 0.0 & 0.3 & 0.9 & 4.3 & 10.4 & 18.2 & 24.7 & 22.1 & 22.1 & 19.3 & 11.8 \\
\hline 9 & -- & 0.0 & 0.3 & 1.2 & 4.5 & 10.3 & 18.7 & 24.8 & 22.1 & 22.0 & 19.3 & 11.2 \\
\hline 10 & -- & 0.0 & 0.3 & 1.3 & 4.7 & 10.3 & 19.1 & 24.9 & 22.0 & 21.9 & 19.1 & 10.6 \\
\hline 11 & -- & 0.0 & 0.3 & 1.5 & 4.9 & 10.3 & 19.4 & 24.9 & -- & 21.8 & 19.0 & 9.9 \\
\hline 12 & -- & 0.0 & 0.3 & 1.5 & 5.2 & 10.3 & 19.7 & 24.9 & -. & 21.7 & 18.9 & 9.1 \\
\hline 13 & -- & 0.1 & 0.3 & 1.7 & 5.4 & 10.3 & 20.0 & 24.8 & -- & 21.6 & 18.7 & 8.5 \\
\hline 14 & -- & 0.1 & 0.3 & 2.0 & 5.7 & 10.3 & 20.2 & 24.8 & -- & 21.5 & -- & 7.8 \\
\hline 15 & -- & 0.1 & 0.4 & 2.3 & 6.1 & 9.9 & 20.1 & 24.8 & -- & 21.3 & -- & 7.2 \\
\hline 16 & -- & 0.1 & 0.4 & 2.1 & 6.6 & 10.3 & 20.4 & 24.8 & -- & 21.1 & -- & 6.5 \\
\hline 17 & -- & 0.2 & 0.4 & 2.3 & 7.0 & 10.4 & 20.8 & 24.8 & 22.0 & 20.9 & -- & 6.0 \\
\hline 18 & -- & 0.2 & 0.4 & 2.7 & 7.3 & 10.3 & 21.0 & 24.8 & 22.0 & 20.7 & -- & 5.7 \\
\hline 19 & -- & 0.2 & 0.4 & 3.0 & 7.5 & 10.7 & 21.2 & 24.7 & 22.1 & 20.6 & -- & 5.3 \\
\hline 20 & -- & 0.2 & 0.4 & 3.3 & 7.7 & 10.9 & 21.3 & 24.6 & 22.1 & 20.4 & -- & 5.1 \\
\hline 21 & -- & 0.2 & 0.4 & 3.7 & 7.9 & 11.3 & 21.5 & 24.5 & 22.2 & 20.3 & 16.9 & 5.1 \\
\hline 22 & -- & 0.2 & 0.4 & 4.1 & 8.2 & 11.4 & 21.6 & 24.4 & 22.2 & 20.3 & 16.7 & 4.9 \\
\hline 23 & -- & 0.2 & 0.4 & 4.4 & 8.4 & 11.3 & 21.7 & 24.2 & 22.3 & 20.2 & 16.5 & 4.9 \\
\hline 24 & -0.2 & 0.2 & 0.3 & 4.4 & 8.6 & 11.6 & 21.8 & 24.0 & 22.4 & 20.1 & 16.2 & 4.8 \\
\hline 25 & -0.1 & 0.2 & 0.3 & 4.3 & 8.8 & 11.9 & 21.9 & 23.6 & 22.4 & 20.0 & 16.0 & 4.8 \\
\hline 26 & -0.1 & 0.2 & 0.3 & 4.2 & 9.1 & 12.4 & 22.1 & 23.3 & 22.5 & 20.0 & 15.7 & 4.9 \\
\hline 27 & -0.1 & 0.2 & 0.2 & 4.3 & 9.4 & 12.7 & 22.3 & 23.1 & 22.5 & 20.0 & 15.4 & 4.8 \\
\hline 28 & -0.1 & 0.3 & 0.3 & 4.4 & 9.7 & 13.0 & 22.6 & 23.0 & 22.5 & 19.9 & 15.1 & 4.6 \\
\hline 29 & -0.1 & -- & 0.2 & 4.5 & 9.8 & 13.6 & 22.8 & 23.0 & 22.6 & 19.9 & 14.9 & 4.5 \\
\hline 30 & -0.1 & -- & 0.3 & 4.4 & 9.8 & 14.2 & 23.0 & 23.0 & 22.6 & 19.9 & $14 . \epsilon$ & 4.4 \\
\hline 31 & -0.1 & -- & 0.3 & -. & 10.0 & -- & 23.2 & 22.9 & -- & 19.8 & -- & 4.3 \\
\hline
\end{tabular}


Table 14n. Mean daily ground-water temperature at site CRM-19 (map ID \#16), Seminole Well Field, Cedar Rapids, lowa, January 1997 through February 1999--Continued

\begin{tabular}{|c|c|c|c|c|c|c|c|c|c|c|c|c|}
\hline Day & Jan & Feb & Mar & Apr & May & Jun & Jul & Aug & Sep & Oct & Nov & Dec \\
\hline & & & & & & 1998 & & & & & & \\
\hline 1 & 4.2 & 0.8 & 0.3 & 1.6 & 2.7 & 17.0 & 14.7 & 16.0 & 23.8 & 22.5 & 18.3 & 14.5 \\
\hline 2 & 4.0 & 0.8 & 0.3 & 1.6 & 2.7 & 17.1 & 15.3 & 16.0 & 23.9 & 22.5 & 18.1 & 14.3 \\
\hline 3 & 3.8 & 0.8 & 0.3 & 1.7 & 2.7 & 17.0 & 15.3 & 16.0 & 23.8 & 22.4 & 17.9 & 14.1 \\
\hline 4 & 3.4 & 0.7 & 0.2 & 1.7 & 2.8 & 16.8 & 16.0 & 16.1 & 23.7 & 22.4 & 17.7 & 13.9 \\
\hline 5 & 3.2 & 0.7 & 0.2 & 1.7 & 2.9 & 16.6 & 16.6 & 16.2 & 23.7 & 22.3 & 17.6 & 13.7 \\
\hline 6 & 3.0 & 0.7 & 0.2 & 1.6 & 3.3 & 16.5 & 17.2 & 16.2 & 23.6 & 22.3 & 17.5 & 13.5 \\
\hline 7 & 2.8 & 0.7 & 0.2 & 1.6 & 4.1 & 16.4 & 17.8 & 16.4 & 23.5 & 22.3 & 17.4 & 13.2 \\
\hline 8 & 2.7 & 0.6 & 0.3 & 1.7 & 5.4 & 16.3 & 18.2 & 16.5 & 23.5 & 22.2 & 17.3 & 12.9 \\
\hline 9 & 2.6 & 0.6 & 0.3 & 1.7 & 6.8 & 16.2 & 18.7 & 16.7 & 23.4 & 22.4 & 17.3 & 12.6 \\
\hline 10 & 2.5 & 0.6 & 0.3 & 1.7 & 7.9 & 16.1 & 19.1 & 17.0 & 23.4 & 22.7 & 17.2 & 12.3 \\
\hline 11 & 2.3 & 0.6 & 0.3 & 1.7 & 8.6 & 16.1 & 19.4 & 17.3 & 23.3 & 22.8 & 17.2 & 11.9 \\
\hline 12 & 2.2 & 0.6 & 0.3 & 1.7 & 9.3 & 15.9 & 19.7 & 17.6 & 23.3 & 22.8 & 17.1 & 11.6 \\
\hline 13 & 2.1 & 0.6 & 0.3 & 1.7 & 10.2 & 15.8 & 20.0 & 17.9 & 23.2 & 22.7 & 17.0 & 11.3 \\
\hline 14 & 2.0 & 0.6 & 0.3 & 1.7 & 11.1 & 15.8 & 20.2 & 18.2 & 23.2 & 22.5 & 16.9 & 10.9 \\
\hline 15 & 1.9 & 0.6 & 0.4 & 1.7 & 12.0 & 15.7 & 20.1 & 18.5 & 23.2 & 22.3 & 16.8 & 10.6 \\
\hline 16 & 1.8 & 0.6 & 0.4 & 1.7 & 12.6 & 15.7 & 20.4 & 18.8 & 23.2 & 22.1 & 16.8 & 9.7 \\
\hline 17 & 1.7 & 0.6 & 0.4 & 1.8 & 12.9 & 15.7 & 17.2 & 19.0 & 23.1 & 21.9 & 16.7 & 8.3 \\
\hline 18 & 1.6 & 0.6 & 0.4 & 1.8 & 13.4 & 15.5 & 15.0 & 19.2 & 23.1 & 21.7 & 16.6 & 7.2 \\
\hline 19 & 1.5 & 0.5 & 0.4 & 1.8 & 14.0 & 15.4 & 15.0 & 19.6 & 23.1 & 21.4 & 16.5 & 6.7 \\
\hline 20 & 1.4 & 0.5 & 0.4 & 1.8 & 14.5 & 15.4 & 15.1 & 19.7 & 23.1 & 21.2 & 16.4 & 6.7 \\
\hline 21 & 1.3 & 0.4 & 0.4 & 1.9 & 14.9 & 15.4 & 15.2 & 19.6 & 23.0 & 21.0 & 16.2 & 6.8 \\
\hline 22 & 1.2 & 0.4 & -- & 1.9 & 15.4 & 15.3 & 15.5 & 19.7 & 23.0 & 20.7 & 16.0 & 7.0 \\
\hline 23 & 1.1 & -- & -- & 2.0 & 15.7 & -- & 15.2 & 20.0 & 22.9 & 20.4 & 15.8 & 7.2 \\
\hline 24 & 1.1 & -. & 0.9 & 2.0 & 16.1 & -- & 15.3 & 20.7 & 22.9 & 20.1 & 15.6 & 7.4 \\
\hline 25 & 1.0 & 0.2 & 0.9 & 2.2 & 16.2 & 11.9 & 15.4 & 21.2 & 22.8 & 19.9 & 15.5 & 7.6 \\
\hline 26 & 1.0 & 0.2 & 0.9 & 2.3 & 16.3 & 12.4 & 15.5 & 21.6 & 22.8 & 19.6 & 15.3 & 7.8 \\
\hline 27 & 1.0 & 0.2 & 1.0 & 2.5 & 16.5 & 12.7 & 15.6 & 22.1 & 22.7 & 19.4 & 15.1 & 7.8 \\
\hline 28 & 0.9 & 0.3 & 1.2 & 2.7 & 16.5 & 13.0 & 15.7 & 22.6 & 22.6 & 19.2 & 15.0 & 7.9 \\
\hline 29 & 0.9 & -- & 1.3 & 2.7 & 16.5 & 13.6 & 15.7 & 23.0 & 22.6 & 19.0 & 14.8 & 8.0 \\
\hline 30 & 0.9 & -- & 1.3 & 2.7 & 16.6 & 14.2 & 15.8 & 23.2 & 22.5 & 18.7 & 14.7 & 8.0 \\
\hline 31 & 0.8 & -. & 1.5 & -- & 16.8 & -- & 15.9 & 23.5 & -. & 18.5 & -. & 7.9 \\
\hline
\end{tabular}


Table 14n. Mean daily ground-water temperature at site CRM-19 (map ID \#16), Seminole Well Field, Cedar Rapids, lowa, January 1997 through February 1999--Continued

\begin{tabular}{|c|c|c|c|c|c|c|c|c|c|c|c|c|}
\hline Day & Jan & Feb & Mar & Apr & May & Jun & Jul & Aug & Sep & Oct & Nov & Dec \\
\hline & & & & & & 1999 & & & & & & \\
\hline 1 & 7.8 & 4.4 & -. & -- & -- & -- & -- & -- & -- & -- & -- & -- \\
\hline 2 & 7.8 & 4.4 & -- & -- & -- & -- & -- & -- & -- & -- & -- & -- \\
\hline 3 & 7.7 & 4.5 & -- & -- & -- & -- & -- & -. & -- & -- & -- & -- \\
\hline 4 & 7.5 & 4.4 & -- & -- & -- & -- & -- & -- & -- & -- & -- & -- \\
\hline 5 & 7.4 & 4.0 & -. & -- & - & -. & -- & -- & - & -- & -- & -- \\
\hline 6 & 7.4 & 3.3 & -- & -- & - & -- & -- & -- & -- & -- & -- & -- \\
\hline 7 & 7.2 & 2.8 & -- & -- & -- & -- & -- & -- & -- & -- & -. & -- \\
\hline 8 & 7.1 & 2.7 & -- & -- & -- & -. & -- & -- & -- & -. & - & -- \\
\hline 9 & 6.9 & 2.6 & -. & -- & -- & -. & -- & -- & -- & -- & -. & -. \\
\hline 10 & 6.8 & 2.3 & -- & -- & -- & -- & -- & -- & -- & -- & -- & -- \\
\hline 11 & 6.7 & 1.8 & -- & -- & -- & -- & -- & -- & -- & -- & -- & -- \\
\hline 12 & 6.6 & 1.4 & -- & -- & -- & -- & -- & -- & -- & -- & -- & -- \\
\hline 13 & 6.6 & 1.1 & -- & -- & -- & -- & -- & -- & -- & -- & -- & -- \\
\hline 14 & 6.5 & 0.9 & -- & -- & -- & -- & -- & -- & -- & -- & -- & -- \\
\hline 15 & 6.4 & 0.7 & -- & -- & -- & -- & -- & -- & -- & -- & -- & -- \\
\hline 16 & 6.4 & 0.6 & -- & -- & -- & -- & -- & -- & -- & -- & -- & -- \\
\hline 17 & 6.2 & 0.5 & -- & -- & -- & -- & -- & -- & -- & -- & -- & -- \\
\hline 18 & 6.1 & 0.3 & -- & -. & -- & -- & -- & -- & -- & -- & - & -- \\
\hline 19 & 5.9 & 0.3 & -- & -- & -. & -- & -- & -- & -- & -- & -- & -- \\
\hline 20 & 5.3 & 0.2 & -- & -- & -- & -- & -- & -- & -- & -- & -- & -- \\
\hline 21 & 4.9 & 0.2 & -- & -- & -- & -. & -- & -- & -- & -- & -- & -- \\
\hline 22 & 4.8 & 0.2 & -- & -- & -- & -- & -- & - & -- & -- & -- & -- \\
\hline 23 & 4.8 & 0.4 & -- & -- & -- & -- & -- & -- & -- & -- & -- & -- \\
\hline 24 & 4.7 & 0.7 & -- & -- & -- & -- & -- & -- & -- & - & -- & -- \\
\hline 25 & 4.7 & 0.9 & -- & -- & - & -- & -- & -- & -- & -- & -- & -- \\
\hline 26 & 4.6 & 0.8 & -- & -- & -- & -- & -- & - & -- & -- & -- & -- \\
\hline 27 & 4.6 & 0.7 & -- & -- & -- & -- & -- & -- & -- & -- & -- & -- \\
\hline 28 & 4.6 & 0.8 & -- & -- & - & -- & -- & -- & -- & -- & -- & -- \\
\hline 29 & 4.5 & -- & -- & -- & -- & -- & -- & -- & -- & -- & -- & -- \\
\hline 30 & 4.5 & -- & -- & -- & -- & -- & -- & -- & -- & -- & -- & -- \\
\hline 31 & 4.4 & -. & -- & -- & -- & -- & -- & -- & -- & -- & -- & -- \\
\hline
\end{tabular}


Table 140. Mean daily ground-water temperature at site CRM-20 (map ID \#17), Northwest Well Field, Cedar Rapids, lowa, February 1998 through March 1999

[Temperature in degrees Centigrade; --, value not measured or not recorded]

\begin{tabular}{|c|c|c|c|c|c|c|c|c|c|c|c|c|}
\hline Day & Jan & Feb & Mar & Apr & May & Jun & Jul & Aug & Sep & Oct & Nov & $\mathrm{Dec}$ \\
\hline & & & & & & 1998 & & & & & & \\
\hline 1 & -- & -- & 0.0 & 2.4 & 11.7 & 18.3 & 20.9 & 27.5 & -- & 22.5 & 15.0 & 11.8 \\
\hline 2 & -. & -- & 0.0 & 2.5 & 11.2 & 19.5 & 21.3 & 27.5 & -- & 22.4 & 14.8 & 11.1 \\
\hline 3 & -. & -- & 0.0 & 2.6 & 10.7 & 19.9 & 21.6 & 27.2 & -. & 22.5 & 14.6 & 10.2 \\
\hline 4 & -. & -- & 0.0 & 3.0 & 9.7 & 20.2 & 21.9 & 26.8 & -- & 22.7 & 14.6 & 9.4 \\
\hline 5 & -. & -. & -0.1 & 4.9 & 8.0 & 20.2 & 22.4 & 26.4 & -- & 22.9 & 14.4 & 8.5 \\
\hline 6 & -- & -- & -0.1 & 7.2 & 6.0 & 20.3 & 23.1 & 26.1 & -- & 22.9 & 14.2 & 7.7 \\
\hline 7 & -- & -- & -0.1 & 8.4 & 4.6 & 20.5 & 23.8 & 25.7 & -- & 22.8 & 14.1 & 7.0 \\
\hline 8 & -- & -- & -0.1 & 9.0 & 4.2 & 20.6 & 24.3 & 25.4 & -- & 22.4 & 14.0 & 6.5 \\
\hline 9 & -- & -- & 0.0 & 8.9 & 4.3 & 20.8 & 24.4 & 25.1 & -- & 21.6 & 13.9 & 6.0 \\
\hline 10 & -- & 0.0 & 0.0 & 8.3 & 4.4 & 20.8 & 24.2 & 24.9 & -- & 20.6 & 13.9 & 5.6 \\
\hline 11 & -- & 0.0 & 0.0 & 7.9 & 4.6 & 20.7 & 24.0 & 24.9 & -- & 20.0 & 13.9 & 5.4 \\
\hline 12 & -- & 0.0 & 0.0 & 8.0 & 4.9 & 20.5 & 23.7 & 25.0 & -- & 19.6 & 13.8 & 5.3 \\
\hline 13 & -- & 0.0 & 0.0 & 8.6 & 5.2 & 20.2 & 23.4 & 25.1 & -- & 19.5 & 13.6 & 5.1 \\
\hline 14 & -- & 0.0 & 0.0 & 9.3 & 5.5 & 20.1 & 22.7 & 25.2 & -- & 19.9 & 13.5 & 5.0 \\
\hline 15 & -. & 0.0 & 0.1 & 9.6 & 5.9 & 20.2 & 21.5 & 25.3 & -- & 20.5 & 13.5 & 4.8 \\
\hline 16 & -- & 0.0 & 0.1 & 9.5 & 6.5 & 20.3 & 20.0 & 25.3 & -- & 20.8 & 13.5 & 4.8 \\
\hline 17 & -- & 0.0 & 0.1 & 9.4 & 7.1 & 20.0 & 20.3 & -- & -- & 21.0 & 13.5 & 4.8 \\
\hline 18 & -- & 0.0 & 0.2 & 9.7 & 7.6 & 19.4 & 20.6 & -. & -. & 20.9 & 13.5 & 4.8 \\
\hline 19 & -- & 0.0 & 0.2 & 10.2 & 8.2 & 19.3 & 20.7 & -- & -- & 20.6 & 13.5 & 4.9 \\
\hline 20 & -- & -0.1 & 0.4 & 10.8 & 8.6 & 19.2 & 20.9 & -- & -- & 20.4 & 13.4 & 5.2 \\
\hline 21 & -- & -0.1 & 0.5 & 11.3 & 9.0 & 19.1 & 21.5 & -- & -- & 20.0 & 13.2 & 5.7 \\
\hline 22 & -- & -0.1 & 0.7 & 11.3 & 9.4 & 19.1 & 22.4 & -- & -- & 19.5 & 13.0 & 6.3 \\
\hline 23 & -- & 0.0 & 1.0 & 10.8 & 9.8 & 19.1 & 23.2 & -- & 23.8 & 18.9 & 13.0 & 7.0 \\
\hline 24 & -. & 0.0 & 1.2 & 10.6 & 10.2 & 19.0 & 23.4 & -- & 23.8 & 18.2 & 13.0 & 7.6 \\
\hline 25 & -- & 0.0 & 1.4 & 10.8 & 10.7 & 19.0 & 23.8 & -. & 23.6 & 17.6 & 13.1 & 8.1 \\
\hline 26 & -- & 0.0 & 1.6 & 11.1 & 11.4 & 19.4 & 24.7 & -- & 23.1 & 16.9 & 13.2 & 8.5 \\
\hline 27 & -- & 0.0 & 1.9 & 11.3 & 12.7 & 19.7 & 25.6 & -- & 22.7 & 16.5 & 13.2 & 8.9 \\
\hline 28 & -- & 0.0 & 2.1 & 11.3 & 14.2 & 20.0 & 26.4 & -- & 22.5 & 16.2 & 13.1 & 9.1 \\
\hline 29 & -- & -- & 2.4 & 11.5 & 15.4 & 20.2 & 27.0 & -- & 22.5 & 15.9 & 12.8 & 9.1 \\
\hline 30 & -- & -- & 2.5 & 11.8 & 16.3 & 20.5 & 27.2 & -- & 22.5 & 15.5 & 12.4 & 8.9 \\
\hline 31 & -- & -- & 2.3 & .. & 17.1 & -- & 27.4 & -- & -. & 15.3 & -- & 8.5 \\
\hline
\end{tabular}


Table 140. Mean daily ground-water temperature at site CRM-20 (map ID \#17), Northwest Well Field, Cedar Rapids, lowa, February 1998 through February 1999--Continued

\begin{tabular}{|c|c|c|c|c|c|c|c|c|c|c|c|c|}
\hline Day & Jan & Feb & Mar & Apr & May & Jun & Jul & Aug & Sep & Oct & Nov & Dec \\
\hline & & & & & & 1999 & & & & & & \\
\hline 1 & 8.0 & -0.1 & -0.1 & -- & -. & .- & -- & -- & -- & -- & -- & -- \\
\hline 2 & 7.4 & -0.1 & -0.1 & -. & -- & -- & -- & -- & -- & -- & -- & -- \\
\hline 3 & 6.8 & -0.1 & -0.1 & -- & -- & -- & -- & -- & -- & -- & -- & -- \\
\hline 4 & 6.2 & -0.1 & -0.1 & -- & -- & -- & -- & -- & -- & -- & -- & -- \\
\hline 5 & 5.5 & -0.1 & 0.0 & -- & -- & -- & -- & -- & -. & -- & -- & -- \\
\hline 6 & 4.6 & -0.1 & 0.0 & -- & -. & -- & -- & -- & -- & -- & -- & -- \\
\hline 7 & 4.1 & -0.1 & 0.1 & -- & -- & -- & -- & -- & -- & -- & -- & -- \\
\hline 8 & 3.7 & -0.1 & 0.2 & -- & - & -- & -- & -- & -- & -- & -- & -- \\
\hline 9 & 3.4 & -0.1 & 0.4 & -- & -- & -- & -- & -- & -- & -- & -- & -- \\
\hline 10 & 3.2 & -0.1 & 0.6 & -- & -- & -- & -- & -- & -- & -- & -- & -- \\
\hline 11 & 3.0 & -0.1 & 0.8 & -- & -- & -- & -- & -- & -- & -- & -- & -- \\
\hline 12 & 2.7 & -0.1 & 1.0 & -- & -- & -- & -- & -- & -- & -- & -- & -- \\
\hline 13 & 2.3 & -0.1 & 1.2 & -- & -- & -- & -- & -- & -- & -- & -- & -- \\
\hline 14 & 1.9 & -0.1 & 1.3 & -- & -- & -- & -- & -- & -. & -- & -- & -- \\
\hline 15 & 1.4 & -0.1 & 1.4 & -- & -- & -- & -- & -- & -- & - & -- & -- \\
\hline 16 & 1.0 & -0.1 & 1.3 & -- & -- & -- & -- & -- & -- & -- & -- & -- \\
\hline 17 & 0.7 & -0.1 & 1.2 & -- & -- & -- & -- & -- & -- & -- & -- & -- \\
\hline 18 & 0.4 & -0.1 & 1.2 & -- & -- & -- & -- & -- & -- & -- & -- & -- \\
\hline 19 & 0.3 & -0.1 & 1.2 & -- & -- & -- & -- & -- & -- & -- & -- & -- \\
\hline 20 & 0.2 & -0.1 & 1.3 & -. & -- & -- & -- & -- & -- & - & -- & -- \\
\hline 21 & 0.1 & -0.1 & 1.6 & -- & -- & -- & -- & -- & -- & -- & -- & -- \\
\hline 22 & 0.0 & -0.1 & 1.9 & -- & -. & -- & -- & -- & -- & -- & -- & -- \\
\hline 23 & 0.0 & -0.1 & 2.3 & -- & -- & -- & -- & -- & -- & -- & -- & -- \\
\hline 24 & 0.0 & -0.1 & 2.5 & -- & -- & -- & -- & -- & -- & -- & -- & -- \\
\hline 25 & -0.1 & -0.1 & 2.6 & -- & -- & -- & -- & -- & -- & -- & -- & -- \\
\hline 26 & -0.1 & -0.1 & 2.6 & $-\cdot$ & -- & -- & -- & -. & -. & $-\cdot$ & -- & -- \\
\hline 27 & -0.1 & -0.1 & 2.6 & -- & -- & -- & -- & -- & -- & -- & -- & -- \\
\hline 28 & -0.1 & -0.1 & 2.5 & -- & -- & -. & -- & -- & -- & -- & -- & -. \\
\hline 29 & -0.1 & -- & 2.3 & -- & -- & -- & -- & -- & -- & -- & -- & -- \\
\hline 30 & -0.1 & -- & 2.1 & -- & -- & -- & -- & -- & -- & -- & -- & -- \\
\hline 31 & -0.1 & -- & 1.8 & -- & -- & -- & -- & -- & -- & -- & -- & -- \\
\hline
\end{tabular}


Table 14p. Mean daily ground-water temperature at site CRM-21 (map ID \#18), Northwest Well Field, Cedar Rapids, lowa, February 1998 through March 1999

[Temperature in degrees Centigrade; --, value not measured or not recorded]

\begin{tabular}{|c|c|c|c|c|c|c|c|c|c|c|c|c|}
\hline Day & Jan & Feb & Mar & Apr & May & Jun & Jul & Aug & Sep & Oct & Nov & Dec \\
\hline \multicolumn{13}{|c|}{1998} \\
\hline 1 & -- & -- & 5.8 & 6.4 & 8.0 & 9.9 & 14.1 & 12.8 & -- & 12.3 & 13.1 & 11.5 \\
\hline 2 & -- & -- & 5.7 & 5.1 & 8.1 & 10.4 & 15.3 & 12.8 & -- & 12.3 & 13.1 & 11.4 \\
\hline 3 & -- & -- & 5.8 & 4.4 & 8.2 & 10.8 & 19.9 & 12.7 & -- & 12.3 & 13.2 & 11.2 \\
\hline 4 & -- & -- & 5.6 & 3.9 & 8.3 & 11.2 & 20.0 & 12.6 & -- & 12.3 & 13.4 & 11.0 \\
\hline 5 & -- & -- & 5.6 & 3.6 & 8.3 & 11.6 & 14.8 & 12.5 & -- & 12.3 & 13.6 & 10.7 \\
\hline 6 & -- & -- & 5.8 & 3.6 & 8.2 & 12.0 & 14.5 & 12.4 & -- & 12.2 & 14.0 & 10.5 \\
\hline 7 & -. & -- & 5.8 & 3.9 & 8.2 & 12.4 & 14.5 & 12.3 & -- & 12.2 & 14.3 & 10.4 \\
\hline 8 & -. & -- & 6.0 & 4.5 & 8.1 & 12.7 & 14.6 & 12.1 & -- & 12.3 & 14.5 & 10.2 \\
\hline 9 & -- & -- & 6.1 & 5.3 & 8.0 & 12.9 & 14.6 & 12.0 & -- & 12.3 & 14.7 & 10.1 \\
\hline 10 & -- & 7.1 & 6.2 & 6.3 & 8.0 & 13.0 & 14.6 & 11.8 & -- & 12.3 & 14.7 & 9.9 \\
\hline 11 & -. & 7.0 & 6.1 & 7.0 & 8.0 & 13.4 & 14.6 & 11.7 & -- & 12.4 & 14.7 & 9.7 \\
\hline 12 & -- & 6.9 & 6.1 & 7.6 & 7.9 & 13.6 & 14.7 & 11.6 & -- & 12.4 & 14.7 & 9.5 \\
\hline 13 & -. & 6.9 & 6.3 & 8.1 & 7.9 & 13.8 & 14.6 & 11.6 & -- & 12.4 & 14.7 & 9.4 \\
\hline 14 & -- & 6.9 & 6.0 & 7.1 & 7.8 & 13.9 & 14.3 & 11.5 & -- & 12.4 & 14.6 & 9.3 \\
\hline 15 & -- & 6.9 & 6.1 & 5.7 & 7.8 & 14.1 & 14.1 & 11.5 & -- & 12.4 & 14.5 & 9.3 \\
\hline 16 & -- & 6.9 & 6.1 & 5.9 & 7.7 & 14.2 & 14.0 & 11.5 & -- & 12.4 & 14.5 & 9.5 \\
\hline 17 & -- & 6.8 & 6.1 & 6.1 & 7.7 & 14.4 & 14.0 & -- & -- & 12.5 & 14.5 & 9.3 \\
\hline 18 & -. & 6.8 & 6.3 & 6.3 & 7.7 & 14.5 & 14.0 & -- & -- & 12.4 & 14.0 & 9.3 \\
\hline 19 & -- & 6.9 & 6.2 & 6.5 & 7.7 & 14.5 & 14.0 & -- & -- & 12.4 & 13.8 & 9.3 \\
\hline 20 & -- & 6.6 & 6.1 & 6.6 & 7.8 & 14.5 & 14.0 & -- & -- & 12.4 & 13.6 & 9.3 \\
\hline 21 & -- & 6.0 & 6.0 & 6.8 & 7.8 & 14.4 & 14.0 & -- & -- & 12.4 & 13.6 & 9.3 \\
\hline 22 & -- & 5.7 & 6.0 & 6.9 & 7.9 & 14.4 & 14.0 & -- & -- & 12.5 & 13.5 & 9.5 \\
\hline 23 & -- & 5.8 & 6.2 & 7.0 & 8.0 & 14.3 & 14.0 & -- & 12.2 & 12.5 & 13.4 & 9.5 \\
\hline 24 & -- & 5.8 & 6.3 & 7.2 & 8.1 & 15.5 & 14.0 & -- & 12.2 & 12.6 & 13.2 & 9.6 \\
\hline 25 & -- & 5.8 & 6.4 & 7.3 & 8.2 & 21.4 & 13.9 & -- & 12.2 & 12.7 & 13.0 & 9.7 \\
\hline 26 & -- & 5.8 & 6.4 & 7.4 & 8.3 & 23.0 & 13.7 & -- & 12.2 & 12.8 & 12.7 & 9.8 \\
\hline 27 & -- & 5.8 & 6.2 & 7.5 & 8.4 & 24.2 & 13.5 & -- & 12.2 & 12.9 & 12.4 & 9.9 \\
\hline 28 & -. & 5.9 & 6.1 & 7.6 & 8.7 & 24.8 & 13.3 & -- & 12.3 & 13.0 & 12.2 & 10.0 \\
\hline 29 & -- & -- & 6.0 & 7.7 & 9.1 & 24.2 & 13.2 & -- & 12.3 & 13.0 & 11.9 & 10.0 \\
\hline 30 & -- & -. & 6.0 & 7.8 & 9.3 & 15.9 & 13.0 & -- & 12.3 & 13.0 & 11.7 & 10.1 \\
\hline 31 & -. & -- & 7.1 & -- & 9.6 & -- & 12.8 & -- & -- & 13.0 & -- & 10.1 \\
\hline
\end{tabular}


Table 14p. Mean daily ground-water temperature at site CRM-21 (map ID \#18), Northwest Well Field, Cedar Rapids, lowa, February 1998 through February 1999--Continued

\begin{tabular}{|c|c|c|c|c|c|c|c|c|c|c|c|c|}
\hline Day & Jan & Feb & Mar & Apr & May & Jun & Jul & Aug & Sep & Oct & Nov & Dec \\
\hline & & & & & & 1999 & & & & & & \\
\hline 1 & 10.2 & 11.2 & 6.1 & - & -- & -- & -- & -- & -. & -- & -- & -. \\
\hline 2 & 10.3 & 11.2 & 6.1 & -- & -- & -- & -- & -- & -- & -- & -- & -- \\
\hline 3 & 10.3 & 11.1 & 6.1 & -- & -- & -- & -- & -- & -. & -- & -- & -- \\
\hline 4 & 10.4 & 11.1 & 5.8 & -- & -- & -- & -- & -- & -- & -- & -- & -- \\
\hline 5 & 10.5 & 11.1 & 5.8 & -- & -- & -- & -- & - & -- & -- & -- & -- \\
\hline 6 & 10.8 & 11.1 & 5.8 & -- & -- & -- & -- & -- & -- & -- & -- & - \\
\hline 7 & 10.9 & 11.1 & 6.0 & -- & -- & -- & -- & -- & -- & - & -- & -- \\
\hline 8 & 10.9 & 11.0 & 6.1 & -- & -- & -- & -- & -- & -- & -- & -- & -- \\
\hline 9 & 11.0 & 11.0 & 5.9 & -- & -- & -- & -- & -- & -- & -- & -- & - \\
\hline 10 & 11.0 & 10.9 & 5.8 & -- & -- & -- & -- & -- & -- & -- & -- & -- \\
\hline 11 & 11.0 & 10.9 & 5.6 & -- & -- & -- & - & -- & -- & -- & -- & -- \\
\hline 12 & 11.0 & 10.9 & 5.6 & -- & -- & -- & -- & -- & -- & -- & -- & -- \\
\hline 13 & 11.1 & 10.8 & 5.7 & -- & -- & -- & -- & -- & -- & -- & -- & -- \\
\hline 14 & 11.1 & 10.8 & 5.8 & -- & -- & -- & -- & -- & -- & -- & -- & -- \\
\hline 15 & 11.2 & 10.7 & 6.1 & -- & -- & -- & -- & -- & - & -- & -- & -- \\
\hline 16 & 11.2 & 10.6 & 6.3 & -- & -- & -- & -- & -- & -- & -- & -- & -- \\
\hline 17 & 11.2 & 10.5 & 6.7 & -- & -- & -- & -- & -- & -- & - & -- & -- \\
\hline 18 & 11.2 & 10.5 & 7.0 & -- & - & -- & -- & -- & -- & -- & -- & -- \\
\hline 19 & 11.3 & 10.3 & 7.2 & -- & -- & -- & -- & -- & - & -- & - & -- \\
\hline 20 & 11.2 & 10.1 & 7.5 & -- & -- & -- & -- & -- & -- & -- & -- & -- \\
\hline 21 & 11.3 & 9.7 & 7.6 & -- & -- & -- & -- & -- & - & - & - & -- \\
\hline 22 & 11.2 & 9.1 & 7.6 & -- & -- & -- & -- & -- & -- & -- & -- & -- \\
\hline 23 & 11.2 & 8.3 & 7.6 & -- & -- & -- & -- & -- & -- & -- & -- & -- \\
\hline 24 & 11.1 & 7.4 & 7.6 & -. & -- & -- & -- & -- & -- & -- & -- & -- \\
\hline 25 & 11.2 & 6.8 & 7.6 & -- & -- & -- & -- & -- & -- & -- & -- & -- \\
\hline 26 & 11.2 & 6.4 & 7.8 & -- & -- & -- & -- & -- & -- & -- & -- & -- \\
\hline 27 & 11.2 & 6.1 & 7.9 & -- & -- & -- & -- & -- & -- & -- & -- & -- \\
\hline 28 & 11.2 & 6.0 & 8.0 & -- & -- & -- & -- & - & -- & -- & -- & -- \\
\hline 29 & 11.2 & -- & 7.9 & -- & -- & -- & -- & -- & -- & -- & -- & -- \\
\hline 30 & 11.2 & - & 7.9 & -- & -- & -- & -- & -- & -- & -- & -- & -- \\
\hline 31 & 11.2 & -- & 8.1 & -- & -- & -- & -- & -- & -- & -- & -- & -- \\
\hline
\end{tabular}


Table 14q. Mean daily ground-water temperature at site CRM-22 (map ID \#19), Seminole Well Field, Cedar Rapids, lowa, February 1998 through February 1999

[Temperature in degrees Centigrade; --, value not measured or not recorded]

\begin{tabular}{|c|c|c|c|c|c|c|c|c|c|c|c|c|}
\hline Day & Jan & Feb & Mar & Apr & May & Jun & Jul & Aug & Sep & Oct & Nov & Dec \\
\hline & & & & & & 1998 & & & & & & \\
\hline 1 & -- & -- & 5.4 & 6.2 & 10.9 & 19.8 & -- & -- & 26.3 & 20.7 & 15.0 & 9.1 \\
\hline 2 & -- & -- & 5.4 & -- & 10.9 & 20.3 & -- & -- & 25.8 & 20.1 & 15.0 & 8.9 \\
\hline 3 & -- & -- & 5.5 & -- & 10.8 & 20.8 & -- & -- & 25.2 & 19.9 & 15.1 & -- \\
\hline 4 & -- & -- & 5.4 & 3.9 & -- & 20.6 & -- & -- & 24.8 & 19.9 & 15.2 & -- \\
\hline 5 & -- & -- & 5.4 & 3.6 & 10.4 & 20.5 & - & -- & 24.7 & 20.2 & 15.3 & 8.5 \\
\hline 6 & -- & -- & 5.6 & 3.6 & 10.4 & 20.5 & -- & -- & 24.7 & 20.5 & 15.3 & 8.2 \\
\hline 7 & -- & -- & 5.6 & 3.9 & 10.4 & 20.4 & -- & -- & 24.6 & 20.7 & 15.3 & 8.0 \\
\hline 8 & $\cdots$ & -- & 5.8 & 4.5 & 10.4 & 20.4 & -- & -- & 24.5 & 20.9 & 15.2 & 8.0 \\
\hline 9 & -- & -- & 5.9 & 5.3 & 10.3 & 20.3 & -- & -- & 24.4 & 21.1 & 15.0 & 8.0 \\
\hline 10 & -- & 7.1 & 6.0 & 6.3 & 10.3 & 20.3 & -- & -- & 24.2 & 21.0 & 14.9 & 8.0 \\
\hline 11 & -- & 7.0 & 6.0 & 7.0 & 10.3 & 20.7 & -. & -- & 23.9 & 20.6 & 14.9 & 8.0 \\
\hline 12 & -- & 6.9 & 6.0 & 7.6 & 10.3 & 20.8 & -- & -- & 23.7 & 20.4 & 15.0 & 8.0 \\
\hline 13 & -- & 6.9 & 6.0 & 8.1 & 10.1 & 20.8 & -- & 23.9 & 23.6 & 20.1 & 14.9 & 7.9 \\
\hline 14 & -- & 6.9 & 5.9 & 8.1 & 9.9 & 19.8 & -- & 23.6 & 23.5 & 19.5 & 14.9 & 7.8 \\
\hline 15 & -- & 6.9 & 5.7 & 8.5 & 9.6 & 20.0 & -- & 23.4 & 23.5 & 18.8 & 14.8 & 7.7 \\
\hline 16 & -- & 6.9 & 5.5 & 8.9 & 9.3 & 19.8 & -- & 23.5 & 23.4 & 18.1 & 14.6 & 7.7 \\
\hline 17 & -- & 6.8 & 5.7 & 9.2 & 9.1 & 19.8 & -. & 23.8 & 23.0 & 17.6 & 14.3 & 7.7 \\
\hline 18 & -- & 6.8 & 5.8 & 9.5 & 9.4 & 19.8 & -- & 24.3 & 22.5 & 17.1 & 13.9 & 7.7 \\
\hline 19 & -- & 6.7 & 5.9 & 9.7 & 10.8 & 19.7 & -- & 24.6 & 22.3 & 16.6 & 13.4 & 7.6 \\
\hline 20 & -- & 6.1 & 5.8 & 9.9 & 12.9 & 19.6 & -- & 24.8 & 22.2 & 16.2 & 12.9 & 7.6 \\
\hline 21 & -- & 5.5 & 5.9 & 9.9 & 15.1 & 19.6 & -- & 24.9 & 22.1 & 16.0 & 12.3 & 7.5 \\
\hline 22 & -- & 5.1 & 5.9 & 9.9 & 16.2 & 19.6 & -- & 25.0 & 22.2 & 15.8 & 11.6 & 7.2 \\
\hline 23 & -- & 5.5 & 6.0 & 10.0 & 16.3 & -- & -- & 25.2 & 22.3 & 15.5 & 10.9 & 6.9 \\
\hline 24 & -- & 5.5 & 6.0 & 11.7 & 16.3 & - & -- & 25.3 & 22.2 & 15.4 & 10.5 & 6.4 \\
\hline 25 & -- & 5.4 & 6.1 & 11.1 & 16.4 & -- & -- & 25.2 & 22.2 & 15.2 & 10.2 & 6.0 \\
\hline 26 & -- & 5.5 & 6.1 & 11.0 & 16.6 & -- & -- & 25.0 & 22.3 & 15.1 & 9.8 & 5.7 \\
\hline 27 & -- & 5.5 & 5.8 & 11.0 & 16.8 & -- & -. & 25.2 & 22.5 & 15.0 & 9.6 & 5.5 \\
\hline 28 & - & 5.5 & 5.8 & 11.0 & 17.0 & -- & -- & 25.6 & 22.4 & 15.0 & 9.4 & 5.4 \\
\hline 29 & -- & -- & 5.7 & 11.0 & 17.9 & -- & -- & 26.1 & 22.0 & 15.0 & 9.4 & 5.5 \\
\hline 30 & -- & -- & 5.7 & 10.9 & 18.6 & -- & -- & 26.4 & 21.3 & 15.0 & 9.3 & 5.5 \\
\hline 31 & -- & -- & 6.8 & - & 19.3 & -- & -- & 26.5 & -- & 15.0 & -- & 5.6 \\
\hline
\end{tabular}


Table 14q. Mean daily ground-water temperature at site CRM-22 (map ID \#19), Seminole Well Field, Cedar Rapids, lowa, February 1998 through February 1999--Continued

\begin{tabular}{|c|c|c|c|c|c|c|c|c|c|c|c|c|}
\hline Day & Jan & Feb & Mar & Apr & May & Jun & Jul & Aug & Sep & Oct & Nov & Dec \\
\hline & & & & & & 1999 & & & & & & \\
\hline 1 & 5.7 & 0.1 & 0.0 & -. & -. & -- & -. & -- & -- & -- & -- & -- \\
\hline 2 & 5.7 & 0.1 & 0.0 & -- & -- & -- & -- & -- & -- & -- & -- & -- \\
\hline 3 & 5.7 & 0.0 & 0.0 & -. & -- & -- & -- & -- & -- & -- & -- & -- \\
\hline 4 & 5.7 & 0.0 & 0.0 & -- & -- & -- & -- & -- & -- & -- & -- & -. \\
\hline 5 & 5.7 & 0.0 & 0.0 & -- & -- & -- & -- & -- & -- & -- & -. & -- \\
\hline 6 & 5.7 & -0.1 & 0.0 & -- & -. & .. & -. & -. & -- & -- & -. & -- \\
\hline 7 & 5.6 & -0.1 & 0.1 & -- & -- & -- & -- & -- & -- & -- & -. & .. \\
\hline 8 & 5.5 & -0.1 & 0.1 & -- & -- & -- & -- & -- & -- & -. & -- & -- \\
\hline 9 & 5.3 & -0.1 & 0.2 & -. & .- & -- & -. & -- & -- & -- & -- & -- \\
\hline 10 & 5.0 & -0.1 & 0.4 & -- & -- & -- & -- & -- & -- & -- & -- & -- \\
\hline 11 & 4.8 & -0.1 & 0.6 & -- & -- & -. & -- & -- & -- & -- & -- & -- \\
\hline 12 & 4.6 & -0.1 & 0.7 & -- & -- & -- & -. & - & -- & -- & -- & -- \\
\hline 13 & 4.5 & -0.2 & 0.9 & -- & -- & -- & -- & -- & -- & -- & -- & -- \\
\hline 14 & 4.2 & -0.2 & 1.0 & -- & -- & -- & -- & -- & -- & -- & -- & -- \\
\hline 15 & 3.9 & -0.2 & 1.1 & -- & -- & -- & -- & -- & -- & -- & -- & -- \\
\hline 16 & 3.6 & -0.2 & 1.1 & -- & -- & -- & -- & -- & -- & -- & -- & -- \\
\hline 17 & 3.2 & -0.2 & 1.1 & -- & -- & -. & -. & -- & -- & -- & -. & -. \\
\hline 18 & 2.8 & -0.2 & 1.2 & -. & -- & -- & -. & -- & -- & -- & -- & -- \\
\hline 19 & 2.5 & -0.2 & 1.2 & -- & -- & -- & -- & -- & -- & -- & -- & -- \\
\hline 20 & 2.2 & -0.2 & 1.2 & -. & -- & -- & -- & -. & -- & -- & -- & -- \\
\hline 21 & 1.8 & -0.2 & 1.2 & -- & -- & -- & -- & -- & -- & -- & -- & -- \\
\hline 22 & 1.7 & -0.1 & 1.3 & -- & -- & -- & -- & -- & -- & -- & -- & -- \\
\hline 23 & 1.3 & -0.1 & 1.3 & -- & -- & -- & -- & -- & -- & -- & -- & -- \\
\hline 24 & 1.1 & -0.1 & 1.3 & -- & -- & -- & -- & -- & -- & -- & -- & -- \\
\hline 25 & 0.9 & -0.1 & 1.3 & -- & -- & -. & -- & -- & -- & -- & -- & -- \\
\hline 26 & 0.7 & -0.1 & 1.4 & -- & -- & -- & -- & -- & -- & -- & -- & -- \\
\hline 27 & 0.6 & 0.0 & 1.5 & -- & -- & -- & -- & -- & -- & -- & -- & -- \\
\hline 28 & 0.4 & 0.0 & 1.7 & -- & -- & -- & -- & -- & -- & -- & -- & -- \\
\hline 29 & 0.4 & -- & 2.0 & -- & -- & -- & -- & -- & -- & -- & -- & -- \\
\hline 30 & 0.2 & -- & 2.2 & -- & -- & -. & -- & -- & -- & -- & -- & -- \\
\hline 31 & 0.2 & -- & 2.4 & -- & -- & -- & -- & -- & -- & -- & -- & -- \\
\hline
\end{tabular}


Table 14r. Mean daily ground-water temperature at site CRM-23 (map ID \#20), Seminole Well Field, Cedar Rapids, lov February 1998 through March 1999

[Temperature in degrees Centigrade; --, value not measured or not recorded]

\begin{tabular}{|c|c|c|c|c|c|c|c|c|c|c|c|c|}
\hline Day & Jan & Feb & Mar & Apr & May & Jun & Jul & Aug & Sep & Oct & Nov & Dec \\
\hline & & & & & & 1998 & & & & & & \\
\hline 1 & -- & -- & 13.2 & 10.2 & 9.1 & 9.9 & 16.6 & 18.3 & 16.7 & 16.4 & 16.0 & 13.8 \\
\hline 2 & -- & -- & 13.3 & -- & 9.0 & 10.1 & 21.0 & 18.3 & -- & 16.3 & 16.0 & 13.7 \\
\hline 3 & -. & -- & 12.9 & -- & 9.0 & 10.1 & 21.8 & 18.3 & -- & 16.3 & 15.9 & 13.5 \\
\hline 4 & - & -- & 12.6 & 9.5 & -- & 9.9 & 18.1 & 18.4 & -- & 16.3 & 15.9 & 13.4 \\
\hline 5 & -. & -. & 12.4 & 9.4 & 8.7 & 10.0 & 16.7 & 18.4 & -- & 16.3 & 15.9 & 13.3 \\
\hline 6 & -- & -- & 12.2 & 9.4 & 8.6 & 10.0 & 15.8 & 18.4 & -- & 16.3 & 15.9 & 13.3 \\
\hline 7 & -- & -- & 12.1 & 9.5 & 8.7 & 10.1 & 14.7 & 18.4 & -- & 16.3 & 15.8 & 13.3 \\
\hline 8 & -- & -- & 12.0 & 9.5 & 8.8 & 10.2 & 14.6 & 18.3 & -- & 16.1 & 15.8 & 13.3 \\
\hline 9 & -- & -- & 12.0 & 9.5 & 8.7 & 10.2 & 14.8 & 18.2 & -- & 16.3 & 15.8 & 13.3 \\
\hline 10 & -- & 13.1 & 11.9 & 9.4 & 8.7 & 10.1 & 15.0 & 18.2 & -- & 16.3 & 15.8 & 13.3 \\
\hline 11 & -- & 13.2 & 11.8 & 9.4 & 8.8 & 11.3 & 15.2 & 18.1 & -- & 16.3 & 15.8 & 13.2 \\
\hline 12 & -- & 13.2 & 11.8 & 9.5 & 8.9 & 11.6 & 15.4 & 18.1 & -- & 16.2 & 15.5 & 13.1 \\
\hline 13 & -- & 13.4 & 11.8 & 9.4 & 8.8 & 11.5 & 15.6 & 18.1 & -- & 16.3 & 15.4 & 13.0 \\
\hline 14 & -- & 13.7 & 11.9 & 9.4 & 8.6 & 11.5 & 15.9 & 18.2 & -- & 16.5 & 15.2 & 12.9 \\
\hline 15 & -- & 13.7 & 11.7 & 9.3 & 8.5 & 11.7 & 16.1 & 18.2 & -- & 16.7 & 15.1 & 12.8 \\
\hline 16 & -- & 13.8 & 11.4 & 9.2 & 8.4 & 11.9 & 16.3 & 18.2 & -- & 16.8 & 15.0 & 12.7 \\
\hline 17 & -- & 13.8 & 11.3 & 9.2 & 8.4 & 12.1 & 16.4 & -- & -. & 16.8 & 14.9 & 12.6 \\
\hline 18 & -- & 13.8 & 11.3 & 9.2 & -- & 12.4 & 16.4 & -- & -- & 16.9 & 14.7 & 12.5 \\
\hline 19 & -. & 13.8 & 11.2 & 9.2 & -- & 12.6 & 16.5 & 17.1 & -- & 16.9 & 14.6 & 12.3 \\
\hline 20 & -- & 13.9 & 11.3 & 9.0 & 8.6 & 11.9 & 16.6 & 17.0 & -- & 17.0 & 14.5 & 12.5 \\
\hline 21 & -- & 13.3 & 11.3 & 8.8 & 8.7 & 11.4 & 16.7 & 17.0 & -- & 16.8 & 14.3 & 12.6 \\
\hline 22 & -- & 12.9 & 11.2 & 8.8 & 8.7 & 11.5 & 16.7 & 17.0 & -- & 16.6 & 14.2 & 12.1 \\
\hline 23 & -- & 12.6 & 11.3 & 9.0 & 8.7 & -- & 16.8 & 17.0 & 16.9 & 16.4 & 14.1 & 11.9 \\
\hline 24 & -- & 12.6 & 11.4 & 9.2 & 8.7 & -- & 17.0 & 17.0 & 16.8 & 16.3 & 14.1 & 11.9 \\
\hline 25 & -- & 12.7 & 11.5 & 9.3 & 8.8 & 19.2 & 17.1 & 17.0 & 16.7 & 16.2 & 14.1 & 11.8 \\
\hline 26 & -- & 12.9 & 11.5 & 9.4 & 8.8 & 23.8 & 17.3 & 17.0 & 16.6 & 16.1 & 14.0 & 11.7 \\
\hline 27 & -- & 13.0 & 11.4 & 9.1 & 8.8 & 24.5 & 17.4 & 17.0 & 16.5 & 16.1 & 14.0 & 11.7 \\
\hline 28 & -- & 13.1 & 11.4 & 9.1 & 8.8 & 24.8 & 17.6 & 16.9 & 16.4 & 16.1 & 14.0 & 11.6 \\
\hline 29 & -- & -- & 11.1 & 9.2 & 9.1 & 24.2 & 17.8 & 16.9 & 16.4 & 16.0 & 13.9 & 11.5 \\
\hline 30 & -- & -- & 10.9 & 9.1 & 9.2 & 21.5 & 18.0 & 16.8 & 16.4 & 16.1 & 13.9 & 11.4 \\
\hline 31 & -- & -- & 10.5 & -- & 9.6 & -- & 18.2 & 16.8 & -- & 16.1 & -- & 11.3 \\
\hline
\end{tabular}


Table 14r. Mean daily ground-water temperature at site CRM-23 (map ID \#20), Seminole Well Field, Cedar Rapids, lowa, February 1998 through February 1999--Continued

\begin{tabular}{|c|c|c|c|c|c|c|c|c|c|c|c|c|}
\hline Day & Jan & Feb & Mar & Apr & May & Jun & Jul & Aug & Sep & Oct & Nov & $\overline{\mathrm{Dec}}$ \\
\hline & & & & & & 1999 & & & & & & \\
\hline 1 & 11.3 & 10.1 & 10.3 & -- & -- & -- & -- & -- & -- & -- & -- & -- \\
\hline 2 & 11.2 & 10.1 & 10.4 & -- & -- & -- & -- & -- & -- & -- & - & -- \\
\hline 3 & 11.1 & 10.1 & 10.4 & -- & -- & -- & -- & -- & -- & -- & -- & -- \\
\hline 4 & 11.0 & 10.1 & 10.4 & -- & -- & -- & -- & -- & -- & -- & -- & -- \\
\hline 5 & 10.9 & 10.1 & 10.4 & -- & -- & -. & -- & -- & -- & -- & -- & -- \\
\hline 6 & 10.8 & 10.1 & 10.4 & -- & -- & -. & -- & -- & -- & -- & -- & -- \\
\hline 7 & 10.8 & 10.1 & 10.4 & -- & -- & -- & -- & -- & - & -- & -- & -. \\
\hline 8 & 11.1 & 10.1 & 10.5 & -- & -- & -- & -- & -. & -. & -- & -- & -- \\
\hline 9 & 10.9 & 10.0 & 10.5 & -- & -- & -- & -- & -- & -- & -- & -- & -- \\
\hline 10 & 10.7 & 10.1 & 10.5 & -- & -- & -- & -- & - & -- & - & -- & -- \\
\hline 11 & 10.7 & 10.1 & 10.5 & -- & -- & -- & -- & - & -- & -- & -- & -- \\
\hline 12 & 10.6 & 10.1 & 10.6 & -- & -- & -- & -- & -- & -. & -- & -- & -- \\
\hline 13 & 10.5 & 10.1 & 10.6 & -- & -- & -- & -- & -- & -- & -- & -- & - \\
\hline 14 & 10.5 & 10.1 & 10.6 & -- & -- & -- & -- & -- & -- & -- & -- & -- \\
\hline 15 & 10.6 & 10.1 & 10.7 & -- & -- & -- & -- & -- & -- & -- & -- & -- \\
\hline 16 & 10.5 & 10.1 & 10.7 & -- & -- & -- & -- & -. & -. & -- & -- & - \\
\hline 17 & 10.3 & 10.1 & 10.7 & -. & -- & -- & -- & -- & -. & -- & -- & -- \\
\hline 18 & 10.3 & 10.1 & 10.7 & -- & -- & -- & -- & -- & -- & -- & -- & -- \\
\hline 19 & 10.2 & 10.2 & 10.7 & -- & -- & -- & -- & -- & -- & -- & -- & -- \\
\hline 20 & 10.2 & 10.2 & 10.9 & -- & -- & -- & -- & -- & -- & -- & -- & -- \\
\hline 21 & 10.2 & 10.2 & 11.1 & -- & -- & -- & -- & -. & - & -- & -- & -- \\
\hline 22 & 10.2 & 10.2 & 11.3 & -- & -- & -- & -- & -. & -- & -- & -- & - \\
\hline 23 & 10.3 & 10.2 & 11.4 & -- & -- & -- & -- & -- & -- & -- & -- & -- \\
\hline 24 & 10.3 & 10.2 & 11.5 & -- & -- & -- & -- & -. & - & -- & -- & -- \\
\hline 25 & 10.2 & 10.2 & 11.6 & -- & -- & -- & -- & -- & -. & -- & -- & -- \\
\hline 26 & 10.2 & 10.3 & 11.6 & -- & -- & -- & -- & -- & -- & -- & -- & -- \\
\hline 27 & 10.2 & 10.3 & 11.6 & -- & -- & -- & -- & -- & -- & -- & -- & -- \\
\hline 28 & 10.1 & 10.3 & 11.4 & -- & -- & -- & -- & -- & -- & -- & -- & -- \\
\hline 29 & 10.1 & -- & 11.2 & -- & -- & -- & -- & -- & -- & -- & -- & -- \\
\hline 30 & 10.1 & -- & 11.2 & -- & -- & -- & -. & -- & -- & -- & -- & -. \\
\hline 31 & 10.1 & -- & 11.1 & -- & -- & -- & -- & -- & -- & -- & -- & -- \\
\hline
\end{tabular}


Table 14s. Mean daily ground-water temperature at site CRM-24 (map ID \#21), Seminole Well Field, Cedar Rapids, lowa, February 1998 through February 1999

[Temperature in degrees Centigrade; --, value not measured or not recorded]

\begin{tabular}{|c|c|c|c|c|c|c|c|c|c|c|c|c|}
\hline Day & $\operatorname{Jan}$ & Feb & Mar & Apr & May & Jun & Jul & Aug & Sep & Oct & Nov & Dec \\
\hline & & & & & & 1998 & & & & & & \\
\hline 1 & -- & -- & 21.3 & 10.1 & -- & 6.6 & -- & -- & 23.9 & 24.9 & 22.5 & 19.4 \\
\hline 2 & -- & -- & 21.3 & 9.6 & -. & 6.6 & -. & -- & 23.9 & 24.8 & 22.5 & -- \\
\hline 3 & -. & -- & 21.3 & -- & -- & 6.7 & -- & -- & 23.9 & 24.7 & 22.5 & -. \\
\hline 4 & -- & -- & 21.3 & -- & -. & 6.8 & -- & -- & 23.8 & 24.6 & 22.5 & -- \\
\hline 5 & -- & -- & 21.2 & -- & 8.4 & 6.9 & - & -- & 23.8 & 24.4 & 22.4 & 19.2 \\
\hline 6 & $\cdots$ & -- & 21.0 & -- & 8.4 & 6.9 & -- & -- & 23.6 & 24.3 & 22.4 & 19.0 \\
\hline 7 & -- & -- & 20.7 & -- & 8.3 & 6.9 & -- & -- & 23.5 & 24.2 & 22.4 & 18.9 \\
\hline 8 & -- & -- & 20.4 & -- & 8.3 & 7.0 & -- & -- & 23.4 & 24.1 & 22.3 & 18.8 \\
\hline 9 & -- & -- & 19.9 & -- & 8.3 & 7.0 & -- & -- & 23.6 & 24.1 & 22.2 & 18.8 \\
\hline 10 & -- & -- & 19.2 & -- & 8.2 & 7.0 & -- & -- & 23.7 & 24.0 & 22.2 & 18.7 \\
\hline 11 & -- & -- & 18.5 & -- & 8.2 & 7.1 & -- & 19.8 & 23.8 & 23.9 & 22.2 & 18.7 \\
\hline 12 & -- & -- & 17.7 & -- & 8.1 & 7.3 & -- & 20.0 & 23.9 & 23.7 & 22.2 & 18.8 \\
\hline 13 & -- & -- & 16.9 & -- & 8.0 & 7.6 & -- & 20.2 & 24.0 & 23.7 & 22.2 & 18.6 \\
\hline 14 & -- & -- & 16.0 & -- & 8.0 & 8.1 & -- & 20.7 & 24.1 & 23.7 & 22.2 & 18.4 \\
\hline 15 & -- & -- & 15.3 & -- & 8.0 & 17.6 & -- & 21.0 & 24.2 & 23.6 & 22.1 & 18.3 \\
\hline 16 & -- & -- & 14.8 & -- & 8.0 & 12.5 & -- & 21.2 & 24.2 & 23.4 & 22.0 & 18.0 \\
\hline 17 & -- & -- & 14.7 & -- & 7.9 & 9.0 & -- & 21.5 & 24.3 & 23.4 & 21.8 & 17.8 \\
\hline 18 & -- & -- & 14.8 & -- & 7.7 & 9.5 & -- & 21.8 & 24.4 & 23.3 & 21.6 & 17.5 \\
\hline 19 & -- & -- & 14.8 & -- & 7.5 & 10.1 & - & 21.9 & 24.5 & 23.2 & 21.4 & 17.3 \\
\hline 20 & -- & -- & 14.8 & -- & 7.4 & 10.5 & -- & 22.2 & 24.4 & 23.1 & 21.2 & 17.0 \\
\hline 21 & -- & -. & 14.8 & -- & 7.1 & 10.5 & -- & 22.4 & 24.4 & 23.0 & 21.0 & 16.7 \\
\hline 22 & -- & 21.6 & 14.5 & -. & 6.8 & 10.6 & -- & 22.6 & 24.6 & 23.0 & 20.7 & 16.3 \\
\hline 23 & -. & 21.7 & 14.5 & -- & 6.8 & -- & -- & 22.8 & 24.8 & 22.9 & 20.6 & 16.0 \\
\hline 24 & -- & 21.6 & 14.5 & -- & 6.9 & -- & -- & 23.0 & 24.9 & 22.9 & 20.4 & 15.7 \\
\hline 25 & -- & 21.5 & 14.3 & -- & 6.9 & -- & -- & 23.2 & 25.0 & 22.8 & 20.2 & 15.3 \\
\hline 26 & -- & 21.5 & 13.6 & -- & 6.9 & -- & -. & 23.4 & 25.0 & 22.7 & 20.0 & 14.9 \\
\hline 27 & -- & 21.4 & 12.9 & -- & 6.9 & -- & -- & 23.6 & 25.1 & 22.7 & 19.9 & 14.5 \\
\hline 28 & -- & 21.4 & 12.3 & -- & 6.9 & -- & -. & 23.7 & 25.1 & 22.6 & 19.8 & 14.1 \\
\hline 29 & -- & -- & 11.7 & -- & 6.8 & - & -- & 23.8 & 25.0 & 22.6 & 19.7 & 13.8 \\
\hline 30 & -- & -- & 11.3 & -- & 6.7 & -- & -- & 23.9 & 25.0 & 22.6 & 19.5 & 13.5 \\
\hline 31 & -- & -- & 10.7 & .. & 6.6 & -- & -- & 23.9 & -- & 22.5 & -- & 13.1 \\
\hline
\end{tabular}


Table 14s. Mean daily ground-water temperature at site CRM-24 (map ID \#21), Seminole Well Field, Cedar Rapids, lowa, February 1998 through February 1999--Continued

\begin{tabular}{|c|c|c|c|c|c|c|c|c|c|c|c|c|}
\hline Day & Jan & Feb & Mar & Apr & May & Jun & Jul & Aug & Sep & Oct & Nov & Dec \\
\hline & & & & & & 1999 & & & & & & \\
\hline 1 & 12.8 & 6.2 & -- & -- & -- & -- & -- & -- & -- & -- & -- & -- \\
\hline 2 & 12.5 & 6.2 & -- & -- & -- & -- & -- & -- & -- & -- & -- & -- \\
\hline 3 & 12.2 & 6.1 & -. & -- & -- & -- & -- & -- & -- & -- & -- & -- \\
\hline 4 & 11.9 & 6.1 & -- & -- & - & -- & -- & -- & -- & -- & -- & -- \\
\hline 5 & 11.6 & 6.0 & -- & -- & -- & -- & -- & -- & -- & -- & -- & -- \\
\hline 6 & 11.3 & 5.9 & -- & -- & -- & -- & -- & -- & -- & -- & -- & -- \\
\hline 7 & 11.1 & 5.7 & - & -- & -- & -- & -. & -- & - & -- & -- & -- \\
\hline 8 & 10.8 & 5.5 & -- & -- & -- & -- & -- & -- & -- & -- & -- & -- \\
\hline 9 & 10.6 & 5.4 & - & -- & -- & -- & -- & -- & -- & -- & -. & -- \\
\hline 10 & 10.4 & 5.3 & -- & -- & -- & -- & -- & -- & -- & -- & -- & -- \\
\hline 11 & 10.1 & 5.2 & -- & -- & - & -- & -- & -- & -- & -- & -- & -. \\
\hline 12 & 9.9 & 5.1 & -- & -- & -- & -- & -- & -- & -- & -- & -- & -- \\
\hline 13 & 9.7 & 4.9 & -- & -- & -- & -- & -- & -- & -- & -- & -- & -. \\
\hline 14 & 9.4 & 4.6 & -- & -- & -- & -- & -- & - & -- & -- & -- & -- \\
\hline 15 & 9.1 & 4.3 & -. & -- & -- & - & -. & -- & -- & -- & -- & -. \\
\hline 16 & 8.8 & 4.0 & -. & -- & -- & -- & -- & -- & -- & -- & -- & -- \\
\hline 17 & 8.5 & 3.8 & -- & -- & -- & -- & -- & -- & -- & -- & -- & -. \\
\hline 18 & 8.3 & 3.5 & -- & -- & -- & -- & -- & -. & -- & -- & -- & -- \\
\hline 19 & 8.0 & 3.2 & -- & -- & -- & -- & -- & -- & -- & -- & -- & -- \\
\hline 20 & 7.7 & 3.0 & -- & -- & -- & -- & -- & -- & -- & -- & -- & -- \\
\hline 21 & 7.3 & 2.7 & -- & -- & -- & -- & -- & -- & -- & -- & -- & -- \\
\hline 22 & 7.0 & 2.5 & -- & -- & -- & -- & -- & -- & -- & -- & -- & -- \\
\hline 23 & 6.8 & 2.2 & -- & - & -- & -- & -- & -- & -- & -- & -- & -- \\
\hline 24 & 6.6 & 2.0 & -- & -- & -- & -- & -- & -- & -- & -- & -- & -- \\
\hline 25 & 6.5 & 1.8 & -- & -- & -- & -. & -- & -- & -- & -- & -- & -- \\
\hline 26 & 6.4 & 1.6 & -. & -- & -- & -. & -- & -- & -- & -- & -- & -- \\
\hline 27 & 6.4 & 1.4 & -- & -- & -- & -- & -- & -- & -- & -- & -- & -. \\
\hline 28 & 6.3 & 1.2 & -- & -- & -- & -- & -- & -- & - & -- & -- & -- \\
\hline 29 & 6.2 & -- & -- & -- & -- & -- & -- & -. & -- & -- & -- & -- \\
\hline 30 & 6.2 & -- & -- & -- & -- & -- & -- & -- & -- & -- & -- & - \\
\hline 31 & 6.1 & -. & -- & -. & -- & -- & -- & -- & -- & -- & -- & -- \\
\hline
\end{tabular}


Table 14t. Mean daily ground-water temperature at site CRM-25 (map ID \#22), Seminole Well Field, Cedar Rapids, low February 1998 through February 1999

[Temperature in degrees Centigrade; --, value not measured or not recorded]

\begin{tabular}{|c|c|c|c|c|c|c|c|c|c|c|c|c|}
\hline Day & Jan & Feb & Mar & Apr & May & Jun & Jul & Aug & Sep & Oct & Nov & Dec \\
\hline \multicolumn{13}{|c|}{1998} \\
\hline 1 & -- & -- & 9.3 & 7.0 & 8.7 & 9.9 & 11.1 & 13.6 & 15.0 & 16.1 & 15.4 & 14.0 \\
\hline 2 & -- & - & 9.4 & 7.1 & 8.8 & 9.9 & 11.2 & 13.7 & 15.1 & 16.1 & 15.4 & 14.0 \\
\hline 3 & -- & -. & 9.3 & 7.3 & 8.8 & 10.0 & 11.2 & 13.8 & 15.2 & 16.1 & 15.3 & 13.9 \\
\hline 4 & -- & -- & 9.3 & 8.1 & 8.8 & 10.0 & 11.2 & 13.7 & 15.3 & 16.0 & 15.3 & 13.8 \\
\hline 5 & -- & -- & 9.3 & 8.3 & 8.8 & 10.1 & 11.1 & 13.7 & 15.3 & 15.8 & 15.3 & 13.7 \\
\hline 6 & -- & -. & 9.3 & 7.8 & 8.8 & 10.2 & 11.1 & 13.6 & 15.4 & 16.0 & 15.3 & 13.6 \\
\hline 7 & -- & -- & 9.2 & 8.5 & 8.8 & 10.2 & 11.1 & 13.9 & 15.5 & 16.1 & 15.3 & 13.6 \\
\hline 8 & -- & - & 9.1 & 8.6 & 8.9 & 10.3 & 11.2 & 14.1 & 15.5 & 16.0 & 15.3 & 13.5 \\
\hline 9 & -- & -- & 9.1 & 8.6 & 8.9 & 10.3 & 11.2 & 14.1 & 15.6 & 16.0 & 15.2 & 13.5 \\
\hline 10 & -- & -- & 9.1 & 8.5 & 8.9 & 10.3 & 11.3 & 14.1 & 15.6 & 16.0 & 15.1 & 13.4 \\
\hline 11 & -- & -. & 9.0 & 8.4 & 9.0 & 10.3 & 11.3 & 14.2 & 15.6 & 16.1 & 15.1 & 13.3 \\
\hline 12 & -- & -- & 8.9 & 8.4 & 9.0 & 10.5 & 11.4 & 14.3 & 15.6 & 16.1 & 15.1 & 13.4 \\
\hline 13 & -- & -. & 8.6 & 8.5 & 9.0 & 11.2 & 11.4 & 14.4 & 15.6 & 16.1 & 15.0 & 12.7 \\
\hline 14 & -- & 9.1 & 8.3 & 8.4 & 9.1 & 11.1 & 11.5 & 14.4 & 15.4 & 16.0 & 15.0 & 12.5 \\
\hline 15 & -- & 9.0 & 8.2 & 8.5 & 9.1 & 10.8 & 11.6 & 14.4 & 15.6 & 15.9 & 15.0 & 12.5 \\
\hline 16 & -- & 9.0 & 8.1 & 8.4 & 9.2 & 10.6 & 11.7 & 14.5 & 15.6 & 16.0 & 14.9 & 12.4 \\
\hline 17 & -- & 9.0 & 8.1 & 8.4 & 9.2 & 10.5 & 11.8 & 14.4 & 15.7 & 15.8 & 14.9 & 12.4 \\
\hline 18 & -- & 9.0 & 8.0 & 8.4 & 9.3 & 10.6 & 11.9 & 14.6 & 15.7 & 15.8 & 14.9 & 12.5 \\
\hline 19 & -- & 9.0 & 8.0 & 8.4 & 9.3 & 10.6 & 12.1 & 14.7 & 15.7 & 15.6 & 14.8 & 12.3 \\
\hline 20 &.- & 8.9 & 8.0 & 8.4 & 9.4 & 10.7 & 12.2 & 14.7 & 15.8 & 15.7 & 14.8 & 12.1 \\
\hline 21 & -- & 8.9 & 8.0 & 8.5 & 9.4 & 10.7 & 12.3 & 14.7 & 15.9 & 15.8 & 14.7 & 12.2 \\
\hline 22 & -- & 9.0 & 7.8 & 8.6 & 9.5 & 11.0 & 12.4 & 14.8 & 15.9 & 15.7 & 14.7 & 12.8 \\
\hline 23 & - & 9.0 & 7.7 & 8.7 & 9.5 & 11.0 & 12.6 & 14.8 & 15.9 & 15.6 & 14.6 & 12.7 \\
\hline 24 & -- & 9.0 & 8.1 & 8.7 & 9.6 & 11.0 & 12.7 & 14.9 & 15.9 & 15.6 & 14.6 & 12.4 \\
\hline 25 & -- & 8.6 & 8.6 & 8.7 & 9.6 & 11.0 & 12.9 & 14.9 & 15.9 & 15.7 & 14.5 & 12.6 \\
\hline 26 & -- & 8.8 & 8.3 & 8.7 & 9.7 & 11.0 & 13.0 & 14.8 & 16.0 & 15.7 & 14.5 & 12.6 \\
\hline 27 & -. & 9.4 & 8.0 & 8.7 & 9.7 & 11.0 & 13.1 & 14.8 & 16.0 & 15.6 & 14.4 & 12.6 \\
\hline 28 & -- & 8.7 & 8.0 & 8.7 & 9.8 & 11.0 & 13.0 & 14.7 & 16.0 & 15.6 & 14.3 & 12.5 \\
\hline 29 & -- & -- & 8.1 & 8.7 & 9.8 & 11.0 & 13.0 & 14.9 & 16.0 & 15.5 & 14.2 & 11.9 \\
\hline 30 & -. & -- & 8.3 & 8.7 & 9.8 & 11.0 & 13.1 & 15.0 & 16.0 & 15.5 & 14.1 & 12.2 \\
\hline 31 & -- & -- & 7.3 & -- & 9.9 & -- & 13.4 & 15.0 & -- & 15.5 & -- & 11.3 \\
\hline
\end{tabular}


Table 14t. Mean daily ground-water temperature at site CRM-25 (map ID \#22), Seminole Well Field, Cedar Rapids, lowa, February 1998 through February 1999--Continued

\begin{tabular}{|c|c|c|c|c|c|c|c|c|c|c|c|c|}
\hline Day & Jan & Feb & Mar & Apr & May & Jun & Jul & Aug & Sep & Oct & Nov & Dec \\
\hline & & & & & & 1999 & & & & & & \\
\hline 1 & 11.4 & 10.1 & -- & -- & -- & -- & -- & -- & -- & -- & -- & -- \\
\hline 2 & 12.1 & 10.1 & -- & -- & -- & -- & -- & -- & -- & -- & -- & -- \\
\hline 3 & 11.2 & 9.9 & -- & -- & -- & -. & -- & -- & -- & -- & -- & -- \\
\hline 4 & 11.1 & 9.8 & - & -- & -- & -- & -- & -- & -- & -- & -- & -- \\
\hline 5 & 11.5 & 9.9 & -- & -- & -- & -- & -- & -- & -- & -- & -- & -- \\
\hline 6 & 11.0 & 9.8 & -- & - & -- & -- & -- & -- & -- & -- & -- & -. \\
\hline 7 & 11.8 & 9.8 & -- & -- & - & -- & -- & +- & -- & -- & -- & -- \\
\hline 8 & 11.8 & 9.8 & -- & - & -- & -- & -- & -- & - & -- & -- & -- \\
\hline 9 & 11.7 & 9.7 & -- & -- & -- & -- & -- & -- & -- & -- & -- & -- \\
\hline 10 & 11.7 & 9.7 & -- & -- & -- & -- & -- & -- & -- & -- & -- & -- \\
\hline 11 & 11.7 & 9.8 & -- & -- & -- & -- & -- & -- & -- & -- & -- & -- \\
\hline 12 & 11.3 & 9.5 & -- & - & -- & -- & -- & -- & -- & -- & -- & -- \\
\hline 13 & 11.0 & 9.4 & -- & -- & -- & -- & -- & -- & -- & -- & -- & -- \\
\hline 14 & 11.0 & 9.5 & -- & -- & -- & -- & -- & -- & -- & - & -- & -- \\
\hline 15 & 11.0 & 9.4 & -- & -- & -- & -- & -- & -- & -- & -- & -- & -- \\
\hline 16 & 10.9 & 9.4 & -- & -- & -- & -- & - & -- & -. & -- & -- & -- \\
\hline 17 & 10.9 & 9.3 & -- & -- & -- & -- & -- & -- & -- & -- & -- & -- \\
\hline 18 & 10.8 & 9.3 & -- & -- & -- & -- & -- & -- & -- & -- & -- & -- \\
\hline 19 & 10.7 & 9.3 & -- & -- & -- & -- & -- & -- & -- & -- & -- & -- \\
\hline 20 & 10.7 & 9.2 & -- & -- & -- & -- & -- & -- & -- & -- & -- & -- \\
\hline 21 & 10.6 & 9.1 & -- & -- & -- & -- & -- & -. & -- & -- & -- & -- \\
\hline 22 & 10.6 & 9.1 & -- & -- & -- & -- & -- & -- & -- & -- & -- & -- \\
\hline 23 & 10.6 & 9.1 & -- & -- & -- & -- & -- & -- & -- & -- & -- & -- \\
\hline 24 & 10.5 & 9.2 & -- & -- & -- & -- & -- & -- & -- & -- & -- & -- \\
\hline 25 & 10.4 & 9.5 & -- & -- & -- & - & -- & -- & -- & -- & -- & -- \\
\hline 26 & 10.3 & 9.3 & -- & -- & -- & -- & -- & -- & -- & -- & -- & -- \\
\hline 27 & 10.3 & 9.3 & -- & -- & -- & -. & -- & -- & -- & -- & -- & -- \\
\hline 28 & 10.2 & 9.4 & -- & -- & -- & -- & -- & -- & -- & -- & -- & -- \\
\hline 29 & 10.0 & -- & -- & -- & -- & -- & -- & -- & -- & -- & -- & -- \\
\hline 30 & 10.1 & -- & -- & -- & -- & -- & -- & -- & -- & -- & -- & -- \\
\hline 31 & 10.1 & -- & -- & -- & -- & -- & -- & -- & -- & -- & -- & -- \\
\hline
\end{tabular}


Table 14u. Mean daily ground-water temperature at site CRM-27 (map ID \#24), Seminole Well Field, Cedar Rapids, lowa, February 1998 through February 1999

[Temperature in degrees Centigrade; --, value not measured or not recorded]

\begin{tabular}{|c|c|c|c|c|c|c|c|c|c|c|c|c|}
\hline Day & Jan & Feb & Mar & Apr & May & Jun & Jul & Aug & Sep & Oct & Nov & Dec \\
\hline \multicolumn{13}{|c|}{1998} \\
\hline 1 & -- & -- & 13.0 & 5.8 & 5.5 & 7.5 & 14.5 & 12.9 & 14.2 & 15.2 & 17.2 & 16.3 \\
\hline 2 & -- & -- & 12.4 & 6.9 & 5.6 & 7.6 & 14.4 & 12.9 & 14.3 & 15.2 & 17.2 & 16.3 \\
\hline 3 & -- & -- & 12.4 & 6.8 & 5.6 & 7.6 & 13.5 & 13.0 & 14.4 & 15.3 & 17.3 & 16.2 \\
\hline 4 & -- & -- & 12.4 & 6.6 & 5.6 & 7.7 & 13.2 & 13.0 & 14.4 & 15.3 & 17.3 & 16.2 \\
\hline 5 & -- & -- & 12.3 & 6.6 & 5.7 & 7.9 & 12.0 & 13.1 & 14.5 & 15.3 & 17.3 & 16.1 \\
\hline 6 & -- & -- & 12.4 & 6.6 & 5.7 & 8.1 & 11.8 & 13.1 & 14.6 & 15.2 & 17.3 & 16.1 \\
\hline 7 & -- & -- & 12.3 & 6.5 & 5.7 & 8.3 & 11.8 & 13.2 & 14.6 & 15.8 & 17.2 & 16.0 \\
\hline 8 & -- & -- & 12.2 & 6.5 & 5.7 & 8.5 & 11.8 & 13.2 & 14.7 & 15.3 & 17.2 & 16.0 \\
\hline 9 & -- & -- & 12.0 & 6.3 & 5.8 & 8.7 & 11.8 & 13.2 & 14.7 & 15.3 & 17.1 & 16.0 \\
\hline 10 & -- & -- & 12.0 & 6.2 & 5.9 & 8.9 & 11.8 & 13.3 & 14.7 & 15.7 & 17.0 & 16.0 \\
\hline 11 & -- & -- & 12.0 & 6.1 & 6.0 & 9.1 & 11.8 & 13.3 & 14.7 & 15.6 & 16.9 & 15.9 \\
\hline 12 & -- & -- & 12.1 & 6.0 & 6.0 & 9.3 & 11.9 & 13.4 & 14.8 & 15.6 & 16.9 & 15.9 \\
\hline 13 & -- & -- & 10.3 & 5.9 & 6.1 & 9.5 & 12.0 & 13.4 & 14.8 & 15.7 & 16.8 & 15.8 \\
\hline 14 & -- & 13.2 & 9.2 & 5.8 & 6.3 & 9.8 & 12.1 & 13.5 & 14.9 & 15.7 & 16.8 & 15.8 \\
\hline 15 & -- & 13.2 & 8.9 & 5.7 & 6.4 & 11.0 & 12.2 & 13.5 & 14.9 & 15.7 & 16.8 & 15.7 \\
\hline 16 & -- & 13.1 & 8.6 & 5.6 & 6.5 & 10.7 & 12.3 & 13.6 & 14.9 & 15.7 & 16.8 & 15.7 \\
\hline 17 & -- & 13.1 & 8.4 & 5.5 & 6.6 & 10.3 & 12.4 & 13.7 & 14.9 & 15.8 & 16.7 & 15.6 \\
\hline 18 & -- & 13.1 & 8.2 & 5.5 & 6.7 & 10.4 & 12.5 & 13.8 & 14.9 & 15.7 & 16.7 & 15.6 \\
\hline 19 & -- & 12.8 & 8.0 & 5.4 & 6.7 & 10.5 & 12.6 & 13.8 & 15.0 & 16.0 & 16.7 & 15.6 \\
\hline 20 & -- & 12.4 & 7.9 & 5.4 & 6.7 & 10.5 & 12.6 & 13.9 & 15.1 & 17.5 & 16.6 & 15.5 \\
\hline 21 & .- & 12.4 & 7.9 & 5.4 & 6.7 & 10.6 & 12.7 & 14.0 & 15.1 & 17.6 & 16.6 & 15.5 \\
\hline 22 & -- & 12.6 & 7.9 & 5.4 & 6.8 & 10.6 & 12.7 & 14.0 & 15.1 & 18.0 & 16.6 & 15.5 \\
\hline 23 & -- & 12.8 & 7.9 & 5.4 & 6.8 & 10.6 & 12.8 & 14.0 & 15.1 & 18.3 & 16.6 & 15.3 \\
\hline 24 & -- & 12.9 & 7.9 & 5.4 & 6.9 & 10.7 & 12.8 & 14.1 & 15.1 & 18.3 & 16.6 & 15.3 \\
\hline 25 & -- & 12.8 & 7.9 & 5.4 & 6.9 & 10.7 & 12.9 & 14.0 & 15.1 & 18.1 & 16.6 & 15.3 \\
\hline 26 & -- & 12.7 & 7.9 & 5.4 & 7.0 & 10.7 & 12.9 & 14.0 & 15.1 & 17.8 & 16.5 & 15.4 \\
\hline 27 & -- & 12.4 & 8.1 & 5.4 & 7.1 & 10.7 & 12.9 & 14.0 & 15.1 & 17.5 & 16.5 & 15.3 \\
\hline 28 & -- & 12.7 & 7.4 & 5.4 & 7.3 & 10.8 & 12.9 & 14.0 & 15.1 & 17.4 & 16.4 & 15.2 \\
\hline 29 & -- & -- & 7.1 & 5.4 & 7.4 & 10.8 & 12.9 & 14.0 & 15.1 & 17.4 & 16.4 & 15.1 \\
\hline 30 & -- & -- & 5.5 & 5.5 & 7.4 & 10.8 & 12.9 & 14.0 & 15.1 & 17.3 & 16.4 & 15.0 \\
\hline 31 & -- & -- & 5.4 & -- & 7.5 & -- & 12.9 & 14.0 & -- & 17.4 & -- & 15.0 \\
\hline
\end{tabular}


Table 14u. Mean daily ground-water temperature at site CRM-27 (map ID \#24), Seminole Well Field, Cedar Rapids, lowa, February 1998 through February 1999--Continued

\begin{tabular}{|c|c|c|c|c|c|c|c|c|c|c|c|c|}
\hline Day & Jan & Feb & Mar & Apr & May & Jun & Jul & Aug & Sep & Oct & Nov & $\overline{\text { Dec }}$ \\
\hline & & & & & & 1999 & & & & & & \\
\hline 1 & 14.9 & 14.5 & -- & -- & -- & -- & -- & -- & -- & -- & -- & -- \\
\hline 2 & 14.9 & 14.5 & -- & -- & -- & -- & -- & -- & -- & -- & -- & -- \\
\hline 3 & 14.8 & 14.5 & -- & -- & -- & -- & -- & -- & -- & -- & -- & -- \\
\hline 4 & 14.8 & 14.5 & -- & .- & -. & -- & -- & -- & -- & -- & -- & -- \\
\hline 5 & 14.7 & 14.5 & -- & -- & -- & -- & -- & -- & -- & -- & -- & -- \\
\hline 6 & 14.7 & 14.5 & -- & -- & -- & -- & - & -- & -. & -- & -- & -- \\
\hline 7 & 14.7 & 14.5 & -- & -- & -- & -- & -- & -- & -- & -- & -- & -- \\
\hline 8 & 14.6 & 14.5 & -- & -- & -- & -- & -- & -- & -- & -- & -- & -- \\
\hline 9 & 14.7 & 14.5 & -- & -- & -- & -- & -- & -- & -- & -- & -- & -- \\
\hline 10 & 14.6 & 14.5 & -- & - & -- & -- & -- & -- & -- & -- & -- & -- \\
\hline 11 & 14.6 & 14.4 & -- & -- & -- & -- & -- & -- & -- & - & -- & -- \\
\hline 12 & 14.6 & 14.3 & .- & -- & -- & -- & -- & -- & -- & -- & -- & -. \\
\hline 13 & 14.6 & 14.4 & -- & -. & -- & -- & -- & -- & -- & -- & - & -- \\
\hline 14 & 14.6 & 14.4 & - & -- & -- & -- & -- & -- & -- & -- & -- & -- \\
\hline 15 & 14.6 & 14.3 & -- & - & -- & - & -- & -- & -- & -- & -- & -- \\
\hline 16 & 14.6 & 14.3 & -- & - & -- & -- & -- & -- & -. & -- & - & -- \\
\hline 17 & 14.6 & 14.3 & -- & -- & -- & -- & -- & -- & -- & -- & -- & -- \\
\hline 18 & 14.6 & 14.3 & -- & -- & -- & -- & -- & -- & -- & -- & -- & -- \\
\hline 19 & 14.6 & 14.3 & -- & -- & -- & -- & - & -- & -- & -- & -- & -- \\
\hline 20 & 14.6 & 14.2 & -- & -- & -- & -- & -- & -- & -- & -- & -- & -- \\
\hline 21 & 14.6 & 14.3 & -- & -- & -- & -- & -- & -- & -- & -- & -- & -- \\
\hline 22 & 14.6 & 14.2 & -- & -- & -- & -- & -- & -- & -- & -- & -- & -- \\
\hline 23 & 14.6 & 14.2 & -- & -- & -- & -- & -- & -- & -- & -- & -- & -- \\
\hline 24 & 14.6 & 14.2 & -- & -- & -- & -- & -- & -- & -- & -- & -- & -- \\
\hline 25 & 14.6 & 14.2 & -- & -- & -- & -- & -- & -- & -- & -- & -- & -- \\
\hline 26 & 14.5 & 14.3 & -- & -- & -- & -- & -- & -- & -- & -- & -- & -- \\
\hline 27 & 14.5 & 14.1 & -- & -- & - & -- & -- & -- & -- & -- & -- & -- \\
\hline 28 & 14.5 & 14.0 & -- & -- & -- & -- & - & -- & -- & -- & -. & -- \\
\hline 29 & 14.5 & -- & -- & -- & -- & -- & -- & -- & -- & -- & -- & -- \\
\hline 30 & 14.5 & -- & -- & -- & -- & -- & -- & - & -- & -- & -- & -- \\
\hline 31 & 14.5 & -- & -- & -- & -- & -- & -- & -- & - & -- & -- & -- \\
\hline
\end{tabular}


Table 14v. Mean daily water temperature in the Cedar River at site SW-2 (map ID \#32), Seminole Well Field, Cedar Rapids, lowa, April 1996 through March 1999

[Temperature in degrees Centigrade; --, value not measured or not recorded]

\begin{tabular}{|c|c|c|c|c|c|c|c|c|c|c|c|c|}
\hline Day & Jan & Feb & Mar & Apr & May & Jun & Jul & Aug & Sep & Oct & Nov & Dec \\
\hline \multicolumn{13}{|c|}{1996} \\
\hline 1 & -- & -- & -- & 6.0 & 11.6 & -- & 27.0 & 24.1 & 24.3 & 17.4 & 3.9 & 0.1 \\
\hline 2 & -- & -- & -- & 6.7 & 11.7 & -- & 26.8 & 24.8 & 24.8 & 17.2 & 3.1 & -0.1 \\
\hline 3 & -- & -- & -- & 8.1 & 11.0 & -- & 26.2 & 25.3 & 24.8 & 14.6 & 3.2 & -- \\
\hline 4 & -- & -- & -- & 7.4 & 12.4 & -- & 26.0 & 24.1 & 25.2 & 13.7 & 4.4 & -0.1 \\
\hline 5 & -- & -- & -- & 6.9 & 13.6 & -- & 25.7 & 24.3 & 25.5 & 13.9 & 5.4 & -0.1 \\
\hline 6 & -- & -- & -- & 7.3 & 12.9 & -- & 25.6 & 26.8 & 25.4 & 15.8 & 6.6 & -0.1 \\
\hline 7 & -- & -- & -- & 6.8 & -- & -- & 26.3 & 28.0 & 24.9 & 15.6 & 6.5 & -0.1 \\
\hline 8 & -- & -- & - & 7.0 & -- & -- & 25.8 & 27.0 & 24.2 & 13.5 & 5.6 & -0.1 \\
\hline 9 & -- & -- & -- & 8.0 & -. & -- & 23.6 & 26.6 & 22.9 & 13.6 & 4.4 & -0.1 \\
\hline 10 & -- & -- & -- & 9.3 & -- & -- & 23.0 & 25.6 & 23.0 & 12.3 & 2.8 & -0.1 \\
\hline 11 & - & -- & -- & 11.4 & -- & -- & 23.5 & 24.3 & 23.3 & 11.6 & 1.5 & -0.1 \\
\hline 12 & -- & -- & -- & 13.2 & -- & -- & 24.2 & 25.0 & 20.0 & 12.7 & 0.8 & -0.1 \\
\hline 13 & -- & -- & -- & 11.7 & -- & -- & 24.6 & 25.8 & 17.8 & 15.2 & 0.6 & -0.1 \\
\hline 14 & -- & -- & -- & 9.3 & -- & -- & 24.5 & 26.4 & 17.3 & 16.6 & 0.1 & -0.1 \\
\hline 15 & -- & -- & -- & 7.5 & -- & -- & 24.6 & 25.3 & 17.8 & 16.9 & 0.0 & -0.1 \\
\hline 16 & -- & -- & -- & 8.6 & -- & -- & 25.4 & 24.4 & 18.3 & 17.2 & 3.2 & -0.1 \\
\hline 17 & -- & -- & -- & 10.7 & -- & -- & 25.4 & 24.7 & 18.4 & 16.5 & 5.3 & -0.1 \\
\hline 18 & -- & -- & -- & 12.1 & -- & -- & 26.9 & 23.7 & 18.5 & 12.2 & 3.2 & -0.1 \\
\hline 19 & -- & -- & -- & 12.0 & -- & -- & 28.3 & 22.6 & 18.3 & 10.4 & 2.0 & -0.1 \\
\hline 20 & -. & -- & -- & 11.2 & -- & -- & 25.3 & 23.5 & 18.1 & 10.3 & 1.6 & -0.1 \\
\hline 21 & - & -- & -- & 11.0 & -- & -- & 23.3 & 25.9 & 17.5 & 11.0 & 1.3 & -0.1 \\
\hline 22 & -- & -- & -- & 12.5 & -- & -- & 23.4 & 26.7 & 17.6 & 10.4 & 0.9 & -0.1 \\
\hline 23 & -- & -- & -- & 12.5 & -- & -- & 24.3 & 25.6 & 18.0 & 8.7 & 1.2 & -0.1 \\
\hline 24 & -- & -- & -- & 13.4 & -- & -- & 25.0 & 24.6 & 17.5 & 8.0 & 1.4 & -0.1 \\
\hline 25 & -- & -- & -. & 13.8 & -- & -- & 24.3 & 24.6 & 16.0 & 9.3 & 0.2 & -0.1 \\
\hline 26 & -- & -- & -- & 12.0 & -- & -- & 24.6 & 24.8 & 14.2 & 11.8 & 0.1 & -0.1 \\
\hline 27 & -- & -- & -- & 12.2 & -- & -- & 24.5 & 24.4 & 13.6 & 12.9 & 0.1 & -0.1 \\
\hline 28 & -- & -- & -- & 12.6 & -- & 25.0 & 24.1 & 24.0 & 14.1 & 11.5 & 0.1 & -0.1 \\
\hline 29 & -- & -- & -- & 10.0 & -- & 26.3 & 24.6 & 24.5 & 14.7 & 11.1 & 0.1 & -0.1 \\
\hline 30 & -- & -- & -- & 9.4 & -- & 27.1 & 24.8 & 24.4 & 16.0 & -- & 0.1 & -0.1 \\
\hline 31 & -- & -- & -- & -- & -- & -- & 24.2 & 24.2 & -- & 5.9 & -- & -0.1 \\
\hline
\end{tabular}


Table 14v. Mean daily water temperature in the Cedar River at site SW-2 (map ID \#32), Seminole Well Field, Cedar Rapids, lowa, April 1996 through March 1999

\begin{tabular}{|c|c|c|c|c|c|c|c|c|c|c|c|c|}
\hline Day & Jan & Feb & Mar & Apr & May & Jun & Jul & Aug & Sep & Oct & Nov & Dec \\
\hline & & & & & & 1997 & & & & & & \\
\hline 1 & -0.1 & -0.1 & 0.0 & -- & 12.4 & 17.9 & 26.0 & 24.4 & 25.9 & 16.6 & 8.5 & 4.0 \\
\hline 2 & -0.1 & -0.1 & 0.0 & -- & 12.1 & 19.4 & 25.7 & 25.3 & 25.0 & 17.3 & 6.8 & 3.5 \\
\hline 3 & -0.1 & -0.1 & -- & -- & 11.0 & 20.5 & 23.4 & 26.3 & 22.3 & 19.7 & 4.8 & 2.9 \\
\hline 4 & -0.1 & -0.1 & -- & -- & 11.6 & 21.3 & 21.5 & 26.8 & 21.3 & 20.5 & 4.1 & 1.9 \\
\hline 5 & -0.1 & -0.1 & -- & 11.4 & 13.1 & 21.2 & 20.6 & 25.9 & 20.8 & 20.2 & 3.9 & 0.5 \\
\hline 6 & -0.1 & -0.1 & -- & 9.7 & 14.1 & 20.8 & 21.5 & 24.2 & 22.1 & 21.1 & 4.2 & -0.3 \\
\hline 7 & -0.1 & -0.1 & -- & 7.1 & 14.2 & 20.5 & 21.9 & 24.0 & 23.0 & 21.6 & 5.2 & -0.3 \\
\hline 8 & -0.1 & -0.1 & -- & 6.4 & 14.0 & 20.8 & 23.2 & 24.2 & 21.5 & 21.1 & 6.0 & -0.3 \\
\hline 9 & -0.1 & -0.1 & -- & 5.7 & 13.7 & 21.2 & 23.4 & 23.9 & 21.0 & 19.6 & 6.0 & -0.1 \\
\hline 10 & -0.1 & -0.1 & -- & 5.2 & 13.8 & 21.9 & 22.5 & 23.6 & 20.5 & 17.4 & 4.8 & 0.4 \\
\hline 11 & -0.1 & -0.1 & -- & 3.9 & 14.3 & 22.3 & 23.2 & 22.2 & 20.2 & 17.2 & 3.2 & 1.0 \\
\hline 12 & -0.1 & -0.1 & -- & 3.2 & 13.7 & 22.2 & 24.4 & 20.9 & 20.3 & 18.0 & 2.1 & 0.6 \\
\hline 13 & -0.1 & -0.1 & -- & 4.5 & 13.4 & 23.3 & 25.2 & 21.8 & 19.9 & 15.9 & 2.1 & -0.3 \\
\hline 14 & -0.1 & -0.1 & -- & 6.3 & 12.5 & 23.4 & 26.2 & 22.1 & 20.5 & 12.7 & 2.0 & -0.4 \\
\hline 15 & -0.1 & -0.1 & -- & 7.7 & 11.5 & 23.7 & 26.8 & 23.2 & 21.3 & 11.7 & 1.5 & -0.2 \\
\hline 16 & -0.1 & -0.1 & -- & 8.3 & 11.8 & 23.7 & 27.4 & 25.5 & 22.2 & 11.6 & 0.3 & 0.0 \\
\hline 17 & -0.1 & -0.1 & -- & 8.6 & 13.6 & 23.3 & 27.3 & 25.2 & 22.6 & 11.5 & -0.1 & 0.4 \\
\hline 18 & -0.1 & -0.1 & -- & 9.4 & 15.4 & 24.3 & 27.7 & 23.3 & 21.7 & 11.3 & 0.2 & 0.7 \\
\hline 19 & -0.1 & -0.1 & -- & 10.6 & 16.0 & 24.2 & 27.6 & 21.9 & 21.8 & 11.2 & 0.5 & 1.1 \\
\hline 20 & -0.1 & -0.1 & -- & 11.6 & 16.0 & 24.8 & 27.2 & 21.7 & 19.8 & 10.6 & 1.3 & 1.4 \\
\hline 21 & -0.1 & -0.1 & -- & 11.6 & 16.6 & 25.5 & 27.1 & 21.7 & 18.3 & 9.8 & 1.9 & 0.3 \\
\hline 22 & -0.1 & -0.1 & -- & 12.2 & 17.2 & 25.5 & 25.6 & 22.0 & 17.9 & 8.7 & 1.6 & 0.1 \\
\hline 23 & -0.1 & -0.1 & -- & 12.6 & 17.8 & 26.2 & 25.2 & 22.0 & 17.2 & 8.9 & 0.7 & 0.4 \\
\hline 24 & -0.1 & -0.1 & -- & 12.7 & 18.3 & 25.5 & 25.6 & 23.2 & 16.9 & 9.4 & 0.0 & 0.1 \\
\hline 25 & -0.1 & -0.1 & -- & 13.2 & 17.5 & 25.0 & 26.2 & 23.6 & 17.6 & 8.7 & 0.9 & -0.2 \\
\hline 26 & -0.1 & -0.1 & -- & 13.7 & 15.0 & 24.6 & 28.2 & 23.1 & 18.5 & 5.9 & 2.3 & -0.2 \\
\hline 27 & -0.1 & -0.1 & -- & 14.1 & 13.5 & 24.7 & 29.2 & 23.0 & 18.9 & 4.0 & 2.3 & -0.4 \\
\hline 28 & -0.1 & 0.0 & -- & 14.8 & 13.1 & 25.0 & 28.1 & 24.0 & 19.2 & 4.4 & 3.4 & -0.4 \\
\hline 29 & -0.1 & -- & -- & 15.8 & 13.0 & 25.2 & 26.7 & 24.0 & 17.6 & 5.4 & 4.4 & -0.4 \\
\hline 30 & -0.1 & -- & -- & 15.0 & 13.7 & 24.4 & 25.4 & 24.0 & 16.7 & 6.5 & 4.6 & -0.4 \\
\hline 31 & -0.1 & -- & -- & -- & 15.8 & -- & 24.7 & 25.4 & -. & 8.1 & -- & -0.4 \\
\hline
\end{tabular}


Table 14v. Mean daily water temperature in the Cedar River at site SW-2 (map ID \#32), Seminole Well Field, Cedar Rapids, lowa, April 1996 through March 1999

\begin{tabular}{|c|c|c|c|c|c|c|c|c|c|c|c|c|}
\hline Day & Jan & Feb & Mar & Apr & May & Jun & Jul & Aug & Sep & Oct & Nov & Dec \\
\hline & & & & & & 1998 & & & & & & \\
\hline 1 & -0.4 & -0.4 & 3.8 & 10.3 & 14.3 & 21.0 & 24.1 & 24.9 & -- & 18.4 & 12.1 & 8.7 \\
\hline 2 & -0.4 & -0.4 & 3.5 & 8.6 & 14.9 & 20.2 & 23.9 & 24.5 & -- & 15.8 & 11.0 & 8.9 \\
\hline 3 & -0.4 & -0.4 & 3.2 & 7.7 & 14.1 & 18.9 & 23.8 & 24.1 & -- & 13.5 & 9.0 & 9.7 \\
\hline 4 & -0.4 & -0.4 & 3.2 & 7.5 & 15.4 & 17.3 & 23.3 & 24.6 & -- & 12.9 & 7.6 & 10.5 \\
\hline 5 & -0.4 & -0.4 & 3.1 & 8.4 & 17.3 & 16.7 & 22.4 & 24.1 & -. & 14.1 & 6.3 & 11.1 \\
\hline 6 & -0.4 & -0.4 & 3.2 & 9.6 & 17.5 & 16.6 & 22.5 & 22.9 & -- & 15.0 & 5.6 & 9.9 \\
\hline 7 & 1.1 & -0.4 & 3.1 & 10.4 & 16.4 & 17.4 & 23.6 & 22.7 & -- & 13.8 & 4.6 & 6.8 \\
\hline 8 & 0.8 & -0.4 & 2.1 & 10.4 & 16.7 & 17.4 & 24.3 & 23.5 & -- & 13.3 & 4.5 & 4.8 \\
\hline 9 & 0.4 & -0.4 & 0.8 & 9.3 & 17.8 & 16.1 & 25.0 & 24.4 & -- & 13.3 & 4.7 & 4.1 \\
\hline 10 & -0.4 & 0.0 & 0.2 & 9.2 & 18.4 & 16.8 & 25.4 & 25.1 & -- & 13.4 & 5.3 & 3.5 \\
\hline 11 & -0.4 & 0.5 & 0.1 & 9.8 & 18.8 & 17.9 & 25.6 & 25.3 & -- & 13.8 & 4.6 & 2.8 \\
\hline 12 & -0.4 & 1.0 & -0.1 & 10.9 & 19.4 & 18.5 & 25.5 & 25.3 & -- & 14.2 & 4.7 & 2.7 \\
\hline 13 & -0.4 & 2.0 & 0.5 & 11.6 & 19.9 & 19.6 & 25.8 & 24.9 & -- & 13.4 & 4.4 & 2.6 \\
\hline 14 & -0.4 & 3.0 & 1.0 & 12.2 & 20.6 & 19.7 & 26.4 & 25.4 & -- & 12.3 & 4.8 & 2.5 \\
\hline 15 & -0.4 & 2.9 & 1.5 & 12.5 & 21.5 & 19.1 & 26.9 & 25.8 & -- & 12.3 & 5.1 & 2.8 \\
\hline 16 & -0.4 & 4.2 & 2.0 & 10.8 & 21.0 & 19.1 & 27.0 & 25.9 & -- & 13.9 & 5.0 & 3.3 \\
\hline 17 & -0.4 & 4.4 & 2.2 & 10.0 & 21.3 & 19.0 & 26.6 & -- & -- & 15.7 & 4.9 & 2.9 \\
\hline 18 & -0.4 & 3.8 & 2.2 & 10.8 & 22.3 & 19.0 & 27.1 & -- & -- & 14.6 & 5.5 & 2.9 \\
\hline 19 & -0.4 & 3.6 & 2.4 & 11.4 & 23.8 & 19.2 & 27.8 & -- & -- & 13.3 & 5.3 & 2.3 \\
\hline 20 & -0.4 & 3.6 & 2.9 & 11.6 & 22.8 & 20.7 & 28.3 & -- & -- & 12.4 & 3.8 & 0.8 \\
\hline 21 & -0.4 & 3.2 & 3.7 & 11.2 & 21.8 & 21.4 & 28.6 & -- & -- & 11.6 & 3.1 & -0.1 \\
\hline 22 & -0.4 & 2.7 & 5.0 & 11.6 & 19.7 & 21.4 & 27.5 & -- & -- & 10.9 & 3.9 & -0.2 \\
\hline 23 & -0.4 & 3.5 & 5.5 & 12.6 & 18.3 & 21.7 & 26.4 & -- & 18.1 & 10.7 & 4.8 & -0.2 \\
\hline 24 & -0.4 & 4.4 & 4.9 & 13.8 & 17.3 & 22.4 & 25.5 & -- & 18.0 & 11.1 & 4.7 & -0.2 \\
\hline 25 & -0.4 & 5.3 & 5.7 & 14.6 & 17.5 & 23.4 & 24.3 & -- & 18.9 & 12.1 & 5.7 & -0.2 \\
\hline 26 & -0.4 & 6.2 & 8.6 & 14.5 & 18.4 & 24.4 & 24.0 & -- & 21.1 & 13.0 & 5.9 & -0.2 \\
\hline 27 & -0.4 & 6.1 & 10.6 & 13.0 & 19.3 & 25.7 & 24.7 & -- & 22.4 & 13.9 & 6.1 & -0.2 \\
\hline 28 & -0.4 & 4.7 & 10.9 & 12.4 & 21.0 & 26.1 & 25.5 & -- & 21.7 & 14.4 & 6.8 & -0.2 \\
\hline 29 & -0.4 & -- & 11.8 & 12.3 & 22.1 & 25.4 & 26.0 & -- & 21.8 & 14.7 & 8.9 & -0.2 \\
\hline 30 & -0.4 & -. & 13.1 & 12.6 & 22.4 & 24.4 & 25.4 & -- & 21.3 & 14.0 & 10.2 & -0.2 \\
\hline 31 & -0.4 & -- & 12.5 & -- & 22.5 & -. & 24.8 & -- & -- & 12.8 & -- & -0.2 \\
\hline
\end{tabular}


Table 14v. Mean daily water temperature in the Cedar River at site SW-2 (map ID \#32), Seminole Well Field, Cedar Rapids, lowa, April 1996 through March 1999

\begin{tabular}{|c|c|c|c|c|c|c|c|c|c|c|c|c|}
\hline Day & Jan & Feb & Mar & Apr & May & Jun & Jul & Aug & Sep & Oct & Nov & Dec \\
\hline & & & & & & 1999 & & & & & & \\
\hline 1 & -0.2 & -0.1 & -- & -- & -- & -- & -- & -- & -- & -- & -- & -- \\
\hline 2 & -0.2 & -0.1 & 4.0 & -- & -- & -- & -- & .. & -- & -- & -- & -. \\
\hline 3 & -0.2 & -0.1 & 2.7 & -- & -- & -- & -- & -- & -- & -- & -- & -- \\
\hline 4 & -0.2 & -0.1 & 2.2 & -- & -- & -- & -- & -- & -- & -- & -- & -- \\
\hline 5 & -0.2 & -0.1 & 2.2 & -- & -- & -- & -- & -- & -- & - & -. & -- \\
\hline 6 & -0.2 & -0.1 & 1.9 & -- & -- & -. & -- & -- & -- & -- & -- & -- \\
\hline 7 & -0.2 & -0.1 & 1.8 & -- & -- & -- & -- & -- & -- & -- & -- & -- \\
\hline 8 & -0.2 & -0.1 & 0.6 & -- & -- & -. & -- & -- & -- & -- & .. & .. \\
\hline 9 & -0.2 & 0.2 & 0.0 & -- & -- & -. & -- & -- & -- & -- & -- & -. \\
\hline 10 & -0.2 & 0.4 & 0.9 & -- & -- & -- & -- & -- & -- & -- & -- & -- \\
\hline 11 & -0.2 & 2.3 & 2.9 & -- & -- & -- & -- & -- & -- & -- & -- & -- \\
\hline 12 & -0.2 & 1.7 & 3.8 & -- & -- & -. & -- & -- & -- & -. & -- & - \\
\hline 13 & -0.2 & 0.0 & 4.3 & -- & -- & -- & -- & -- & -- & -. & -- & -- \\
\hline 14 & -0.2 & 0.2 & 4.6 & -- & -- & -- & -- & -- & -- & -- & -- & -- \\
\hline 15 & -0.2 & 1.7 & 5.3 & -- & -- & -- & -- & -- & -- & -- & -- & -- \\
\hline 16 & -0.2 & 2.7 & 6.5 & -- & -- & -- & -- & -- & -- & -- & -- & -- \\
\hline 17 & -0.2 & 2.1 & 8.3 & -- & -- & -- & -- & -- & -- & -- & -. & -- \\
\hline 18 & -0.2 & 2.0 & 7.6 & -- & -- & -- & -- & -- & -- & -- & -- & -- \\
\hline 19 & -0.2 & 1.7 & 7.5 & -- & -- & -- & -. & -- & -- & -- & -- & -- \\
\hline 20 & -0.2 & 1.2 & 7.9 & -- & -- & -- & -- & -- & -- & -- & -- & -- \\
\hline 21 & -0.2 & 1.0 & 7.9 & -- & -- & -- & -- & -- & -- & -- & -- & -- \\
\hline 22 & -0.2 & 1.0 & 7.3 & -- & -. & -- & -- & -- & -- & -- & -- & -- \\
\hline 23 & -0.2 & 0.7 & 7.2 & -- & -- & -- & -- & -- & -- & -- & -- & -- \\
\hline 24 & -0.2 & 0.5 & 8.0 & -- & -- & -- & -- & -- & - & -- & -- & -- \\
\hline 25 & -0.1 & 0.7 & 8.0 & - & -- & -- & -- & -- & -- & -- & -- & -- \\
\hline 26 & -0.2 & 1.7 & 8.1 & -- & -- & -- & -- & -- & -- & -- & -- & -- \\
\hline 27 & -0.2 & 3.0 & 8.6 & -- & -- & -- & -- & -- & -- & -- & -- & -- \\
\hline 28 & -0.1 & 3.3 & 9.0 & -- & -- & -- & -- & -. & -- & -- & -- & -- \\
\hline 29 & -0.1 & -. & 10.0 & -- & -- & -- & -- & -. & -- & -- & -- & -- \\
\hline 30 & -0.1 & -- & 11.5 & -- & -- & -- & -- & -- & -- & -- & -- & -- \\
\hline 31 & -0.1 & -- & 12.6 & -- & -- & -- & -- & -- & -- & -- & -- & -- \\
\hline
\end{tabular}

